\title{
Germanistische Dialektlexikographie zu Beginn des 21. Jahrhunderts
}

Herausgegeben von

Alexandra N. Lenz und Philipp Stöckle

Germanistik

Franz Steiner Verlag

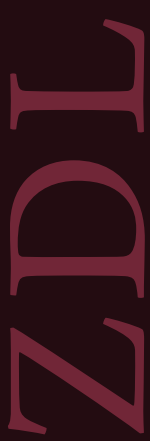

\section{ZEITSCHRIFT \\ FÜR DIALEKTOLOGIE \\ UND LINGUISTIK}

BEIHEFTE 
8 


\section{Zeitschrift für Dialektologie und Linguistik}

In Verbindung mit

MICHAEL ELMENTALER, JÜRG FLEISCHER Und MARK L. LOUDEN

Herausgegeben von

JÜRGEN ERICH SCHMIDT

Beiheft 181 


\title{
Germanistische Dialektlexikographie zu Beginn des 21. Jahrhunderts
}

\author{
Herausgegeben von \\ Alexandra N. Lenz und Philipp Stöckle
}

Unter Mitarbeit von

Angela Bergermayer, Andreas Gellan, Sabine Wahl, Eva-Maria Wahlmüller und Patrick Zeitlhuber 
Gedruckt mit freundlicher Unterstützung der Österreichischen Akademie der Wissenschaften (ÖAW)

Dieses Buch ist eine Open-Access-Publikation.

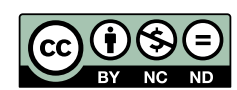

Dieses Werk ist lizenziert unter einer Creative Commons Namensnennung -

Nicht kommerziell - Keine Bearbeitung 4.o International Lizenz.

https://creativecommons.org/licenses/by-nc-nd/4.o/deed.de

Bibliografische Information der Deutschen Nationalbibliothek:

Die Deutsche Nationalbibliothek verzeichnet diese Publikation in der Deutschen

Nationalbibliografie; detaillierte bibliografische Daten sind im Internet über <http://dnb.d-nb.de> abrufbar.

Dieses Werk einschließlich aller seiner Teile ist urheberrechtlich geschützt. Jede Verwertung außerhalb der engen Grenzen des Urheberrechtsgesetzes ist unzulässig und strafbar.

(C) Franz Steiner Verlag, Stuttgart 2021

Druck: Beltz Grafische Betriebe, Bad Langensalza

Gedruckt auf säurefreiem, alterungsbeständigem Papier.

Printed in Germany.

ISBN 978-3-515-12911-4 (Print)

ISBN 978-3-515-12920-6 (E-Book)

https://doi.org/10.25162/9783515129206 


\title{
VORWORT DER HERAUSGEBERIN UND DES HERAUSGEBERS
}

\author{
Alexandra N. Lenz, / Philipp Stöckle
}

\section{ZU DIESEM BAND}

Der vorliegende Band ist der germanistischen Dialektlexikographie zu Beginn des 21. Jahrhunderts gewidmet. Er gründet auf dem ersten Treffen des „Netzwerk[s] großlandschaftlicher Dialektwörterbücher des Deutschen“ (LexikoNet 1), das 2017 vom Team des 2016 wiederaufgenommenen ,Wörterbuch[s] der bairischen Mundarten in Österreich (WBÖ)“ in Wien ausgerichtet wurde. Insgesamt 14 großlandschaftliche Dialektwörterbücher werden im Folgenden in selbstständigen Kapiteln präsentiert. Von einer Ausnahme ${ }^{1}$ abgesehen folgen die Kapitel einer weitgehend standardisierten Struktur, die dem Handbuchcharakter dieses Bandes gerecht werden soll.

Mit den thematischen Schwerpunktsetzungen, die in den Wörterbuchkapiteln Berücksichtigung finden, verfolgt dieser Band drei zentrale Ziele:

\section{1. Übersicht zu lexikographischen Aspekten}

Wir intendieren eine aktuelle Übersicht über die laufenden großlandschaftlichen Dialektwörterbücher des Deutschen. Diese umfasst Informationen zur meist langjährigen Geschichte der Wörterbücher, ihren wissenschaftshistorischen Kontexten sowie den institutionellen Rahmenbedingungen der Projektgruppen vom Beginn der Wörterbucharbeit bis in die Gegenwart. Auch werden die empirischen Daten und Materialien, auf denen die Wörterbucharbeit aufbaut, ausführlich vorgestellt, unter Berücksichtigung der durch das jeweilige Wörterbuch abgedeckten Sprachräume, Art und Umfang der „Rohdaten“, Erhebungs- bzw. Sammlungsverfahren sowie der Daten spendenden Personen und Gruppen. Eine zentrale Rolle kommt in den Projektbeschreibungen auch konzeptionellen Aspekten der Wörterbuchartikel zu (Inhalt und Struktur, „Straffungskonzepte“ u. a.), die anhand von zitierten Beispielsartikeln illustriert werden.

1 Bei der Darstellung des „Sudetendeutschen Wörterbuchs“ handelt es sich lediglich um eine Kurzpräsentation als Abriss einer ausführlichen Vorstellung des Projekts, die in einem eigenen Tagungsband zu der 2018 von dieser Arbeitsstelle selbst ausgerichteten LexikoNet 2 vorgenommen wird. 


\section{2. Übersicht zu texttechnologischen Aspekten}

Auch wenn die Arbeit an den meisten der in diesem Band vertretenen Wörterbüchern in vordigitalisierter Zeit begonnen wurde, haben viele Projekte in den letzten Jahrzehnten oder Jahren insofern eine texttechnologische Wende erlebt, als digitale Methoden und Werkzeuge die Projektarbeit mehr oder weniger stark ergänzt, beeinflusst und verändert haben. Auch diese innovativen Pfade werden in den Wörterbuchkapiteln dieses Bandes vorgestellt und diskutiert.

Neben analog vorliegenden Fragebogensammlungen, die häufig händisch sortiert, exzerpiert und interpretiert wurden, können nicht wenige Wörterbuchprojekte mittlerweile auf digital verarbeitete Bestände ihrer Daten zugreifen - sei es in Form von digitalisierten „Bildern“ (z. B. gescannte Fragebögen), die das Arbeiten mit den Daten forschungspraktisch erleichtern, oder sei es sogar in Form von als ,digitale Volltexte" erfassten Daten, die in verschiedenen Datenbankformaten vorliegen.

Die lexikographische Arbeit selbst wird nicht selten dadurch erleichtert, dass neben gängigen Textverarbeitungsprogrammen eigens für das individuelle Wörterbuchprojekt entwickelte Redaktionssysteme zum Schreiben, Formatieren und schließlich Publizieren der Wörterbuchartikel genutzt werden. Sie erleichtern auch neue, texttechnologische Formen der digitalen Publikation, die sich zunehmend zur klassischen Publikation in Form gedruckter Wörterbücher gesellen.

Schließlich und endlich unterstützen und gewährleisten digitale Werkzeuge auch die Langzeitarchivierung der Wörterbuchmaterialien über die Projektlaufzeiten hinaus. Nur eine Langzeitarchivierung ermöglicht es, die Projektdaten auch für zukünftige Forschergenerationen nutzbar zu machen.

\section{Einblick in linguistische Potenziale}

Nicht nur durch die Berücksichtigung texttechnologischer Aspekte geht dieser Band über die Beschreibung der lexikographischen Kernarbeit der Wörterbuchprojekte hinaus. Er will zudem die Potenziale aufzeigen, die die Wörterbuchartikel und mehr noch die ihnen zugrundeliegenden Daten im Hinblick auf linguistische Forschung ganz allgemein mit sich bringen. Dies geschieht, indem dieser Band auch exemplarische Fallstudien beinhaltet, die in überzeugender Art und Weise illustrieren, welche Möglichkeiten die Wörterbuchkorpora auch über die lexikalische Systemebene hinaus bieten, etwa im Hinblick auf quantitative und qualitative Analysen lautlicher und grammatischer Phänomene. 


\section{DANK}

Schließen möchten wir dieses Vorwort nicht, ohne unseren herzlichen Dank auszusprechen

- der Österreichischen Akademie der Wissenschaften für die Finanzierung der Drucklegung dieses Bandes, aber vielmehr noch für die Wiederaufnahme des „Wörterbuchs der bairischen Mundarten in Österreich“,

- unserem WBÖ-Team für die tatkräftige Unterstützung bei der Ausrichtung der ersten LexikoNet-Veranstaltung in Wien 2017, aber mehr noch für die sorgfältige Begutachtung dieses Bandes, und Theresa Ziegler für die Hilfe bei der umfangreichen Formatierung desselben,

- dem Herausgebergremium der ZDL für die Aufnahme dieses Bandes in ihren Beiheften,

- allen Autorinnen und Autoren, die dazu beigetragen haben, dass dieser Band möglich wurde,

- und schließlich allen Kolleginnen und Kollegen, die mit ihrer unermüdlichen Arbeit und leidenschaftlichen Forschung einen wertvollen Beitrag zur germanistischen Dialektlexikographie leisten. 



\section{INHALTSVERZEICHNIS}

Vorwort der Herausgeberin und des Herausgebers ...........................................5

Philipp Stöckle

Wörterbuch der bairischen Mundarten in Österreich (WBÖ) ............................. 11

Michael Schnabel / Manuel Raaf / Daniel Schwarz

Bayerisches Wörterbuch (BWB)

Almut König / Manuel Raaf / Alfred Klepsch

Das Fränkische Wörterbuch (WBF).... .77

Brigitte Schwarz / Edith Funk / Manuel Raaf / Ursula Welsch

Dialektologisches Informationssystem von Bayerisch-Schwaben (DIBS) 105

Christoph Landolt / Tobias Roth

Schweizerisches Idiotikon -

Wörterbuch der schweizerdeutschen Sprache

Tobias Streck

Badisches Wörterbuch 175

Alexander Werth / Bernd Vielsmeier / Stefan Aumann

Hessen-Nassauisches Wörterbuch (HNWb) 201

Robert Damme

Das Westfälische Wörterbuch (WWb) .223

Maik Lehmberg

Das Niedersächsische Wörterbuch

auf dem Wege zu seiner Fertigstellung. 251

Ulrich Wenner

Das Mittelelbische Wörterbuch .275

Matthias Vollmer

Das Pommersche Wörterbuch 303

Isabelle Hardt

Kurzpräsentation des Sudetendeutschen Wörterbuchs 
Elisabeth Knipf-Komlósi / Márta Müller

Wörterbuch der Ungarndeutschen Mundarten (WUM)

Sigrid Haldenwang

Siebenbürgisch-Sächsisches Wörterbuch 351 


\title{
WÖRTERBUCH DER BAIRISCHEN MUNDARTEN IN ÖSTERREICH (WBÖ) ${ }^{1}$
}

\author{
Philipp Stöckle

\section{GESCHICHTE UND MATERIALGRUNDLAGE}

\subsection{Geschichtlicher Abriss}

Das „Wörterbuch der bairischen Mundarten in Österreich“ (WBÖ) ist, wie viele andere großlandschaftliche Dialektwörterbücher, ein Langzeitprojekt, das auf eine mittlerweile mehr als hundertjährige Geschichte zurückblickt. Seine Initiierung fällt ins Jahr 1910, als der Münchener Indogermanist ERNST KUHN dem Wiener Germanisten JOSEPH SEEMÜLLER in einem Brief den Vorschlag für ein gemeinsames bayerisch-österreichisches Dialektwörterbuch unterbreitete. Nachdem es zuvor Überlegungen gegeben hatte, eine Überarbeitung von SCHMELLERS (1872-77) „Bayerischem Wörterbuch“vorzunehmen, wurde dieser Plan zugunsten eines gänzlich neuen, modernisierten Wörterbuchprojekts fallen gelassen, das den gesamten bairischen Sprachraum im Königreich Bayern sowie in der österreichisch-ungarischen Monarchie umfassen sollte. ,[N]eben dem heutigen Österreich (ohne Vorarlberg) [sollten somit] auch Südtirol, Westungarn, die damalige Untersteiermark und Unterkärnten, die bairischen Sudetengebiete und eine größere Anzahl von Sprachinseln in das Bearbeitungsgebiet" des WBÖ einbezogen werden (BAUER / KÜHN 1998: 369). Zur Verwirklichung dieses Vorhabens wurden im Jahr 1911 in München und Wien Wörterbuchkommissionen eingesetzt, in den beiden folgenden Jahren ,wurden die Beschlüsse von den beiden Akademien offiziell genehmigt und jeweils eigene Wörterbuchkanzleien eingerichtet, in Wien am 12. Februar 1913““ (REIFFENSTEIN 2005: 2). Dieser Tag kann somit als Gründungsdatum des WBÖ betrachtet werden (vgl. MEISTER 1963: VI).

Die Pläne zur Erstellung des Wörterbuchs sahen vor, dass die Arbeitsstellen die Materialerhebung in ihren jeweiligen Gebieten organisierten, die eigentliche Wörterbucharbeit aber, d. h. die Archivierung des Materials sowie das Verfassen der Wortartikel nach einem einheitlichen Konzept, in Wien durchgeführt werden sollte. Im Jahr 1913 wurde ein gedruckter Aufruf verbreitet, mittels dessen freiwillige

1 Mein herzlicher Dank gilt Ingeborg Geyer, SAbine Wahl, Patrick Zeitlhuber und AleXANDRA N. LENZ für ihre wertvollen Anmerkungen, die zur Verbesserung dieses Textes beigetragen haben. Alle verbliebenen Unzulänglichkeiten liegen ausschließlich in meiner Verantwortung. 
Sammler/-innen gesucht wurden. Der Aufruf stieß auf große Resonanz in der Bevölkerung, sodass umgehend mit dem Versand der ersten Fragebögen gemeinsam mit einer „Belehrung für die Sammler“ begonnen werden konnte.

In den folgenden Jahrzehnten erhoben die Sammler/-innen, bei denen es sich „zum größten Teil [um] Angehörige der Lehrerschaft wie des geistlichen Standes, z. T. auch Ärzte“" (HoRNUNG 1976: 42) handelte, mithilfe von ca. 500 Gewährspersonen, die ,vorwiegend aus dem Bauernstand“ (HoRNUNG 1976: 43) stammten, einen großen Teil des Belegmaterials. Die Antworten auf die Fragen aus den Fragebögen wurden auf Handzetteln notiert, welche wiederum an die Wörterbuchkanzlei in Wien zurückgeschickt wurden. Insgesamt wurden in den Jahren 1913 bis 1933 mit vierjähriger Unterbrechung durch den Ersten Weltkrieg - 109 Fragebögen an die Sammler/-innen ausgesandt. ${ }^{2}$ Im Zusammenhang mit den Erhebungen für ein geplantes Atlasprojekt ${ }^{3}$ von EBERHARD KRANZMAYER, der im Jahr 1926 zum Wörterbuch hinzustieß, wurden von 1927 bis 1937 neun weitere dialektgeographische Fragebögen (die sog. „Ergänzungsfragebögen“) verschickt, mittels derer das Material beträchtlich erweitert werden konnte. Parallel wurden zur weiteren Aufstockung und zum Schließen von Lücken im Belegmaterial seit 1927 direkte Erhebungen durch geschulte Dialektolog/-innen in Form von Kundfahrten (bis 1965) und Fragebucherhebungen (bis 1990) durchgeführt (vgl. HoRNUNG 1976: 43). Neben der flächendeckenden Sammlung von Sprachmaterial wurden Exzerpte aus dialektologischer Fachliteratur (z. B. aus Regionalwörterbüchern und Dissertationen) und aus weiteren schriftlichen Quellen (z. B. aus Mundartliteratur und historischen Texten) erstellt. Insgesamt verteilt sich das Belegmaterial zu folgenden Anteilen auf die verschiedenen Quellen: Mit ca. $55 \%$ bildet das von den Sammler/-innen erhobene Material den größten Bestandteil, während das auf Kundfahrten erhobene Material etwa $35 \%$ ausmacht und die Literaturexzerpte ca. $10 \%$.

Nachdem die Arbeitsstellen in München und Wien seit der Gründung des Wörterbuchunternehmens mit Blick auf ein gemeinsames Wörterbuch mehrere Jahrzehnte zusammengearbeitet hatten, gestaltete sich die Beziehung in den 1950erJahren zunehmend schwieriger. Da man in Wien zügig mit der Publikation beginnen wollte, in München jedoch noch Lücken im Belegmaterial sah, wurde 1961 die institutionelle Trennung der beiden Arbeitsstellen beschlossen. Seitdem erscheinen beide Wörterbücher getrennt, jedoch unter dem gemeinsamen Reihentitel „Bayerisch-österreichisches Wörterbuch“. Im Jahr 1963 erschien die erste Lieferung des

2 Abschriften der Fragebögen sind auf den Seiten des „Bayerischen Wörterbuchs“ unter der folgenden URL einsehbar: <www.bwb.badw.de/fragebogen/systematischer.html>; Stand: 27.11.2019.

3 Das Atlasprojekt wurde nie abgeschlossen, die von EBERHARD KRANZMAYER handgezeichneten Dialektkarten sind jedoch Gegenstand des von der ÖAW geförderten GO!DIGITAL-Projekts „Österreichische Dialektkartographie 1924-1956. Digitalisierung, Kontextualisierung, Visualisierung“ (Projektleitung: MANFRED GLAUNINGER; Projektmitarbeiter: JAN DAVID ZIMMERMANN), in dem sie einer historisch-wissenschaftskritischen Betrachtung unterzogen werden. Ein großer Teil der Karten liegt auch in gescannter Form vor und ist über die Seiten des „Bayerischen Wörterbuchs" unter folgender URL abrufbar: <www.bwb.badw.de/ sprachkarten.html>; Stand: 27.11.2019 
WBÖ, 1970 wurde der erste Band $(A-A z o r)$ publiziert, bis zum Jahr 2015 sind die ersten fünf Bände in insgesamt 41 Lieferungen erschienen, die die Lemmastrecken zu den Buchstaben $A, B / P, C, D / T$ und $E$ enthalten (vgl. WBÖ 1-5). Aktuell wird im Rahmen der Neukonzeption die Arbeit ab dem Buchstaben $F$ fortgeführt.

\subsection{Institutionelle Rahmenbedingungen}

Was die institutionelle Einbettung betrifft, war das WBÖ von 1911 bis 1999 eine Kommission der Österreichischen Akademie der Wissenschaften (ÖAW), der folgende Obmänner vorstanden: JOSEPH SEEMÜLLER (1911-1920), PAUL KRETSCHMER (1920-1956), DIETRICH KRALIK (1956-1959), RICHARD MEISTER (19591964), OTTO HÖFLER (1964-1967), EBERHARD KRANZMAYER (1967-1975) und INGO REIFFENSTEIN (1975-1999) (vgl. GEYER 2019: 480). Im Rahmen struktureller Änderungen an der ÖAW wurde die Wörterbuchkanzlei im Jahr 1994 in das Institut für Dialekt- und Namenlexika (DINAMLEX) umgewandelt, das in den folgenden Jahren von WERNER BAUER (1994-1998), ISOLDE HAUSNER (1998-2005) und INGEBORG GEYER (2005-2013) geleitet wurde. Von 1999 bis 2006 war PETER WIESINGER Vorsitzender des begleitenden wissenschaftlichen Kuratoriums. Im Jahr 2013 wurde das DINAMLEX als Forschungsgruppe in das Institut für Corpuslinguistik und Texttechnologie (ICLTT) integriert. Aufgrund interner Schwierigkeiten, die letztendlich dazu führten, dass INGEBORG GEYER als einzige Mitarbeiterin die Weiterarbeit am WBÖ fortsetzen konnte, wurde das Projekt im Oktober 2014 vorübergehend eingestellt. Eine „Reihe von Anfragen und Protestbriefen“ (GEYER 2019: 483) sowie anschließende positive Gutachten zur Wissenschaftlichkeit führten schließlich zur Wiederaufnahme des WBÖ. Seit April 2016 ist es in der von ALEXANDRA N. LENZ geleiteten Forschungsabteilung ,Variation und Wandel des Deutschen in Österreich (VaWaDiÖ) ${ }^{* 4}$ am Austrian Centre for Digital Humanities and Cultural Heritage (ACDH-CH $)^{5}$ angesiedelt, wo es mit einem neuen Team von Lexikograph/-innen unter der redaktionellen Leitung von PHILIPP STÖCKLE fortgeführt wird. ${ }^{6}$

\subsection{Datengrundlage}

Wie bereits in Kapitel 1.1 erläutert wurde, verrichteten die freiwilligen Sammler/-innen zwar häufig mit großem Eifer ihre Arbeit, waren jedoch keine wissenschaftlich geschulten Dialektolog/-innen. Das Resultat ihrer Arbeit (sowie der

4 URL: <https://vawadioe.acdh.oeaw.ac.at/>; Stand: 27.11.2019.

5 Bis zur Umstrukturierung und gleichzeitigen Erweiterung der Institution Anfang 2020 lautete der Name Austrian Centre for Digital Humanities (ACDH).

6 Eine ausführliche Darstellung der Geschichte (bis 2014), auch im Hinblick auf die institutionelle Einbettung, findet sich in GEYER (2019). 
Kundfahrten und zusätzlichen Literaturexzerpte) ist daher eine einerseits 3,6 Millionen Handzettel umfassende und damit sehr umfangreiche Materialsammlung, die andererseits im Detail sowohl bezüglich der dargestellten Inhalte wie auch deren Qualität häufig recht heterogen ist.

Die für die Wörterbucharbeit zentralen Informationen umfassen Angaben zum abgefragten Lemma, zur Bedeutung, zur Lautung und zum Erhebungsort und sind auf den meisten Belegzetteln zu finden. Daneben gibt es häufig Beispielsätze (oftmals mit einer standardsprachlichen Übersetzung), grammatische Angaben (z. B. zur Wortart oder zur Flexion), Informationen zum Sammler bzw. zur Sammlerin, zum Fragebogen sowie zur Fragenummer, zur Etymologie oder zur literarischen Quelle. Abbildung 1 zeigt anhand einiger Beispiele den großen Variantenreichtum der Handzettel.

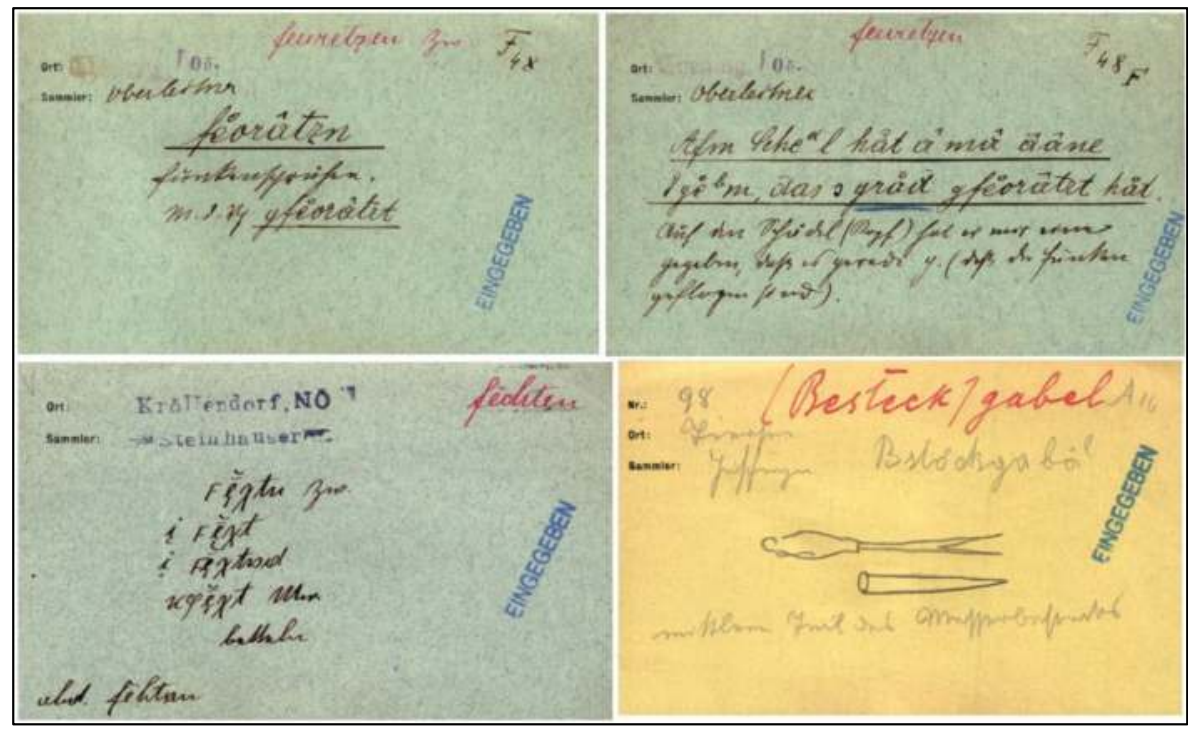

Abb. 1: Beispiele für Belegzettel

Nach dem Eintreffen in der Arbeitsstelle wurden die Belegzettel lemmatisiert - in Abbildung 1 durch die Lemmata in roter Schrift ersichtlich - und durch Informationen zur Herkunft bzw. Quelle ergänzt. Ab 1993 wurden die Zettel abgetippt und in eine TUSTEP-Datenbank eingegeben (siehe Kapitel 3.1). Nach diesem Arbeitsschritt erhielt jeder Zettel den Stempel „eingegeben“. In manchen Fällen kam es vor, dass die Informationen zu einem Lemma aus Platzgründen auf mehrere Zettel verteilt wurden, wie die beiden Belegzettel zum Lemma feuretzen illustrieren. Der linke Zettel enthält die Angaben zur Aussprache des Infinitivs, zum Partizip 2 sowie zur Bedeutung ('Funken sprühen'), während der rechte Zettel die Bedeutung durch einen Beispielsatz inklusive einer standardsprachlichen Übersetzung verdeutlicht. Der Belegzettel zum Lemma fechten zeigt neben der Bedeutung ('betteln') und der althochdeutschen Form zusätzlich Angaben zur Flexion, im Fall von Besteckgabel 
wurde die Bedeutung durch eine Zeichnung illustriert. Informationen zur Lautung lassen sich allen vier Zetteln entnehmen, wobei hier die in vielen Fällen uneinheitliche Umsetzung der Lautschrift-Konventionen durch die Sammler/-innen berücksichtigt werden muss.

Die Handzettel sind im sog. Hauptkatalog alphabetisch nach Lemma sortiert. Wichtigstes Vorbild für „die innere Anlage des Hauptkataloges“ (MEISTER 1963: X) war seit Beginn des Wörterbuchunternehmens das Schweizerische Idiotikon (vgl. dazu den Beitrag von LANDOLT / ROTH in diesem Band), an dem man sich sowohl bei der Einordnung der Wortzusammensetzungen als auch bei der Lemmatisierung orientierte. Komposita, Partikel- und Präfixwörter werden stets nach dem Grundwort bzw. der Basis einsortiert und im Artikel zum entsprechenden Simplex behandelt. Pfinztag wird demnach unter Tag behandelt, einfädeln unter fädeln. Was die Lemmatisierung betrifft, wurde ursprünglich ein historisch-etymologischer Ansatz verfolgt, d.h. es wurde ein „Kompromiß zwischen der schriftsprachlichen Orthographie und jener etymologischen Form, von der die Mundartlautungen ausgehen, angestrebt“" (MEISTER 1963: X). Dieser Ansatz ist nach wie vor im Belegmaterial vorzufinden und wurde in den Bänden eins bis fünf für die Artikel verwendet. In der Neukonzeption des WBÖ wird er jedoch durch einen an der gesamtdeutschen Standardsprache orientierten Ansatz abgelöst (vgl. dazu ausführlicher Kapitel 2). ${ }^{7}$

Zur Beschleunigung der Artikelarbeit und zur Eröffnung neuer Forschungsperspektiven durch erleichterten Zugriff auf die Daten wurde in den 1990er Jahren damit begonnen, den gesamten Hauptkatalog ab dem Buchstaben $D$ - die Lemmata zu den Buchstaben $A, B / P$ und $C$ waren bereits in den ersten drei Bänden bearbeitet worden - zu digitalisieren (vgl. BAUER / KÜHN 1998: 371). Ab dem Jahr 1993 wurden die Belegzettel manuell in TUSTEP (Tübinger System von TextverarbeitungsProgrammen $)^{8}$ eingegeben. Diese Arbeit war mit zahlreichen Schwierigkeiten und Herausforderungen verbunden, die zu einem beträchtlichen Teil auf die Tatsache zurückzuführen waren, dass die Sammler/-innen linguistische Laien gewesen waren und die Belegzettel eine große Heterogenität hinsichtlich der dargestellten Inhalte sowie der Qualität und Lesbarkeit aufwiesen. Für alle Informationen mussten in TUSTEP entsprechende Felder definiert werden, und für die möglichst originalgetreue Repräsentation der Lautschrift mussten einheitliche Codierungsrichtlinien gefunden werden (vgl. dazu auch BARABAS et al. 2010). Eine weitere Herausforderung an die Digitalisierung lag in der schieren Größe des Handzettelkatalogs. Obwohl erst ab dem Buchstaben $D$ begonnen wurde, dauerte die Volltexterfassung bis ins Jahr 2011, und an ihrem Ende stand die „Datenbank der bairischen Mundarten

7 Für die Zukunft ist zudem in Zusammenarbeit mit dem Verlag der Österreichischen Akademie der Wissenschaften eine Retro-Ditigalisierung der ersten fünf WBÖ-Bände sowie eine Normalisierung der darin enthaltenen Lemmata nach dem neuen, standardsprachlichen Ansatz geplant, die dann über das Lexikographische Informationssystem Österreich (LIÖ) gemeinsam mit den neuen Artikeln zugänglich gemacht werden. URL: <https://lioe.dioe.at/> (Stand: 01.07.2019; vgl. dazu ausführlicher Kapitel 3).

8 URL: <http://www.tustep.uni-tuebingen.de>; Stand: 01.07.2019. 
in Österreich (DBÖ) ${ }^{‘ 9}$ mit einem Umfang von ca. 2,4 Millionen Einträgen als Ergebnis.

Während sich TUSTEP zu Beginn der Digitalisierungsphase sicherlich als eines der Datenverarbeitungsprogramme erwies, die den Bedürfnissen der WBÖ-Redaktion - insbesondere hinsichtlich der Möglichkeiten der Darstellung der vielen in der Lautschrift verwendeten diakritischen Zeichen - am meisten entsprachen, wurden in den letzten Jahren die Defizite im Hinblick auf moderne technische Anforderungen deutlich. Die Computertechnologie hatte seit den 1990er-Jahren rasante Entwicklungen vollzogen, von denen die Etablierung des Internets sicherlich als eine der weitreichendsten bezeichnet werden kann. Im Jahr 2014 wurde daher damit begonnen, die gesamte TUSTEP-Belegdatenbank in das plattformunabhängige und leicht handhabbare Format XML/TEI zu konvertieren (vgl. dazu BOWERS / STÖCKLE 2018). Auch dieser Schritt war nicht frei von Problemen und erforderte umfangreiche Korrekturarbeiten der in TUSTEP vorliegenden Daten sowie die Erstellung zahlreicher Skripte zur korrekten Konvertierung der Daten nach Unicode ${ }^{10}$, sodass erst zum gegenwärtigen Zeitpunkt eine vollumfänglich nutzbare Belegdatenbank in XML/TEI vorliegt, die sowohl als Grundlage für die Artikelarbeit verwendet wird (vgl. Kapitel 2) als auch - seit Dezember 2018 - externen Nutzer/innen über das Lexikalische Informationssystem Österreich (LIÖ) für eigene Recherchen zur Verfügung gestellt wird (vgl. Kapitel 3 und 4).

\section{KONZEPTION, STRUKTUR UND INHALT DER WÖRTERBUCHARTIKEL}

\subsection{Neukonzeption und Straffungen}

Als Ende 2016 die neue Redaktion des WBÖ vor der Aufgabe stand, die Wörterbucharbeit wiederaufzunehmen und eine neue Konzeption zu erarbeiten, sah sie sich mit mehreren Herausforderungen ${ }^{11}$ konfrontiert: Zum einen war klar, dass die grundsätzliche Aufgabe des Wörterbuchs fortgeführt werden sollte, den Wortschatz der gesprochenen Mundart in lexikographisch aufbereiteter Form in Wortartikeln zu präsentieren, und dass dabei ein gewisses Maß an Kontinuität zu den vorausgegangenen Arbeiten erkennbar sein sollte. Zum anderen war jedoch ebenso deutlich geworden, dass grundlegende Änderungen notwendig waren, die sowohl Struktur und Inhalt der Artikel betreffen als auch Maßnahmen zur Reduktion der Bearbeitungsdauer. Das WBÖ sollte dabei noch leserfreundlicher als bisher und dabei auch medial an die Gewohnheiten eines zeitgenössischen Publikums angepasst werden; es sollte gleichzeitig weiterhin den hohen wissenschaftlichen Standards gerecht werden und als Fortführung des „bisherigen“ WBÖ erkennbar sein. Und all dies

9 Die Datenbank ist ebenfalls über das Lexikalische Informationssystem Österreich (LIÖ) online zugänglich

10 Diese Arbeiten wurden von SONJA SCHWAIGER und JOHANN DERKITS durchgeführt.

$11 \mathrm{Zu}$ diesen Herausforderungen zählten neben inhaltlich-konzeptionellen Aufgaben auch die personelle Neuaufstellung sowie der gesamte infrastrukturelle Aufbau der Abteilung. 
sollte - nach Maßgabe der ÖAW - zudem mit einem realistischen Plan zur Fertigstellung des WBÖ in einer absehbaren Zeit verknüpft sein.

Zur Umsetzung des letzten Punktes wurde eine Reihe von Straffungsmaßnahmen durchgeführt, die teilweise eine konsequentere Durchführung von in früheren Straffungskonzepten angekündigten Maßnahmen vorsieht, teilweise jedoch noch deutlich über diese hinausgeht. Neben einer Überarbeitung und Vereinheitlichung der Artikelstruktur betreffen diese Maßnahmen besonders das Datenvolumen, das der Artikelschreibung zugrunde gelegt wird. Die Beschränkungen beziehen sich v. a. auf das Bearbeitungsgebiet, auf den dargestellten Zeitraum und auf die Mindestanzahl von Belegen für ein Lemma.

- Zum Bearbeitungsgebiet: Wie in Kapitel 1.1 beschrieben, deckte das WBÖ in seiner ursprünglichen Konzeption einen Raum ab, der deutlich über das heutige Österreich hinausging und der Ausdehnung des bairischen Sprachgebiets im damaligen Habsburgerreich entsprach. Nachdem bereits im Rahmen des Straffungskonzepts von 1998 beschlossen wurde, von einer Bearbeitung des nordbairischen Egerlands, des mittleren und oberen Böhmerwalds sowie einiger Sprachinseln abzusehen, werden nach dem neuen Konzept nur noch Belege aus dem bairischsprachigen Österreich (d. h. aus allen Bundesländern außer dem alemannischsprachigen Vorarlberg) sowie aus Südtirol in die Artikel aufgenommen. Diese Entscheidung liegt auch darin begründet, dass große Teile der außerhalb des heutigen Österreich liegenden bairischsprachigen Gebiete in eigenen Wörterbuchprojekten ${ }^{12}$ bearbeitet werden, während Südtirol als einzige Großregion von keinem eigenen Wörterbuch abgedeckt wird.

- Zum Zeitraum der berücksichtigten Materialbasis: In der Neubearbeitung des WBÖ werden keine Belege mehr aufgenommen, die aus der Zeit vor 1860 stammen. Der dargestellte Zeitraum umfasst daher nun etwa ein Jahrhundert (von 1860 bis 1960, sieht man von dem kleinen Anteil an Belegen ab, die nach 1960 erhoben wurden). Da es sich bei den exzerpierten historischen Belegen zu einem beträchtlichen Teil um Rechtstexte (Weistümer, Urbare) handelt und gleichzeitig die durch die Sammlerinnen und Sammler empirisch erhobenen Daten den Großteil des Korpus ausmachen (vgl. Kapitel 1.1), verlagert sich dadurch der Schwerpunkt des WBÖ noch stärker auf die gesprochene Sprache in der ersten Hälfte des 20. Jahrhunderts.

- Zur Mindestbeleganzahl: Es werden zukünftig nur noch Artikel zu Lemmata erstellt, für die es (inkl. Wortbildungen) eine Mindestanzahl an Belegen gibt, die für die weitere Verarbeitung hinsichtlich des Bearbeitungsgebiets und des Zeitraums relevant sind.

Neben der - durch die beschriebenen Straffungsmaßnahmen erreichten - Reduktion der zu bearbeitenden Daten wird als weitere Maßnahme - einerseits zur Beschleunigung der Bearbeitungszeit, andererseits jedoch auch zur Qualitätssicherung - der Fokus stärker als bisher auf eine empirische, datengestützte Arbeitsweise gelenkt. Das bedeutet, dass sämtliche Inhalte und Informationen in den Artikeln (sei es zur

12 Vgl. dazu auch die Beiträge zum „Sudetendeutschen Wörterbuch“ und zum „Wörterbuch der ungarndeutschen Mundarten" in diesem Band. 
Aussprache, zur Bedeutung oder auch zu Verwendungskontexten) in der Datenbank bzw. in der dort zitierten Literatur belegt sein müssen. Empirisch nicht nachgewiesene (Re-)Konstruktionen von Lautvarianten, die nicht im WBÖ-Datenmaterial aufzufinden sind, sind dabei ebenso unzulässig wie Ergänzungen von Bedeutungen, die sich (lediglich introspektiv) aus der individuellen Sprachkompetenz und dem Sprachgebrauch der Lexikograph/-innen ergeben. Interpretationen und Zusammenfassungen von Bedeutungen sind nur insofern erlaubt, als sie sich im Gesamtkontext eines Bedeutungsfeldes durch weitere Datenbankbelege oder Quellen empirisch stützen lassen.

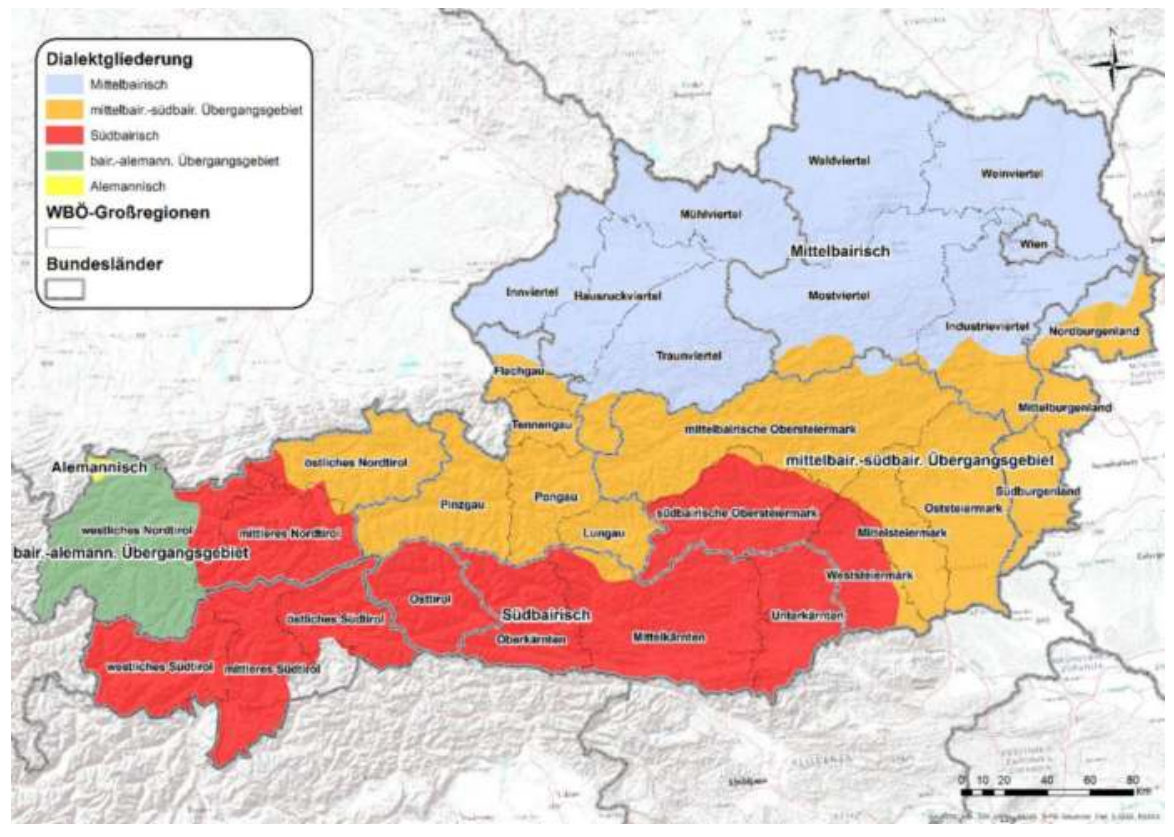

Abb. 2: Karte des WBÖ-Bearbeitungsgebiets (Grundlage für die Dialekteinteilung und die Angabe der Großregionen: WBÖ-Beiheft 2)

Die vorgestellten Straffungsmaßnahmen führen zu einer deutlichen Beschleunigung der lexikographischen Bearbeitungszeit. Zugleich erhöht die streng datengestützte Vorgehensweise die Qualität der nun noch stärker empirisch fundierten WBÖ-Artikel. Da die neue Gesamtkonzeption neben den Wörterbuchartikeln gleichzeitig auch die erstmalige Online-Publikation der Belegdatenbank vorsieht, stehen aber sämtliche Informationen, die aufgrund der Straffungen nicht in die Artikel eingehen können, allen Interessierten zur Verfügung, indem sie über das Lexikalische Informationssystem Österreich (LIÖ) einsehbar werden (vgl. dazu die Kapitel 3 und 4). 


\subsection{Lemmaansatz}

In Bezug auf die Artikel selbst betrifft eine der wichtigsten grundlegenden Neuerungen den Lemmaansatz. Während das WBÖ bislang einen historisch-etymologischen Ansatz verfolgte, der i. d. R. nur für ein philologisch geschultes Fachpublikum ohne Weiteres nachvollziehbar ist, rücken bei der Neubearbeitung des WBÖ die Leserfreundlichkeit und die Adressierung eines breiteren Publikums stärker in den Vordergrund. Der neue Lemmaansatz orientiert sich daher an der Standardsprache. Falls zu einem Stichwort eine gemeindeutsche Entsprechung existiert, wird diese als Lemma gewählt. Die Entscheidung darüber, ob ein Wort als gemeindeutsch klassifiziert werden kann, wird anhand einschlägiger Wörterbücher wie dem Duden (2015), dem Österreichischen Wörterbuch (PABST et al. 2016), dem Variantenwörterbuch des Deutschen (AMMON/ BICKEL/LENZ 2016) und dem Wortauskunftssystem zur deutschen Sprache in Geschichte und Gegenwart (DWDS

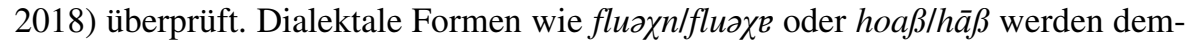
nach als fluchen oder hei $\beta$ angesetzt. Existiert $\mathrm{zu}$ einem Lemma keine standardsprachliche Entsprechung, wird nach historisch-etymologischen Regeln eine Form konstruiert, die so in der Standardsprache auch vorkommen könnte. Dialektale Varianten wie $\mathrm{Fa}(\mathrm{a}) \mathrm{m}$ oder Foam werden entsprechend als Feim lemmatisiert.

\subsection{Artikelstruktur und Beispielartikel}

Auch bezüglich der Artikelstruktur wurden einige grundlegende Veränderungen vorgenommen. Während im WBÖ bisher zwischen Kopf-, Kurz-, Verweisungsund Heteronymieartikel unterschieden wurde (vgl. WANDL-VoGT 2005b), wurde die Artikelstruktur im „neuen“ WBÖ vereinheitlicht. Diese soll im Folgenden anhand des Artikels feuretzen vorgestellt und erläutert werden. Da die Artikel bislang lediglich online im Lexikalischen Informationssystem Österreich publiziert werden (vgl. dazu Kapitel 3), wird der Beispielartikel in einer an die Online-Darstellung angelehnten Form angezeigt.

\section{feuretzen}

Verb (schwach)

\section{Verbreitung}

öSTir.; Ktn.; Stmk.; Sbg.; OÖ; Mostv., Waldv., Weinv.; Wien; MBgl.

\section{Lautung}

Überblick: Wie bei allen Wörtern mit auf mhd. iu zurückgehendem Stammvokal zeigt sich hohe areale Variation, die nur teilweise auch diachrone Zustände widerspiegelt. Durch die Angleichung an langes ü sind bereits im Spätalthochdeutschen regional gestreute pseudomethatetische Formen entstanden, die im Mittelhochdeutschen als oi/ui und $e u / i u$ vorliegen. Reste der iu-Formen finden sich als eo-Dipthonge im konservativen westlichen Ostmittelbairischen (fęorbdsñ), ansonsten dominieren (auch im Mittelbairischen) 
die fallenden Diphthonge mit gesenktem Onset (fgiradsn) und daraus entstandene jüngere sog. „rückgebildete“ Formen mit ui-Diphthong (fuiradsn). Verkehrssprachliche aiDiphthonge (fairadsn) und sogar $a$-Monophthonge (foradsn) repräsentieren in Teilen des östlichen Ostmittelbairischen den modernsten Lautstand. Das Intensivierungssuffix -etzen erscheint teilweise mit $a$-haltigem Anlaut, manchmal ist mit dem Suffix ein I-Einschub bei gleichzeitigem Schwund von $r$ verbunden (foilodsn.) Anlautendes $f$ tritt teilweise lenisiert auf (vuiradsno).

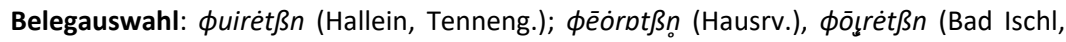
Traunv.); фẩirenton (Bad Großpertholz, Waldv.), -foiladsn (Allentsteig, Waldv.); fpratsn (Pilgersdorf, MBgl.)

\section{Etymologie}

Ableitung von $\rightarrow$ feuern

\section{Bedeutung}

I. 1. 'intensiv einheizen; Feuer machen' Sbg.; Innv.

2. 'Funken sprühen' öSTir; Ktn.; OÖ; Waldv.; MBgl. - Gfăhr $n$ bin e̊, das's gråd gfe̊orâtzt håt (Kirchberg-Thening, Hausrv.)

3. 'zündeln; mit dem Feuer spielen' Stmk.; Hausrv., Mühlv.

4. 'die Zündhölzer unnötig verbrauchen' Waldv.

II. 1. 'glänzen; funkeln' sbair.ObStmk. Weinv.

2. 'flimmern; flackern' sbair.ObStmk.; Hausrv.

III. 1. 'blitzen bei einem Gewitter' Sbg.; Hausrv.; Waldv.; MBgl. - Schiach gfuiretzt hats und doacht (SALZBURGER WB. 1995: 68; Sbg.)

2. 'wetterleuchten' Waldv.

IV. 'Blitze vor den Augen sehen, wenn man von jemandem geschlagen wird' Innv., Traunv., Mühlv. - $o^{o ̈ I s ~ g f o ̈ ̈ r o ̈ t z t ~ h a ̊ d s ' ~ f a ̊ r n ~ A u g ~ y a n ~-~ a ~ s o ̄ ~ h a ̊ d ~}$ $a^{r} h \bar{e}$ khaud (Gmunden, Traunv.)

\section{Wortbildung}

anfeuretzen

'anzünden' Waldv.

ausfeuretzen

'ausschlagen mit den Hinterbeinen (von Pferden)' Hausrv.; Innv.

danherfeuretzen

'ausschlagen mit den Hinterbeinen (von Pferden)' Waldv. - er fairizt dauna

(Zwettl-Niederösterreich, Waldv.)

einfeuretzen

'intensiv einheizen; Feuer machen' Weinv. 


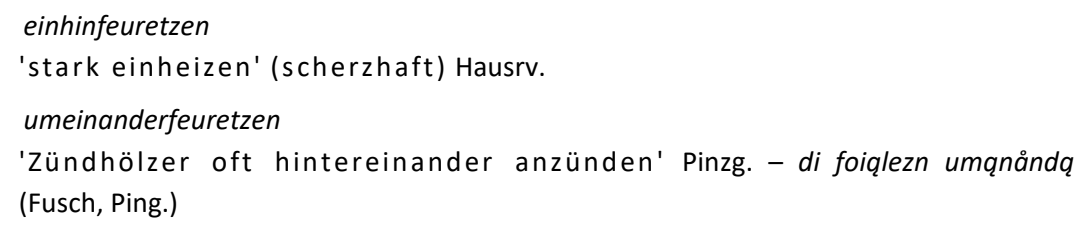

\section{Redewendungen}

die Augen feuretzen 'die Augen sprühen vor Zorn' Innv. - d augク foérétsn (Mühlheim am Inn, Innv.)

A.G.

Abb. 3: Beispielartikel feuretzen

Neben den vielen Vorteilen, die sich unmittelbar aus dem Internet als (vorerst) primärem Publikationsmedium heraus ergeben und auch bei der Darstellung des Artikels feuretzen offenkundig werden, ist auch der zur Verfügung stehende Platz ein Aspekt. Während die Wörterbuchartikel im WBÖ im (auch in den meisten anderen gedruckten Wörterbüchern vorherrschenden) klassischen Spaltenformat dargestellt wurden und zusätzlich zur Platzersparnis eine große Anzahl an Abkürzungen verwendet wurde, spielen derlei Aspekte bei der elektronischen Publikation keine Rolle mehr. Dadurch werden die Artikel wiederum leserfreundlicher, und es ergeben sich eine Reihe weiterer Möglichkeiten der Darstellung und Vernetzung, auf die in Kapitel 3 genauer eingegangen wird.

Nach der neuen Konzeption liegt allen WBÖ-Artikeln dieselbe, im Artikel feuretzen illustrierte Struktur zugrunde, deren einzelne Positionen im Folgenden erläutert werden sollen.

\subsubsection{Artikelkopf}

Im Artikelkopf stehen die wichtigsten Informationen, die das Lemma an sich betreffen. Dazu gehört der in Kapitel 2.2 vorgestellte Lemmaansatz, der möglichst knapp gehalten werden soll. Im Fall eines standardsprachlichen Pendants wird dieses angegeben, bei rein dialektalen Wörtern soll ebenfalls eine Form ausgewählt werden. Existieren zwei Varianten gleichwertig nebeneinander (wie beispielsweise Fack, Facke oder fert, ferten), können beide in den Lemmaansatz aufgenommen werden.

Als grammatische Information wird jeweils die Wortart sowie bei Substantiven das Genus und bei Verben der Flexionstyp angegeben. Gibt es mehrere Genera (wie z. B. beim Substantiv Falbe ${ }^{2}$, das als Maskulinum und Femininum belegt ist) oder mehrere Flexionstypen (wie z. B. beim Verb fechten, das sowohl stark als auch schwach flektiert werden kann), werden die Varianten gemäß der Reihenfolge ihrer in den Daten belegten Häufigkeit angegeben. 
In vielen Fällen kommt es vor, dass Diminutive nicht nur spontan gebildete Formen darstellen, sondern zu einem gewissen Grade lexikalisiert vorkommen und häufig mit einer bestimmten Bedeutung verknüpft sind. Diese lexikalisierten Diminuierungen werden ebenfalls im Artikelkopf angegeben, wobei zwischen folgenden Typen unterschieden wird:

- $\quad$ el: für Diminutiva auf - el und synkopiertes - $l$ (z. B. Fäckel)

- -elein: für Diminutiva auf -ele und -le (besonders im Südbairischen) (z. B. Fäckelein)

- - erl: für Diminutiva auf -al, -erl, -ai (z. B. Fäckerl)

- $\quad-i$ : für Diminutiva auf $-i$, meist kindersprachlich (z. B. Fäcki)

- $\quad$-tsch-: Dabei handelt es sich um ein Infix (z. B. Fätschel zum Lemma Farch), das in Kombination mit verschiedenen Diminutiv-Suffixen auftreten kann und ebenfalls unter den Diminuierungen genannt wird.

Weitere Sonderformen zum Lemma betreffen Movierungen (d. h. geschlechtsspezifische Ableitungen wie z. B. Fasserin zum Lemma Fasser), Kurzwörter (z. B. Foto zum Lemma Fotografie) sowie Nebenformen (z. B. Peienfalter zum Lemma Feifalter), die ebenfalls in den Artikelkopf aufgenommen werden.

\subsubsection{Verbreitung}

Das Verbreitungsfeld ergibt sich als eine Konsequenz aus der streng datengestützten Vorgehensweise, die der Artikelschreibung zugrunde liegt. In vielen Fällen kommt es vor, dass einzelnen Belegen weder eine Bedeutung zugeschrieben werden kann - z. B. im Fall fehlender oder tautologischer Angaben wie 'fechten' als Bedeutung des Lemmas fechten -, noch eine empirisch gesicherte Angabe zur Lautung in den Artikel übernommen werden kann (vgl. dazu Kapitel 2.3.3). Um trotzdem die Gesamtverbreitung eines Lemmas in Übereinstimmung mit der Beleglage abzubilden, werden in der Verbreitung sämtliche Einträge zu dem entsprechenden Lemma berücksichtigt, die sich hinsichtlich Bearbeitungszeitraum und -gebiet (vgl. Kapitel 2.1) zur Aufnahme in den Artikel qualifizieren (d. h. es werden nur Belege aus der Zeit nach 1860 sowie aus Österreich und Südtirol aufgenommen). Im Artikel feuretzen etwa betrifft dies die Stadt Wien, für die es zwar einen Beleg im Datenmaterial gibt, der jedoch im weiteren Artikel nicht enthalten ist.

Den Angaben der Verbreitungsgebiete liegt eine mehrstufige geographische Klassifikation zugrunde, die für den fünften WBÖ-Band erarbeitet wurde und im WBÖ-Beiheft 2 ausführlich dargestellt wird. Diese Klassifikation umfasst auf der untersten Ebene die Gemeinden, welche dann von Kleinregionen über Großregionen bis hin zu Bundesländern zu immer größeren geographischen Einheiten zusammengefasst werden. In der Neukonzeption des WBÖ stellen die Großregionen die zentrale Ebene für geographische Angaben dar. Diese decken sich in einigen Bundesländern mit historisch gewachsenen Kulturregionen (in Oberösterreich und Niederösterreich sind es die Viertel, z. B. Traunviertel OÖ oder Mostviertel NÖ, in Salzburg sind es die Gaue, z. B. Flachgau), in anderen Bundesländern besitzen sie 
keine kulturell-geographische Entsprechung (z. B. mittelbairische Obersteiermark oder südbairische Obersteiermark). Ist ein Lemma in allen Großregionen eines Bundeslands belegt, wird das Bundesland genannt. Die Gebietseinteilung ist in Abbildung 2 ersichtlich.

\subsubsection{Lautung}

Dieser Artikel-Abschnitt besteht aus zwei Teilen: einem Überblick, der die wichtigsten lautlichen Verhältnisse zusammenfasst, sprachhistorisch einordnet und sprachgeographisch darstellt, sowie einer ergänzenden Belegauswahl, die eine repräsentative Auswahl an Lautungsbelegen aus den verschiedenen durch den Artikel abgedeckten Regionen darstellt. Im Folgenden sollen die Prinzipien bei der Erstellung der Überblicksdarstellung sowie der Auswahl der Lautungsbelege erläutert werden.

Wie in Kapitel 1.1 aufgeführt, beruht der größte Teil des Datenmaterials auf den empirischen Erhebungen der Sammler/-innen. Die Dialektverschriftlichungen sollten in einer Vorform bzw. Variante des Schriftsystems Teuthonista vorgenommen werden, einer in der deutschsprachigen Dialektologie weit verbreiteten Lautschrift. Obwohl die Sammler/-innen zur Vorbereitung auf ihre Arbeit eine Belehrung erhielten, die vor allem die korrekte Verschriftlichung der Dialektlaute behandelt, ist die Darstellung der Lautungen im Belegmaterial nicht immer einheitlich, in zahlreichen Fällen sind gar keine oder nur phonetisch unzureichend transkribierte Lautungsbelege vorhanden.

Für den Überblick zu den Lautverhältnissen ergeben sich daraus verschiedene Konsequenzen. Einerseits beruht die Überblicksdarstellung gemäß dem empirischen Zugang der WBÖ-Neukonzeption auf den Lautungsbelegen im Datenmaterial, d. h. es werden nur Aussagen zu Regionen getroffen, für die auch entsprechende Belege vorhanden sind. Andererseits erfordern die Daten aus den oben genannten Gründen eine sorgfältige Interpretation und entsprechende Einordnung in die lautlichen bzw. lautgeschichtlichen Verhältnisse. Für diese Arbeit wird daher auf entsprechende Literatur zurückgegriffen (insbesondere KRANZMAYER 1956), für die Darstellung der verschiedenen Lautungen werden Leitformen in einer vereinheitlichten Teuthonista-Transkription angeführt, wobei sich die Konventionen der Verschriftlichung an den bisherigen WBÖ-Bänden orientieren.

In der Belegauswahl werden nur Lautungsvarianten angeführt, die im Material belegt sind, d. h. es werden keine Formen (re-)konstruiert, und es werden weiterhin nur Belege zitiert, die in ausreichender Qualität transkribiert sind. Grundsätzlich eignen sich eher Exzerpte aus wissenschaftlicher Literatur oder valide Sammlerbelege für die Aufnahme in die Artikel, während Dialektwörterbücher oder Dialektliteratur häufig eher ungeeignete Quellen darstellen. Die Beurteilung obliegt aber letztendlich den Lexikograph/-innen. Generell ausgeschlossen werden Belege, die Di- oder Trigraphen (wie ch oder sch) enthalten oder Belege, die Zeichen aus der standardsprachlichen Orthographie enthalten, die für Lautkombinationen stehen (z. B. $x$ in Faxe für die Lautung /ks/). Weiterhin gilt der Grundsatz, dass die Belege 
genauso zitiert werden, wie sie im Original notiert wurden, es werden also auch keine Vereinheitlichungen bei der Lautschrift vorgenommen. Als einzige Ausnahme gilt lediglich, dass am Wortanfang (auch bei Substantiven) nur Kleinbuchstaben verwendet werden. Bei der Auswahl der im Artikel zitierten Belege wird darauf geachtet, dass diese möglichst repräsentativ für ein Gebiet sein sollen und dass zudem im Idealfall das gesamte Verbreitungsgebiet abgedeckt sein soll. Um das Lautungsfeld nicht zu umfangreich werden zu lassen (was besonders bei Artikeln mit hoher Beleganzahl vorkommen kann), werden pro Großregion maximal drei Lautungsbelege angegeben. Wie im Artikel feuretzen ersichtlich, kann es jedoch vorkommen, dass die Datenbasis nur für einen Teil der Gebiete Lautungsbelege ermöglicht. Da bei der Belegauswahl keine Lauttypen konstruiert, sondern Belege aus dem Datenmaterial zitiert werden, wird bei der regionalen Verortung jeweils die kleinstmögliche geographische Einheit (i. d. R. die Gemeinde) gemeinsam mit der entsprechenden Großregion angegeben.

\subsubsection{Etymologie}

In diesem Feld werden die wichtigsten etymologischen Informationen angegeben. Handelt es sich um ein Lemma mit standardsprachlicher Entsprechung, orientiert sich die Darstellung in erster Linie an KLUGE (2011) und PFEIFER (2014), bei rein dialektalen Wörtern werden zusätzlich Wörterbücher älterer Sprachstufen des Deutschen (z. B. LEXER 1872-1878; SCHÜTZEICHEL 2012) konsultiert. Die Angabe der Sprachstufen erfolgt beim Deutschen chronologisch (angefangen beim Althochdeutschen), bei Fremd- oder Lehnwörtern wird zuerst die Sprache genannt, aus der ein Wort entlehnt wurde (z. B. Französisch), danach folgen ältere Sprachstufen (z. B. Latein). Handelt es sich bei einem Lemma um eine Ableitung von einem anderen Lemma, das in einem eigenen Wörterbuchartikel behandelt wird, wird lediglich auf dieses Lemma verwiesen. Dies ist auch bei unserem Beispiellemma feuretzen, einer Ableitung von feuern, der Fall.

\subsubsection{Bedeutung}

Die Bedeutungsangaben stellen die zentrale Komponente der Wörterbuchartikel dar und haben daher auch meist den größten Umfang. Die Struktur des Bedeutungsfelds folgt einem hierarchischen Schema, nach dem semantisch eng beieinanderstehende Bedeutungen gruppiert und innerhalb der einzelnen Gruppen noch einmal differenziert werden. Es können maximal vier Hierarchieebenen unterschieden werden, wobei in den meisten Fällen zwei Ebenen für die Bedeutungsdifferenzierung ausreichen.

Auf der obersten Ebene werden die Bedeutungen durch römische Ziffern zu Blöcken zusammengefasst bzw. voneinander unterschieden, auf der nächsten Ebene folgen arabische Ziffern, danach lateinische Kleinbuchstaben und schließlich griechische Kleinbuchstaben. Im Fall des Artikels feuretzen umfasst der erste Block 
Bedeutungen, die einen direkten Bezug zu 'Feuer' oder 'Funken' haben während der zweite Block Bedeutungen enthält, die sich auf die optischen Reflexe beziehen. Die Bedeutungen unter III. beziehen sich auf das Wetter, der vierte Block schließlich enthält noch eine übertragene Bedeutung. Innerhalb der Bedeutungsblöcke findet sich zudem in diesem Artikel noch eine weitere Ebene (etwa II. 1. 'glänzen; funkeln' [...] 2. 'flimmern; flackern'), darunter werden keine Bedeutungen mehr differenziert.

Wie anhand dieses Artikels deutlich wird, orientiert sich die Reihenfolge der Bedeutungsangaben an der Ursprungsbedeutung. Dieser Grundsatz wird auch dann beibehalten, wenn diejenige Bedeutung, die der Etymologie am nächsten steht, nur selten belegt ist und innerhalb des Datenmaterials auch synchron nicht mehr als die Hauptbedeutung angesehen werden kann.

Auch bei der Beschreibung der Bedeutungsvarianten selbst gilt der Grundsatz einer streng empirischen Herangehensweise, d. h. es wird eine enge Orientierung am Datenmaterial angestrebt, wobei - besonders bei in der Standardsprache vorkommenden Wörtern - stets der Duden und das DWDS zum Vergleich konsultiert werden.

Bedeutungsangaben werden gesperrt gedruckt und unter einfache Anführungszeichen gesetzt. In vielen Fällen werden zusätzlich zu den Bedeutungen Angaben zur Stilebene gemacht. Diese werden in Klammern gesetzt und außerhalb der Anführungszeichen platziert, z. B. Fisch 'Seidentuch' (Gaunersprache). Bezieht sich eine Bedeutung ausschließlich oder auch auf eine Variante des Lemmas - etwa eine Diminutivform oder ein bestimmtes Genus -, so wird diese ebenfalls vermerkt (z. B. nur Fänkerl (Diminutiv) 'ein wenig; ein bisschen' beim Lemma Fanken). Liefern die Quellen im Belegmaterial nur unklare bzw. unzureichende Informationen und sind deshalb nicht eindeutig interpretierbar, werden die Bedeutungsangaben aus dem Original zitiert und in doppelte Anführungszeichen gesetzt, z. B. Föhn',"übler Wind“"

Im Idealfall soll zu jeder Bedeutung mindestens ein Belegsatz angeführt werden, was jedoch leider aufgrund der Datenlage oft nicht möglich ist. Da es sich bei den Belegsätzen ebenfalls um Zitate handelt, wird hier - wie bei den Lautungsbelegen - stets die genaueste geographische Verortung angegeben, i. d. R. also die Gemeinde (inkl. Großregion). Handelt es sich beim Belegsatz um ein Literaturexzerpt, wird zusätzlich die Quelle zitiert.

\subsubsection{Wortbildung}

Dieses Feld orientiert sich am „Grundwortprinzip“, das auch schon früher angewandt wurde, d. h. es umfasst sowohl Komposita als auch Partikel- und Präfixwörter, in denen das entsprechende Simplex als Grundwort bzw. als Basis enthalten ist. Die Partikelverben anfeuretzen und ausfeuretzen erscheinen dementsprechend als Bestandteil des Artikels feuretzen, das Kompositum Liebesfaxe wird im Artikel Faxe behandelt. Die Darstellung der Bedeutung(en) inkl. geographischer Angaben und Beispielsätze entspricht der Struktur des Bedeutungsteils (siehe Kapitel 2.3.5). 


\subsubsection{Redewendungen}

Dieser Artikel-Abschnitt umfasst idiomatische Wendungen wie Sprüche, Sprichwörter oder Redensarten, in denen das entsprechende Lemma enthalten ist. Zur Aufnahme einer Mehrwortäußerung in das Feld müssen mehrere Bedingungen erfüllt sein: Zum einen muss zu einer Redewendung eine Bedeutung erkennbar sein, zum anderen muss sie in einer dialektal transkribierten Form belegt sein. Eine weitere Voraussetzung ist, dass Informationen zur regionalen Verortung vorliegen. Die Wortfolge orientiert sich an der dialektalen Vorlage, lediglich in der Standardsprache ungebräuchliche oder ungrammatische Konstruktionen werden korrigiert (z. B. er gibt ihr's zu er gibt es ihr).

Enthält ein Artikel mehrere Redewendungen, die zudem unterschiedliche Varianten des Lemmas enthalten - beim Artikel Fack, Facke wären dies neben dem Lemma selbst die Diminutive Fäckel und Fäckelein sowie die Komposita Spanfäckel, Stubenfäckel und Tuttenfäckel -, werden diese zunächst nach der entsprechenden Lemmavariante geordnet und innerhalb dieser dann alphabetisch nach dem ersten Buchstaben des Satzes bzw. der Phrase sortiert.

\section{TEXTTECHNOLOGISCHE ASPEKTE}

Eine der umfassendsten Modernisierungsmaßnahmen der WBÖ-Neukonzeption sieht eine intensive Nutzung digitaler Methoden und Ressourcen vor, die sich auf alle Bereiche der Wörterbucharbeit erstreckt. Darunter fallen u. a. die sukzessive Optimierung der Datenbank durch redaktionelle Eingriffe, der Ausbau und die Optimierung der sprachkartographischen Visualisierungen des Materials, die Verknüpfung der Datenbankeinträge mit Bilddigitalisaten zu Handzetteln und Fragebögen, die Erstellung einer eigenen Schriftart zur adäquaten Darstellung der Lautzeichen $^{13}$ sowie die Speicherung und Archivierung der zugrundeliegenden Belegmaterialien inklusive der Zugriff darauf. Insbesondere soll im Folgenden auf die Überarbeitung der Belegdatenbank und die Erstellung der Wörterbuchartikel (Kapitel 3.1) sowie die Publikation und Präsentation der Ergebnisse der lexikographischen Arbeit im Internet (Kapitel 3.2) eingegangen werden.

\subsection{WBÖ-Belegdatenbank und Redaktionssystem}

Im Vergleich mit den meisten anderen großräumigen Dialektwörterbüchern sieht sich die Redaktion in der glücklichen Lage, praktisch den gesamten für die Artikelarbeit erforderlichen Datenbestand in digitaler Form zur Verfügung zu haben, wobei wesentliche Optimierungsschritte im Zuge der Neukonzeption seit Ende 2016 durchgeführt wurden. Wie bereits in Kapitel 1.2 dargestellt wurde, ist dies auf die

13 Die Schriftart mit dem Namen „Fiduz“ wurde in enger Kooperation mit dem Schriftlabor (<http://www.schriftlabor.at>; Stand: 20.05.2020) erstellt. 
höchst aufwändige und nahezu zwei Jahrzehnte dauernde Digitalisierungsarbeit zurückzuführen, die zwischen 1993 und 2011 durchgeführt wurde. Nachdem zu Beginn der 1990er-Jahre das System TUSTEP als Format gewählt worden war und auf dieser Grundlage der fünfte WBÖ-Band erarbeitet wurde, zeigte sich in den letzten Jahren immer deutlicher, dass diese Technologie vielen Anforderungen in Bezug auf Zugriffsmöglichkeiten, Plattformunabhängigkeit und Weiterverwertbarkeit nicht mehr genügte. Aus diesem Grund wurde die gesamte Datenbank in mehreren iterativen Schritten, die jeweils (vor allem in den Jahren 2017 und 2019) von umfangreichen Korrekturen und Nachbearbeitungen begleitet waren, in das plattformunabhängige Format XML/TEI konvertiert (vgl. dazu Kapitel 1.2 sowie ausführlicher BOWERS / STÖCKLE 2018).

Der erste Schritt bei der Erstellung eines Wörterbuchartikels besteht stets in einer Datenbankabfrage nach dem entsprechenden Hauptlemma. Um sämtliche für den Artikel relevanten Belege inklusive aller Komposita und Wortbildungsvarianten zu finden, wird das Lemma sowohl in Groß- als auch in Kleinschreibung in den Suchbefehl eingegeben. Da die Lemmatisierung der Handzettel und dementsprechend auch der Belegdatenbank nach dem historisch-etymologischen Prinzip erfolgte, muss zusätzlich darauf geachtet werden, die entsprechende Schreibvariante auszuwählen. Für das Lemma Feim (Grundbedeutung 'Schaum') liefert daher eine Suche nach den Zeichenfolgen <Fēim> und <feeim> alle relevanten Belege. In der Anfangsphase der WBÖ-Neukonzeption (d. h. in den Jahren 2017 bis 2019) wurde für die Abfragen das XML-Datenbankmanagementsystem BaseX (vgl. BaseX 2018) verwendet. Anfang 2020 erfolgte jedoch die Umstellung auf ein eigens für das WBÖ entwickeltes browserbasiertes Suchinterface, das Elasticsearch (vgl. Elasticsearch 2018) als Suchmaschine im Hintergrund verwendet.

Das Resultat einer solchen Abfrage ist stets eine Tabelle, in der sämtliche Informationen zu einem Lemma (neben dem Lemma selbst z. B. Aussprache, Bedeutung, Verwendungskontext, Quellenangaben, Belegort/-region, Anmerkungen etc.), die in XML durch verschiedene Tags gekennzeichnet sind, in Spaltenform dargestellt werden. Da es sich bei XML um ein Format handelt, in dem Daten hierarchisch strukturiert sind und potentiell beliebig tief verschachtelt sein können (vgl. Abbildung 4) und darüber hinaus nicht für jeden Beleg stets alle Informationen in der gleichen Weise und Anzahl vorhanden sind - beispielsweise gibt es nicht zu allen Einträgen Lautungsbelege oder Beispielsätze -, ist stets ein Teil der Zellen (häufig auch Spalten) in der Ergebnistabelle leer (vgl. Tabelle 1). Die folgende Abbildung zeigt einen Ausschnitt eines Eintrags zum Lemma feuretzen aus der Gemeinde Kirchberg-Thening in Oberösterreich im TEI-Format. 
1 <entry xmlns="http://www.tei-c.org/ns/1.0" xml:id="f255_qdb-d1e101174" n="253897" source="\#orig-f255_qdb-d1e101174">

Abb. 4: TEI-Eintrag zum Lemma feuretzen aus Kirchberg-Thening (Hausruckviertel, OÖ) (Ausschnitt) 
Es handelt sich um einen Eintrag, der auf der Grundlage zweier zusammengehörender und aufeinander folgender Handzettel erstellt wurde (vgl. Abbildung 1). Sämtliche Informationen der Belegzettel finden sich in dem Eintrag wieder und sind mit entsprechenden TEI-Tags versehen. Die oberste Zeile im TEI-Eintrag enthält Informationen zur genauen Identifikation des Eintrags innerhalb der Datenbank, darauf folgen Angaben zum Lemma (Zeilen 2-4), zur Wortart (Zeilen 5-7) und zu den verschiedenen Lautungsvarianten (Zeilen 8-16), wobei die Form gfeorâtzt zusätzlich noch als Partizip II gekennzeichnet ist (Zeilen 13-15). Auf die Bedeutungsangabe 'Funken sprühen' in den Zeilen 17-19 folgt der Belegsatz samt standarddeutscher Übertragung und Zusatzangaben zur Grammatik und zum verwendeten Fragebogen (Zeilen 20-25). Weiterhin werden in dem Ausschnitt Angaben zum Sammler (Zeilen 27-29) und zum Erhebungsort (Zeilen 29-33) dargestellt. In Tabelle 1 ist der entsprechende Eintrag durch graue Unterlegung hervorgehoben.

\begin{tabular}{|c|c|c|c|c|c|c|}
\hline HL & Lautung & Bedeutung & Kontext & $\ldots$ & Ort & GR \\
\hline feuretzen & foiratsn & $\begin{array}{l}\text { "feuretzen"; } \\
\text { funken }\end{array}$ & & $\ldots$ & Ahrntal & öSTir. \\
\hline feuretzen & fōịrẹtsn & $\begin{array}{l}\text { Funken er- } \\
\text { zeugen }\end{array}$ & $\begin{array}{l}\text { [einen Stoß aufs Auge } \\
\text { erhalten], dass gfoịrẹtst } \\
\text { [P2] hǫd }\end{array}$ & $\ldots$ & $\begin{array}{l}\text { Mühlheim } \\
\text { am Inn }\end{array}$ & Innv. \\
\hline feuretzen & foírètsn & $\begin{array}{l}\text { Funken sprü- } \\
\text { hen }\end{array}$ & $\begin{array}{l}\text { i ho }{ }^{\mathrm{b}} \text { mè }{ }^{\mathrm{h}} \text { əsó } \text { ą }^{\mathrm{n}} \text { gręnd } \\
\text { das s nəgrǫd gfoirətst } \\
{[\mathrm{P} 2] \text { hǫd }}\end{array}$ & $\ldots$ & $\begin{array}{l}\text { Mühlheim } \\
\text { am Inn }\end{array}$ & Innv. \\
\hline feuretzen & $\begin{array}{l}\text { fe̊orâtzn, } \\
\text { gfe̊orâtzt }\end{array}$ & $\begin{array}{l}\text { Funken sprü- } \\
\text { hen }\end{array}$ & $\begin{array}{l}\text { Afm Sched l håt â mâ } \\
\text { ââne 'ge̊ }{ }^{\mathrm{b}} \mathrm{m} \text {, das s gråd } \\
\text { gfe̊orâtzt [P2] håt }\end{array}$ & $\ldots$ & $\begin{array}{l}\text { Kirchberg- } \\
\text { Thening }\end{array}$ & Hausrv. \\
\hline feuretzen & vėorptßñ & $\begin{array}{l}\text { flackern, blit- } \\
\text { zen }\end{array}$ & & $\ldots$ & Schlatt & Hausrv. \\
\hline feuretzen & föoötzn & $\begin{array}{l}\text { Feuerschein } \\
\text { geben }\end{array}$ & $\begin{array}{l}\text { ols gförötzt [P2] håds' } \\
\text { fårn Augnan, - a sō håd ar } \\
\text { hę-khaud }\end{array}$ & $\ldots$ & Gmunden & Traunv. \\
\hline feuretzen & föorötzn & $\begin{array}{l}\text { Funken sprü- } \\
\text { hen }\end{array}$ & & $\ldots$ & Gmunden & Traunv. \\
\hline $\begin{array}{l}\cdots \\
\text { (ein)feuret- } \\
\text { zen }\end{array}$ & $\begin{array}{l}\text {... } \\
\text { ẹфärntßn }\end{array}$ & $\begin{array}{l}\text { einheizen; } \\
\text { unterzünden }\end{array}$ & $\ldots$ & $\begin{array}{l}\cdots \\
\cdots\end{array}$ & $\begin{array}{l}\text {... } \\
\text { Pulkau }\end{array}$ & $\begin{array}{l}\cdots \\
\text { Weinv. }\end{array}$ \\
\hline $\begin{array}{l}\text { (ein)feuret- } \\
\text { zen }\end{array}$ & $\begin{array}{l}\text { eıñ-fei- } \\
\text { razn }\end{array}$ & einheizen & & $\ldots$ & & Weinv. \\
\hline $\begin{array}{l}\text { (um-ēin-an- } \\
\text { der)feu-ret- } \\
\text { zen }\end{array}$ & $\ldots$ & $\ldots$ & $\begin{array}{l}\text { di foiąlezn [pl3] } \\
\text { umąnåndą }\end{array}$ & $\begin{array}{l}\cdots \\
\cdots\end{array}$ & $\begin{array}{l}\ldots \\
\text { Fusch }\end{array}$ & $\begin{array}{l}\cdots \\
\text { Pinzg. }\end{array}$ \\
\hline
\end{tabular}

Tab. 1: Ergebnistabelle der Abfrage zum Hauptlemma feuretzen (Ausschnitt)

(HL= ,Hauptlemma“, GR= ,Großregion“) 
Die Tabelle zeigt einen Ausschnitt der Abfrageergebnisse zum Hauptlemma feuretzen. Aus Platzgründen werden nicht alle Ergebnisse angezeigt, ebenso wird ein Großteil der Spalten ausgeblendet (wie etwa Angaben zu weiteren Lautungen, Bedeutungen oder Beispielsätzen, aber auch zur Quelle, zur Fragebogennummer etc.).

Tabellen dieser Art stellen - zusammen mit den Scans der Belegzettel und der in Kapitel 2.3 angeführten einschlägigen Fachliteratur - die Grundlage für die lexikographische Alltagsarbeit beim WBÖ dar. Für die Bearbeitung der Belege steht den Lexikograph/-innen ein eigens entwickeltes Tagging-System zur Verfügung, mit dessen Hilfe jedem Beleg beliebig viele Tags zugeordnet werden können. Auf diese Weise werden alle Belege mit verschiedenen Informationen (z. B. zur Bedeutung, zur geographischen und zeitlichen Verortung oder zur Wortbildung) angereichert, was wiederum die Grundlage für die Erstellung sog. „Collections“ - also Gruppen bzw. Sammlungen von Belegen, die nach bestimmten Kategorien zusammengefasst werden - bildet. Die Collections stellen einerseits einen wichtigen Teil der der lexikographischen Arbeit dar, andererseits sind sie technische Voraussetzung für die spätere Verknüpfung der Einzelbelege mit den entsprechenden Inhalten der Wörterbuchartikel (siehe unten).

Zum Verfassen der Artikel wurde von HANS CHRISTIAN BREUER ${ }^{14}$ ein speziell auf die Bedürfnisse der WBÖ-Redaktion und die Artikelstruktur abgestimmtes Redaktionssystem entwickelt. Es handelt sich dabei um eine auf Electron ${ }^{15}$ basierende Open-Source-Software, die plattformübergreifend verwendet und potenziell auch von anderen Wörterbuchprojekten adaptiert werden kann. Die Artikel werden in einem Git-Repositorium ${ }^{16}$ gespeichert, was eine einfache Versionsverwaltung erlaubt und zusätzlich die Möglichkeit schafft, die Artikel, sobald sie den Status zur Freigabe erhalten, automatisch in die Publikationsplattform im Lexikalischen Informationssystem (siehe Kapitel 3.2) hochzuladen. Abbildung 5 zeigt die Eingabemaske des WBÖ-Redaktionstools in der Startansicht.

14 Siehe auch <www.hcb.de>; Stand: 12.12.2018.

15 Siehe auch <https://electronjs.org>; Stand: 12.12.2018.

16 Siehe auch <https://git-scm.com>; Stand: 12.12.2018. 


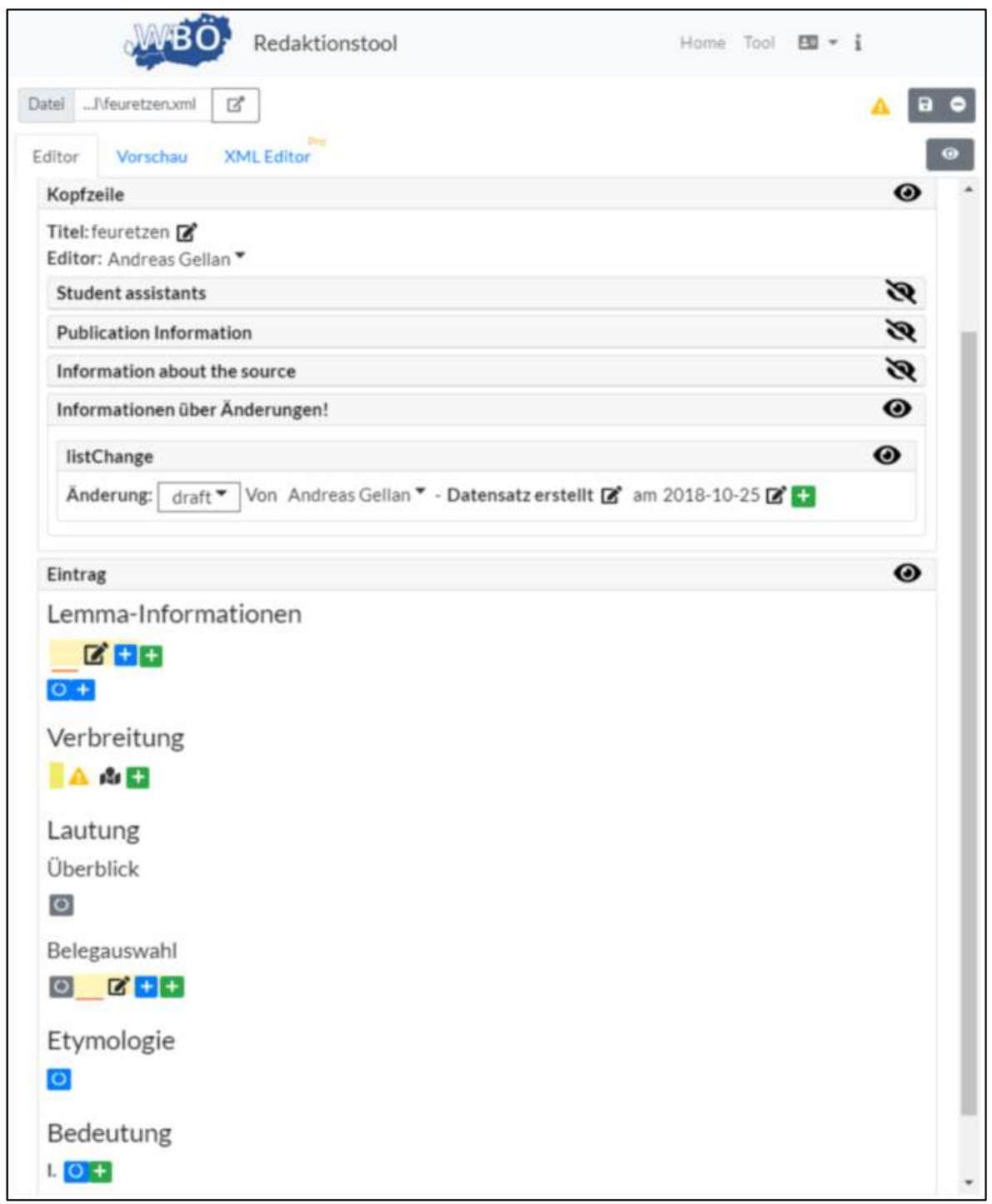

Abb. 5: Startansicht der Eingabemaske des WBÖ-Redaktionstools 
Die Kopfzeile enthält Informationen zum Namen des Artikels (Titel „feuretzen“), zum Autor bzw. zur Autorin sowie zum jeweiligen Stand der Bearbeitung. Die verschiedenen Bearbeitungsstände werden separat gespeichert und können bei Bedarf abgerufen werden. Daneben enthält die Kopfzeile Informationen zum Publikationsstand sowie zur zugrundeliegenden Quelle. Neben der Editor-Ansicht stellt das Redaktionstool zusätzlich eine Vorschau-Funktion zur Verfügung, in der der Artikel ohne Tags betrachtet werden kann. Daneben bietet es die Möglichkeit zur Ansicht des Artikels im XML-Format.

Im Feld „Eintrag“ sind sämtliche Artikelpositionen angelegt und mit verschiedenen Eingabehilfen versehen. Neben der Erstellung einer wohlgeformten XML/TEI-Struktur, die sowohl für digitale als auch für Print-Publikationsformate verwendet werden kann, besteht der Hauptzweck des Redaktionstools in einer maximalen Arbeitserleichterung beim Artikelschreiben, d. h. Arbeitsschritte sollen, wo immer möglich, automatisiert werden. Dazu zählt auch, dass Inhalte, die wiederkehrend in den Artikeln erscheinen (wie z. B. grammatische Informationen oder Angaben zur geographischen Lokalisation), direkt im Redaktionstool hinterlegt sind und einfach über Listen abgerufen werden können. In Bezug auf das Lemma selbst betrifft dies etwa die Wortart (inkl. weiterer grammatischer Merkmale wie Genus bei Substantiven oder Flexionsklasse bei Verben) sowie die Angabe von Sonderfällen wie Diminuierungen, movierten Formen oder Kurzformen. Weiterhin sind sämtliche Informationen zu geographischen Angaben auf den Ebenen der Gemeinde, der Kleinregion, der Großregion und der Bundesländer hinterlegt, was neben der Erleichterung des Artikelschreibens und der Vermeidung von Tippfehlern den weiteren Vorteil hat, dass diese Informationen direkt mit den entsprechenden Geodaten verknüpft sind. Geographische Angaben in den Wörterbuchartikeln können somit in der Publikationsplattform LIÖ (siehe Kapitel 3.2) per Mausklick auf der Karte angezeigt werden. Eine weitere Erleichterung bei der Eingabe von Lautungsbelegen stellt die phonetische Tastatur dar, die bei der Eingabe eines Buchstabens eine Reihe weiterer Lautzeichen vorschlägt. Im Fall des Buchstabens $a$ umfasst sie beispielsweise die Zeichen ${ }^{\mathrm{a}},{ }^{\mathfrak{p}},{ }^{\mathrm{a}},{ }^{\text {}}{ }^{\mathfrak{p}},{ }^{\mathrm{p}}$, a, a und $\mathrm{e}$.

Daneben verfügt das Programm über eine Kommentarfunktion sowie über die Möglichkeit, Verweise auf andere Artikel einzubauen. Letzteres wird insbesondere im Zusammenhang mit der Etymologie beim Verweis auf Lexeme aus der gleichen Wortfamilie häufig verwendet (siehe dazu Kapitel 2.3.4). Abbildung 6 zeigt einen Ausschnitt des Redaktionstools mit dem vollständig eingegebenen Artikel feuretzen. 


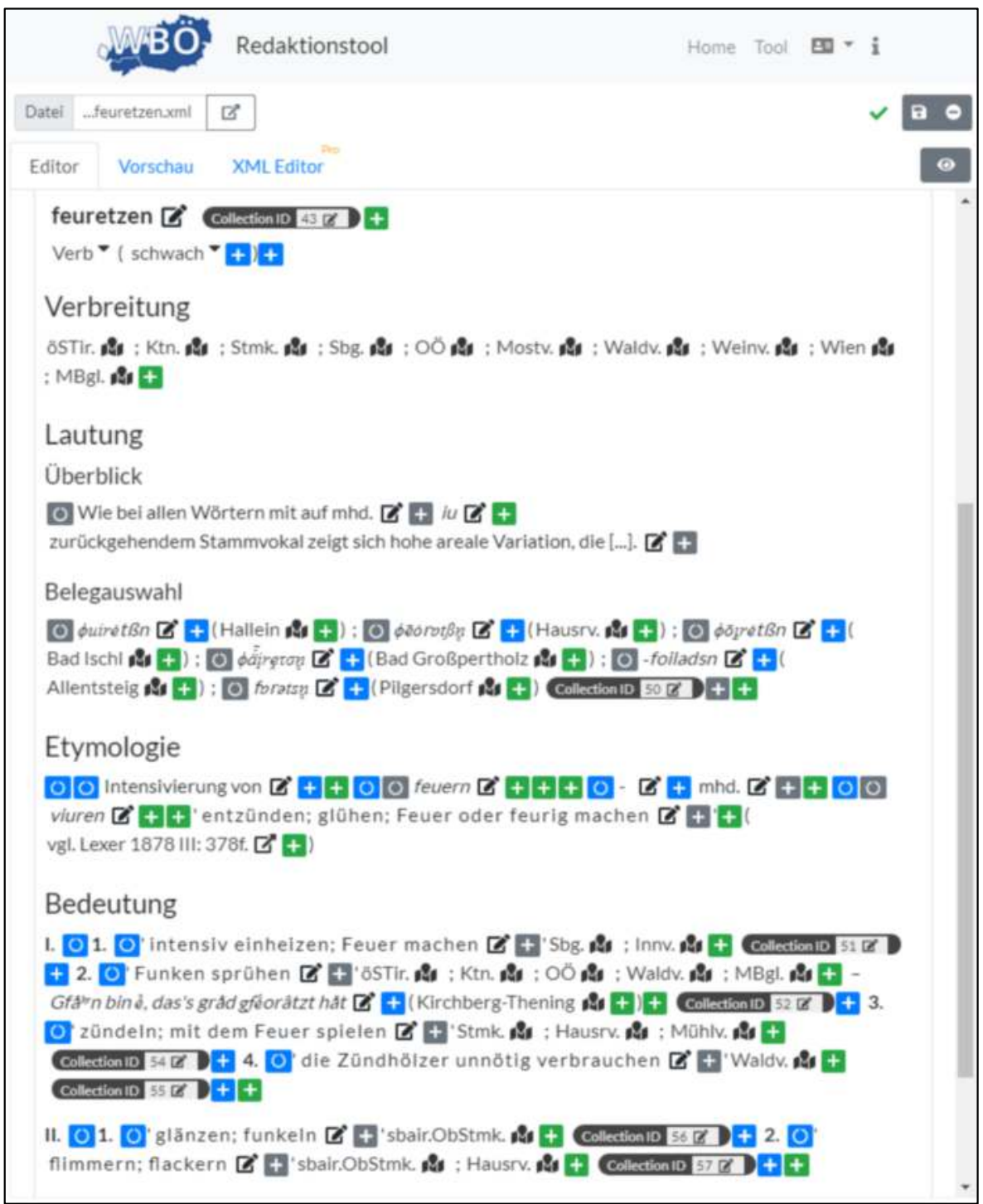

Abb. 6: Artikel feuretzen im WBÖ-Redaktionstool ${ }^{17}$

Neben den in der Publikationsansicht dargestellten Inhalten (siehe Kapitel 2.3) enthält das Redaktionstool an verschiedenen Stellen Informationen zu den „Collections". Die mit dem Lemma feuretzen verknüpfte Collection mit der ID 43 umfasst beispielsweise alle Belege, die für den Artikel herangezogen wurden, während etwa

17 Aus Gründen der Übersichtlichkeit wurde der Abschnitt „Überblick“ in der Lautungsposition für die Darstellung gekürzt. 
Collection 51 alle Belege enthält, die der Bedeutung I.1 'intensiv einheizen; Feuer machen' zugrunde liegen. Über eine Schnittstelle werden sämtliche Informationen aus den Artikeln, den Collections und der Belegdatenbank in die Publikationsplattform LIÖ übernommen, wodurch eine direkte Verknüpfung von Artikelinhalten mit den entsprechenden Belegen möglich wird, was wiederum eine genaue Kartierung oder eine Einsicht in die zugrundeliegenden Handzettel erlaubt. Im Folgenden wird das Lexikalische Informationssystem Österreich (LIÖ) ausführlich vorgestellt.

\subsection{Online-Publikation im Lexikalischen Informationssystem Österreich (LIÖ)}

Ein zentraler Aspekt der Neuaufstellung und Neukonzeption des WBÖ seit dem Jahr 2016 betrifft nicht nur eine Reihe grundlegender lexikographisch-inhaltlicher und konzeptioneller Neuerungen, sondern bezieht sich auch auf die Publikationsformen. Mehr als die meisten anderen Textsorten eignen sich gerade Wörterbuchartikel für die digitale Publikation. Dies hat einmal mit dem Charakter von Wörterbüchern als Nachschlagewerke zu tun, in denen gezielt bestimmte Informationen gesucht werden, die sich wiederum über Suchfunktionen schnell finden lassen; daneben stellen elektronische Texte Möglichkeiten der Verknüpfung mit weiteren Texten und sonstigen digital verfügbaren Materialen zur Verfügung. Im Gegensatz zu Print-Publikationen sind Internet-Publikationen grundsätzlich von überall aus zugänglich, und auch hinsichtlich der Darstellung bieten sie entscheidende Vorteile, da der zur Verfügung stehende Platz prinzipiell nicht begrenzt ist und daher bei der Gestaltung des Layouts auf Übersichtlichkeit geachtet und gleichzeitig auf die Verwendung von Abkürzung weitgehend verzichtet werden kann. Eine gedruckte Version des WBÖ ist auch für die Zukunft weiterhin geplant, da auch diese wesentliche Vorteile mit sich bringt (wie z. B. die langfristige Archivierung bei gleichzeitig minimalem Wartungsaufwand), jedoch orientiert sich die Konzeption der Artikel zunächst an den Möglichkeiten des Internets.

Neben den Wörterbuchartikeln enthält LIÖ weitere Module, deren Kern aktuell das WBÖ darstellt, die in Zukunft aber auch auf andere lexikalische bzw. lexikographische Projekte und Materialien erweitert und ausgebaut werden sollen. Zu diesen Modulen gehören ein Kartentool, die Belegdatenbank sowie verschiedene Behelfsmaterialien (z. B. ein Überblick über die verwendeten Lautzeichen, eine Karte des Bearbeitungsgebiets oder ein Abkürzungsverzeichnis). Abbildung 7 zeigt den Artikel feuretzen in der Default-Ansicht in LIÖ. Da die zentrale Information eines Wörterbuchartikels in der Angabe der verschiedenen Bedeutungen liegt, erscheint dieses Feld in dieser Ansicht ausgeklappt, alle anderen Felder lassen sich aber ebenfalls beliebig ein- und ausblenden. 


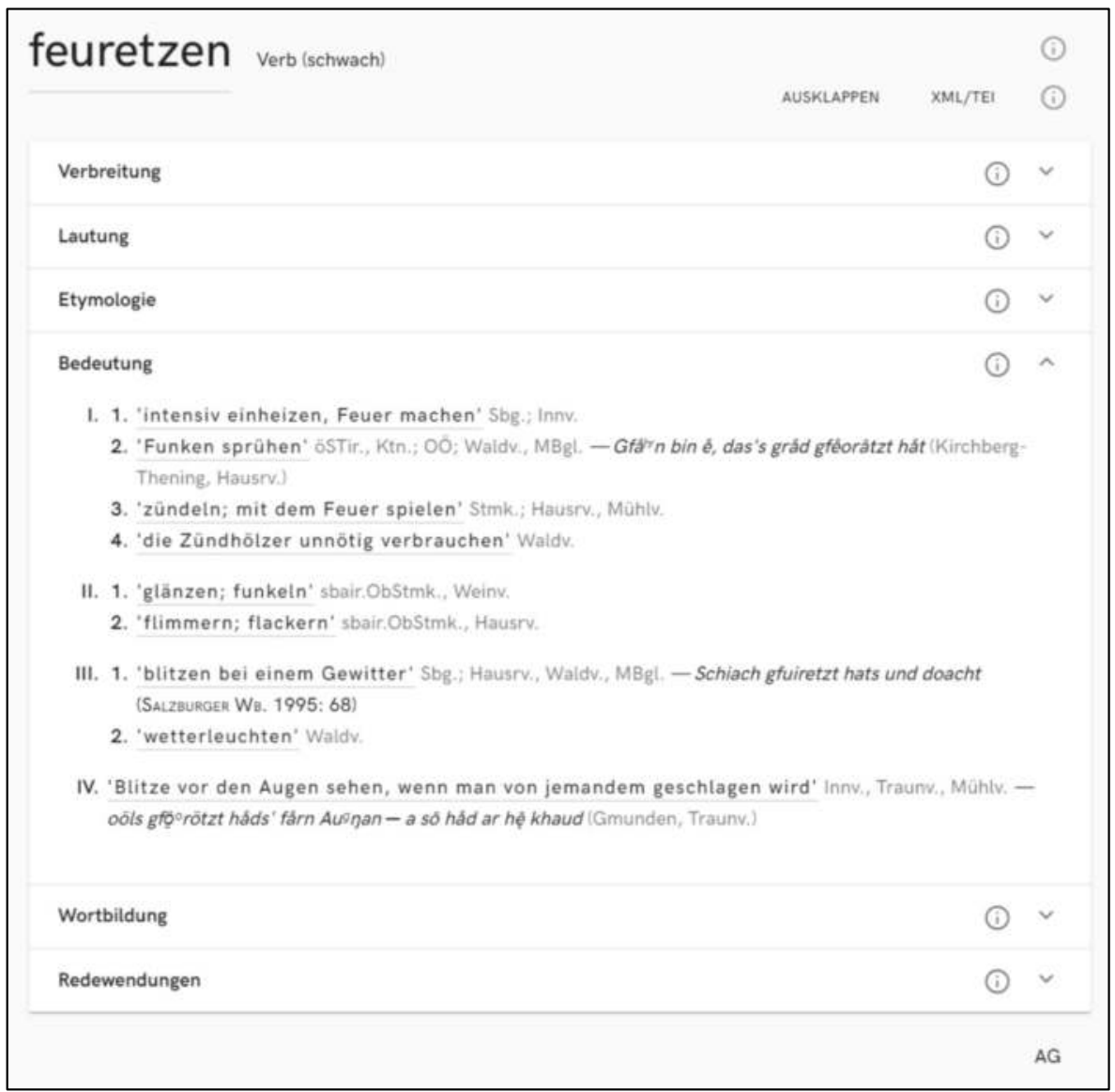

Abb. 7: Default-Ansicht des Artikels feuretzen in LIÖ

Jedes Artikelfeld enthält am rechten Rand ein kleines Informations-Icon, das per Mouse-over-Funktion die wichtigsten Informationen zu den jeweiligen Inhalten anzeigt und auf Wunsch per zusätzlichem Mausklick eine ausführlichere Beschreibung des Felds liefert. Nutzer/-innen, die sich besonders für die texttechnologischen Aspekte der Wörterbuchartikel interessieren, haben außerdem die Möglichkeit, den gesamten Artikel in der XML/TEI-Version anzusehen und herunterzuladen.

Eine der wichtigsten Funktionen, die das Internet als Publikationsmedium ermöglicht, ist die direkte Verknüpfung von Inhalten der Wörterbuchartikel mit den zugrundeliegenden Belegdaten. Wie im vorausgehenden Kapitel 3.1 beschrieben wurde, wird diese Zuordnung während des Artikelschreibens über sog. „Collections" durchgeführt, im online publizierten Artikel wird sie durch Unterstreichung der entsprechenden Stelle markiert. Im Artikel feuretzen betrifft dies - in der in Abbildung 7 dargestellten Ansicht - das Lemma selbst sowie die verschiedenen Bedeutungen. Per Mausklick gelangt man zur entsprechenden Belegauswahl in der 
Belegdatenbank. Abbildung 8 zeigt die der Bedeutung I.3 'zündeln; mit dem Feuer spielen' zugrundeliegenden Belege.

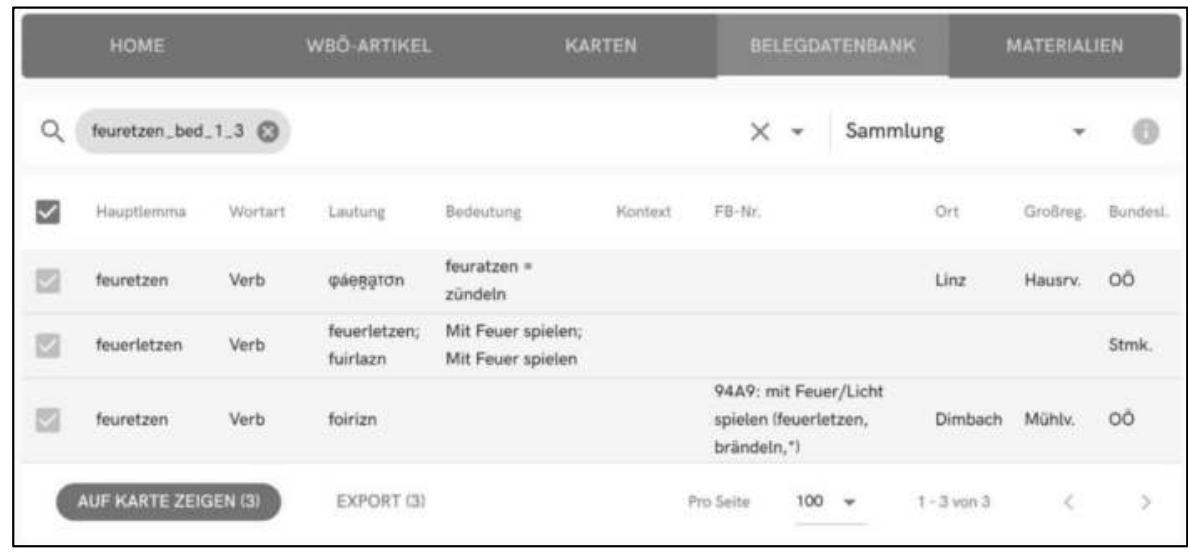

Abb. 8: Collection zur Bedeutung 'zündeln; mit dem Feuer spielen' des Lemmas feuretzen

Neben dem Einblick in die Einzelbelege - und zukünftig auch in die Scans der entsprechenden Handzettel - stellt das Programm auch die Möglichkeit bereit, die Herkunftsorte bzw. -regionen der Belege über ein dynamisches Kartensystem direkt zu kartieren. Eine ausführlichere Beschreibung der Belegdatenbank und ihrer Funktionen erfolgt in Kapitel 4.

Für die Zukunft ist ein zunehmender Ausbau des Lexikalischen Informationssystems Österreich geplant. Auf der einen Seite sollen WBÖ-eigene Inhalte und Materialien - wie die bereits angesprochenen Scans der Handzettel, aber auch Scans von Fragebögen und weiterer Archivmaterialien - sukzessive in die Plattform integriert werden. Daneben wird die Belegdatenbank stetig verbessert und angereichert (etwa durch fehlende geographische Daten), und auch die Kartierfunktionen sollen nutzerfreundlicher und flexibler gestaltet werden. Auf der anderen Seite soll das Informationssystem dazu genutzt werden, lexikographische Inhalte und Informationen verschiedener Art langfristig einem breiten Publikum zur Verfügung zu stellen. Dazu zählen beispielsweise Ergebnisse des geplanten Lexik-Schwerpunkts im SFB „Deutsch in Österreich“, aber auch Daten und Materialien, die im Kontext des WBÖ zur Lexik des Bairischen in Österreich erhoben und aufbereitet werden. 


\section{LINGUISTISCHES POTENZIAL}

Auch wenn das Belegmaterial für das WBÖ als Grundlage für die Wörterbuchartikel gesammelt wurde und auch weiterhin in erster Linie dafür verwendet wird, sind in der WBÖ-Geschichte zahlreiche Publikationen entstanden, in denen WBÖ-Material in anderer Form für Untersuchungen herangezogen wurde. Neben größtenteils lexikographischen Aspekten betrifft dies auch Fragen zu den Bereichen Sprachkontakt (z. B. BERGMANN 2009; 2011), Kulturgeschichte (z. B. WANDL-VOGT 2005a, HAUSNER / WIESINGER 2005), Dialektgeographie (z. B. GEYER 2008), zu GenderAspekten (z. B. POBER et al. 2016), zur Morphologie (z. B. WAHL / LENZ eingreicht) sowie zu Schnittstellen von Lexik mit den Ebenen der Pragmatik (z. B. GEYER 2012) oder Syntax (z. B. STÖCKLE im Druck sowie Kapitel 4.1; LENZ 2013).

Ein großer Mehrwert besonders für zukünftige Forschung ergibt sich aus der Überarbeitung der Datenbank und der Konvertierung ins XML/TEI-Format, in dem die Abschriften der Originaldaten nahezu frei von technischen, formalen oder inhaltlichen Fehlern dargestellt werden. Dies erlaubt die Verwendung der Belegdatenbank als Korpus, das mittels gezielter Abfragemöglichkeiten durchsucht werden kann und das die Untersuchung verschiedener linguistischer Systemebenen sowie das Filtern nach unterschiedlichen sprachlichen und außersprachlichen Parametern erlaubt. Zu den sprachlichen Parametern gehören neben dem Lemma selbst etwa die Angabe der Wortart, während die wichtigsten außersprachlichen Parameter Angaben zur Quelle - entweder zum Sammler bzw. zur Sammlerin und der zugrundeliegenden Frage aus dem Fragebuch oder zur literarischen Quelle - sowie zur genauen geographischen Herkunft eines Belegs liefern. Ein weiterer Mehrwert der Datenbank gegenüber den Handzetteln besteht in der Informationsanreicherung, die im Zuge der Digitalisierung durchgeführt wurde und sich $u$. a. in grammatischen Zusatzinformationen äußert, die an verschiedenen Stellen zu den Daten hinzugefügt wurden. Neben der Wortart, die bei allen Belegen angegeben wird, betrifft dies besonders die Felder zur Lautung und zu den Belegsätzen. Hier wurden bei Varianten, die von der Nennform abweichen, häufig grammatische Angaben zur Flexion (z. B. Numerus, Tempus, Modus, Kasus), aber auch zur Wortbildung (z. B. Diminutiv, Genus) hinzugefügt. Die Belegdatenbank ist somit zwar nicht systematisch grammatisch annotiert, enthält aber viele Informationen, die bei der Analyse bestimmter grammatischer Phänomene (siehe Kapitel 4.1) hilfreich sein können.

Im Folgenden soll das Potenzial der Belegdatenbank zur Beantwortung linguistischer Fragestellungen, die über die lexikographische Arbeit hinausgehen, anhand zweier Beispiele dargestellt werden.

\subsection{Fallbeispiel Grammatik: Der Konjunktiv II im Bairischen}

Beim Konjunktiv II handelt es sich um ein Phänomen, das als besonders charakteristisch für das Bairische betrachtet werden kann (vgl. DONHAUSER 1992). Ähnlich wie im Standarddeutschen existieren auch hier eine synthetische und eine analytische Variante, wobei die synthetische Form basisdialektal mit -ad gebildet wird 
(z. B. dos wĩntšed e khãen hưnd nẹd 'das wünschte ich keinem Hund', Ungenach/Oberösterreich), während die analytische Konstruktion das Hilfsverb tun (und in seltenen Fällen werden) verwendet (z. B. de neigiari Moasn tāt am gean ausfratschln 'die neugierige Meise täte einen gern ausfragen', Poysdorf/Niederösterreich). ${ }^{18}$ Sprachhistorisch bemerkenswert im Zusammenhang mit der synthetischen Form ist, dass diese zwar ursprünglich mit schwachen Verben gebildet wurde, sich das Bildungsmuster mit -ad jedoch auch auf starke Verben übertragen hat (wenn $i$ nu öippas sö́chat 'wenn ich nur etwas sähe', Schwaz/Nordtirol) und dort neben einer starken Flexionsform mit Wechsel des Stammvokal existiert (wen i nu öpas

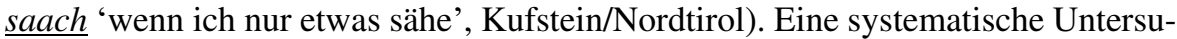
chung und sprachgeographische Beschreibung der verschiedenen Konjunktivvarianten in den bairischen Basisdialekten Österreichs auf empirischer Grundlage steht allerdings bislang noch aus. Aktuelle Entwicklungen werden im Rahmen des SFB „Deutsch in Österreich“ in BREUER / WITTIBSCHLAGER (2020) beschrieben, die im Folgenden vorgestellten Analysen beruhen auf STÖCKLE (im Druck).

Zur Analyse der Konjunktivvariation wurden die Belegsätze in der WBÖ-Datenbank herangezogen. Wie bereits an anderer Stelle angemerkt, wurden im Kontext der Digitalisierung zahlreiche grammatische Zusatzangaben zu den Daten hinzugefügt. Im Fall des Konjunktivs handelt es sich um das Kürzel „kj“, das sich als Zeichenfolge über eine einfache Abfrage suchen lässt. Da diese grammatische Angabe vor allem bei analytischen Formen hinzugefügt wurde, wurde eine zusätzliche Abfrage nach den entsprechenden Formen der Hilfsverben (täte und würde) durchgeführt. Zwar sind die Belegsätze i. d. R. in der Lautschrift Teuthonista transkribiert, was eine Suche nach Wortformen erschwert, allerdings finden sich bereits auf den Handzetteln in ca. 2/3 der Fälle zusätzliche standardsprachliche Übersetzungen bzw. Paraphrasierungen. Auf diese Weise lassen sich ohne großen Aufwand zwar nicht alle Beispielsätze, aber doch ein großer Teil davon nach entsprechenden Wortformen durchsuchen.

Betrachten wir zunächst die Ergebnisse zu den als konjunktivisch markierten Verbformen in den Belegsätzen. Für die Analyse konnten insgesamt 1986 Belege herangezogen werden, wobei einige Vorbereinigungen notwendig waren. Dazu zählen z. B. das Aussortieren von Belegen wie Lockjagd, in denen zwar die Zeichenfolge „,kj“ enthalten ist, jedoch nicht zur Konjunktivmarkierung verwendet wird, oder von Gruß- bzw. Abschiedsformeln wie grias ti gót ('grüße dich Gott', Imst/Nordtirol) oder bvîat aínk gôud ('behüte euch Gott', Natschbach-Loipersbach/Niederösterreich), die im Konjunktiv I stehen und zudem im gesamten bairischen Sprachraum verbreitet sind.

Bei den zu analysierenden Verben wurde einerseits unterschieden, zu welcher Flexionsklasse diese gehören (d. h. stark, schwach oder unregelmäßig), andererseits wurde geprüft, ob sie den Konjunktiv mit oder ohne das Suffix -ad bilden. Zusätz-

18 Für ausführlichere Informationen zur sprachhistorischen Entwicklung, geographischen Verbreitung sowie zur Verwendung vgl. GLAUNINGER (2008), WIESINGER (1989) und STÖCKLE (im Druck). 
lich wurde zwischen synthetischen und analytischen Konjunktivformen unterschieden. Die Verteilung der Konjunktiv-Markierungen auf die verschiedenen Verbtypen ist in Abbildung 9 dargestellt.

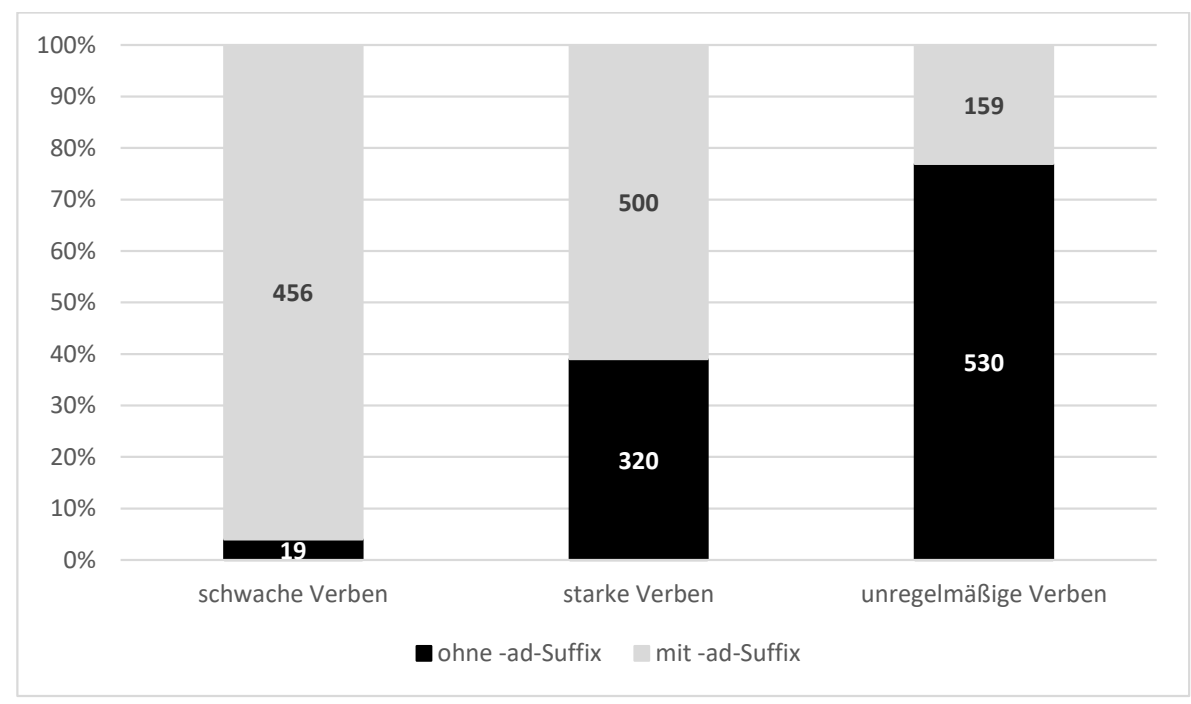

Abb. 9: Verteilung der Konjunktiv II-Markierung auf Flexionstypen

Wie zu erwarten, finden sich unter den insgesamt 475 schwachen Verben 456 (96\%), die den Konjunktiv mit dem Suffix -ad bilden. Bei den unregelmäßigen Verben handelt es sich hingegen größtenteils um Hilfs- und Modalverben, die hochfrequent sind und daher den Konjunktiv II ohne - $a d$ bilden. Bemerkenswert ist besonders die Gruppe der starken Verben, und das aus zwei Gründen: Erstens stellt sie mit 820 Belegen die umfangreichste Gruppe dar, zweitens bildet auch in dieser Gruppe die Mehrzahl der Verben (insgesamt 500, d. h. $61 \%$ ) den Konjunktiv mit - $a d$, also nach einem ursprünglich schwachen Muster ( $i$ gęwat auf 'ich gäbe auf', Lesachtal/Kärnten), während die stark gebildeten Varianten (dēar gab no sei Haut hear 'der gäbe noch seine Haut her', Hall in Tirol/Nordtirol) lediglich $39 \%$ ausmachen.

Neben der Verteilung der Konjunktivmarkierung auf die verschiedenen Verbtypen zeigen auch die sprachgeographische Distribution sowie die Variation zwischen synthetischen Konjunktiv-II-Formen ein differenziertes Bild der bairisch-österreichischen Dialektlandschaft. Zu den Details sei jedoch an dieser Stelle auf die entsprechende Publikation (STÖCKLE im Druck) verwiesen. 


\subsection{Fallbeispiel Lautgeographie: mhd. ei}

Bei dem zweiten hier präsentierten Fallbeispiel handelt es sich um ein Phänomen, das in der bairischen Dialektologie ausführlich beschrieben und diskutiert wurde (vgl. z. B. KRANZMAYER 1956, WIESINGER 1990 oder FANTA-JENDE im Druck) und sich daher besonders eignet, die Qualität und das linguistische Potenzial der WBÖDaten zu prüfen. Es handelt sich um die Reflexe von mhd. $e i$, die standardsprachlich als /ei/ realisiert werden (z. B. klein, heiß, Seife), im Bairischen größtenteils als fallender Diphthong /ov/ (kl/ov/n, h/ov/ß, S/ov/fe) sowie in manchen Regionen als Monophthong /a:/ (kl/a:/n, h/a:/ß, S/a:/fe). Die Notwendigkeit für eine breite empirische Datenbasis zur Dokumentation der lautgeographischen Verhältnisse und als Vergleichsgrundlage für neuere Forschungen (wie derzeit im SFB „Deutsch in Österreich“, vgl. LENZ 2019) ergibt sich in besonderem Maße daraus, dass Österreich - im Gegensatz zu den meisten anderen Teilen des deutschen Sprachraums - nur für einige Teilregionen durch Sprachatlanten abgedeckt wird ${ }^{19}$, eine flächendeckende Dokumentation der dialektalen Verhältnisse auf einer einheitlichen Grundlage jedoch fehlt. Vorhandene sprachgeographische Beschreibungen beruhen auf den Materialien Wenkers (vgl. WIESINGER 1962-1969) oder auf WBÖ-Material (vgl. KRANZMAYER 1956), das jedoch nur zu einem begrenzten Teil ausgewertet wurde. Über das Lexikalische Informationssystem LIÖ sind diese Materialien in Zukunft für jede/n Interessierte/n zugänglich und können nach individuellen Vorgaben ausgewählt und auch kartiert werden.

Gemäß der gängigen dialektologischen Praxis wurde die Abfrage für mhd. $e i$ anhand verschiedener Lexeme durchgeführt. Im Folgenden sollen einige Ergebnisse für das Lexem klein vorgestellt werden. Über eine Suche im HauptlemmaFeld konnten insgesamt 1863 Belege herangezogen werden. Die häufigsten Varianten, die stets aus dem Feld „Lautung“ exzerpiert wurden, sind in Tabelle 2 zusammengefasst.

\begin{tabular}{|c|c|c|}
\hline Тур & Anzahl & Varianten \\
\hline /kloen/ & 1131 & kloa $^{\mathrm{n}}$, klǫa, kxlọ̃ $\tilde{\mathrm{p}}$, kloan, klõãn, glǫa ${ }^{\mathrm{n}}, \mathrm{kloqa}^{\mathrm{n}}, \ldots$ \\
\hline /kla:n/ & 278 & 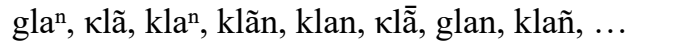 \\
\hline /kleen/ & 258 & 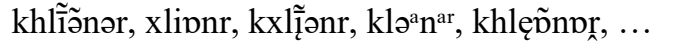 \\
\hline /kluen/ & 109 & 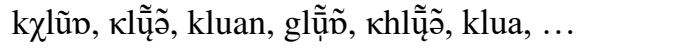 \\
\hline /kli(:)n/ & 22 & 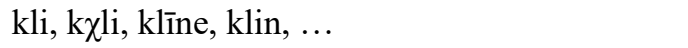 \\
\hline /klain/ & 19 & klęin, khlęin, klein, kléin, klain, ... \\
\hline
\end{tabular}

Tab. 2: Zusammenfassung der häufigsten Varianten des Lexems klein ${ }^{20}$

19 Vgl. dazu die Dialektatlasprojekte SAO, TSA und VALTS.

20 Insgesamt wurden 1835 Belege (von 1863 ) in die Tabelle aufgenommen. Bei den restlichen 28 Belegen handelt es sich um verschiedene Einzelvarianten, die sich nicht in die hier aufgeführten Kategorien einordnen ließen. 
Wie bereits zuvor erwähnt, so wird auch aus Tabelle 2 die große Bandbreite und Variation an Aussprache-Verschriftlichungen deutlich. Eine Herausforderung besteht also zunächst in der Kategorisierung der Varianten zu Typen. Für die Zwecke dieser Analyse wurde die Kategorisierung relativ grob durchgeführt, es ließen sich sicherlich im Detail noch feinere Abstufungen unterscheiden.

Vergleicht man die in der Tabelle ersichtlichen Varianten, so zeigt sich eine hohe Übereinstimmung mit den in der Literatur dargestellten Beschreibungen: Die Variante mit fallendem Diphthong /op/ ist am häufigsten, gefolgt von der Monophthong-Variante /a:/. Daneben zeigt sich jedoch noch eine weitere Form, die den Diphthong /ex/ enthält. Wie die entsprechenden Belege jedoch suggerieren, handelt es sich bei diesen Varianten um Formen des Komparativs, der deshalb im Folgenden aus der Analyse ausgeschlossen wird. Betrachtet man nur noch die Varianten des Lexems klein in der Grundform, ergibt sich die in Abbildung 10 dargestellte geographische Verteilung.

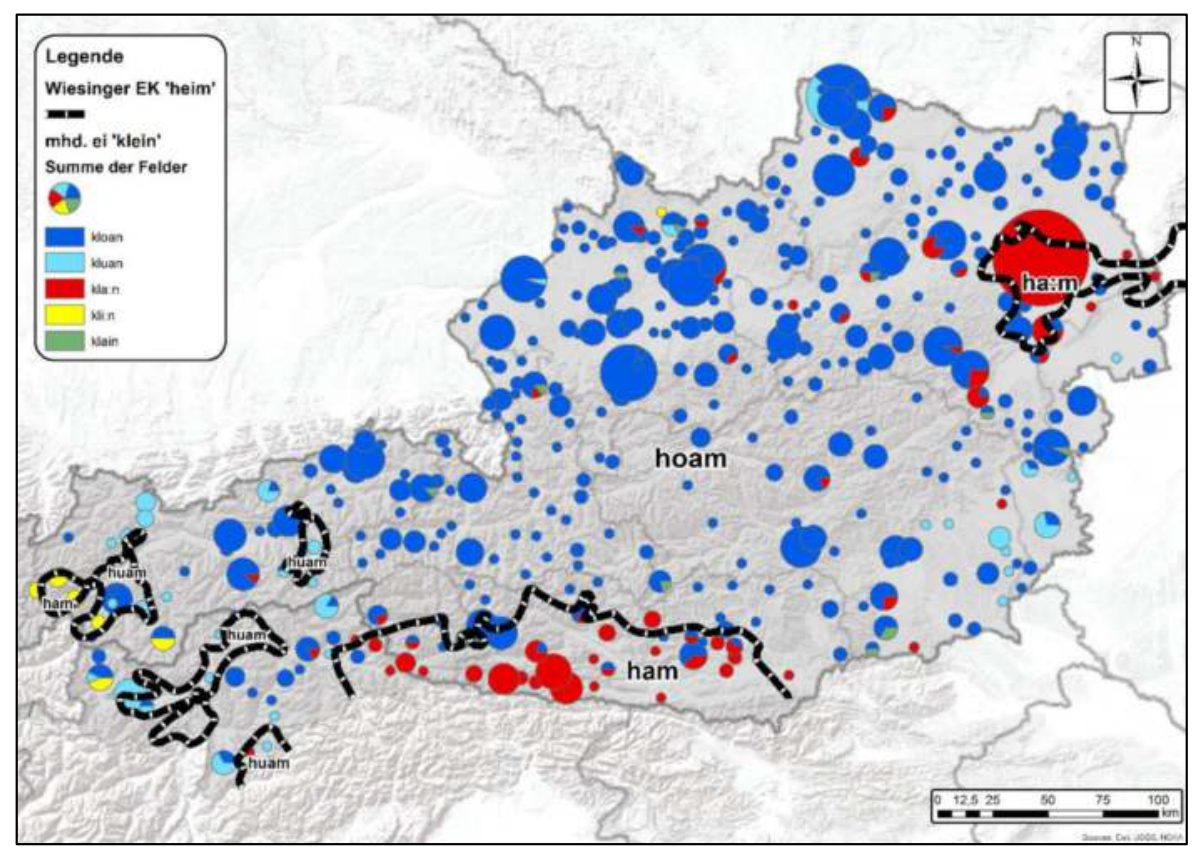

Abb. 10: Sprachgeographische Verteilung der Varianten von klein. Hintergrundkarte basierend auf WIESINGER (1962-1969) (Ergänzungskarte , heim “)

Die verschiedenfarbigen Punkte repräsentieren die unterschiedlichen Lauttypen (vgl. Tabelle 2), wobei die Größe der Symbole relativ zur jeweiligen Anzahl der 
Belege pro Ort ist. Die eingezeichneten Isoglossen basieren auf der Ergänzungskarte „heim“ zum Deutschen Sprachatlas von WIESINGER (1962-1969) ${ }^{21}$. Der Einfachheit wurden nur die Leitformen in die Karte übernommen.

Die Abbildung offenbart die Qualität und das linguistische Potenzial in den WBÖ-Daten: Beide Datenbestände - d. h. die auf den Wenkerbögen beruhende Ergänzungskarte sowie die WBÖ-Daten - zeigen eine große Übereinstimmung hinsichtlich der Verbreitung sowohl der Hauptvariante mit dem Diphthong /or/ als auch der Formen mit Monophthong (/a:/ bzw. /a/) sowie größtenteils der Variante mit Diphthong /ひe/. Die Abweichungen im Westen, für den die WBÖ-Daten eine Verbreitung der Form kli:n aufweisen (im Gegensatz zu Varianten mit Monophthong ham oder fallendem Diphthong huam), sind sicherlich lexikalisch bedingt, und auch die zahlreichen Belege mit /ü/ lassen sich bei genauerer Betrachtung in der originalen Ergänzungskarte wiederfinden.

Neben den Vorzügen zeigt Abbildung 10 aber auch die Schwierigkeiten, die sich im Zusammenhang mit den WBÖ-Daten ergeben und die bei der Verarbeitung stets berücksichtigt werden müssen. Dazu zählen u. a. die ungleichmäßige geographische Abdeckung der Belege, die offenbar auf standardsprachlichen Einfluss zurückzuführenden Varianten vom Typ klain sowie einzelne Abweichungen (wie etwa die im Norden von Oberösterreich nahe der tschechischen Grenze belegte Form kli:n).

\subsection{Zusammenfassung}

Wie die Fallbeispiele gezeigt haben, steckt in den Belegdaten des WBÖ ein Potenzial für sprachwissenschaftliche Analysen, das weit über die reine Verwendung in der Wörterbucharbeit hinausgeht. Gerade vor dem Hintergrund, dass Österreich bislang noch nicht flächendeckend durch Dialektatlanten erfasst ist, erweist sich das basisdialektale Sprachmaterial des WBÖ daher als höchst relevant. Auch wenn bereits an verschiedenen Stellen auf die Uneinheitlichkeit der Daten - sowohl im Hinblick auf Herkunft und Qualität als auch auf die geographische Abdeckung - und damit verbundene Schwierigkeiten hingewiesen wurde, so besticht die Materialsammlung schon alleine durch ihre riesige Menge. Sie eignet sich daher nicht nur für Analysen aus dem Bereich der Lexik, sondern auch zu Phänomenen der Lautung oder Grammatik. Ein ,behutsamer“ Umgang mit den Daten inklusive kritischem Blick auf die zugrundeliegenden Quellen muss dabei jedoch vorausgesetzt werden. Dies gilt nicht nur für die Vorbearbeitung und Bereinigung der Daten, sondern auch für die Interpretation der Ergebnisse - eine ungleichmäßige geographische Verteilung sprachlicher Varianten muss nicht bedeuten, dass diese in bestimmten Regionen nicht vorkommen, sondern kann genauso gut auf die Motivation oder Vorlieben der dortigen Sammler/-innen zurückzuführen sein.

21 Auf die Karte wurde über das REDE-SprachGIS zugegriffen. URL: <https://regionalsprache.de>; Stand: 13.05.2019 
Sieht man von diesen Einwänden und Vorsichtsmaßnahmen ab (die in ähnlicher Form ja für jede Arbeit mit linguistischen Daten gelten), lässt sich die WBÖMaterialbasis als wichtige und umfassende Vergleichsgrundlage auffassen, mittels derer sich eine Lücke in der empirischen Dokumentation der bairischen Basisdialekte Österreichs und Südtirols schließen lässt. Über das Lexikalische Informationssystem Österreich (LIÖ) ist sie zudem nicht nur für Mitarbeiter/-innen des WBÖ, sondern für alle interessierten Nutzer/-innen zugänglich.

\section{KONTAKTADRESSE, HOMEPAGE, WICHTIGE LINKS}

Österreichische Akademie der Wissenschaften (ÖAW)

Austrian Centre for Digital Humanities and Cultural Heritage (ACDH-CH)

Abteilung „Variation und Wandel des Deutschen in Österreich“

Postgasse 7-9

1010 Wien

Österreich

E-Mail:wboe@oeaw.ac.at

Homepages:

WBÖ: <https://www.oeaw.ac.at/acdh/vawadioe/projekte/wboe/>

LIÖ: <https://lioe.dioe.at/>

\section{LITERATURVERZEICHNIS}

AMMON, UlRich / HANS BicKel / ALEXANDRA N. LENZ (Hg.) (2016): Variantenwörterbuch des Deutschen. Die Standardsprache in Österreich, der Schweiz, Deutschland, Liechtenstein, Luxemburg, Ostbelgien und Südtirol sowie Rumänien, Namibia und Mennonitensiedlungen. 2., völlig neu bearbeitete und erweiterte Auflage. Berlin/Boston: de Gruyter.

BARABAs, BetTina / Claudia HARETER-Kroiss / Birgit HofstetTER / LANA MAYER / BARBARA PIRINGER / SONJA SCHWAIGER (2010): Digitalisierung handschriftlicher Mundartbelege. Herausforderungen einer Datenbank. In: Germanistische Linguistik 199-201. Fokus Dialekt. Festschrift für Ingeborg Geyer zum 60. Geburtstag, 47-64.

BaseX (2018): Webseite des Unternehmens. URL: <http://www.basex.org>; Stand: 15.12.2018.

BAUER, WERNER / ERIKA KÜHN (1998): Vom Zettelkatalog zur Datenbank. Neue Wege der Datenverwaltung und Datenbearbeitung im „Wörterbuch der bairischen Mundarten in Österreich“. In: Hutterer (†), Claus JÜrgen / GerTrude PAURITSCh (Hg.): Beiträge zur Dialektologie des ostoberdeutschen Raumes. Referate der 6. Arbeitstagung für bayerisch-österreichische Dialektologie, 20.-24.9.1995 in Graz. Göppingen: Kümmerle, 369-382.

BERGMANN, HUBERT (2009): Randstücke - slowenisches dialektales Material im Belegarchiv zum Wörterbuch der bairischen Mundarten in Österreich (WBÖ). In: SMOLE, VERA (Hg.): Slovenska narečja med sistemom in rabo. Ljubljana (Obdobja. 26), 83-100.

BOWERS, JACK / PHILIPP STÖCKLE (2018): TEI and Bavarian dialect resources in Austria: updates from the DBÖ and WBÖ. In: FrANK, ANDREW U. / Christine IVANOVIC / FrANCESCO MAM- 
Brini / Marco Passarotti / CARoline Sporleder (Hg.): Proceedings of the Second Workshop on Corpus-Based Research in the Humanities (CRH-2), 25-26 January 2018 Vienna, Austria. Wien: Gerastree Proceedings, 45-54.

BREUER, LUDWIG MAXIMILIAN / ANJA WiTTIBSCHLAGER (2020): On the variation of the subjunctive II in Austria - Evidence from urban and rural analyses. In: Linguistic Variation 20/1, 136-171.

DONHAUSER, KARIN (1992): Der Konjunktiv im Bairischen. In: WEISS, ANDREA (Hg.): Dialekte im Wandel. Referate der 4. Tagung zur bayerisch-österreichischen Dialektologie, Salzburg, 5. bis 7. Oktober 1989. Göppingen: Kümmerle, 226-242.

Duden $(2015)$ = Dudenredaktion (Hg.): Duden. Deutsches Universalwörterbuch. 8., überarbeitete und erweiterte Auflage. Berlin: Dudenverlag.

DWDS (2018): Das Wortauskunftssystem zur deutschen Sprache in Geschichte und Gegenwart. URL: <https://www.dwds.de>; Stand: 20.11.2018.

Elasticsearch (2018): Webseite des Unternehmens. URL: <https://www.elastic.co>; Stand: 15.12.2018.

FANTA-JENDE, JOHANNA (im Druck): Varieties in contact. Horizontal and vertical dimensions of phonological variation in Austria. In: LENZ, ALEXANDRA N. / MATEUSZ MASELKO (Hg.): Variationist linguistics meets contact linguistics. Göttingen: Vienna University Press (Wiener Arbeiten zur Linguistik).

GEYER, INGEBORG (2008): Zur Entwicklung von Laut- und Wortgrenzen im Bairisch-österreichischen Raum an Hand ausgewählter Beispiele aus dem Wörterbuch der bairischen Mundarten in Österreich (WBÖ) und der Datenbank der bairischen Mundarten in Österreich (DBÖ). In: ERnst, Peter / Franz PATOCKA (Hg.): Dialektgeographie der Zukunft. Akten des 2. Kongresses der Internationalen Gesellschaft für Dialektologie des Deutschen (IGDD) am Institut für Germanistik der Universität Wien, 20. bis 23. September 2006. Stuttgart: Steiner (Zeitschrift für Dialektologie und Linguistik. Beihefte. 135), 193-202.

GEYER, INGEBORG (2012): Zur Entwicklung des Sprachgebrauchs von $e h$ in Partikelfunktion. In:

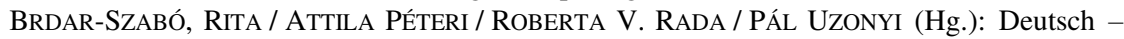
grenzenlos. Festschrift für Elisabeth Knipf zum 60. Geburtstag. Budapest: Universitas Budapestinensis de Rolando Eötvös nominata, ELTE Germanistisches Institut (Budapester Beiträge zur Germanistik. 58), 138-150.

GEYER, INGEBORG (2019): Wörterbuch der bairischen Mundarten in Österreich: Rückblick auf 105 Jahre Erheben, Aufbereiten und Auswerten im institutionellen Rahmen der ÖAW. In: KÜRSCHNer, Sebastian / Mechthild Habermann / Peter O. Müller (Hg.): Methodik moderner Dialektforschung. Erhebung, Aufbereitung und Auswertung von Daten am Beispiel des Oberdeutschen. Hildesheim: Olms (Germanistische Linguistik. 241-243), 471-488.

GLAUNINGER, MANFRED (2008): Synthetische und analytische „Konjunktiv 2“-Formen im Wiener Nonstandard-Deutsch. In: PATOCKA, FrAnz / GuIDO SEILER (Hg.): Dialektale Morphologie, dialektale Syntax. Beiträge zum 2. Kongress der Internationalen Gesellschaft für Dialektologie des Deutschen, Wien, 20.-23. September 2006. Wien: Praesens, 233-247.

HAUSNER, IsOldE / WIESINGER, PETER (Hg.) (2005): Deutsche Wortforschung als Kulturgeschichte: Beiträge des Internationalen Symposiums aus Anlass des 90-jährigen Bestandes der Wörterbuchkanzlei der Österreichischen Akademie der Wissenschaften, Wien, 25.-27. September 2003. Wien: Verlag der Österreichischen Akademie der Wissenschaften.

HORNUNG, MARIA (1976): Wörterbuch der bairischen Mundarten in Österreich. In: FRIEBERTSHÄUSER, HANS (Hg.): Dialektlexikographie. Berichte über Stand und Methoden deutscher Dialektwörterbücher. Festgabe für Luise Berthold zum 85. Geburtstag am 27.1.1976. Wiesbaden: Steiner, 37-47.

KLUGE, FRIEDRICH (2011): Etymologisches Wörterbuch der deutschen Sprache. Bearbeitet von Elmar Seebold. 25., durchgesehene und erweiterte Auflage. Berlin/Boston: de Gruyter.

KRANZMAYER, EBERHARD (1956): Historische Lautgeographie des gesamtbairischen Dialektraumes. Mit 27 Laut- und 4 Hilfskarten in besonderer Mappe. Graz/Wien: Hermann Böhlaus Nachf. 
LENZ, ALEXANDRA N. (2013): Vom ,kriegen“ und ,bekommen“. Kognitiv-semantische, variationslinguistische und sprachgeschichtliche Perspektiven. Berlin/Boston: de Gruyter (Linguistik Impulse \& Tendenzen. Band 53).

LENZ, AlEXANDRA N. (2019): Der SFB „Deutsch in Österreich. Variation - Kontakt - Perzeption“. In: EICHINGER, LUDWIG / ALBRECHT PlEWNIA (Hg.): Neues vom heutigen Deutsch. Empirisch - methodisch - theoretisch. Berlin/Boston: de Gruyter (Jahrbuch des Instituts für Deutsche Sprache 2018), 335-338.

LEXER, MATTHIAS (1872-1878): Mittelhochdeutsches Handwörterbuch. Zugleich als Supplement und alphabetischer Index zum Mittelhochdeutschen Wörterbuch von Benecke-Müller-Zarncke. 3 Bände. Leipzig: Hirzel.

MEISTER, RICHARD (1963): Vorwort. In: KRANZMAYER, EBERHARD (Hg.): Wörterbuch der bairischen Mundarten in Österreich (WBÖ). Band 1: $A-$ Azor. Wien: Verlag der Österreichischen Akademie der Wissenschaften, V-XVI.

PABst, Christiane M. / Herbert Fussy / Ulrike Steiner (Hg.) (2016): Österreichisches Wörterbuch. 43. Auflage. Wien: Österreichischer Bundesverlag Schulbuch GmbH \& Co. KG.

PFEIFER, WOLFGANG (2014): Etymologisches Wörterbuch des Deutschen. Koblenz: Edition Kramer.

Pober, Maria / Ingeborg GeYer / Eveline WAndL-Vogt / BARbara Piringer (2016): Von vielrednerischen Weibern und alles erforschenden Männern - Genderasymmetrien in der Datenbank der bairischen Mundarten in Österreich (DBÖ) im Vergleich mit Großwörterbüchern der Gegenwart. In: Margalitadze, Tinatin / George Meladze (Hg.): Proceedings of the $17^{\text {th }}$ EURALEX International Congress. Ivane Javakhishvili Tbilisi University Press, 810-822.

REIFFENSTEIN, INGO (2005): Die Geschichte des „Wörterbuchs der bairischen Mundarten in Österreich“ (WBÖ). Wörter und Sachen im Lichte der Kulturgeschichte. In: HAUSNER, ISOLDE / PETER WIESINGER (Hg.): Deutsche Wortforschung als Kulturgeschichte. Beiträge zum Symposium ,,90 Jahre Wörterbuchkanzlei“ der Österreichischen Akademie der Wissenschaften, Wien, 25.-27. September 2003. Wien: Verlag der Österreichischen Akademie der Wissenschaften, 1-13.

$\mathrm{SAO}=$ Adalbert-Stifter-Institut des Landes Oberösterreich (Hg.): Sprachatlas von Oberösterreich . Linz: Adalbert-Stifter-Institut des Landes Oberösterreich

SCHMELLER, JOHANN ANDREAS (1872-77): Bayerisches Wörterbuch. In 2 Bänden. Zweite, mit des Verfassers Nachträgen vermehrte Ausgabe. Bearbeitet von G. KARL FROMMANN. München: Rudolf Oldenbourg.

SCHÜTZEICHEL, RUDOLF (2012): Althochdeutsches Wörterbuch. 7., durchgesehene und verbesserte Auflage. Berlin / Boston: de Gruyter.

STÖCKLE, PHILIPP (im Druck): Dialektdynamik an der Schnittstelle von Syntax, Morphologie und Lexik - der Konjunktiv II in den bairischen Dialekten Österreichs und Südtirols. In: CHRISTEN, Helen / BrigitTe GAnswindt / JOACHIM HERRGEN / JÜRGEN ERICH SCHMIDT (Hg.): Regiolekt - Der neue Dialekt? Akten des 6. Kongresses der Internationalen Gesellschaft für Dialektologie des Deutschen (IGDD). Stuttgart: Steiner.

TSA = KLEIN, KARL KURT / SCHMITT, LUDWIG ERICH / KÜHEBACHER, EGON (1965-1971): Tirolischer Sprachatlas, 3 Bde. Marburg: Elwert (Deutscher Sprachatlas. Regionale Sprachatlanten 3).

VALTS = Gabriel, Eugen (1985 ff.): Vorarlberger Sprachatlas mit Einschluss des Fürstentums Liechentstein, Westtirols und des Allgäus. Bregenz: Vorarlberger Landesbibliothek.

WAHL, SABINE / ALEXANDRA N. LENZ (eingereicht): 'Diminutive' verbs in the Austrian language area - a diachronic and corpus linguistic approach. In: WERNER, MARTINA / WOLFGANG U. DRESSLER (Hg.): Between Derivation and Inflection. Wien: Verlag der ÖAW (Veröffentlichungen zur Linguistik und Kommunikationsforschung).

WANDL-VoGT, Eveline (2005a): ... was nicht im Wörterbuch steht. Die Datenbank der bairischen Mundarten in Österreich (DBÖ) als digitales Archiv am Beispiel kulturgeschichtlicher Fragestellungen. In: EGGERS, ECKHARD / JÜRGEN ERICH SCHMIDT / DIETER STELLMACHER (Hg.): Moderne Dialekte - Neue Dialektologie. Akten des 1. Kongresses der Internationalen Gesellschaft für Dialektologie des Deutschen (IGDD) am Forschungsinstitut für deutsche Sprache 
„Deutscher Sprachatlas“ der Philipps-Universität Marburg vom 5.-8. März 2003. Stuttgart: Steiner (Zeitschrift für Dialektologie und Linguistik. Beihefte. 130), 589-612.

WANDL-VoGT, EVELINE (2005b): Überlegungen zur Artikelstruktur im Wörterbuch der bairischen Mundarten in Österreich (WBÖ) unter Berücksichtigung des Neuen Straffungskonzeptes von 1998. Dargestellt anhand ausgewählter Wörterbuchartikel von Günter Lipold. In: PABST, Christiane N. (Hg.): Sprache als System und Prozess. Festschrift für Günter Lipold zum 60. Geburtstag. Wien: Edition Praesens, 69-94.

WIESINGER, PETER (1962-1969): Ergänzungskarten zum Deutschen Sprachatlas. Nacherhebungen in Süd- und Osteuropa. Marburg: Handgezeichnet.

WIESINGER, PETER (1989): Die Flexionsmorphologie des Verbums im Bairischen. Wien: Verlag der Österreichischen Akademie der Wissenschaften.

WIESINGER, PETER (1990): The Central and Southern Bavarian Dialects in Bavaria and Austria. In: Russ, Charles V. J. (Hg.): The Dialects of Modern German: A Linguistic Survey. London: Routledge, $438-519$.

WBÖ 1 = KRANZMAYER, EBERHARD; unter Mitwirkung von VIKTOR DOLlMAYR, MARIA HORNUNG, FrANZ RoITINGER, ALOIS PISCHINGER, WERNER BAUER, ALBRECHT ETZ (1970): Wörterbuch der bairischen Mundarten in Österreich (WBÖ). Band 1: A-Azor. Wien: Kommissionsverlag der Österreichischen Akademie der Wissenschaften.

WBÖ 2 = KRANZMAYER, EBERHARD; unter Mitwirkung von MARIA HORNUNG (Redaktion), WERNer Bauer, Albrecht EtZ, Ingeborg Geyer, Elisabeth Groschopf, ERIKA KÜHN, WILFRIED SCHABUS (1976): Wörterbuch der bairischen Mundarten in Österreich (WBÖ). Band 2: $B(P)$ - Bezirk. Wien: Verlag der Österreichischen Akademie der Wissenschaften.

WBÖ 3 = HORNUNG, MARIA \& WERNER BAUER; unter Mitarbeit von INGEBORG GEYER, ELISABETH GROSCHOPF, ERIKA KÜHN, GÜNTER LIPOLD, INGRID MARSCHALL, WILFRIED SCHABUS, ELISABETH SCHUSTER, HERBERT TATZREITER (1983): Wörterbuch der bairischen Mundarten in Österreich (WBÖ). Band 3: $P f-C$. Wien: Verlag der Österreichischen Akademie der Wissenschaften.

WBÖ 4 = BAUER, WERNER; unter Mitarbeit von INGEBORG GEYER, ELISABETH GROSCHOPF, ERIKA KÜHN, WILFRIED SCHABUS, HERBERT TATZREITER (1998): Wörterbuch der bairischen Mundarten in Österreich (WBÖ). Band 4: D,T-tętzig. Wien: Verlag der Österreichischen Akademie der Wissenschaften.

WBÖ 5 = BAUER, WERNER \& INGEBORG GEYER; unter Mitarbeit von BETTINA BARABAS, HubERT BERGMANN, MANFred GlaUninger, Elisabeth Groschopf, BIRgIT HOFSTETTER, ERIKA KÜhn, Barbara Piringer, Christina Schrödl, Ulrike Thumberger, EVEline WANDLVoGT (2015): Wörterbuch der bairischen Mundarten in Österreich (WBÖ). Band 5: deuEzzes. Wien: Verlag der Österreichischen Akademie der Wissenschaften.

WBÖ-Beiheft 2 = Institut für österreichische Dialekt- und Namenlexika (I Dinamlex) (Hg.) (2005): Wörterbuch der bairischen Mundarten in Österreich. Beiheft 2: Erläuterungen zum Wörterbuch. Lautschrift, Abkürzungsverzeichnis, Literatur- und Quellenverzeichnis, Gebietsverzeichnis, Gemeindeverzeichnis (mit einer Übersichtskarte und 6 Detailkarten zum Gebietsverzeichnis. Wien: Verlag der Österreichischen Akademie der Wissenschaften. 


\title{
BAYERISCHES WÖRTERBUCH (BWB)
}

\author{
Michael Schnabel / Manuel Raaf / Daniel Schwarz ${ }^{1}$
}

\section{ORGANISATION}

Das „Bayerische Wörterbuch“ hat seinen Sitz an der Bayerischen Akademie der Wissenschaften in München und ist eines der ältesten Forschungsprojekte an dieser Institution. Es erforscht und dokumentiert den Wortschatz der im Freistaat Bayern gesprochenen bairischen Dialekte. Darüber hinaus wird auch die literarische Überlieferung aus (Alt-)Bayern seit ihren Anfängen im 8. Jahrhundert berücksichtigt.

Die mit der Herausgabe des BWB beauftragte „Kommission für Mundartforschung" wurde bereits im Jahr 1911 gegründet. Im Zuge einer Neustrukturierung der Akademie im Jahr 2015 wurden die bis dahin „Kommissionen“ genannten Forschungsgruppen in „Projekte“ umgewandelt. Ein Ausschuss mit neun Mitgliedern leitet das Projekt „Bayerisches Wörterbuch“. Ein Beirat mit dreizehn Mitgliedern begleitet und berät den Ausschuss.

Das Redaktionsteam des Projekts besteht derzeit aus ANDREA SCHAMBERGERHIRT (Leitung ab Mai 2019), EDITH BURKHART-FunK (halbe Stelle), FELICITAS ERHARD (halbe Stelle), MichAEL SCHNABEL und VinCENZ SCHWAB. Unterstützt wird das Redaktionsteam durch den seit März 2019 im Ruhestand befindlichen ehemaligen Projektleiter ANTHONY RowLEY, der weiterhin als Vorsitzender des Projektausschusses fungiert, und den ehemaligen Redaktor JOSEF DENZ.

\section{PUBLIKATIONEN}

Die erste Heftlieferung des BWB (Artikelstrecke A-Acker) erschien im Jahr 1995, der erste Band ( $A-B a z i)$, der die Lieferungen 1 bis 8 umfasst, 2002. Im Jahr 2012 wurde der zweite Band (be-Boxhamer; Lieferung 9-17), im Oktober 2019 der dritte Band (prä-törmisch; Lieferung 18-27) veröffentlicht. Die ersten beiden Hefte des vierten Bandes werden im Laufe des Jahres 2020 erscheinen.

Der erste und zweite Band sowie die Lieferungen 18-23 stehen als PDFDateien online zur Verfügung und können über die Homepage des BWB (<bwb.badw.de>) eingesehen werden. Gleiches gilt für das Beiheft mit dem Ortsund Quellenverzeichnis, das 1995 publiziert wurde.

Das Gesamtwerk soll zehn bis zwölf Bände umfassen.

1 Michael Schnabel zeichnet für die Kapitel 1 bis 6 verantwortlich, MANUEL RAAF und DANIEL SCHWARZ für das Kapitel 7. 


\section{ZUR GESCHICHTE DES BWB}

Die Mundartforschung hat eine lange Tradition an der Bayerischen Akademie der Wissenschaften, die schon JOHANN ANDREAS SCHMELLERS Arbeiten gefördert hat. Die im Jahr 1911 gegründete Kommission für Mundartforschung hatte den Auftrag, Wörterbücher vorzubereiten, die sich mit den bairischen, ostfränkischen und rheinpfälzischen Mundarten im damaligen Königreich Bayern befassten. Die schwäbische Mundart in Bayern zählte nicht zum Aufgabengebiet, denn sie wurde damals bereits im „Schwäbischen Wörterbuch“ von HERMANN FISCHER bearbeitet.

Das Wörterbuch-Projekt an der Bayerischen Akademie der Wissenschaften hat mit dem „Bayerischen Wörterbuch“ von JOHANN ANDREAS SCHMELLER (*1785 Tirschenreuth, $\uparrow 1852$ München) einen berühmten Vorläufer. SCHMELLER hatte in den Jahren 1827-1837 das erste großräumige wissenschaftliche Dialektwörterbuch verfasst, 1872-1877 erschien eine von GEORG KARL FROMMANN bearbeitete Neuauflage. Doch SCHMELLERs Wörterbuch war zu Beginn des 20. Jahrhunderts vergriffen. Heute ist es als Reprint-Ausgabe wieder neu aufgelegt, es genügt aber den Anforderungen der modernen Mundartforschung nicht mehr.

Der gesamte bairische Wortschatz sollte ursprünglich in Zusammenarbeit mit der Österreichischen Akademie der Wissenschaften in einem gemeinsamen Wörterbuch dargestellt werden. Die Österreichische Akademie der Wissenschaften war in besonderem Maße an dem neuen Wörterbuchprojekt interessiert, denn Schmeller hatte in seinem Wörterbuch den Wortschatz der Dialekte Österreichs kaum berücksichtigt.

Die Arbeit am Wörterbuch begann 1912, zeitgleich an den Akademien in Wien und München, teilweise mit gemeinsamen Befragungen. Die Münchner Arbeitsstelle sollte sich ursprünglich auf das Sammeln und Ordnen des Dialektmaterials für ihr Gebiet beschränken, die Publikation dann in einem gemeinsamen BayerischÖsterreichischen Wörterbuch in Wien erfolgen.

Nach den schwierigen Jahren des Zweiten Weltkriegs, in denen es zum Stillstand der Arbeiten gekommen war, nahm man die Arbeiten in München erst 1947 wieder auf. Glücklicherweise war die Materialsammlung fast komplett erhalten. Da München und Wien in unterschiedlichen Besatzungszonen lagen, war es schwierig, engen Kontakt zu halten. Organisatorische, politische und persönliche Probleme führten dazu, dass man das ursprüngliche Konzept eines gemeinsamen Wörterbuchs für Bayern und Österreich aufgab. Zu Beginn der 1960er Jahre einigte man sich darauf, die Wörterbücher für die bairischen Dialekte Österreichs und Bayerns getrennt erscheinen zu lassen. Die Münchner Arbeitsstelle, die in den ersten Jahrzehnten und insbesondere in den Kriegsjahren nur einen oder zwei Mitarbeiter hatte, wurde im Laufe der 1960er Jahre auf den gegenwärtigen Stand von vier hauptamtlichen wissenschaftlichen Mitarbeitern ausgebaut.

Die beiden Akademien in München und Wien vereinbarten, dass das BWB den Wortschatz aller bairischen Mundarten erfassen sollte, die im heutigen Freistaat Bayern gesprochen werden. Die außerhalb Bayerns gesprochenen bairischen Mundarten behandelt das von der Wiener Akademie herausgegebene „Wörterbuch 
der bairischen Mundarten in Österreich“ (WBÖ), also auch jene, die in den an Österreich angrenzenden Nachbarländern gesprochen werden wie beispielsweise in Südtirol.

Die Trennung hat sich vorteilhaft ausgewirkt, da die gewaltigen dialektalen Unterschiede des Bairischen in zwei getrennten, kleineren Werken leichter zu bewältigen sind. Heute pflegen die beiden Arbeitsstellen der Akademien in Wien und München einen regen kollegialen und freundschaftlichen Austausch. ${ }^{2}$

\section{ARBEITSGEBIET}

Die Grenzen des Arbeitsgebiets des BWB, das die Regierungsbezirke Oberbayern, Niederbayern und Oberpfalz sowie die bairischsprachigen Teile der Regierungsbezirke Oberfranken, Mittelfranken und Schwaben umfasst (s. Abbildung 1), sind im Osten und Süden durch die heutige deutsche Staatsgrenze vorgegeben. Im Westen und Nordwesten aber verläuft die Grenze mitten durch den Freistaat Bayern. So reicht das Arbeitsgebiet im Südwesten bis zum Lech, der als Grenze zum schwäbisch-alemannischen Dialektgebiet gilt. Mit einbezogen wird jedoch das westlich des Lechs gelegene Gebiet im oberbayerischen Altlandkreis Schongau. Die rechtslechischen Orte des schwäbischen Altlandkreises Füssen werden dagegen nicht berücksichtigt. Zum ostfränkischen Dialektgebiet hin umfasst das Arbeitsgebiet die Dialekte, die die nordbairischen ,gestürzten“ Diphthonge in $k^{h} o u$ 'Kuh', breiv 'Brief' (mhd. kuo, brief) und die Diphthongierung des mhd. â zu ou in blousn 'blasen' (mhd. blâsen) aufweisen (s. Abbildung 2). ${ }^{3}$

2 Zu einer umfassenden Darstellung der Geschichte des BWB s. S. VII-XXIX des ersten Bandes des „Bayerischen Wörterbuchs“.

3 Die innerhalb des Freistaats Bayerns gesprochenen ostfränkischen Dialekte werden vom „Fränkischen Wörterbuch“ (WBF; ehemals „Ostfränkisches Wörterbuch“; s. S.77) bearbeitet, die am Südwestrand Bayerns gesprochenen ostschwäbischen Dialekte, die auch im „Schwäbischen Wörterbuch“ von HERMANN FISCHER erfasst sind, vom „Digitalen Informationssystem von Bayerisch-Schwaben“ (DIBS; s. S.105). 


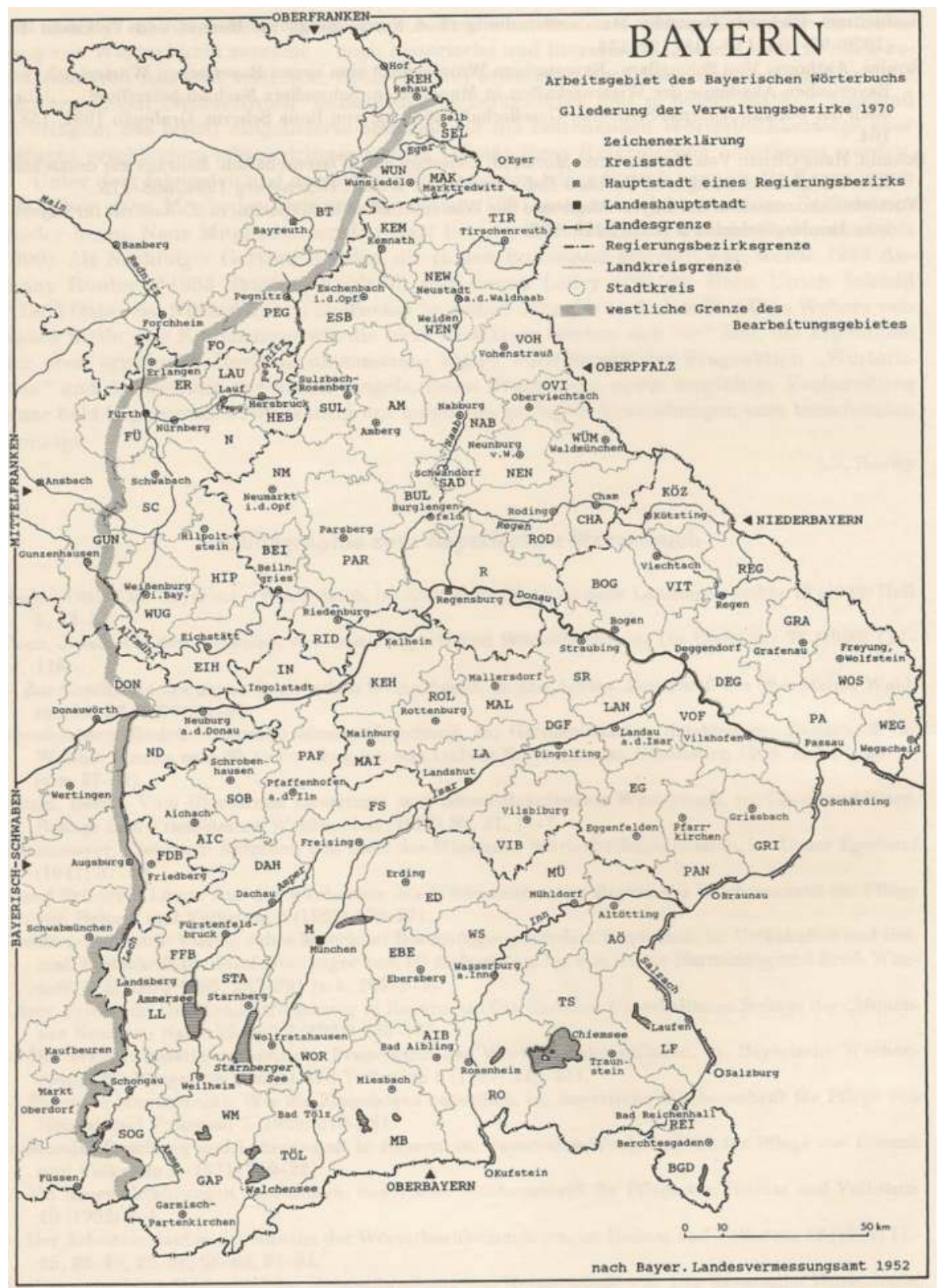

Abb. 1: Arbeitsgebiet, Karte mit Verwaltungsgliederung vor der Gebietsreform von 1972 (aus BWB, Band 1: $X X X)$ 


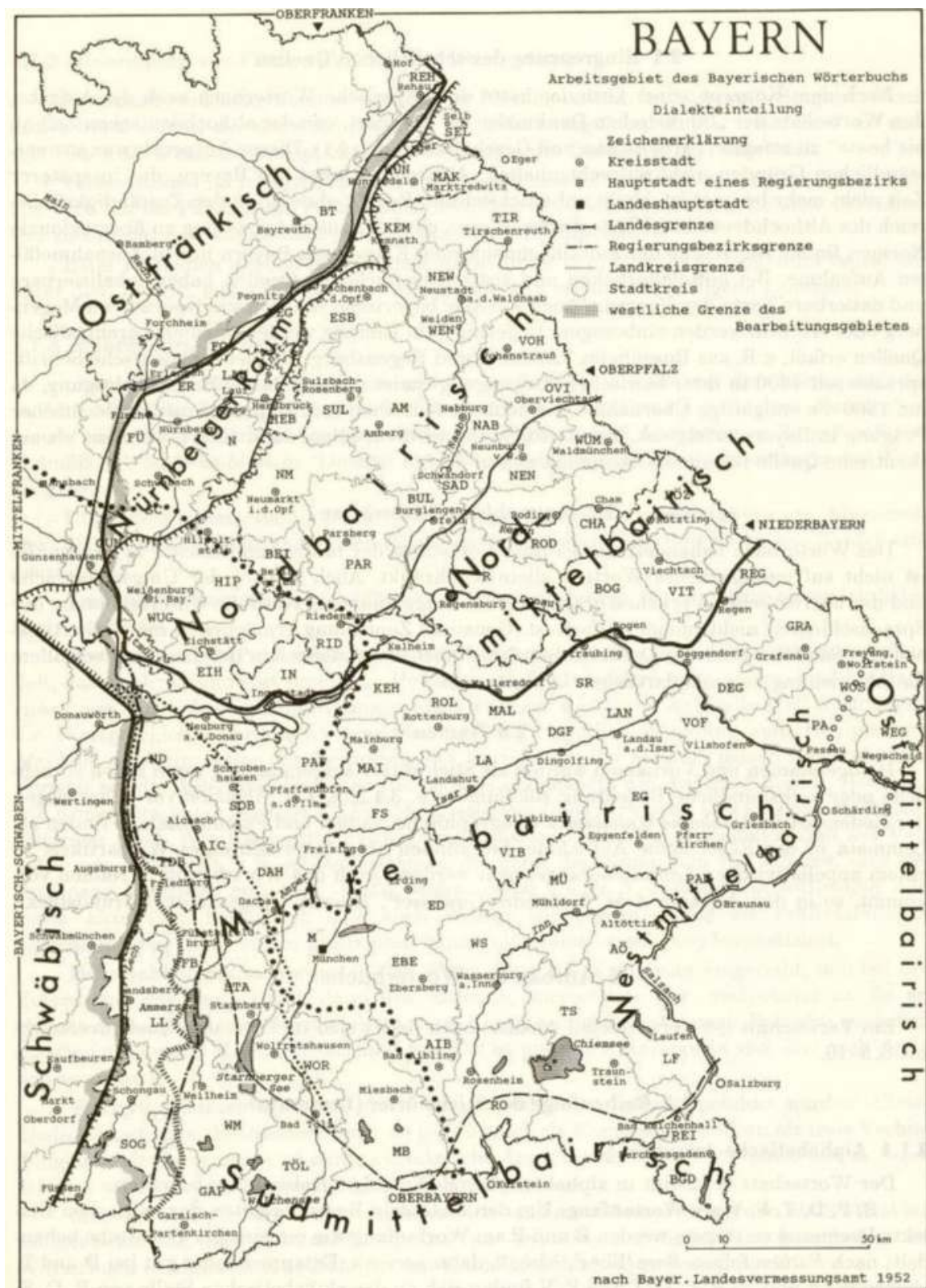

\footnotetext{
— nördl. ou, sudl. $u \alpha$ fur mhd, wo in 'Kuh". ........ abweichender Verlauf nach $\mathrm{N}$ bei mhd. - $\mathrm{k}$ - im Inlaut TIm ostl. $\rho x$, westl. pi, ai far mhd. ei in 'breit'

ताrm sö. pi, nw, a fur mhd, ei in Mehrsilbern ('Seife'). ('trocken').

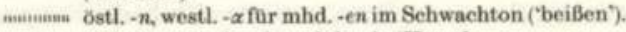

-... ostl. d. $\rho$, westl. $a$ fur mhd. $a$ in 'Katze'.

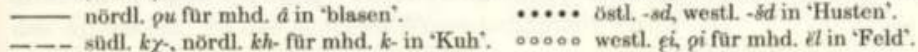

Abb. 2: Dialektale Gliederung des Bairischen in Bayern nach lautlichen Kriterien (aus BWB, Band 1: XXXV) 


\section{MATERIALGRUNDLAGE}

Das Archiv des BWB enthält ca. sieben Millionen Belege aus unterschiedlichen Erhebungsphasen und aus der exzerpierten Literatur. Den Hauptbestandteil bilden die Beiträge der Gewährspersonen, die Fragebögen beantwortet oder eigenständige Wortsammlungen zur Verfügung gestellt haben. Derzeit sammeln ca. 350 Mundartsprecherinnen und -sprecher in ganz Altbayern Belege für das BWB, indem sie an sie versandte Fragebögen (,Wörterlisten“; s. u.) bearbeiten.

\subsection{Das Sammlermaterial}

Seit 1913 sind mehrere Erhebungen durchgeführt worden, und zwar:

- 1913-1933: Systematischer Fragebogen

- 1927-1933: Kundfahrtenkatalog

- 1927-1940: Mundartgeographischer Fragebogen

- 1934: Fragebogen Maurer

- 1958 bis heute: Wörterlisten

\subsubsection{Systematischer Fragebogen}

Bereits 1913 begann in Zusammenarbeit mit der Wiener Wörterbuchkanzlei (heute WBÖ) der Versand sogenannter „Systematischer Fragebögen“, von denen bis 1930 insgesamt 109 Serien verschickt wurden. Jeder dieser Fragebögen hatte ein Leitthema (z. B. „Kopf“; s. Abbildung 3). Es wurde nicht nur nach einem bestimmten Grundwort und dessen Bedeutungsspektrum gefragt, sondern auch nach Zusammensetzungen, Vergleichen, Redensarten und Bräuchen, die in Zusammenhang mit dem thematisierten Wort stehen. Die Belege wurden von den Probanden auf mitgelieferte Karteizettel geschrieben (s. Abbildung 4). Die „Sammler“, die diese Fragen beantworten sollten, waren in der Mehrzahl Volksschullehrer, die wiederum alteingesessene Personen aus ihrem jeweiligen Schulort befragten. In den Jahren 1913 bis 1915 wurden bayernweit 41 unterschiedliche Fragebögen an jeweils etwa 350 Sammler versandt. 1915 mussten die Erhebungen kriegsbedingt eingestellt werden. Als nach dem Ersten Weltkrieg der Versand wieder aufgenommen wurde, konnte das Sammlernetz nicht mehr auf diese Dichte gebracht werden. Am Schluss der Aktion waren insgesamt nur noch 38 Sammler beteiligt. Etwa zwei Drittel der Sammler stammten aus Altbayern. Das Ergebnis der „Systematischen Fragebögen“ waren etwa 780000 Belegzettel. Davon gingen gegen Ende des Zweiten Weltkriegs etwa 64000 verloren. 


\section{' Kopf (1).}

1. Ausdrïcke für Kopf, Haupt (gewöhnliche und scherzhafte Beneunungen; man achte besonders auch auf die Verwendung des Wortes in ubertragenem, bildlichem Sinno); 2. Vergleiche und Redensarten mit Kopf in eigentlicher Bedeutung («. B. Kopf stehn, der Kopf möchte einem zerspringen usw.); 3. solche mit Kopf in tibertragener Bedeutung (z. B. das ist ein feiner, offener Kopf, jemand den Kopf verdrehen); 4. schöner, ebenmäßig gebauter Kopf (Christus-, Tituskopf); $4^{\mathrm{a}}$. häßlicher, unebenmäßiger K.; 5 . großer K. (man achte hier wic im folgenden auf die zugehürigen Eigenschaftswörter, vgl. D2); 5". Wasserkopf; 6. kleiner K.; 7 . oben zugespitzter K.; 8. langer K.; 9. kurzer, platter K.; 10. runder K.; 11 . breiter K.; 12. schmaler K.; 13. sehiefer K.; 14. K. mit dunkler Gesiehtsfarbe und krausem Haar (Molhrenkopf); 15. mißgestalter K., z. B. Fischkopf (Kindskopf mit fischartig anfgezogener Nase und Oberlippe), Hasenkopf (K. mit Hasenscharte), Vogelkopf; 16. K. mit Ungeziefer behaftet (Laus, Nißkopf); 17. Krankheiten des Kopfes (Kopfweh, -typhus usw.); 17ª durch Krankheiten entstellter K. (Kritizen-, Grindkopf u. a.); 17 ${ }^{\text {}}$. Beule, Auswuchs am K. (Redensarten); 18. auf den Kopf schlagen (,schïdeln"), Schlag auf den Kopf; 19. enthaupten, köpfen; 20. sonstige Zusammensetzungen mit Kopf, Schädel usw., an zweiter, 20". erster Stelle (\%. B. Weiberkopf, Kopfstiick).

Abb. 3: Systematischer Fragebogen Nr. 1, Leitthema ,Kopf“

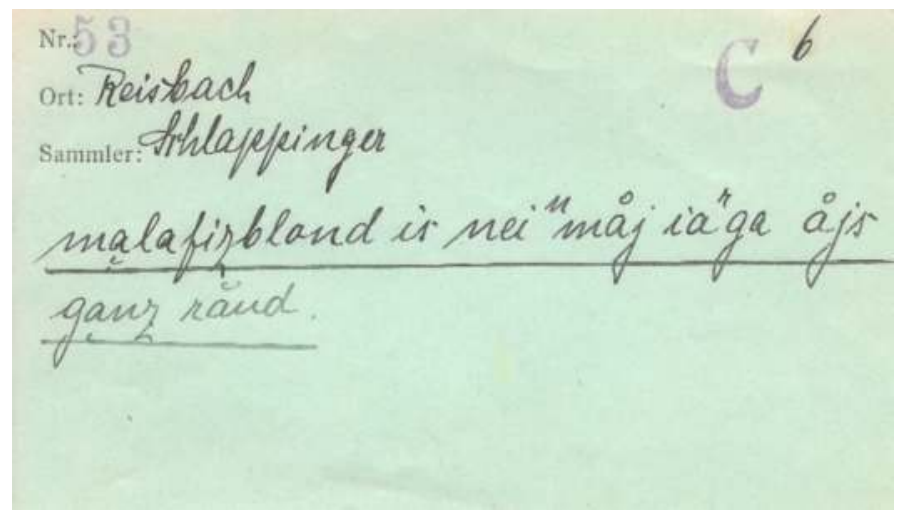

Abb. 4: Belegzettel aus Reisbach, Altlandkreis Dingolfing; Antwort auf Frage C6 des Systematischen Fragebogens Nr. 53 (,, sonstige Ausdrücke für rot und seine Abstufungen "): malafizblond is nei ${ }^{\mathrm{n}}$ måj ia ${ }^{\mathrm{r}} \mathrm{ga}$ åjs ganz raud 'malefizblond ist neunmal ärger als ganz rot' 


\subsubsection{Kundfahrtenkatalog}

Von 1927 an unternahmen die damaligen Redaktoren EBERHARD KRANZMAYER und FRIEDRICH LÜERS in Bayern mehrere Kundfahrten. Das dadurch gewonnene Material („Kundfahrtenkatalog“) ist die einzige Materialschicht des BWB, die durch direkte Befragung vor Ort entstanden ist. Hauptsächliche Zielsetzung war die Erfassung von Lautungen zur Zeichnung von Lautkarten, demgemäß erfolgte die Verschriftung der Belege in phonetischer Transkription (s. Abbildung 5). Diese Materialschicht ist für die lexikographische Arbeit nur eingeschränkt verwendbar, sie ist aber nützlich für Angaben zur Lautung eines Lemmas, die bei Stichwörtern mit interessanter sprachgeographischer Lautvarianz im Anschluss an den Bedeutungsteil gegeben werden. Insgesamt enthält der Kundfahrtenkatalog etwa 108000 Belegzettel.

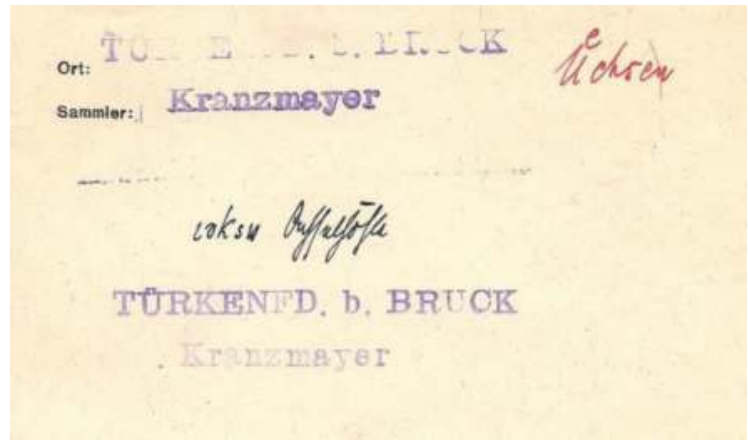

Abb. 5: Belegzettel zum Stichwort Üchse (Achselhöhle) aus Türkenfeld, Landkreis Fürstenfeldbruck; der Explorator EBERHARD KRANZMAYER notierte die konkrete Ausspracheform in phonetischer Transkription: ıəksp

\subsubsection{Mundartgeographischer Fragebogen}

Der „Mundartgeographische Fragebogen“ wurde zwischen 1927 und 1940 verschickt. Kurze und prägnante Fragen nach den Ausdrücken für bestimmte Bedeutungen (im Durchschnitt etwa 20-30 pro Bogen), versandt an eine möglichst hohe Zahl von Gewährspersonen, sollten in relativ kurzer Zeit zu einem Belegnetz führen, das für die Erstellung sprachgeographischer Karten im Hinblick auf die Veröffentlichung eines Sprachatlasses tauglich war. Die insgesamt 291 verschickten Bogenserien lagen der „Wochenschrift zur Pflege von Heimat und Volkstum“ bei. Die Zeitschrift wurde von Amts wegen an alle Schulleitungen versandt. Die Antworten wurden direkt in die Bögen eingetragen (s. Abbildung 6). Mithilfe dieses Fragebogens wurden ca. 1,3 Millionen Mundartbelege gesammelt. Die insgesamt ca. 80000 eingegangenen Fragebögen liegen auch als Scans vor. 


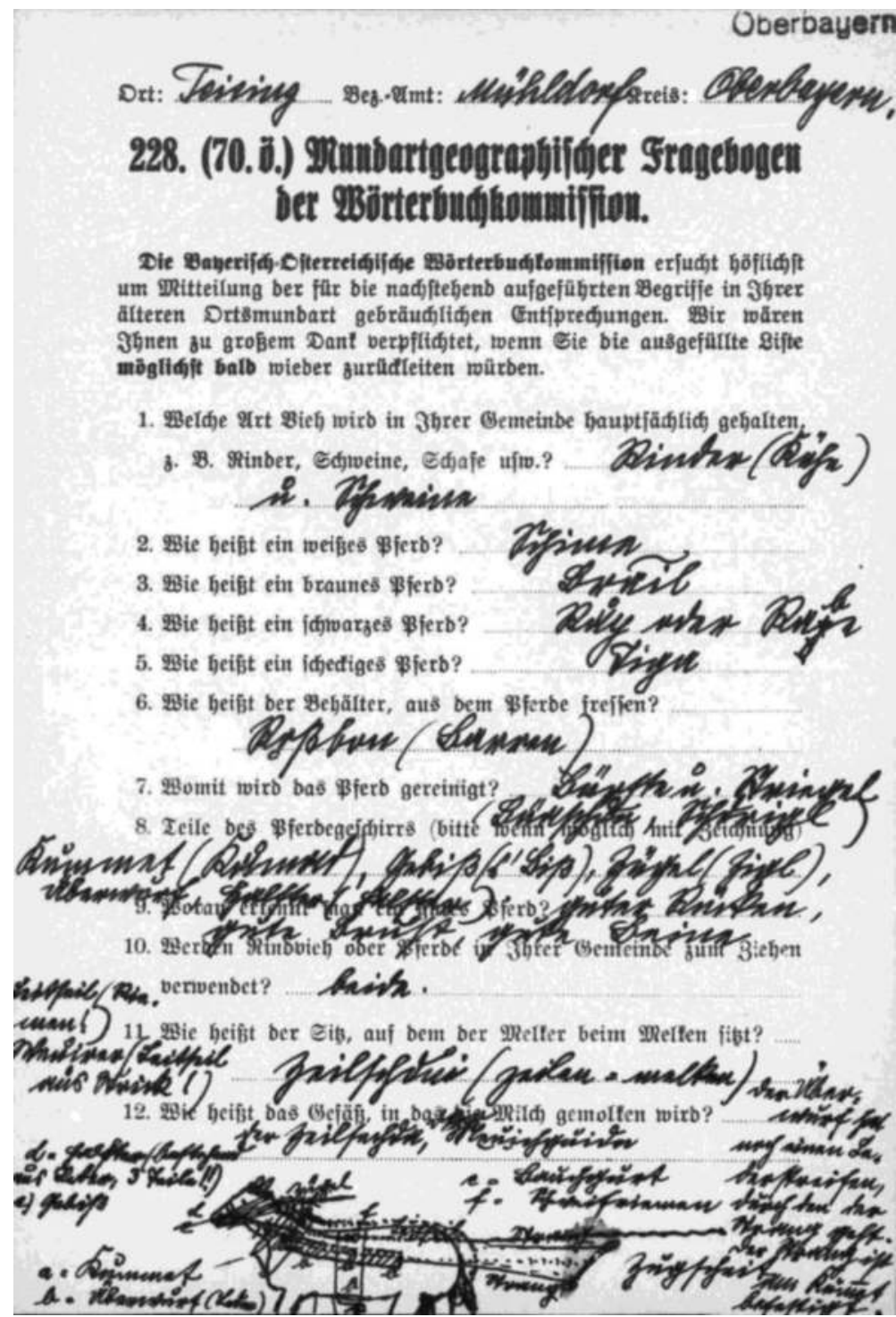

Abb. 6: Erste Seite des Mundartgeographischen Fragebogens Nr. 228 aus Teising, Landkreis Mühldorf; als Antworten auf die Fragen 2, 3, 4 und 5 notierte die Gewährsperson in lautnaher Orthographie Schime (Stichwort ,,Schimmel“), Brãil (,,Braunel“), Råp oder Rabe (,,Rappe“), Tiga (,, Tiger") 


\subsubsection{Fragebogen Maurer}

Im Jahr 1934 versandte der Germanist FRIEDRICH MAURER in Erlangen einen für das geplante „Ostfränkische Wörterbuch“ ausgearbeiteten Fragebogen mit 90 Fragen zu Lautung und Wortschatz. Dieser so genannte „Maurer-Bogen“, in den die Gewährspersonen die Antworten wie beim „Mundartgeographischen Fragebogen“ direkt eintrugen (s. Abbildung 7), wurde als Beilage zum „Bayerischen Schulanzeiger“ an alle Schulen Bayerns versandt. Selbst WENKERs „Deutscher Sprachatlas“ erreichte kein Ortsnetz von dieser Dichte.

Die Bögen aus den über 2800 bairischen Orten enthalten ca. 250000 Belege. Sie liegen auch als Scans vor.

\subsubsection{Wörterlisten}

Seit 1958 werden als „Wörterlisten“ bezeichnete Fragebögen verschickt. Sie ergänzen das vorhandene Material der früheren Erhebungen, das teilweise erhebliche Lücken aufweist. Im Gegensatz zur onomasiologischen Ausrichtung der älteren Fragebögen ist die Fragestellung hier semasiologisch, d. h. es wird nicht ausgehend vom Objekt nach der Bezeichnung gefragt, sondern es werden umgekehrt mundartliche Ausdrücke oder auch Redewendungen vorgegeben und danach gefragt, was sie in dem betreffenden Ort bedeuten. Auch wird explizit darum gebeten, dass die Gewährspersonen Satzbeispiele liefern (s. Abbildung 8). Der Fokus dieser Erhebung liegt also auf der Erfassung der Bedeutungsgeographie und der Verwendung der Mundartausdrücke in bestimmten Äußerungskontexten. Auf diese Weise gewinnt man Belege, die den typischen Gebrauch des Wortes illustrieren und nicht nur isolierte Dialektausdrücke, was bei den älteren Erhebungen häufig der Fall war.

Aktuell sind über 270 Wörterlisten mit je 60 Fragen verschickt worden, was weit über vier Millionen Belege einbrachte. Diese Befragungsaktion steht kurz vor dem Abschluss, es sind lediglich noch Teile der Stichwörter mit den Anfangsbuchstaben $R, S$ und $W$ zu erheben. 


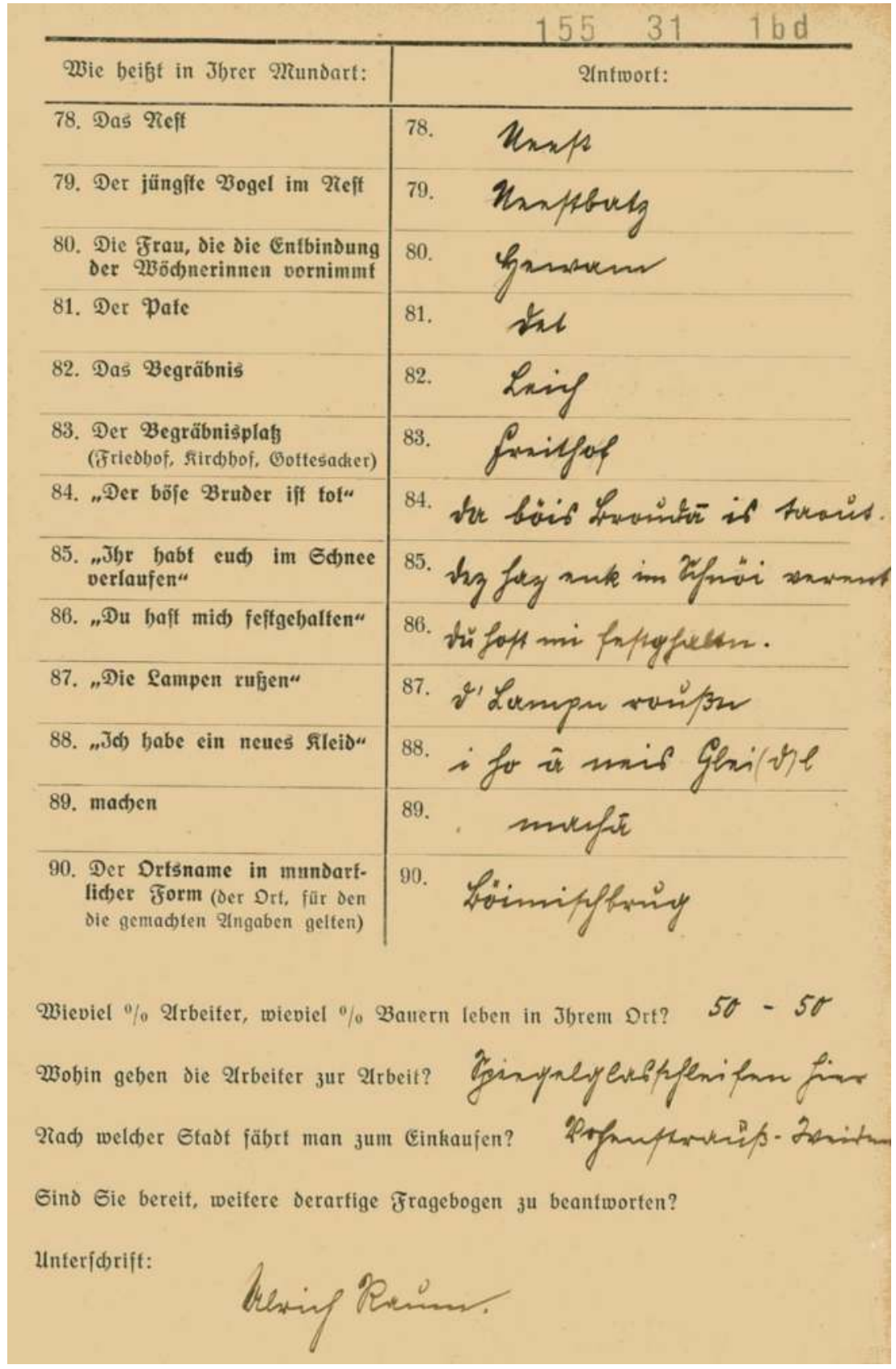

Abb. 7: Sechste (und letzte) Seite des Maurer-Bogens aus Böhmischbruck, Altlandkreis Vohenstrau $\beta$ 


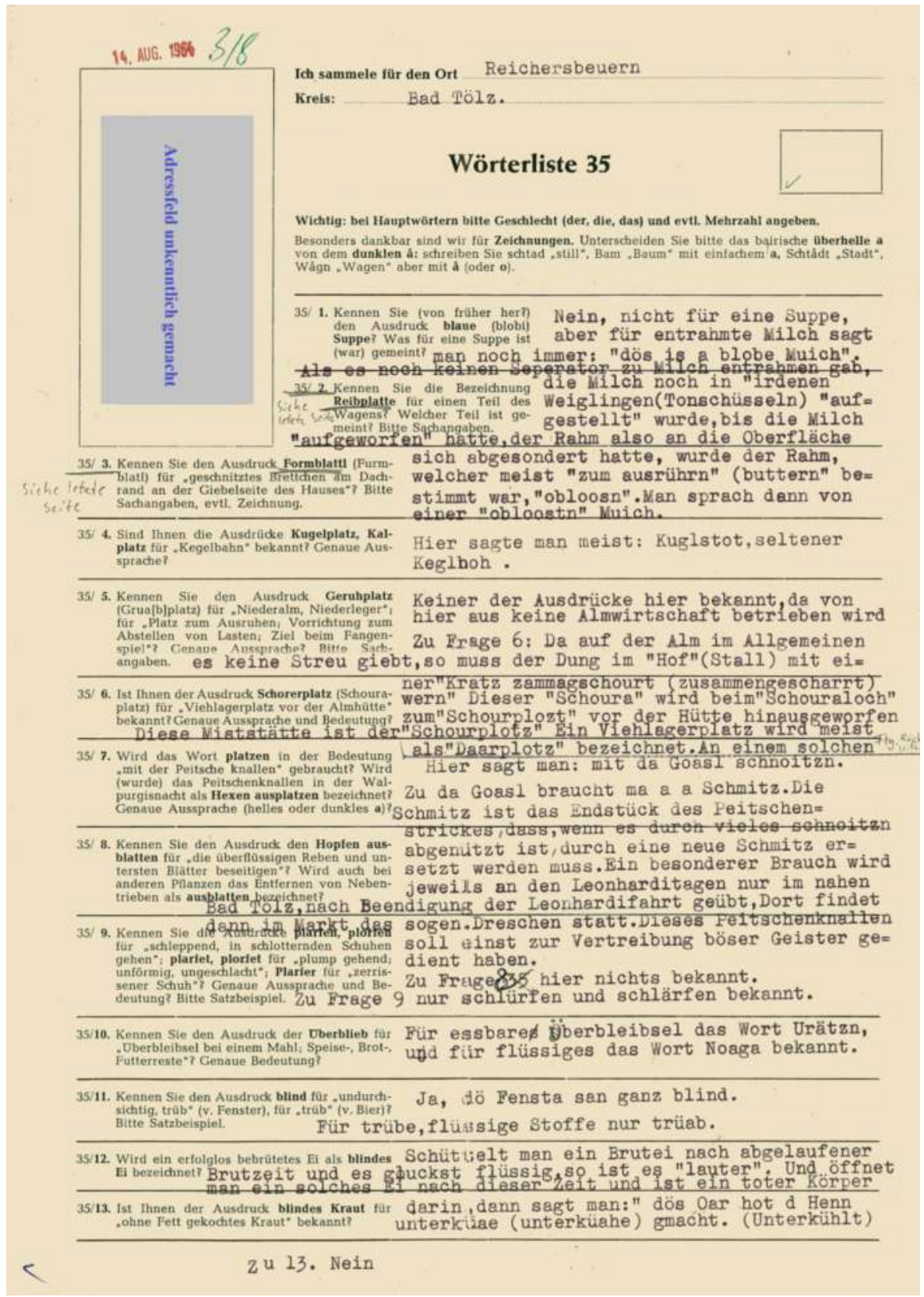

Abb. 8: Wörterliste 35 aus Reichersbeuern, Altlandkreis Bad Tölz; eine für die Wörterlisten typische Fragestellung ist in Frage 11 (,,Kennen Sie den Ausdruck blind für 'undurchsichtig, trüb'? ... Bitte Satzbeispiel “) gegeben, die die Gewährsperson mit dem Satz dö Fensta san ganz blind beantwortete. 


\subsection{Literarische Quellen}

Eine wichtige Materialgrundlage stellen auch bairische Belege aus literarischen Quellen dar. Dabei bezieht sich ,literarisch“ nicht ausschließlich auf belletristische Literatur wie etwa die im BWB berücksichtigten Werke von LENA CHRIST, LUDWIG THOMA und OSKAR MARIA GRAF, sondern allgemein auf Spracherzeugnisse in geschriebener Form, d. h. hierunter fallen auch Grammatiken und Wortsammlungen zum Bairischen sowie sach- und volkskundliche Literatur.

Nach dem Konzept seiner Gründer hatte das BWB auch die Aufgabe, den Wortschatz von der althochdeutschen Zeit bis in die Gegenwart zu erfassen. Althochdeutsches aus Bayern, das in späterer Zeit nicht mehr bezeugt ist, bleibt allerdings unberücksichtigt. Es fällt ohnehin in den Zuständigkeitsbereich des Althochdeutschen Wörterbuchs. Wegen der bekannten Anpassung an überregionale Normen finden Belege aus bairischen Werken der mittelhochdeutschen Klassik auch nur dann Berücksichtigung, wenn sich das betreffende Wort in jüngerer Zeit noch belegen lässt. Häufig zitiert werden die Werke von KONRAD VON MEGENBERG und AVENTIN, die für das Bairische der frühneuhochdeutschen Sprachperiode eine ergiebige Belegquelle darstellen. Bei mittelalterlichen und spätmittelalterlichen Quellen werden vorzugsweise lokalisierbare und datierbare Texte berücksichtigt, in geringem Umfang auch unedierte archivalische Quellen, z. B. aus Rosenheim, Landshut und Regensburg. Die neuhochdeutsche Schriftsprache seit 1800 in ihrer bairischen Ausprägung findet nur am Rande Berücksichtigung, da um 1800 die endgültige Übernahme der neuhochdeutschen Schriftsprache ostmitteldeutscher Prägung in Bayern erfolgt ist. Mundartdichtung wird vor allem dann zitiert, wenn sie als authentische Quelle für einen Ortsdialekt gelten darf.

Das Material aus literarischen Quellen umfasst ca. 800000 Belege.

\section{WÖRTERBUCHARTIKEL: KONZEPTION, STRUKTUR, INHALT}

Die Grundfunktion des BWB besteht darin, den Wortschatz des Bairischen beleggestützt zu dokumentieren. Es ist ein Nachschlagewerk, das Auskunft gibt über die - oft vom Standarddeutschen abweichenden - grammatikalischen und semantischen Eigenschaften der Lemmata. Im Vordergrund steht die differenzierte Beschreibung der Wortbedeutungen und deren Illustration anhand konkreter Sprachbelege. In besonderen Fällen erfolgen auch Angaben zur speziellen Lautung der Dialektwörter. Über die lexikographische Erfassung des Wortschatzes hinaus bietet es aber auch eine materialreiche Grundlage für Studien mit anderem linguistischen Schwerpunkt. So ermöglichen z. B. Satzbelege Untersuchungen zu spezifisch bairischen syntaktischen Erscheinungen, durch die Berücksichtigung historischer Belege finden Sprachhistoriker/-innen reichhaltiges Material für die diachrone Analyse des bairischen Wortschatzes und die in den Wortartikeln dargestellten festen Fügungen, Redensarten und Sprichwörter bilden ein umfangreiches Corpus für die Phraseologieforschung. Da in den Artikeln häufig auch aufgezeigt wird, welche Rolle das betreffende Lemma im bäuerlichen Alltagsleben und Brauchtum spielt, 
ist das BWB nicht nur für Linguist/-innen, sondern auch für Volkskundler/-innen eine wertvolle Belegquelle.

Die Prinzipien des Aufbaus der Wörterbuchartikel sind auf S. XXXVI-XLIV des ersten Bandes des „Bayerischen Wörterbuchs“ detailliert dargelegt. Im Rahmen dieses Aufsatzes beschränken wir uns auf wesentliche Hinweise.

\subsection{Reihenfolge der Lemmata}

Der Wortschatz wird in alphabetischer Reihenfolge präsentiert. Dabei ist Folgendes zu beachten: Wortinitiales $B$ und $P, D$ und $T$ sowie $F$ und $V$ werden jeweils wie ein einziger Buchstabe behandelt, da diese Buchstabenpaare in den meisten Dialekten Bayerns im Anlaut gleich ausgesprochen werden und auch historisch nicht immer zu trennen sind. So steht z. B. das Stichwort plombieren vor blond und Defler 'Vielredner, Schwätzer' vor Tegel 'Lehm, Ton' und Tegel 'Tiegel'.

$\mathrm{Zu}$ beachten ist auch: Komposita sowie Bildungen mit Partikeln und Präfixen werden im Anschluss an das jeweilige Grundwort behandelt. Haustür erscheint also nicht in der Buchstabenstrecke $H$, sondern nach Tür. Entsprechend steht abblatten nach blatten und verstehen nach stehen. Nach diesem Einordnungsprinzip wird zur leichteren Auffindbarkeit auch gegen die Wortgeschichte verfahren: maulaffen 'gaffen', eigentlich Ableitung zum Kompositum Maulaffe, erscheint unter den Komposita des Verbs affen. Bei Maulaffe findet sich aber ein Verweis auf die Ableitung. Ebenfalls stehen Scheinkomposita wie Armbrust, Hebamme unter [Arm]brust, [Heb]amme (mit Verweisen unter Armbrust, Hebamme). ${ }^{4}$ Wie Komposita werden auch substantivische Zusammenbildungen aus Verbalformen behandelt, z. B. Kehraus, das unter den Komposita von -aus ([Kehr]aus) steht; Gleiches gilt für Adverbien mit -halb, -mäßig, -weise u. a. ([außer]halb, [gerichts]mäßig usw.). Synchron simplizische Wörter wie gesund, genug werden wie Präfixbildungen ([ge]sund, [ge]nug) behandelt und unter den Konstruktlemmata -sund und -nug gelistet.

Mehrfachkomposita werden in der Weise unter die Komposita eingereiht, dass bei der Einordnung das letzte Glied, dann das vorletzte usw. maßgebend ist, d. h. [Brat-erd] apfel erscheint nach [Erd] apfel, [Ge-leget]brett 'Querbrett einer Holzbrücke' nach gleichbedeutendem [Leg]brett.

Wortgruppenlexeme, die aus einem Objekt und einem Infinitiv gebildet sind, gelten nicht als Komposita, sondern als freie Verbindungen zweier Lexeme. So wird das Spiel Arschboßen 'Schinkenklopfen' als Fügung (den) Arsch boßen unter dem Verb boßen 'klopfend schlagen' behandelt.

Mit -her, -hin gebildete Richtungsadverbien (aufher, aufhin usw.) werden als Suffixableitungen betrachtet (und nicht als Komposita von -her, -hin). Auf diese Weise können sie in der alphabetischen Listung in die Nähe der Konkurrenzbildungen [her]auf, [hin] auf gerückt werden.

4 Demgegenüber werden verdunkelte Komposita (z. B. Adler, Grummet) wie Simplizia normalalphabetisch eingereiht (mit Verweis unter Aar und [Grün]mahd). 


\subsection{Lemmatisierung}

Es werden drei verschiedene Arten von Lemmata unterschieden: Haupt-, Nebenund Verweislemmata. Das Hauptlemma leitet einen Wortartikel ein. Nebenlemmata, die hinzutreten können, nennen wichtige Varianten des Hauptlemmas. Verweislemmata führen an die Stelle des Alphabets, wo das gesuchte Wort behandelt wird.

Ausgangspunkt für die Ansetzung des Hauptlemmas ist die standardsprachliche Schreibweise, d.h. sie erfolgt gemäß der im „Duden-Wörterbuch“ oder „Grimm’schen Wörterbuch“ (DWB bzw. ${ }^{2} \mathrm{DWB}$ ) angegebenen Form. Ist das betreffende Wort in diesen Wörterbüchern nicht verzeichnet, orientieren wir uns an ScHMELlER, Schwäb.Wb. und WBÖ. Fehlt das Wort auch dort, richtet sich die Lemmatisierung nach der mundartlichen Lautung, z. B. znifig 'schwächlich, zurückgeblieben'.

Dem Hauptlemma folgen gegebenenfalls Nebenlemmata, die stellvertretend für jene vom Standardsprachlichen abweichenden etymologischen Formen eingesetzt werden, aus denen sich die Mundartentsprechungen eines Worts entwickelt haben. Als solche finden die Benutzer/-innen sowohl die von der Schriftsprache abweichenden ostoberdeutschen Grundformen wie bachen für 'backen', Bruck für 'Brücke', als auch Formen, die - regional bedingt - aus unterschiedlichen etymologischen Grundformen herzuleiten sind, wie äfer neben aper 'schneefrei' oder Bein neben Biene.

Die Verweislemmata weisen die Benutzer/-innen auf das Stichwort, unter dem das gesuchte Wort behandelt wird. Als Verweislemmata werden z. B. die Nebenlemmata und volkstümlichen Verschriftungen, die eine gewisse Verbreitung erlangt haben, aufgenommen (Irxen 'Achselhöhle' mit Verweis auf Üchse).

\subsection{Artikelstruktur}

Der Wörterbuchartikel enthält maximal folgende Positionen:

- Artikelkopf mit Hauptlemma und knappen grammatikalischen Angaben ${ }^{5}$

- Bedeutungsteil

- etymologische und wortgeschichtliche Hinweise ${ }^{6}$

- Angaben zu Lautung, Formen und Verwendung im Satz (nur in interessanten Fällen)

5 Am Beginn des Artikels steht, durch Fettdruck hervorgehoben, das Hauptlemma mit eventuellen Nebenlemmata. Im Anschluss erfolgt die Angabe der Wortart, bei Substantiven nur des Genus.

6 Eine etymologische Angabe erfolgt obligatorisch bei Simplizia, wobei, falls vorhanden, die althochdeutschen und mittelhochdeutschen Formen angeführt werden. Bei Ableitungen erfolgen etymologische Angaben nur, wenn mittelhochdeutsche (und althochdeutsche) Formen gegeben sind, bei Komposita nur, wenn das entsprechende Grundwort alleine nicht belegt ist, so z. B. bei -dacht ${ }^{1}$, das nur in Formen wie [An]dacht, [Ver]dacht vorkommt: „Ahd. -dâht, mhd. dâht stf. 'Denken', Abl. zur Wz. von $\rightarrow$ denken" oder bei -täckern, das nur in Verbindung mit $a b-, a n$ - und be- belegt ist: „Gleicher Herkunft wie $\rightarrow$ täckeln ${ }^{2 “}$. 
- Hinweise auf Literatur und wörterbucheigene Sammelaktionen ${ }^{7}$

- Verweise auf Ableitungen

- Komposita und Präfixbildungen mit dem Lemma als Grundwort

- Name der Redaktorin oder des Redaktors

Im Bedeutungsteil werden die Bedeutungen eines Wortes, gegebenenfalls mit Untergliederungen, genau aufgeschlüsselt. Die einzelnen Gliederungspunkte weisen maximal folgende Struktur auf:

- Bedeutungsangabe ${ }^{8}$

- Zuordnung zu bestimmten Sprachbereichen (Umgangssprache, Fachsprache, ältere Sprachstufen usw.)

- geographische Verbreitung

- Belegteil, in dem auch Redensarten, Sprichwörter usw. aufgenommen werden

- sach- und volkskundliche Angaben

Den Angaben zur geographischen Verbreitung liegt das Material der verschiedenen Sammelperioden zugrunde. Sie richtet sich nach der Gliederung der Regierungsbezirke und Landkreise, wie sie vor der Gebietsreform im Jahr 1972 gegeben war (s. Abbildung 1). Literarische Belege werden dabei nicht berücksichtigt. Angaben über Schwaben und die fränkischen Regierungsbezirke beziehen sich nur auf die Teile, in denen bairische Dialekte gesprochen werden. Durch die Angabe „Gesamtgebiet" (im Artikel: Gesamtgeb.) wird die im Material nachgewiesene Verbreitung in allen Regionen angezeigt; wenn keine Sammlerbelege vorliegen, eine allgemeine Verbreitung aber dennoch angenommen werden muss, steht ,allgemein verbreitet" (allg.verbr.). Die Angaben „vielfach“ (vielf.), „mehrfach“ (mehrf.), „vereinzelt“ (vereinz.) sind als knappe Zusammenfassungen der Beleglage aufzufassen, sie sind nicht Angaben zur Worthäufigkeit. „Vielfach“ bedeutet, dass Sammlermeldungen aus allen oder vielen Teilen der Bezugsregion vorliegen; „mehrfach“, dass Sammlermeldungen zumindest in lockerer Dichte über die Bezugsregion gestreut sind; „vereinzelt", dass nur wenige Belege vorliegen. Einzelbelege werden nur unter Angabe des Belegorts angeführt (vgl. Abbildung 9, Stichwort achten, Bedeutung 3).

7 Dies umfasste ursprünglich Verweise auf die Behandlung des Hauptlemmas (1) in den historischen Dialektwörterbüchern des Bairischen (DELling, HÄSSLEIN, PRASCH, SCHMELLER, WESTENRIEDER, ZAUPSER), (2) in den Wörterbüchern der benachbarten oberdeutschen Mundartlandschaften (vor allem WBÖ, Schwäb.Wb., Schw.Id., Suddt.Wb.), (3) in den Wörterbüchern zu historischen Sprachstufen des Deutschen, (4) in neueren Orts- und Landschaftswörterbüchern, (5) Fragenummern, unter denen in Befragungsaktionen explizit nach dem Lemma gefragt wurde. Dabei verweist vorgesetzes S- auf die „Systematischen Fragebögen“ (1912-1933), M- auf die „Mundartgeographischen Fragebögen“ (1928-1940), W- auf die „Wörterlisten“ (von 1958 an) und FM- auf den Fragebogen MAURER (1934). Entsprechend einer Anregung der Evaluation des BWB im Jahr 2015 werden ab Heft 21 nur noch die unter (1) genannten Wörterbücher sowie das WBÖ angegeben.

8 Bei Einzelbelegen erfolgt die Bedeutungsangabe z. T. nicht an erster Stelle, sondern erst im Belegteil als Zitat des Sammlers (vgl. Abbildung 9, Stichwort Achtement) bzw. des Autors (vgl. Abbildung 9, Stichwort [aus]achteln). 
Wenn eine Verbreitungsangabe fehlt und nur literarische Belege zitiert werden, liegen keine Sammlerbelege vor (vgl. Abbildung 9, Stichwörter achteln und [aus]achteln].

Im Belegteil wird das Material des Wörterbucharchivs in Auswahl zum Nachweis und zur Veranschaulichung der angesetzten Bedeutungen dargeboten. Belege erscheinen stets in Kursivdruck. Sie sind folgendermaßen angeordnet: Zuerst stehen Sammlerbelege mit Ortsangabe. Belegorten, die keine Kreisstädte sind, werden zur leichteren Lokalisierung die üblichen Siglen der Altlandkreise beigefügt. ${ }^{9}$ Die Sammlerbelege werden in der Reihenfolge Oberbayern (OB), Niederbayern (NB), Oberpfalz (OP), Oberfranken (OF), Mittelfranken (MF), Schwaben (SCH) zitiert. Vor Sammlerbelegen nach 1958 steht das Zeichen ${ }^{\circ}$ (vgl. Abbildung 9, Stichwort Achtel, Bedeutung 1). Erinnerungsform (Ef.) bedeutet, dass die Gewährsperson das betreffende Wort nur noch aus der Erinnerung an die Sprache älterer Generationen kennt. ${ }^{10}$ Redaktionelle Verständnishilfen und Ergänzungen werden durch Einklammerung mit [...] gekennzeichnet (vgl. Abbildung 9, Stichwort Achtel, Bedeutung 1 und 2), Angaben des Sammlers durch doppelte Anführungszeichen (vgl. ebd., den Beleg aus Taching im Stichwort -ächteln und den Beleg im Stichwort Achtement). Bedeutungsangaben, die sich aus der Fragestellung ergeben, die dem Sammler vorlag, erscheinen ohne weitere Kennzeichnung, so z. B. bei „der Nobad Zeit der Dämmerung" im Stichwort Abend (die Bedeutungsangabe entspricht hier Frage 18 des 103. „Mundartgeographischen Fragebogens“).

Die Wiedergabe der Sammlerbelege erfolgt möglichst buchstabengetreu, jedoch werden Interpunktion, Großschreibung sowie Zusammen- bzw. Getrenntschreibung normalisiert. Ein weitergehender Eingriff in die Orthographie der Sammlerbelege ist undurchführbar, da jeder Schreibung dem Bearbeiter unbekannte lautliche Gegebenheiten zugrunde liegen können. Zudem würde das Umschreiben in eine phonetisch präzisere Schreibweise einen unvertretbar hohen Arbeitsaufwand bedeuten.

9 Z. B. ,a Achtl Milli Waging LF [= Landkreis Laufen]“ (s. Abbildung 9, Stichwort Achtel, Bedeutung 2) im Gegensatz zu ,hoscht des ned gåchd, wia dea Schteanschnubbn owa is? Dachau [= Kreisstadt des Landkreises Dachau]" (s. Abbildung 9, Stichwort achten, Bedeutung 2).

10 So z. B. bei [Kummet]tasche, Bedeutung 4: „Stirnjoch: ${ }^{\circ}$ Kummertaschn (Ef.) Dietfurt RID“. 


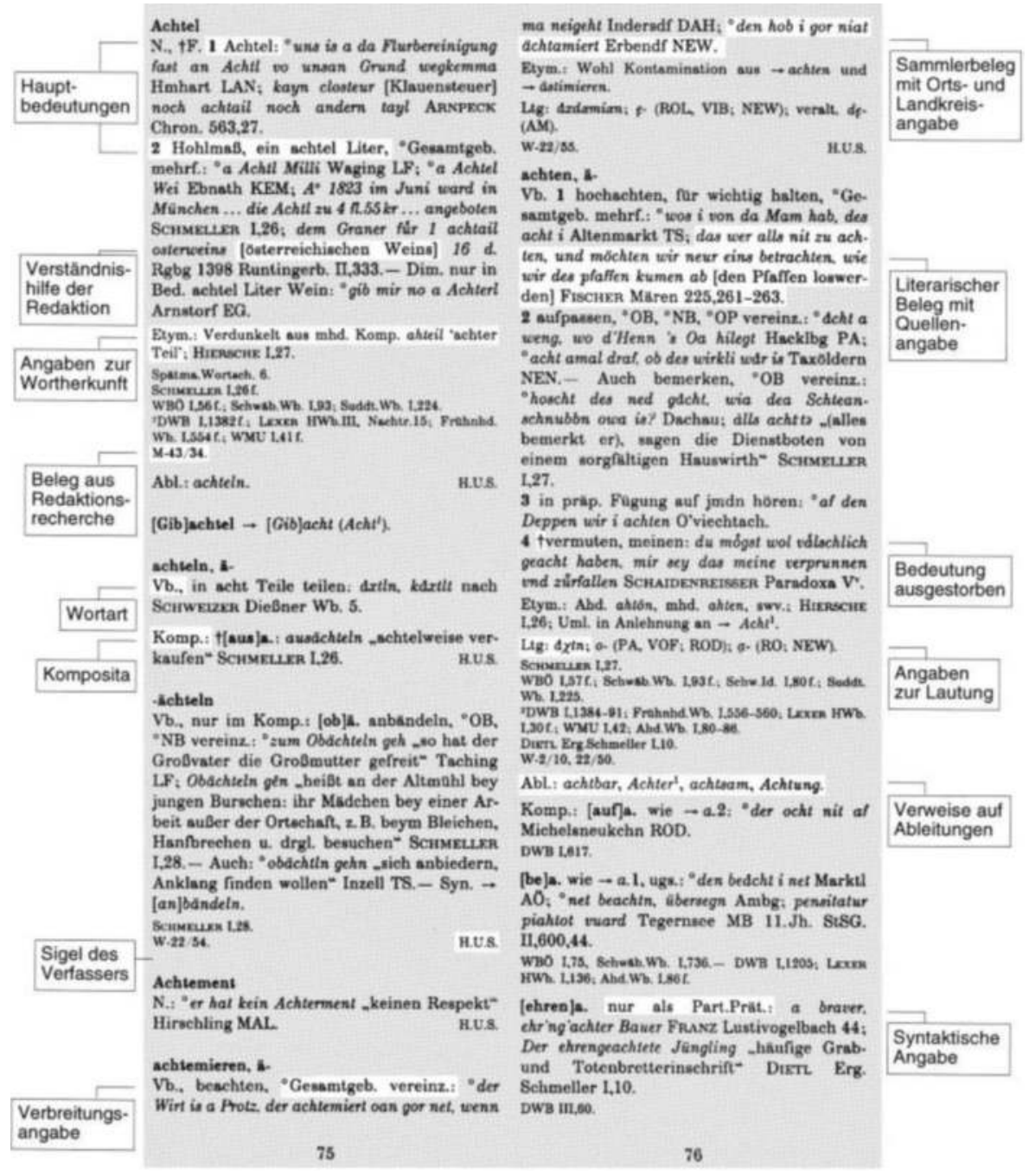

Abb. 9: Wortartikel (aus BWB, Band 1: XL)

Den Sammlerbelegen folgen Belege aus literarischen Quellen seit 1800, dann in chronologischer Reihenfolge historische Belege vom Anfang der Überlieferung bis 1799. Nach den literarischen Quellen werden, soweit vorhanden,

- feste Fügungen $^{11}$

- Vergleiche ${ }^{12}$

11 Z. B. Stichwort Preis: „In festen Fügungen um (einen) jeden / keinen P. u. ä. auf jeden / keinen Fall, ${ }^{\circ} \mathrm{OB},{ }^{\circ} \mathrm{NB},{ }^{\circ} \mathrm{OP}, \mathrm{SCH}$ vereinz.: um koan Preis tua i dös wieda Ascholding WOR.“

12 Z. B. Stichwort [Kraut(s)]brente 'Krautfaß': „Im Vergleich wie eine $K$. sehr dick u. groß, OB vereinz.: dear håt an Schedl wia a Krautbrente Hofhegnenberg FFB.“ 
- Redensarten $^{13}$

- Sprichwörter $^{14}$

- Rätsel ${ }^{15}$

- Wetterregeln ${ }^{16}$ u. a.

angeführt, wenn das Lemma darin sinntragendes Element ist. ${ }^{17}$

\section{TEXTTECHNOLOGISCHE ASPEKTE}

Dieser Abschnitt behandelt die Digitalisierungsarbeiten des BWB, die seit Februar 2015 mithilfe der IT-Abteilung der Bayerischen Akademie der Wissenschaften durchgeführt werden. Die folgenden Seiten dokumentieren diese ausführlich, stellen deren wissenschaftlichen Mehrwert detailliert dar, liefern Informationen zur Realisierung sowie zur verwendeten Infrastruktur und spiegeln die bisherige Nutzung - auch seitens der interessierten Öffentlichkeit - wider.

Teile dieses Abschnitts, die die allgemeinen Eigenschaften des „LexHelfers“ behandeln, finden Sie im Artikel zum „Fränkischen Wörterbuch“. An entsprechenden Stellen wird gesondert darauf verwiesen.

\subsection{Datenbasis und Redaktionssystem}

\subsubsection{Datenbasis}

Die Datenbasis bilden über 450000 Seiten „Wörterlisten“ (s. Kapitel 5.1.5) und über 17000 Seiten „Maurerbögen“( $($ s. Kapitel 5.1.4), die 2015 durch einen externen Dienstleister digitalisiert wurden. ${ }^{18}$ Durch die semantische Dateinamensvergabe nach dem Muster „Typ-Nummer-Ort-Landkreis-Gewährsperson-Seitennummer“

13 Z. B. Stichwort Brett: „Ra.: keine dicken B.er bohren / schneiden u. ä. sich nicht anstrengen, nicht viel leisten: er hat koi dicke Breda boahrt Altglashütte TIR.“

14 Z. B. Stichwort Praller: „Sprichw.: die größten Prahler tun die kleinsten Knaller Eigenlob stinkt Wildenranna WEG.“

15 Z. B. Sichwort brennen: „Rätsel: Was brennt um's Haus rum und verbrennt doch net? [Brennessel] STADLBAUER Heilpflanzen Opf. 72.“

16 Z. B. Stichwort [Ge]burt: „Wetterregel: Mårie Gebuät fläign d Schwålbm fuät Bruck ROD.“

$17 \mathrm{Ab}$ Heft 21 werden feste Fügungen, Vergleiche, Redensarten und Sprichwörter nicht mehr als eigene Rubriken unterschieden, sondern unter einer übergreifenden Rubrik „Phraseologie“ (Phras.) zusammengefasst.

18 Die vierseitigen Bögen der Wörterlisten wurden zuerst an der BAdW durch Hilfskräfte des BWB vorbereitet, damit alle in gleicher Ausrichtung vorlagen und von Objekten (z. B. beigegebenen Nägeln, getrockneten Pflanzen, festgehefteten Fotos) befreit waren. Sie wurden anschließend aufgeklappt eingescannt, sodass die Seiten 1 und 4 sowie die Seiten 2 und 3 in jeweils einer Datei landeten, welche anschließend automatisch in je zwei Dateien geteilt wurde, die die entsprechend korrekte Seitenzahl im Dateinamen enthielten. Die sechsseitigen Maurerbögen wurden aufgeklappt mit den Seiten 1, 5 und 6 in einem Scan sowie 2, 3 und 4 im anderen Scan digitalisiert. Anschließend erfolgte ebenso eine automatische Trennung in Einzeldateien. 
kann jedes Exemplar zielgenau aufgerufen werden. Der Typ gibt an, ob es sich um eine Wörterliste, einen Maurerbogen oder eine womöglich künftig weitere Quelle handelt. Die Datenmenge der Wörterlisten allein beläuft sich auf 1,1 Terrabyte im JPEG-Format. Weitere Daten, z. B. Lemmazuordnungen oder Belegabschriften, waren nicht vorhanden. Eine automatische Transkription der Antworten via Texterkennungsmethoden war und ist nicht möglich, da es zu viele unterschiedliche Handschriften gibt, als dass eine Software in akzeptabler Zeit darauf trainiert werden könnte, ausreichend fehlerfreien, maschinenlesbaren Text zu generieren.

Die Metadaten der Digitalisate und die Dateipfade zu ihnen sind ein wichtiger Bestandteil der Datenbank des Redaktionssystems, da die Lemmatisierung auf diesen Daten aufsetzt.

Zusätzlich zu den beiden genannten Quelltypen werden derzeit Zettelkästen digitalisiert, die ebenfalls wichtiges Belegmaterial enthalten und künftig auch über das Redaktionssystem abrufbar sein sollen. Seit April 2017 können zudem neu erfasste Fragebögen online ausgefüllt werden (s. Kapitel 7.1.3).

\subsubsection{Redaktionssystem}

Nach dem positiv verlaufenen ersten Test des Redaktionssystems „LexHelfer“ des „Fränkischen Wörterbuchs“ (WBF) und dessen Präsentation beim BWB im zweiten Quartal 2015 wurde mit der Entwicklung einer gesonderten Variante der Software für das BWB begonnen, um die projektspezifischen Anforderungen der dortigen Redaktion beim Exzerpieren digital abbilden zu können. ${ }^{19}$

In stetigem Austausch mit der Redaktion wurde die Programmierung bis Anfang 2016 durchgeführt; nach einer Testphase und anschließenden Fehlerkorrekturen begann die produktive Arbeit mit LexHelfer dann im April 2016. Seither werden Belege mit dessen Hilfe und somit ausschließlich digital lemmatisiert. Seit Januar 2017 liefert das System zudem die öffentliche Einsicht in die Forschungsprimärdaten des Wörterbuchprojekts. ${ }^{20}$

Für das BWB bestand die Anforderung anfänglich darin, Einzelbelege lemmatisieren und diese Daten zielgenau suchen sowie exportieren zu können. Hierzu wurde als besonders wichtig erachtet, Belege ausfiltern zu können, wenn diese z. B. keine (relevanten) Antworten enthielten, damit die Ergebnisliste entsprechend kleiner ausfällt und Nullbelege das virtuelle Durchblättern nicht unnötig verlangsamen. Durch Eingabe der Wörterliste und der Fragennummer kann die Redaktion innerhalb weniger Sekunden alle zugehörigen Belege abrufen. Die Einschränkung auf Orte oder Regierungsbezirke erleichtert zudem die Suche nach Daten einzelner Regionen. Die Belege werden als Bildausschnitte angezeigt und enthalten nur die gewünschten Bereiche (s. Kapitel 7.2.1.3).

20 S. <http://www.bwb.badw.de/bwb-digital/datenbank>; Stand: 07.01.2019. 
Die anfänglichen Funktionen von LexHelfer für das BWB wurden seit April 2016 weiterentwickelt, um die Arbeitsabläufe weiter zu optimieren. Sie umfassen zum Januar 2019:

- anonyme Nutzungsstatistiken

- Datenstatistiken

- Markierung eines Belegs als relevant/irrelevant, erledigt/nicht erledigt

- Markierung eines Belegs als „,im Wörterbuch enthalten“

- Suche nach Wörterliste, Fragenummer, Ort, Landkreis, Regierungsbezirk, Lemma

- Suche nach Schlagwort in Fragestellung

- Suche nach Belegen mit bzw. ohne Lemmazuweisung

- Suche nach Belegen gemäß ihrer Markierung

- Lemmatisierung

- Hinzufügen von Notizen pro Beleg

- Neuzeichnung eines Beleg-Schnipsels

- Export der Schnipsel eines Lemmas bzw. einer Liste in druckbare PDF

Des Weiteren liefert das Tool ohne Mehraufwand für die Wörterbuchredaktion eine im Funktionsumfang reduzierte und lediglich auf die Datenausgabe beschränkte Version, sodass die interessierte Öffentlichkeit wie auch projekt-externe Fachkreise den gesamten Bestand ohne Login einsehen können.

Im Folgenden werden die besonderen Merkmale der Anwendung dargestellt; die allgemeinen und in vielen Online-Wörterbüchern eher als Standard betrachteten Funktionen (z. B. Suchoptionen mit Platzhaltern) werden nicht gesondert beschrieben, können jedoch online unter 〈http://www.bwb.badw.de/bwb-digital.html> (Stand: 07.01.2019) eingesehen werden.

\subsubsection{Online-Erfassung neuer Fragebögen}

Die Wörterlisten des BWB sind seit dem Start der neuen Webseite zusätzlich online über eine Eingabemaske ausfüllbar. Begonnen wurde mit der Wörterliste 252. Teilnehmerinnen und Teilnehmer, die die Listen mit der neuen Technik ausfüllen möchten, melden sich dazu per E-Mail oder telefonisch im Sekretariat des BWB und erhalten den persönlichen Zugang anschließend in Form eines Links, eines Benutzernamens und eines Passwortes zugeschickt. Über den Link gelangen die Bearbeiterinnen und Bearbeiter zur Anmeldung und anschließend zu einem Überblick über die noch zu bearbeitenden sowie abgeschlossenen Wörterlisten. Entsprechend bezeichnete Knöpfe und Formularfelder ermöglichen es, die Fragen der Wörterlisten zu beantworten, zur späteren Weiterbearbeitung zu speichern und abzuschlieBen.

Das Werkzeug zur Wörterlistenerfassung wurde direkt in die Webseite des BWB eingebaut und nutzt dazu als Grundlage, wie auch die Webseite selbst, das PHP-Content-Management-System TYPO3. Das Erfassungstool wurde dabei als Erweiterung (fachlich: extension) innerhalb der Extbase-Konventionen in TYPO3 
mit PHP realisiert. Gehostet wird das ganze System im Webhosting des an der Bayerischen Akademie der Wissenschaften ansässigen Leibniz-Rechenzentrums als klassischer LAMP-Stack (Linux, Apache, MySQL, PHP).

Der Frontendbereich (Ansicht für die Teilnehmerinnen und Teilnehmer; s. Abbildung 10) besteht aus einer Listenansicht für den Überblick, jeweils einer Detailansicht zum Beantworten der Fragen sowie einer Druckansicht:

a) Direkt nach der Anmeldung gelangt man in die Listenansicht, welche dem Überblick über die abgeschlossenen und noch offenen Wörterlisten dient. Mit entsprechenden Knöpfen können die Benutzerinnen und Benutzer hier neue Listen beginnen, an gespeicherten Listen weiterarbeiten oder abgeschlossene Listen in der Druckansicht anzeigen.

b) Die Detailansicht einer Wörterliste besteht aus 60 Fragen sowie den entsprechenden Formularfeldern zum Beantworten der Fragen und zum Hochladen von Dateien. Der Eingabebereich wurde durch den quelloffenen JavaScriptEditor „TinyMCE“ mit der Möglichkeit zur bequemen Eingabe von dialektalen Lautungen, die mit den gängigen Zeichen des Alphabets nur unzureichend wiedergegeben werden können (z. B. å), erweitert.

c) Die Druckansicht ist eine reine HTML-Ansicht einer Wörterliste (ohne Webseitenlayout) zum Ausdrucken.

Der „Backendbereich“ besteht aus einer Datenbank zur Speicherung der Antworten und einem Dateisystem für die Ablage von hochgeladenen Dateien, wie z. B. Bildern oder gescannten Zeichnungen. Zur Persistierung der Daten dient eine MySQLDatenbank. ${ }^{21}$ In ihr werden Benutzerdaten und Wörterlisten in Relation zueinander gespeichert. Von den Benutzerinnen und Benutzern hochgeladene Dateien werden auf dem Dateisystem des Webservers ebenfalls in Relation zu den Wörterlisten bzw. Benutzerinnen und Benutzern gespeichert. Die persistierten Daten werden später in den LexHelfer integriert und über diesen in gleicher Weise zugänglich gemacht und lemmatisierbar, wie es aktuell mit den gescannten Fragebögen der Fall ist.

Um den Redaktionsmitgliedern des BWB einen Überblick über die aktuell von den Sammlern abgeschlossenen Wörterlisten zu geben, wurde ein zeitgesteuertes Script implementiert, welches die Daten aus der Datenbank ausliest, eine ExcelListe erstellt und automatisch an das Projekt versendet. So erhält die Redaktion in regelmäßigen Intervallen Informationen dazu, welche Personen welche Listen bereits abgeschlossen haben, sowie die Änderungen bzw. Listenabschlüsse des letzten Monats.

Die Speicherung und Verarbeitung personenbezogener Daten wird gemäß den Datenschutzbestimmungen und dadurch mit expliziter Zustimmung der Gewährspersonen vorgenommen.

21 Unter Persistierung versteht man in der Informatik die Speicherung von Daten auch über das Programmende hinaus, damit sie später wieder aufgerufen werden können. Sie sagt nichts über die Art der Daten oder die Art des Speichermediums aus, legt für Letzteres jedoch klar fest, dass es Daten ohne dauerhafte Stromquelle halten muss. 


\section{Erfassung der aktuellen Wörterlisten}

Abmelden Max.Mustermann

Worterliste speichern

Zuruck zur Listenauswahl

Wörterliste 267

(i) Fragen, die Sie nicht beantworten, bitte leer lassen.

$>\quad$ Frage 1:

Wenn wir zu schauen und Zusammensetzungen mehr Fragen stellen und dabei um Ihre Geduld und besondere Sorgfalt bitten müssen, so liegt das neben dem Mangel an Belegen daran, dass schauen bei uns vielfach hochsprachliches „sehen" vertritt.

Ist Ihnen der Ausdruck das schaut einem gleich, ähnlich für „das ist für jemandem typisch" bekannt? Bitte Satzbeispiel.

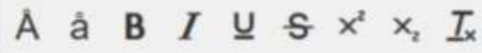

Abb. 10: Ansicht des Frontendbereichs des Online-Erfassungstools neuer Wörterlisten am Beispiel der Wörterliste 267, Frage 1 


\subsection{Mehrwert eines digitalen Workflows}

\subsubsection{Geschwindigkeit}

\subsubsection{Suche}

Eine Datenbank liefert das gewünschte Suchergebnis in aller Regel um ein Vielfaches zügiger als eine manuelle Suche innerhalb der originalen Wörterlisten. Wie schnell sie dabei ist, hängt von verschiedenen Faktoren ab - im Datenbestand des BWB existieren jedoch vergleichsweise wenige Datensätze, die zudem allesamt indiziert sind, sodass ein zufällig gewähltes Belegexemplar vom Computer immer schneller gefunden wird als vom Menschen. ${ }^{22}$ Möchte man z. B. die Antwort der Gewährsperson 5 zu Frage 13 aus Wörterliste 82 in Passau, muss man als Redaktionsmitglied den entsprechenden Karton aus dem Schrank holen, alle Bögen entnehmen und diese nach dem gewünschten Exemplar durchsuchen. Man gelangt relativ schnell zum Ergebnis, falls der Inhalt des Kartons alphabetisch sortiert ist, und benötigt bestenfalls wohl rund eine Minute für den Vorgang. Andernfalls muss man sich durch alle Bögen blättern, nur um letztlich festzustellen, dass die Gewährsperson 5 in Passau keine Antwort zu dieser Frage gegeben hat. Bearbeitet man die Frage nicht nur für einen Einzelbeleg, sondern für alle Gewährspersonen eines Landkreises oder gar Regierungsbezirks, wird die Sucherei unabhängig einer etwaigen Sortierung der Kartoninhalte zur Tages- oder gar Wochenaufgabe. Der Computer hingegen liefert die Ergebnisse in jedem der genannten Szenarien in wenigen Sekunden und kann diese auf Wunsch entsprechend nach z. B. Ort oder Kreis sortieren.

\subsubsection{Lemmatisierung}

Auch die Lemmatisierung erfolgt mithilfe des Redaktionssystems schneller als zuvor, da ein Lemma aus der Liste bereits angelegter Lemmata mit wenigen Klicks ausgewählt und zugeordnet werden kann. Existiert z. B. das Lemma Dach für eine bestimmte Frage eines Bogens, kann es bei anderen Belegen dieser Frage mit nur einem Klick zugeordnet werden. Andernfalls kann es über die systemweite Lemmaliste ausgewählt oder neu angelegt werden. Dies verhindert zum einen das schnelle Ermüden der Hand durch die Verringerung unnötiger Mausbewegungen. Zum anderen vermeidet es Tippfehler, wie sie bei der Arbeit mit Textverarbeitungsprogrammen durch das stetig erneut notwendige Tippen des Wortes entstehen können, und sorgt für handschriftunabhängige, gute Lesbarkeit.

22 Millionen Datensätze, die primär numerische Werte enthalten, sind für moderne Datenbanksysteme keine Herausforderung. Selbst Millionen Wörter sind mithilfe eines Indexes schnell dursuchbar. Daher sind Schnipsel-Koordinaten wie auch Lemmata innerhalb der Datenbank des BWB schnell auffindbar. 
Ebenfalls von Bedeutung für die Redaktion ist die Zusammenstellung aller Belege eines ausgewählten Lemmas. Durch die vorangehend erwähnte Suchgeschwindigkeit ist eine Belegliste innerhalb weniger Sekunden zusammengestellt und nach Wahl online einsehbar oder für den Druck in ein PDF exportierbar.

\subsubsection{Schnipselgenerierung}

Um gezielt einen Einzelbeleg oder eine Liste von allen Belegen einer Frage eines Bogens aufzurufen, bedarf es der genauen Koordinaten der jeweiligen Bildbereiche. Der Aufruf des ganzen Scans würde andernfalls alle darin abgebildeten Belege und damit nicht erwünschte Informationen darstellen sowie zu viel Platz auf dem Bildschirm einnehmen. Bei über 100000 ausgefüllten Bögen mit jeweils 60 Fragen und oftmals zusätzlichen Angaben auf Beiblättern erhält man allein für den Typ „Wörterliste“ über 6000000 Belege. Deren graphische Erfassung würde trotz Scharen emsiger Hilfskräfte Monate der Arbeit und entsprechende finanzielle Mittel bedeuten.

Da jedoch alle Fragen einer Wörterliste die gleichen Koordinaten haben, muss man sie nur einmalig erfassen, um sie anschließend für alle Vorkommnisse in die Datenbank eintragen zu können. Dies wurde mithilfe eines gesonderten Tools zur Schnipselgenerierung von einer Hilfskraft in ca. 16 Stunden Arbeitszeit vorgenommen. Anschließend erfolgte die Eintragung der erfassten Bildausschnitte in allen Einzelfällen vollautomatisch in ca. 24 Stunden durch ein Script.

Später ergab sich durch dieses Tool noch der Mehrwert, personenbezogene und damit datenschutzrechtlich relevante Daten auf gleiche Art unkenntlich zu machen: Die Pixel-Bereiche der Adressdaten wurden pro Wörterliste einmal erfasst und im Anschluss durch ein gesondertes Script ausgegraut. Die Öffentlichkeit erhält diese Dateien zur Ansicht; die Redaktion greift intern weiterhin auf die unbearbeiteten Original-Scandateien zu. Arbeitsschritte, die bei manueller Bearbeitung Monate in Anspruch genommen hätten, wurden somit auch hier mithilfe der technischen Möglichkeiten innerhalb weniger Stunden durchgeführt.

\subsubsection{Normalisierte und nativ digitale Neuerfassungen}

Ein Vorteil des in Kapitel 7.1.3. beschriebenen Erfassungstools besteht darin, dass die von den Gewährspersonen gelieferten Belege in Bezug auf die Verschriftung einheitlicher (,normalisierter") sind als zuvor. Die Vorgabe dialektspezifischer Zeichen oder Diakritika (um beispielsweise die Länge des Vokals anzugeben) verhindert eine Vielzahl verschiedener, jedoch bedeutungsgleicher Angaben, wie sie im Verlauf der Jahrzehnte bei einer nicht geringen Anzahl von Gewährspersonen handschriftlich getätigt wurden. Durch diese Einschränkung der Eingabemöglichkeiten entfällt beim Exzerpieren die Normalisierung, die letztlich im Artikel verwendet wird. Ferner ist es den Gewährspersonen nicht möglich, durch Über-/Unterschreiten der Fragebereiche in die Antwort einer anderen Frage hineinzuschreiben oder 
gar im Querformat den Rand des Bogens zu beschriften. Die Felder sind groß genug, um ausreichend große Texte $\mathrm{zu}$ fassen und erleichtern dadurch der Redaktion hinterher die Auswertung.

Des Weiteren können neue Daten nativ digital erfasst werden. Der Verlust der Belege wird dadurch quasi ausgeschlossen, da sie auf gesicherten Laufwerken gespeichert werden. ${ }^{23}$ Ebenso werden die sonst irgendwann nötigen Digitalisierungskosten vermieden.

\subsection{Technik}

Die allgemeine Beschreibung der Serverumgebung ist identisch mit der des WBF. Bitte lesen Sie diese dort in Kapitel 3.3 nach.

\subsubsection{Probleme}

\subsubsection{Texterkennung in Digitalisaten}

Leider ist es aufgrund der Vielzahl unterschiedlicher Handschriften bisher nicht möglich, Texterkennungstechniken (OCR, ICR) zu verwenden, um die Originalbelege in akkurate computerlesbare Form zu bringen. Geeignete Programme zu trainieren und deren Ausgabe zu korrigieren wäre so aufwändig, dass das Abtippen letztlich schneller ist. Es gab im Verlauf der letzten Jahre zwar Fortschritte in diesem Bereich, jedoch keine, die Kurrentschrift ausreichend fehlerfrei in maschinenlesbaren Text umzuwandeln. Die per Schreibmaschine beantworteten Fragen könnten zwar relativ zuverlässig eingelesen werden, aber hier ergibt sich anschließend das Problem, dass die Exemplare mit Kurrentschrift manuell aus der Ergebnisliste ausgefiltert werden müssten. Auch die nicht seltenen Fälle mit Über-/Unterschreitung der Fragengrenzen (z. B. aufgrund sehr langer Antworten, die mehr Platz als das eigentlich vorgesehene Antwortfeld benötigten; Kommentare, die der Antwort hinzugefügt wurden) müssten ebenfalls manuell begutachtet und korrigiert werden, sodass die gesamte Korrekturphase keinen zeitlichen Vorteil mehr liefern dürfte.

Durch den enormen personellen und zeitlichen Aufwand für das Abtippen bzw. für nötige Korrekturen nach einer automatischen Texterkennung ist es daher bis auf Weiteres leider nicht möglich, Belegtexte ebenfalls in der Datenbank abzulegen.

23 Ein Verlust kann niemals zu $100 \%$ ausgeschlossen werden, jedoch müsste dafür das LeibnizRechenzentrum oder zumindest die dortige Technik komplett zerstört werden. Dies ist aufgrund der dortigen, vielschichtigen Sicherheits- und Sicherungsmaßnahmen äußerst unwahrscheinlich. 


\subsubsection{Inkonsistente bzw. fehlerhafte Ortsnamen}

Wie bereits in Kapitel 7.1.1 erwähnt, fand die Digitalisierung der Wörterlisten durch einen Dienstleister statt. Die Originalbögen wurden in München gescannt und die Dateien anschließend in Vietnam mit Dateinamen versehen. Hierfür gab es als Hilfsmittel Listen mit Orts- und Landkreisnamen, sodass im Zweifelsfall nachgeschaut werden konnte, wie etwas zu schreiben ist bzw. was überhaupt existiert. Bei Unklarheiten sollten $X$-e als Platzhalter verwendet werden. Insgesamt funktionierte das Vorgehen sehr gut. Die Unklarheiten wurden vor dem Einspeisen der Scans in die Datenbank korrigiert, ebenso wie wenige augenscheinliche Tippfehler.

Es gab jedoch bei manchen Orten Fehler, die erst spät auffielen, und bei anderen den ebenfalls später aufgetretenen Wunsch der Umbenennung. So wurde z. B. entschieden, dass alle Orte, die ein Gewässer im Namen tragen, dieses nicht mehr im Dateinamen enthalten sollen. Aus „Mühldorf am Inn“ wurde somit „Mühldorf“. Dadurch wurde u. a. das Problem der Inkonsistenz beseitigt, dass verschiedene Schreibungen für ein und denselben Ort im Datenbestand existieren: „Mühldorf“, „Mühldorf a. I.“, „Mühldorf am Inn“, „Mühldorf a. Inn“, oder Schreibungen mit fehlendem Leerzeichen wurden alle normalisiert zu „Mühldorf“. Diese Bezeichnung ist ausreichend, da es im (hier: gleichnamigen) Landkreis keinen weiteren Ort mit diesem Namen gibt.

Aufgrund der bereits bestandenen Durchmischung verschiedener Schreibungen musste sehr genau geprüft werden, ob es den neuen Dateinamen bereits gibt. Ferner war es unabdingbar, die Datenbankeinträge ebenfalls dahingehend zu prüfen und abzuändern, damit nach Änderung der Dateinamen nicht Datensätze auf die falschen bzw. nicht mehr existente Dateien verweisen. Die Kontrollen nahmen jeweils viel Zeit in Anspruch, da Dateisystem und Datenbank jeweils gegengeprüft werden mussten, was bei der Daten-/Dateimenge langwierig war.

\subsubsection{Generizität}

Der Inhalt dieses Kapitels ist mit Kapitel 3.3.1.2 des Artikels des „Fränkischen Wörterbuchs“ identisch.

\subsection{Nachhaltigkeit}

Der Inhalt dieses Kapitel ist mit Kapitel 3.4 des Artikels des „Fränkischen Wörterbuchs" identisch. 


\section{5 Öffentliche Sichtbarkeit und Nutzung}

Durch die automatische Bereitstellung einer auf den Lesezugriff beschränkten Version des Redaktionssystems ist es der breiten Öffentlichkeit möglich, auf alle Inhalte der Datenbank zuzugreifen und dabei den jeweils letzten Bearbeitungsstand einzusehen. Von der Einführung der Zugriffsstatistik im Februar 2017 bis Dezember 2018 gab es fast 100000 öffentliche Zugriffe. ${ }^{24}$ Dadurch zeigt sich, dass reges Interesse am Projekt und dessen Arbeit besteht sowie offenkundig interessierte Laien nachschlagen, ob bzw. welche Dialektbegriffe z. B. in ihrer Heimatregion attestiert sind. Dies unterstreicht die Wichtigkeit des Projekts auch außerhalb der Fach-Community.

\subsection{Ausblick}

Die Datenbestände der an der Bayerischen Akademie der Wissenschaften ansässigen Dialektwörterbuchprojekte BWB, WBF und DIBS (,Dialektologisches Informationssystem von Bayerisch-Schwaben") werden aller Voraussicht nach zusammen mit dem Datenbestand der „Bayerischen Dialektdatenbank“ (BayDat) Anfang 2019 in einer gemeinsamen Anwendung namens „Bayerns Dialekte Online“ (BDO) online gehen, sodass Fachwissenschaftlerinnen und Fachwissenschaftler wie auch interessierte Laien alle Materialquellen und Wörterbucheinträge durchsuchen und miteinander vergleichen können. Für Letzteres steht insbesondere die Sachgruppenzuordnung nach POST (1998) im Vordergrund, um unabhängig vom Lemma eine verlässliche semantische Verknüpfung von Dialektwörtern über die jeweiligen Dialektgrenzen hinweg zu ermöglichen.

Für das BWB werden derzeit noch keine Sachgruppen erfasst. Für 2019 ist allerdings geplant, in der Artikelverfassung von Word auf XML mittels Oxygen umzusteigen. Im Zuge dieser Umstellung sollen auch die Sachgruppenzuordnungen eingeführt und für die bereits fertiggestellten Artikel in semi-automatischer Weise nachgetragen werden. Hierfür sollen die Daten des WBF, des DIBS sowie des „Pfälzischen Wörterbuchs“ herangezogen werden. Unabhängig von der Sachgruppenzuordnung können die einzelnen Datenquellen stets über das Lemma oder die Bedeutung miteinander verbunden werden. Nach und nach sollen außerdem weitere Belegquellen digitalisiert und ins System eingespeist werden.

24 Der Vollzugriff auf das Redaktionssystem ist nur User/-innen möglich, die sich via Benutzername und Passwort authentifizieren. Der eingeschränkte Lesezugriff findet ohne Login statt und signalisiert dies dem System. Dadurch kann ohne weitere Datenabfrage und -speicherung datenschutzkonform erfasst werden, wie viele Anfragen aus welchem Nutzerkreis stammen (d. h. Redaktion vs. Öffentlichkeit). Die hier gezählten 100000 Zugriffe stammen nicht von jeweils einer einzelnen Person, sondern stellen die Summe aller Aufrufe aller Personen dar. 


\title{
8 KONTAKTADRESSE, HOMEPAGE
}

\author{
Bayerisches Wörterbuch \\ Bayerische Akademie der Wissenschaften \\ Alfons-Goppel-Straße 11 \\ 80539 München \\ Deutschland
}

E-Mail: post@kmf.badw.de

Homepage: <bwb.badw.de>

\section{LITERATURVERZEICHNIS}

BWB = Bayerisches Wörterbuch (1995 ff.). Hg. von der Kommission für Mundartforschung. München: Oldenbourg (1995-2013), Berlin/Boston: de Gruyter (2014 ff.). (Bayerisch-österreichisches Wörterbuch. II. Bayern).

DELLING, JOHANN VON (1820): Beiträge zu einem baierischen Idiotikon. 2 Bände. München: Lentner.

Duden (1993): Das große Wörterbuch der deutschen Sprache. 3. Aufl. 10 Bände. Mannheim [u. a.]: Dudenverlag.

DWB = GRIMM, JAKOB / WILHELM GRIMM (1854-1954): Deutsches Wörterbuch. 16 Bände. Leipzig: Hirzel (Nachdruck München 1984).

${ }^{2}$ DWB = Deutsches Wörterbuch von Jacob Grimm und Wilhelm Grimm (1965 ff.). Neubearbeitung. 2. Auflage. Leipzig: Hirzel.

FUNK, EDITH / ANDREA SCHAMBERGER-HIRT (2016): BWB-Digital. URL: <http://bwb.badw.de/ bwb-digital.html>; Stand: 07.01.2019.

HÄSSLEIN = OSWALD-MÜLLER, GABI (Hg.) (1993): Das Nürnberger Wörterbuch des Johann Heinrich Häßlein (1737-1796) Und seine Benutzung durch Johann Andreas Schmeller. Grafenau: Morsak (Jahrbuch der Johann-Andreas-Schmeller-Gesellschaft 1991).

Post, RudolF (1998): Möglichkeiten der elektronischen Strukturierung, Vernetzung und Verfügbarmachung von lexikographischen Daten bei der Arbeit am Pfälzischen Wörterbuch. In: GROSSE, RudOLF (Hg.): Bedeutungserfassung und Bedeutungsbeschreibung in historischen und dialektologischen Wörterbüchern. Leipzig: Verlag der Sächsischen Akademie der Wissenschaften.

PRASCH, J. LudWIG (1689): Dissertatio altera, De Origine Germanica Latinae Linguae ... Accedit Glossarium Bavaricum. Regensburg: Hofmann.

RAAF, MANUEL (2016 bis heute): LexHelfer BWB. Online-Datenbank der Forschungsprimärdaten des Bayerischen Wörterbuchs. URL: <https://bwb.badw.de/bwb-digital/datenbank>; Stand: 07.01.2019.

SCHMELLER, JOHANN ANDREAS (1872-1877): Bayerisches Wörterbuch. 2., von G. KARL FrOMMANN überarbeitete Aufl. 2 Bände. München: Oldenbourg.

Schw.Id. = Schweizerisches Idiotikon (1881 ff.). Wörterbuch der schweizerdeutschen Sprache. Frauenfeld: Huber.

Schwäb.Wb. = Schwäbisches Wörterbuch (1904-1936). Bearb. von HERMANN FISCHER (Band 6 von WILHELM PFLEIDERER). 6 Bände. Tübingen: Laupp'sche Buchhandlung.

SCHWARZ, DANIEL (2017 bis heute): Erfassungstool für neue Wörterlisten des BWB. URL: <http:// bwb.badw.de/bwb-digital/zur-erfassung.html>; Stand: 08.01.2019. 
Suddt.Wb. = Sudetendeutsches Wörterbuch (1988 ff.). Hg. von Heinz Engels. München: Oldenbourg.

WESTENRIEDER, LORENZ VON (1816): Glossarium Gemanico-Latinum vocum obsoletarum primi et medii aevi, inprimis Bavaricarum. München: Zangl.

WBÖ = Wörterbuch der bairischen Mundarten in Österreich (1963 ff.). Hg. vom Institut für Österreichische Dialekt- und Namenlexika (vormals Kommission für Mundartkunde und Namenforschung). Wien: Österreichische Akademie der Wissenschaften (Bayerisch-österreichisches Wörterbuch. I. Österreich).

ZAUPSER, ANDREAS (1789): Versuch eines baierischen und oberpfälzischen Idiotikons. München: Lentner (Nachdruck Grafenau 1986: Morsak). 


\title{
DAS FRÄNKISCHE WÖRTERBUCH (WBF)
}

\author{
Almut König / Manuel Raaf / Alfred Klepsch
}

Das „Fränkische Wörterbuch“ sammelt, dokumentiert und beschreibt den mundartlichen Wortschatz der Menschen in den bayerischen Regierungsbezirken Ober-, Mittel- und Unterfranken. Das Projekt entstand 1933 als Ableger des „Bayerischen Wörterbuchs“, als die Kommission für Mundartforschung der Bayerischen Akademie der Wissenschaften (BAdW) eine Wörterbuchkanzlei in Erlangen mit dem Ziel einrichtete, ein Ostfränkisches Wörterbuch zu erarbeiten. 2012 wurde das Wörterbuch von „Ostfränkisches Wörterbuch“ in „Fränkisches Wörterbuch“ umbenannt, womit man dem in Bayern allgegenwärtigen laienlinguistischen Usus entsprach, die in den drei fränkischen Regierungsbezirken gesprochenen Dialekte einfach als Fränkisch zu bezeichnen.

Die Geschichte des Wörterbuchs prägten Forscherpersönlichkeiten wie FRIEDRICH MAURER, ERICH STRASSNER oder EBERHARD WAGNER, die im Wesentlichen für verschiedene Phasen der Datenerhebung stehen. Erst seit 2001, mit dem Antritt von ALFRED KLEPSCH als leitendem Redaktor, liegt der Fokus auf der Aufbereitung (siehe Kapitel 2.2) und Publikation der in 90 Jahren Sammlertätigkeit zusammengetragenen Sprachdaten. Seit einer Kooperationsvereinbarung zwischen der BAdW und der Friedrich-Alexander-Universität Erlangen-Nürnberg (FAU) im Jahr 2012 wird das Projekt von MeChTHILD HabeRMANN, der Inhaberin des Lehrstuhls für Germanistische Sprachwissenschaft an der FAU, geleitet. Die neue Organisationsstruktur führte zu grundsätzlichen konzeptionellen Veränderungen. Man entschied, ausschließlich online zu publizieren und konsequent auf Digitalisierung zu setzen, indem man das Archiv vollständig einscannte und mit dem Aufbau einer Volltextdatenbank begann. Auf diese Weise generiert man am WBF eine hochwertige Quelle, die den Datenbestand sowohl für dialektinteressierte Nutzer/-innen, als auch für moderne Methoden der Informationsverarbeitung, wie systematische statistische Auswertungen und die Mustererkennung, zugänglich macht.

Dieser Beitrag behandelt verschiedene Aspekte der Arbeit am WBF, von denen manche bereits an anderen Stellen publiziert wurden. Zum Beispiel findet man die Projektgeschichte und die Datensammlung ausführlich auf der WBF-Homepage beschrieben. Die Texte dort stammen in weiten Teilen von ALFRED KLEPSCH. ${ }^{1}$ Das Kapitel zur Datenaufbereitung fasst wesentliche Inhalte aus der Handreichung zur Datenerfassung und Datenbeschreibung am WBF zusammen, die ALFRED KLEPSCH

1 Zur ausführlichen Darstellung der Geschichte des Fränkischen Wörterbuchs siehe KLEPSCH / KÖNIG (2016) unter <http://www.wbf.badw.de/geschichte.html>; Stand: 07.01.2019. 
und ALMUT KÖNIG gemeinsam verfasst haben. ${ }^{2}$ Die Ausführungen zu texttechnologischen Aspekten stammen von MANUEL RAAF, der als Mitarbeiter der IT-Abteilung an der BAdW die Redaktionsumgebung des WBF entwickelt.

\section{DIE SAMMLUNG}

Das in der Wörterbuchkanzlei versammelte Material wurde in fünf Erhebungsserien (Systematische Fragebogen 1913-1915, Mundartgeographische Fragebogen 19281940, Maurer-Bogen 1933-1934, Mündliche Befragungen 1966-1967, Nachkriegsbogen 1960-2001) zusammengetragen. Mit Ausnahme der mündlichen Befragungen, für die Exploratoren in über 800 Erhebungsorten einen festen Kanon an 125 Wörtern aufzeichneten, wurden alle Erhebungen indirekt mittels Fragebogen durchgeführt. Insgesamt lagern über 100000 ausgefüllte Fragebogen im Archiv der Forschungsstelle. Sie liegen vollständig als Scans vor. ${ }^{3}$

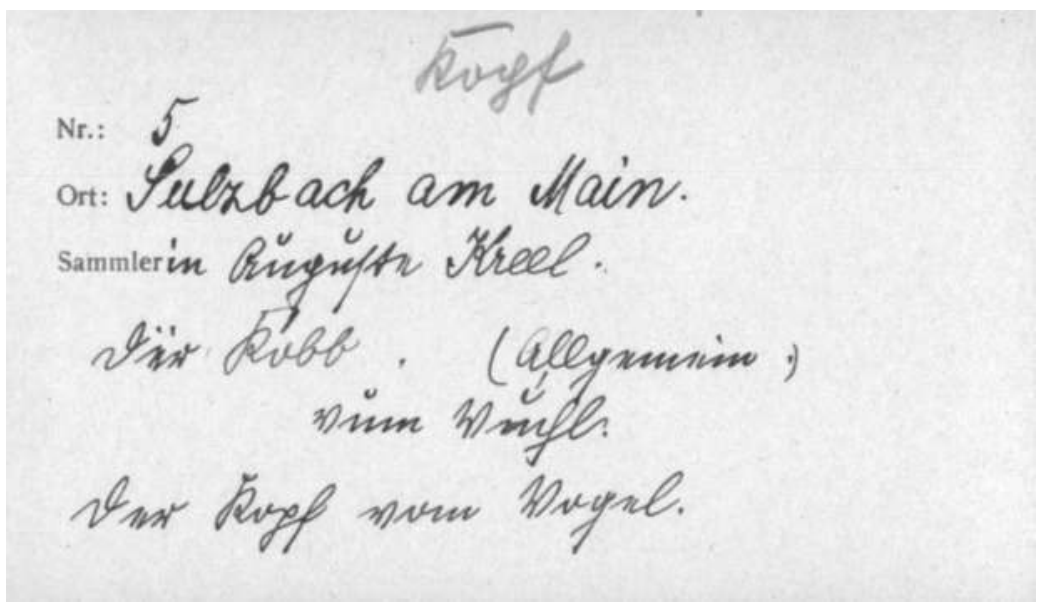

Abb. 1: Systematische Fragebogen (Sulzbach am Main)

Mit den Systematischen Fragebogen, die von 1913 bis 1915 von München aus verschickt wurden, sollte der dialektale Wortschatz in seiner ganzen Breite abgefragt werden. Als Sammler dienten meist Volksschullehrer, die die alteingesessene Bevölkerung befragten. Sie schrieben die Antworten auf kleine Abreißzettel. Diese Abreißzettel wurden von den Mitarbeitern der Wörterbuchredaktion in München

2 KLEPSCH / KÖNIG (2016) unter <http://www.wbf.badw.de/geschichte.html>; Stand: 07.01.2019.

3 Das Einscannen wurde durch finanzielle Zuwendungen aus der Oberfrankenstiftung, der Unterfränkischen Kulturstiftung und der Mittelfrankenstiftung ermöglicht, für die wir uns an dieser Stelle bedanken. 
mit einem Lemma versehen und, nachdem die Forschungsstelle in Erlangen eingerichtet worden war, in der Zentralkartei des Fränkischen Wörterbuchs archiviert.

Wiederum von München ausgehend wurden zwischen 1927 und 1940 die Dialekte in Franken mittels der Mundartgeographischen Fragebogen erhoben. Ziel dieser Erhebungen war ein Kartenwerk, ein Sprachatlas der Dialekte in Bayern und Österreich. Um eine hohe Belegdichte zu erzielen, wurden die Fragebogen der „Wochenschrift zur Pflege von Heimat und Brauchtum“ beigegeben, die an alle Schulen in Bayern versendet wurde. Die Fragebogen aus Franken, die der fränkischen Wörterbuchkanzlei sowohl als Originale als auch als Scans vorliegen, wurden bislang nicht ausgewertet.

Die erste Dialekterhebung, die von Franken ausging, fand 1932 statt. Unter der Leitung von FRIEDRICH MAURER, der 1931 an die Universität Erlangen berufen worden war, unternahm man zunächst Kundfahrten und entwarf daraufhin den sogenannten Maurer-Bogen mit knapp 100 Fragen. Gefragt wurde vor allem nach dem Dialektwortschatz der Sachbereiche „Tier“ (z. B. Wie heißt in Ihrer Mundart: die Ameise, der Star, das jüngste Tier im Nest), „Pflanze“ (z. B. Wie heißt in Ihrer Mundart: die Kartoffel, die Herbstzeitlose, die Stachelbeere) und „Mensch, seine Arbeit und Umwelt“" (z. B. Wie heißt in Ihrer Mundart: die Dachrinne, arbeiten, die hängende Schaukel, die Frau, die die Entbindung der Wöchnerin vornimmt). Der mehrseitige Fragebogen ist in zwei Spalten unterteilt. In der linken Spalte sind die Fragen abgedruckt, die rechte Spalte ist der Antwort vorbehalten. Als Sozialdaten werden Alter, Beruf und Geburtsort der Gewährspersonen sowie der Geburtsort ihrer Eltern erhoben. Drei Fragen zielen auf die Sozialstruktur des Erhebungsortes. Gefragt wird nach dem Anteil an Bauern und Arbeitern sowie nach den Orten, wohin man zur Arbeit und zum Einkaufen geht. Der Fragebogen enthält unter der Überschrift „Anmerkung“ einen kurzen Text, in dem das Vorhaben erläutert sowie eine Anleitung gegeben wird, wie der Bogen auszufüllen ist. Diese „fränkische“ Erhebung war sehr erfolgreich. Innerhalb von zwei Jahren erreichten nahezu 3000 ausgefüllte Fragebogen aus Ober-, Mittel- und Unterfranken sowie aus Nordbaden und Nordwürttemberg die Redaktion, auch sie wurden bislang nicht aufbereitet. 


\section{Kintempels \\ z 35,2

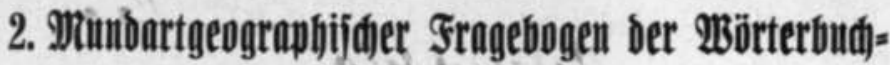 Rommififion.}

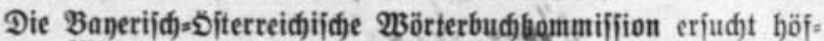
lidjit ium Dritteilung Der für Die nadjitebend aufgefübrten Begriffe in Shrer älteren Drtsmun๖art gebrüuddliden Entipred)ungen. Sollten

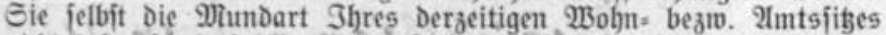
nidjt beberridjen, jo bitten wir Sie, Dieje Begriffe bei eimem älteren,

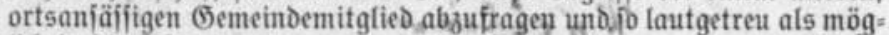
lid) in Die Eifte einzutragen; in Diejem Falle wäre es notwendig, Name

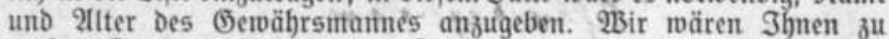
grobem Dank verpflid)tet, werm Sie Die ausgefüllte Sị̂te möglidjit balb wieber jurïdkleiten würgen.

- Edjubkarten

2. Siehridjt

3. Epreu beim Flad)s

4. Epreu bei Der Eerite

5. Spreu beim Koggen

6. Spreu beiur Sdajer

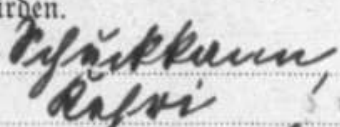

7. Spreu beim Budweizen

8. Budjweĭen

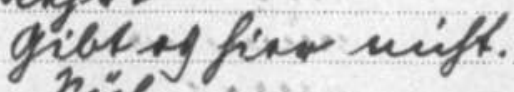

9. anjteigende $\mathfrak{W i e j e}$ (Seite, S)enge, Ranke, S)aldos)
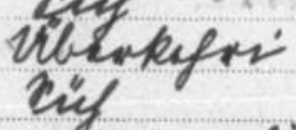

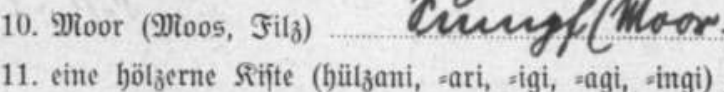

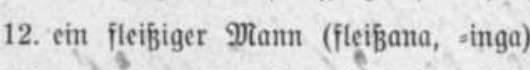

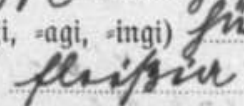

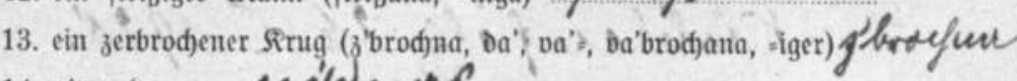

14. nirgends

15. gebabt (khabt, khat, kla'j ' fect

16. Sีnäuel $\mathfrak{W}$ olle

ousielet thelew

17. S)uiten (\$)uajtut ober S)uajditn?)

18. $\mathfrak{B i e h}$

19. breit

20. Peiter

21. Flad)s

22. Fladjs bredjeln
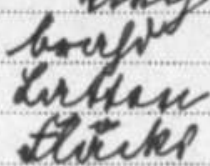

bregeter

Abb. 2: Mundartgeographischer Fragebogen (Kühlenfels) 


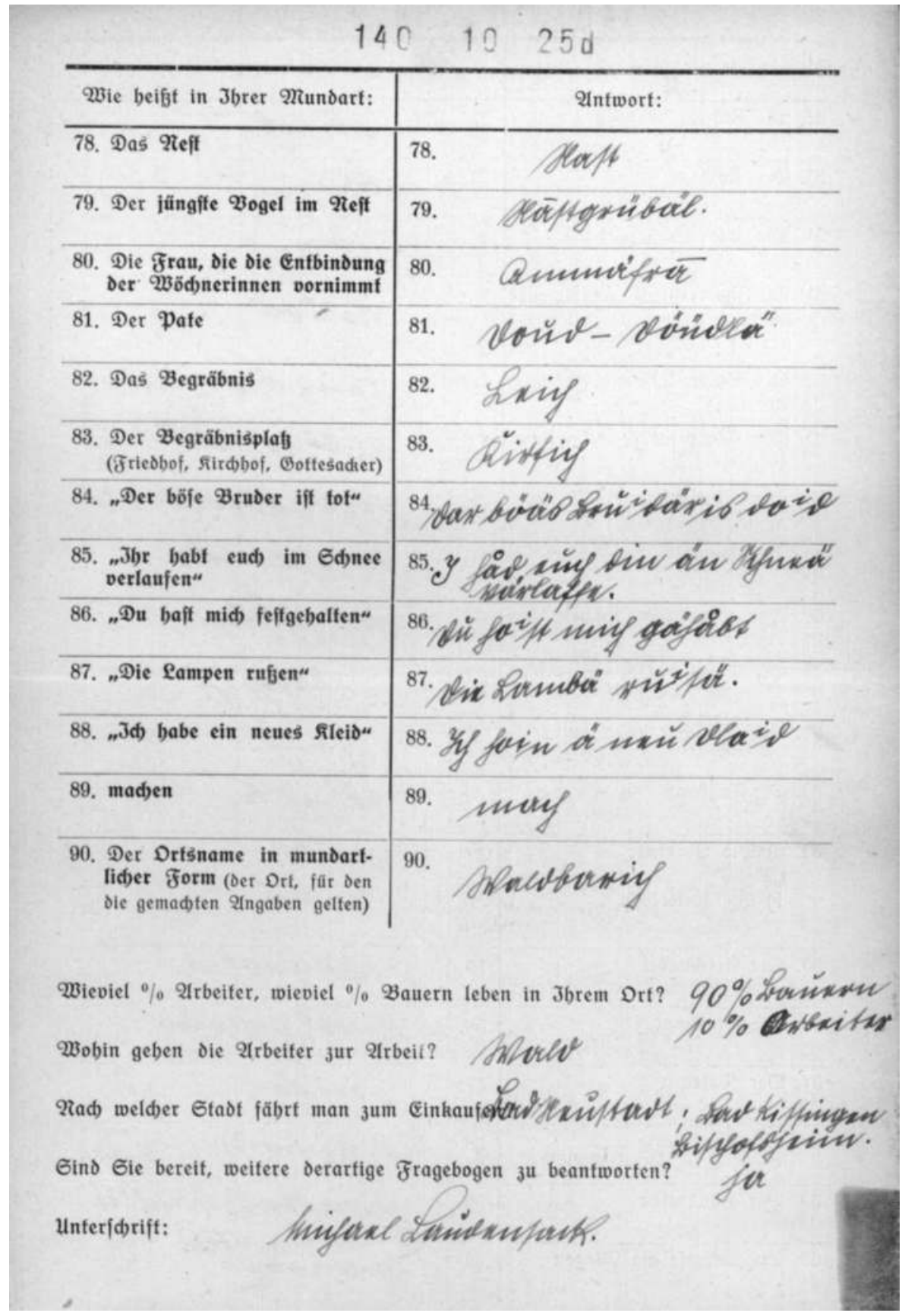

Abb. 3: Maurer-Bogen (Waldberg) 


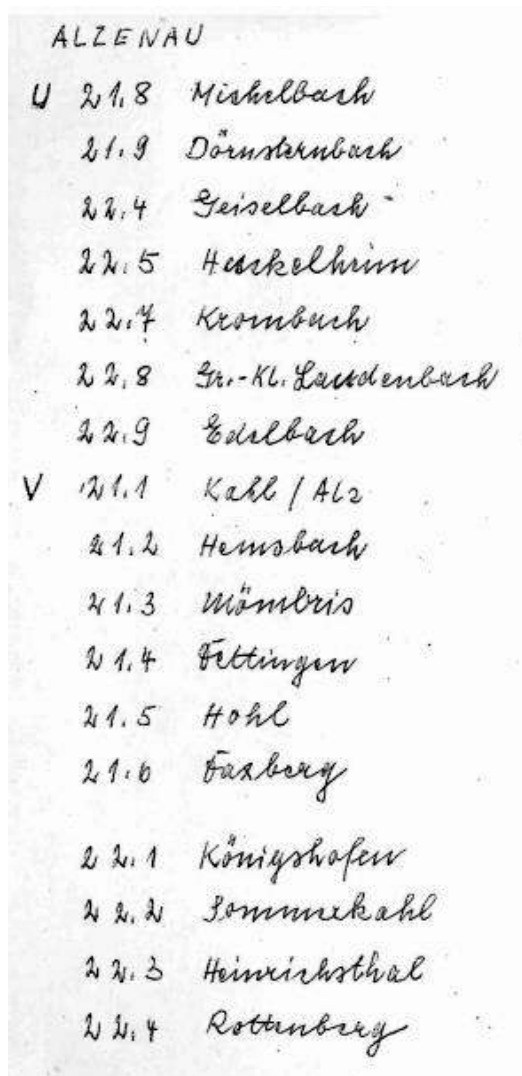

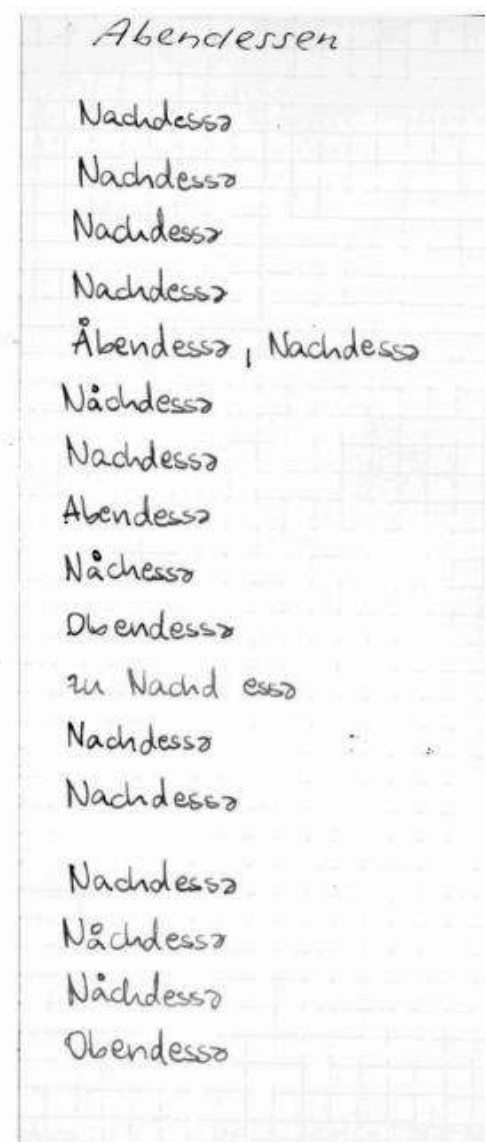

Abb. 4: Direkterhebung (Landkreis Alzenau)

Anlass für die Direkterhebung war EBERHARD WAGNERS Idee einen kleinen fränkischen Sprachatlas zu erarbeiten. Bei der Auswahl der Fragen orientierte sich Wagner, um an bestehende Projekte anzuschließen, an den Wortkarten des „Südhessischen Wörterbuchs“ und dem „Sudetendeutschen Sprachatlas“. In insgesamt 825 Orten fragten studentische Hilfskräfte nach Dialektwörtern z. B. für die Regenpfütze, den Schnittlauch, die Hundepfote, den Fastnachtskrapfen, das stumpfe Messer oder den Scheuerlappen. Die Antworten notierten sie direkt teils in Lautschrift, teils in lateinischer Schrift unter der Verwendung von Diakritika in Hefte. Diese Hefte wurden alle eingescannt und die Antworten abgetippt. Die Ergebnisse dieser Befragung sind noch nicht online verfügbar und müssen, bevor sie in die Datenbank eingebunden werden, für das WBF aufbereitet werden. 


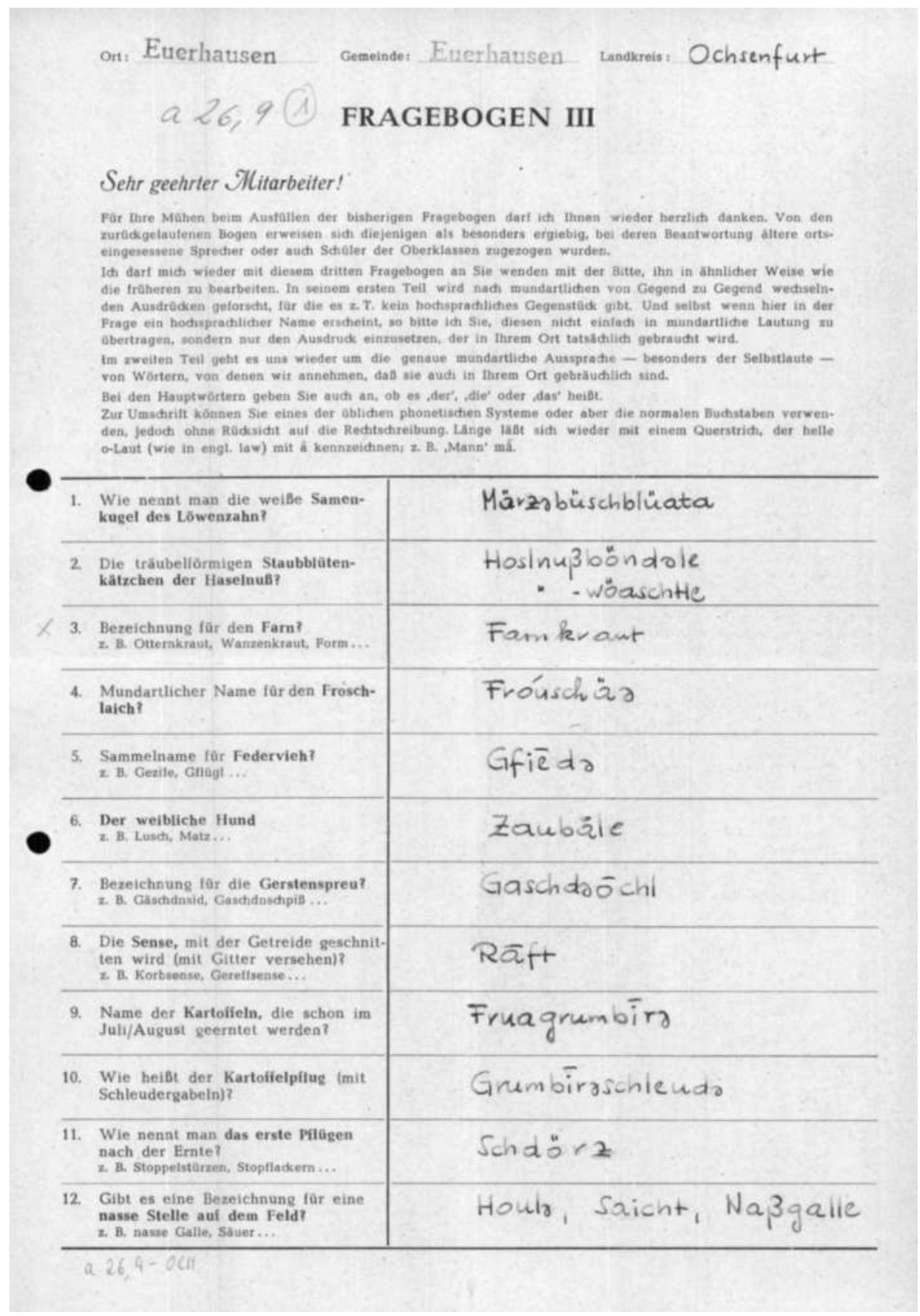


Die umfangreichste Sammlung stellt die Nachkriegserhebung dar. Über einen Zeitraum von 40 Jahren wurden im Jahresdurchschnitt drei Fragebogenserien mit jeweils knapp 60 Fragen verschickt. Der Rücklauf schwankte über die Jahre hinweg stark. Den größten Rücklauf hatte der erste aus dem Jahr 1960 mit 1 268, den niedrigsten der vorletzte Bogen im Jahr 2001 mit 177 zurückgesendeten Fragebogen. Insgesamt erreichten über 48000 ausgefüllte Bogen die Wörterbuchredaktion. Die Gegenstände der Fragestellungen änderten sich im Laufe der Zeit. Sie bilden auch die Interessen des jeweiligen Redaktors ab. Zum Beispiel stellte der Volkskundler ERICH STRASSNER viele Fragen zu fränkischen Bräuchen bei Kirchweih, Weihnachten und Hausschlachtung. Ein Fragebogen der Nachkriegsserie umfasst in der Regel vier Seiten. Wie schon der Maurer-Bogen sind die Seiten in zwei Spalten unterteilt. Links stehen die Fragen, die rechte Spalte ist den Antworten vorbehalten (siehe Abbildung 5). Eine Liste mit allen 6793 gestellten Fragen ist über die Datenbank des Fränkischen Wörterbuchs verfügbar. Die Nachkriegserhebung ist bislang als einzige Erhebungsserie in die Datenbank des „Fränkischen Wörterbuchs“ eingeflossen. Der Arbeitsstand zum jetzigen Zeitpunkt (Juli 2018) stellt sich wie folgt dar: Alle Fragebogen sind gescannt, etwas mehr als die Hälfte der 48000 Fragebogen ist von studentischen Hilfskräften abgetippt, ca. 12000 Fragebogen sind von den Redaktoren annotiert, lemmatisiert und in die Datenbank eingestellt.

Im Archiv des „Fränkischen Wörterbuchs“ lagern auch weitere Einsendungen wie Wortschatzsammlungen oder Dialekttexte, die aus Eigeninitiative von Laien unabhängig von den Fragebogenerhebungen entstanden. Handschriftliche Einsendungen wie ein mehrere hundert Seiten umfassendes „Erlanger Wörterbuch“ wurden ebenfalls gescannt. Auch diese Bilddaten sind bislang nicht online verfügbar. Einsendungen, die als Druckerzeugnisse vorliegen, wurden bislang nicht gescannt.

\section{DATENAUFBEREITUNG FÜR EIN ONLINEWÖRTERBUCH}

\subsection{Modell des Onlinewörterbuchs}

Das WBF ist ein Online-Informationssystem, das auf den Säulen Daten, Zugriffswerkzeuge und Dokumentation beruht (siehe Abbildung 6). Im Folgenden werde ich die philologische Arbeit bei der Datenaufbereitung erläutern, texttechnologische Aspekte stellt Manuel Raaf in Kapitel 3 vor.

Mit den Scans verfügt das WBF über ein Archiv an Bilddateien. Um die digitale Weiterverarbeitung zu gewährleisten, begann man mit dem Aufbau einer Volltextdatenbank. In dieser erscheint jeder Originalbeleg - Zweifelsfälle und unsichere Belege eingeschlossen - in einer Abschrift, einer Umschrift sowie einer Grundform. Jeder Originalbeleg ist zudem mindestens einem Lemma zugeordnet und mit Annotationen versehen. Jeder Datensatz ist mit einem Scan des Originalbelegs sowie mit den Sozialdaten der Gewährsperson, von der der Beleg stammt, verknüpft. Mit Ausnahme der Links zu den Sozialdaten, die nur mit besonderen Rechten abrufbar sind, sind alle Daten öffentlich zugänglich (siehe KLEPSCH / KÖNIG 2016). 
Forschungsergebnisse können so unter Rückgriff auf die Datenbank vollständig transparent dargestellt werden und sind jederzeit bis zu den Quelldaten nachvollziehbar.

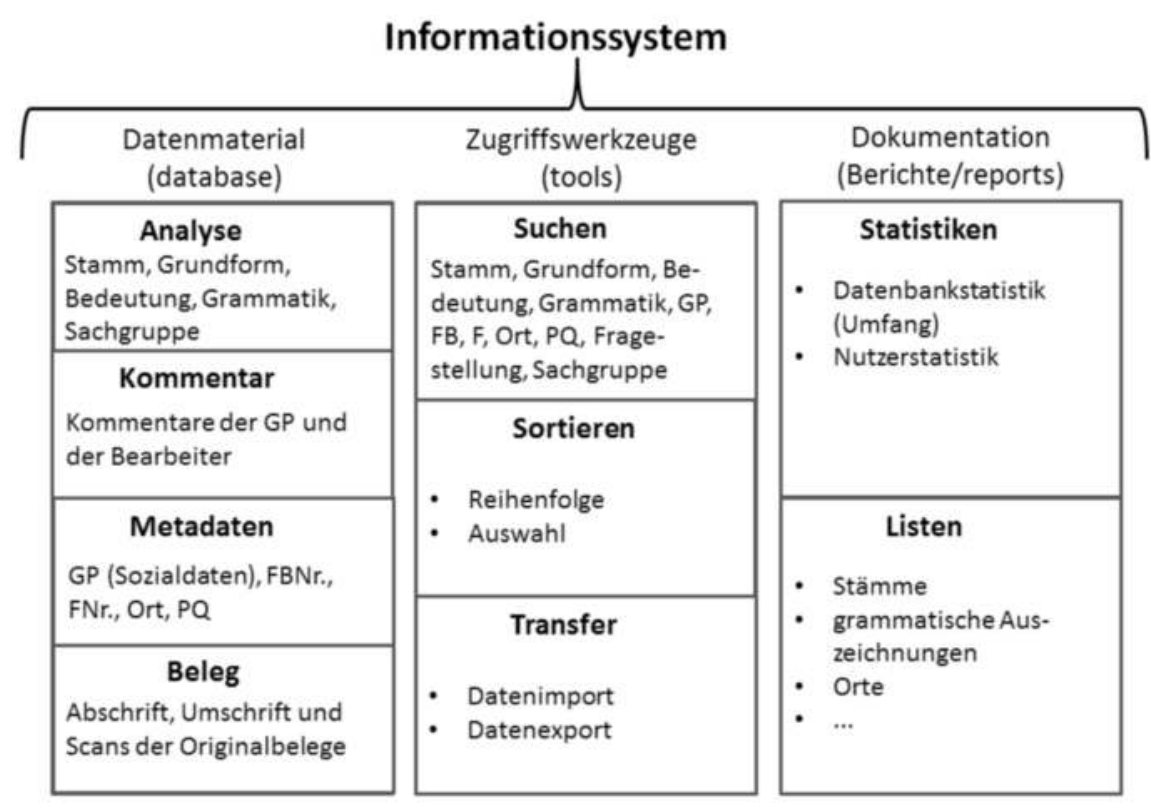

Abb. 6: Modell des Online-Wörterbuchs

\subsection{Datenaufbereitung}

Form und Inhalt von Wörterbüchern stehen seit jeher im Fokus der wissenschaftlichen Wörterbuchkritik und der wissenschaftlichen Untersuchung von Wörterbüchern. Form und Inhalt sind auch leicht von außen zugänglich, weil sowohl Gestalt wie Inhalt im gedruckten Wörterbuch auf Papier oder beim elektronischen Wörterbuch auf einem Bildschirm sichtbar sind. Eher im Hintergrund läuft dagegen der Prozess ab, in dessen Verlauf ein Wörterbuch entsteht. (KLOSA / TIBERIUS 2018: 67)

Die Prozesse, die ein Wort vom Fragebogeneintrag bis hin zum im WBF publizierten Datensatz durchläuft, werden in der Handreichung „Datenerfassung und Datenbeschreibung im Fränkischen Wörterbuch“" ausführlich dargestellt und dokumentiert. Diese Handreichung soll die Entscheidungen der Wörterbuchredaktion nachvollziehbar und überprüfbar machen. Entsprechend dem Fortschritt der lexikographischen Arbeit an der Datenbank wird diese Handreichung immer wieder überarbeitet und ergänzt. Im Folgenden werde ich wesentliche Punkte des Datenaufbereitungsprozesses knapp erläutern, für am Detail Interessierte verweise ich auf die Handreichung, die über die Homepage des WBF einseh- und abrufbar ist. 
Die Gewährspersonen füllten die Originalfragebogen sowohl handschriftlich als auch maschinenschriftlich aus, wobei die mit Hand ausgefüllten Bogen überwiegen (siehe auch Abbildungen 1 bis 5). Die späteren Befragungen zeigen meist lateinische Schrift, die frühen in der Regel Sütterlin. Sonderzeichen aus phonetischen Schriften sind selten belegt. Am häufigsten findet man $<\vartheta>$ als Zeichen für einen Schwa-Laut oder <å>, das für einen Laut steht, der zwischen [a] und [o] anzusetzen ist. Ebenfalls selten verwendet werden Diakritika. Gängigstes Zeichen ist ein Überstrich, der über Vokalen stehend als Dehnung, über Konsonanten als Gemination interpretiert werden kann. Die Datenaufbereitung beinhaltet mehrere Arbeitsschritte, hierzu gehören das Erstellen einer Abschrift, einer Umschrift und einer Grundform, die Zuordnung zu einem Lemma, die semantische Beschreibung mit Zuordnung zu einer Sachgruppe sowie die Annotation mit grammatischen und Sachinformationen.

Die Abschrift der Fragebogen erfolgt originalgetreu. Das heißt, es werden keine Veränderungen oder Korrekturen vorgenommen. Auch offensichtliche Verschreiber tippen wir ab und versehen sie im Bearbeiterkommentar mit der Kennung \#evtl. verschrieben. Sonderzeichen und Diakritika werden in der Regel wiedergegeben. Nicht erfasst werden Zeichen ohne phonetischen Inhalt, wie z. B. der Strich über $\breve{u}$, der $\langle\mathrm{n}\rangle$ und $\langle\mathrm{u}\rangle$ graphisch voneinander differenziert und in der Regel der besseren Lesbarkeit dient. Die Umschrift ist als erste Standardannäherung zu verstehen, bei der phonetische Unterschiede zur Standardsprache ausgeglichen, morphologische jedoch beibehalten werden. Originalschriftlich basst wird daher mit „passt“, Baam mit „Baum“, des hot sich reihgsleicht [sic] mit „das hat sich hereingeschleicht" wiedergegeben, wobei die schwache Flexion des Verbs schleichen beibehalten wird. Abschrift und Umschrift werden von studentischen Hilfskräften und Praktikantinnen und Praktikanten angefertigt, die im Rahmen ihres Praktikums ECTS-Punkte erwerben. Die weitere Bearbeitung der Daten erfolgt durch die Redaktoren und studentische Hilfskräfte, die sich bereits am WBF qualifiziert haben.

Am WBF verstehen wir unter Grundform ungefähr das, was in anderen Wörterbüchern einem Stichwort entspricht. Grundformen sind die Nennformen bzw. Zitatformen eines Originalbelegs. Als Lemma hingegen bezeichnen wir die am einfachsten gebaute, semantisch durchsichtige Form einer Wortfamilie. So gilt das Belegwort Ziefer synchronisch als Rückbildung aus Ungeziefer, andererseits ist das Simplex schon althochdeutsch als ziobar belegt (vgl. KLUGE 2011: 942 und 1009). Aufgrund seiner geringeren Komplexität wird Ziefer als Lemma angesetzt, unter dem wiederum Belege wie Federgeziefer, verziefern, Ungeziefer und Zieferzeug versammelt sind. Die Zuordnung der Grundformen zu einem Lemma ermöglicht neben der glattalphabetischen Darstellung auch nischen- oder nestalphabetische Sortierungen der Stichwörter. Viele Wörter werden, obwohl sie historisch aus ein und demselben Etymon hervorgegangen sind, nicht mehr als zusammengehörig verstanden. Hierzu zählen neben Ableitungen mit nicht mehr produktiven und intransparenten Suffixen auch die historischen ti-Ableitungen wie Zucht, Fahrt und Tracht. Diese Ableitungen führen wir als eigene Lemmata und ordnen sie nicht den Lemmata ziehen, fahren und tragen zu. Ähnlich verfahren wir mit Derivationen von 
Nomina auf Basis von Verben mit Ablautparadigmen, deren historische Stammform im Verbparadigma nicht mehr vorhanden ist. Zum Beispiel ist das Substantiv Zug ein bereits auf westgermanischer Stufe gebildetes Verbalabstraktum. Auf neuhochdeutscher Stufe enthält das Paradigma des Verbs ziehen keine Form mit dem Stammvokal - $u$ - mehr, die das Mittelhochdeutsche noch im Plural des Präteritums (si zugen 'sie zogen') aufweist. Die Präteritalform fuhr des Verbs fahren ist im neuhochdeutschen Standard üblich, aber in der rezenten Mundart nicht mehr gebräuchlich, weshalb Belege wie Fuhre oder Furt als eigenständige Lemmata behandelt und nicht dem Lemma fahren zugeschlagen werden.

Als mögliche Bedeutungsangaben sehen wir standarddeutsche Entsprechung, Synonym bzw. Heteronym und Paraphrase vor. Die standarddeutsche Entsprechung wählen wir v. a. bei Belegen, bei denen Dialektwort und Standardwort inhaltsseitig vollkommen übereinstimmen, z. B. 'Kuh' als Bedeutungsangabe für Kou in a drocherder Kou 'eine tragete Kuh'. Synonyme bzw. Heteronyme werden gewählt, soweit sie das Standardlexikon vorhält, z. B. 'Kaminkehrer' für Schlotfeger. Eine Paraphrase wählen wir nur dann, wenn das Standardlexikon kein Lexem zur Verfügung stellt, z. B. tandern 'sich spielend balgen von jungen Katzen'. Um einen zusätzlichen semantischen Zugriff auf das in der Datenbank gespeicherte Material zu ermöglichen, klassifizieren wir die Dialektdaten nach dem Schema, das RUDOLF POST, der ehemalige Leiter des „Badischen Wörterbuchs“, zur Erfassung von Sachgruppen entwickelt hat. Dieses Schema basiert auf dem von RUDOLF HALLIG und WALTHER VON WARTBURG entworfenen Begriffssystem (RUDOLF HALlig / WALTHER VON WARTBURG 1963). Nach dem POST'schen-Schema wird jeder Wortbedeutung ein vierstelliger Zahlencode mit einem absteigendem Abstraktionsgrad zugewiesen. Zum Beispiel ist das Lemma Stücht mit der Bedeutung 'oben offenes zylindrisches Holzfass' der Kategorie „7000 Der Mensch sozial (Arbeit und Umwelt)/7700 Wohnung/7730 Einrichtung/7735 Gefäße“ zugeordnet. Die Übernahme der Sachgruppenklassifikation nach POST bietet sich für das WBF an, da das „Pfälzische“ und das „Südhessische Wörterbuch“ entsprechend ausgezeichnet wurden. Beim „Schweizerischen Idiotikon“ ist man gerade dabei, den gesamten Wörterbuchbestand nach diesem Schema zu klassifizieren (siehe BICKEL 2013: 130-131). Mit der Übernahme dieser Klassifikation reiht sich das WBF in die Riege dieser Wörterbücher ein und schafft die Grundlage für einen Sprach- und Landesgrenzen übergreifenden onomasiologischen Zugriff auf Sprachdaten in einem zukünftigen digitalen Verbund von Dialektwörterbüchern.

Grammatisch bestimmt wird das zu betrachtende Wort in der Form, in der es in der Originalschrift belegt ist. Angegeben werden die Wortart und je nach Wortart Flexion, Rektion oder Funktion. Die grammatische Auszeichnung unterbleibt, wo aufgrund von gebietsbedingten Schwankungen und fehlenden Angaben der Gewährsleute keine klare Zuordnung möglich ist. Ein Beispiel: Im Oberostfränkischen und in den Übergangsgebieten zum Nordbairischen schwankt das Genus des Belegwortes Asche zwischen Maskulinum und Femininum. Dort begegnen Belege wie die Aschn (Oberkotzau im Altlandkreis Hof), der Aschn (Bieswang im Altlandkreis Weißenburg), die Ascher (Wüstenselbitz im Altlandkreis Münchberg), der Ascher 
(Nagel im Altlandkreis Wunsiedel). Es gibt diesbezüglich jedoch keine klar umrissenen Areale. Belege ohne Artikel sind demzufolge nicht mit Sicherheit einem der beiden Genera zuzuordnen. Daher wird östlich der Linie Dinkelsbühl - Uffenheim - Schweinfurt - Mellrichstadt bei fehlendem Artikel das Genus des Belegwortes Asche nicht angegeben.

Manchmal notierten die Gewährsleute auch ganz unterschiedlich geartete Kommentierungen zu den Belegen. Zum Beispiel liefert eine Gewährsperson die Bedeutungsangabe „vollbeladener Wagen“ zum Beleg a Fuhr, eine andere macht Angaben zur Aussprache von Grumbiern ('Grundbirnen'), „bei = birn, ie sprechen also = biern" und wieder eine andere schätzt die Vorkommenshäufigkeit des Wortbelegs Gronbir ('Grundbirne') mit „selten“ ein. Alle Kommentierungen der Gewährsleute werden in die Spalte „Kommentar Gewährsperson“ eingetragen. Kommentierungen der Bearbeiter/-innen sind ebenfalls vorgesehen (Spalte „Kommentar Bearbeiter"). Bearbeiter/-innen-Kommentare behandeln meist den Beleg selbst, z. B. dessen Leserlichkeit, aber auch Verweise auf das Vorkommen in anderen Dialektwörterbüchern. Wie alle anderen Datenbankeinträge sind auch die Kommentierungen durchsuchbar und sortierbar. Auch sie können für systematische linguistische Untersuchungen abgerufen werden.

\section{TEXTTECHNOLOGISCHE ASPEKTE}

Dieses Kapitel behandelt die Digitalisierungsarbeiten des WBF, die seit Februar 2015 mithilfe der IT-Abteilung der Bayerischen Akademie der Wissenschaften durchgeführt werden. Die folgenden Seiten dokumentieren diese ausführlich, stellen deren wissenschaftlichen Mehrwert detailliert dar, liefern Informationen zur Realisierung sowie zur verwendeten Infrastruktur und spiegeln die bisherige Nutzung - auch seitens der interessierten Öffentlichkeit - wider.

\subsection{Datenbasis und Redaktionssystem}

\subsubsection{Datenbasis}

Die Dateneingabe findet seit 2004 in Excel-Dateien statt, in denen zeilenweise alle Daten eines jeden Belegs erfasst werden. Im ersten Schritt werden die Belege durch Hilfskräfte und Praktikantinnen und Praktikanten abgeschrieben; anschließend wird durch die Redaktion lemmatisiert sowie Grammatik und Sachgruppe eingetragen. 
Das Quellmaterial - die Fragebogen - ist nach seiner Digitalisierung durch einen externen Dienstleister seitenweise ${ }^{4}$ als JPEGs vorhanden und aufgrund der semantischen Dateinamen nach dem Muster Bogen-Planquadrat-Ortsname-Gewährsperson-Seite eindeutig mit den Daten der Excel-Dateien verknüpfbar: jede Belegzeile in Excel enthält die Bogennummer, das Planquadrat, über welches der Ortsname aus einer gesonderten Liste gelesen werden kann, die Gewährsperson und die Seitenangabe.

\subsubsection{Redaktionssystem}

Um den Arbeitsablauf des WBF weiter zu digitalisieren und vor allem möglichst direkt auf den Beleg zu einer bestimmten Frage eines Ortes zugreifen zu können, wurde das Redaktionstool „LexHelfer“ entwickelt, das kurz nach Einführung im WBF und nach projektspezifischen Anpassungen seit April 2016 auch beim „Bayerischen Wörterbuch“ (nachfolgend: BWB) sowie seit Juni 2018 beim „Dialektologischen Informationssystem für Bayerisch-Schwaben“ (nachfolgend: DIBS) Verwendung findet. Die Entwicklung begann im Februar 2015 in seither engem Kontakt mit der Wörterbuchredaktion, um deren Anforderungen und Wünsche bestmöglich umsetzen zu können. Seit Frühjahr 2016 ist das Redaktionssystem im produktiven Einsatz und steht seit Anfang des Jahres 2017 auch der Öffentlichkeit zur Verfügung. ${ }^{5}$

LexHelfer kann als Basisprogramm beschrieben werden, das für jedes der drei Wörterbuchprojekte eigene Funktionen bereitstellt, um den spezifischen Anforderungen der jeweiligen Redaktion entsprechen zu können. ${ }^{6}$ Die Entwicklung war bereits von Beginn an so konzipiert, dass Erweiterungen jederzeit durch entsprechende Module oder anderweitige Code-Anpassungen möglich sind. Für das WBF bestand die anfängliche Anforderung darin, die Originalbelege über die Scans grafisch und punktgenau aufrufen sowie die Inhalte der Excel-Listen online durchsuchen und abrufen zu können. Daher stellt die Variante des Programms für das WBF seit seiner ersten Version die Daten der Excel-Listen in tabellarischer Form dar und ermöglicht deren Bearbeitung, ähnlich wie dies in Excel möglich ist. Zusätzlich ist über einen Button der zugehörige Originalbeleg als Bild abrufbar; eine gesonderte Ansicht, die nur die Originalbelege lädt, kann via Suchoption ebenfalls aufgerufen werden (siehe Kapitel 7.2.1.3 Schnipselgenerierung). Durch die Eingabe der Wörterliste, der Fragennummer und des Ortes bzw. Regierungsbezirks kann die Redaktion nun innerhalb weniger Sekunden alle gewünschten Belege zur Anzeige auf den

4 Die Bogen wurden aufgeklappt eingescannt. Die so entstandenen Dateien enthielten jeweils die Seiten 1 und 4 sowie die Seiten 2 und 3. Diese Dateien wurden anschließend automatisch in je zwei Dateien mit der entsprechenden korrekten Seitenzahl im Dateinamen geteilt.

5 Siehe <http://www.wbf.badw.de/wbf-digital/wörterbuch>; Stand: 07.01.2019.

6 Siehe hierzu auch Kapitel 7.3.1.3 Generizität. 
Bildschirm laden und benötigt hierfür lediglich einen Computer mit Internetverbindung. Davor war die Einsicht in die Bogen nur durch deren händisches Heraussuchen aus Kartons möglich, die in den Redaktionsräumen des Projekts gelagert sind.

Inzwischen bietet LexHelfer für das WBF mehr Funktionen, die künftig noch weiter ausgebaut werden. Der momentane Funktionsumfang (Stand: Mai 2018) beinhaltet:

- anonyme Nutzungsstatistiken $^{7}$

- Datenstatistiken

- Import der Excel-Dateien mit der Möglichkeit des Ersetzens (für Korrekturen) oder Hinzufügens der Importinhalte

- anschließender Zugriff auf alle importierten Inhalte (Beleg- und Metadaten) und gezielte Suche nach diesen

- Löschen eines Importvorgangs

- Änderung der Feldinhalte je Feld oder je Spalte

- Zuweisung einer Sachgruppe für alle Bedeutungen oder je Beleg

- Listen zu Lemmata, Grammatikangaben, Grundformen und Belegorten

- Beschränkung der Suche auf ausgewählte Felder (Lemma, Grundform, Bedeutung, Ort, Bezirk, Landkreis, Fragebogen etc.)

- Sortierung der Suchergebnisse nach gewünschten Feldern

- automatische Artikelerstellung

- Export der Suchergebnisse in CSV

- Benutzerverwaltung

Außerdem liefert das Tool ohne Mehraufwand für die Wörterbuchredaktion eine im Funktionsumfang reduzierte und lediglich auf die Datenausgabe beschränkte Version, die automatisch geladen wird, wenn kein Login stattfindet, sodass die interessierte Öffentlichkeit wie auch projekt-externe Fachwissenschaftler/-innen im gesamten Bestand suchen und ihn sich bei Bedarf als CSV exportieren können.

Im Folgenden werden die besonderen Merkmale der Anwendung dargestellt; die allgemeinen und in vielen Online-Wörterbüchern eher als Standard betrachteten Funktionen (z. B. Suchoptionen mit Platzhaltern) werden nicht gesondert beschrieben, können jedoch online unter <http://www.wbf.badw.de/wbf-digital.html> (Stand: 07.01.2019) eingesehen werden.

7 Datenschutzkonform werden keine personenbezogenen Daten erhoben, sondern lediglich der Zugriff auf eine Seite sowie die bloße Aktion des Suchens (d. h. ohne Suchparameter etc.) gezählt. 


\subsection{Mehrwert eines digitalen Workflows}

\subsubsection{Datenbank vs. Excel: Geschwindigkeit}

In Excel ist es zwar möglich, alle Spalten anhand der Inhalte mindestens einer von ihnen zu sortieren, allerdings ist dies dateiübergreifend nicht ohne Weiteres möglich und zudem auf manchen Arbeitsrechnern sogar so langsam, dass es zum Programmabsturz kommt. Das ist vor allem dann problematisch, wenn zuvor getätigte Änderungen nicht gespeichert wurden und durch den Absturz verloren gehen. Bezüglich der Suche innerhalb einer oder mehrerer Excel-Dateien stehen Nutzer/-innen vor dem Problem, die Treffer nicht in einer Übersicht zu erhalten, sondern diese nacheinander per Tastendruck anspringen zu müssen.

Die Verwendung einer relationalen Datenbank leistet in beiden Fällen zuverlässig Abhilfe: Die Art und Weise der Speicherung der Daten ermöglicht nicht zuletzt aufgrund der verwendeten Indizes schnelle Suchen, sondern auch schnelle Sortierungen, selbst über mehrere Spalten. Das Absetzen einer Such- und/oder Sortieranforderung und die anschließende Übermittlung des Ergebnisses ist deutlich schneller, als dies innerhalb von Excel trotz etwaiger Makros oder Programme realisierbar wäre. ${ }^{8}$ Darüber hinaus erfordert der Vorgang keinerlei Rechenressourcen des Benutzercomputers, da alles auf dem leistungsfähigeren Server ausgeführt wird. Aus diesem Grund besteht auch kein potentielles Sicherheitsrisiko (vgl. Fußnote 8). Die Erstellung von Listen über den gesamten Datenbestand hinweg erlaubt es der Redaktion, schnell und zielgenau zu überprüfen, ob eine bestimmte grammatische Annotation bereits erfasst wurde und welche Belege diese enthalten. Auch Fragestellungen der Art ,Welche besonderen morphologischen Phänomene gibt es im Fränkischen“ können so effizienter mit Belegen versehen werden, da alle Inhalte des Grammatikfeldes gruppiert dargestellt besser durchsuchbar sind und somit seltenere oder ungewöhnlichere Angaben und zugehörige Belege leichter aufgefunden werden können, als dies in der tabellarischen Ansicht aller Belege der Fall ist diese müssten bei dieser Fragestellung nämlich alle durchgesehen werden. Für die Redaktion ergibt sich aus den Listen zudem der Vorteil, dass vereinheitlichende Korrekturen der Art „diverse Werte ähnlich A“ zu „Wert B“ durch das gezielte Auffinden des zu korrigierenden Musters einfacher vorgenommen werden können.

8 Programme, die innerhalb Excel selbst laufen, können in der von Microsoft entwickelten Programmiersprache Visual Basic for Applications (kurz: VBA) geschrieben werden. Sie ist eine für Office-Programme abgespeckte Variante der Sprache Visual Basic und ermöglicht die Manipulation von Excel-Arbeitsblättern sowie die Darstellung von individuellen Benutzeroberflächen mit Auswahllisten, Textfeldern, Optionsbuttons u. Ä. Bei strikten Sicherheitseinstellungen des Computers ist die Ausführung dieser Programme jedoch nicht möglich, da durch den möglichen Zugriff auf das Dateisystem ein potentielles Sicherheitsrisiko besteht. 


\subsubsection{Automatismen}

Unser Gehirn ist im Abstrakten noch wesentlich leistungsfähiger als ein Computerprozessor, kann jedoch deutlich langsamer rechnen und einzelne Aufgaben schlecht parallelisieren. ${ }^{9}$ Aus diesem Grund eignen sich Prozessoren hervorragend dafür, Millionen von Wörtern miteinander zu vergleichen, Quersummen oder Zeichenentfernungen zu berechnen, ähnliche Wörter zu finden oder tausende Seiten Text in morphologische Einheiten zu zerlegen. Die Regeln, die hierfür nötig sind, können in einer Programmiersprache formuliert und anschließend per Tastendruck ohne weiteres Eingreifen der Reihe nach durchlaufen werden, um innerhalb weniger Minuten oder Stunden automatisiert das auszuführen, wozu der Mensch Monate oder gar Jahre benötigt. Im WBF sind derzeit folgende Automatismen implementiert:

\subsubsection{Schnipselgenerierung}

Bislang sind lediglich die Scans der Nachkriegsbogen (1961-2001) online abrufbar. Im Rahmen dieser Erhebung wurden 122 Fragebogen mit jeweils bis zu 60 Fragen an mehrere Hundert Sammler verschickt. Als Rücklauf erreichten fast 48000 Fragebogen das Wörterbuch. Die Zahl der einzeln darzustellenden Ausschnitte beläuft sich auf rund 2,8 Millionen, die so aufzubereiten bzw. darzustellen sind, dass via Suchoption direkt zur gewünschten Frage gesprungen werden kann. Diese Menge manuell zu bearbeiten, war zeitlich wie auch finanziell nicht möglich. Deshalb wurde ein Zusatz für LexHelfer entwickelt, der es ermöglichte, für jeden Fragebogen bei jeweils einem Exemplar die Koordinaten der Fragen zu erfassen. Da die Platzierung der Fragen innerhalb eines Fragebogens mit einer vernachlässigbaren Abweichung weniger Pixel über alle entsprechenden Scans hinweg identisch ist, konnte anhand dieser jedes Vorkommnis einer Frage damit versehen werden.

Die Erfassung der Koordinaten wurde in ca. acht Stunden von Hilfskräften vorgenommen; die Entwicklungs- und Testphase des Tools dauerte ca. zwei Arbeitstage. Die automatisierte Eintragung der Daten aller Scans lief im Anschluss an die Erfassung in wenigen Stunden auf dem Server bei geringer Auslastung. Die dadurch erzielte Zeitersparnis ist enorm.

Personenbezogene Daten, die aus datenschutzrechtlichen Gründen nicht öffentlich zugänglich sein dürfen und sich unserer bisherigen Kenntnis nach auf der ersten oder letzten Seite eines Bogens im dortigen Adressfeld befinden, konnten mittels selbiger Methode wirkungsvoll geschwärzt werden: Die Koordinaten der Adress-

9 Ein Mensch kann z. B. ein Gesicht in Sekundenbruchteilen erkennen und den Namen sowie weitere Informationen zur betreffenden Person abrufen, wohingegen eine Maschine allein für den Analysevorgang der Gesichtszüge vergleichsweise lange benötigt. Liegen der Maschine letztlich alle Informationen vor, sind diese allerdings deutlich schneller verarbeitet, als das menschliche Gehirn es zu leisten vermag. Siehe hierzu HoRNYAK (2013), Human Brain Project (2017) und JOHN (2016). 
feldbereiche wurden im Zusatztool erfasst und anschließend automatisiert via Bildbearbeitung mit einem passenden, farblich gefüllten Rechteck überschrieben. ${ }^{10} \mathrm{Die}$ Originaldatei ist für Redakteur/-innen weiterhin im System einsehbar; lediglich die öffentlichen User/-innen haben keine Möglichkeit, die ungeschwärzten Originale abzurufen. Auch in diesem Fall hätte eine manuelle Schwärzung der Adressbereiche einen enormen Zeitaufwand bedeutet.

\subsubsection{Sachgruppenzuordnung}

„Welche Dialektausdrücke gibt es bei uns im Bereich der Landwirtschaft“ oder „Welche Wörter haben wir rund um die Taufe“" sind denkbare Fragen von Wissenschaftler/-innen und der Allgemeinheit, die allein über Suchanfragen, die nur das Lemma berücksichtigen, nicht beantwortet werden können, da zu diesem keine Verbindung zum jeweiligen Themengebiet besteht. Auch über die Bedeutung lassen sich nur wenige Belege auffinden und auch nur dann, wenn explizit nach ihr gesucht wird (z. B. „Taufe“ als Bedeutung von Kinsschenk, 'Kindsschenke'). Eine von Bedeutung, Grundform und Lemma unabhängige Suche muss sich daher eines anderen Feldes bedienen. Im WBF wird hierfür die dankenswerterweise von RUDOLF POST (1998) in digitaler Form bereitgestellte und auf der Arbeit von HALLIG / V. WARTBURG (1963) basierende, leicht erweiterte Sachgruppenliste und -zuordnung verwendet. ${ }^{11}$

Ausgehend von der Zuordnung, die innerhalb des „Pfälzischen Wörterbuchs“ (nachfolgend: PfWB) vorgenommen wurde, verglich ein Skript dessen vorhandene Einträge mit den Belegen des WBF und wies bei vollständiger Übereinstimmung von Grundform und Bedeutung die im PfWB genannte Sachgruppe zu. Der Vorgang wurde im Frühjahr 2017 testweise mit dem damals aktuellen Daten-Set von ca. 750000 Belegen durchgeführt. Von diesen 750000 Einträgen wurden lediglich ca. 78000 eindeutig zugewiesen, da es bei diesen eine 1:1-Zuweisung beider Datenquellen bezüglich der Feldinhalte von Grundform und Bedeutung gab. Aus diesem Grund wurde der Versuch unternommen, mit der sog. Levenshtein-Distanz eine Verbesserung zu erwirken. Bei diesem Algorithmus wird mit einer definierbaren Gewichtung eine Aussage über die Ähnlichkeit zweier Zeichenketten getroffen, anhand derer man die Wahrscheinlichkeit der Übereinstimmung angeben kann. ${ }^{12}$

10 Via PHP-interner Bibliothek GD.

11 Die Sachgruppenzuordnung nach HALLIG / V. WARTBURG (1963) wird in (teils erweiterter) Form im „Schweizerisches Idiotikon“ verwendet sowie im „Badischen Wörterbuch“, im „Pfälzischen Wörterbuch“ und im „Dialektologischen Informationssystem für Bayerisch-Schwaben“. Im „Badischen“ und „Pfälzischen Wörterbuch“ findet die Sachgruppenliste jedoch eine bisher ausschließlich interne Verwendung; die Zuordnungen sind unseres Kenntnisstandes nach bis dato nicht veröffentlicht.

12 Der Levenshtein-Distanz liegt keine künstliche Intelligenz o. Ä. zugrunde, sodass sehr strikt und ausnahmslos auf die Schriftzeichen einer Zeichenkette geachtet wird, nicht jedoch auf die 
Die erste Zeichenkette für den Vergleich war die Bedeutung des WBF-Belegs; die zweite der Reihe nach jeder Eintrag des PfWB und seiner Sachgruppenzuordnung. Das beste Ergebnis und somit die geringste Distanz wurden hierbei gewählt. Andernfalls wurde in der einfacheren Variante keine Sachgruppe zugewiesen; in der erweiterten ein zweiter Durchlauf mit allen Grundformen des PfWB unternommen.

Die Gewichtung in unserem Test betrug jeweils den Wert 1 für das Ersetzen eines Zeichens, das Hinzufügen und das Löschen eines Zeichens. Die Länge der Wörter wurde stets mit in den Vergleichswert eingerechnet. Wie in Tabelle 1 dargestellt, lieferte die Zuordnung bei Feuermännchen je nach Wahl der Methode (einfach/erweitert) einmal 7350 (Brauchtum [auch Schreckgestalten]) und einmal 8810 (Volksglaube, Aberglaube) - beides korrekte und semantisch sehr nahstehende Felder. Bei Schnecke im Sinne von 'Gebäcksorte' ist die Sachgruppe 5911 (fertig zubereitete Speisen) ebenfalls treffend.

\begin{tabular}{|c|c|c|c|}
\hline Lemma & Grundform & Bedeutung & Sachgruppe \\
\hline Ysop & Joseferer & $\begin{array}{l}\text { Pflanze, die Duft- } \\
\text { blatt für das } \\
\text { Gebettuch [sic] }\end{array}$ & 3000 \\
\hline Mann & Feuermännchen & Traumgespenst & $7350 / 8810$ \\
\hline Schnecke & Schnecke & Gebäcksorte & 5911 \\
\hline Schnecke & Schnecke & Schnecke & 5210 \\
\hline Falle & Mausefalle & Bett & --- \\
\hline
\end{tabular}

Tab. 1: Beispielhafte Ergebnisse der Anwendung der Levenshtein-Distanz

Für das Tier Schnecke würde man zweifelsohne einen eindeutigen Treffer erwarten, aber da Schnecke im PfWB mit dem zoologischen Fachbegriff Gastropoda gelistet ist, erzielt die beschriebene Methode aufgrund des Fehlens dieses Wortes im DatenSet einen fehlerhaften Treffer. Ähnlich verhält es sich bei Mausefalle, die keine Zuordnung erreichte. Dieses Problem könnte folgendermaßen gelöst werden: Die Sachgruppenzuweisung wird um einen dritten Schritt erweitert, in dem die Sachgruppe des ersten Lemma-Eintrags aus dem PfWB verwendet wird, falls dieser mit der Bedeutung des WBF-Eintrags übereinstimmt, d. h. beide Zeichenketten identisch sind. So bekäme man im Beispiel der Mausefalle mit der Bedeutung 'Bett' aus dem WBF die Sachgruppe des ersten Eintrags des PfWB zu Bett, nämlich 7730 (Einrichtung). Für Schnecke erhielte man auf diese Weise die ebenfalls korrekte Sachgruppe 4500 (Weichtier), da der erste Eintrag des PfWB mit der Zeichenabfolge Schnecke diesem zugeordnet ist. Diesen dritten Vergleich als Letztes zu nutzen, ist wichtig, da andernfalls bei einer Vielzahl von Bedeutungen immer nur die erste, primäre Be-

Bedeutung. Die beiden Lexeme Haus und Heim ergeben eine hohe Abweichung von 3 (maximal wäre 4); die Lexeme Haus und Maus hingegen lediglich eine Abweichung von 1. Inhaltlich sind sich allerdings Haus und Heim deutlich näher als Minimalpaare wie Haus und Maus. 
deutung des Lemmas für die Sachgruppenzuordnung verwendet wird und diese oftmals nicht korrekt ist. Der vorherige Abgleich der Bedeutungsfelder beider Wörterbücher sowie der zwischen Grundform des WBF und Bedeutung des PfWB muss daher Vorrang haben.

Die Zuordnung von Josefer zur Sachgruppe 3000 (Pflanze und ihre Frucht) ist aufgrund der 1:1 Zuordnung des ersten Teils der Bedeutungsangabe 'Pflanze' zustande gekommen, da in einer Optimierung der Zeichenketten versucht wird, attributive Nebensätze auszufiltern.

Insgesamt beträgt die Fehlerquote bei Verwendung der Levenshtein-Distanz rund $50 \%$ und ist damit in der Tat sehr hoch. Die Untersuchung der ersten tausend Einträge des Buchstabens $F$, die im Rahmen einer Bachelor-Arbeit ausgeführt wurde, gibt ebenfalls nahezu $50 \%$ korrekte Zuweisungen an (siehe CHRISTIANI 2017: 27-29). Man muss allerdings bedenken, dass die korrekt gesetzten Sachgruppen mehrere 100000 Einträge bedeuten, die nach einer kurzen Prüfung nicht weiter korrigiert werden müssen. Je mehr korrekte Zuordnungen erfasst sind, desto besser verlaufen künftige Durchläufe mit neuem Datenmaterial, da zu erwarten ist, dass in diesem mehrere Zuordnungen direkt vorgenommen werden können. Die Annahme wird gestützt durch den Umstand, dass die direkten Treffer zu $90 \%$ korrekt waren. Letztlich hängt es davon ab, ob im Import Vorkommnisse von Grundform und Bedeutung gegeben sind, die im korrigierten Sachgruppenzuordnungsbestand, der als primärer Vergleichswert dient, enthalten sind. Bei größtmöglicher fehlerfreier Liste sinkt die Wahrscheinlichkeit, dass im neuen Daten-Set zu viele Lücken sind.

\subsubsection{Artikelkompilierung}

Dank der granularen Erfassung der Beleginformationen können Wörterbuchartikel automatisch generiert werden. Die aktuellen Artikelkompilate dienen zum einen der Redaktion zur Kontrolle der vorhandenen Daten eines Lemmas, da sich durch die feldbasierte Gruppierung aller Einträge Fehler schneller auffinden lassen als dies bei der Durchsicht aller (meist hunderter) Zeilen möglich ist. Die klassische Ansicht des Artikels erleichtert das Lesen deutlich. Sind aus Sicht der Redaktion keine Fehler mehr im Datenbestand enthalten, kann der generierte Artikel freigegeben werden, damit er der Öffentlichkeit zur Verfügung steht und alle Informationen der Datenbank minimalisiert enthält, die zum jeweiligen Lemma vorhanden sind. Durch den URL-Kürzungsdienst der Bayerischen Akademie der Wissenschaften kann zudem eine persistente Kurz-URL zum Artikel erzeugt werden, um diesen platzsparend in Publikationen zu verwenden. ${ }^{13}$ Die jeweilige Freigabe bleibt unveränderbar im System gespeichert und ist damit zitierfähig - bei inhaltlichen Änderungen kann jederzeit eine neue Version des Artikels erstellt werden, die wiederum zitierfähig und persistent abrufbar ist.

13 <https://purl.badw.de> (Stand: 07.01.2019) stellt für BAdW-Projekte gekürzte, persistente URLs bereit. 
Zum anderen ist ein solcher Artikel insbesondere für fachfremde Nutzer/-innen aus ähnlichen Gründen angenehmer zu verwenden als die tabellarische Auflistung aller Belege: Die für Wörterbücher typische und vertraute Struktur bietet eine bessere Lesbarkeit der Inhalte. Im Rahmen der Projektmöglichkeiten und unter Einbeziehung der Erfahrungen ähnlicher Projekte wird die Struktur weiter optimiert, um etwaige Hürden bezüglich der Benutzerfreundlichkeit $\mathrm{zu}$ minimieren (siehe GEYKEN / WIEGAND / WÜRZNER (2017) und ZIMMER (2017). Außerdem soll erprobt werden, ob z. B. die Etymologie (semi-)automatisch nachgepflegt werden kann, um einen zusätzlichen wissenschaftlichen Mehrwert erzielen zu können (siehe SAGOT 2017).

Ausgehend vom automatisch generierten Inhalt kann durch mehrere Verlinkungen jederzeit auf die tabellarische Ansicht zugegriffen werden, um bei Bedarf ohne manuelle Formulierung der entsprechenden Suche die Minimalisierung, die durch die Zusammenfassung entsteht, aufzuheben und alle Belegdaten einsehen zu können. Durch diese Möglichkeit kann ein freigegebener Artikel auch der Fach-Community als Übersichtsseite eines Lemmas dienen - dies ist insbesondere dann nützlich, wenn keine tiefergehenden Suchanfragen Gegenstand des Zugriffs sind, sondern lediglich kurz etwas im Belegbestand eines Lemmas nachgelesen werden soll.

Ein weiterer Mehrwert eines Artikelkompilats ist dadurch gegeben, dass es in XML gefasst ist und künftig sowohl das BWB als auch das DIBS Artikel nativ in XML veröffentlichen, sodass letztlich alle bayerischen Wörterbücher eine einheitliche, aus einer gemeinsamen XML-Basis hervorgehenden Online-Ansicht bereitstellen können. Abweichungen, die durch unterschiedliche Strukturen der Wörterbücher bedingt sind, behindern diesen Ansatz nicht, da das XML an vielen Stellen fakultative Elemente bzw. Attribute enthält und die Basis bei allen identisch ist.

\subsubsection{Duplikate entfernen}

Im Laufe der Zeit haben sich in den Excel-Listen vergleichsweise wenige Duplikate eingeschlichen, die dennoch störend waren und manche Ergebnisse unnötig vervielfacht haben. Die Duplikate können effizient durch eine einzelne SQL-Abfrage aufgefunden werden. In ihr werden alle Einträge geprüft, indem die einzelnen Felder der Eintragstabelle in Kombination mit der Sachgruppe gruppiert und anschließend jene gelöscht werden, die in der Gruppenzählung einen Wert größer 1 aufweisen. Müsste eine einzelne Person diese Aufgabe ausführen, bräuchte sie aufgrund der großen Datenmenge und der Anforderung, buchstabengetreu zu vergleichen und zu gruppieren, nicht weniger als ein Jahr. Auf dem Server hingegen sind die Abfrage sowie die anschließende Löschung in weniger als einer Minute abgeschlossen. Der Vorgang wird nun automatisch nach jedem Datenimport ausgeführt, um etwaige vorhandene Duplikate neuer Dateien ohne Mehraufwand direkt zu entfernen. 


\subsection{Technik}

Die Anwendung läuft auf einem virtuellen Server im Leibniz-Rechenzentrum, auf dem Suse Linux Enterprise Server in Version 12 als Betriebssystem eingesetzt wird sowie Apache 2.4 als Webserver mit Einbindung von PHP 7.0 und darüber angebunden MariaDB ${ }^{14}$ 10.0.32 als SQL-Datenbankmanagementsystem. Ferner werden wenige Skripte mittels Perl 5.18 ausgeführt. Das Setup des Servers ist demnach ein klassischer LAMP, in welchem die Servervariablen der einzelnen Komponenten bedarfsentsprechend gesetzt wurden. ${ }^{15}$ Die Speicherung aller Daten findet auf virtualisierten Magnetspeicherplatten statt, die einen Datendurchsatz von durchschnittlich ca. $600 \mathrm{MB} / \mathrm{s}$ beim Schreiben sowie über $2 \mathrm{~GB} / \mathrm{s}$ beim Lesen liefern. Das ist vollkommen ausreichend, vor allem vor dem Hintergrund, dass in den Anwendungen vergleichsweise geringe Datenmengen gelesen/geschrieben werden müssen. Die zwei Prozessorkerne sowie die acht Gigabyte Arbeitsspeicher sind aktuell ebenfalls noch mehr als ausreichend, um alle Aufgaben in angemessener Zeit auszuführen. Bei der Übertragung der Scans profitiert LexHelfer von der schnellen Gigabit-Anbindung, die das Leibniz-Rechenzentrum bereitstellt. Login-Mechanismen im gesamten System bedienen sich aktueller, sicherer Verschlüsselungsalgorithmen und -verfahren, sodass Unbefugte keinen Zugriff auf die einzelnen nichtöffentlichen Bereiche oder gar das ganze System erhalten.

\subsubsection{Probleme}

\subsubsection{Belegerfassung}

Leider ist es aufgrund der Vielzahl unterschiedlicher Handschriften bisher nicht möglich, Texterkennungstechniken (OCR, ICR) zu verwenden, um die Originalbelege in akkurate computerlesbare Form zu bringen. Geeignete Programme zu trainieren und deren Ausgabe zu korrigieren, wäre so aufwändig, dass das Abtippen letztlich schneller ist. Es gab im Verlauf der letzten Jahre zwar Fortschritte in diesem Bereich, jedoch keine, die Kurrentschrift ausreichend fehlerfrei in maschinenlesbaren Text umwandeln. Die via Schreibmaschine beantworteten Fragen könnten zwar relativ zuverlässig eingelesen werden, aber hier ergibt sich anschließend das Problem, dass die Exemplare mit Kurrentschrift manuell aus der Ergebnisliste ausgefiltert werden müssten. Auch die nicht seltenen Fälle mit Über-/Unterschreitung der Fragengrenzen (z. B. aufgrund sehr langer Antworten, die mehr Platz als das eigentlich vorgesehene Antwortfeld benötigten; Kommentare, die der Antwort hinzugefügt wurden) müssten ebenfalls manuell begutachtet und korrigiert werden, sodass die gesamte Korrekturphase keinen zeitlichen Vorteil mehr liefern dürfte. Die

14 Anstatt des SQL-Dialekts MySQL, der bis vor wenigen Jahren standardmäßig innerhalb eines LAMPs verwendet wurde, fiel die Wahl aufgrund der höheren Leistungsfähigkeit und offeneren Lizenz auf dessen Derivat MariaDB.

15 LAMP = Linux, Apache, MariaDB, PHP. 
Texterkennung selbst wäre auch bei 48000 Seiten innerhalb einiger Tage selbstständig vom Computer erledigt, aber die anschließende Korrektur würde enormen personellen und zeitlichen Aufwand erfordern.

\subsubsection{Generizität}

Seit vielen Jahren haben Redaktionssysteme zunehmend den Anspruch, generisch zu sein, dadurch eine Vielzahl ähnlicher oder gar gänzlich unterschiedlicher Projekte bedienen zu können und alle damit behandelten Projektdaten untereinander vernetzbar zu machen. Generizität der Programmierung erfordert allerdings im Vergleich zur projektspezifischen Softwareentwicklung einen deutlich höheren Aufwand und ist letztlich in vielen Fällen trotzdem nicht hundertprozentig gegeben falls das Ergebnis überhaupt als generisch bezeichnet werden kann. Die Versuche, eine Software für ,alles“ zu entwickeln, sind grundsätzlich nicht uninteressant und eine reizvolle Herausforderung, scheitern aber meist schon in der Planung an der Logik: Ein Programm kann nicht ohne Grenzen grundsätzlich verschiedene Daten bzw. Funktionsanforderungen gleichermaßen zufriedenstellend verarbeiten bzw. erfüllen. In der Theorie klingt das oft noch machbar, doch die Praxis zeigt immer wieder ernüchternd, dass es nicht (annähernd) zu $100 \%$ realisierbar ist und sich der Mehraufwand letztlich nicht lohnt. Auf der Seite der Daten funktioniert der generische Ansatz gut, wenn sie in XML vorliegen. Die benötigten Funktionen allerdings bleiben das Manko, das durch Erweiterungen (Plug-Ins) ausgeglichen wird und dies wieder einiger Entwicklungszeit bedarf. Falls keine zwingende Notwendigkeit an einer generischen Lösung besteht bzw. sie sich nicht ohnehin nahezu automatisch aus der Aufgabenstellung heraus ergibt, ist es im Hinblick auf die meist sehr begrenzten Ressourcen ratsam, darauf zu verzichten - auch wenn dies gerne Kritik hervorbringt.

Aus diesem Grund ist LexHelfer eine Nischenlösung, die bewusst nur für die drei Wörterbuchprojekte verwendet wird, für diese allerdings maßgeschneidert programmiert wurde und die Bedürfnisse der Redaktionen vollständig berücksichtigt. Projekte, die ähnliche Datenbasen und Ansprüche an den Funktionsumfang haben, könnten die Anwendung nach Anpassungen ebenfalls verwenden. Daher wird eine Veröffentlichung des Quellcodes auf Github erfolgen. ${ }^{16}$

\subsection{Nachhaltigkeit}

Im Bereich der Digital Humanities steht der Begriff der Nachhaltigkeit primär in Zusammenhang mit verwendeten Programmen, Programmiersprachen und Datenformaten, die allesamt auch in vielen Jahren noch zugänglich und weitgehend etabliert sein sollten, um Anpassungen oder Migrationsvorhaben auf künftige Systeme 
oder Formate durchführen zu können. Grundsätzlich eignen sich quelloffene Software sowie offene Datenformate deutlich besser als proprietäre. Insbesondere eignen sich jene, die in der Informatik als Standards angesehen werden und im jeweiligen Anwendungsgebiet weit verbreitet sind. Aus diesen Gründen wurde LexHelfer als Client-Server-Webanwendung konzipiert, die serverseitig auf die Scriptsprache PHP sowie das SQL-Datenbankmanagementsystem MariaDB setzt und clientseitig lediglich einen modernen Browser mit aktiviertem JavaScript benötigt. Einige Skripte zum Datenimport wurden zudem in Perl 5 geschrieben.

Eine verlässliche Aussage darüber, ob die gewählten Sprachen und Formate auch in zehn Jahren noch gängig sind, lässt sich offen gesagt nicht mit Sicherheit treffen, da Änderungen in der IT schnell voranschreiten können. Aufgrund der enorm starken Verbreitung von PHP und SQL sowie deren Bestehen seit über 20 Jahren ist es jedoch äußerst unwahrscheinlich, dass sich in zehn Jahren niemand mehr für Anpassungen oder Migrationsvorhaben finden lässt - falls diese dann überhaupt nötig sein sollten. Gleiches gilt für das immer stärker werdende JavaScript. In Bezug auf Perl 5 ist dies ähnlich, obgleich die Sprache in den letzten Jahren zunehmend von Python überholt wurde. Langfristig könnte es also schwieriger werden, IT-Unterstützung für Perl zu finden. Allerdings ist der vorhandene Perl-Code sauber unter Einhaltung gängiger Konventionen geschrieben sowie dokumentiert, sodass dank der syntaktischen Ähnlichkeit aller drei Sprachen auch ein/-e PHP- oder Python-Entwickler/-in damit zurechtkäme.

Die Forschungsprimärdaten liegen als schwach komprimierte JPEG-Bilddateien vor. Es wurde kein verlustfreies Format gewählt, da die Datenmenge andernfalls nicht mehr handhabbar geworden wäre und im Falle eines Bitfehlers im ursprünglichen Scan, der das Bild vollständig unzugänglich macht, ohnehin der Originalbogen nachgescannt werden kann. ${ }^{17}$ Die schwache Komprimierung reduziert die Dateigröße ohne erkennbare Einbußen der Qualität. Die Dateien sind in einem professionellen Backup- und Archivierungssystem am Leibniz-Rechenzentrum gespeichert und im Falle eines Datenverlusts auf dem Webserver schnell wiederherstellbar. Bisher sind keine Bitfehler in automatischen Verarbeitungen der Dateien aufgetreten, sodass davon ausgegangen werden kann, im Backup am Rechenzentrum zu $100 \%$ integre Daten zu haben.

17 Bei verlustbehaftet komprimierten Bildformaten kann im schlimmsten Fall bereits ein fehlerhaftes Bit dafür sorgen, dass die Datei nicht mehr lesbar ist und Reparatursoftware diesen $\mathrm{Zu}$ stand nicht korrigieren kann, sodass alle Bildinformationen der Datei letztlich unwiderruflich verloren sind. Die Scans des WBF sind alle soweit fehlerfrei, dass die Bilder geöffnet und dargestellt werden können. Hierfür wurden sie alle automatisiert geöffnet, um bei fatalen Fehlern eine entsprechende Meldung zu erhalten. Scanfehler können dadurch nicht erkannt werden, jedoch hängen diese (im Sinne eines Übertragungsfehlers beim Speichern) nicht mit dem Format zusammen und stellen somit allein auch keinen Grund für ein verlustfreies Verfahren bzw. Format dar. 


\section{5 Öffentliche Sichtbarkeit und Nutzung}

Durch die automatische Bereitstellung einer auf den Lesezugriff beschränkten Version des Redaktionssystems ist es der breiten Öffentlichkeit möglich, auf alle Inhalte der Datenbank zuzugreifen und dabei den jeweils letzten Bearbeitungsstand einsehen zu können. Der granularen Erfassung der Beleginformationen wird es zu verdanken sein, dass regelmäßig Suchabfragen getätigt werden, die nicht von den Redaktionsmitgliedern oder Studierenden stammen. ${ }^{18}$ Es zeigt sich dadurch, dass reges Interesse am Projekt und dessen Arbeit besteht sowie offenkundig interessierte Laien nachschlagen, ob und welche Dialektbegriffe in z. B. ihrer Heimatregion attestiert sind. Dies unterstreicht die Wichtigkeit des Projekts auch außerhalb der Fach-Community.

Seit öffentlicher Onlinestellung der Datenbank im Februar 2017 gab es bis einschließlich 31. 05. 2018 insgesamt rund 400000 Aufrufe, von denen $99 \%$ die Suche betreffen und der geringe Rest die Listenansichten oder die Hilfeseite. Monatlich sind rund 1500 bis 2000 einzelne Besucher/-innen (unique visitors) in der Anwendung aktiv. ${ }^{19}$ Die Datenbankinhalte können außerdem durch eine öffentlich zugängliche API abgefragt werden. ${ }^{20}$ Von weiteren Funktionen und einer multimedialen Anreicherung der Belege verspricht sich die Redaktion wie auch die IT-Abteilung der BAdW eine noch intensivere Nutzung und eine noch größere öffentliche Sichtbarkeit des Wörterbuchs.

18 Der Vollzugriff auf das Redaktionssystem ist nur Usern möglich, die sich via Benutzername und Passwort authentifizieren. Der eingeschränkte Lesezugriff findet ohne Login statt und signalisiert dies dem System. Dadurch kann ohne weitere Datenabfrage und -speicherung datenschutzkonform erfasst werden, wie viele Anfragen aus welchem Nutzerkreis stammen (d. h. Redaktion vs. Öffentlichkeit).

19 Die Genauigkeit der Erfassung eines einzelnen Besuchs prüft - insbesondere im Hinblick auf den Datenschutz - nicht exakt durch Cookies o. Ä., sondern basierend auf Webserver-Logdateien, welche Zugriffe einer Maschine zugeordnet werden können, hinter der sich zumeist eine einzelne Person befindet. Weder IP-Adresse noch Host des Zugriffs werden dabei aufgenommen, sondern lediglich der Zähler iteriert. Dies geschieht tagesaktuell, da die Webserver-Logs nicht dauerhaft gespeichert werden (dürfen). Vor dem Hintergrund, dass bei den meisten Internet-Serviceprovidern eine kurzzeitige Trennung der Verbindung nach 24 Stunden stattfindet und im Anschluss meist eine neue IP-Adresse zugewiesen wird, kann man eine Zahl von z. B. 1000 eindeutigen Besuchern und Besucherinnen pro Tag so deuten, dass dies tatsächlich nahezu 1000 einzelne Personen sind. Im Zeitraum von einem Monat hingegen kann nur noch eine sehr vage Schätzung der eindeutig einzelnen Besucher/-innen gegeben werden. Bei einer tagesaktuellen Betrachtung zeigt sich für das WBF für März, April und Mai 2018, dass die Datenbankanwendung an jedem Tag des jeweiligen Monats von durchschnittlich 73 Benutzer/innen aufgerufen wurde.

$20<$ http://www.wbf.badw.de/wbf-digital/wörterbuch/api/showInfo=yes>; Stand: 07.01.2019. 


\subsection{Ausblick}

Die Datenbestände der an der Bayerischen Akademie der Wissenschaften ansässigen Dialektwörterbuchprojekte BWB, WBF und DIBS werden aller Voraussicht nach zusammen mit dem Datenbestand der BayDat Ende 2018/Anfang 2019 in einer gemeinsamen Anwendung namens Bayerns Dialekte Online (BDO) online gehen, sodass Fachwissenschaftler/-innen wie auch interessierte Laien alle Materialquellen und Wörterbucheinträge durchsuchen und miteinander vergleichen können. Für Letzteres steht insbesondere die Sachgruppenzuordnung im Vordergrund, die weiter ausgebaut werden soll, um unabhängig des Lemmas eine verlässliche Verknüpfung von Dialektwörtern über die jeweiligen Dialektgrenzen hinweg anbieten zu können. Davon unabhängig können die einzelnen Datenquellen stets über das Lemma oder die Bedeutung miteinander verbunden werden. Durch eine Sachgruppenvernetzung wäre es zudem ohne Mehraufwand möglich, ein in seiner Form und Informationsfülle bis dato einzigartiges deutsches Sachgruppenwörterbuch bereitzustellen (vgl. hierzu Arbeiten von DORNSEIFF 1934 und HALLIG / VON WARTBURG 1963), das online durchsuchbar ist und im Gegensatz zu anderen Ansätzen auf Millionen empirischer Belege basiert, zu welchen stets der jeweilige Kontext der gestellten Frage sowie alle Metadaten online nachverfolgt werden können. Dadurch eröffnet sich die Möglichkeit, Fragestellungen zu semantischen Feldern auch geokontrastiv in nativ-digitaler Form zu bearbeiten.

Die Belegdaten der fränkischen Bezirke innerhalb der BayDat werden zudem mittels eigens erstelltem Tool innerhalb der nächsten zweieinhalb bis drei Jahre lemmatisiert, annotiert und korrigiert, sodass diese Daten anschließend in den Bestand des WBF-LexHelfer aufgenommen werden können. ${ }^{21}$ Das Tool ist bezogen auf diese Datenquelle sozusagen generisch und kann somit auch zur Lemmatisierung und Informationsanreicherung der Belege der anderen Regierungsbezirke genutzt werden.

Ferner sind weitere Exportfunktionen, Visualisierung (Kartierung) und Anreicherung mit multimedialen Inhalten geplant. Die bereits erwähnte Einbindung eines etymologischen Wörterbuchs in die Artikelkompilierung, eine unscharfe/phonetische Suche, die Migration der Perl-Skripte auf Python oder PHP, eine erweiterte index-basierte Speicherung der für die Suche relevantesten Datenbankfelder oder die nur für die Redaktion sichtbare Einbindung aller Informationen zu Gewährspersonen sind weitere denkbare Entwicklungen, zu denen es aktuell allerdings noch keine konkrete Planung gibt.

21 Die Integration der Daten wird von der Oberfrankenstiftung, der Unterfränkischen Kulturstiftung und der Stiftung des Bezirks Mittelfranken finanziell unterstützt. 


\section{LINGUISTISCHES POTENTIAL}

Als Erstes der großräumigen Dialektwörterbücher im deutschen Sprachraum, macht das WBF über seine Volltextdatenbank alle Belege mit Sozialdaten versehen ortspunktgenau abruf- und auswertbar. Forschungsergebnisse können unter Rückgriff auf die Datenbank vollständig transparent dargestellt werden und sind jederzeit bis zu den Quelldaten nachvollziehbar. Damit liefert das WBF eine Datenquelle, deren linguistisches Potential kaum abgeschätzt werden kann. Neben Material für Fragestellungen zur Phonem-Graphem-Beziehung, Morphologie, Lexik, Syntax, Grammatikalisierung, Sprachwandel usw. bietet die Datenbank ausreichend Material für statistisch signifikante Untersuchungen, die sich der Methoden der Mustererkennung bedienen. Zusammen mit der IT-Abteilung der BAdW wollen wir die Datenbestände des WBF für den von April bis Mai 2019 stattfindenden Coding Da Vinci Hackathon-Süd zur Verfügung stellen, bei dem es darum geht, „technikaffine und kulturbegeisterte Communities mit deutschen Kulturinstitutionen“ [zu vernetzen] (<https://codingdavinci.de/about/index-de.html>; Stand: 10.10.2018).

An der FAU nutzen wir das noch junge Online-Informationssystem - das WBF ist seit 2016 öffentlich zugänglich - bereits als Datengrundlage für den universitären Unterricht in Seminaren zur Dialektologie. Fragen zur Lexik und zur Datenmodellierung stellen wir in unregelmäßigen Abständen im Blog 22 und in Publikationen aus dem Kreis der Projektmitarbeiter vor. So zeichnet HABERMANN (2018) den Gebrauch von lokalen Adverbien als Präpositionen nach, KLEPSCH (2018) diskutiert die Zuordnung von grätschen, Grät und Gratel zu einer Wortfamilie. Als Arbeiten von Dritten liegen v. a. Untersuchungen vor, die sich mit Fragen der Wortbildung auseinandersetzen. Zum Beispiel legt NICKEL (2016) eine Studie zum Fugen-s im Ostfränkischen vor, GANSLMAYER / MÜLLER (2019) diskutieren Fragen der arealen Wortbildung am Beispiel des Adjektivsuffixes -icht/-et. Dialektale Wort- und Bedeutungsbildung untersucht das Projekt „Produktivität und Kreativität in der Lexik des Ostfränkischen“ das, gefördert von der Fritz Thyssen Stiftung, seit Oktober 2018 an der FAU angesiedelt ist. Die Projektleiter CHRISTINE GANSLMAYER, PETER OTTO MÜLLER, HoRST HAIDER MunSKE und ihr Projektmitarbeiter Uli RING nutzen erstmals die Datenbank des WBF, um am Beispiel des Ostfränkischen Strategien und aktuelle Entwicklungen dialektal mündlicher Wortschatzerweiterung durch Wortbildung, Bedeutungsbildung und Entlehnung aufzudecken. ${ }^{23}$ Bezüge zu anderen Sprachen stellt der SLAwIST MiKHAIL KONDRATENKO (Jaroslawl) her, der uns kürzlich in der Forschungsstelle besuchte, um Material aus dem WBF für eine Untersuchung zu deutschen Entlehnungen im Wortschatz für die Gestalten des Volksglaubens in den slawischen Mundarten zu nutzen. 


\section{KONTAKTADRESSE, HOMEPAGE}

Fränkisches Wörterbuch

Friedrich-Alexander-Universität Erlangen-Nürnberg

Arbeitsstelle „Fränkisches Wörterbuch““

Am Weichselgarten 9

91058 Erlangen

Deutschland

E-Mail: fraenkisches-woerterbuch@fau.de

Homepage: <http://www.wbf.badw.de/>

\section{LITERATURVERZEICHNIS}

BICKEL, HANS (2013): Fortschreitende Digitalisierung. Neue Zugriffe auf das Idiotikon. In: 150 Jahre Schweizerisches Idiotikon: Beiträge zum Jubiläumskolloquium in Bern, 15. Juni 2012. Bern, 121-134. URL: <https://www.idiotikon.ch/redaktion/mitarbeitende/bickel>; Stand: 26.09.2016.

CHRISTIANI, CHRISTIAN (2017, unveröffentlicht): Ontologischer Zugriff auf digitale Wörterbücher am Beispiel des Fränkischen Wörterbuchs. Bachelorarbeit an der Universität Würzburg.

DORNSEIFF, FRANZ (1934): Der deutsche Wortschatz nach Sachgruppen. Berlin/Leipzig: de Gruyter.

GanslmaYer, Christine / Peter O. MÜLler (2019): Areale Wortbildung - Das Adjektivsuffix icht/-et. In: Kürschner, Sebastian / Mechthild Habermann / Peter O. MÜller (Hg.): Methodik moderner Dialektforschung. Erhebung, Aufbereitung und Auswertung von Daten am Beispiel des Oberdeutschen. Hildesheim: Olms, 397-426.

GEYKEN, ALEXANDER / FRANK WIEGAND / KAY-MICHAEL WÜRZNER (2017): On-the-fly Generation of Dictionary Articles for the DWDS Website. In: KosEM, IZTOK / MILOŠ JAKUBíČEK / JELENA KALLAS / SiMON KREK (Hg.): Electronic lexicography in the 21st century. Proceedings of eLex 2017 conference. Brno: Lexical Computing CZ s.r.o. URL: <https://elex.link/elex2017/wpcontent/uploads/2017/09/paper34.pdf>; Stand: 24.05.2018.

HABERMANN, MeChTHILD (2018): Lokale Adverbien als Präpositionen - Beobachtungen zum Ostfränkischen. In: FunK, EDITH / ANDREA SCHAMBERGER-HIRT / MiCHAEL SCHNABEL / FELICITAS ERHARD (Hg.): Dialects are Forever. Die unbandige Lust an der Wortklauberey. Festschrift für Anthony R. Rowley zum 65. Geburtstag. Regensburg: edition vulpes, 111-122.

HALLIG, RUDOLF / WALTHER VON WARTBURG (1963): Begriffssystem als Grundlage für die Lexikographie. Versuch eines Ordnungssystems. 2. Auflage. Berlin: Akademie-Verlag.

HORNYAK, TIM (2013): Fujitsu supercomputer simulates 1 second of brain activity. URL: <https://www.cnet.com/news/fujitsu-supercomputer-simulates-1-second-of-brain-activity>; Stand: 22.05.2018.

Human Brain Project (2017): Projektwebseite. URL: <https://www.humanbrainproject.eu/en/brainsimulation>; Stand: 22.05.2018.

JoHn, YoHAN (2016): How Powerful Is The Human Brain Compared To A Computer?. URL: <https://www.forbes.com/sites/quora/2016/03/02/how-powerful-is-the-human-brain-compared-to-a-computer/\#4a15e7f2628e>; Stand: 22.05.2018.

KLEPSCH, ALFRED (2018): Grätschen, Grät und Gratel - Eine mundartliche Wortfamilie mit verwickelten Innen- und Außenbeziehungen. In: FunK, EDITH / ANDREA SCHAMBERGER-HIRT / MICHAEL SchnABEl / Felicitas ERHARD (Hg.): Dialects are Forever. Die unbandige Lust an der 
Wortklauberey. Festschrift für Anthony R. Rowley zum 65. Geburtstag. Regensburg: edition vulpes, $153-164$.

KLEPSCH, ALFRED / ALMUT KÖNIG (2018): Datenerfassung und Datenbeschreibung im WBF. Ein Regelwerk. URL: <http://www.wbf.badw.de>; Stand: 10.10.2018.

KLEPSCH, ALFRED / ALMUT KÖNIG (2016): WBF-Digital. URL: <http://www.wbf.badw.de/wbf-digital.html>; Stand: 10.10.2018.

KLOSA, ANNETTE / CAROLE TiBERIUS (2018): Der lexikographische Prozess. In: DFG-Netzwerk „Internetlexikographie" unter Leitung von KLOSA, ANNETTE und CAROLIN MÜLLER-SPITZER (Hg.): Internetlexikographie. Ein Kompendium. Unter Mitarbeit von MARTIN LODER. Berlin/Boston: de Gruyter, 65-110.

KLUGE, FRIEDRICH (2011): Etymologisches Wörterbuch der deutschen Sprache. Bearbeitet von ELMAR SEEBOLD. 25., durchgesehene und erweiterte Auflage. Berlin/Boston: de Gruyter.

KÖNIG, ALMUT (2019): Wörterbuch im Wandel. In: KÜRSCHNER SEBASTIAN / MECHTHILD HABERMANN / PETER O. MÜLLER (Hg.): Methodik moderner Dialektforschung. Erhebung, Aufbereitung und Auswertung von Daten am Beispiel des Oberdeutschen. Hildesheim: Olms, 499-510.

NICKEL, GRIT (2016): Von Gäul-s-bauer, April-s-narr und Getreid-s-gabel. Die Verwendung und Verbreitung des Fugen-s im Ostfränkischen. In: HENTSCHEL, ELKE (Hg.): Wortbildung im Deutschen. Aktuelle Perspektiven. Tübingen: Narr, 214-243.

PfWB = CHRISTMANN, ERNST / JULIUS KRÄMER (Hg.) (1965-1997): Pfälzisches Wörterbuch. Wiesbaden: Steiner.

POST, RUDOLF (1998): Möglichkeiten der elektronischen Strukturierung, Vernetzung und Verfügbarmachung von lexikographischen Daten bei der Arbeit am Pfälzischen Wörterbuch. In: GROSSE, RUDOLF (Hg.): Bedeutungserfassung und Bedeutungsbeschreibung in historischen und dialektologischen Wörterbüchern. Leipzig: Verlag der Sächsischen Akademie der Wissenschaften, 211-220.

RAAF, MANUEL (2016 bis heute): LexHelfer WBF: Online-Datenbank der Forschungsprimärdaten des Fränkischen Wörterbuchs. URL: <http://www.wbf.badw.de/wbf-digital/wörterbuch>; Stand: 20.06.2018.

SAGOT, BENOÎT (2017): Extracting an Etymological Database from Wiktionary. In: KosEM, IZTOK TANARA ZINGANO KUHN (Hg.): Electronic lexicography in the 21st century. Proceedings of eLex 2017 conference. Brno: Lexical Computing CZ s.r.o. URL: <https://elex.link/elex2017/wp-content/uploads/2017/09/paper44.pdf>; Stand: 24.05.2018.

ZIMMER, BEN: Defining the Digital Dictionary - How to Build More Useful Online Lexical References. Keynote auf der eLex 2017, Leiden. URL: <https://www.youtube.com/watch? v=q2mqx Ylvtlg >; Stand: 24.05.2018. 


\title{
DIALEKTOLOGISCHES INFORMATIONSSYSTEM VON BAYERISCH-SCHWABEN (DIBS)
}

\author{
Brigitte Schwarz / Edith Funk / Manuel Raaf / Ursula Welsch ${ }^{l}$
}

\section{DAS PROJEKT}

Das Projekt „Dialektologisches Informationssystem von Bayerisch-Schwaben“ (DIBS) begann im August 2017 als „Digitales Wörterbuch von BayerischSchwaben“ (WBS) an der Bayerischen Akademie der Wissenschaften (BAdW). Auf der Basis einer bereits 1998 von BRIGITTE SCHWARZ begonnenen Datenbank soll der schwäbisch-alemannische Wortschatz in Bayern dokumentiert und im Internet allen Interessierten zur Verfügung gestellt werden. Das Bearbeitungsgebiet entspricht in etwa dem Regierungsbezirk Schwaben, im Osten jedoch bildet der Lech die Grenze. Das Projekt ist auf 20 Jahre konzipiert und die Finanzierung bis 2021 gesichert. Anfang dieses Jahres gab WERNER KÖNIG die Leitung an ALFRED WILDFEUER ab. Derzeit teilen sich die Redaktorinnen BRIGITTE SCHWARZ und EDITH FUNK eine Stelle. Eine studentische Hilfskraft arbeitet den beiden zu. Für die Informationstechnologie sind URSULA WELSCH und MANUEL RAAF zuständig. Sollte dem Projekt wie beantragt eine weitere Redaktorenstelle zugeteilt werden, dann kann das digitale Wörterbuch um Materialien unterschiedlicher Medienarten erweitert, mit zahlreichen Zusatzfunktionen ausgestattet und dem Projekttitel entsprechend zu einem ,Dialektologischen Informationssystem von Bayerisch-Schwaben“ (DIBS) ausgebaut werden.

\subsection{Der Vorläufer: Das Dialektwörterbuch von Bayerisch-Schwaben}

Das Vorläuferwerk, das „Dialektwörterbuch von Bayerisch-Schwaben“, geht auf eine Initiative von WERNER KÖNIG zurück. Die Arbeiten dazu begannen im Februar 1998 mit dem Ziel, den Dialektwortschatz vom Allgäu bis zum Ries und von der Iller bis zum Lech umfassend zu beschreiben und als Printversion zu veröffentlichen. Von Anfang an stand fest, dass das Wörterbuch ausschließlich am PC erstellt werden sollte, vom Sammeln des gesamten Sprachmaterials bis zum Layout. Als Software wurde Microsoft-Access gewählt, das als kommerzielles Datenbanksys-

1 BRIGITTE SchwarZ verantwortet Kapitel 1, EdTH FunK Kapitel 2, ManUEL RaAF Kapitel 3 und URSULA WELSCH Kapitel 4. 
tem den Vorteil hatte, immer wieder an die Neuerungen des Softwaremarktes angepasst zu werden. Dies ist besonders bei Projekten mit längeren Laufzeiten entscheidend, um nicht von den technischen Entwicklungen abgeschnitten zu sein.

\subsubsection{Die Sammelphase}

Die Sammelphase begann im Frühjahr 1998 mit der Eingabe von Belegen in die Datenbank. ${ }^{2}$ Wie früher Dialektwörter und ihre Bedeutungen auf Karteikarten notiert wurden, so wurde in der Datenbank Datensatz für Datensatz angelegt und alle zu einem Lemma gehörenden Sprachdaten dem jeweiligen Stichwort zugeordnet. Schon bei der Eingabe wurde das Sprachmaterial entsprechend den Vorgaben der Datenbank strukturiert. Die Zahl der Bedeutungsangaben zu einem Lemma war beliebig erweiterbar, jeweils mit Angabe der Quelle, aus der die Bedeutung stammt, und mit Angabe des Ortes bzw. der Region. Darüber hinaus konnten Lautungen und grammatikalische Angaben in dafür vorgesehenen Feldern notiert werden, und zwar in unbegrenzter Anzahl. Volkskundliche und sachkundliche Angaben wurden in einem eigens dafür vorgesehenen Datenfeld bei der zutreffenden Bedeutung notiert. Bedeutungsangaben und Belegbeispiele aus den Quellen wurden einander zugeordnet. Das Sammeln von Belegen wurde nach dem Abschluss des ,,Sprachatlasses von Bayerisch-Schwaben“ (SBS) im Jahr 2006 beendet.

\subsubsection{Die Bearbeitungsphase}

Noch im selben Jahr begann die Bearbeitung des Sprachmaterials in der Datenbank. In dieser Phase des Projekts wurde die Datenbank nochmals um einige Datenfelder erweitert. Das bearbeitete und für den Druck vorgesehene Sprachmaterial musste vom Rohmaterial getrennt werden, denn beides, das Rohmaterial und das bearbeitete Sprachmaterial, standen in der Datenbank nebeneinander. Gleichzeitig wurde das Layout für die Wörterbuchartikel konzipiert. Parallel dazu entwickelte ein Informatikstudent Programme, mit deren Hilfe die äußerst komplizierte und aufwändige Transformation der in Access vorbereiteten Artikel in das Textverarbeitungsprogramm Word bewerkstelligt werden sollte. Im Mai 2008 war das Programm nach zahlreichen Testläufen fertig programmiert, so dass aus der Datenbank Wortartikel mit allen gewünschten Auszeichnungen erstellt werden konnten. Die Umwandlung, die Transformation der Wortartikel aus der Access-Datenbank nach Word, wurde am PC durchgeführt. Die Makrostruktur des Wörterbuchs und die Mikrostruktur eines Wortartikels waren durch das Transformationsprogramm festgelegt. Es regelte, welcher Sprachinhalt auf welche Weise und an welcher Stelle im Wörterbuch bzw. im Wortartikel erscheinen sollte.

2 Für die Datenbank wurde eine bereits vorhandene, für eine Ortsmundart erstellte Access-Datenbank erweitert und entsprechend den variantenreicheren und komplexeren Strukturen der Dialektwörter einer Region angepasst. 


\subsubsection{Die Fertigstellung}

Die Ergebnisse der Testtransformation hatten aber auch gezeigt, dass das in der Access-Datenbank liegende Sprachmaterial (insgesamt ca. 36000 Lemmata) bei einer Veröffentlichung in der konzipierten Form mehrere tausend Seiten umfassen und die Bearbeitung der gesamten Datenbank die Projektlaufzeit weit überschreiten würde. Da das Projekt auf zehn Jahre begrenzt war, wurde beschlossen, vorerst ein Wörterbuch mit ca. 3000 Stichwörtern herauszugeben. Ende 2013 erschien schließlich das von ScHWARZ verfasste „Dialektwörterbuch von Bayerisch-Schwaben. Vom Allgäu bis zum Ries“.

Im Jahr 2017 gelang es, das Wörterbuch an der BAdW anzusiedeln mit dem Ziel, aus dem gesammelten Sprachmaterial Wortartikel für eine Onlineversion zu erstellen.

\subsection{Die Quellen}

\subsubsection{Bereits integrierte Quellen}

In der Datenbank befinden sich ca. 36000 Stichwörter und ca. 300000 Belege. Die Belege stammen von Exzerpten aus regionalen literarischen Quellen (Belletristik, Sach- und Gebrauchsliteratur), aus veröffentlichten und unveröffentlichten Wörtersammlungen sowie dem „Sprachatlas von Bayerisch-Schwaben“ (SBS) und dem „Vorarlberger Sprachatlas“(VALTS). Es handelt sich um etwas mehr als 400 Quellen.

Die älteste Quelle ist ein schwäbisches Idiotikon von GoTTSCHED und stammt aus dem Jahr 1737. Fünf weitere Wortlisten datieren aus den 80er Jahren des 18. Jahrhunderts. 24 Quellen haben ihren Ursprung im 19. Jahrhundert, der Rest der Quellen im 20. bzw. 21. Jahrhundert. Die große Masse der Belege stammt also aus dem 20. Jahrhundert. Sie spiegeln die Dialekte so wider, wie sie vor allem in der ersten Hälfte dieses Jahrhunderts noch als lebendige Alltagssprache gebraucht wurden.

Ungefähr ein Viertel der Quellen sind unveröffentlicht, 50 davon sind von Laien zusammengestellte und dem Wörterbuch überlassene Wortlisten. Ihr Umfang reicht von einigen wenigen Wörtern bis hin zu Sammlungen mit über 2500 Wörtern. Eine ganz besondere Stellung nimmt eine umfangreiche, unveröffentlichte Mundartkartei für das Allgäu ein, in der in 14 großen Schubladen hand- und maschinengeschriebene Karteikarten gesammelt sind. Neben Wortlisten wurden dialektale Prosatexte, Dialektgedichte, Spruchsammlungen, Texte über das Brauchtum, über alte Handwerke etc. für die Datenbank exzerpiert und aufgenommen.

Besondere Bedeutung kommt dem SBS und den für das Allgäu relevanten Teilen des VALTS zu. Es wurde von Sprachwissenschaftlern erhoben und in genauer Lautschrift (Teuthonista) notiert. Da das für den VALTS erhobene Sprachmaterial 
nur zu einem Teil veröffentlicht ist, wird immer wieder auf die Bayerische Dialektdatenbank (BayDat) zurückgegriffen, in der das gesamte für das bayerische Allgäu relevante Sprachmaterial aus dem VALTS online vorliegt.

Auf eine eigene umfangreiche direkte Befragung von Gewährspersonen wurde verzichtet, weil diese den zeitlichen Rahmen des Projekts gesprengt hätte. In manchen Fällen aber werden Dialektsprecher/-innen befragt, z. B. um bei der Bearbeitung der Wortartikel auftretende Unklarheiten auf diese Weise schnell beseitigen zu können. Insbesondere Personen, die dem Wörterbuch schriftliches Material überlassen haben, aber auch andere dialektsichere Personen, sind gerne bereit, mündlich Auskunft zu geben.

\subsubsection{Noch zu integrierende Quellen}

Die in der Datenbank gespeicherte Materialbasis des Projekts wird ergänzt durch bislang erst teilweise erschlossenes Sprachmaterial. Zu diesem zählen 660 Fragebögen von FRIEDRICH MAURER aus dem Jahre 1934 mit je 90 Fragen, die in gescannter Form vorliegen, jedoch noch nicht in die Datenbank integriert sind. Im Archiv des „Bayerischen Wörterbuchs“ lagern ferner ca. 300 sog. Mundartgeographische Fragebögen, die in den Jahren 1927-1940 von Gewährspersonen in einer großen Zahl von Orten aus Bayerisch-Schwaben ausgefüllt wurden. Insgesamt sind es fast 15000 Fragebögen mit etwa 300000 Belegen, die bereits gescannt vorliegen, aber noch sprachwissenschaftlich erschlossen werden müssen. Alle MaurerFragebögen sind noch zu lemmatisieren und die Scans in Einzelbelege zu zerteilen, um sie in der Datenbank berücksichtigen zu können. Die Scans der Mundartgeographischen Fragebögen müssen in Einzelbelege zerteilt werden, wie es mit den Wörterlisten des „Bayerischen Wörterbuchs“ geschehen ist, um für die Bearbeitung der Wortartikel zur Verfügung zu stehen. ${ }^{3}$ Sie sollen dann über ein semantisches System, das auf den Fragestellungen in den Fragebögen basiert, in die Datenbank integriert werden. Weiteres noch nicht in der Datenbank vorhandenes Sprachmaterial sind die Spontanbelege des SBS, die von Gewährspersonen bei der Exploration genannt und von den Explorator/-innen als zusätzliche Sprachbelege notiert wurden. Sie liegen lemmatisiert in digitaler Form vor, bieten eine wertvolle Zusatzinformation und erlauben einen schnellen Zugriff. Im Archiv der Universität Augsburg wurden kürzlich ca. 4000 bis 5000 Fragebögen aus dem Nachlass von EDUARD FRIEDRICH NÜBLING entdeckt. Sie müssen zunächst gescannt und dem Projekt zugänglich gemacht werden. 


\subsection{Lemmatisierung}

Die Lemmatisierung des gesamten sich in der Datenbank befindenden Sprachmaterials erfolgte bereits bei der Eingabe in die Datenbank. Jeder der ca. 300000 dialektalen Belege ist einem Lemma zugeordnet. Die in der Lexikographie übliche Form, bei Substantiven und Pronomina der Nominativ Singular, bei Verben der Infinitiv und bei Adjektiven die unflektierte starke Form des Nominativs Singular, ist als Lemmaform gewählt.

Die Lemmatisierung der Dialektwörter geschieht zumeist nach sprachhistorischen, aber auch nach pragmatischen Gesichtspunkten. Im Hinblick auf einen gemeinsamen Internetauftritt orientiert sich DIBS bei der Lemmatisierung im Wesentlichen an den beim „Bayerischen Wörterbuch“ (BWB) angewendeten Regeln. ${ }^{4}$ Einige Abweichungen ergeben sich jedoch unter anderem durch Unterschiede bei Wortbildungsmorphemen in den beiden Dialektgruppen (das im Schwäbischen übliche heisala ‘spielen' wird z. B. mit „häuselen“ lemmatisiert, das Bairische haisln dagegen mit ,häuseln“').

Wenn es möglich ist, wird die standardsprachliche Form von Duden (1999) übernommen. Ausnahme hiervon bilden Wörter, bei denen kleine lautliche Abwandlungen fast im gesamten Gebiet belegt sind. So wird etwa die häufig belegte nicht-umgelautete Form als Hauptlemma, eine selten belegte umgelautete Form als Variante notiert.

Beispiel: Dialektale Lautform: kuxe 'Küche'; Lemma: Kuche; Variante: Küche.

Bei Dialektwörtern, die im Standarddeutschen nicht vorkommen, können die Schreibregeln der entsprechenden Wortfamilie im Standard übernommen werden.

Beispiel: Dialektale Lautform: bluə, mhd. bluo, blüe (abgeleitet vom Verb blühen); Lemma: Bluhe.

Fehlt ein schriftsprachliches Pendant für ein Dialektwort, sind aber historische Vorformen belegt, so wird das Lemma den Regeln der regionalen historischen Lautlehre entsprechend angesetzt.

Beispiel: Dialektale Lautform: breisa, mhd. brîsen 'schnüren, einschnüren, einfassen'; Lemma: breisen.

Lehnwörter, die keine standardsprachliche Entsprechung haben, also nicht im Duden stehen, werden lautnah lemmatisiert.

Beispiel: Dialektale Lautform: vadsənędle aus ital. fazzoletto 'Taschentuch'; Lemma: Fazenettlein.

Bei vielen Mundartwörtern ist ein etymologischer Anschluss nicht möglich. In solchen Fällen wird lautnah lemmatisiert, unter Beachtung der in der entsprechenden Region geltenden historischen Lautlehre. 
Beispiel: Dialektale Lautform: bvrebvlar 'nicht fachgerecht arbeitender Mensch'; 'armer Mensch'; Lemma: Pfrepfler.

Generell wird besonders dann lautnah lemmatisiert, wenn mit lautlichen oder morphologischen Unterschieden auch Unterschiede in der Bedeutung einhergehen.

Beispiel: Dialektale Lautform: raufa 'mit der Wurzel aus der Erde ziehen'; 'streiten'; Lemma: raufen.

Dialektale Lautung: raifa 'auskämmen'; 'beim Kämmen schmerzhaft an den Haaren ziehen'; 'mit der Sichel mähen'; Lemma: räufen.

Belege mit kleineren lautlichen oder morphologischen Unterschieden können unter einem Stichwort zusammengefasst werden, wenn sich ihre Bedeutungen decken.

Beispiel: Dialektale Lautformen: mụhāgl, mụhakl, mụhęgl; Lemma: Muhhagel 'wortkarge Person'; 'Tölpel'; 'Rüpel'.

In solchen Fällen ist es sinnvoll, beim entsprechenden Wortartikel mehrere Lemmavarianten anzusetzen. Im zitierten Beispiel wären Muhhäckel, Muhhägel als Lemmavarianten zu notieren.

Auch bei stark verschliffenen Formen werden bei einem Wortartikel in der Regel mehrere Lemmavarianten vermerkt.

Beispiel: Dialektale Lautformen: hambvōl, hambvl, hęmbvl; Lemma: Handvoll. Lemmavarianten: Händvoll, Hampfel, Hämpfel.

Unterschiedliche morphologische Formen werden in unterschiedlichen Artikeln mit unterschiedlichen Lemmata dargestellt.

Beispiel: Dialektale Form: hoppə ‘hüpfen'; 'hinken’; ‘rüttelnd fahren'; 'hapern, nicht in genügendem Ausmaß vorhanden sein'; Lemma: hoppen.

Dialektale Form: hoppra 'rüttelnd fahren'; Lemma: hopperen.

Kommt in einem Kompositum ein Glied nur in einer bestimmten lautlichen oder morphologischen Variante vor, so wird dies bei der Lemmatisierung berücksichtigt.

Beispiel: Lemma Schnupftabaksduse 'Schnupftabaksdose'.

Wörter, die ausschließlich in der Diminutivform oder im Plural belegt sind, werden in dieser Form im Wörterbuch aufgenommen.

Beispiel: Ablassglöcklein 'kleine Kirchenglocke, die beim Versehgang geläutet wird' (eine nichtdiminuierte Form ist nicht belegt).

Homonyme Lemmata werden durch Hochzahlen unterschieden.

Beispiel: abreden ${ }^{1}$ 'jemanden durch eindringliches Reden davon zu überzeugen suchen, etwas nicht zu tun'; abreden 'durchsieben'. 


\subsection{Die Datenbank}

Im Hinblick auf die geplante Onlinestellung der Wortartikel entwickelte MANUEL RAAF von der IT-Abteilung der BAdW auf der Basis der Access-Datenbank eine neue Datenbank im MySQL-Format. Im Juli 2018 wurden die Daten in die neue Datenbank transferiert.

Wie dies bereits in der Access-Datenbank für das Printwörterbuch geschehen ist, werden auch in der MySQL-Datenbank die Wortartikel für die Onlineversion geschrieben. Das bedeutet, dass sich das in den Artikeln zitierte Sprachmaterial neben dem nicht zitierten Rohmaterial befindet. Jedoch sind die fertigen Teile eines Wortartikels speziell gekennzeichnet. Damit ist die Voraussetzung für einen zielgerichteten Online-Zugriff geschaffen. Im Folgenden werden einige Ansichten der Datenbank vorgestellt.

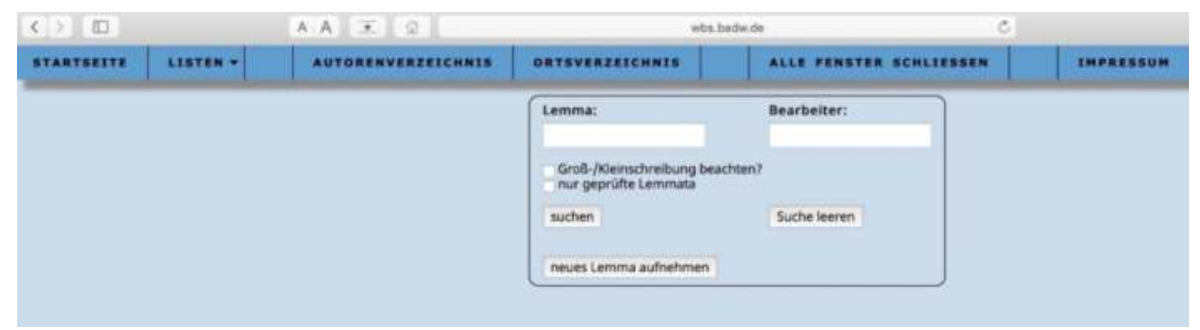

Abb. 1: Eingangsmaske der Datenbank

Nach dem Aufrufen der Datenbank öffnet sich die Startseite (Abbildung 1). Im Datenfeld „Lemma“ wird nach einem vorhandenen Lemma gesucht oder ein neues Lemma aufgenommen. Durch Anklicken von „Groß-/Kleinschreibung beachten?“ oder „,nur geprüfte Lemmata“ kann die Suche eingeengt werden.

Die Suche nach dem Lemma abwinden öffnet den Datensatz zu diesem Lemma (Abbildung 2). Über dem Lemma abwinden sind in den grau hinterlegten Feldern „Grammatikbelege“, „Lautungsbelege“ und „Lemmavarianten“ typische oder besondere grammatische Formen bzw. Lautformen sowie Varianten zum Lemma zu finden. Unter dem Lemma im Feld „Wortfamilie“ steht das Stichwort der betreffenden Wortfamilie. Im Feld „Etymologie“ ist bei der Präfixbildung abwinden auf die Artikel der entsprechenden Simplizia verwiesen. Bei den Simplizia stehen eine kurze Etymologie und der Hinweis auf die dazu herangezogene Quelle. 


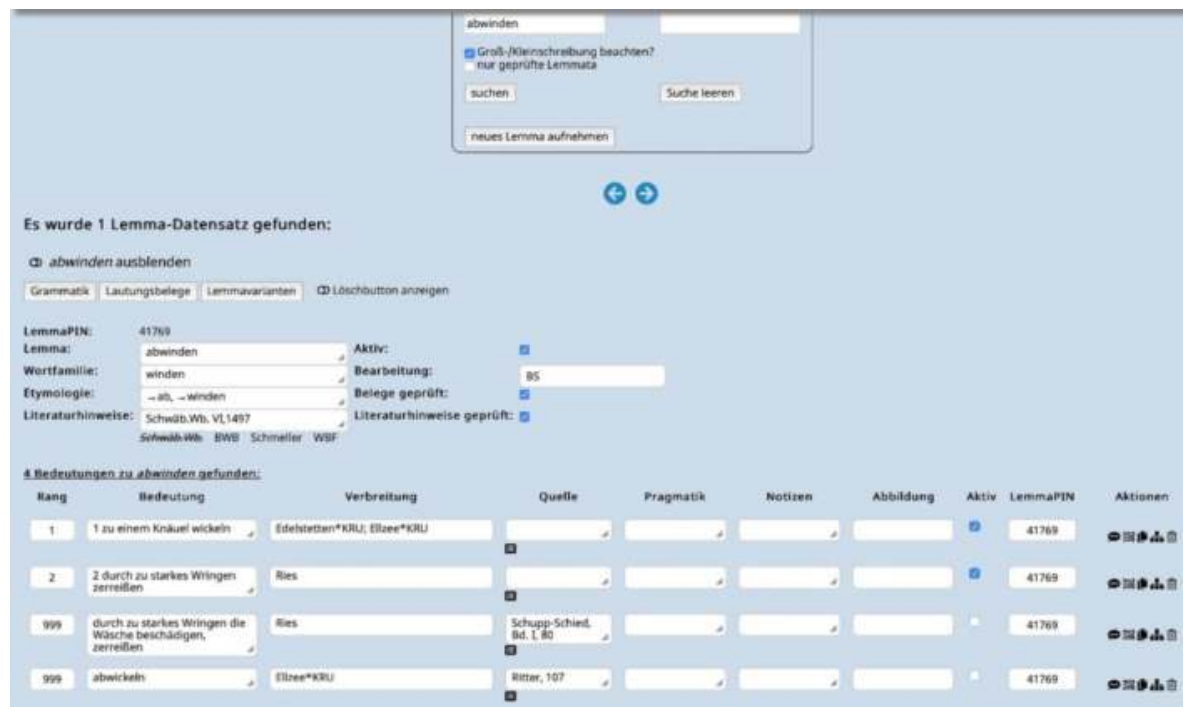

Abb. 2: Das Lemma ,abwinden“

Das Feld „Literaturhinweise“ verweist auf das „Schwäbische Wörterbuch“ von HERMANN FISCHER (1904-1936). In den übrigen Referenzwörterbüchern, „Bayerisches Wörterbuch“(BWB), JOHANN ANDREAS SCHMELLERS (1872-1877), Bayerisches Wörterbuch“, „Handwörterbuch von Bayerisch-Franken“ (WBF) von EBERHARD WAGNER und ALFRED KLEPSCH (2007), lässt sich zu diesem Lemma kein Eintrag finden. Im Feld „Rang“ wird die Reihenfolge der einzelnen Bedeutungen festgelegt, so wie sie im Onlinewörterbuch erscheinen sollen. Die Datensätze mit der Rangnummer 999 werden im online gestellten Wortartikel nicht zitiert, sollen aber als Rohdaten eingesehen werden können. Rechts neben der Bedeutung wird die Verbreitung des Wortes in Form von Orts- oder Gebietsangaben notiert. Klickt man mit dem Cursor in das Verbreitungsfeld, dann öffnet sich eine Auswahlliste mit allen Orts- und Gebietsnamen und ermöglicht so deren korrekte Eingabe. Das folgende Datenfeld „Quelle“ enthält bei bearbeiteten Bedeutungsangaben keine Einträge, lediglich bei den nicht zitierten Primärdaten (unter dem Rang 999). ${ }^{5}$ Unter „Pragmatik“ folgt ggf. ein Hinweis zur Wortverwendung, z. B. ,scherzhaft“, „,fachsprachlich“. Das Feld „Notizen“ dient den Redaktorinnen der Wortartikel als Merkzettel und unter „Abbildungen“ sind Quellenangaben zu Bildern oder Zeichnungen vermerkt.

Klickt man auf die am rechten Rand stehenden Symbole, die unter „Aktionen“ stehen, so öffnen sich weitere Masken.

5 Die bei einer Bedeutung zitierten Belege mitsamt ihren Quellenangaben sind ja in einer eigenen Liste ausgelagert. 


\section{Aktionen \\ …ㅁㅁㅁㄴㄷㄴ 甶}

Abb. 3: Symbole zu Aktionen

Beim ersten Symbol (von links nach rechts gesehen) öffnet sich eine Maske mit allen zur entsprechenden Bedeutung gehörigen Belegen. Nach dem Anklicken dieses Symbols erscheint die Maske für die Belege. Für das Lemma abwinden sind zwei Belegbeispiele notiert (Abbildung 4). Der „Rang“ gibt die Reihenfolge der Belege im Artikel an. Im Kommentarfeld können Bedeutungsangaben aus den Quellen und Bedeutungsangaben oder Kommentare der Redaktorinnen eingetragen werden. Unter „Belegort“ ist die entsprechende Orts- oder Gebietsangabe genannt, unter "Quelle“, woher der Beleg stammt. Bleibt das Feld „Quelle“ leer, dann handelt es sich um eine mündliche Angabe.

\section{Belege}

Lemma-IdentNr: 41769
BedeutungspiN: 127339

1 zu einem Knäuel wickeln

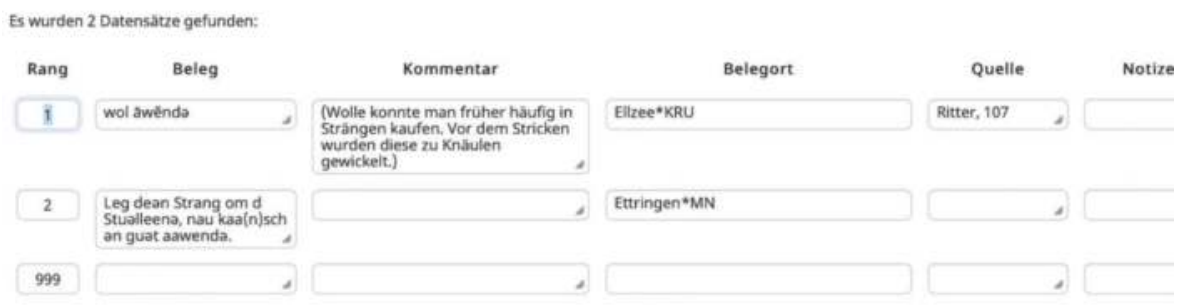

Abb. 4: Belege zu „abwinden“

Sach- und volkskundliche sowie sprachliche Hinweise sind unter dem zweiten Symbol abgelegt (Abbildung 3). Das dritte Symbol ermöglicht die Kopie der jeweiligen Datenzeile. Die onomasiologische Eingruppierung der Bedeutungsangabe kann in einer Maske, die hinter dem vierten Symbol verborgen liegt, für jede Einzelbedeutung vorgenommen werden. Das letzte Symbol erlaubt das Löschen der Datenzeile.

Die neue Datenbank bietet eine benutzerfreundliche Oberfläche. Die Schreibfelder passen sich automatisch der Größe des Eintrags an. In Feldern, in denen dies nötig ist, öffnet sich nach dem Anklicken eine Symbolleiste, die unterschiedliche Formatierungen ermöglicht und Unicode-Sonderzeichen anbietet. Die Datenbank 
verfügt über eine Reihe von Tools für schnelles Suchen, z. B. den Asterisk* als Auslassungszeichen, um bestimmte Buchstabenfolgen oder Wortteile zu suchen.

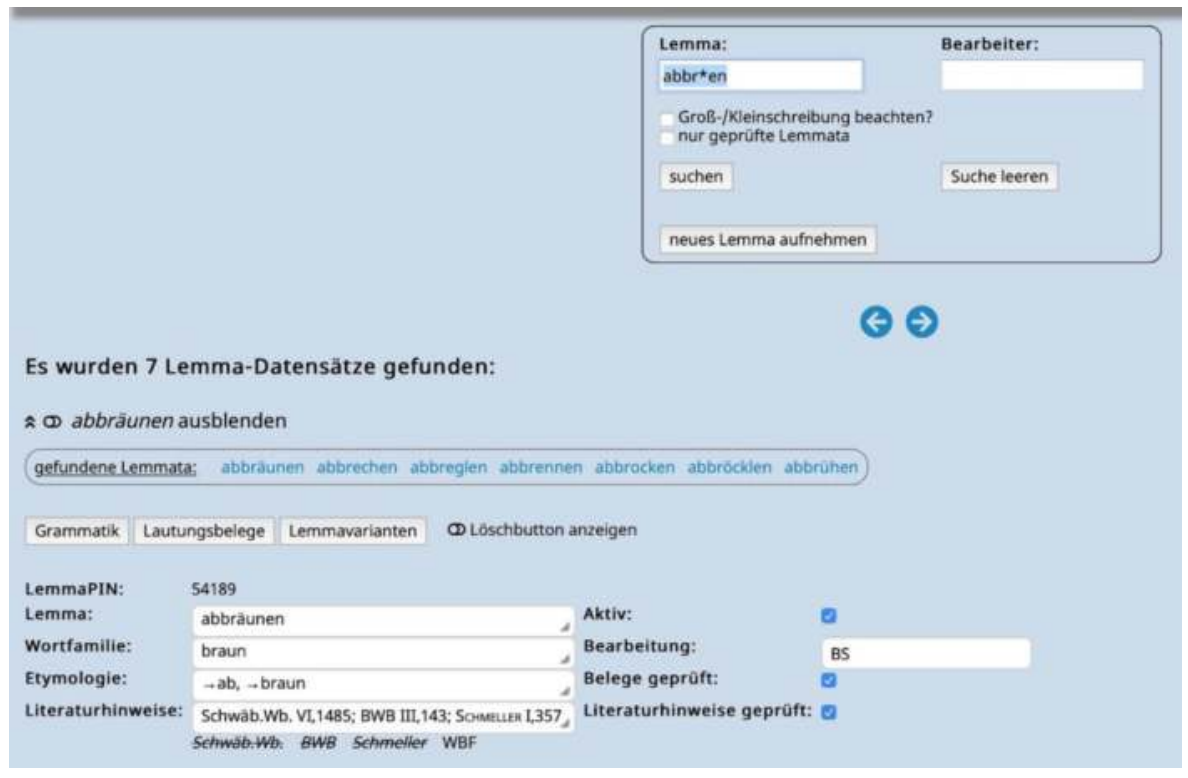

Abb. 5: Suche nach „abbr*en“

Im obigen Beispiel wird im Feld „Lemma“ nach ,abbr*en“ gesucht. Der Asterisk steht für eine beliebige Folge von Buchstaben. Die Datenbank listet unter ,gefundene Lemmata“" sieben Lemmata aus der Datenbank auf. Jedes dieser sieben Lemmata kann angeklickt werden. Sofort gelangt man in den entsprechenden Datensatz und kann darin arbeiten. Auf die gleiche Weise können die Felder mit den Bedeutungsangaben und den Belegen nach einer Buchstabenfolge oder einem Wort durchsucht werden. Mit einem einzigen Mausklick lassen sich überdies zu einem Lemma alle Belege, geordnet nach ihren Bedeutungen, grammatischen Formen und unterschiedlichen Lautungen, auflisten. Dieser Schnellzugriff ermöglicht einen Überblick über die gesamte Beleglage zu einem Lemma.

Eine Lemmaliste, in der die bereits bearbeiteten Lemmata farbig unterlegt sind, zeigt die Lemmata im Überblick und verlinkt jedes Lemma mit dem dazugehörigen Datensatz. 


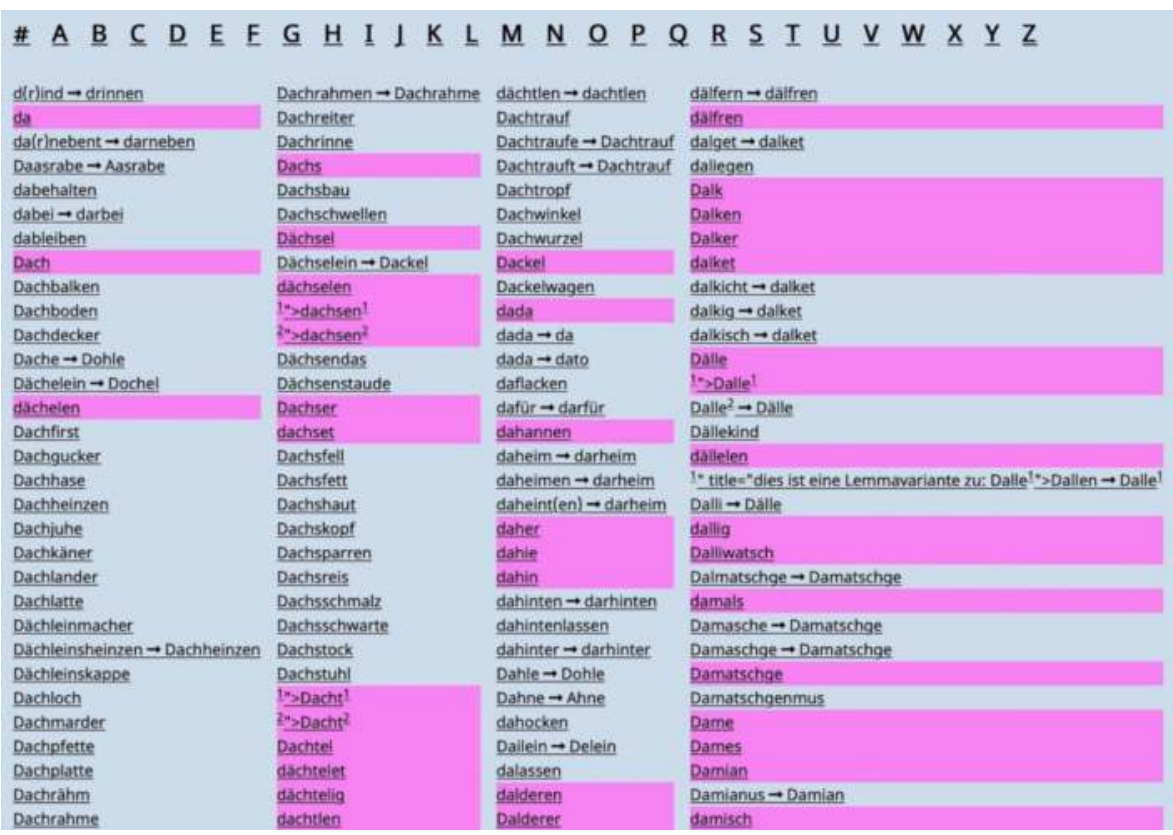

Abb. 6: Ausschnitt aus der Lemmaliste

Hier wird auch automatisch die Anzahl der bereits bearbeiteten Lemmata gezählt.

Es ist geplant, die Datenbank mit all ihren bearbeiteten und nicht bearbeiteten Daten in nächster Zeit im Netz zugänglich zu machen.

\section{DIBS - (MEHR ALS) EIN WÖRTERBUCH UND SEINE ARTIKEL}

Die digitale Erarbeitung von DIBS erlaubt erstens unzählige Möglichkeiten der Vernetzung innerhalb des Wörterbuchs und auch außerhalb mit anderen (nicht nur lexikographischen) Projekten. Zweitens eröffnet der fortlaufende Einbezug von mehr als nur schriftlichen Medien, nämlich darüber hinaus auch von Bild- und Tondokumenten, neue Dimensionen der Rezeption über die sinnliche Wahrnehmung. Damit reift das ursprünglich projektierte „Digitale Wörterbuch von BayerischSchwaben“ (WBS) zum „Dialektologischen Informationssystem von BayerischSchwaben“ (DIBS) aus und geht in seinen Funktionalitäten und Leistungen über das, was ein Wörterbuch, auch ein „born digital“ Wörterbuch, zu bieten hat, weit hinaus. Drittens wird die permanente Fortschreibung und Anpassung der einzelnen Artikel bzw. des gesamten Projekts möglich: nämlich an eine erweiterte Datenlage, aber auch an Verbesserungswünsche, die der Nutzer / die Nutzerin an das System stellt. Denn es ist beabsichtigt, mit den Nutzer/-innen in einen Dialog zu treten und ihnen die Beteiligung am Projekt als Forscher/-innen zu ermöglichen. Damit verändern sich die Bedeutungen der Begriffe „Artikel“ und „Wörterbuch“ natürlich 
grundlegend, wenn sie sich in solchermaßen dynamisierten Strukturen nicht gar völlig auflösen.

All dies hindert DIBS nicht daran, auch als Wörterbuch im klassischen Sinn aufzutreten, mit Artikeln, die wie in einem traditionellen Print-Wörterbuch rezipiert werden können. Damit sollen auch Benutzer/-innen zufrieden gestellt werden, die gewohnt sind, mit gedruckten oder retrodigitalisierten Wörterbüchern umzugehen und wünschen, ein Wörterbuch durchblättern und durchschmökern zu können.

DIBS ist formal und inhaltlich so angelegt, dass auch eine klassische PrintVersion möglich ist, wenn einmal zu allen Lemmata Artikel verfasst sind. Eine zusätzliche gedruckte Version war von Anfang des Projekts an bereits geplant, auch die Datenbank wurde entsprechend konzipiert.

\subsection{Vernetzung als Strukturprinzip}

Das in DIBS gesammelte Sprachmaterial ist durch seine sprachwissenschaftliche Kategorisierung und die Gliederung in unterschiedliche Felder der Datenbank in vielerlei Hinsicht bereits ausgezeichnet. Dies erlaubt unterschiedlichste Ordnungsmuster, die dementsprechend unterschiedlichste Abfragen ermöglichen. Während in klassischen Wörterbüchern gezielt und strukturiert nur nach Lemmata, also (alphabetisch angeordneten) Stichwörtern, gesucht werden kann, eröffnet DIBS völlig neue Zugänge, z. B. über Bedeutungen, Bedeutungsmerkmale, Sachgruppen- bzw. Themenzugehörigkeiten (wie etwa zum Fachwortschatz der Gerberei). Gezielte Suchen z. B. nach grammatischen Kategorien (etwa alle Maskulina oder alle Wörter, in denen bestimmte Lautfolgen vorkommen), nach Phraseologien, Wetter- und Bauernregeln, Sprichwörtern, Kinderspielen werden möglich. Es kann der Wortschatz nur eines bestimmten Ortes, eines Landkreises oder einer bestimmten Region (z. B. Allgäu, Ries) abgefragt werden. Möglich sind auch kombinatorische Suchprozesse (z. B. alle Maskulina auf -er im Allgäu im Fachwortschatz der Käserei). All dies ist jetzt schon möglich.

Darüber hinaus ist geplant, Materialien unterschiedlichster Medientypen durch Verlinkungen mit den entsprechenden Stichwörtern einzubinden, wie z. B. Sprachkarten, Foto-, Film- und Tondokumente, um die Inhalte der Wortartikel auch auditiv und visuell erfahrbar zu machen. Möglich wären Auszeichnungen dieser Medieninhalte etwa nach Sachgruppen, nach der Provenienz, nach der Entstehungszeit u. Ä. Dann könnten diese Materialien nicht nur über das entsprechende Lemma gefunden, sondern darüber hinaus in (komplexe) Suchen einbezogen werden.

Ausdrücklich gewünscht ist auch die Vernetzung mit anderen Projekten, insbesondere den anderen beiden an der BAdW angesiedelten Mundartwörterbüchern (vgl. Kapitel 4), aber auch weiteren Projekten mundartlexikographischer, volkskundlicher oder ähnlicher Thematik. Wir gehen davon aus, dass für diese erweiterten Forschungsziele künftig die personelle Ausstattung entsprechend angepasst werden kann. 


\subsubsection{Artikel mit open end}

Bei DIBS werden die Wörterbuchartikel direkt in der Datenbank erarbeitet, anschließend in eine XML-Struktur überführt und online gestellt. Dieser Online-Gang ist als fortlaufender Prozess geplant: sobald ein Lemma bearbeitet ist, geht der entsprechende Artikel ins Netz. Damit ist der Artikel zwar (vorläufig) fertig, aber nicht dauerhaft abgeschlossen. Jedes lexikographische Projekt macht die Erfahrung, dass mit dem fortschreitenden Arbeitsprozess, mit dem Auftauchen neuer Materialien, mit der Anpassung oder Veränderung der Konventionen u. Ä. Änderungen an bereits geschriebenen Artikeln wünschenswert wären. Das führt dann zu verbesserten Auflagen. Erst recht sind solche Veränderungen bzw. Verbesserungen notwendig, wenn der Nutzer / die Nutzerin die Möglichkeit hat, eigenes Material zur Verfügung zu stellen oder Kommentare abzugeben. Bei DIBS ist die permanente Fortschreibung der Artikel von Anfang an einkalkuliert, sodass jederzeit Ergänzungen vorgenommen werden können. Die einzelnen Versionen und ihre URLs werden automatisch datiert, damit ist ein eindeutiges Zitieren möglich.

\subsubsection{Auflösung gewohnter Strukturen}

Eine Reihe von Fragen zum Aufbau der Makrostruktur, die zu Beginn jeder Arbeit an einer gedruckten Version stehen, sind für DIBS wie für alle ,born digital“" (Wörterbuch-)Projekte unerheblich. Das betrifft z. B. die Anordnung und die Bearbeitungsreihenfolge der Artikel wie auch der Außenteile. Man braucht sich z. B. keine Gedanken über die Alphabetstrecke machen, etwa ob $B$ - und $P$-, $D$ - und $T$-, $V$ - und $F$ - in einer gemeinsamen Strecke stehen sollen oder nicht oder ob die Komposita direkt auf das mit dem Grundwort identische Simplex folgen oder glattalphabetisch angeordnet werden sollen. Schon bei der Bearbeitung der Artikel braucht man die alphabetische Reihenfolge oder überhaupt ein Ordnungsprinzip nicht einzuhalten.

Auch in seinen Mikrostrukturen ist DIBS nicht festgelegt. Ein „Artikel“ setzt sich aus Bausteinen zusammen, deren Anordnung und optische Gestaltung vor allem in der Bearbeitungsphase keine Rolle spielen. Auch ob sie stets an der Oberfläche liegen oder unter einem Pop-up-Menü verborgen liegen, braucht nicht von Anfang an festgelegt zu werden. Selbst dann, wenn diese Bausteine in der XMLFassung in bestimmter Reihenfolge vorliegen, kann ihre Anordnung und Gestaltung jederzeit verändert werden.

\subsection{Die Inhalte von DIBS}

Zunächst war beabsichtigt, die Sammeltätigkeit in einem Stadium abzuschließen, in dem so viel Material vorhanden ist, dass nur noch mit einem unverhältnismäßigen Aufwand weitere Wörter, einzelne Bedeutungen oder zusätzliche Phraseologien zu einem Wort zu ermitteln wären. Nach der neuen Planung sollen aber die 
zukünftigen Nutzer/-innen auch als Sammler/-innen und Forscher/-innen einbezogen, weitere Materialien unterschiedlicher Medienarten eingebunden und Vernetzungen mit anderen lexikographischen, volkskundlichen und ähnlichen Projekten hergestellt werden.

\subsubsection{Inhalte wie gewohnt}

Das bisher in der Datenbank vorliegende Material ist dem anderer großräumiger Mundartwörterbücher sehr ähnlich. Gesammelt wurde der gesamte mundartliche Wortschatz der Gegenwart im Untersuchungsgebiet (vgl. Kapitel 1.2). Personenund Örtlichkeitsnamen sind berücksichtigt, sofern sie auch für den appellativischen Wortschatz von Bedeutung sind, etwa als Wortbildungselemente oder in Phraseologien oder wenn sie von volkskundlichem Interesse sind. Da die digitale Version keine räumlichen Einschränkungen nötig macht und etwaige Überfrachtungen kein Thema sind (was nicht interessiert, wird nicht aufgerufen und was nicht aufgerufen wird, ist nicht präsent), werden alle Stichwörter bearbeitet und online gestellt, etwa auch solche Komposita, die rein ,,additiver" Natur sind, sich also aus den einzelnen Gliedern selber erklären, wie z. B. hinaufgehen, Zwetschgenmarmelade u. Ä., und auch sonst unauffällig, also z. B. in keinen Phraseologien belegt sind.

\subsection{2 ... aber auch ungewohnt}

Es ist beabsichtigt, nicht nur ein Mundartwörterbuch, sondern ein multimediales Informationssystem für Bayerisch-Schwaben zu erarbeiten und zusammen mit der IT-Abteilung bis Oktober 2021 dafür ein tragfähiges Konzept auszuarbeiten. Wir möchten bis dahin die ersten Materialien eingebunden und mit Bearbeitungswerkzeugen für die Benutzer/-innen ausgestattet haben.

Den Anfang soll der „Sprachatlas von Bayerisch-Schwaben“ (SBS) machen, nicht nur, weil er die umfangreichste Quelle der Datenbank ist, sondern weil vor Jahren bereits eine digitale Fassung, ausgestattet mit Suchfunktionen und einfachen Bearbeitungstools erarbeitet wurde. Sie muss auf den neuesten Stand der Technik überführt, die Tools entweder überarbeitet oder neugestaltet und das System insgesamt noch durchgetestet werden. Denkbar ist die Einbindung über die Lemmata, denn der SBS hat ein umfangreiches Register, das alle im Atlas vorkommenden Wörter enthält.

Im Umfeld des SBS entstanden Sammlungen sowie Publikationen unterschiedlicher Medienarten, mit denen die Einbindung solcher Medien gut erprobt werden kann: 
- Der „Sprechende Sprachatlas von Bayerisch-Schwaben“6 mit seinen Tondokumenten sowie die schwäbischen Teile des „Sprechenden Sprachatlasses von Bayern". 7

- Die populäre Ausgabe „Kleiner Sprachatlas von Bayerisch-Schwaben“ von WERNER KÖNIG und MANFRED RENN (2002).

- Das gesamte Primärmaterial des SBS, also die Originalmitschriften der Exploratoren, die auf Mikrofiches verfilmt sind. Sie könnten als Bilddateien mit den entsprechenden Lemmata verknüpft werden.

- Die beim SBS gesammelten Tondokumente zu jedem Aufnahmeort (insgesamt ca. 400 Stunden). Sie sind erschlossen und genau protokolliert. Es handelt sich großteils um Mitschnitte der Erhebungen, die mit den jeweiligen Lemmata verlinkt werden könnten. Der Rest sind freie Gespräche mit Erzählungen. Deren Protokolle erleichtern die Einbindung über die Inhalte.

- Das Fotoarchiv des SBS, dessen Einbindung einfach sein dürfte, da jedes Foto mit dem Aufnahmeort und dem entsprechenden Lemma, d. h. mit der Bezeichnung für das fotografierte Objekt, versehen ist.

Anschließend daran oder auch parallel dazu sollen die ins Primärmaterial eingegangenen Quellen, wie z. B. von Laien verfasste Wortsammlungen oder Wörterbücher zu bestimmten Orten oder Regionen, gescannt und als Faksimiles mit den entsprechenden Lemmata verlinkt und einsehbar werden.

Wenn all dies einmal funktioniert, wenn quasi die Pilotversuche gut gelaufen sein werden, können weitere Materialien, auch Filmdokumente, eingebunden werden. Da die nötigen Auszeichnungen und Verlinkungen wohl nicht automatisiert werden können, ist dies mit nur einer Mitarbeiterstelle nicht zu bewältigen.

\subsection{Die Bausteine eines Artikels}

Die einzelnen in der Datenbank vorhandenen Elemente können bei der Online-Präsentation beliebig angeordnet und gestaltet sowie ein- oder ausgeblendet werden und ähnlich wie die mit einem Artikel verknüpften weiteren Materialien aktiviert werden, z. B. durch Pop-up-Fenster. Inhaltlich entsprechen die meisten Bausteine den Artikelteilen klassischer Wörterbücher. Über die optische Darstellung der Artikel wie über die Präsentation des gesamten Informationssystems im Netz wurden noch keine Entscheidungen getroffen. Soweit es möglich ist und die digitale Form es zulässt, werden bei der Gestaltung der einzelnen Teile die Konventionen des BWB übernommen, die damit dann auch für den gemeinsamen Auftritt in BDO (Bayerns Dialekte Online) gelten (vgl. Kapitel 4).

6 <https://www.bayerische-landesbibliothek-online.de/sprachatlas-schwaben>; Stand: 12.03.2019.

7 <https://www.bayerische-landesbibliothek-online.de/sprachatlas>; Stand: 12.03.2019. 


\subsubsection{Lemmablock}

Der Lemmablock kann mehrere Einträge enthalten. Prinzipiell sind alle Varianten gleichwertig. Gründe für solche Varianten sind etwa, dass Formen mit und ohne Umlaut oder mit minimalen morphologischen Unterschieden in einem Artikel zusammengefasst werden oder dass einem etymologisch orientierten ein lautnah verschriftetes Lemma zur Seite gestellt wird (vgl. das Kapitel 1.3 Lemmatisierung). Die Absicht dabei ist, dass der Artikel auch bei unterschiedlichem Suchverhalten seitens der Nutzer/-innen gefunden werden kann. Sogenannte weiche Suchen sollen möglich sein, ähnlich wie beim Schweizerdeutschen Idiotikon. So führen Suchen mit Anfangsbuchstaben $D$ - auch zu den entsprechenden Formen mit $T$ - (z. B. führt die Eingabe Dockel auch zu Tockel 'Puppe'). Von sprachwissenschaftlicher Seite sind die Kriterien für eine weiche Suche entsprechend den lautlichen Verhältnissen in Bayerisch-Schwaben noch festzulegen. Bei digitalen Wörterbüchern muss der Umfang des Lemmablocks nicht eingeschränkt werden. Bei den Lemmaeinträgen werden keine Abkürzungen (etwa der Grundwörter bei Komposita) verwendet, da in einer digitalen Version kein Platz gespart werden muss, vor allem aber, da solche Abkürzungen automatisierte Vernetzungen erschweren. Deshalb wird auch darauf verzichtet, morphologische Strukturen hervorzuheben (z. B. bei Komposita etwa durch Einklammerungen oder Bindestriche), da Suchen dadurch eher behindert als unterstützt werden; und eine Suche nach Wortteilen ist in digitalen Fassungen selbstverständlich.

\subsubsection{Grammatikblock}

Die Datenbanklisten mit grammatischen Angaben zu einzelnen Belegen werden in der Artikelstruktur nicht berücksichtigt. Wie auch bei anderen vergleichbaren Wörterbüchern, etwa dem BWB, wird hier die Wortart, bei Substantiven auch das Genus, angegeben. Darüber hinaus können weitere notwendige Angaben erfolgen, z. B. ob von einem Verb nur Partizip-Präteritum-Formen, von einem Substantiv nur Diminutiv- bzw. Pluralformen belegt sind.

\subsubsection{Bedeutungsblock}

Die einzelnen Felder des Bedeutungsblocks in der Datenbank wurden bereits in Kapitel 1.4 erklärt.

\subsubsection{Bedeutungsangabe}

Im Unterschied zu manchen gedruckten Wörterbüchern muss in jedem Fall eine Bedeutungsangabe stehen und kann nicht etwa dadurch kompensiert werden, dass 
aus einem zitierten Beleg selbst die Bedeutung ganz offensichtlich hervorgeht. Das ist für den reibungs- und verlustlosen Verlauf von Anfragen und Suchen nötig.

Ist mehr als eine Bedeutung belegt, kann hierarchisch gegliedert werden, was sich in der Wahl unterschiedlicher Zahlen- und Ziffernarten für die Durchnummerierung widerspiegelt. In diesem Punkt wurden die Konventionen des BWB übernommen. Diese Ziffern werden der Bedeutungsangabe im gleichen Feld vorangestellt, ihr folgen etwaige grammatische Angaben, die sich nicht auf das ganze Lemma beziehen (und daher nicht im Grammatikblock stehen), sondern nur auf diese jeweilige Bedeutung.

\subsubsection{Geographische Verbreitung}

Jede Bedeutung ist mit Angaben zu ihrer räumlichen Verbreitung verknüpft. Das können Orte oder ganze Regionen sein, die aus einem Menü ausgewählt werden müssen, damit über standardisierte lokale Angaben Suchen möglich sind. Über die Häufigkeit können, wenn bei größerer Verbreitung nicht alle entsprechenden Orte aufgezählt werden, wie im BWB grobe Angaben gemacht werden (,,vereinzelt“", „mehrfach“, ,vielfach“, z. B. „Ries vielfach“).

\subsubsection{Belegzitate}

Jede Bedeutung, auch jede übertragene, muss mit mindestens einem Beleg anschaulich gemacht werden. Die dazu aus der Datenbank ausgewählten Belege werden mit Nummern versehen, um ihre Reihenfolge festzulegen. Bei ihrer Darstellung gelten die gleichen Konventionen wie im BWB (Kursivdruck für Mundartformen, doppelte Anführungszeichen, wenn recte gesetzte Kontexte vorhanden sind u. Ä.). Die in der Datenbank vorhandenen Angaben zur Lokalisierung und die Quellenangabe sowie etwaige Angaben zur Grammatik, zur Wortverwendung etc. werden übernommen.

In diesem Block und jeweils einer bestimmten Bedeutung zugeordnet, stehen auch Phraseologien, Sprichwörter, Bauern- und Wetterregeln, Reime, Kinderverse, Schnellsprechverse u. Ä. Sie werden jeweils eigens ausgezeichnet, sodass differenzierte Suchen möglich sind. Phraseologien, also feste Fügungen, Redensarten, Vergleiche u. Ä. haben jeweils eigene Bedeutungen und müssen deshalb mit einer entsprechenden Angabe versehen werden. Sind für eine Phraseologie, ein Sprichwort etc. jeweils mehrere Belege vorhanden, muss eine lemmatisierte Form und eine Verbreitungsangabe aufgeführt werden. Zur Veranschaulichung muss mindestens ein Beleg, ebenfalls versehen mit Lokalisierung und Quellenangabe, zitiert werden. Die in der Quelle verwendete Lautschrift wird in aller Regel beibehalten. Sehr stark differenzierte Notationen, wie sie vor allem bei den Teuthonista-Schreibungen in den Sprachatlanten verwendet wurden, werden vereinfacht. Sollte es einmal auf genaue Notationen ankommen, kann DIBS, so wie es geplant ist, einmal durch seine 
Verlinkungen mit den entsprechenden Sprachatlaskarten bzw. mit den Faksimiles der übrigen Quellen helfen.

\subsubsection{Angaben zur Pragmatik}

In einem eigenen Feld stehen eventuell nötige pragmatische Angaben wie „scherzhaft“, ,abwertend“, ,,alt“ (was bedeutet, dass der Beleg aus einer Zeit vor 1900 stammt), ,jägersprachlich“ u. Ä. Die Angaben sind stark konventionalisiert, um systematische Suchen zu erleichtern.

\subsubsection{Sach- und Volkskunde}

Wenn nötig, können bei einer Bedeutung sach- und volkskundliche Angaben gemacht werden.

\subsubsection{Semantische Auszeichnung}

Zwingend ist die semantische Auszeichnung jeder Bedeutung nach dem sog. PosTschen Sachgruppensystem. ${ }^{8}$ Da dieses System auch in anderen Projekten wie dem „Pfälzischen Wörterbuch“, dem „Schweizerdeutschen Idiotikon“ oder auch im WBF angewandt wird und zukünftig wohl auch im BWB, sind projektübergreifende semantische Suchen möglich.

\subsubsection{Wortfamilie}

Bei jedem Artikel wird verzeichnet, zu welcher Wortfamilie das behandelte Wort gehört. Bei Komposita wird die entsprechende Wortfamilie jedes Gliedes notiert. Das erlaubt einfache Suchen nach allen Ableitungen und Komposita zu einem Wortstamm, was ein Gewinn für alle morphologisch Interessierten ist.

\subsubsection{Hinweise zu Lautungen, Formen und syntaktischem Verhalten}

Kommen bei einem Wort besondere Lautungen vor, die nicht der üblichen historischen Lautlehre der jeweiligen Region entsprechen, so kann in einem eigenen Abschnitt darauf eingegangen werden. Das gleiche gilt für morphologische oder syntaktische Besonderheiten. Von hier aus kann eine Vernetzung mit z. B. den entsprechenden Listen der Datenbank oder mit Sprachatlaskarten besonders lohnend sein. 


\subsubsection{Etymologie}

Sofern vorhanden, werden bei Simplizia und Ableitungen die alt- und mittelhochdeutschen Vorformen zitiert sowie die weitere Wortherkunft, z. B. aus dem Indogermanischen, in sehr knapper Form vermerkt, wenn dies möglich ist. Bei Lehnwörtern wird ohne Erklärung der weiteren Herkunft nur auf die Gebersprache eingegangen. Etymologische Angaben orientieren sich immer an der einschlägigen Fachliteratur, die stets auch zitiert wird.

\subsubsection{Literaturhinweise}

Derzeit wird bei der Bearbeitung der Artikel in einem eigenen Abschnitt vermerkt, ob ein Wort in den benachbarten Mundartwörterbüchern (,Schwäbisches Wörterbuch“ von HERMANN FISCHER, BWB, „Bayerisches Wörterbuch“ von JOHANN ANDREAS SCHMELLER, WBF) verzeichnet ist. Dieser Block wird möglicherweise überflüssig, wenn einmal die drei Wörterbuchprojekte der BAdW im gemeinsamen Portal BDO zusammen auftreten und auch die älteren Wörterbücher von SCHMELLER bzw. FISCHER (als Faksimiles oder als digitale Versionen) integriert sind.

\subsubsection{Bearbeiterinnen}

Bei jedem Artikel ist mit dem entsprechenden Kürzel vermerkt, welche Redaktorin ihn verfasst hat. Derzeit sind EDITH FunK (EF) und BRIGITTE SCHWARZ (BS) die Bearbeiterinnen.

\subsection{Die Potenziale von DIBS}

Was DIBS ist, entscheidet der Nutzer / die Nutzerin selbst. Es kann ein modernes Online-Wörterbuch sein, ausgestattet mit beliebig kombinierbaren Suchfunktionen, bei dem er/sie z. B. durch die Möglichkeit von Aus- und Einblendungen eigenständig Schwerpunkte setzen kann. DIBS kann aber auch ein Publikum bedienen, das wie in einem traditionellen, gedruckten Wörterbuch mit dem gewohnten Layout schmökern möchte.

DIBS soll aber mehr sein: Sprachatlas, sprechender Sprachatlas, Sammlung von Sprachdaten aus unterschiedlichen Erhebungen, aber auch von längeren zusammenhängenden Mundarttexten wie Erzählungen, Berichten, Beschreibungen, Bild-, Film- und Tonsammlung z. B. mit volks- und sachkundlichen Inhalten u. v. A. m. - und dies alles miteinander vernetzt und verwoben.

Freilich kann DIBS immer nur sein, was potentiell in ihm angelegt ist. Für ein brauchbares Wörterbuch müssen also aus dem in der Datenbank bereits vorhandenen Material Artikel verfasst werden, was nicht automatisch geschehen kann, da es ohne Interpretation nicht geht. Die zusätzlichen Materialien, die noch nicht in die 
Datenbank eingespeist sind, müssen erst noch integriert werden. Dies allein genügt aber nicht. Auch dieses Material muss interpretiert werden, um ausgezeichnet werden zu können, damit es für sinnvolle Suchen nutzbar ist. Und es müssen für die Redaktorinnen und Bearbeiterinnen sowie für die Nutzer/-innen Bearbeitungsbzw. Nutzungstools erarbeitet und bereitgestellt werden.

\subsubsection{DIBS: was schon möglich ist}

Bis vor ca. einem Jahr war geplant, in die Datenbank kein weiteres Material außer die das Untersuchungsgebiet betreffenden oben genannten Mundartgeographischen Fragebögen und Maurerbögen aufzunehmen. Das Material ist linguistisch aufbereitet und die Bearbeitungstools sind soweit fertig und getestet, dass bereits Artikel geschrieben werden konnten (bislang über tausend). Was die Werkzeuge betrifft, so sind noch ein paar Kinderkrankheiten auszumerzen und einzelne Wünsche der Redaktorinnen zu erfüllen. Derzeit wird die XML-Struktur für die Artikel erarbeitet. Dann können in diesem Jahr die Datenbank sowie die ersten Artikel des Wörterbuchs ins Netz gestellt werden. Denn jeder Artikel soll unmittelbar nach seiner Erarbeitung online gestellt werden und das Wörterbuch auf diese Weise allmählich wachsen.

\subsubsection{DIBS als Wörterbuch}

Die primären Aufgaben von DIBS als Wörterbuch sind es, über die Bedeutungen eines Wortes, seine regionale Verbreitung, seine Verwendung in Phraseologien und ggf. übertragene Bedeutungen zu informieren. Grammatische oder lautliche Besonderheiten und die Wortgeschichte werden thematisiert; auch die Wortfamilie wird genannt, zu der ein Wort gehört. Darüber hinaus bietet DIBS durch die zahlreichen Belege ein reichhaltiges Corpus, das als Materialbasis für linguistische Studien dienen kann, zu Lautung, Morphologie, aber auch zur Syntax, da häufig Satzbelege zitiert werden, die eine Verwendungsmöglichkeit eines Wortes im Satz demonstrieren sollen. In den Mundarten sind zahlreiche Wörter lebendig, die in der Hochsprache veraltet oder ausgestorben sind oder gar nie in sie vordringen konnten; daher ist DIBS eine Fundgrube für sprachhistorische Studien. Besonders reichhaltiges Material bietet sich der Phraseologieforschung, Untersuchungen zu Reimen, Sprichwörtern, Wetter- und Bauernregeln und allen möglichen Sentenzen. Durch die Kennzeichnung besonderer Verwendungen wie ,abwertend“, „,kindersprachlich“, ,scherzhaft“" etc. sind pragmalinguistische Studien möglich. Untersuchungen von Gruppen- oder Fachsprachen werden bedient durch Auszeichnungen wie z. B. „gaunersprachlich“, ,jägersprachlich“.

Aber nicht nur die Sprachwissenschaft kann profitieren. Die Volkskunde etwa findet zahlreiche Hinweise und Beschreibungen zu Alltagsleben und Brauchtum, zu religiösen Traditionen, zu Volksmusik und Spielen. Es finden sich Beschreibun- 
gen von Arbeitsgeräten und Arbeitsweisen der Landwirtschaft sowie des traditionellen Handwerks und v. A. m. Selbst die Tier- und Pflanzenkunde kann wertvolle Hinweise erhalten z. B. wo welche Apfel-, Birnen-, Rübensorten üblich waren, wo welche Acker(un)kräuter vorkamen, wo welche Rinder- oder Pferderassen, welche Insekten, welche Wildtiere etc.

\subsubsection{DIBS und seine Datenbank online}

Nicht alle Felder der Datenbank werden in den Artikeln abgebildet. Die Listen mit Belegen etwa, die besondere lautliche oder morphologische Formen enthalten, werden nicht aufgenommen. Auch die nicht zitierten, nur für die geographischen Verbreitungsangaben gezählten Belege sind im Artikel nicht sichtbar. Da der Nutzer / die Nutzerin auf die Datenbank zugreifen kann, ist dieses Material für ihn jedoch nicht verloren. Nicht einsehbar sind selbstverständlich die Felder mit arbeitsorganisatorischen oder automatensteuerungstechnischen Inhalten, wie z. B. „Notizen“ oder „Rang“. Dem Nutzer / der Nutzerin werden Bearbeitungstools zur Verfügung gestellt, sodass er/sie das Basismaterial in ähnlicher Weise durchsuchen, zusammenstellen, herunterladen und bearbeiten kann wie die Redaktorinnen. Selbstverständlich ist die Datenbank mit einem Schreibschutz versehen.

\subsubsection{Was DIBS noch werden soll: ein dialektologisches Informationssystem}

Geplant ist die Integration vielfältiger Materialien unterschiedlicher Medien in die Datenbank, deren Vernetzung und deren Ausstattung mit Bearbeitungstools (vgl. dazu Kapitel 2.2.2) sowie die Einbindung des Nutzers / der Nutzerin als Forscher/ -in. DIBS soll auch mit anderen Projekten vernetzt werden, zum einen durch den gemeinsamen Online-Auftritt mit den beiden verwandten Projekten der Akademie BWB und WBF in BDO (vgl. dazu Kapitel 4), zum anderen durch die Vernetzung mit weiteren Projekten wie Online-Wörterbüchern, ganzen Wörterbuchnetzen aber auch nichtlexikographischen Projekten wie Verba Alpina, heimat-, volks- und sachkundlichen Projekten und digitalen Sammlungen.

Die Pläne einer praktischen Umsetzung der hier beschriebenen Ziele sind noch nicht weit gediehen, sodass man nichts Genaueres darüber sagen kann. Die Unterschiede der Materialien, der Medienarten und Präsentationsformen sind zudem so groß, dass bei der Integration wohl jeweils eigene Herangehensweisen erforderlich sind. Deshalb kann hier nicht differenziert darauf eingegangen werden. Anhand des SBS soll aber beispielhaft angedeutet werden, wie solche Materialien nutzbar zu machen sind. 


\subsubsection{Ein Beispiel: DIBS als aktiver Sprachatlas}

Der SBS enthält etwa 11000 Lemmata auf ca. 2700 Karten mit den dazugehörigen Legenden und Kommentaren; den Wortkarten sind darüber hinaus die Beleglisten mitgegeben. Durch Verlinkungen der Lemmata mit den Stichwörtern der Datenbank bzw. des entsprechenden Wortartikels könnte man an die Stellen im SBS gelangen, in denen das Wort vorkommt. Wenn das Archiv des SBS zu einem Lemma auch ein Foto oder eine Tonaufnahme enthält, könnte man sich das Dokument ansehen bzw. anhören. (Zu den Möglichkeiten einer Einbindung vgl. Kapitel 2.2.2)

In der nicht publizierten digitalen Fassung des $\mathrm{SBS}^{9}$ sind die Einzelkarten (also nicht die Kombinationskarten), zu denen auch die Wortkarten gehören, über die Symbole auf der Karte mit nichtlinguistischen Daten bereits vernetzt (etwa Alter, Geschlecht, Religionszugehörigkeit, Geburtsort der Gewährspersonen, Herkunft deren Eltern und Großeltern sowie des Ehepartners, Größe des Ortes, überwiegende Religionszugehörigkeit der Bewohner). Dabei ist es nicht nur möglich, sich die einem bestimmten Symbol auf der Karte zugeordneten Daten anzusehen. Vielmehr kann man je nach Belieben ganze Gruppen von Symbolen mit bestimmten Merkmalen optisch verändern oder ein- und ausblenden. So könnte man sich nur jene Symbole zeigen lassen, die Orten mit weniger als 500 Einwohnern zugeordnet sind, nur männlichen oder nur weiblichen Gewährspersonen, oder den unter 60jährigen, oder den über 80jährigen, oder auch kombiniert nur den über 80jährigen Frauen u. v. A. m. Auch die Symbole selbst könnte man verändern, z. B. alle weiblichen Gewährspersonen zugeordneten Symbole rot einfärben lassen oder durch ein ganz anderes Symbol ersetzen.

Anhand von ca. 2300 Karten des SBS wurde mit aufwändigen mathematischen und statistischen Verfahren eine Dialektgliederung mittels dialektometrischer Faktoren von SIMON PRÖLL (2015) erarbeitet. Denkbar wäre, dass der Nutzer / die Nutzerin Karten dieses Projekts mit den Karten des SBS überblenden bzw. sie als Layer verwenden kann.

Da das Basismaterial des SBS, die Beleglisten, ebenfalls integriert werden können, kann der Nutzer / die Nutzerin je nach Wunsch und Interesse, selber Karten zeichnen. Er / sie könnte mit den bereitgestellten Kartierwerkzeugen sogar Karten mit projektfremdem Material erzeugen.

\subsubsection{DIBS und Citizen Science}

Wir möchten die Öffentlichkeit nicht nur passiv am Projekt teilhaben, sondern aktiv daran teilnehmen lassen: „Citizen Science“ oder „Nutzer/-innen als Forscher/-innen“. Dies ist in der Dialektologie (anders als z. B. in den Naturwissenschaften) überhaupt nichts Neues, sie hat den gemeinen Mann, die Frau vom Lande (und der

9 WERnER KÖNIG, Digitaler Sprachatlas von Bayerisch-Schwaben. Programmiert von CHRISTOPH BunZ, STEFAN MAIER, MARKUS STROHMEIER, JÜRGEN WERSINGER. Inhaltlich gefüllt von SABINE KLUG. Nicht publiziert. 
Stadt) immer schon als Gewährspersonen einbezogen. Die Sprecher/-innen sind unsere Materialquelle. Die Qualität ihrer Angaben entscheidet über die Qualität dialektologischer Forschung.

Für die praktische Durchführung dieser Interaktionsmöglichkeiten wurde noch kein detailliertes Konzept erarbeitet. Dies ist aber notwendig, damit die Resonanz nicht bloß in sporadischen und nur schwer überprüfbaren Mitteilungen oder fragwürdigen Kommentaren besteht. $\mathrm{Zu}$ überlegen ist, wie man die flüchtigen Besucher/-innen zu Dauergästen macht und animiert, auch eigene Kommentare zu Artikeln oder anderem Material abzugeben sowie die Datenlage zu verbessern. Zu überlegen ist, wie es um die Zuverlässigkeit der Sammler/-innen und ihrer Materialien steht. Die Ermittlung der Sozialdaten ist unerlässlich, wobei die Zusammenarbeit mit Datenschutzbeauftragten wohl nötig sein wird. Vielleicht kann man von den Erfahrungen anderer profitieren: Das BWB unterhält seit Jahrzehnten ein Sammlernetz von mehreren Hundert Sammler/-innen. Vom „Atlas zur deutschen Alltagssprache“ $(\mathrm{AdA})^{10}$ könnte man lernen, wie man über digitale Medien kompetente Sammler/-innen rekrutiert.

\section{TEXTTECHNOLOGISCHE ASPEKTE}

Dieser Abschnitt behandelt die Digitalisierungsarbeiten von DIBS, die seit Dezember 2017 mithilfe der IT-Abteilung der BAdW durchgeführt werden. Die folgenden Seiten dokumentieren diese ausführlich, stellen deren wissenschaftlichen Mehrwert detailliert dar und liefern Informationen zur Realisierung sowie zur verwendeten Infrastruktur.

Teile dieses Abschnitts, die die allgemeinen Eigenschaften des „LexHelfers“ behandeln, findet man im Beitrag zum „Fränkischen Wörterbuch“. An entsprechenden Stellen wird gesondert darauf verwiesen.

\subsection{Datenbasis und Redaktionssystem}

\subsubsection{Datenbasis}

In DIBS stellt die Datenbasis eine im Jahr 1998 erstellte und seither konstant verwendete und befüllte Access-Datenbank dar. Die Daten selbst liegen dort im proprietären Jet-Format vor; die Oberfläche ist eine mit den Formularmöglichkeiten der Anwendung gestaltete Eingabe- und Suchmaske. ${ }^{11}$ Der Export der Wörterbuchartikel in eine XML-Struktur wurde mithilfe eines in C\# geschriebenen Programms und via XSLT vorgenommen. Da es in Access unter Umständen bei mehreren gleichzeitigen Zugriffen zu Schreibproblemen und damit zu Datenverlust kommen kann, wurde zunächst die Möglichkeit der Konvertierbarkeit in ein diesbezügliches

$10<$ http://www.atlas-alltagssprache.de>; Stand: 12.03.2019.

$11<$ https://en.wikipedia.org/wiki/Microsoft_Jet_Database_Engine>; Stand: 12.03.2019. 
geeigneteres Datenformat bzw. -system untersucht, damit parallel darauf zugegriffen werden kann. ${ }^{12}$ Es zeigte sich schnell, dass ein Wechsel zu MariaDB möglich war. Dieses Datenbankmanagementsystem wird in der Softwareentwicklung vielfach in Webanwendungen eingesetzt, sodass die Entscheidung für eine Neuentwicklung der bisherigen Access-Datenbank als Webanwendung fiel. $\mathrm{Zu}$ diesem Zweck wurde das nach MariaDB konvertierte SQL bezüglich der verwendeten Datentypen etwas optimiert, sonst aber zum Großteil strukturell nicht verändert. Lediglich gemeinsam genutzte Felder (z. B. Ortsangaben) wurden in Verknüpfungstabellen aufgespalten, damit die Werte einheitlich in einer Haupttabelle gepflegt werden können.

\subsubsection{Redaktionssystem}

Obwohl die Redaktionssysteme des BWB und des WBF denselben Ursprung haben, welcher jedoch in aktuell keiner Weise Berührungspunkte mit dem von DIBS hat, ist dessen Name dennoch ebenfalls LexHelfer, da es sich auch hier um ein Hilfsprogramm zur lexikographischen Arbeit handelt. Mit der geplanten Einspeisung von Digitalisaten verschiedener Fragebögen wird zudem auch der DIBS-LexHelfer die in den beiden anderen Programmversionen enthaltene Ansicht und Lemma-Verknüpfung von Belegausschnitten erhalten.

Die Entwicklungsarbeiten der Anwendung begannen im Januar 2018 in engem Kontakt mit den beiden Redaktorinnen des Wörterbuchs und auf Basis der Funktionen der bis dato verwendeten Access-Datenbank. Nach einigen Tests und anschließenden Korrekturen wurde schließlich im Juli 2018 der Umstieg von Access auf LexHelfer vollzogen. Seither werden alle Daten eines Wörterbuchartikels (u. a. Lemma, Etymologie, Bedeutung, Grammatik, Belege, Quellen, Sachgruppe) in diesem erfasst und durch einen Export nach XML geschrieben. Somit dient LexHelfer für dieses Projekt nicht der Exzerpierung oder Erfassung von Metadaten zu digitalisierten Belegen, sondern direkt zur Erstellung des fertigen Artikels. Dabei wurde beachtet, dass später ggf. Mediendateien eingefügt werden können, um Artikel bzw. Artikelteile damit aufwerten zu können. Zum Januar 2019 bietet die Anwendung folgende Funktionen:

- Erfassung aller im Artikel erscheinenden Felder

- Verwendung von Unicode

- Erfassung interner Felder, die die kollaborative Arbeit erleichtern

- Quellenverzeichnis

- Ortsverzeichnis

- Suche nach Lemma oder Beleg

- Suche nach Bedeutungen und Suche nach Sachgruppen

12 Während der Entwicklung des neuen Redaktionssystems traten ein paar dieser Probleme auf, die zwar durch Backups gelöst werden konnten, auf Dauer jedoch kein komfortables und sicheres Arbeiten ermöglichen. 
Für 2019 sind die Entwicklungen weiterer Funktionen geplant, ebenso wie die Onlinestellung des gesamten Datenbestandes für die interessierte Öffentlichkeit.

\subsection{Mehrwert eines digitalen Workflows}

DIBS setzt von Anfang an auf digitale Methoden zur Erarbeitung seiner Inhalte. Dadurch liegen seit jeher alle Artikeldaten des Projekts nativ digital vor - ein vorteilhafter Umstand insbesondere im Hinblick auf die internetgestützte Veröffentlichung der erarbeiteten Daten sowie die Möglichkeit der Vernetzung mit anderen Projekten. Die Struktur der Datenbasis ist zudem seit Beginn relational, sodass viele sonst anfallenden Redundanzen vermieden wurden. Auch die Weiterverarbeitung der Daten ist dadurch besser realisierbar, zum einen zu anderen relationalen Formaten, zum anderen aber auch zu XML.

Neben dem nachfolgenden Mehrwert der Unicode-Dateneingabe liefert der DIBS-LexHelfer auch den für solche Systeme üblichen Vorteil der Geschwindigkeit in der Suche sowie der Datenverarbeitung. Siehe hierzu vergleichend jeweils die Kapitel 3.2.1 der Artikel zum „Bayerischen Wörterbuch“ und zum „Fränkischen Wörterbuch".

\subsubsection{Dateneingabe in Unicode}

In Access wurden noch Kodierungen verwendet, die auf ASCII-Zeichen basierten und für projektfremde Nutzer/-innen unleserlich erschienen. ${ }^{13}$ So wurde z. B. die Kodierung „O5O5“ für $\bar{o}$ verwendet. Mithilfe des quelloffenen und leistungsstarken Texteditors TinyMCE ist es möglich, durch eine frei definierbare Zeichentabelle unicode-basierte Buchstaben und Diakritika mit nur einem Klick hinzuzufügen. ${ }^{14}$ Durch eine Änderung des offenen Quellcodes des Editors wurde zudem erreicht, beliebig viele Zeichen kombinieren zu können, sodass auch einer sehr granularen Angabe lautlicher Phänomene nichts im Wege steht (sofern Unicode Zeichen dafür bereitstellt). ${ }^{15}$ Die zuvor verwendete ASCII-Kodierung wurde durch ein Script automatisch ersetzt. In Kombination mit HTML-Auszeichnungen, z. B. für Hoch-/Tiefstellung oder Kursivierung, können im DIBS-LexHelfer somit Artikelinhalte deutlich genauer als zuvor in Access formatiert werden. Einige dieser Auszeichnungen werden in der Weiterverarbeitung zu XML verwendet, um Elemente zu generieren.

13 ASCII = American Standard Code for Information Interchange; das Amerikanische Alphabet.

14 <https://www.tiny.cloud>; Stand: 12.03.2019.

15 Siehe Fork des Charmap-Plugins unter <https://github.com/tinymce/tinymce/pull/4225> bzw. <https:/github.com/manuelraaf/tinymce/tree/master/src/plugins/charmap>; Stand: 12.03.2019. 


\subsection{Technik}

Die allgemeine Beschreibung der Serverumgebung ist identisch mit der des WBF (vgl. dazu Kapitel 3.3 im Beitrag zum WBF).

\subsubsection{Probleme}

\subsubsection{Modernisierung: mit Access ins Web?}

Es war grundsätzlich nicht schwer, die Daten aus Access in eine MariaDB-Datenbank einzuspeisen, da es hierfür freie Tools gibt. ${ }^{16}$ Problematisch an der Migration gestaltete sich die Notwendigkeit der vollständigen Neuentwicklung der Oberfläche, da aus Access keine einzige Zeile Code übernommen werden konnte. Visual Basic und HTML/JavaScript sind grundsätzlich verschiedene Sprachen, die in unterschiedlichen Domänen arbeiten und daher nicht ineinander überführt/ umgewandelt werden können. ${ }^{17}$ Eine Access-Anwendung kann daher auch nicht direkt ins Web gestellt werden.

Dieses Problem betrifft jedoch weder speziell noch allein DIBS, sondern alle, insbesondere älteren Anwendungen, die im Laufe der Jahre in ein moderneres System überführt werden sollen bzw. müssen. Es soll hier lediglich der Vollständigkeit halber erwähnt sein, da viel Zeit für eine solche Migration bzw. Modernisierung eingeplant werden muss. Außerdem soll die Wichtigkeit etablierter sowie offener Sprachen und Systeme ebenso unterstrichen werden wie die Möglichkeiten des Internets: Durch sie können Aufwände für künftige Modernisierungsmaßnahmen auf ein Minimum reduziert und grundsätzlich eine nachhaltigere Bereitstellung des Systems und seiner Daten erreicht werden. Insbesondere durch die stetig steigende Leistungsfähigkeit internetfähiger Endgeräte und der ebenfalls steigenden Leitungsgeschwindigkeiten ist es zudem ratsam, kollaborative Systeme als Webanwendungen zu konzipieren, sofern keine gewichtigen Gründe dagegensprechen.

16 <https://github.com/brianb/mdbtools>; Stand: 12.03.2019.

17 Visual Basic ist eine von Microsoft entwickelte Sprache zur Erstellung eigenständiger Desktopanwendungen oder diesen ähnelnden Formularen innerhalb der MS-Office-Programme (in diesen heißt die Sprache aufgrund des geringeren Funktionsumfangs Visual Basic for Applications, kurz VBA). Sie wird insbesondere in Access verwendet, um Formulare zu definieren und Aktionen auszuführen, da dies der Weg der Benutzeroberflächengenerierung in Access ist. Ersteres geschieht vorwiegend durch Drag \& Drop, sodass unter Umständen keine Zeile Code geschrieben werden muss, um ein funktionierendes Formular zu erhalten. Aus diesem Grund gilt Access als einfache Datenbankanwendung, mit der auch Laien schnell umgehen können. HTML und JavaScript hingegen sind Sprachen, die für Webseiten gedacht sind und durch ein geeignetes Programm erst korrekt angezeigt werden können (primär via Browser). Sie unterscheiden sich beide syntaktisch voneinander und jeweils zu Visual Basic. HTML legt als Seitenbeschreibungssprache das Layout der Webseite fest; JavaScript modifiziert die Inhalte ohne Neuladen selbiger. 
Durch das Internet ergeben sich u. a. die Vorteile der allgegenwärtigen Verfügbarkeit, der öffentlichen Sichtbarkeit und der damit auch stärkeren Verbreitungsmöglichkeit als dies jeweils bei Offline-Anwendungen der Fall ist.

\subsubsection{Generizität}

Vgl. dazu das entsprechende Kapitel im Artikel des WBF.

\subsubsection{Dateninkonsistenz und -redundanz}

In Access-Anwendungen kann durch verschiedene Methoden, jedoch immer programmseitig, verhindert werden, dass Inkonsistenzen oder Redundanzen im Datenbestand entstehen. In der Access-Datenbank von DIBS war dies jedoch nicht ausreichend der Fall, sodass insbesondere bei Ortsnamen und den Feldern, die sie enthielten, diese beiden Probleme auftraten. Insbesondere in den Feldern, in denen die Eintragung mehrerer Orte erlaubt waren, konnte keine automatisierte Normalisierung der Fehlschreibungen vorgenommen werden, da diese nicht immer eindeutig durch Textmuster erkennbar waren.

Zur Lösung wurden die eindeutigen Fehler in eine Excel-Liste geschrieben, dort korrigiert und anschließend in die Datenbank eingespeist. Übrig gebliebene Fehler werden sukzessive bei Auffinden über das angelegte Ortsverzeichnis im Redaktionssystem direkt und manuell behoben. Um erneute Inkonsistenzen oder Redundanzen im Redaktionstool zu verhindern, wird beim Speichern eines neuen bzw. beim Umbenennen eines bereits existenten Ortes geprüft, ob dieser bereits im Bestand enthalten ist.

\subsection{Nachhaltigkeit}

Auch die Informationen zur Nachhaltigkeit des Redaktionssystems sind identisch mit denen des WBF und daher dem entsprechenden Kapitel dort zu entnehmen.

\subsection{Ausblick}

Die Datenbestände der an der BAdW ansässigen Dialektwörterbuchprojekte BWB, WBF und DIBS werden aller Voraussicht nach zusammen mit dem Datenbestand der BayDat im Laufe des Jahres 2019 in einer gemeinsamen Anwendung namens „Bayerns Dialekte Online“ online gehen, sodass Fachwissenschaftler/-innen wie auch interessierte Laien alle Materialquellen und Wörterbucheinträge durchsuchen und miteinander vergleichen können. Für Letzteres steht insbesondere die Sach- 
gruppenzuordnung nach POST (1998) im Vordergrund, um unabhängig vom Lemma eine verlässliche Verknüpfung von Dialektwörtern über die jeweiligen Dialektgrenzen hinweg anbieten zu können.

In DIBS werden Sachgruppen bei der Artikelerstellung handverlesen eingetragen. Davon unabhängig können die einzelnen Datenquellen stets über das Lemma oder die Bedeutung miteinander verbunden werden.

Die fertigen Artikel sollen zudem über eine Exportfunktion als XML heruntergeladen werden können, um diese eigens weiterzuverarbeiten und ggf. zu drucken.

Es stehen für 2019 außerdem Weiterentwicklungen des Funktionsumfangs im DIBS-LexHelfer an, um den Komfort der redaktionellen Arbeit weiter zu erhöhen und diese dadurch zu beschleunigen, sowie die Onlinestellung aller Datenbankinhalte für die interessierte Öffentlichkeit. In den Jahren 2020 sowie 2021 wird zudem geprüft, welche externen Datenquellen auf welche Weise zur Anreicherung der Artikel verwendet werden können (z. B. Audioaufnahmen oder Bildmaterial). Ferner ist die Einbindung digitalisierter Fragebögen geplant.

Die fertigen Artikel von DIBS sollen im seit Februar 2019 entstehenden Sprachinformationssystem „Bayerns Dialekte Online“ (kurz: BDO) publiziert werden und innerhalb dieses Systems auch granular recherchierbar sein, sodass Fachwissenschaft und interessierte Laien bequem und in wählbaren Graden alle Inhalte durchsuchen können. Über BDO wird zudem die Verknüpfung der Inhalte von DIBS mit denen des BWB und WBF erfolgen.

\section{BAYERNS DIALEKTE ONLINE (BDO): DIE INTEGRATION DER BAYERISCHEN DIALEKT-WÖRTERBÜCHER}

Mit der Abrundung der Dialektprojekte der BAdW durch die Aufnahme von DIBS in den Akademie-Kanon entstand erstmals die Möglichkeit, ein gemeinsames Online-Portal für alle bayerischen Dialekte zu schaffen. Ausgehend von der für DIBS geplanten, ausgefeilten Online-Anwendung können nun in der Konzeption auch die für BWB und WBF notwendigen Erfordernisse berücksichtigt werden.

Ziel ist es, ein umfassendes Informationssystem rund um die bayerischen Dialekte zu schaffen, in dem die Dialekt-Substanzen miteinander auf vielfältige Weise vernetzt sind, auf Expertenlevel gemeinsam durchsucht werden können und die mit gemeinsam nutzbarem Anschauungsmaterial aller Art angereichert werden können.

Die zentralen Funktionen der Online-Anwendung können dabei dieselben sein für alle drei Wörterbücher. Eine deutlich schwierigere Aufgabe ist die Frage, wie die für eine gemeinsame Online-Stellung benötigten granularen Informationseinheiten aus den bestehenden Substanzen des BWB zu extrahieren bzw. die fehlenden redaktionellen Artikel des WBF zu ersetzen sind und dann alles in einen „Topf“ resp. eine Datenbank zu bringen ist, denn die Projektkonzeptionen könnten unterschiedlicher nicht sein.

So ist die Hauptaufgabe des BWB die Erstellung einer Printausgabe, von der bereits drei komplette Bände vorliegen. Diese müssen jedoch für die BDO retrodi- 
gitalisiert werden, d. h. von einer intensiv mit Verdichtungen arbeitenden Printversion muss eine online-fähige Datenvariante erstellt werden, die den Suchbedarf der Nutzer/-innen befriedigt und daher ebenso granuliert vorliegen muss wie die Daten aus DIBS.

Beim WBF dagegen ist vorerst keine Publikation und damit auch keine Artikelerstellung vorgesehen, sondern das Hauptaugenmerk liegt auf der kompletten Erfassung und Bereitstellung der Belege.

Insofern sind auch die im Redaktionsprozess benutzten Tools verschiedene: Nutzt z. B. das BWB für die Artikelerstellung bislang ausschließlich MS Word mit nur minimalem Einsatz von Formatvorlagen (v. a. zur typografischen Auszeichnung, nicht jedoch für die semantisch-granulare Kennzeichnung der einzelnen Informationen in einem Artikel), so werden die fränkischen Belege in MS Excel erfasst, lemmatisiert und in LexHelfer eingespielt, wo sie in tabellarischer Form zur Verfügung stehen und zu Korrekturzwecken lemmaweise in HTML ausgespielt werden können.

Dementsprechend unterschiedlich sind die granularen Mikrostrukturen von DIBS, BWB und WBF in ihrer Abfolge und Tiefe der Informationserschließung. Trotzdem bleibt es die Hauptaufgabe von BDO, die Inhalte von DIBS, BWB und WBF gemeinsam nutzbar zu machen.

\subsection{XML als „Datendrehscheibe“}

Die Lösung, die den größtmöglichen Nutzen für die Online-Anwendung verspricht, ist der Einsatz von XML als „Datendrehscheibe“ - als neutrales Format, dem alle zu publizierenden Artikel genügen müssen.

Wie auch in anderen Wörterbuchprojekten bereits üblich (vgl. z. B. die Strukturierungen des Trierer Wörterbuchnetzes), wird die Artikelstruktur in ihrem semantischen Aufbau explizit gemacht, indem die Artikel nach ihren linguistischen Informationsarten segmentiert werden - und nicht auf der Basis ihrer typografischen Gestaltung. Diese kann ja jederzeit aus den semantischen Informationsarten erzeugt werden. Bei der Segmentierung lehnen wir uns an die Medio- und Mikrostrukturen von WIEGAND (1988-1999), in der Fortführung von MüLLER-SPITZER (2007) vom IDS an.

An dieser Stelle soll noch einmal betont werden, dass diese semantische Struktur den Artikel ausschließlich in seinen linguistisch relevanten Informationsteilen abbildet - und nicht in seinen typografischen, geschweige denn in medienspezifischen wie der Printform mit ihren intensiven Verdichtungen.

Bei der Wahl des XML-Dialekts haben wir uns für eine Eigenentwicklung entschieden, die ganz exakt auf die Erfordernisse der drei Wörterbücher und ihrer funktionalen Integration ineinander abgestimmt ist. Das hat den Vorteil, dass bei der Wahl der Auszeichnungsnamen die Begrifflichkeit der Redaktionen berücksichtigt werden kann, um eine gewisse Vertrautheit bei allem sonst Neuen zu schaf- 
fen, und dass im Arbeitsfluss bei der Artikelerstellung stets nur die (wenigen) Auszeichnungen zur Verfügung stehen, die auch benötigt werden, was einen Gutteil zur frühzeitigen Qualitätssicherung der Artikelstrukturen beiträgt.

Dass wir uns dabei nicht für den in wissenschaftlichen Projekten weit verbreiteten XML-Dialekt TEI P5 entschieden haben, liegt daran, dass dort zum einen nicht für alle Informationseinheiten eines BWB-Artikels semantische Auszeichnungen existieren und wir auf die sehr fehlerträchtige Qualifizierung über das typeAttribut oder andere Workarounds hätten zugreifen müssen. Zum anderen bietet der Einsatz der TEI ebenso wenig die Garantie der Interoperabilität mit „fremden“ Wörterbuchbeständen, wie es auch unsere Eigenentwicklung tut. Sollte eine TEIFassung des Datenbestands benötigt werden, z. B. für die Langzeitarchivierung, so kann sie jederzeit per Transformation hergestellt werden. Ebenso wie auch eine Printausgabe einschließlich ihrer Verdichtungen jederzeit aus den XML-Dokumenten generiert werden kann.

\subsection{Die konkreten Strukturmodelle}

Die konkreten Strukturmodelle bilden die Medio- und Makrostrukturen der jeweiligen Wörterbücher ab. Am klarsten und eindeutigsten ist dies an der Struktur für DIBS abzulesen (vgl. zum Folgenden Abbildung 7):

Ein jeder Wörterbuchartikel muss zumindest bestehen aus: Lemmaposition (lemma-position), Bedeutungsposition (bedeutung-position) und einer Verfasserangabe (verfasser). Außerdem können Angaben zu Etymologie (etymologie-position; vgl. Kapitel 2.3.6), Lautung und Formen (lautung-position; vgl. Kapitel 2.3.5), Literatur (literatur-position; vgl. Kapitel 2.3.7) gemacht und weitere Erläuterungen (erlaeuterung-position) angefügt werden.

Die Lemmaposition setzt sich zusammen aus dem Lemmablock mit Lemma, Lemmavarianten (lemma, lemma-variante; vgl. Kapitel 2.3.1) und Lemmaverweisen, die im Fall einer Printausgabe als Verweisartikel generiert werden können, dem Grammatikblock (grammatik mit den Attributen wortart, genus, numerus; vgl. Kapitel 2.3.2) und der Wortfamilie (vgl. Kapitel 2.3.4).

In der Bedeutungsposition werden alle Bedeutungen zusammengefasst, in ihren hierarchischen Bezügen verschachtelt angeordnet und jeweils mit Angaben zur Verbreitung (verbreitung-position; vgl. Kapitel 2.3.3.2), zur semantischen Verschlagwortung (sachgruppen; vgl. Kapitel 2.3.3.6), zur sach- bzw. volkskundlichen Einordnung (sachkunde-position, volkskunde-position; vgl. Kapitel 2.3.3.5) und den jeweils zugehörigen Belegen (beleg-position) versehen.

In der Belegposition stehen stets die Wortbelege zuerst - jeweils mit Belegtext, ggf. einem redaktionellen Kontext und der Quellenangabe (beleg-text, beleg-kontext, beleg-quelle; vgl. Kapitel 2.3.3.3) - gefolgt von Satzbelegen und Phraseologien (phraseologie, phrase; vgl. Kapitel 2.3.3.3).

Parallel zur Bedeutungsangabe (bedeutung; vgl. Kapitel 2.3.3.1) können auch Angaben zur Pragmatik (pragmatik; vgl. Kapitel 2.3.3.4) gemacht und weitere Erklärungen, wie grammatische Besonderheiten (erklaerung), ergänzt werden. 
Die XML-Ausgabe eines DIBS-Artikels wird durch Export aus der LexHelferDatenbank erzeugt und für die Online-Publikation in der Artikel-Datenbank abgelegt. Etwaige Fehler und Ungereimtheiten werden jeweils in LexHelfer bereinigt und der Artikel erneut in XML ausgespielt - solange bis eine (vorläufig) fertige Version des Artikels zur Publikation frei gegeben wird.

Spätere Ergänzungen des Artikels führen zu einer weiteren Version des Artikels in der Artikel-Datenbank, die dann als neueste Fassung in die Suchen einbezogen wird, während die ältere Fassung ,nur“ noch als Beleg für direkte Adressierungen zur Verfügung steht.

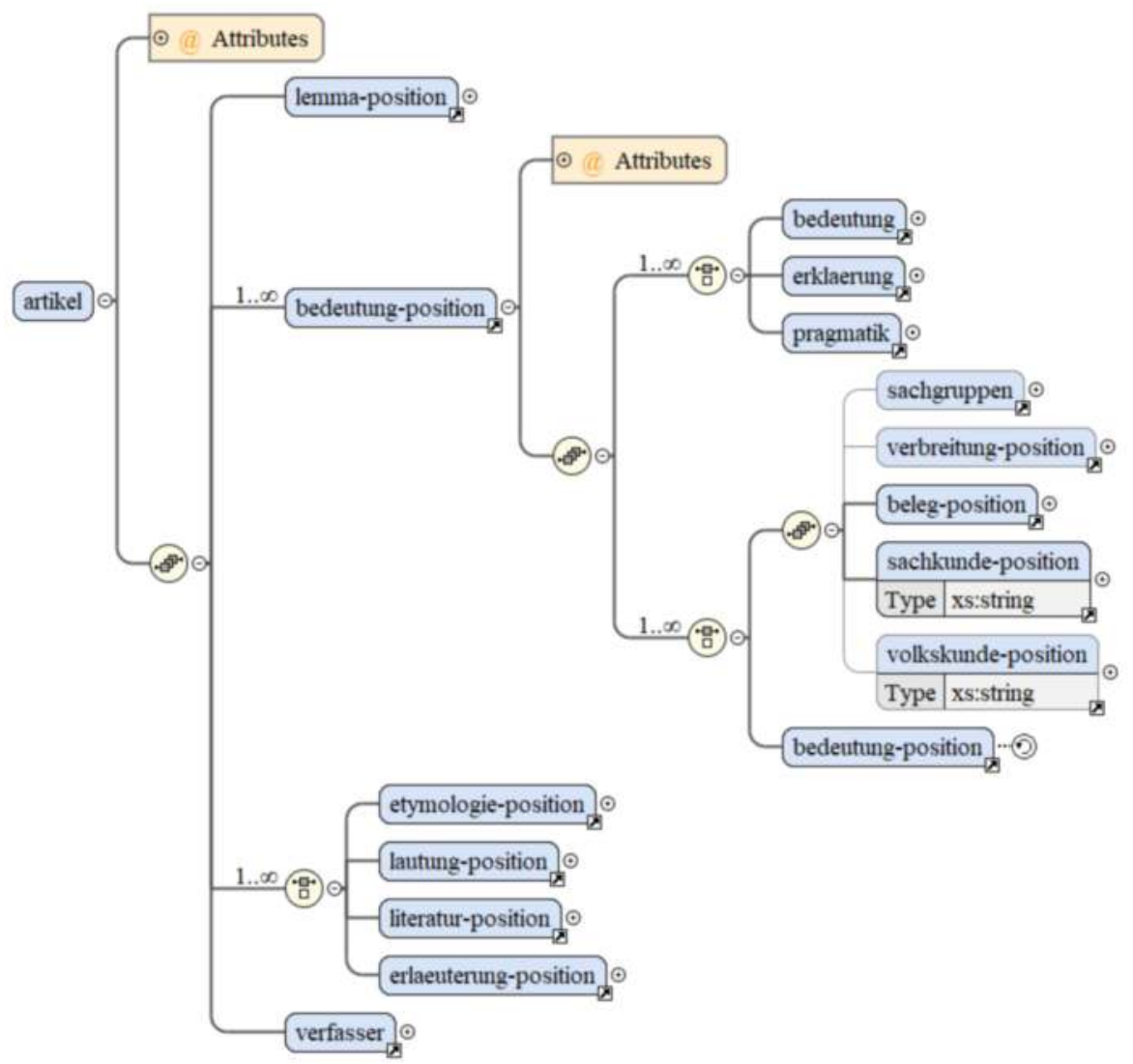

Abb. 7: Die Artikelstruktur von DIBS mit den wichtigsten Informationspositionen und Abhängigkeiten in der XML-Struktur 
Die Struktur des BWB vertieft diejenige des DIBS an verschiedenen Stellen. Augenfällig wird in Abbildung 8, dass die Struktur am Ende ergänzt wird um einen Komposita-Anhang (komposita-position) und um die Möglichkeit, Unikale zu beschreiben, die zwar lemmatisiert werden, jedoch nur in einem Kompositum vorkommen und daher keine Bedeutung (beim Lemma) aufweisen. Auch bei den Phraseologien stehen komplexere Möglichkeiten zur Verfügung, da diese hierarchisiert (geschachtelt) werden können (vgl. Abbildung 9).

Hier ist auch zu erkennen, dass die Auszeichnung pragmatik an zwei verschiedenen Stellen in der Struktur vorkommt. Dies ist den inhaltlichen Gepflogenheiten des BWB geschuldet. In der Online-Anwendung sollen sich jedoch die Angaben zur Pragmatik - egal an welcher Stelle im Artikel sie stehen - in einem gemeinsamen Suchregister wiederfinden.

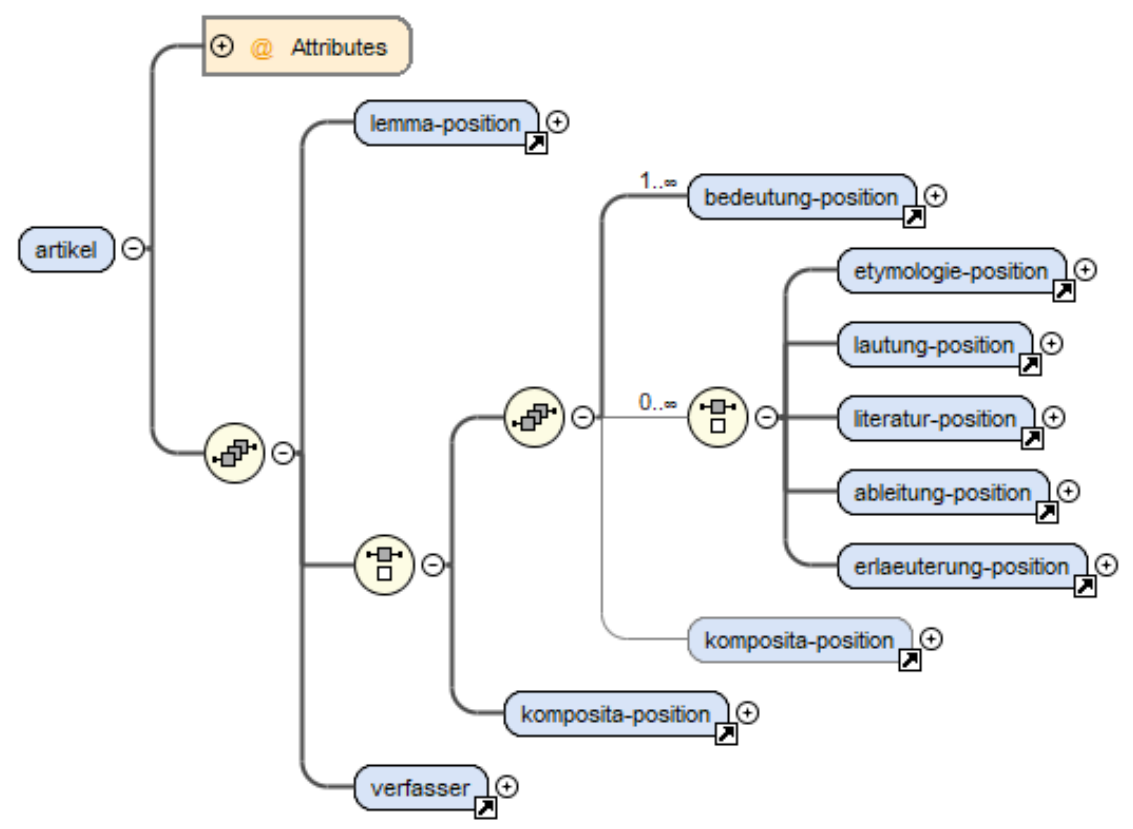

Abb. 8: Die Grundstruktur des „Bayerischen Wörterbuchs“ mit den wichtigsten Informationspositionen und Abhängigkeiten in der XML-Struktur 


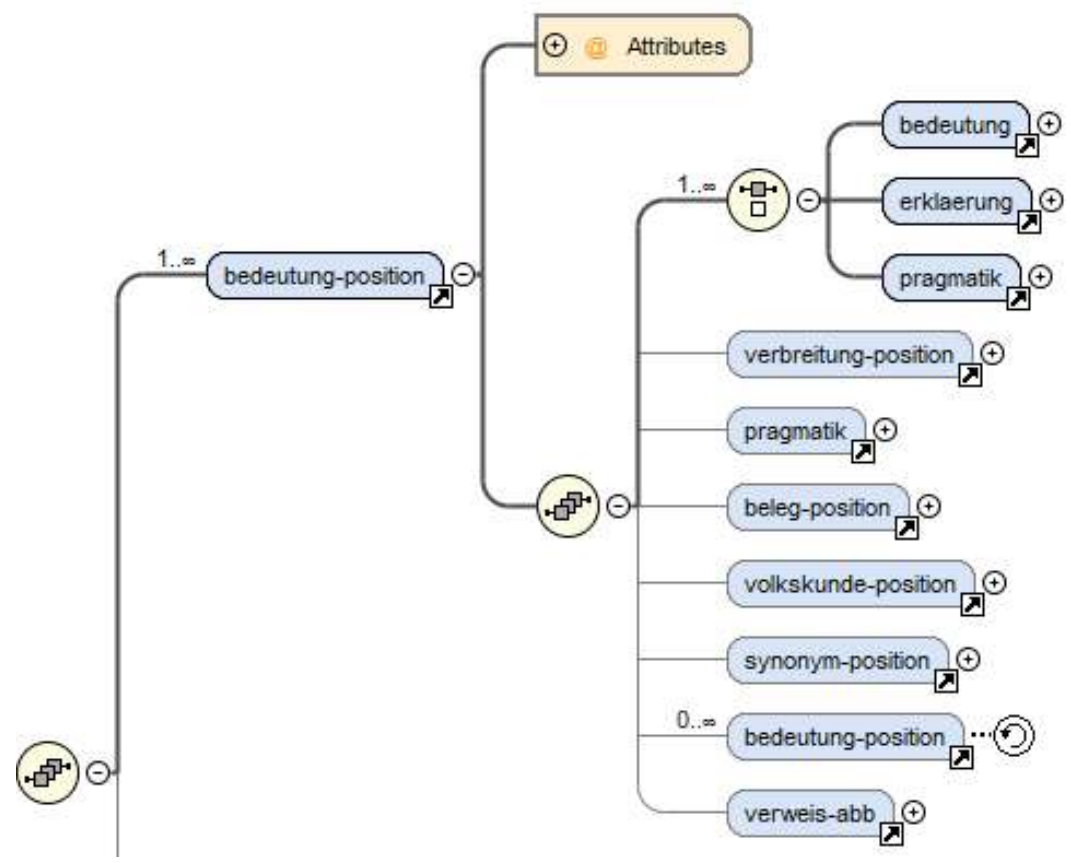

Abb. 9: Die Bedeutungsposition des BWB mit bedeutungsspezifischen Angaben, die zum einen die Informationsarten rund um die Bedeutung selbst und zum anderen die Möglichkeit zu ergänzenden Angaben zeigt, wobei die Bedeutung(sposition)en je nach ihrer Position in der Bedeutungshierarchie geschachtelt und/oder gereiht angeordnet werden können

Beim fränkischen Wörterbuch wurde vereinbart, deren zu Prüfzwecken erfolgenden HTML-Export in XML umzusetzen, um die Inhalte in dieser Form in die Artikel-Datenbank einzubinden. Aus der Struktur ist abzulesen, dass mit einem anderen Lemmakonzept gearbeitet wird, das sich aus einem übergeordneten Begriff (superlemma) und zugehörigen Wortnutzungen und Wortbildungen inkl. Komposita (grundform) zusammensetzt. Jeder Grundform sind dann wieder Bedeutungen mit ihren Belegen zugeordnet. 


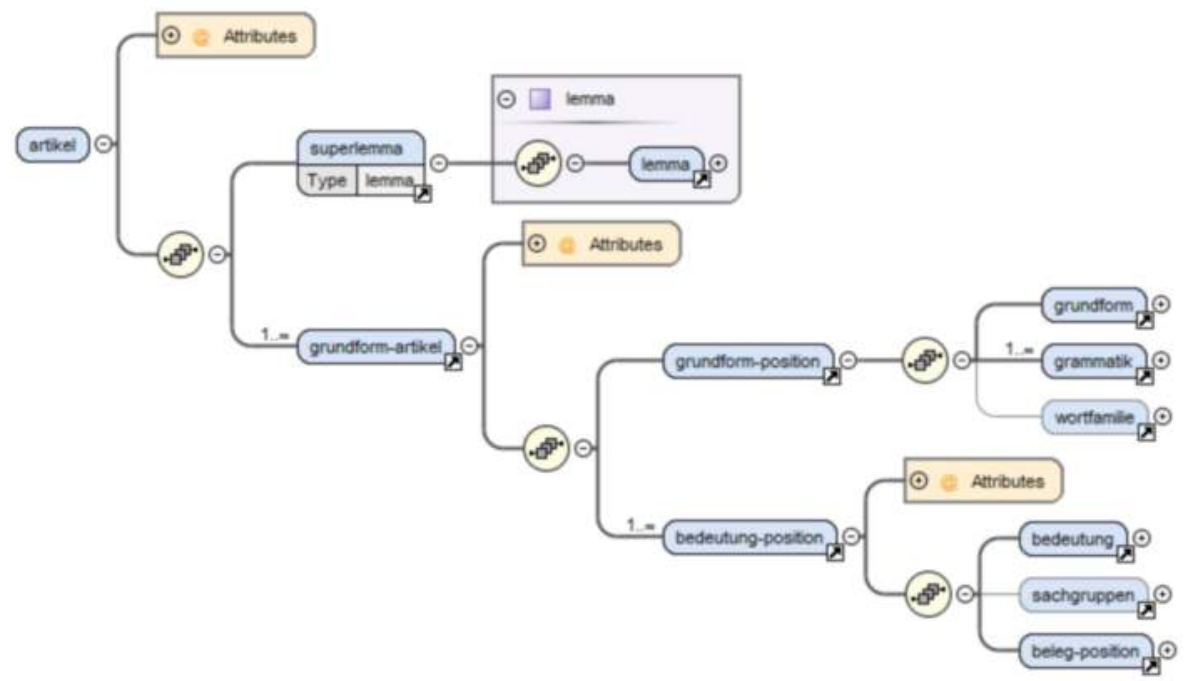

Abb. 10: Die Struktur des ,Fränkischen Wörterbuchs“ mit seinen generierten Artikeln

$\mathrm{Ab}$ Band 4 des BWB wird die Redaktion die Artikel mit Oxygen direkt in XML erfassen. Abbildung 11 zeigt einen Screenshot von der Erfassungsansicht.

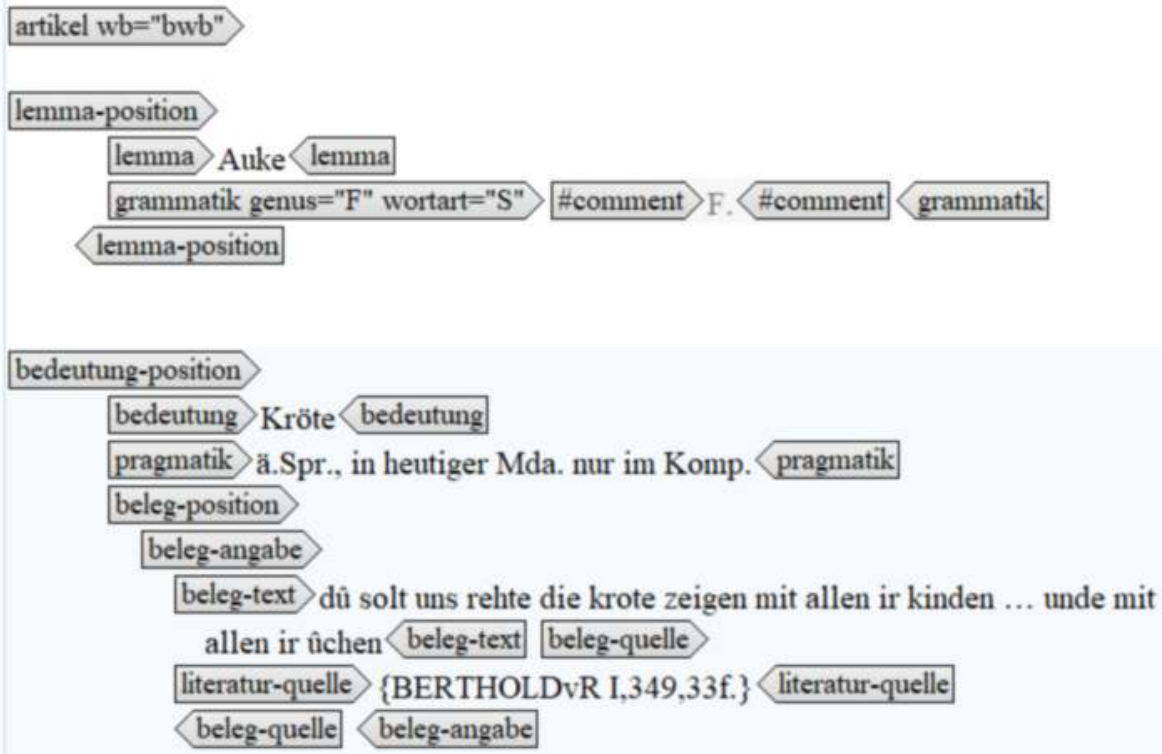

Abb. 11: Die Erfassungsansicht für die Artikel des BWB 


\subsection{Zur Datenaufbereitung resp. Retrodigitalisierung}

Die Aufbereitung der Wörterbuchdaten für eine Online-Bereitstellung gehorcht im Vergleich zu einer Printausgabe - ganz anderen Regeln für die Präsentation der Daten, da weder Platz gespart werden muss, um so wenige Seiten wie möglich zu „verbrauchen“, noch an Farbe oder Detailtreue der präsentierten Angaben geknausert werden muss.

Viel wichtiger ist es, dass sämtliche Angaben in einer Ausführlichkeit vorhanden sind, die die Eindeutigkeit der Inhalte zum Ergebnis hat und die dafür sorgt, dass Begriffe komplett in die jeweiligen Suchregister der Online-Anwendung eingehen können. Ein typisches Beispiel ist die Abkürzung des Lemmabegriffs an allen Stellen im Artikel - außer im Lemma selbst. Rezipient/-innen eines Artikels wissen natürlich, in welchem Artikel sie sich befinden und damit auch, was die Abkürzung „A.“ bedeutet. Eine Suchroutine dagegen findet hunderte Male diese Abkürzung und weiß nicht, um welches Lemma es sich handelt. Ähnlich geht es mit einem leer gelassenen Bedeutungsfeld, weil der Begriff gleichlautend im folgenden Belegtext oder redaktionellen Kommentar vorkommt. Die Suchroutine sieht nur, dass nichts da steht.

Ein weiterer Fall der lexikographischen Verdichtungspraxis sind zusammenfassende Formulierungen, wie sie v. a. im BWB vorkommen, z. B. „Oberbayern mehrfach", das die konkreten Belegorte verschweigt, nach denen der Nutzer / die Nutzerin wahrscheinlich sucht, oder auch die Formulierung „Restgebiet“, die aus der Sicht der Artikelverfasser ganz eindeutig ist, im Kontext mit den anderen beiden Wörterbüchern jedoch Unsicherheit über den konkreten regionalen Bezug aufkommen lässt. In DIBS als ,,born digital“ Wörterbuch werden diese Fälle von vorneherein berücksichtigt, in den anderen Fällen werden - soweit es vernünftig ist - die Angaben im Artikel auf die konkreteren Formulierungen zurückgeführt, wobei sowohl die Ursprungs- als auch der ergänzte Zustand über entsprechende Permalinks eindeutig adressierbar sein werden.

Aber nicht nur die der Printausgabe geschuldeten Verdichtungen benötigen Lösungen, sondern es gibt auch Strukturunterschiede, deren Kompatibilität mit den anderen Wörterbüchern nicht einfach nur durch gleiche Auszeichnungsnamen gelöst werden können. Hierzu zählen z. B. die Grammatikangaben, die üblicherweise direkt beim Lemma stehen und sich auch direkt auf dessen Grundform beziehen. In den WBF-Tabellen hingegen gibt es nur Grammatikangaben mit Bezug zum Belegtext, um dessen grammatische Eigenschaften exakt festzuhalten. Deshalb werden für die BDO die grammatischen Basisangaben beim Lemma aus diesen ausführlicheren Angaben extrahiert, um sie im gemeinsamen Suchpool ebenso suchbar und auffindbar machen zu können, wie die Angaben von DIBS und des BWB.

Was hier alles recht mühselig klingt - und zum Teil auch ist -, hat jedoch seine befreienden Momente immer dann, wenn es gelungen ist, einen derartigen Stolperstein per Routine in eine gleichwertige Information für die gesamte Anwendung umzuwandeln.

Ähnlich beglückende Momente wird es auch geben, wenn es gelungen sein wird, aus dem wenigen, was jetzt schon in DIBS und WBF mit den PosTschen 
Sachgruppen korrekt verschlagwortet ist, eine qualitativ ähnliche Verschlagwortung mit minimalem redaktionellen Aufwand auf die BWB-Daten zu übertragen. Insofern dürfen wir uns auf weitere befruchtende Auswirkungen der Integration der drei Dialektwörterbücher der BAdW - und vielleicht auch weiterer Wörterbücher - freuen!

\section{KONTAKTADRESSE, HOMEPAGE}

Dialektologisches Informationssystem von Bayerisch-Schwaben c/o Bayerische Akademie der Wissenschaften

Alfons-Goppel-Str. 11

80539 München

Deutschland

E-Mail: schwarz@swb.badw.de

Homepage: <https://dibs.badw.de/>

\section{LITERATURVERZEICHNIS}

AdA = Atlas zur deutschen Alltagssprache. URL: 〈http://www.atlas-alltagssprache.de>; Stand: 12.03.2019.

BayDat = Bayerische Dialektdatenbank. URL: <https://baydat.badw.de>; Stand: 28.02.2019.

BWB = Kommission für Mundartforschung (Hg.) (1995 ff.): Bayerisches Wörterbuch. München: Oldenbourg (Bände 1-3,1), Berlin/Boston: de Gruyter (Bände 3,2-3,26). URL: <https://www.bwb.badw.de/das-projekt.html>; Stand: 12.03.2019.

Duden (1999): Das große Wörterbuch der deutschen Sprache. 10 Bände. 3. Auflage. Mannheim [u. a.]: Dudenverlag.

FISCHER, HERMANN (1904-1936): Schwäbisches Wörterbuch. 6 Bände. Tübingen: Laupp.

GOTTSCHED, JOHANN CHRISTOPH (1737-1738): Anmerkung über die Erklärung altdeutscher Schriften, aus noch übrigen Provinzialwörtern, und besonderer Mundart, zumal des Oberschwabens. In: GotTSCHED, JOHANN CHRISTOPH (Hg.): Beyträge zur Critischen Historie der Deutschen Sprache, Poesie und Beredsamkeit. Band 5. Leipzig: Breitkopf, 270-287.

KÖNIG, WERNER (nicht publiziert): Digitaler Sprachatlas von Bayerisch-Schwaben. Programmiert von CHRISTOPH BUNZ, STEFAN MAIER, MARKUS STROHMEIER, JÜRGEN WERSINGER. Inhaltlich gefüllt von SABINE KLUG.

KÖNIG, WERNER / MANFRED RENN (2007): Kleiner Sprachatlas von Bayerisch-Schwaben. Augsburg: Wißner.

MÜLLER-SPITZER, CAROLIN (2007): Der lexikografische Prozess. Konzeption für die Modellierung der Datenbasis. Tübingen: Narr.

Post, RUDOLF (1998): Möglichkeiten der elektronischen Strukturierung, Vernetzung und Verfügbarmachung von lexikographischen Daten bei der Arbeit am Pfälzischen Wörterbuch. In: GROSSE, RUDOLF (Hg.): Bedeutungserfassung und Bedeutungsbeschreibung in historischen und dialektologischen Wörterbüchern. Leipzig: Verlag der Sächsischen Akademie der Wissenschaften, 211-220. 
PRÖLL, SIMON (2015): Raumvariation zwischen Muster und Zufall. Geostatistische Analysen am Beispiel des Sprachatlas von Bayerisch-Schwaben. Stuttgart: Steiner (Zeitschrift für Dialektologie und Linguistik. Beihefte. 160).

SBS = KÖNIG, WERNER (Hg.) (1996-2009): Sprachatlas von Bayerisch-Schwaben. 14 Bände. Heidelberg: Winter.

SCHMELLER, JOHANN ANDREAS (1872-1877): Bayerisches Wörterbuch. Bearb. von GEORG CARL FROMMANN. 2 Bände. Nachdruck: München 1985. URL: 〈https://publikationen.badw.de>; Stand: 12.03.2019.

SCHNABEL, MiCHAEL: Mausklicks statt Gänge zum Regal - das Bayerische Wörterbuch auf dem Weg in die Digitalisierung. Weblog des dhmuc (Arbeitskreis Digital Humanities München). URL: <http://dhmuc.hypotheses.org/669\#more-669>; Stand: 14.12.2016.

SCHWARZ, BRIGITTE (2013): Dialektwörterbuch von Bayerisch-Schwaben. Vom Allgäu bis zum Ries. Augsburg: Wißner.

Sprechender Sprachatlas von Bayerisch-Schwaben. URL: <https://www.bayerische-landesbibliothek-online.de/sprachatlas-schwaben>; Stand: 12.03.2019.

Sprechender Sprachatlas von Bayern. URL: <https://www.bayerische-landesbibliothek-online.de/ sprachatlas >; Stand: 12.03.2019.

VALTS = GABRIEL, EUGEN (1985-2007): Vorarlberger Sprachatlas mit Einschluß des Fürstentums Liechtenstein, Westtirols und des Allgäus. 5 Bände. Bregenz: Vorarlberger Landesbibliothek.

WAGNER, EBERHARD / ALFRED KLEPSCH (2007): Handwörterbuch von Bayerisch-Franken. Bamberg: Fränkischer Tag.

WBF = Fränkisches Wörterbuch. URL:<https://wbf.badw.de/das-projekt.html>; Stand: 12.03.2019.

WIEGAND, HERBERT ERNST (1988-1999): Kleine Schriften. Eine Auswahl aus den Jahren 1970 bis 1999. 2 Bände. Berlin/New York: de Gruyter. 



\title{
SCHWEIZERISCHES IDIOTIKON - WÖRTERBUCH DER SCHWEIZERDEUTSCHEN SPRACHE
}

\author{
Christoph Landolt / Tobias Roth
}

\section{KONZEPT, GESCHICHTE, RAHMENBEDINGUNGEN}

\subsection{Lexikographisches Konzept}

Das „Schweizerische Idiotikon“2 oder „Wörterbuch der schweizerdeutschen Sprache“ oder auch „Schweizerdeutsche Wörterbuch“ ist neben dem „Glossaire des patois de la Suisse romande“, dem „Vocabolario dei dialetti della Svizzera italiana“ und dem „Dicziunari Rumantsch Grischun“ eines der vier nationalen Wörterbücher der Schweiz. Es dokumentiert die alemannische Sprache des Landes vom Spätmittelalter bis in die Gegenwart und ist damit sowohl ein großlandschaftliches Mundartwörterbuch als auch ein spätmittelhochdeutsches und frühneuhochdeutsches Wörterbuch. Nach dem von den Brüdern GRIMM begründeten „Deutschen Wörterbuch" ist es das zweitgrößte bedeutungsgeschichtliche Wörterbuch des Deutschen. Die Darstellung des Stoffes ist ab der zweiten Hälfte des vierten Bandes von einer solchen Ausführlichkeit, Breite und Tiefe, dass sich das „Schweizerische Idiotikon“ in die Reihe der vielbändigen nationalen historischen Wörterbücher anderer germanischsprachiger Länder gesellt.

Der vom Wörterbuch abgedeckte geographische Raum ist die Deutschschweiz gemäß den Sprachgrenzen des 19. Jahrhunderts, ergänzt um die im Hochmittelalter vom Wallis her gegründeten, im heutigen Nordwestitalien liegenden Walsersiedlungen. ${ }^{3}$ Ausgeschlossen sind damit diejenigen Gebiete im Kanton Graubünden, die erst ab dem 19. Jahrhundert germanisiert wurden, darunter das vom Tirol her eingedeutschte und damit bairischsprachige Samnaun.

1 Christoph Landolt verantwortet die Kapitel 1, 2 und 4, Tobias Roth das Kapitel 3.

2 Das Wort „Idiotikon“ ist ein im 18. Jahrhundert gebildeter, von griechisch idios 'abgesondert, eigen, privat' abgeleiteter Begriff für ein Wörterbuch, das den für eine bestimmte Landschaft „eigentümlichen“ Wortschatz verzeichnet (vgl. HAAS 1994: xxv-xxx). - In Stellenangaben wird „Schweizerisches Idiotikon“ im Folgenden mit „Id.“ abgekürzt.

3 Vereinzelt findet sich ein Ausgreifen auf Südbaden, wie die Quellenangabe ,[Johann Peter] Hebel“, die Verbreitungsangaben ,jens(eits) AAK[aiserstuhl]“ und „BAD[en] bei Kaiserst.“ "sowie die Ortssiglen Bergösch[ingen], Berw[angen], Bühl, Günzg[en], Gurtw[eil] und Kadel$\mathrm{b}[\mathrm{urg}]$ - die in den frühen Bänden alle dem Kanton Aargau zugeordnet wurden - verraten. Auch Belege aus rechtssprachlichen Quellen können sich ausnahmsweise auf die engere Nachbarschaft der Schweiz beziehen. 
Das Quellenmaterial des „Idiotikons“ teilt sich in zwei Hauptgruppen, nämlich diejenige der jüngeren, hauptsächlich mundartlichen Sprache (ab 1800) und diejenige der älteren, ganz überwiegend kanzleisprachlichen Sprache (13. Jahrhundert bis 1799). Das mundartliche Material besteht vornehmlich aus Einsendungen von Korrespondenten aus allen Teilen der Deutschschweiz sowie aus Exzerpten der umfangreichen Dialektbelletristik und schließt die Auswertung der dialektologischen, volkskundlichen und sachkulturellen Fachliteratur sowie von über sechzig Mundartwörterbüchern ein; als Quelle für die Gegenwartsmundart fungieren neuerdings auch Chat-Foren und andere Texte im Internet. Das sprachliche Material vor 1800 gründet in der Exzerption von Chroniken, literarischen Quellen verschiedenster Gattungen, Urkunden, Rechtsquellen und Gerichtsprotokollen, religiösem Schrifttum (mit Schwergewicht Reformationszeit), Arzneibüchern, naturwissenschaftlicher und weiterer Sachliteratur, Autobiographien, Reiseliteratur, Tagebüchern, Briefeditionen, Pamphleten, Flugschriften, Schmähreden usw., wobei nicht allein gedruckte Editionen, sondern auch Manuskripte ausgewertet werden. Da die ersten acht Bände zwischen 1881 und 1920 publiziert worden sind, ist die dokumentierte „Gegenwartssprache“ in wesentlichen Teilen diejenige der zweiten Hälfte des 19. und des frühen 20. Jahrhunderts. Das Korpus ist aber bis heute offen, sodass in den jüngsten Bänden auch Quellen aus dem 21. Jahrhundert berücksichtigt werden können. Angesichts dessen, dass der beschriebene Zeitraum schon um etwa 1300 anfängt, fällt diese Ungleichheit aber weniger ins Gewicht, als es auf den ersten Blick scheinen mag.

Das „Schweizerische Idiotikon“ ist damit synchrones und diachrones Wörterbuch, dialektal-schweizerdeutsches und kanzleisprachlich-spätmittelhochdeutsches/frühneuhochdeutsches Wörterbuch sowie allgemeinsprachliches und fachsprachliches Wörterbuch in einem. Obwohl in der Präsentation weitgehend an die politischen Grenzen der Schweiz gebunden, ist es auch ein Referenzwerk für den gesamten alemannisch-schwäbischen und überhaupt deutschsprachigen Raum.

\subsection{Geschichte}

Der Beschluss, ein neues Wörterbuch zu erarbeiten und damit dasjenige „Schweizerische Idiotikon“ zu ersetzen, das FRANZ JOSEPH STALDER 1806 und 1812 in zwei Bänden herausgegeben hatte, fiel 1862: FRIEDRICH STAUB bewirkte mit einem Vortrag vor der Antiquarischen Gesellschaft in Zürich, dass diese eine Kommission zur Förderung eines schweizerdeutschen Wörterbuchs bildete. Ein Aufruf zur Mitarbeit mobilisierte weite Kreise, und es bildete sich innert Kürze ein ausgedehntes Korrespondentennetz. Bis heute in vielerlei Hinsicht konzeptionell grundlegend sind die 1863 von LUDWIG TOBLER niedergeschriebenen „Unmaßgeblichen Gedanken über die Methode des schweizerischen Wörterbuchs“"

„Neben dieser der Laut- und Formenangabe zu widmenden Sorgfalt sollte im Ganzen höheres Gewicht auf Sammlung, Angabe und gehörige Entwicklung der Bedeutungen aller einzelnen Wörter und ihrer phraseologischen Verbindungen gelegt werden; hierin wird, für die Wissenschaft wie für das weitere Publikum, für Gegenwart und Zukunft der werthvollste Gehalt des 
Wörterbuchs bestehen. Zur „Bedeutung“ zählen wir aber auch eine Reihe bisher allzusehr vernachlässigter syntactischer Erscheinungen in Congruenz, Rection, Wortstellung, eigenthümlichem Gebrauch von Pronomina und Präpositionen, endlich das Vorherrschen gewisser Triebe in der Wortbildung (besonders beliebte Ableitungssylben und Zusammensetzungen).“

Das historische Prinzip war anfänglich nicht unumstritten, wurde dann aber im von GEORG VON WYSS unterzeichneten, 1874 versandten „Aufruf betreffend Anfertigung von Auszügen aus der ältern schweizerdeutschen Litteratur für das Idiotikon“ deutlich formuliert:

\begin{abstract}
„Das schweizerische Idiotikon muss, um seinem Zwecke zu entsprechen und sich mit Ehren neben die schon bestehenden Werke ähnlicher Art stellen zu können, auf wissenschaftlicher Grundlage aufgebaut werden. Hiezu gehört ganz besonders, dass es den historischen Zusammenhang der heutigen Sprache mit derjenigen der früheren Perioden darlege. Erst durch die Beiziehung der ursprünglichen Formen und Bedeutungen und dem Nachweis der allmäligen Wandlungen erhält die jetzige Mundart ihre Beleuchtung und damit ein allgemeineres Interesse. Die Nothwendigkeit, auf unsere alten Ueberlieferungen zurückzugehen, drängt sich aber noch aus andern Gesichtspunkten auf. Unser Idiotikon soll nicht bloss gelehrten Zwecken dienen, es übernimmt die Aufgabe, auch ein Handbuch zur Befriedigung praktischer Bedürfnisse zu sein. Der Historiker, der Jurist, überhaupt der Fachmann soll darin Aufschluss über ehemalige Verhältnisse und Ausdrücke finden. Und endlich verlangt es die Ehre der Schweiz, dass der sprachliche und kulturhistorische Reichthum, welcher in ihrer Litteratur verborgen ist, so weit wenigstens unsere Mittel reichen, zu Tage gefördert und zu Jedermanns Gebrauch geordnet und erläutert werde.“
\end{abstract}

Seit jeher finden auch volkskundliche Aspekte ihre Berücksichtigung im Wörterbuch. Im 1874 veröffentlichten „Plan des schweizerischen Wörterbuchs“ hieß es:

\begin{abstract}
„Aberglaube, Bräuche, Sitten, Spiele, Räthsel, Sprichwörter, Lieder und Sagen können im Wörterbuch nur zur Behandlung kommen, soweit die Erklärung einzelner Wörter es mit sich bringt. Redensarten, Formeln, Reime, welche die Form der Volkssprache tragen, sollen möglichst aufgenommen werden, besonders so weit sie als Belege für die Bedeutung einzelner Wörter und auch zugleich als Proben der einzelnen Mundarten dienen können.“
\end{abstract}

Die erste Lieferung des „Schweizerischen Idiotikons“ erschien nach einer keineswegs überlangen Vorbereitungszeit 1881 - ,gedrängt durch die Ungeduld eines Publikums [...] und zugleich beengt durch mancherlei andere äussere Rücksichten“, wie STAUB und TOBLER im Vorwort schrieben (Id. I xxx).

Die Anfänge des Wörterbuchs fielen noch in die Frühzeit der Germanistik. Gegen Ende des 19. Jahrhunderts hatte sich die Situation aber völlig verändert, denn die Schule der Junggrammatiker brachte strenge wissenschaftliche Anforderungen mit sich. Universitätsprofessor ALBERT BACHMANN, der 1896 STAUBS Nachfolge als Chefredaktor antrat, leitete deshalb einen eigentlichen Paradigmenwechsel ein. Auslöser war zweifellos HERMANN PAUls Aufsatz „Ueber die Aufgaben der wissenschaftlichen Lexikographie“. Der Münchner Philologe forderte darin, dass wissenschaftliche Wörterbücher „,den Aufbau einer wirklichen Wortgeschichte“ zum Ziel haben sollten. Dazu brauche es „eine möglichst genaue Abgrenzung der Sphäre des Gebrauchs für jedes Wort und jede Verwendungsweise desselben“, wozu „eine genügende Ausnutzung der Quellen“ die wichtigste Voraussetzung sei (PAUL 1895: 54). So wurden unter BACHMANN lexikalische, geographische und zeitliche Lücken 
geschlossen, die Wortartikel erfuhren eine umfassende Erweiterung, Verdichtung und Vertiefung, die Darstellung erhielt eine verstärkt diachronische Ausrichtung und das „Idiotikon“ wandelte sich zum „Thesaurus“ ${ }^{4}$ Dies hatte die unausweichliche Folge, dass die ersten dreieinhalb Bände sich deutlich von den seither erschienenen unterscheiden.

In der Zeit nach ALBERT BACHMANN wurde das Wörterbuch kaum mehr konzeptionellen Neuerungen unterzogen. ${ }^{5}$ Was sich seit der Jahrtausendwende hingegen stark geändert hat, ist die Nutzerorientierung. Zum einen betrifft dies den verstärkten Auftritt der Redaktion in der Öffentlichkeit, sei es an Veranstaltungen, am Radio oder in den Social Media. Besonders beliebt sind die zweimal wöchentlich ausgestrahlten Erklärungen von Familiennamen auf Radio SRF 1 und die über mehrere Kanäle publizierten „Wortgeschichten“, welche die Herkunft von Wörtern und mitunter auch von Brauchtum in laienfreundlicher Sprache erläutern. Zum andern steht das Wörterbuch seit 2010 gratis online zur Verfügung. Die Erarbeitung eines Semantikregisters hat in der Zwischenzeit zur besseren Erschließung der (digitalen) Wörterbuchartikel geführt, indem nun von jedem Artikel eine Bedeutungsübersicht aufgerufen werden kann, die den direkten Zugriff auf eine gesuchte Bedeutung erlaubt. Gleichzeitig wurden die Grundlagen gelegt, um Kurzwörterbücher, Zeitstufenwörterbücher und Umkehrwörterbücher generieren sowie einen onomasiologischen Zugriff ermöglichen zu können. In den nächsten Jahren wird die Online-Ausgabe des „Schweizerischen Idiotikons“ zu einem vollständig digitalen Wörterbuch ausgebaut werden, was unter anderem die Voraussetzung für gezielte Textergänzungen schaffen soll.

\subsection{Institut, Redaktion und Vernetzung}

Die in Zürich domizilierte Wörterbuchstelle ist ein selbständiges Institut, für das sich ebenfalls der Name Schweizerisches Idiotikon eingebürgert hat. Es wird vom privatrechtlichen, 1950 gegründeten Verein für das Schweizerdeutsche Wörterbuch getragen, dessen Mitglieder die ganz- und teilweise deutschsprachigen Kantone sowie einige natürliche Personen sind. Die Finanzierung geschieht weitestgehend durch die Schweizerische Eidgenossenschaft über die Schweizerische Akademie der Geistes- und Sozialwissenschaften (SAGW) sowie durch die genannten Kantone.

Das Redaktionsteam setzt sich in der Regel aus sechs (aktuell sieben) Redaktoren und Redaktorinnen zusammen, die sich etwas mehr als fünf (aktuell sechs) Stellen teilen. Dieses Team wird durch derzeit 22 weitere wissenschaftliche, studentische und administrative Mitarbeiter und Mitarbeiterinnen ergänzt, die für

4 Zur Leistung BACHMANNS siehe HAAS (1981: 66-72).

5 Zur seither ,zunehmend breitere[n] und noch stärker belegorientierte[n] Darstellung“, aber auch zur damit verbundenen weiteren Qualitätssteigerung sowie dem im ersten Jahrzehnt des 21. Jahrhunderts redaktionsintern initiierten Straffungskonzept siehe SCHIFFERLE (2017: 297$300)$. 
IT/Sprachtechnologie, redaktionelle Assistenzarbeiten, weitere Projektaufgaben und die Verwaltung zuständig sind.

Am Institut befinden sich auch die gesamten Materialien des zwischen 1935 und 2003 erarbeiteten „Sprachatlasses der deutschen Schweiz“, dessen Bestände gegenwärtig gesichert, digitalisiert und online verfügbar gemacht werden (Sprachatlas der deutschen Schweiz 2019). Seit 2016 ist es am von swissuniversities finanzierten Projekt „histHub“ (2019) beteiligt, das vernetzte und normierte Daten für die historischen Wissenschaften zur Verfügung stellen will. 2019 ging zudem das vom Schweizerischen Nationalfonds finanzierte Projekt ,Siedlungsnamen des Kantons Zürich“ (2019) von der Universität Zürich an das Schweizerische Idiotikon über. Mit dem Institut assoziiert ist das 2019 gestartete Projekt „Hunziker 2020“ (2019), das zum Zweck hat, JAKOB HUNZIKERS (1877) „Aargauer Wörterbuch“ digital zugänglich zu machen und der Bevölkerung für Aktualisierungen und Ergänzungen zur Verfügung zu stellen. Und schließlich unterhält es das „Portal der schweizerischen Ortsnamenforschung“ (ortsnamen.ch 2019), das „Schweizer Textkorpus“ (2019) sowie die Websites des „Kleinen Sprachatlasses der deutschen Schweiz“ (2019), des „Kollokationenwörterbuchs“ (2019) und des „Online-Lexikons zur diachronen Phraseologie“ (2019).

Der Universität Zürich ist das Institut seit jeher eng verbunden; so waren mehrere frühere Wörterbuchredaktoren zugleich Lehrstuhlinhaber oder Titularprofessoren, und bis heute hat es einen regelmäßigen Lehrauftrag inne. Derzeit bestehen auch persönliche Beziehungen zur Universität Basel, wo ein Redaktionsmitglied als Titularprofessor lehrt. Mit der Universität Freiburg (Schweiz) gibt es seit 2008 ein Abkommen, dass Studierende des Masterstudiengangs „Germanistische Linguistik mit dialektologischem Profil“" auf der Redaktion des "Schweizerischen Idiotikons“ ihr Praktikum absolvieren können. Institutionalisierte Vernetzungen finden überdies seit langer Zeit durch die Vertretung der Redaktion in der Nomenklaturkommission des Kantons Zürich sowie im Schweizerischen Dudenausschuss statt, und seit Neuerem hat sie auch im Ständigen Ausschuss für geographische Namen (StAGN; Frankfurt am Main) sowie im Beirat des Zentrums für digitale Lexikographie der deutschen Sprache (ZDL; Berlin) Einsitz. Seit 2016 ist das Schweizerische Idiotikon überdies Mitglied des Consortium Historicum, einer Arbeitsgruppe selbständiger Forschungsprojekte, die den Informationsaustausch und die Förderung der Vernetzung digitaler historisch ausgerichteter Projekte verfolgt.

Mit seinen drei Schwesterwörterbüchern aus der lateinischen Schweiz tauscht sich die Redaktion an gegenwärtig im Zweijahresturnus stattfindenden, von der Schweizerischen Akademie der Geistes- und Sozialwissenschaften initiierten Treffen aus. Aktiv beteiligt ist sie auch an dem 2012 von verschiedenen Schweizer Forschungsstellen begründeten „Kolloquium Namenforschung“. Auf gesamtdeutschsprachiger Ebene pflegt sie den Kontakt mit den großlandschaftlichen Mundartund den Sprachstadienwörterbüchern auf verschiedenen Plattformen. Zu nennen sind etwa die Arbeitstagungen für alemannische Dialektologie (seit 1963), die Tagungen der deutschsprachigen Akademie-Wörterbücher (1993-2009), die Kongresse der Internationalen Gesellschaft für Dialektologie des Deutschen (seit 2003), die Arbeitsgespräche zur historischen Lexikographie (seit 2007) sowie die Netz- 
werktreffen für oberdeutsche und bündnerromanische Dialektlexikographie (ab 2010) beziehungsweise nun von LexikoNet, einer Plattform für alle großlandschaftlichen Wörterbücher des Deutschen (seit 2017). Eine konkrete Zusammenarbeit besteht mit dem Kompetenzzentrum für elektronische Erschließungs- und Publikationsverfahren in den Geisteswissenschaften an der Universität Trier, in dessen Online-Wörterbuchnetz (Wörterbuchnetz 2019) auch die Lemmaliste des „Schweizerischen Idiotikons“ integriert ist. Zu einer Vernetzung über den deutschsprachigen Raum hinaus hat die Teilnahme an der COST-Aktion IS1305 „European Network of e-Lexicography“ (ENeL) geführt (2013-2017), die einen systematischeren Austausch von Know-how zwischen den verschiedenen europäischen Wörterbuchprojekten zum Ziel hatte.

\subsection{Gedruckte und digitale Publikation}

Die erste Lieferung des „Schweizerischen Idiotikons“ erschien 1881. Die frühen Bände, die noch auf vergleichsweise wenig Material basierten, kamen in rascher Folge heraus; BACHMANNS neues Konzept (siehe unter „Geschichte“) und die stete Materialergänzung führten im 20. Jahrhundert zu einer deutlichen Verlangsamung der Publikation. Derzeit wird am 17. Band (Z) gearbeitet. Verlegt wurde das Werk von 1881 bis 2012 vom Verlag Huber in Frauenfeld, seit 2013 wird es vom Schwabe Verlag in Basel betreut.

Seit 2010 ist das Wörterbuch über die Website des Instituts online abrufbar (Schweizerisches Idiotikon digital 2019). Dieser Zugang wird laufend ausgebaut, um das Wörterbuch im Sinne der Digital Humanities als digitale Infrastruktur weiterentwickeln zu können.

\subsection{Zettelmaterial und Bibliothek}

Dem 1862 versandten „Aufruf betreffend Sammlung eines Schweizerdeutschen Wörterbuchs" war ein unerwarteter Erfolg beschieden. Bis 1880 hatten gegen 400 Korrespondenten aus fast allen Gegenden der deutschen Schweiz Beiträge nach Zürich geschickt, und mehrere Personen wurden gar dazu bewogen, eigens Dialektwörterbücher und Mundartliteratur zu verfassen und diese der Redaktion zur Verfügung zu stellen. Gleichzeitig begann man, die mundartliche Belletristik auszuwerten, und bald schon folgte die Exzerption der älteren Schweizer Literatur, der Rechtsquellen und vielerlei sonstiger Texte aus der Zeit vor 1800 - darunter Handschriften in großer Zahl. Systematische Enquêten, wie sie andere Wörterbücher kennen, führte die „Idiotikon“-Redaktion dagegen nie durch.

Zuverlässige Schätzungen über den Umfang der Belegsammlung liegen keine vor, da sehr viele Zettel mehrere Belege enthalten und überdies nicht selten später umgedreht und wiederverwendet wurden. Dass 1880, als die Publikation des ersten Faszikels kurz bevorstand, die Belegsammlung schon „eine runde Million Zettel aus zerschnittenem Abfallpapier aller Farben und Formate“ umfasst haben soll 
(HAAS 1981: 31), dürfte jedoch zu hoch gegriffen sein. Außer Zweifel steht aber, dass sich die Zahl der Belege seither vervielfacht hat, da der Hauptharst der Mundartbelletristik, der Texteditionen, der Fach- und der Sachliteratur erst nach 1900 publiziert wurde und das Korpus bis heute offen ist. Nicht verändert hat sich die Beschaffenheit der Exzerpte: Bei der täglichen Arbeit hält das Redaktionsmitglied Papierzettel in der Hand, die zwischen den 1860er-Jahren und der Gegenwart beschriftet worden sind.

Die Institutsbibliothek enthält rund 12000 Einheiten. Sie umfasst den größten Teil der einleitend umrissenen Quellen sowie verschiedenste Sekundärliteratur. Teil der Bibliothek sind Drucke ab dem frühen 16. Jahrhundert sowie handschriftliche Unikate aus fünf Jahrhunderten. Hinzu kommen die archivierten großen und kleinen Einsendungen ,aus allen Kreisen des Schweizervolkes“ (wie es auf dem Titelblatt jedes Wörterbuchbandes heißt), die ab 1862 an die Redaktion geschickt worden sind. Bibliothek, Zettelsammlung und Archiv sind nicht öffentlich, jedoch wird Interessierten auf Anfrage Zugang gewährt.

\section{DIE WÖRTERBUCHARTIKEL: KONZEPTION, STRUKTUR, INHALT}

\subsection{Lemma, Schreibung}

Die Lemmaform ist in der Regel die Grundform, auf die sich die verschiedenen Mundartlautungen zurückführen lassen (Id. I xiv):

\footnotetext{
„Wo [...] die verschiedenen Mundarten verschiedene Wortformen darbieten, welche sich auf eine Grundform vereinigen lassen, wird diese ältere und richtigere Form zum Stichwort und Hauptträger des Artikels gewählt, gleichviel welche der concurrierenden Formen jetzt das numerische Übergewicht habe. In den weitaus meisten Fällen ist diese Form die mhd.“
}

In der Schreibung der Mundart geht das „Schweizerische Idiotikon“ eigene Wege, was seine Ursachen in dessen früher Gründung hat. Andere Wörterbücher, die Mundart behandeln, schreiben diese entweder in einer Version der Teuthonista oder bringen die Belege in der originalen Schreibweise der Quelle. Als das „Idiotikon“ begonnen wurde, gab es aber weder eine breit anerkannte Schreibweise für Dialekte noch eine breit rezipierte Lautschrift („Die mundartliche Phonetik ist eine Disciplin, welche sich erst seit kurzem aus dem Stadium der Empirie zur Wissenschaft herauszuarbeiten begonnen hat.“ Id. I xv; verfasst 1881). Dazu kam, dass die Qualität des sowohl handschriftlich eingereichten wie gedruckt vorliegenden mundartlichen Materials in phonetischer Hinsicht höchst unterschiedlich war und damals weder die „Beiträge zur Schweizerdeutschen Grammatik“ (ab 1910 erschienene, junggrammatisch ausgerichtete Ortsgrammatiken ${ }^{6}$ ) noch die Bände des „Sprach-

6 Die im Auftrag des Leitenden Ausschuss des Schweizerischen Idiotikons von AlBERT BACHMANN herausgegebenen „Beiträge zur Schweizerdeutschen Grammatik“ wurden „zur Ergänzung der Sammlungen des Idiotikons, insbesondere aber zur Vorbereitung der nach Abschluß des Wörterbuchs auszuarbeitenden Grammatik des Schweizerdeutschen" erarbeitet. Nach BACHMANNS Tod 1934 wurde die Reihe aus Kostengründen eingestellt. 
atlasses der deutschen Schweiz“ (erschienen ab 1962) vorlagen, die bei der Interpretation der Laienschreibungen hätten helfen können. Die - in kursiver Form stehende - Wiedergabe der dialektalen Daten erfolgte in den frühen Bänden deshalb in einer stark vereinfachten Form. Mit dem zunehmenden Wissen über die genauen Lautungen wurde auch deren Wiedergabe mit der Zeit exakter; die jüngere Transkriptionspraxis steht deshalb der effektiven Lautung recht nahe. ${ }^{7}$ Typisch für das „Schweizerische Idiotikon“ ist die Hochstellung stumm gewordener, nur etymologisch relevanter Laute und Lautgruppen. In den ersten Bänden wurde diese nur zurückhaltend angewandt; mit dem Erstarken der historischen und dialektologischen Forschungsrichtung setzte man die stumm gewordenen Laute aber häufiger ein, weshalb die vielen Hochstellungen inzwischen ein Markenzeichen des „Idiotikons“ sind. ${ }^{8}$ Umgeschrieben werden übrigens seit jeher auch die Belege aus der gedruckt vorliegenden Dialektliteratur.

Die Wiedergabe der Belege aus historischen Quellen - in der Regel recte gedruckt - war anfänglich recht frei; so wurde die Schreibung stark normalisiert, und Sätze wurden ohne weiteres umgestellt, wenn der Zitatausschnitt eine andere Syntax verlangte. Auch hier führte die Entwicklung der Germanistik und der Geschichtswissenschaft zu einem zunehmend quellennahen Umgang. Heute werden Belege aus der Zeit vor 1800 weitgehend so wiedergegeben, wie sie in der Quelle stehen; Ausnahmen stellen etwa die Normalisierung der Groß- und Kleinschreibung (Großschreibung der Substantive in Belegen ab 1600) sowie die prinzipielle Restituierung der Diphthonge 〈uo〉 und 〈üe〉 dar, die in älteren Editionen oft vernachlässigt wurden.

\subsection{Alphabetische Anordnung}

Ein Merkmal des „Schweizerischen Idiotikons“ ist die Anordnung der Lemmata: Die Ansätze sind nicht nach dem üblichen Alphabet, sondern erstens nach dem Konsonantengerüst der Stammsilbe angeordnet (hier steht das „Idiotikon“ neben dem alten „Bayerischen Wörterbuch“ sowie dem „Wörterbuch der elsässischen Mundarten"), und zweitens werden zusammengesetzte und präfigierte Wörter unter dem Grundwort eingereiht (Letzteres trifft außer auf die genannten Wörterbücher auch auf das neue „Bayerische Wörterbuch“ und das „Wörterbuch der bairischen Mundarten in Österreich“ zu). Dieses Vorgehen führt drittens dazu, dass ganze (Teil-)Wortfamilien im Zusammenhang präsentiert werden können.

Das Prinzip, die einzelnen Wörter in den Lexemverband einzubetten, hat seine Anfänge in den Wörterbüchern der Barockzeit (vgl. REICHMANN 2012: 451), und

7 Wichtigste Ausnahmen sind bis heute die Nichtwiedergabe der sekundären Dehnung (außer in der Lautübersicht), der Unterscheidung von /æi/ und /ei/ (anders als diejenige von /æu/ und /ou/, die in der Umschrift berücksichtigt wird) und der ///-Vokalisierung.

8 Die Hochstellungen wurden später von den andern großlandschaftlichen Wörterbüchern der Alemannia - dem „Badischen Wörterbuch“, dem „Schwäbischen Wörterbuch“, dem „Vorarlbergischen Wörterbuch“ und dem „Wörterbuch der elsässischen Mundarten“ - übernommen, zumeist aber viel zurückhaltender umgesetzt. 
dass es auch im 19. Jahrhundert noch durchaus anerkannt war, zeigen etwa das „Bayerische Wörterbuch“ von ANDREAS SCHMELLER (1827-1837), der „Althochdeutsche Sprachschatz“ von EBERHARD GRAFF (1834-1842) und das „Mittelhochdeutsche Wörterbuch" von GEORG BENECKE, WILHELM MÜLLER und FRIEDRICH ZARNCKE (1854-1866). SCHMELLER sah in dieser ,etymologisch-alphabetische[n] Ordnung“ einerseits den wissenschaftlicheren Zugang, ,über welchen kein Freund der Sprachvergleichung und Sprachforschung im Zweifel seyn kann“, und glaubte anderseits so die Frage, unter welcher Lautung ein Dialektwort angesetzt werden sollte, entschärfen zu können, da Konsonanten im Allgemeinen viel stabiler sind als Vokale (SCHMELLER 1827: vi). FriedRICH STAUB, der das „schmellersche System“, wie er es nannte, auch für das "Schweizerische Idiotikon“ durchsetzte, interessierte die etymologische Herangehensweise freilich weniger (STAUB 1876: 61):

\begin{abstract}
„Wie verlockend aber auch die Aufgabe ist, die einzelnen Bruchstücke der Sprache in ihrem organischen Zusammenhang einzusetzen, so glaube ich doch dieselbe für das Idiotikon ablehnen [...] zu sollen. [...] Förstemann hat gewiß Recht, wenn er sagt, bei Wörterbüchern [...] müsse man der Sitte mehr als der Wissenschaftlichkeit in der Anordnung der einzelnen Artikel folgen.“
\end{abstract}

Ausschlaggebend waren für ihn praktische Gründe (STAUB 1876: 79):

\begin{abstract}
„Man braucht sich nicht den Kopf zu zerbrechen, ob man Ätter oder Etter, bänd oder bend (behend) schreiben oder aufschlagen wolle, denn die Stellen für die beiden Schreibungen, welche nach der Grimm'schen Anordnung sehr weit aus einander liegen, rücken sich bei Schmeller so nahe, daß sie einander nahezu decken und daß der Aufschlagende und der Redaktor, auch wo sie differieren, einander begegnen müssen."
\end{abstract}

Der massive Ausbau des Wörterbuchs unter ALBERT BACHMANN und seinen Nachfolgern hat diese Nähe der verschiedenen Ansatzmöglichkeiten zunichte gemacht. Rückblickend kann man sagen, dass die Lemmaform großmehrheitlich einer (allerdings etymologisch basierten) durchschnittsschweizerdeutschen „Normallautung“ entspricht, was die - ohnehin wenig nutzerfreundliche - Anordnung nach dem Konsonantengerüst als unnötig erscheinen lässt.

Die Behandlung zusammengesetzter und präfigierter Lemmata unter dem Grundwort ist hingegen von nach wie vor zentraler Bedeutung: Erstens ermöglicht sie die gleichzeitige Bearbeitung ganzer Wortfamilien in ihrem etymologischen, morphologischen und semantischen Zusammenhang anstatt lediglich von singulären, aus ihrer semantischen Nachbarschaft losgelösten Einzelwörtern, was für den Wörterbuchverfasser ein unschätzbarer Vorteil ist. Und zweitens eröffnet sich damit für die Benützerinnen und Benützer die Möglichkeit, eine ganze Wortfamilie an einer zentralen Stelle zu überschauen und auszuwerten. ${ }^{9}$ Nehmen wir das Beispiel Wurz(el), so enthält der sechzehnte Band des „Idiotikons“ (Sp. 1716-1763) nicht allein dieses Wort, sondern auch eine Unmenge von Pflanzennamen wie Imber-, Aron-, Astränze ${ }^{n}$-, Gold-, Harnisch-, Hasel-, Meister-, Bluet-, Schmër ${ }^{w}$-, Walloder Wolfs-Wurz - alles eng beieinander statt über viele Bände verstreut und nicht

9 Zur Leistungsfähigkeit dieses Stammwortprinzips vgl. REICHMANN (2012: 451-455), wo sie anhand des ,Schweizerischen Idiotikons“, Wortfamilie War I 'Wahrnehmung durch Sinnesorgane, Beobachtung' (Id. XVI 821-861), erläutert wird. 
nur für die botanisch Interessierten, sondern auch für die mit Arzneien vergangener Zeiten sich Beschäftigenden eine wahre Fundgrube. Gleiches gilt etwa für die unzähligen Zusammensetzungen mit -Wasser in demselben Band (Sp. 1789-1845), wo sich an einer Stelle vereint neben dem volkskundlich interessanten Wīh-Wasser und dem namenkundlich aufschlussreichen Land-Wasser unter anderem eine ganze Palette alkoholischer Getränke wie Ënziānen ${ }^{-}$, Gigertschi-, Gallöri-, Gurāschi-, Gätzi-, Chümin-, Chirsi-, Nuss-, Bätzi-, Treber-oder Zwätschgen-Wasser, eine Unmenge von (Volks-)Arzneien wie Agelstere $^{n}$-, Ilge ${ }^{n}$-, Arkenbusade ${ }^{n}$-, Fënchel-, $G^{e}$ frörni-, Guldin ${ }^{n}$, Mueter-, Rōse ${ }^{n}$ - oder Schwalme ${ }^{n}$-Wasser oder zahlreiche Bezeichnungen für schlechten Kaffee wie Amsle ${ }^{n}$, Gunggel-, Babeli-, Backseckel-, Schlodi-, Schlïffstei ${ }^{n}$ - oder Tschalöri-Wasser finden. Noch umfassender wirkt sich das Stammwortprinzip bei Verben aus. Die ebenfalls in Band XVI (Sp. 1924-1978) behandelte Wortfamilie wīse $e^{n} I$ 'weisen' umfasst außer diesem Kernwort erstens alle präfigierten Verben von $a b-w \bar{l} s e^{n}$ bis zue-wīse $e^{n}$, zweitens alle zu diesen Zusammensetzungen gebildeten Ableitungen wie Ab-wīser, ab-wīslich, Ab-wīsung, drittens deren Zusammensetzungen und Ableitungen wie Rad-Ab-wīser oder Chuglen$A b-w \bar{\imath} s i^{n} g$, viertens die vom Grundwort aus gebildeten Ableitungen wie Wīser, Wìsi, $W_{\bar{i}} \bar{i}^{n} \mathrm{~g}$, Wīsnus, Wīstum einschließlich fünftens deren Zusammensetzungen und Ableitungen wie $W \ddot{e} g$-Wīser und wëg-wīsere ${ }^{n}$ sowie endlich sechstens - aus alphabetischen Gründen wortfamilieneinleitend - auch die Rückkürzungen vom Typus $W \bar{\imath} s$ inklusive deren Zusammensetzungen wie $\bar{U} s$-wis usw. - alles direkt aufeinanderfolgend (Absatz nach LANDOLT 2010: 411-412).

Die Konzepte „Konsonantengerüst“ und „Wortfamilie“ (oder „Wortsippe“) stehen allerdings in einem gewissen Gegensatz zueinander. Letzteres wird im „Schweizerischen Idiotikon“ nur bei gleichem Vokal (einschließlich dessen Umlauts) angewandt (etwa Wasser und $G^{e}$ wässer), nicht aber bei Ablaut (z. B. Band, binde $^{n}$, Bund) und nicht bei frühem Umlaut (z. B. Wëtter, $G^{e}$ witter); vorkommende Ausnahmen bestätigen die Regel. Dazu kommen viele Inkonsequenzen im Hinblick auf die Einordnung ins Konsonantengerüst. So wurden Wörter mit dem Suffix -sch auf vier verschiedene Arten eingeordnet: mägersch (Id. IV 103) findet sich innerhalb der Sippe mager angesetzt; rötsch (Id. VI 1854) dagegen wurde nicht in der Sippe $r \bar{o} t$ untergebracht, sondern leitete eine eigene Sippe im Konsonantengerüst $r$-tsch ein; hündsch (Id. II 1436) und windsch (Id. XVI 590) wiederum sind Nebenansätze der Lemmata hündisch bzw. windisch; und $b^{e} h a ̈ n d s c h$ wurde nicht eigens lemmatisiert, sondern in den Ansatz $b^{e} h \ddot{a} n d$ integriert (Id. II 1408). Auch andere Suffixe erfuhren eine uneinheitliche Behandlung: - de und -nis(s) etwa im Fall von Bewegde (Id. XV 721) und Bewegniss (Id. XV 727) sind in die Sippe bewege $e^{n}$ eingebunden, -st im Fall von $G^{e}$ winst (Id. XVI 721) bildet dagegen eine eigene Sippe und findet sich nicht in derjenigen von $G^{e}$ winn. Verschieden gehandhabt wurden auch Ableitungen mit -sl-: schrībsle ${ }^{n}$ (Id. IX 1560) findet sich in der Sippe schrībe ${ }^{n}$; walpsle $^{n}$ (Id. XV 1582) hingegen bildet eine eigene Sippe und wurde nicht in diejenige von walbe $e^{n}$ eingeordnet (die in beiden Fällen gleiche Lautung /ps/ wurde also im ersten Fall etymologisch, im zweiten phonetisch interpretiert). Die „Idiotikon“Praxis kann aber auch sehr pragmatisch sein, etwa im Fall von zöche $e^{n}$ (Id. XVII 241), unter welchem Lemma auch die Typen $z \ddot{a} u c h e^{n}, z \overline{o ̈ k} e^{n}, z \ddot{a} u k e^{n}$ abgehandelt 
werden, obwohl sie nicht einfach dessen lautgesetzlich herzuleitenden Varianten sind; die verschiedenen Lautungen werden aber in der Anmerkung adäquat besprochen. Den Widerstreit zwischen Konsonantengerüst und Wortfamilie lösten STAUB und seine Nachfolger somit nicht selten anders als ihr Vorbild: SCHMELLERS „Bayerisches Wörterbuch" handelt beispielsweise die etymologisch eng verwandten Wörter zeihen, Zicht und Bezig alle am gleiche Ort ab, nämlich unter dem erstgenannten Grundwort, und gibt damit dem Lexemverband den Vorzug, wogegen das „Schweizerische Idiotikon“ sich hier streng am Konsonantengerüst orientiert und diese Wortfamilie auf drei Teil-Wortfamilien aufspaltet. SCHMELLER stand damit näher bei der Praxis von GRAFFS „Althochdeutschem Sprachschatz“ (1834-1842) und dem in dessen Nachfolge stehenden „Mittelhochdeutschen Wörterbuch“ von BENECKE, MÜLLER und ZARNCKE (1854-1866), wo das Wurzelprinzip strikte eingehalten wurde und das Konsonantengerüst nur für das jeweilige Kernwort galt.

Die mit dem „schmellerschen System“ verbundene Schwierigkeit, ein gesuchtes Wort zu finden, ist spätestens seit dem ab etwa 1900 stattfindenden Ausbau des Wörterbuchs eklatant geworden. Dem Problem begegnete man, indem die Redaktion 1961 - in Einlösung eines schon früh gegebenen Versprechens - begann, ein normalalphabetisches Register zu veröffentlichen. Dieses liegt nun einerseits als Registerband für die Bände I-XI, anderseits in Form von Bandregistern ab Band XII gedruckt vor. Seit 2008 ist es, in ein einziges Register zusammengeführt, auch online über die Website des Idiotikons abrufbar. Es wird seither laufend um unterschiedliche Schreibweisen, Lautungen und Formen ergänzt.

\subsection{Artikelstruktur}

Der Grundaufbau eines Artikels im „Schweizerischen Idiotikon“ ist dreiteilig: Artikelkopf - Bedeutungsteil - Anmerkung.

Der Artikelkopf wird durch das Lemma eingeleitet. Das erste der (Teil-) Wortfamilie ist fett gedruckt, die weiteren Lemmata innerhalb der Wortfamilie sind in gesperrter Schrift gesetzt. Homophone Stichwörter werden mittels römischer Ziffern voneinander geschieden. Dem Lemma folgen die verschiedenen Lautvarianten, wobei die lautgesetzlichen, im „Sprachatlas der deutschen Schweiz“ und in den „Beiträgen zur Schweizerdeutschen Grammatik“ nachzuschlagenden Varianten lediglich mittels einer mit „bzw.“ eingeleiteten Reihung zum Ausdruck gebracht werden; bei Substantiven folgen Genus, Plural und Diminutiv, bei Adjektiven gegebenenfalls die umlautende Steigerung, bei Verben Angaben zum Präsens, allenfalls zum Präteritum ${ }^{10}$ sowie das Partizip Perfekt.

Der Bedeutungsteil wird von der Definition eingeleitet und enthält die Belege, welche die angeführte Definition nachweisen, wobei innerhalb eines jeden Gliederungsabschnitts die mundartlichen Belege (ab 1800) den historischen (bis 1799) vorangehen. Mundartbelege sind kursiv; das gilt auch für die wenigen Fälle von

10 Von den Mundarten des 20. Jahrhunderts kannte einzig diejenige von Saley/Salecchio im piemontesischen Antigoriotal ein Präteritum. 
Mundart aus der Zeit vor 1800. Belege der historischen (Kanzlei-)Sprache vor 1800 sowie solche aus der Standardsprache ab 1800 stehen in aufrechtem Druck, eingerahmt von einfachen Anführungszeichen. Die Datierung der Belege erfolgt entweder direkt über die eine Jahrzahl enthaltende Quellensigle, oder aber sie wird mittels einer der Sigle vorangehenden Jahrzahl ausgedrückt; die Datierungen gewisser Siglen (wie „Ansh.“ “ für Anshelms „Berner-Chronik“ von 1540) können allerdings nur über das Quellenverzeichnis in Erfahrung gebracht werden. Dialektale Belege, die lediglich mit einer Ortssigle versehen werden, stammen oft aus dem im 19. Jahrhundert eingesandten, ungedruckten Mundartmaterial.

Seit AlBERT BACHMANN, also der zweiten Hälfte des vierten Bandes, werden die Bedeutungen von Simplizia in der Regel historisch angeordnet, das heißt, die älteren Bedeutungen gehen den jüngeren voraus - eine Praxis, die auch anderen historischen Nationalwörterbüchern eignet (REICHMANN 2012: 344). Eine deutliche Anwendung findet sie etwa in den Artikeln Brief (Id. V 435), Reis, reise ${ }^{n}$ (Id. VI 1288, 1303), rāten (Id. VI 1595), Sach (Id. VII 97), Schelm (Id. VIII 692), Schimpf, schimpfen (Id. VIII 782, 787), Ding (Id. XIII 470), tauwe ${ }^{n}$ II (Id. XIII 2224), Trōst (Id. XIV 386), weide lich (Id. XV 541), warten (Id. XVI 1590), wette ${ }^{n}$ II (Id. XVI 2225), Witz (Id. XVI 2380). ${ }^{11}$ Ausgenommen davon können solche jüngere Sonderentwicklungen sein, die semantisch immer noch derart nahe bei der älteren Bedeutung stehen, von der sie ausgegangen sind, dass sie im Wörterbuch auch positionsmäßig eng an diese angeschlossen werden dürfen, statt davon losgelöst viel weiter unten zu figurieren. Zusammensetzungen und Ableitungen werden hingegen nicht selten semantisch parallel zum Simplex angeordnet, auch wenn die jeweilige Bedeutungsgeschichte eine andere ist.

Im Sinne einer Dienstleistung für die Benützerinnen und Benützer wird im Übrigen ab dem ausgehenden zehnten Band in größeren oder komplexeren Artikeln dem Bedeutungsteil eine Inhaltsübersicht vorangestellt. ${ }^{12}$ In der digitalen Version des Wörterbuchs kann eine solche sogar für jeden einzelnen Artikel aufgerufen werden.

Der kleingedruckte Anmerkungsteil schließlich stellt - soweit gegeben - den Bezug zum Alt- und Mittelhochdeutschen her sowie zu einer Reihe Wörterbücher, die das Wort ebenfalls behandeln. Im Weitern können hier je nach Bedarf Ausführungen beispielsweise zur Formen- und Bedeutungsgeschichte oder zur Etymologie

11 Grundsätzliche Abweichungen vom bedeutungsgeschichtlichen Prinzip kommen dann und wann auch vor. Wer das Wörterbuch häufig braucht, scheint allerdings die historische Anordnung zu erwarten, wie in einer Rezension von ALFRED EGLI (2013) deutlich wird: Weil die ursprüngliche Bedeutung von wüest (Id. XVI 2173), nämlich 'unbelebt, öde', im „Idiotikon“ erst an vierter Stelle steht, wogegen die in der heutigen Mundart dominierende, jüngere Bedeutung 'hässlich' den Anfang macht, interpretierte der Rezensent letztere prompt als sprachgeschichtliche ,Ausgangsbedeutung“.

12 Die Idee stammte von EDUARD SCHWYZER, der sie 1938 hinsichtlich des Artikels stā 'stehen' vorschlug. Die Redaktion nahm den Vorschlag sogleich auf und setzte ihn schon bei dem kurz vor dem Druck stehenden Artikel Stëck (Id. X 1605) erstmals um (Stëck geht im schmellerschen Anordnungssystem $s t \bar{a}^{n}$ voraus). 
stehen, und zum Stichwort gehörende Familien- und Örtlichkeitsnamen werden ebenfalls an dieser Stelle aufgeführt.

\subsection{Belege}

Das „Schweizerische Idiotikon“ ist ein Belegwörterbuch. Die Belege haben somit in erster Linie die Funktion, die angeführten Wortdefinitionen nachzuweisen und sie in verschiedenen Kontexten zu dokumentieren, nicht etwa, sie lediglich zu illustrieren. Die Belege werden deshalb so ausgewählt und mit so viel Kontext gegeben, dass aus ihnen die Bedeutung des Stichworts so eindeutig wie möglich hervorgeht. Die Zahl der angeführten Belege wurde nach Jahrzehnten des steten Anwachsens in jüngerer Zeit zwar wieder etwas reduziert. Dennoch gilt die Devise unverändert, dass das Gewicht eines Wortes oder einer Bedeutung sowie ein gehäuftes Auftreten sich auch in einer als angebracht empfundenen Dichte der Belege manifestieren soll. Unverändert gilt auch das Ziel, „den Wortinhalt allseits auszuleuchten [und] auch kleinste Facetten sichtbar zu machen“" (WANNER 1976: 19 [1978: 14]).

Das „Schweizerische Idiotikon“ ist sodann ein semasiologisches Wörterbuch. Ein onomasiologischer Zugang wird zum einen durch zahlreiche Verweise auf Synonyme, Antonyme und Bedeutungsverwandtes gewährleistet (vgl. BURRI 1998), zum andern aber auch dank der oben beschriebenen Anordnung der Lemmata nach Wortfamilien (vgl. SCHIFFERLE 2006 und SCHIFFERLE 2009: 262-268). Eine gewichtige Ausnahme vom semasiologischen Prinzip machte in den älteren Bänden und vereinzelt auch noch später die Darstellung volkskundlicher und sachkultureller Inhalte, indem die „Bräuche und Sitten“ anhand der Quellen in freier Form beschrieben wurden - wie es die Schwesterwörterbücher der lateinischen Schweiz bis heute machen. Der ältere, wortfeldorientierte Zugang ermöglichte es beispielsweise, die Glockensprache, also die in den Klang der Glocke hineingedeuteten Wörter und Sätze, in den Artikel Glogg (Id. II 609) oder die zeitliche Reihenfolge der Arbeiten im Weinberg in den Artikel Rëb (Id. VI 37) zu integrieren, obwohl das Stichwort in den angeführten Zitaten gar nicht vorkommt; der semasiologische Wortartikel wurde also gleichsam aufgebrochen und onomasiologisch erweitert (zum Vorgehen bei Rëb vgl. SCHIFFERLE 2009: 254-256). In den späteren Bänden werden auch die volkskundlichen und sachkulturellen Informationen streng semasiologisch, also mittels der Zitierung originaler Zeugnisse, dargeboten. Immerhin bleiben diese thematisch gruppiert und werden unter passenden Zwischentiteln präsentiert.

\subsection{Verbreitungsangaben}

Die Angaben betreffend die Verbreitung von Lauten, Formen, Bedeutungen, Namen usw. sind eine Kompilation aller Daten von 1800 (wann in der „Idiotikon“Terminologie die „rezente Sprache“ anfängt) bis in die Erscheinungszeit des jewei- 
ligen Bandes und widerspiegeln folglich je länger desto weniger synchronische Verhältnisse.

Grundsätzlich werden Kantone in Kapitälchen, Ortschaften und Regionen in Normalschrift geschrieben; ausnahmsweise in Kapitälchen stehen ferner Angaben, die kantonsübergreifend sind. In der Aufzählung werden Kantone mittels eines Strichpunkts, Orte und Regionen mittels eines Kommas voneinander getrennt, wobei der erste Ort beziehungsweise die erste Region direkt an die Kantonssigle angehängt wird. Die Übersichtlichkeit wird freilich vermindert, indem die Kantonssiglen aus einem bis vier Buchstaben bestehen können - „S“ etwa steht für den Kanton Solothurn, „SCH“ für den Kanton Schaffhausen und „SCHW“ für den Kanton Schwyz -; dass bei Siglen, die aus nur einem Buchstaben bestehen, Kapitälchen nicht zum Ausdruck kommen können, versteht sich von selbst. Auch Orts- und Gebietssiglen können aus einem bis zu etlichen Buchstaben bestehen und ihrerseits mehrdeutig sein, es gilt also aufzupassen, welchem Kanton sie zugeordnet sind. Daneben gibt es Angaben wie ,,allg.“ für ,,allgemein bekannt”, ,,verbr.“ für ,verbreitet bekannt" oder „Fachspr.“ für ein nicht regionalisierbares Wort der Fachsprache.

$\mathrm{Zu}$ bedenken ist, dass dieses etwas opake Abkürzungssystem im 19. Jahrhundert für Artikel geschaffen worden war, die viel einfacher strukturiert waren als jene, wie sie sich ab dem frühen 20. Jahrhundert entwickelten. So wurden in den ersten Bänden für Verbreitungsangaben sehr häufig nur die Kantone beigezogen; Lokalisierungen auf Ortsstufe stellten anfänglich die Ausnahme dar und wurden erst nach und nach üblich.

\section{TEXTTECHNOLOGISCHE ASPEKTE}

\subsection{Online-Publikation}

Die Druckfassung des „Schweizerischen Idiotikons“ ist vollständig und kostenlos online zugänglich. Neue Artikellieferungen für den sich in Bearbeitung befindenden letzten Band werden jeweils innerhalb weniger Monate aufgeschaltet.

Die seit 2009 umgesetzte Digitalstrategie (vgl. BICKEL 2007; überhaupt zum Folgenden RоTн 2020) basiert maßgeblich auf etappenweisem Vorgehen in kleinen Schritten. Dadurch lässt sich das Werk in seinem Umfang und seiner Komplexität besser handhaben. Kleine Schritte bieten den Vorteil, dass schnell Resultate online gebracht werden können, die für die Öffentlichkeit bereits von Nutzen sind, auch wenn es sich im Ganzen noch nicht um ein perfekt abgerundetes Produkt handelt. Benützerrückmeldungen können so ab einem frühen Zeitpunkt und immer wieder in die Weiterentwicklung einfließen. Das Vorgehen entspricht dem in agilen Softwareentwicklungs-Methodologien verbreiteten, von ERIC RAYMOND (2001: 29) geprägten Grundsatz: „Release early. Release often. And listen to your customers.“

Online zugänglich sind zum jetzigen Zeitpunkt die gescannten Seiten des gedruckten „Idiotikons“, erschlossen über digitale Zugriffsstrukturen und präsentiert 
in einer Weboberfläche, die Orientierung und Benutzung erleichtern soll, ohne das Lesen des Wörterbuchtexts zu behindern.

Begonnen wurde die Online-Publikation mit einer digitalisierten und durchsuchbaren Version des alphabetischen Lemmaregisters, gefolgt von den damit verlinkten Wörterbuchscans. Diese erste Version wurde anschließend ergänzt um eine Volltextsuche über den - nicht systematisch korrekturgelesenen - OCR-Text des gesamten Wörterbuchs. Ebenfalls in einer frühen Phase wurde das digitalisierte grammatische Register online veröffentlicht. Das grammatische Register ordnet verschiedenen linguistischen Phänomenen (angesiedelt in unterschiedlichen Gebieten, etwa Phonetik, Morphologie, Phraseologie) einzelne Lemmata als Beispiele zu.

Mit dem sogenannten Semantikregister, das in Teilen bereits online ist, wird das Wörterbuch semantisch erschlossen. Es werden einige Kerninformationen zu den einzelnen Artikeln, etwa die Bedeutungserläuterungen, die Position der Artikel auf der Seite und eine Zusammenfassung der Beleglage, manuell extrahiert. Auf diese Weise kann z. B. zum Lemma direkt eine Bedeutungsübersicht eingeblendet werden, noch bevor man sich den eigentlichen Wörterbuchtext ansieht. Das Semantikregister wird eine wichtige Grundlage sein für das künftige Online-Wörterbuch sowie für allfällige nachgelagerte gedruckte Wörterbücher, z. B. Kurzwörterbücher, Zeitstufen- und Umkehrwörterbücher.

Obwohl im Moment der digitale, korrigierte Volltext des ,Schweizerischen Idiotikons" noch nicht vorliegt, haben wir bereits erste Versuche unternommen, den Text auf Basis des unkorrigierten OCR-Texts automatisiert weiter zu erschließen. So sind die meisten wörterbuchinternen Verweise mit Band- und Spaltenangaben unterdessen aktive Links: Ein Klick darauf öffnet das Wörterbuch direkt auf der entsprechenden Seite. Extern verlinkt ist das „Idiotikon“ mit dem Wörterbuchnetz des Kompetenzzentrums für elektronische Erschließungs- und Publikationsverfahren in den Geisteswissenschaften an der Universität Trier (Wörterbuchnetz 2019), wo das Lemmaregister des „Idiotikons“ als Suchstruktur hinterlegt ist.

Der großen Zunahme internetfähiger Mobilgeräte in den letzten Jahren ist das „Idiotikon“ mit einem spezifisch auf Kleinbildschirme angepassten Layout seiner Wörterbuchseite begegnet. Hauptänderung gegenüber der klassischen Seite ist der Wechsel auf eine ein- statt zweispaltige Darstellung des Wörterbuchs.

Damit das „Schweizerische Idiotikon“ noch besser und vielfältiger als linguistische Ressource eingesetzt werden kann, wurde eine Web-API, also eine Schnittstelle Maschine-zu-Maschine, eingerichtet. Damit kann z. B. ein anderes Projekt Teile des „Idiotikons“ direkt in eigene Software einbauen und darüber nutzen.

Neben dem eigentlichen Wörterbuch betreibt das Schweizerische Idiotikon weitere Online-Dienste:

- „ortsnamen.ch“ (2019), die Online-Plattform der schweizerischen Ortsnamenforschung, auf der Orts- und Flurnamen der meist kantonal organisierten Namenbücher gemeinsam online publiziert werden;

- das „Schweizer Textkorpus“ (2019), ein ausgewogenes linguistisches Korpus zur deutschen Standardsprache in der Schweiz, das aktuell um ein Mundartkorpus ergänzt wird; 
- die digitalisierten Originalaufnahmen des „Sprachatlasses der deutschen Schweiz" (2019);

- die Websites der drei abgeschlossenen Projekte „Kleiner Sprachatlas der deutschen Schweiz“ (2019), „Kollokationenwörterbuch“ (2019) und „Online-Lexikon zur diachronen Phraseologie“" (2019).

\subsection{Digitale Materialien}

Neben den doch umfangreichen digitalen Daten, die über die obengenannten Seiten öffentlich online zugänglich sind und die je nach Perspektive mehr Material oder mehr Resultat sein können, ist wenig weiteres Material in digitaler Form vorhanden.

Insbesondere ist die Sammlung handschriftlicher Belegzettel, die eigentliche Hauptgrundlage des Wörterbuchs, nie digitalisiert worden. Es bestehen momentan auch keine Pläne dazu. Aufgrund der Heterogenität von Papier und Handschriften scheint der Aufwand unverhältnismäßig groß, der zu erwartende Gewinn zu klein.

\subsection{Technische Grundlagen der Redaktionsarbeit}

Die Wörterbuchmanuskripte werden gegenwärtig in einem herkömmlichen Textverarbeitungsprogramm verfasst. Die Artikel werden dabei noch nicht nach dem endgültigen Drucklayout formatiert, sondern unter Verwendung einer einfachen Auszeichnungssprache, die sich an den Formatierungsanweisungen der früher üblichen Typoskripte orientiert. ${ }^{13}$ Nach Korrekturgängen auf Manuskriptstufe wird der Text mithilfe von skriptbasierten Routinen auf formale Konsistenz geprüft und für den externen Satz konvertiert. Der fertig gelayoutete Wörterbuchtext geht nach zweifacher Fahnenkorrektur als PDF an die Druckerei.

Ein eigenes Manuskriptlayout hat den Vorteil, dass eine für den Produktionsprozess optimierte Darstellung gewählt werden kann, während das Drucklayout auf die Rezeption (sowie Platz etc.) fokussiert. Im Manuskript stehen z. B. die Belege je in einem eigenen Absatz - klar übersichtlicher als im gedruckten Wörterbuch, wo Belege lediglich durch geviertbreite Wortabstände voneinander getrennt werden.

Zwei gewichtige Nachteile dieser Art der einfachen Manuskriptauszeichnungssprache zeigen sich vor allem im Hinblick auf die Online-Ausgabe. Zum einen werden keine semantischen Auszeichnungen vorgenommen: Die Auszeichnungen betreffen ausschließlich die Textformatierung, sie tragen in vielen Fällen keine einfach zuordenbare Bedeutung. Man denke etwa an die oben beschriebenen, bei Verbreitungsangaben verwendeten Kapitälchen, die nicht immer sichtbar (und in diesen Fällen auch nicht als solche ausgezeichnet) sind. Für eine elektronische Erschließung sind aber klar gekennzeichnete Informationselemente wichtig. Um z. B.

13 Vor dem Einsatz computergestützter Textverarbeitung wurden mit Schreibmaschine geschriebene Typoskripte und noch früher handschriftliche Manuskripte in den Satz gegeben. 
zu einem Lemma einzig die Bedeutungserläuterung (ohne den Rest des Artikels) anzeigen zu können, muss klar gekennzeichnet sein, welches das Lemma und welches die Bedeutungserläuterung ist.

Zum andern erfolgen umfangreichere Korrekturen öfter auch während der Fahnenkorrektur, also dann, wenn die Artikel aus dem Satz zurückkommen. Die Artikel liegen zu diesem Zeitpunkt erstmals im endgültigen Wörterbuchlayout vor, und da die Artikel für diese Form produziert werden, sind hier auch Korrekturen noch sehr wahrscheinlich. Dies führt aber dazu, dass das Manuskript nicht mehr mit dem gedruckten Text übereinstimmt und nicht mehr für die Online-Ausgabe verwendet werden kann. Der bereits gesetzte Wörterbuchtext schließlich liegt als PDF in nur schwer automatisch interpretierbarer Form vor.

\subsection{Digitalisierungsprojekte und weitere Nutzungsmöglichkeiten}

Umfang und Reichtum an Wissensbeständen und Materialien im „Schweizerischen Idiotikon" bieten sich zusammen mit den sich ständig erweiternden Möglichkeiten der Informationstechnologie geradezu ideal für Digitalisierungspläne an.

Das Wörterbuch selbst hat schon heute eine starke Online-Präsenz. Die gedruckte Ausgabe steht mit den Arbeiten am 17. und letzten Band vor ihrem Abschluss. Die Weiterführung des „Idiotikons“ ist als rein digitales Wörterbuch geplant, wozu aber noch einige weitere Digitalisierungsschritte notwendig sind.

Insbesondere ist vorgesehen, den gesamten Volltext des Wörterbuchs in guter Qualität zu retrodigitalisieren, durch maßgeschneidertes OCR (automatische Zeichenerkennung) oder durch Abschrift im Double-Keying-Verfahren (dabei wird der Text zweimal unabhängig abgetippt - bei Abweichungen erfolgt eine Nachkontrolle). Da künftig bei einer Bearbeitung nicht mehr wie bis anhin nur lesend, sondern auch schreibend auf den bisherigen Wörterbuchtext zugegriffen wird, ist es wichtig, den korrekten Text zur Verfügung zu haben. Bei den bisherigen Digitalisierungsschritten $-z$. B. Semantikregister und interne Verlinkung - wurde stets darauf geachtet, dass diese auch bei Vorliegen des korrekten Volltexts weiterhin verwendet werden können und ihren Wert nicht verlieren. Parallel dazu soll der Wörterbuchtext weiter automatisch erschlossen werden: Aktivierung externer Verweise, Auflösung von Abkürzungen und Quellenkürzeln, Verlinkung mit weiteren Materialien wie etwa den Fotos des „Sprachatlasses der deutschen Schweiz“. Im Hinblick auf das nur mehr digitale „Idiotikon“ ist auch ein neues Redaktionssystem geplant, angepasst an die Möglichkeiten und Erfordernisse digitaler Inhalte.

Von weiteren Digitalisierungsprojekten im Umfeld des Wörterbuchs sind zwei hervorzuheben: der Aufbau eines schweizerdeutschen Mundartkorpus und die weitere Digitalisierung der Materialien des „Sprachatlasses der deutschen Schweiz“. Mit beidem wurde 2019 angefangen.

Das anvisierte Dialektkorpus wird im Rahmen des am Schweizerischen Idiotikon angesiedelten Projekts „Schweizer Textkorpus“ erarbeitet und soll digitale Mundarttexte aller Art enthalten, wobei in einer ersten Phase vorwiegend Texte der Mundartliteratur digitalisiert werden. Dieses Korpus wird einen wichtigen Grund- 
stock für die geplante gezielte Überarbeitung insbesondere der noch im 19. und frühen 20. Jahrhundert verfassten Wortartikel im digitalen „Idiotikon“ liefern.

Im Rahmen der Online-Publikation der Archivalien des „Sprachatlasses der deutschen Schweiz" wird nun das Fotomaterial digitalisiert, nachdem 2017 die von den Exploratoren handschriftlich aufgezeichneten Antworten der Gewährspersonen veröffentlicht worden sind. Die Fotos sollen, soweit passend, mit den zugehörigen „Idiotikon“-Artikeln verlinkt werden, sodass sie dort als Illustrationen zu bestimmten Begriffen verwendet werden können. Häufig handelt es sich um landwirtschaftliche oder handwerkliche Geräte, die heutzutage nicht mehr im Gebrauch sind. Geplant ist zudem, die Karten und Register des gedruckten Werks zu digitalisieren, zu erschließen und zu publizieren.

Ideen für weitere Nutzungsmöglichkeiten des Wörterbuchs und der dazugehörigen Ressourcen für Forschung und Allgemeinheit sind immer willkommen. Wir sehen vor allem in der Verlinkung mit anderen Wissensbeständen aus benachbarten und verwandten Gebieten einiges Potential. Dies drückt sich beispielsweise in der bereits bestehenden Mitarbeit des Schweizerischen Idiotikons am Kooperationsprojekt „histHub“ (2019) aus, das vernetzte und normierte Grunddaten für die Geschichtswissenschaften in der Schweiz bereitstellen möchte. Das Idiotikon pflegt aber ganz allgemein einen offenen Umgang mit seinen Daten und versucht diese möglichst breit abgestützt, z. B. über sein Webangebot, über Web-APIs oder über Kooperationen, anzubieten und nutzbar zu machen.

\section{LINGUISTISCHES UND KULTURHISTORISCHES POTENTIAL}

Wissenschaftlich erarbeitete Wörterbücher dienen gewöhnlich dazu, Bedeutungen nachzuschlagen, und dementsprechend tauchen sie meist nur in Fußnoten auf. Das „Schweizerische Idiotikon“ ist aber nicht allein ein Wörterbuch, es ist viel mehr: Dank seiner wissenschaftlichen Exaktheit, der Breite seiner Belege und seiner historischen Tiefe dient es als Fundgrube für etymologische, lexikologische, morphologische, syntaktische und kulturhistorische Fragestellungen. Wir präsentieren im Folgenden einen bunten Strauß solcher Inhalte, die vom Wörterbuch teils explizit, teils implizit zur Verfügung gestellt werden, und demonstrieren, wie diese für weitere Publikationen nutzbar gemacht werden können.

\subsection{Etymologie}

Erste und grundsätzliche etymologische Hinweise ergeben sich indirekt durch die Einbindung in die Wortfamilie. So fehlt in der Anmerkung zu $\left(u m e^{n}\right)$ blegere $^{n}$ oder - plegere $^{n}$ 'faul herumliegen oder -sitzen, faulenzen' (Id. V 38) jeglicher Hinweis, woher das Wort stammt. Wer jedoch an den Anfang der Wortfamilie zurückblättert, stößt auf das Substantiv Blag 'Tierleiche, Aas' (Id. V 35) - sprachgeschichtlich Interessierte haben also selber den Schluss zu ziehen, dass $\left(u m e^{n}\right.$-) blegere ${ }^{n}$ wortwörtlich 'herumliegen wie ein Kadaver' bedeutet. 
Bei anderen Wörtern werden in der Anmerkung explizite Etymologien präsentiert:

In den Artikeln Bërchta 'Bertha' (Id. IV 1538) und Bërchtele ${ }^{n}$ stag 'Berchtoldstag, 2. Januar' (Id. XII 962) wird das Lemma auf mittelhochdeutsch ze dëm bërhten tage, ze dër bërhten naht 'Epiphanie' zurückgeführt und damit schon vor rund 120 Jahren die richtige Herleitung formuliert - während noch heute im Internet die pseudowissenschaftliche Bezugnahme auf eine Wintergöttin herumgeistert. ${ }^{14}$

Ein weiteres Beispiel ist Stör (Id. XI 1250) in der Bedeutung 'während eines gewissen Zeitraums (bzw. bis zur Fertigstellung) erfolgende Arbeit eines Handwerkers außer dem Haus', wo das „Schweizerische Idiotikon“ die alemannisch als Unterbedeutung von Störr 'Störung' belegte Bedeutung 'Zeitabschnitt, Weile; Tour, Mal' als Ausgangspunkt sieht. Die Bedeutung der Handwerkerstör dürfte damit von einer ursprünglichen Bedeutung 'mit Unterbrechungen, nicht fortlaufend, sondern in einzelnen Abschnitten von beschränkter Dauer geleistete Arbeit' ausgehen. Das „Deutsche Wörterbuch“ (X 3, 361) erklärt die Wortherkunft dagegen mit „störung der zunft [...] ein handwerker, der solche arbeit übernahm, verging sich gegen die handwerksordnung, er störte sie“.

Ietwëder 'jedweder, jeder' wird im „Schweizerische Idiotikon“ (in Übereinstimmung mit dem „Deutschen Wörterbuch“) als Zusammensetzung mit de-wëder 'einer (von beiden)' (Id. XV 486) etymologisiert und in der Folge als ie-de-wëder ‘jeder' (Id. XV 490) angesetzt. Es stellt sich damit gegen die in „Kluge. Etymologisches Wörterbuch der deutschen Sprache“ (2011: 456) vorgeschlagene Herleitung von iegewëder, da dewëder im Material des „Idiotikons“ reich bezeugt ist, iegewëder hingegen nur ganz marginal. Konsequenterweise wird auch $e i^{n}$ twëder 'einer von beiden, jeder von beiden, irgendeiner, entweder' (Id. XV 492) als einde-wëder etymologisiert und lemmatisiert, wogegen „Kluge. Etymologisches Wörterbuch der deutschen Sprache“ (2011: 249) entweder als Zusammensetzung von ein + weder und den Dental als Gleitlaut deutet.

In der Anmerkung zu Winden II (Id. XVI 540) schließlich wird bezüglich der Bedeutung 4a 'Dachboden, Lagerraum unter dem Dach' gegen die von EDUARD KOLB (1957: 97-104) postulierte nordgermanisch-alemannische Verwandtschaft von zürichdeutsch Winde f. und dem weitgehend synonymen dialektal-schwedischen vind $\mathrm{m}$. Stellung bezogen: KOLBS Argumentation wird vor dem Hintergrund der reichen Belege des „Schweizerischen Idiotikons“ widerlegt und die fragliche Bedeutung gut fundiert auf eine Übertragung von Bedeutung 1a 'Vorrichtung zum Heben, Senken, Heranziehen oder Anspannen', also des bei den stadtzürcherischen Häusern im Dachraum befindlichen Geräts auf das ganze Geschoß, zurückgeführt. ${ }^{15}$

Nicht verschwiegen sei aber, dass in etlichen Fällen die Wortherkunft weder über die Einbindung in eine Wortfamilie noch über eine explizite Anmerkung erschlossen ist, sondern diese allein über einen Verweis auf anderweitige Literatur abgetan wird. So ist in der Anmerkung zu dem typisch alemannisch-tirolischen Wort wimme $^{n}$ 'Trauben lesen' (Id. XV 1722), das im Zentrum einer eigenen

14 Siehe hierzu auch LANDOLT (2013b).

15 Siehe hierzu auch SCHIFFERLE (2006: 80-83). 
Wortfamilie steht, nur „Ahd. windemōn, mhd. windemen; vgl. Gr. WB. XIV 2, 222 [usw.]" zu lesen - aber kein Wort dazu, dass hier eines jener aus dem Lateinischen stammenden Lexeme vorliegt, die mit der römischen Kultur in die Alemannia mitgebracht worden sind (vindēmiāre). An Sprachgeschichte Interessierte werden also lediglich auf den einschlägigen Artikel im „Deutschen Wörterbuch“ verwiesen, falls sie sich nicht schon mit der lakonischen Angabe, es komme bereits im Althochdeutschen vor, zufriedengeben.

\subsection{Historische Wortgeographie}

Der Einbezug der bis ins spätere Mittelhochdeutsch zurückreichenden Sprache dient im „Schweizerischen Idiotikon“ dazu, das Aufkommen und Verschwinden von Wörtern und Bedeutungen nachzuzeichnen. Eine gezielte Auswertung dieser Daten kann aber weit darüber hinausgehen, wie HANS WANNER (1960) in einem Aufsatz demonstriert hat. Anhand der Wörter für die Leitersprosse, des Wortpaars flicke $^{n}$ und büeze $e^{n}$ 'ausbessern, flicken', von Frōntag I 'Donnerstag', Guetentag 'Montag' und Tobel 'Schlucht, tiefer Einschnitt' sowie der Synonyma Langsi, Früejār/Früelin $g$ und Ustag 'Frühling' zeigte er auf, was aus dem „Idiotikon“ für die historische Wortlandschaft herauszuholen ist - und was nicht.

Letzteres trifft für die Leitersprosse - Sëdel (Id. VII 296), Seigel (Id. VII 480), Speich I (Id. X 26), Spettel (Id. X 599), Spittel (Id. X 617), Spränzel (Id. X 917), Sprosse $^{n}$ (Id. X 945), Staffel I (Id. X 1407) und Stapfe ${ }^{n}$ (Id. XI 1151) - zu: Historische Belege, das heißt solche aus der Zeit vor 1800, finden sich hiervon allein für Seigel und Spettel, und diese stammen zum Teil aus Orten, die weit von den mundartlichen Verbreitungsgebieten des 19. und frühen 20. Jahrhunderts entfernt sind. Das historische Material des „Idiotikons“ lässt einen also im Stich, und dies aus zwei Gründen: Das unscheinbare Ding fand kaum je Eingang in Akten und Bücher, und wenn, dann in Textsorten, die keinen Rückschluss auf den Ortsgebrauch erlauben.

Aus einem ganz andern Grund ist die Wortgeschichte von flicke ${ }^{n}$ 'etw. wieder ganz machen’ (Id. I 1162) aus dem „Idiotikon“ nicht zu rekonstruieren: Die Darstellung im schon 1885 abgeschlossenen ersten Wörterbuchband ist schlicht ungenügend und überdies nach WANNER zu wenig überzeugend vom wohl nur indirekt verwandten sich inflicken 'sich zudrängen, einschleichen, einmischen, einnisten' (ebd.) geschieden. Damit scheitert auch das Nachzeichnen der Verdrängung des älteren, ursprünglich synonymen (heute aber 'nähen überhaupt' bedeutenden) büezen (Id. IV 2030) durch das in der alemannischen Schweiz einst unbekannte flicke ${ }^{n}$.

Differenziert fällt dagegen der Befund bei Frōntag I 'Donnerstag' (Id. XII 860) und Guetentag 'Montag' (Id. XII 873) aus: Ersteres ist aus den rezenten Mundarten des Wallis und der Südwalser gut bezeugt, fehlt aber in der historischen Sprache vollständig (NOTKERS frôntag steht für den Sonntag). Angesichts dessen, dass die Walliser- und Südwalsermundarten als außerordentlich archaisch gelten, möchte man annehmen, dieses Wort sei auch im mittelalterlichen Alemannisch üblich gewesen, doch weit gefehlt: Die historischen Quellen der übrigen Deutschschweiz 
kennen allein den Donnerstag. Frōntag im Wallis muss zwar alt sein, sonst hätten es die Walser im Hochmittelalter nicht in ihre in den Südtälern der Alpen liegenden Kolonien mitgenommen, aber nördlich des Alpenkamms hat es allem Anschein nach zu keiner Zeit eine große Rolle gespielt.

Anders der Guetentag oder Guentig, wie er in der lebenden Mundart lautet: Heute ist dieses Wort für den Montag zwar nur noch in Appenzell Innerrhoden bekannt; laut den historischen Quellen des „Schweizerischen Idiotikons“ und von FISCHERS „Schwäbischem Wörterbuch“ (III 961) aber galt es bis ins 16. Jahrhundert in weiten Teilen der östlichen Schweiz (einschließlich Zürichs) sowie Schwabens und wurde dann sukzessive von Montag verdrängt. Anders als beim offenbar innovativen Walliser Frōntag haben wir es beim Appenzeller Guentig mit einem ausgeprägten Reliktwort zu tun.

Einen gegenteiligen Befund liefert das Wort Tobel 'Schlucht' (Id. XII 116), dessen historische Belege einen Raum abdecken, der mit den rezenten Angaben praktisch deckungsgleich ist. Die Verbreitung von Tobel hat sich also seit mindestens 500 Jahren nicht wesentlich verändert.

Für 'Frühling' schließlich kannte man in den Jahrzehnten vor und nach 1900 laut „Idiotikon“ drei Worttypen: ${ }^{16}$ Langsi (Id. III 1339) galt im obersten Wallis, in Teilen der Innerschweiz, in Glarus und in Graubünden, Ustag (oder Ustage, Ustig, Hüstag[e]; Id. XII 815) sagte man in der westlichen Deutschschweiz, um Zürich herum sowie ebenfalls zum Teil in der Zentralschweiz, und Früejār oder Früeling herrschte ganz im Norden und im Nordosten (die Arealität des dritten Typus ist indirekt aus den Verbreitungsangaben unter den Synonymen zu erschließen; er wird Id. I 1293 nur in aller Kürze abgehandelt). Während Früeli ${ }^{n}$, Früejār und Langsi im Wörterbuch wegen des Erscheinens in den frühen Bänden I (1885 abgeschlossen) und III (1895 abgeschlossen) mit keinem beziehungsweise nur mit einem einzigen historischen Beleg gebucht sind, weshalb sich ihre Verbreitung im historischen Schweizerdeutsch nicht nachzeichnen lässt, erlaubt das im „Idiotikon“ schon für das Spätmittelalter reich bezeugte Ustag eine Interpretation: WANNER sieht in ihm einen westlichen Worttypus, der sich in der Innerschweiz und entlang der Handelsroute Zürich-Bündnerpässe nach Osten ausgedehnt hat.

\subsection{Morphologie}

Dass Flexionsformen einen eigenen Abschnitt haben, ist nicht weiter auffällig deren Nennung gehört zum Standard eines jeden besseren Wörterbuchs. Doch das "Schweizerische Idiotikon" geht weiter, indem es in vielen Wortartikeln in der jeweiligen Anmerkung auch auf den Flexionswandel eingeht. So teilt es etwa mit, dass die jüngere Pluralform Wälder die älteren Walde und Wälde in den schrift-

16 Dieses Bild wird noch mehr oder weniger vom „Sprachatlas der deutschen Schweiz“ (I 8) bestätigt, dessen Daten um 1950 bei einer weit überwiegend älteren bäuerlichen und handwerklichen Bevölkerung erhoben wurden. Heute dürfte es infolge des Vordringens von Früelin ${ }^{n}$ deutlich anders aussehen. 
lichen Quellen ab dem frühen 17. Jahrhundert abgelöst hat (Id. XV 1475, Anm.; in der gesprochenen Sprache sind die älteren Formen aber auch noch für das 19. und 20. Jahrhundert $\mathrm{zu}$ erheben) oder dass die erstmals 1480 greifbare Pluralform Wölf(e) das ältere Wolf(e) schon in der ersten Hälfte des 16. Jahrhunderts im Verhältnis von über vier zu eins dominierte und bis 1600 ganz verdrängte - und zwar so gründlich, dass sie in der gegenwärtig gesprochenen Sprache lediglich noch in den stark isolierten Walsergemeinden südlich und östlich des Monte Rosa gebraucht wird (Id. XV 1560, Anm.).

Entsprechendes gilt bei Verben: Beim ursprünglich nach der fünften starken Klasse flektierten wëtte ${ }^{n}$ 'ins Joch spannen' wird thematisiert, dass sich im 15. Jahrhundert schwache Formen entwickelten, die ab dem 17. Jahrhundert vorherrschten (Id. XVI 2238, Anm.); umgekehrt wird zum ursprünglich schwachen überzü $g e^{n}$ 'überzeugen' mitgeteilt, dass dessen in der modernen Mundart sehr frequent gewordenes starkes Partizip Perfekt über'zoge $e^{n}$ erstmals in der Burschensprache der ersten Hälfte des 19. Jahrhunderts bezeugt ist (Id. XVII 651, Anm.). Im Artikel wëbe 'weben' wiederum findet sich der Hinweis, dass das im heutigen Schweizerdeutsch allgemein geltende Partizip Perfekt $g$ 'wobe ${ }^{n}$ im Material des „Idiotikons“ erstmals 1605 anstelle des ursprünglichen $g$ 'wëbe $e^{n}$ fassbar ist (Id. XV 97, Anm.) - laut „Deutschem Wörterbuch“ (XIII 2622-2623) treten wob, gewoben in Deutschland erst im 18. Jahrhundert neben das dort herrschende, in der Schweiz aber praktisch unbekannte schwache webte, gewebt. In der Anmerkung zu verzìhe ${ }^{n}$ wird die schon von HERMANN PAUL (1916: 206) als schweizerisches Spezifikum festgehaltene Vermischung von verzeihen und verziehen besprochen: Sie geht auf den ab dem 15. Jahrhundert belegten und in großen Teilen des rezenten Schweizerdeutschs geltenden lautgesetzlichen Zusammenfall der beiden Verben in Infinitiv und Indikativ Präsens Plural zurück, der sich analogisch auf alle anderen Formen des Paradigmas ausgeweitet hat - das in den meisten heutigen Mundarten geltende Homonymenpaar Infinitiv verzieh" 'verziehen' und 'verzeihen' beziehungsweise Partizip Perfekt verzoge $e^{n}$ 'verzogen' und 'verziehen' war also schon vor 600 Jahren Realität (Artikel in Arbeit).

\subsection{Syntax}

Das „Schweizerische Idiotikon“ räumt auch der Syntax viel Platz ein. Während sich die meisten Wörterbücher bei vorwiegend grammatisch und insbesondere syntaktisch relevanten Wörtern mit den allernötigsten Angaben bescheiden, widmet ihnen das „Idiotikon“ Artikel, welche die Bedeutungen und Funktionen dieser Partikeln und Auxiliare umfassend darstellen und auch marginalen historischen oder mundartlichen Gebrauch dokumentieren. Diesen Anspruch hatte, wie oben unter „Geschichte“ im Zitat wiedergegeben, schon 1863 LUDWIG TOBLER formuliert. In den frühen Bänden waren solche Artikel oft noch mehr nach semantischen Kriterien aufgebaut, wie etwa $a b$ (Id. I 26), uber 'über' (Id. I 56), uf/üf 'auf' (Id. I 116), $a^{n}$ (Id. I 249), $i^{n} / \bar{\imath}^{n}$ 'in, ein' (Id. I 286), under I 'unter' (Id. I 324), us/ūs 'aus' (Id. I 550), von (Id. I 839), für (Id. I 952) oder die auch als Auxiliarverben dienenden 
gëbe $^{n}$ (Id. II 71) und chomme $e^{n}$ (Id. III 71) zeigen. Ab dem frühen 20. Jahrhundert, als mit ALBERT BACHMANN die damals moderne Sprachwissenschaft in der Wörterbuchredaktion Einzug gehalten hat, tritt die Orientierung nach grammatischen Kriterien zunehmend stärker hervor; siehe $b \bar{\imath}$ 'bei [usw.]' (Id. IV 900), sō (Id. VII 15), $s \bar{l}^{n}$ III 'sein [usw.]' (Id. VII 1018), dā (Id. XII 1), dann/denn (Id. XIII 18), tue $e^{n}$ 'tun [usw.]' (Id. XIII 290), dër, die, das I 'dieser, diese, dieses [usw.]' (Id. XIII 1028), dër II 'der, die, das [usw.]' (Id. XIII 1122), durch (Id. XIII 1443), dass (Id. XIII 1715), wā 'wo [usw.]' (Id. XV 1), wie (Id. XV 1573), wëder (Id. XV 470), $w a^{n}$ II 'außer, nur, aber, sondern, wiewohl, sodass, als, wie' (Id. XVI 59), wann III / wenn III (Id. XVI 73), wër I (Id. XVI 1043), wërde ${ }^{n}$ II (Id. XVI 1333), was (Id. XVI 1763), zue/ze 'zu [usw.]' (Id. XVII 4). Wer an historischer und dialektaler Grammatik interessiert ist, sollte keinen Bogen um diese Artikel machen. Gerade die Artikel dër und zue/ze sind die wohl umfangreichsten und detailliertesten Studien über die Bedeutungen und Funktionen dieser beiden Kleinwörter, die es im deutschen Sprachraum überhaupt gibt - ja selbst deren Nichtauftreten wird lexikographisch thematisiert. ${ }^{17}$

Auch frühe, im 19. Jahrhundert verfasste „Idiotikon“-Artikel können noch heute für Untersuchungen im Bereich der Syntax nutzbar gemacht werden. ANDREAS LÖTSCHER griff in einem 1993 publizierten Aufsatz über die frequente Verdoppelung der Verben choo 'kommen', gaa/goo 'gehen', aafaa/aafoo 'anfangen' und laa/loo 'lassen' in Fällen wie ga bsueche gaa 'besuchen gehen', cho bsueche choo 'besuchen kommen', la gheie laa 'fallen lassen', afa chochen aafaa/aafange 'zu kochen anfangen' auf den Wörterbuchartikel $g a^{n}$ (Id. II 322) zurück. Während WERNER HODLER (1969: 544) in seiner Berner Syntax das Phänomen als eine „Grammatisierung vom Verb zur Partikel“ (LÖTSCHER 1993: 183-185) interpretierte, knüpfte LÖTSCHER an die kurz nach 1885 im „Schweizerischen Idiotikon“ präsentierte Erklärung an, wonach zumindest ein Teil dieses $g a / g e / g i / g o / g u$ nicht auf altalemannisch gân 'gehen', sondern auf gen 'gegen' zurückgeht. Der Artikel $g a^{n}$ mit seinen beiden Hauptbedeutungen „I. Präposition“ und „II. Partikel“ präsentiert nämlich eine Reihe von Belegen ab dem Spätmittelalter, welche die Vorläufer der heutigen Konstruktion nachvollziehbar machen; wir bringen nur einige Beispiele: Sind geritten gan Einsidlen, 'sind nach (,gegen“) Einsiedeln geritten' (1497); Er ist nit kon gen klagen 'er ist nicht gekommen, um zu (,gegen“) klagen' (1549); So du gen schlafen gon willt 'wenn du ,gegen“ schlafen gehen willst' (1557); Tribschen, so auch gan Horw hört 'Tribschen, das auch zu (,gegen“) Horw gehört' (um 1600); So hoch das Viech gahn weiden gaht 'so hoch das Vieh ,gehen“" weiden geht' (1620). Auf dieser Grundlage beschreibt LÖTSCHER die Entstehung der Verbalverdoppelung im Schweizerdeutschen wie folgt: In einem ersten Schritt wurde die Präposition gen zu einer Verbalpräposition entwickelt, diese produzierte in einem zweiten Schritt über die Reinterpretation als verdoppeltes gân eine neue

17 Siehe unter dër II die Bedeutungen A1b, 3a, b, c, B1b $\beta 8, \mathrm{c} \alpha, 2 \mathrm{~b} \zeta$ sowie Anmerkung D2, E und unter zue die Bedeutungen B2b, E1d, E3c, E4e. 
Verdoppelungsregel, was schließlich drittens eine Ausdehnung der Verdoppelungsregel auf andere Verben zur Folge hatte (LÖTSCHER 1993: 190-193). ${ }^{18}$

\subsection{Das Wörterbuch als Korpus}

Das „Schweizerische Idiotikon“ kann dank seinen zahlreichen Satzbelegen auch als Korpus herangezogen werden. Ein frühes Beispiel für eine solche Nutzung ist MANFRED SZADROWSKYS (1930-1937) Aufsatz über die höchstalemannische Syntax, für den er das Wörterbuch nach Belegen aus dem Walserdeutschen absuchte.

Ein rezenter Aufsatz, dem die PDF-Version des „Idiotikons“ als Korpus diente, ist CHRISTOPH LANDOLTs (2013a) Untersuchung zum Partizip Perfekt der starken Reihe IIIb mit Ablaut $u$. Ausgang hierfür war, dass erstens WEINHOLDS Alemannische Grammatik von 1863, zweitens zahlreiche in der ersten Hälfte des 20. Jahrhunderts erschienene Monographien zu schweizerdeutschen Dialekten und zum Übergang der schweizerischen zur ostmitteldeutsch-ostfränkisch geprägten Schriftsprache und drittens die heutigen Standardwerke zur mittelhochdeutschen und frühneuhochdeutschen Grammatik sich mit Partizipien wie gehulfen statt geholfen schwertun: Während die älteren Werke den $u$-Typus zumeist als ,alt“" (vom $a$-Umlaut unberührt) charakterisieren, bleiben die modernen altsprachlichen Standardgrammatiken neutral und stellen verschiedene Erklärungsmöglichkeiten gleichberechtigt nebeneinander. Um das Auftreten des $u$ - und des $o$-Typus im alemannischen Raum quantifizieren und qualifizieren zu können, bot sich das ,Schweizerische Idiotikon" dank seiner Fülle an Belegen, die einen Zeitraum von über 700 Jahren sowie zahlreiche verschiedene Textsorten abdecken, als zweckdienliches Korpus dar: Danach war Ablaut /u/ im Partizip der Verben der Klasse IIIb, soweit sie stammschließendes /1/ + Konsonant aufweisen, im schweizerisch-alemannischen Deutsch des 14. bis 17. Jahrhunderts hochfrequent und alles in allem schreibsprachlich etwa gleich stark vertreten wie Ablaut/o/, wogegen bei postvokalischem $/ \mathrm{r} /$ + Konsonant (Typus geworfen) Ablaut/o/ nahezu uneingeschränkt galt (lediglich zwei Fälle mit /u/). Eine räumliche Verteilung von Ablaut /o/ gegenüber Ablaut /u/ ließ sich aus den historischen Daten hingegen nicht herauslesen; die meisten Kanzleien brauchten sie offenbar in freier Variation. Die Daten der rezenten Mundarten, für die auch Ortsgrammatiken usw. beigezogen wurden, geben dagegen eine klar definierbare räumliche Differenzierung zu erkennen: Der Typus gehulfen tritt in einem großen zentralen Raum zwischen Berner Seeland und Walensee einerseits und zwischen Südschwarzwald und Gotthard anderseits auf, fehlt aber im Südwesten, Südosten und Nordosten der Deutschschweiz. Die Interpretation der historischen und rezenten Daten lässt den gehulfen-Typus nur als Innovation gelten, und die Analyse aller Hebungen von /o/ und /u/ im Schweizerdeutsch des 19. und 20. Jahrhunderts schließt einen lautgesetzlichen Vorgang aus. Damit kommt allein die Deutung als Analogie zu den Verben der Klasse IIIa (gebunden-Typus) in Frage. Das Ergebnis bestätigt das Bild eines zentralalemannischen Raums, der eine Reihe 
weiterer, schon bekannter Neuerungen hervorgebracht hat, aber bislang von der schweizerischen Dialektologie - die von der Opposition Nord versus Süd und Ost versus West geprägt ist - noch nicht genügend gewürdigt worden ist.

\subsection{Volkskunde, Sachkultur, Rechtskultur}

Das „Schweizerische Idiotikon“ legt wie das „Glossaire des patois de la Suisse romande“, das „Vocabolario dei dialetti della Svizzera italiana“ und das „Dicziunari Rumantsch Grischun“ sein Augenmerk auch auf die Kulturgeschichte (zu „Idiotikon“ und Volkskunde generell WeISS 1954, zu „Idiotikon“ und Rechtsgeschichte generell FEHR 1956).

Anhand des für die historische Volkskunde besonders gewinnbringenden Artikels Chlaus (Id. III 687) zeigte CHRISTOPH LANDOLT (in BUCHELI / LANDOLT 2014: 77-78), wie fruchtbar die Verbindung von Wort und Brauch, wie sie das „Schweizerische Idiotikon“ pflegt, für kulturhistorische Untersuchungen eingesetzt werden kann. Der Artikel Chlaus zeichnet ein primär für die erste Hälfte des 19. Jahrhunderts geltendes Bild über das Nikolaus-, Weihnachts- und Neujahrsbrauchtum, an das heute kaum mehr etwas erinnert. Während heutzutage die Meinung herrscht, das Christkind sei der in der Deutschschweiz alteingesessene Gabenbringer, war es damals vielmehr der Nikolaus, der in vielen Regionen am Nikolaustag (katholisch), an Weihnachten oder um Neujahr (reformiert) die Gaben brachte, wogegen es an anderen Orten das von der reformierten Obrigkeit propagierte Neujahrskind (Neujārchindli, Id. III 346) war. Das angeblich ursprüngliche Christkind (Christchindli, Id. III 346) wird im redaktionellen Kommentar zum Neujärchindli 1892 dagegen als „,noch moderner [als das Neujahrskind] und wohl erst mit der deutschen Einwanderung zu uns gekommen" bezeichnet. Vor diesem kulturgeschichtlichen Hintergrund wäre die heute oft geäußerte Klage, der aus Amerika und Nordeuropa importierte Santa Claus verdränge das gute alte Christkind, zu relativieren. ${ }^{19}$

Umgekehrt machte HANS WANNER (1960: 142) darauf aufmerksam, wie dank kulturhistorisch relevanten Artikeln das hohe Alter eines Brauches nachgewiesen werden kann: Das Mundartwort helse $e^{n}$ I(Id. II 1212) mit der Bedeutung 'Geschenke machen, schenken' bezeugt das Wörterbuch schon aus einer Vorschrift von 1376 aus dem Zürcher Stadtbuch. ${ }^{20}$

Weitere für die Volkskunde interessante, onomasiologisch-beschreibend aufgebaute Artikel sind etwa Glogg (Id. II 609), wo die sogenannte Glockensprache thematisiert wird; Wuetisher (Id. II 1555), wo es um den Geisterzug geht, der zu bestimmten Zeiten übers Land jagt; lü̈te $e^{n}$ (Id. III 1506), wo sich das in vielem längst vergangene Läutebrauchtum besonders des 19. Jahrhunderts dokumentiert findet;

19 Siehe hierzu auch LANDOLT (2013c).

20 Ursprünglich gemeint und bis ins 20. Jahrhundert sachlich bezeugt war, wie unter dem synonymen würgen $1 b$ (Id. XVI 1466) deutlicher wird, 'jmdm. am Namenstag oder Geburtstag die Kehle zudrücken (damit der Beglückwünschte etwas zum Besten gebe)'. Die unter helsen angeführte Erklärung 'jmdm. ein Geschenk um den Hals hängen' ist wohl falsch. Weiteres siehe LANDOLT (2019). 
Girize $^{n}$ mos (Id. IV 470), wo der Schabernack beschrieben wird, den man mit alten Jungfern und Junggesellen trieb; ${ }^{21}$ Pfarrer (Id. V 1170), dessen gesellschaftliche Position sich vielfältig in der Sprache niedergeschlagen hat; oder Tauffi (Id. XII 577) mit einer breiten Schilderung der Bräuche, die mit der Taufhandlung, dem Taufgang und dem Taufmahl einhergehen. Andere volkskundlich relevante Artikel sind streng semasiologisch aufgebaut, beispielsweise Tüfel (Id. XII 638), wo den volkstümlichen Ansichten zu dieser Verkörperung des Bösen viel Platz eingeräumt wird, oder Bërchtele ${ }^{n}$ stag (Id. XII 962) mit all den Traditionen, die mit diesem typisch reformiert-schweizerischen, bis heute am Jahresanfang begangenen und historisch gesehen an die Stelle der Epiphanie getretenen Brauchtumstag verbunden waren oder auch noch sind.

Schwergewichtig der Sachkultur gewidmet sind etwa die Artikel Fochenze 'Brot, das man sich aus eigenem Gut vom Bäcker backen lässt; Brotlaib, den man beim Bäcker kauft' (Id. I 652), dessen lange Anmerkung die verschüttete Geschichte dieses mit dem mittelalterlichen Backwesen verbundenen und später immer beliebiger werdenden Wortes nachzeichnet, ${ }^{22}$ Rëb 'Weinrebe' (Id. VI 37); Trūb 'Traube, Frucht der Weinrebe' (Id. XIV 189); $W \imath^{n}$ 'Wein' (Id. XVI 139; zu diesen drei vgl. SCHIFFERLE 2009) sowie Ziger (Id. XVII 405), ein in den Alpen und Voralpen produziertes Milchprodukt, das in historischen Belegen nicht ohne weiteres mit dem modernen Marktprodukt gleichgesetzt werden darf.

Endlich sei auf Artikel hingewiesen, in denen die mittelalterliche und frühneuzeitliche Rechtskultur eine wichtige Rolle spielt. Hier fungiert das „Schweizerische Idiotikon" als Rechtswörterbuch des alemannischen Raumes. Eigentliche Rechtswortfamilien sind Wer IV 'Innehabung, Nutznießung von Gütern; Besitztitel' (Id. XVI 970, mit weren II 'jmdn. in den Besitz einer Sache einsetzen' usw.) und Wër I 'Garant; Bürge; Sicherheitszeichen' (Id. XVI 992, mit wëre ${ }^{n} I$ 'garantieren, verbürgen; Abgaben entrichten' usw.; hierzu ausführlich SCHIFFERLE 2011). Wichtig sind die rechtsgeschichtlichen Aspekte auch etwa in den Wortfamilien Ding (Id. XIII 470), wo Bedeutungen wie 'gesetzlich festgelegte, regelmäßig abgehaltene Versammlung; Gerichtsbezirk; Gerichtsbarkeit; Rechtshandlung; Streitsache; Vertrag, Satzung' abgehandelt werden, und wisse $e^{n}$ (Id. XVI 1924), das unter anderem die Bedeutung 'etw. mittels Eidesleistung beweisen, etw. bezeugen (lassen); jmdn. mittels Eidesleistung überführen' kennt. Zentrale Begriffe des früheren Rechts sind sodann Zug (Id. XVII 468) und ziehen (in Arbeit): Hier kommen dem Verb drei rechtliche Bedeutungen zu, nämlich erstens 'einen Rechtshandel aufgrund von Uneinigkeit unter den Gerichtsgenossen, den Parteien oder Urteilern bzw. Richtern an ein anderes Rechtssprechungskollegium oder an eine Obrigkeit bringen, später in die Bedeutung an eine übergeordnete Rechtsinstanz appellieren übergehend', zweitens 'als besser Berechtigter an die Stelle des Käufers gegen Erstattung des Kaufgeldes und der aufgelaufenen Kosten eintreten' und drittens 'im Konkursfall als Pfandgläubiger das verpfändete Gut in sein Eigentum übernehmen unter der Verpflichtung, die vorgehenden darauf haftenden Schulden zu übernehmen; auch: als

21 Siehe hierzu auch LANDOLT (2016).

22 Siehe hierzu auch LANDOLT (2017). 
belasteter Grundeigentümer, der nicht persönlicher Schuldner ist, die Belastung durch Zahlung ablösen'.

\subsection{Namenkunde}

Das „Schweizerische Idiotikon“ dokumentiert seit seinen Anfängen neben dem Appellativwortschatz auch Personen- und Örtlichkeitsnamen - meist in der Anmerkung zum jeweiligen Appellativ, im Fall der Taufnamen in Form separater Artikel. Die schweizerische Namenforschung macht sich das seit langem zunutze.

Insbesondere die zahlreichen abgeschlossenen oder sich noch in Arbeit befindenden kantonalen Orts- und Flurnamenbücher wären ohne Beizug des „Idiotikons“ kaum denkbar. Im Fall des „Ortsnamenbuchs des Kantons Bern“ (ONB) gab das Wörterbuch entscheidende Hinweise zu Bedeutung, Etymologie oder Benennungsmotiv beispielsweise bei März (ONB III 241), Mätte (ONB III 249), Mueli (ONB III 339), Bätterich (ONB IV 251), Bilitscher (ONB IV 331), Steibille (ONB IV 332), Bläj- (ONB IV 361), Büf(f)el (ONB IV 702), Rindestampfi (ONB V 179), Schlächt (ONB VI 648), Schlatt (ONB VI 666) und Scho(r)r(e) (ONB VI 792). ${ }^{23}$

Ebenso auf das Wörterbuch angewiesen ist die Familiennamenforschung. Dass der Name Binggeli zu Bingg 'kleiner, verwachsener Mensch; Hinkender' (Id. IV 1377), die Namen Brogle, Brogli zu Brogli 'Prahler' (Id. V 518), der Name Fey zu feie $^{n}$ 'spielen, im Scherz miteinander kämpfen; umherschwärmen' (Id. I 632), die Namen Schiffer, Schifferle, Schifferli zu Schifer 'Splitter' (Id. VIII 377) oder der Name Tschirky zu tschirggen 'langsam, schlurfend gehen' (Id. XIV 1794) zu stellen sind, hätte sich aus anderen Nachschlagewerken nicht so leicht erschließen lassen. ${ }^{24}$ Auch für die gegenwärtig in Bearbeitung befindliche Publikation „Familiennamen der deutschen Schweiz. Ein etymologisches Lexikon"von SiMONE BERCHTOLD und MARTIN H. GRAF bildet das „Schweizerische Idiotikon“ die Grundlage.

\section{KONTAKTADRESSE, HOMEPAGE}

Schweizerisches Idiotikon

Auf der Mauer 5

8001 Zürich

Schweiz

E-Mail: info@idiotikon.ch

Homepage: <https://www.idiotikon.ch>

23 Die Beispiele verdanken wir unserem Kollegen THIS FETZER, der selber längere Zeit am „Ortsnamenbuch des Kantons Bern“" gearbeitet hat.

24 Beispiele aus HürLimann u. a. (2017) sowie Schweizer Radio SRF 1 bzw. 3 (2008 ff.). 


\section{LITERATURVERZEICHNIS}

Badisches Wörterbuch (1940 ff.). Begonnen von ERNST OcHS [...]. Lahr (Schwarzwald): Schauenburg bzw. Berlin: de Gruyter.

Bayerisches Wörterbuch (2002 ff.). München: Oldenbourg.

Beiträge zur Schweizerdeutschen Grammatik (1910-1941). Im Auftrag des Leitenden Ausschusses für das Schweizerdeutsche Idiotikon hg. von Albert Bachmann. Frauenfeld: Huber.

BENECKE, GEORg FrIEDRICH / WiLHELM MÜLLER / FriedRICH ZARNCKE (1854-1866): Mittelhochdeutsches Wörterbuch. Leizpig: Hirzel.

Berchtold, Simone / Martin H. Graf (in Arbeit): Familiennamen der deutschen Schweiz. Ein etymologisches Lexikon.

BICKEL, HANS (2007): Idiotikon digital. Überlegungen zu einer elektronischen Ausgabe des Schweizerdeutschen Wörterbuchs. In: Schweizerdeutsches Wörterbuch. Schweizerisches Idiotikon. Bericht über das Jahr 2006. Zürich, 13-26.

Bucheli, Claudia / Christoph Landolt (2014): Dialekt und Konfession in der Deutschschweiz. In: Frieben, Elisabeth / Ulrich Kanz / Barbara Neuber / Ludwig Zehetner (Hg.): Dialekt und Religion. Beiträge zum 5. dialektologischen Symposium im Bayerischen Wald, Walderbach, Juni 2012. Regensburg: Vulpes (Regensburger Dialektforum. 20), 73-94.

BURRI, ANDREAS (1998): Möglichkeiten der Wortfelderfassung im Schweizerdeutschen Wörterbuch. In: GROSSE, RUDOLF (Hg.): Bedeutungserfassung und Bedeutungsbeschreibung in historischen und dialektologischen Wörterbüchern. Beiträge zu einer Arbeitstagung der deutschsprachigen Wörterbücher, Projekte an Akademien und Universitäten vom 7. bis 9. März 1996. Stuttgart/Leipzig: Hirzel (Abhandlungen der Sächsischen Akademie der Wissenschaften zu Leipzig, Phil.-Hist. Klasse. 75, 1), 107-115.

Deutsches Wörterbuch von JACOB und WILHELM GRIMM (1854 ff.): Leipzig bzw. Stuttgart: Hirzel.

Dicziunari Rumantsch Grischun (1939 ff.). Fundà da RoBert DE Planta, FloRIAn MElcher [...]. Chur: Bischofberger bzw. Institut dal Dicziunari Rumantsch Grischun.

EGLI, ALFRED (2013): Idiotikon Band XVI vollendet! Ein Blick auf die abschliessenden Hefte 220 und 221. In: Schweizerdeutsch 21/1, 18-22.

FEHR, HANS (1956): Das Schweizerdeutsche Wörterbuch im Spiegel der Rechtsgeschichte. In: Schweizerdeutsches Wörterbuch. Schweizerisches Idiotikon. Bericht über das Jahr 1955. Zürich, 13-26.

Glossaire des patois de la Suisse romande (1924 ff.). Neuchâtel/Paris: Attinger bzw. Genève: Droz.

GRAFF, EBERHARD GotTLIEB (1834-1842): Althochdeutscher Sprachschatz oder Wörterbuch der althochdeutschen Sprache. Berlin: Nikolaische Buchhandlung.

HAAS, WALTER (1981): Das Wörterbuch der schweizerdeutschen Sprache. Versuch über eine nationale Institution. Hg. von der Redaktion des Schweizerdeutschen Wörterbuchs. Frauenfeld: Huber.

HAas, WAlter (1994): Provinzialwörter. Deutsche Idiotismensammlungen des 18. Jahrhunderts. Hg. unter Mitarbeit von W. GÜNTHER GANSER, KARIN GERSTNER, HANSPETER VON FLÜE. Berlin / New York: de Gruyter (Historische Wortforschung. 3).

histHub (2019): Plattform für normierte und vernetzte Daten zur Schweizergeschichte. URL: $<$ https://histhub.ch>; Stand: 31.08.2019.

HODLER, WERNER (1969): Berndeutsche Syntax. Bern: Francke.

HUNZIKER, JAKOB (1877): Aargauer Wörterbuch in der Lautform der Leerauer Mundart. Aarau: Sauerländer.

Hunziker 2020 (2019): Interaktives Digitalisat des Aargauer Wörterbuchs. URL: <https://www. hunziker2020.ch>; Stand: 31.08.2019.

HÜrlimann, Esther / Christoph Landolt / Anina Rether / ERIKa Suter / DANiel ZinNenLAUF / Christian SCHOCH (2017): Fleischli, Luder, Schlumpf. Schweizer Namen - Gründlich erklärt. Zürich: Orell Füssli. 
Kleiner Sprachatlas der deutschen Schweiz (2019): Online-Version der gedruckten Ausgabe. URL: $<$ https://www.kleinersprachatlas.ch>; Stand: 31.08.2019.

Kluge. Etymologisches Wörterbuch der deutschen Sprache (2011). Bearbeitet von ELMAR SEEBOLD. 25., durchgesehene und erweiterte Auflage. Berlin/Boston: de Gruyter.

KOLB, EDUARD (1957): Alemannisch-nordgermanisches Wortgut. Frauenfeld: Huber (Beiträge zur schweizerdeutschen Mundartforschung. 6).

Kollokationenwörterbuch (2019): Onlineversion der gedruckten Ausgabe. URL: <https://www. kollokationenwoerterbuch.ch>; Stand: 31.08.2019.

LANDOLT, Christoph (2010): Das Schweizerische Idiotikon - ein diachrones Wörterbuch der schweizerdeutschen Sprache. In: Runow, Holger (Hg.): Mitteilungen des Deutschen Germanistenverbandes 57/4: Historische Lexikographie des Deutschen, 410-418.

LANDOLT, CHRISTOPH (2013a): „Dis gelt ist ouch den burgern genzlich vergulten.“ Die Partizipia Präteriti der Reihe IIIb mit Ablaut $u$ im Alemannischen. In: Zeitschrift für deutsche Philologie $132,401-416$.

LANDOLT, CHRISTOPH (2013b): Berchtoldstag. In: Wortgeschichten. Hg. von der Redaktion des Schweizerischen Idiotikons. URL: <https://www.idiotikon.ch/wortgeschichten/berchtoldstag>; Stand: 31.08.2019.

LANDOLT, CHRISTOPH (2013c): Nikolaus, Christkind, Weihnachtskind, Neujahrskind, Mutti, Pelzer \& Santa Claus. In: Wortgeschichten. Hg. von der Redaktion des Schweizerischen Idiotikons. URL: <https://www.idiotikon.ch/wortgeschichten/gabenbringer>; Stand: 31.08.2019.

LANDOLT, ChRISTOPH (2016): Das Giritzenmoos. In: Wortgeschichten. Hg. von der Redaktion des Schweizerischen Idiotikons. URL: <https://www.idiotikon.ch/wortgeschichten/giritzenmoos>; Stand: 31.08.2019.

LANDOLT, CHRISTOPH (2017): Focaccia und Fogetzebrot - New meets Old. In: Wortgeschichten. Hg. von der Redaktion des Schweizerischen Idiotikons. URL: <https://www.idiotikon.ch/ wortgeschichten/fogetzebrot>; Stand: 31.08.2019.

LANDOLT, CHRISTOPH (2018): Go(ge) schaffe gaa. In: Wortgeschichten. Hg. von der Redaktion des Schweizerischen Idiotikons. URL: <https://www.idiotikon.ch/wortgeschichten/go>; Stand: 31.08.2019.

LANDOLT, CHRISTOPH (2019): Vom Helsen und Würgen - oder warum man den Kuchen besser schon prophylaktisch ins Büro mitbringt. In: Wortgeschichten. Hg. von der Redaktion des Schweizerischen Idiotikons. URL: <https://www.idiotikon.ch/wortgeschichten/helsen-undwuergen>; Stand: 31.08.2019.

LÖTSCHER, ANDREAS (1993): Zur Genese der Verbverdoppelung bei gaa, choo, laa, aafaa (,gehen“, „kommen“, „lassen“, „,anfangen“) im Schweizerdeutschen. In: ABRAHAM, WeRNER / JOSEF BAYER (Hg.): Dialektsyntax. Opladen: Westdeutscher Verlag, 180-200.

Online-Lexikon zur diachronen Phraseologie (2019): Deutsche Sprichwörter und Redewendungen im Sprachwandel. URL: $<$ https://www.oldphras.net $>$; Stand: 31.08.2019.

Ortsnamenbuch des Kantons Bern (alter Kantonsteil). I. Dokumentation und Deutung (1976 ff.). Hg. von PaUl ZinsLi [...]. Bern bzw. Basel/Tübingen: Francke.

ortsnamen.ch (2019): Das Portal der schweizerischen Ortsnamenforschung. URL: $<$ https://www. ortsnamen.ch $>$; Stand: 31.08.2019.

PAUL, HERMANN (1895): Ueber die Aufgaben der wissenschaftlichen Lexikographie mit besonderer Rücksicht auf das deutsche Wörterbuch. In: Sitzungsberichte der philosophisch-philologischen und der historischen Classe der k[öniglich] b[ayerischen] Akademie der Wissenschaften zu München. Jahrgang 1894. München: Akademie, 53-91.

Paul, Hermann (1916): Deutsche Grammatik. Band II, Teil III: Flexionslehre. Halle a/S.: Niemeyer.

Plan des schweizerischen Wörterbuchs (1874). In: Jahresbericht über das schweizerdeutsche Idiotikon, umfassend den Zeitraum vom Weinmonat 1873 bis Ende Herbstmonat 1874, Zürich 1874, 6-7, mit nur wenigen Abweichungen auch in Schweizerisches Idiotikon (wie unten), Band I, vi. 
RAYMOND, ERIC S. (2001): The Cathedral and the Bazaar: Musings on Linux and Open Source by an Accidental Revolutionary. Revised Edition. Cambridge, Mass.: O'Reilly Media.

REICHMANN, OSKAR (2012): Historische Lexikographie. Ideen, Verwirklichungen, Reflexionen an Beispielen des Deutschen, Niederländischen und Englischen. Berlin/Boston: de Gruyter (Studia Linguistica Germanica. 111).

Rотн, TовIAS (2020): Im Übergang zum digitalen Wörterbuch. Zum Stand der Digitalisierung des Schweizerischen Idiotikons: Rückblick und Ausblick. In: AQUINO, DOROTHÉE / YAN GREUB (Hg.): La lexicographie informatisée: les vocabulaires nationaux dans un contexte européen. Bern: Schweizerische Akademie der Geisteswissenschaften, 103-118 (Swiss Academies Reports. 15,1$)$.

SCHIFFERLE, HANS-PETER (2006): „Währschafte“ Lösungen für „währhafte“ Probleme. Lemmatisierung und Etymologie im Schweizerdeutschen Wörterbuch. In: KLAUSMANN, HUBERT (Hg.): Raumstrukturen im Alemannischen. Beiträge der 15. Arbeitstagung zur alemannischen Dialektologie, Schloss Hofen, Lochau (Vorarlberg) vom 19.-21.9.2005. Graz/Feldkirch: Neugebauer (Schriften der Vorarlberger Landesbibliothek. 15), 73-84.

SCHIFFerLe, HANS-PETER (2009): Die Fachsprache des Weinbaus im Schweizerdeutschen Wörterbuch. In: Besse, MARIA / WOLfGANG HAUBrichs / ROLAND PUHL (Hg.): Weinwörter - Weinkultur. Ein europäisches Fachwörterbuch im linguistischen, kulturellen und historischen Kontext. Beiträge des internationalen und interdisziplinären Kolloquiums im Institut für pfälzische Geschichte und Volkskunde in Kaiserslautern, 14./15. September 2007. Stuttgart: Steiner (Akademie der Wissenschaften und der Literatur, Abhandlungen der geistes- und sozialwissenschaftlichen Klasse. 2009, 3), 241-271.

SCHIFFERLE, HANS-PETER (2011): „Nachwähr“, „Viehwährschaft“ und verwandte Garantien. Rechtsgeschichtliche Aspekte einer Wortfamilie im Schweizerischen Idiotikon. In: Signa Iuris 7, 131-147.

SCHIFFERLE, HANS-PETER (2017): Diachrone Dialektlexikographie im Schweizerischen Idiotikon Konzepte und Praxis einer 150-jährigen Geschichte. In: WANDL-Vogt, Eveline / AMELIE DoRN (Hg.): dialekt | dialect 2.0. Langfassungen. 7. Kongress der Internationalen Gesellschaft für Dialektologie und Geolinguistik (SIDG) [2012]. Wien: Praesens, 290-306.

SCHMELLER, ANDREAS (1827-1837). Bayerisches Wörterbuch. Stuttgart: Cotta.

Schwäbisches Wörterbuch (1904-1936). Bearb. von HeRMANN FISCHER [...]. Tübingen: Laupp.

Schweizerisches Idiotikon. Wörterbuch der schweizerdeutschen Sprache (1881 ff.). Begonnen von Friedrich STAub und Ludwig Tobler [...]. Frauenfeld: Huber bzw. Basel: Schwabe.

Schweizerisches Idiotikon digital (2019): Online-Zugriff auf das Schweizerische Idiotikon. URL: $<$ https://www.idiotikon.ch/woerterbuch/idiotikon-digital>; Stand: 31.08.2019.

Schweizer Radio SRF 1 (2015 ff.) / Schweizer Radio SRF 3 (2008-2014): Auf den Spuren eures Namens. URL: <https://www.srf.ch/radio-srf-1/auf-den-spuren-eures-namens $>$; Stand: 31.08 . 2019 (unvollständige Liste).

Schweizer Textkorpus (2019): Referenzkorpus für die deutsche Standardsprache des 20. und 21. Jahrhunderts in der Schweiz. URL: <https://www.chtk.ch>; Stand: 31.08.2019.

Siedlungsnamen des Kantons Zürich (2019): Zürcher Namenbuch. URL: <https://www. idiotikon.ch/projekte/siedlungsnamen-des-kanton-zuerich>; Stand: 31.08.2019.

Sprachatlas der deutschen Schweiz (1962-1997). Begründet von HEINRICH BAUMGARTNER und RUDOLF HOTZENKÖCHERLE. Bern bzw. Basel: Francke.

Sprachatlas der deutschen Schweiz (2019): Originalmaterialien und Werkgeschichte des „Sprachatlasses der deutschen Schweiz“. URL: <https://www.sprachatlas.ch>; Stand: 31.08.2019.

STALDER, FRANZ JOSEPH (1806-1812): Versuch eines Schweizerischen Idiotikons, mit etymologischen Bemerkungen untermischt. Basel/Aarau: Flickische Buchhandlung.

STAUB, FRIEDRICH (1876): Die Reihenfolge in mundartlichen Wörterbüchern und die Revision des Alphabetes. Ein Vorschlag zur Vereinigung; vorgelegt vom Büreau des Schweizerdeutschen Idiotikons. Zürich. 
SZADROWSKY, MANFRED (1930-1937): Zur hochalemannischen Syntax. In: Beiträge zur Geschichte der deutschen Sprache und Literatur 54, 65-137, 281/93; 60, 445-458; 61, 273-288.

TOBLER, LUdWIG (1863): Unmaßgebliche Gedanken über die Methode des schweizerischen Wörterbuchs. (Manuskript im Archiv des Schweizerischen Idiotikons.)

Vocabolario dei dialetti della Svizzera italiana (1952 ff.). Lugano: Commerciale / Natale Mazzuconi bzw. Bellinzona: Cavalli.

Vorarlbergisches Wörterbuch mit Einschluss des Fürstentums Liechtenstein (1960-1965). Bearb. von LEO JuTZ. Wien: Holzhausen.

WANNER, HANS (1960): Das sog. historische Material in landschaftlichen Wörterbüchern. In: Zeitschrift für Mundartforschung 27, 129-143.

WANNER, HANS (1976): Das Schweizerdeutsche Wörterbuch. Schweizerisches Idiotikon. Wörterbuch der schweizerdeutschen Sprache. In: Dialektlexikographie. Hrsg. von HANS FRIEBERTSHÄUSER. Steiner: Wiesbaden (Zeitschrift für Dialektologie und Linguistik, Beiheft N. F. 17), Wiesbaden: Steiner, 11-24. Korrigierter Separatdruck: Zug 1978.

WeINHOLD, KARL (1863): Alemannische Grammatik. Berlin: Dümmler.

WEISS, RichARD (1954): Das Schweizerdeutsche Wörterbuch und die Volkskunde. In: Schweizerdeutsches Wörterbuch. Schweizerisches Idiotikon. Bericht über das Jahr 1953. Zürich, 10-23.

Wörterbuch der bairischen Mundarten in Österreich (1963 ff.): Wien: Böhlau bzw. VÖAW.

Wörterbuch der elsässischen Mundarten (1899-1907). Bearb. von ERNST MARTIN und HANS LIENHART. Straßburg: Trübner.

Wörterbuchnetz (2019): Wörterbuchnetz des Kompetenzzentrum für elektronische Erschließungsund Publikationsverfahren in den Geisteswissenschaften an der Universität Trier. URL: $<$ www.woerterbuchnetz.de>; Stand: 31.08.2019.

VON WYSS, GEORG (1874): Aufruf betreffend Anfertigungen von Auszügen aus der ältern schweizerdeutschen Litteratur für das Idiotikon. (Im Archiv des Schweizerischen Idiotikons.) 



\title{
BADISCHES WÖRTERBUCH
}

\author{
Tobias Streck
}

\section{GESCHICHTLICHES UND RAHMENBEDINGUNGEN}

Mit seiner inzwischen rund 100-jährigen Geschichte spiegelt das „Badische Wörterbuch" bereits auch einen langen und durch erheblichen technischen Fortschritt geprägten Abschnitt der Geschichte der deutschen (Dialekt-)Lexikographie wider. Eine so lange Bearbeitungszeit bringt unweigerlich eine gewisse Heterogenität in Bezug auf das verwendete Datenmaterial sowie seiner Aufbereitung und Darstellung mit sich. Mit den Jahren ist das Belegmaterial deutlich angewachsen, was sehr viel genauere Darstellungen, auch in der Fläche, erlaubte. Die Artikeltexte, die selbstverständlich von Beginn an artikelübergreifende Gestaltungsmerkmale hatten, jedoch anfangs mitunter in der Abfolge der einzelnen Informationsebenen variierten und zuweilen Passagen mit eher erzählendem Charakter aufwiesen, wurden mit der Zeit immer systematischer. Nach Abschluss des zweiten Bandes erfolgte mit dem Wechsel von Fraktur zu Antiqua ein typographischer Bruch, der zum damaligen Zeitpunkt (Mitte der 1970er Jahre) kaum vermeidbar, wenn nicht sogar überfällig war. Im vierten Band wurde als Neuerung die Publikation von laut- und wortgeographischen Karten eingeführt, mit dem Beginn des fünften Bandes wurde aus texttechnologischen und typographischen Gründen auf Sperrsatz in den Artikeln verzichtet. Diese und weitere Veränderungen und Entwicklungen werden hier und in den beiden folgenden Kapiteln nochmals aufgegriffen und näher erläutert. Zunächst richtet sich der Blick jedoch auf die Geschichte, das Arbeitsgebiet und die Rahmenbedingungen des „Badischen Wörterbuchs“.

\subsection{Geschichtlicher Abriss und personelle Ausstattung der Arbeitsstelle}

Die Idee zur Schaffung des „Badischen Wörterbuchs“ geht letztlich auf das späte 19. Jahrhundert zurück. Im Jahr 1893 fasste der germanistisch und volkskundlich ausgebildete Universitätsbibliothekar FRIDRICH PFAFF den Plan, im Rahmen einer flächendeckenden Umfrage die volkskundliche Überlieferung Badens zu erheben. Für sein Vorhaben konnte er FRIEDRICH KLUGE, damals gerade neu als ordentlicher Professor für deutsche Sprache und Literatur nach Freiburg berufen, und ELARD Hugo MEYER, Bibliothekar und außerordentlicher Professor für Germanistik und Volkskunde, gewinnen. Noch 1893 wurde ein erster Fragebogen mit einem programmatischen Aufruf in der Zeitschrift „Alemannia“ veröffentlicht, 1894/1895 dann eine erweiterte Version an Lehrer und Pfarrer im ganzen Land versandt. Da 
das umfangreiche und vielfältige Material, das die Fragebogenaktion erbrachte, auch im mundartlichen Bereich so viel Interessantes und Neues beinhaltete, ${ }^{1}$ zogen die drei Initiatoren nun, entgegen früherer Bedenken, die Schaffung eines Wörterbuchs ernsthaft in Betracht. Man war sich allerdings darüber im Klaren, dass das Material aus der Umfrage allein nicht für dieses Vorhaben ausreichen würde. Ab 1901 wurde daher weiter gesammelt, planmäßiger erweitert wurden die Sammlungen dann ab 1907, als der Germanist und Bibliothekar ALFRED GöTZE dem inzwischen erblindeten FRIEDRICH KLUGE an die Seite trat und mit diesem zusammen über Jahre hinweg eine wöchentlich stattfindende Befragung badischer Studenten zu deren Heimatdialekt leitete.

Im Jahr 1914 konnte der aus Ettenheim stammende ERNST OCHS, ein Schüler KLUGES, als erster hauptamtlicher Bearbeiter eingestellt werden. Als seine Aufgabe wurde festgelegt, ein Wörterbuch der alemannischen und fränkischen Dialekte des Landes Baden zu schaffen (zum Arbeitsgebiet vgl. Abbildung 1). ${ }^{2}$ Die Arbeit wurde allerdings bereits nach wenigen Monaten durch Kriegsdienst und Gefangenschaft unterbrochen und konnte erst 1919 wieder aufgenommen werden. In den vorbereitenden Wörterbuchausschuss traten zu KLUGE, GöTZE und OCHS noch der Indogermanist Ludwig SÜTTERLIN und Kluges Nachfolger FriedRICH Wilhelm hinzu. Das Unternehmen kam räumlich im Deutschen Seminar der Universität Freiburg unter und erhielt Mittel von der Freiburger Wissenschaftlichen Gesellschaft. Das badische Kultusministerium gewährte OCHS, der eigentlich Gymnasialprofessor war und von der Schulverwaltung bezahlt wurde, eine Deputatsreduktion auf vier bis sechs Stunden. An der Universität war er zunächst als Lehrbeauftragter, ab 1946 dann als Honorarprofessor tätig. Eine feste Universitätsstelle konnte erst im Jahre 1961 für OCHS' Nachfolger KARL FRIEDRICH MÜLLER eingerichtet werden. Nach MÜLLERS Pensionierung wurde diese 1968 mit GERHARD WOLFRAM BAUR besetzt, ihm folgte von 1998 bis 2009 RUdOLF POST. Seit 2009 schließlich leitet TOBIAS STRECK die zur Abteilung Germanistische Linguistik am Deutschen Seminar gehörende Arbeitsstelle. Sie ist also von Beginn an bis heute (abgesehen von den Jahren 1935-1939 und 1946-1948 sowie 1973, vgl. BAUR 1983: 390) ein „Einmannbetrieb" geblieben.

1 Für ausführlichere Informationen zu den Erhebungen zur Sammlung der Volksüberlieferungen Badens 1894/1895 sowie deren Leistungen für die Mundartforschung siehe POST (2001).

2 Bei der ersten Mundart-Wörterbuch-Konferenz in Marburg hatte man sich 1913 dafür entschieden, sich bei den Bearbeitungsgebieten der großen wissenschaftlichen Dialektwörterbücher an die damaligen politischen Grenzen zu halten, was auch schon die früher ins Leben gerufenen Nachbarwörterbücher des „Badischen Wörterbuchs“ (,Schweizerisches Idiotikon“, „Wörterbuch der elsässischen Mundarten“, „Schwäbisches Wörterbuch“) so gehandhabt hatten (vgl. BAUR 1983: 387). 


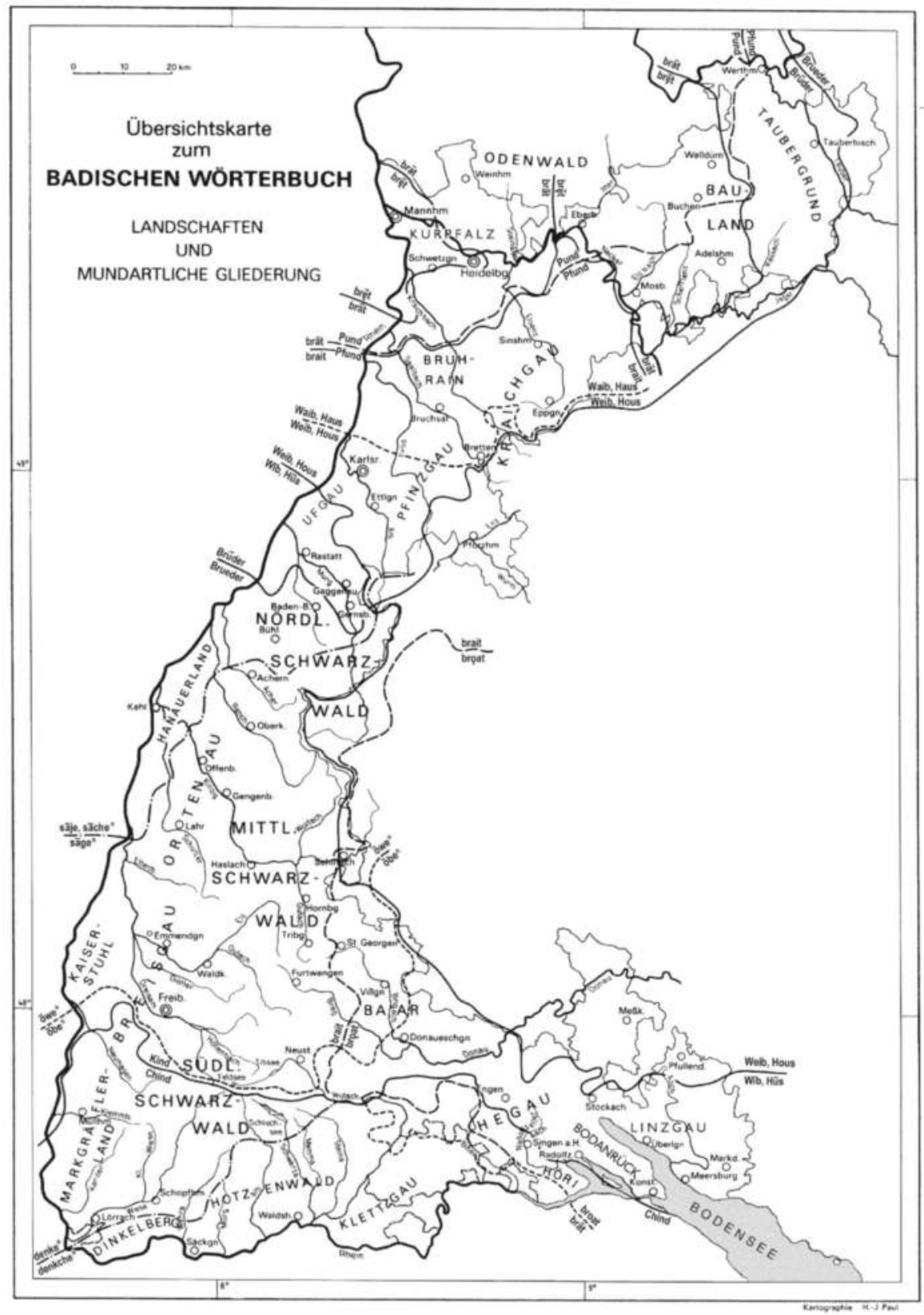

Abb. 1: Karte des Arbeitsgebiets des „Badischen Wörterbuchs" (= politisches Gebiet des ehemaligen Großherzogtums Baden) mit wichtigen Isoglossen und der Lage häufig erwähnter Städte und Landschaften 
Nachdem in den früheren Jahren lediglich studentische Hilfskräfte oder freiwillige Mitarbeiter (wie pensionierte Lehrer) Unterstützung beim Exzerpieren und bei anderen Schreibarbeiten leisteten, wurde 1973 vom Land immerhin eine Sekretariatsbzw. Schreibkraftstelle bewilligt, die auch heute noch - als Halbtagesstelle - vorhanden ist. Weiterhin stehen Mittel für studentische Hilfskräfte zur Verfügung, die in der Regel gerade ausreichen, um ein bis zwei Studierende für einige Stunden in der Woche zu beschäftigen. Damit gilt wohl auch heute noch, was bereits BAUR (1983: 390) schrieb: „Verglichen mit den übrigen deutschsprachigen Wörterbuchkanzleien gehört die unsere zu den personell am schlechtesten ausgestatteten Unternehmen.“ Dass der derzeitige Bearbeiter des „Badischen Wörterbuchs“ bis 2019 ein Lehrdeputat von fünf Semesterwochenstunden zu erfüllen hat, bewirkte außerdem leider einen nur langsamen Fortgang der Publikation neuer Lieferungen.

\subsection{Konzeption und Materialien}

Den Begründern war von Anfang an klar, dass man sich aus Kostengründen auf eine Darstellung der „lebenden Volkssprache“ (BAUR 1976b: 65) beschränken musste. Historischer Wortschatz konnte nur aufgenommen werden, wenn , man ihn in kurzer Zeit mit freiwilligen oder spärlich besoldeten Helfern verarbeiten konnte oder durch besondere Interessen von Mitarbeitern quasi gratis geliefert bekam“ (BAUR 1976b: 65). Letzteres war beispielsweise bei der landschaftlichen Urkundensprache durch die Arbeit von FrIEDRICH WILHELM an seinem „Corpus der altdeutschen Originalurkunden vor 1300“ der Fall. Weiterhin wurden Belege aus den „Oberrheinischen Stadtrechten“ sowie aus Urbaren und Weistümern exzerpiert (vgl. BAUR 1976a: 29) und in Auswahl auch Orts- und Flurnamen, insbesondere von MÜLLER und BAUR, ins Material aufgenommen (vgl. BAUR 1983: 390).

Obwohl man also im „Badischen Wörterbuch“ deutlich weniger Historisches findet als beispielsweise im „Schweizerischen Idiotikon“ oder im „Schwäbischen Wörterbuch", kann auch nicht von einem synchronen Ansatz die Rede sein, da seit Beginn der Materialsammlung inzwischen 125 Jahre vergangen und immer wieder neue Quellen/Belege hinzugekommen sind. Man kann hier somit nicht mehr von einer einheitlichen Mundart sprechen und muss bei der lexikographischen Darstellung den Wortgebrauch möglichst durch Datierungsangaben nachweisen.

Wie bereits in Kapitel 1.1 erwähnt, war man sich früh darüber im Klaren, dass das Material aus der Volkskundeumfrage allein nicht für die Erarbeitung eines Wörterbuchs ausreichen würde. Es wurde daher ein neuer Aufruf zur Mitarbeit mit genauen Anweisungen zur Transkription veröffentlicht, der eine Fülle neuen Materials erbrachte - insbesondere aus dem fränkischen Norden, der 1894 vergleichsweise schwach vertreten war (vgl. BAUR 1976b: 65). Darüber hinaus gelang es, einige verdiente Mundartforscher, wie OtTo HeILIG, PhILIPP LENZ und OTHMAR MEISINGER, dazu zu bewegen, ihre umfangreichen Wortsammlungen in den Fundus des „Badischen Wörterbuchs“ einzubringen und zu dessen Gunsten auf eigene Vorhaben zu verzichten (vgl. BAUR 1976b: 65 sowie BAUR 1983: 387). Die oben genannten, an der Vorbereitung des „Badischen Wörterbuchs“ beteiligten Freiburger 
Germanisten sowie später auch FrIEDRICH MAURER, BRUNO BOESCH und EUGEN GABRIEL regten immer wieder Studierende zum Abfassen von Staatsexamensarbeiten oder Dissertationen über dialektologische Themen (überwiegend im Bereich der Laut- und Formengeographie) an, die schließlich auch für das „Badische Wörterbuch“ lautgetreu transkribierte Belege aus direkter Erhebung erbrachten. In Auswahl wurde auch Mundartliteratur ins Material aufgenommen. Besonders intensiv wurden Werke von JOHANN PETER HeBEl und HERMANN BuRTE ausgewertet, jedoch auch Literatur von OSKAR FURTWÄNGLER, AUGUST GANTHER, KARL GOTTFRIED NADLER und einigen anderen Autorinnen und Autoren.

Mit der Auswertung von Arbeiten zur Wortgeographie, Wortbildung und Syntax, zu einzelnen Fachsprachen bzw. -terminologien (Land- und Waldwirtschaft, Weinbau, verschiedene Handwerke) und zum Rotwelschen sowie von Wörterbüchern zu einzelnen Orten oder Landschaften wurde das Material weiter abgerundet. Seit etwa 20 Jahren bietet sich dem „Badischen Wörterbuch“ zudem die Möglichkeit, auch auf das Fragebuchmaterial des „Südwestdeutschen Sprachatlasses“ in digitaler Form zuzugreifen, was für den südlichen Teil des Arbeitsgebiets oftmals die Belegdichte deutlich erhöht und insbesondere für die Erstellung von Karten von großem Wert ist. Wie schon früh ERNST OCHS, hat später auch GERHARD W. BAUR noch viel selbst exploriert. Mittels eines Fragebogens führte er in 179 Orten in Nord- und Südbaden direkte Erhebungen durch und machte dabei häufig auch zusätzlich noch Tonbandaufnahmen von freien Gesprächen und Erzählungen. Zusammen mit einigen Aufnahmen aus dem Deutschen Spracharchiv können die Tonaufnahmen BAURS viele Spontan- und Satzbelege liefern.

Insgesamt entstammt der größte Teil des Materials den Jahren 1894-1986. Jüngere Belege wurden und werden zwar bisweilen durchaus auch berücksichtigt, allerdings nicht systematisch und in großem Umfang, sondern eher sporadisch, wenn ein bestimmter Wortartikel in den Augen des Bearbeiters inhaltlich davon profitiert.

\subsection{Datenaufbereitungen}

Die aus den in Kapitel 1.2 umrissenen Quellen exzerpierten Belege wurden, wie bei den allermeisten Wörterbuchunternehmen üblich, klassisch verzettelt. Das Zettelmaterial „,im früheren Kanzleiformat (16,5 × 5,5 cm)“ (BAUR 1976a: 27) umfasst insgesamt schätzungsweise zwei Millionen Belegzettel (vgl. Post 2003: 319). Es ist alphabetisch sortiert, in der Arbeitsstelle in hölzernen Bibliotheks-Karteischüben archiviert und Interessierten nach Absprache zugänglich. Die zu großen Teilen handschriftlichen (sehr häufig in Sütterlin- bzw. Kurrentschrift), später auch mit Kopieschnipseln (teils auch Karten etc.) aus den Quellen beklebten oder in jüngerer Zeit in Textverarbeitungsprogrammen erstellten Belegzettel enthalten bestenfalls alle in der jeweiligen Quelle vorhandenen Angaben zum Stichwort. Die Quellen selbst sind überwiegend in der Bibliothek oder dem Archiv der Arbeitsstelle vorhanden. Einige Quellen sind lediglich in anderen Freiburger Bibliotheken verfügbar. Nur in wenigen Ausnahmefällen sind Quellen derzeit nicht ohne Weiteres zugänglich, da sie sich beispielsweise in Archiv-/Privatbesitz befinden. 
In welchem Umfang das Material aus der Volkskundeumfrage verzettelt wurde, ist schwer zu sagen, da man es offenbar in den ersten Jahren des Sammelns versäumte, dies festzuhalten. Es scheint jedenfalls bei weitem nicht alles zu sein (vgl. PosT 2001: 272). Die Originalantworten zu den Fragebögen befinden sich seit vielen Jahren an unterschiedlichen Aufbewahrungsorten (wie es dazu kam, lässt sich heute nicht mehr ohne Weiteres rekonstruieren). Der weitaus größte Teil (aus ca. 400 Orten) ist im Badischen Landesmuseum - Außenstelle Südbaden / Landesstelle für Volkskunde in Staufen im Breisgau archiviert, der kleinere Teil in der Arbeitsstelle „Badisches Wörterbuch“ (aus 112 Orten) und in dem von der Universitätsbibliothek Freiburg verwalteten Nachlass von FRIDRICH PFAFF (aus 18 Orten). Die Wörterbucharbeitsstelle besitzt Kopien der bei der Landesstelle für Volkskunde archivierten Originalantworten und vice versa. ${ }^{3}$

Der Teil des Zettelmaterials, der die Grundlage für den fünften (und zugleich letzten) Band des „Badischen Wörterbuchs“ bildet, wurde in den Jahren 2010 und 2011 gescannt. Die Digitalisate sind im PDF-Format auf einem Server des Freiburger Lehrstuhls für Germanistische Linguistik sowie auf Bandlaufwerken des Rechenzentrums der Universität archiviert und im JPEG-Format über eine im Jahr 2010 speziell für das „Badische Wörterbuch“ entwickelte Online-Datenbank verfügbar (vgl. Kapitel 3). Die auf den Belegzetteln vorhandenen Angaben werden seither (überwiegend von studentischen Hilfskräften) sukzessive von Hand in die Datenbank eingegeben, da der Einsatz einer Texterkennungssoftware bei der enormen Heterogenität des Materials (diverse sehr unterschiedliche [Hand-] Schriften, verschiedenste Transkriptions-/Sonderzeichen usw.) wenig Erfolg verspricht.

Alle Belegzettel wurden vor dem Scanvorgang durchnummeriert und erhielten somit eine eindeutige Identifikationsnummer. Die Eingabemaske der Datenbank bietet für alle Informationen, die die Belegzettel potenziell enthalten, ein eigenes Feld (vgl. Abbildung 2). Weiterhin wird bei der Eingabe durch das Setzen eines Häkchens angegeben, ob es sich um einen Satzbeleg handelt, und ob der Bearbeiter den entsprechenden Zettel unbedingt nochmals anschauen muss (weil beispielsweise unleserliche Angaben oder weitere Informationen etc. auf dem Zettel vorhanden sind). Wie in Abbildung 2 zu erkennen ist, bietet die Eingabemaske darüber hinaus noch die Möglichkeit, etwaige auf den Zetteln vorhandene Verweise auf andere Lemmata (insbesondere Synonyme, Komposita) und auf andere Wörterbücher einzugeben. Außerdem können Anmerkungen verschiedenster Art in ein dafür vorgesehenes Feld eingegeben werden. Bei einer Recherche in der Datenbank sind dann alle diese Felder grundsätzlich durchsuchbar, und Suchergebnisse können anhand der einzelnen Informationstypen in unterschiedlicher Weise sortiert werden (vgl. dazu auch Kapitel 3).

3 Aktuell werden alle Originalantworten zu den Volkskundefragebögen digitalisiert, in „LEOBW“, dem landeskundlichen Informationssystem des Bundeslandes Baden-Württemberg (<https://www.leo-bw.de>), zusammengeführt und öffentlich verfügbar gemacht. 


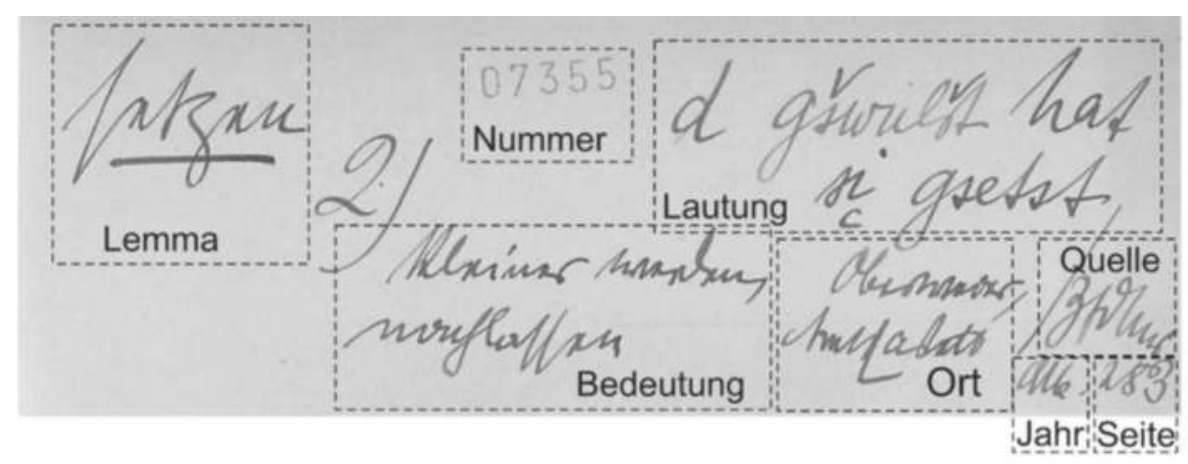

\section{Beleg-Eintrag hinzufügen}
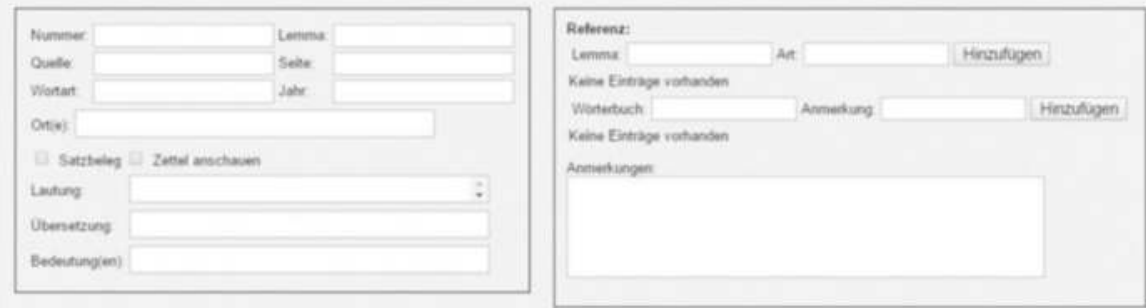

Never Beteg Never Zetel Neves Lemma

Abb. 2: Originalbelegzettel (oben, mit Markierung und Beschriftung der einzelnen Informationen) und Eingabemaske in der Datenbank (unten)

Die in Kapitel 1.2 erwähnte Mundartliteratur wurde nur in Auswahl verzettelt. Für die Auswertung von „Hebels Werke in vier Teilen“, FURTWÄNGLERS „Die Uhrenmacher im Schwefeldobel“", NADLERS ,Fröhlich Palz, Gott erhalts!“ und GANTHERS „Stechpalmen“ wurde ein elektronischer Volltext erstellt, der mit einem von RUDOLF POST entwickelten Programm einfach durchsucht werden kann. Auch für die Arbeit mit den Daten aus den Fragebucherhebungen für den „Südwestdeutschen Sprachatlas" hat RUdOLF POST ein Programm entwickelt, mit dessen Hilfe in dem umfangreichen Material recherchiert werden kann. Mit einem separaten, ebenfalls von POST entwickelten Kartierprogramm können auf der Grundlage dieser und anderer Daten übersichtliche Arbeitskarten erstellt werden.

Den weiter oben bereits genannten Tonbandaufnahmen misst BAUR (1983: 391) einen hohen Stellenwert bei und bedauert, dass ihre Auswertung seinerzeit noch nicht in von ihm erwünschtem Maße erfolgen konnte: „Weil diese relativ junge Quelle zwar viele der in jedem Wörterbuchmaterial selteneren Satzbelege liefern kann, ihre Auswertung aber sehr zeitaufwendig ist, konnte das bisher leider nicht in wünschenswertem Umfang geschehen." Es ist somit sicherlich in BAURS Sinne, dass seine Aufnahmen nun seit einigen Jahren allesamt digitalisiert und transkribiert (in Form einer „Übersetzung“ ins Standarddeutsche, was unter anderem eine einfache Suche in den Transkripten auf Wortebene ermöglicht) sind und 
gemeinsam mit Tonaufnahmen, die im Rahmen der Erhebungen für den Südwestdeutschen Sprachatlas entstanden, in der Online-Datenbank „MOCA“ des Freiburger Lehrstuhls für Germanistische Linguistik verfügbar sind. ${ }^{4}$ Anders als 1983 ist die Suche nach möglichen (Satz-)Belegen in den Tonaufnahmen über eine Recherche in dieser Datenbank heutzutage recht komfortabel und mit einem deutlich geringeren zeitlichen Aufwand verbunden als damals.

\subsection{Publikationen}

Als Vorarbeit für das Wörterbuch und auch für seine Vorschläge zur Gliederung des Alemannischen (OCHS 1921) erarbeitete ERNST OCHS eine „Gliederung der badischen Mundarten“, die 1921 in erster und 1923 in zweiter, erweiterter Auflage erschien und „Proben des Badischen Wörterbuchs“ enthielt (OCHS 1923). Ein Jahr später publizierte OCHS auch in der „Zeitschrift für deutsche Mundarten“ Proben von Wörterbuchartikeln (OCHS 1924), bevor 1925 schließlich die erste Lieferung des „Badischen Wörterbuchs“ erschien. Den ersten Band $(A, B / P, D / T, E)$, der insgesamt 725 Seiten umfasst, konnte OCHS 1942 abschließen. Inzwischen schätzte der Bearbeiter den künftigen Umfang des ursprünglich auf zwei Bände konzipierten Wörterbuchs auf drei Bände. Für OcHS zeichnete sich nun auch ab, dass er das Werk, anders als er lange Zeit dachte, nicht mehr selbst vollenden würde. Bis zu seinem Tod im Jahr 1961 arbeitete er jedoch am zweiten Band und veröffentlichte die Lieferungen 10-26. KARL-FRIEDRICH MÜLLER, der bereits langjähriger ehrenamtlicher Mitarbeiter von OCHS gewesen war, wurde sein Nachfolger und erarbeitete bis zu seiner Pensionierung im Jahr 1968 weitere Lieferungen des zweiten Bandes. MÜLLER wurde von GERHARD W. BAUR abgelöst, der die letzten beiden Lieferungen (33/34) des zweiten Bandes erarbeitete und diesen ( $F / V, G, H / 806$ Seiten) im Jahr 1974 vollendete. Auch der dritte Band (I, J, K, L, M / 712 Seiten) wurde vollständig von BAUR erarbeitet und 1997 mit seiner Pensionierung abgeschlossen. Auf BAUR folgte 1998 RUdOLF POST, ein als früherer Bearbeiter des „Pfälzischen Wörterbuchs“" sehr erfahrener Lexikograph. Er erarbeitete den vierten Band $(N, O$, $Q, R, S a, S c h$ / 806 Seiten), ab Buchstabe $S$ unterstützt von FrIEDEL SCHEERNAHOR, und konnte diesen mit seinem Eintritt in den Ruhestand 2009 abschließen. Nachfolger von POST wurde schließlich TOBIAS STRECK, der aktuelle Bearbeiter des „Badischen Wörterbuchs“. Seit er die Arbeitsstelle leitet, wurde ein neues Redaktionssystem entwickelt und schrittweise ausgebaut (vgl. Kapitel 3). In diesem neuen System wurden bislang die ersten vier Lieferungen des fünften Bandes (Lieferung 82, I se - Seichschupfe, erschienen im Dezember 2012 / Lieferung 83, Seichstande - Sellerie, erschienen im Dezember 2014 / Lieferung 84, Sellerieknollen -

4 Die Digitalisierung und Transkription der Tonaufnahmen aus dem Archiv des „Badischen Wörterbuchs" und ihre Integration in die Datenbank MOCA wurde durch eine für beide Seiten gewinnbringende Kooperation zwischen der Arbeitsstelle „Badisches Wörterbuch“ und dem DFG-Projekt „Phonologischer Wandel am Beispiel der alemannischen Dialekte Südwestdeutschlands im 20. Jahrhundert" (vgl. hierzu zusammenfassend STRECK 2015) ermöglicht. 
siebenunddreißig, erschienen im Juni 2017 / Lieferung 85, siebenundfünfzig - II Sol, erschienen im November 2019) erarbeitet.

Über die lexikographischen Artikel hinaus erschienen bisweilen auch weitere, in der Regel kleinere Publikationen zum Material (z. B. SCHEER-NAHOR 2001). PHILIPP LENZ veröffentlichte bereits kurz nach dem Erscheinen der Lieferung 1 des „Badischen Wörterbuchs“ einige Nachträge zu dieser Lieferung (LENZ 1925/1926). Mit dem Material aus den Erhebungen zur Sammlung der Volksüberlieferungen Badens und dessen Wert für die Dialektologie und das „Badische Wörterbuch“ befasst sich POST (2001). Sondersprachliches im „Badischen Wörterbuch“ wird in POST (2002) beleuchtet, Hebraismen in SCHEER-NAHOR (1998). STRECK (2008) beschäftigt sich mit französischen Entlehnungen in den badischen Mundarten und geht dabei ebenfalls vom Material des „Badischen Wörterbuchs“ aus.

Das „Badische Wörterbuch“ erschien zunächst 1925-1999 im Moritz Schauenburg Verlag (Lahr/Schwarzwald). Nach dessen Liquidation ging es an den R. Oldenbourg Verlag in München über, bei dem es 2000-2012 erschien. Zu einem erneuten Verlagswechsel kam es durch den (rückwirkend zum 1. Januar 2013 vollzogenen) Verkauf des Oldenbourg-Verlags an die Walter de Gruyter GmbH. Seither erscheint das „Badische Wörterbuch“ bei De Gruyter (Berlin/München/Boston).

Für den weiteren Fortgang der Publikation von Lieferungen für den fünften (= letzten) Band gilt auch heute noch - und umso mehr in Anbetracht des im Vergleich zu Mitarbeiterinnen und Mitarbeitern anderer Wörterbuchprojekte hohen Lehrdeputats des aktuellen Bearbeiters (vgl. Kapitel 1.1) - die Einschätzung von BAUR (1976b: 71): „Es läßt sich indessen absehen, daß das Badische Wörterbuch nur mit einem weiteren hauptamtlichen Bearbeiter in vertretbarer Zeit abgeschlossen werden kann $[\ldots]^{“}$.

\section{DIE WÖRTERBUCHARTIKEL: KONZEPTION, STRUKTUR, INHALT}

\subsection{Makrostruktur}

Die Artikel des „Badischen Wörterbuchs“ sind strikt alphabetisch angeordnet. Das Lemma wird in seiner standarddeutschen Form angesetzt. Falls es nur in einer mundartlichen Form existiert, wird für das Stichwort eine neuhochdeutsche Form konstruiert, die „der historischen Entwicklung des mittel- oder althochdeutschen Lautstands in unserem Gebiet entspricht" (BAUR 1983: 391). In einer solchen konstruierten neuhochdeutschen Form wird also zum Beispiel ein im Mundartwort vorkommender Stammvokal, der auf mhd. $\hat{\imath}$ beruht, als <ei> umgesetzt etc.

Von sehr verbreiteten Ausspracheformen eines Wortes wird auf das schriftsprachlich angesetzte Lemma verwiesen, also beispielsweise von Bigi auf Beige (vgl. Abbildung 3, linke Spalte, Zeile 66), von ieme(s) auf jemand, von na auf hinab, hinan und von sett auf selt usw.

Wie bei oberdeutschen Wörterbüchern üblich, wurden auch beim „Badischen Wörterbuch" die lautlich oft schwer zu trennenden Anlaute $b$ und $p, d$ und $t$ sowie 
$f$ und $v$ zusammengenommen; $c$ steht bei $k$ (vgl. dazu auch die Erläuterungen in Band 3, Seite 43). Die Bände 1 und 2 umfassen somit Stichwörter mit den folgenden Anfangsbuchstaben: $A, B / P, D / T, E, F / V, G, H$ (vgl. auch Kapitel 1.4). Im Inlaut ist dagegen das alphabetische Prinzip strikt durchgehalten. Wie Abbildung 3 zeigt, steht also Pietist (linke Spalte, Zeile 21) zwischen Bietingen und Bietnaze; Bilanz (rechte Spalte, Zeile 56) ist zwischen Pikkolo und Pilatus zu finden etc.

In seltenen Fällen wurde aus Platzgründen von der glattalphabetischen Lemmaanordnung mit fett gedrucktem und leicht eingerücktem Lemma abgewichen und stattdessen für kurze Wortstrecken eine nischenalphabetische oder nestartige Anordnung gewählt. Abbildung 4 zeigt auf der linken Seite ein Beispiel aus dem dritten Band, wo eine solche Wortstrecke aus Flurnamen mit dem Bestimmungswort Mühle(n)- besteht. Hier wurde nicht für jeden Flurnamen ein eigener Wortartikel mit eingerücktem Lemma erstellt, sondern diese wurden nischenalphabetisch als Sublemmata eingeordnet. Sie sind zwar auch fett gedruckt, jedoch wurde kein Bindestrich zwischen Bestimmungswort und Grundwort eingefügt.

Auf der rechten Seite zeigt Abbildung 4, wie in Lieferung 84 die Flurnamen auf Sieben- unter II sieben in Gliederungspunkt 3 behandelt wurden. Auch hier wurde also, um Platz zu sparen, zu diesen Stichwörtern nicht jeweils ein eigener Wortartikel erstellt, sondern sie haben jeweils einen eigenen Unter-Gliederungspunkt unter II sieben 3 erhalten. Ein Verweis (siehe Zeile 57) informiert die Benutzer/-innen darüber, dass die Flurnamen dort gesammelt - und ausnahmsweise nicht strikt alphabetisch nach dem Artikel II sieben - zu finden sind.

Der Aufbau der Seiten im Artikelteil entspricht grundsätzlich bis heute weitgehend dem der Beispielseite aus dem ersten Band (Abbildung 3). Es wird ein zweispaltiger Satz mit einer zwischen den beiden Spalten stehenden Zeilennummerierung in Fünferschritten verwendet. Während in den Bänden 1 und 2 die Spalten noch jeweils 72 Zeilen umfassen, enthält seit der Umstellung von Fraktur auf Antiqua (vgl. die Abbildungen 3 und 4) ab Band 3 jede Spalte nur noch 64 Zeilen. Im Kolumnentitel jeder Seite werden innen (d. h. bei geraden Seiten über der rechten Spalte und bei ungeraden Seiten über der linken Spalte) das erste und das letzte auf der jeweiligen Seite behandelte Stichwort und außen die Seitenzahl aufgeführt.

Als Außentexte enthalten die bisher erschienenen Bände neben einem Vorwort des Bearbeiters ein Verzeichnis der Quellen- und Abkürzungen (bzw. Ergänzungen zum entsprechenden Verzeichnis aus dem vorherigen Band) und eine Erklärung bestimmter Schriftzeichen sowie der verwendeten Lautschrift. Die Bände 3 und 4 enthalten außerdem jeweils zwei Übersichtskarten zum Bearbeitungsgebiet. Auch die Lieferungen des fünften Bandes enthalten - jeweils als Teil des Einbandes - die Erklärung der Schriftzeichen und der Lautschrift sowie die oben in Abbildung 1 dargestellte Übersichtskarte. Das Verzeichnis der Quellen- und Abkürzungen wird bei Bedarf ergänzt und ist in seiner jeweils aktuellsten Fassung als PDF-Dokument über die Internetseite der Arbeitsstelle öffentlich zugänglich. 
biet em haim! $\mathrm{cb} .49,37$; fírowä bjäda \& $\mathrm{ttbm}$ (vgl. abbieten 2). I hawan a $p^{c}$ ârmôl dsur rü gabods oberídefft. Er bat gabods bie Bereinser: fammlung angejagt th terỏ wis $5 \mathrm{~m} / 23$ a a g Beb. 578 (ogl. Gebot, umbieten). (5r is bi (a)da gaya, er labet 3um Begräbrtis ein' 9 ( ) ern; vgt. Leich(en)bieterin, Hochzeitbieten, Antlingentbes unter beten 3. - Bithe $1,1106 \mathrm{ff}$.

Bieter m. (2) rung mitbietet; in Ratbausfprade $X X$ g in no neier.

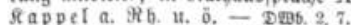

Bietshols i.: jur Eritellung eines $I I$ Biet taugliøes

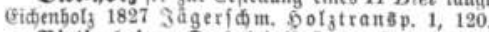

Bietigaheim, Dorf bei Raftatt. Das erfte Slieb berubt auf einem Mănnermamen; vgf, cinerieits Bietingen, anberieits Bödig-heim. - stieger "1, 159. giffer 1, 1108,

Bietingen beibt ein Dorf bei 9Rebtird unb eines (oit Biethingen gefidrieben) weftlic) von Cingen a. 5 . bazu bet jamilienname Biethinger. Bgl. Bietigheim. - Siteger 21, 190.

Bietiît m.: 1) als Settierer ober refigiōer Giferer;

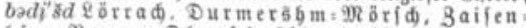
biu, $R$ app.. Oberide fift. Deibefb. (Settaue Sdeibung von Baptist 2 ift niфt möglid. Das solt bentt guveilen an beten, feltener an Bett; in $8 \mathrm{riefen}$.

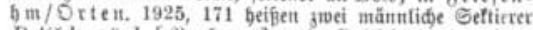
Betisda-päul [नqaul, m.] uno Bettkist(e) w. (ogl.

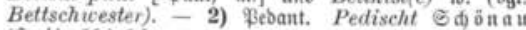
(5)eibelb.) b. - Bifder 1, 1108,

Bietsnaze m.: biadnâdse merben bie Bewobner bes III Biet 2 befonbers vou ben proteftantifíd wâtttem: bergij̄jen Paфbarn genannt. Das zweite SItieb gebört 3u Ignatius. $23 \mathrm{gl}$. Bietschenkel.

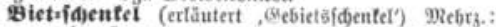
lubername ber qeute von 5obentwart (bas zum III Bief 2 gebört) in \$oude enfelo (bas nidbt zu biejem Biet gebort). BgI. Bietnaze.

Bietung $(=$ Bettung $t+I I$ Biet $)$ w.: eidgene

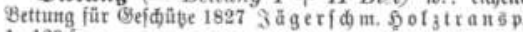
1. $129 \mathrm{f}$.

\footnotetext{
A bieze fiebe baßen.
}

A Biezig-bofen, Dorf bei greiburg. Das erite ङtié berubt auf einem MRämernamen, wobt verwanbt mit Al-biez unb bilßen. - sricger "1, 190.

Bifoet: in beibelb. it bifdek 斤., Beefiteat, ba:

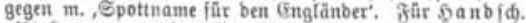
gibt $q e n 34,13$ in beiben Pebeutungen männtides (Me:

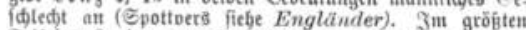
Teit bes Qanbes bertílt bifdek f. in ber eriten Bebeu. tung (fiebe oben $\mathcal{S} .19^{\mathrm{b}} 3.66$ ); in $\Re$ beinbifd. wurbe baraus befdẹk gemã ben bottigen e-Rauten. Bur Bes banblung ber inneren Ronjonantengruppe ogl. obstinat unb franaöf. bifteck, - ๓ा, 2, 17.

bifi, Qtustuf; bif 9adjabmung eines furzen, feinent Sinalls, 3. B. einer 9 inberpiftole oberfde ffit.; bif baf buf bezeidnet ben Flintenfidus E tab r g n. Be: gegnet aud in शlbjäblverien, fiege oben ङ. 108 a 3. 18, äbnlid piff paff draus Tiengen ( 58 a / b 5 b.); anber: roärts eber tick-tack - ediscis. 4, 1038f. Buffer 1, 1508 .

9iñg fiebe Beifang 2

8igamie ift tein Bolfōmort.

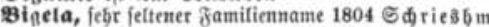
(zugezogen aus os ro $1926,172$.

Bissaber fiebe Bippapper.

8 igi w. fiebe Beige.

Bigument if tein Bolfsmort.

9igäger (m.) Mebrz.: bie Bewobnter von sajel werben im naben Eđđjopfbut i. 23. bigögar genant, ba gegen im naben Dofienbad als Frösch(e) bezeidnet, weil in feudtem Talgrunb wobnent. Der Bogelname Pip.

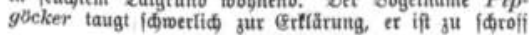

fräntijø. Bielleid)t gebt ber Sopott auf eine auffallenb verbüllenbe Iušfpradje ber Beteuerung bei Gott; vgL. Bigott. - ediveis. 2, 5192178 ?

girgott, Familienname 1925 if reib.; fdon (Enbe 5 bes 16 . Yabrbunberts wirb bier ein Hans Adam Bey. gott ermäbnt. 3 u bei Golt, fiebe oben 5. 139 a 3. 19. Zgl. aud Bigöger. Dagegen finb Bicko unb bas Eิigíd. bigott fernzubaiten. - Miè ह̂teib. 10.

bigott (Figf $\phi$ : : fromm bis zur libertreibung' hat mit

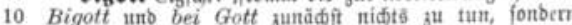
frammt aus bem Jranjōifiden. Srommt in llmgangs ipradje vor; in edter Manbart betig ober heilig, forvie (in sanbid.) bie eingebeutifjte frorm bigóttisch. weinanb $51,237$.

\$ite , bie jweite Farbe im Sartenfpiel foumt unter (afabemifiden) Elatipielern juweilen vor; bafür aud „picus, der Grilnspecht“ (I) 1907 G 1 tb m. Die 9 tumb artipreder fagen Schippen, jumal bei ben cintbeimifijeren Spielent. - wrigant $\$ 2,426$.

$20 \quad R$ bifan fiebe bekanum.

vifant -' Gigíd.: priđelnb. Sommt, meipt auf Speifen bezogen, it ber llmgangs̆prađe oor. Sgl. $p i$. kieren 1. - Ediqanb $52,426$.

Bife w. Ianger Spie $\beta^{\prime}$ ift fein 23olfs̆rnort.

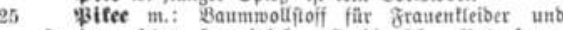

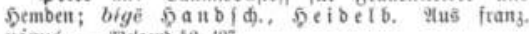
pique. - weigand 12,497

\$ifeesbruit w.: Бembbruịt aนร ßifee; bigêbrust 5) eibetb.

कifeesunterroo m.: Unterrod aus gilfee (f. b.) Biequé-Unnerrock 9 a o let 142 .

Bifett - (f.): beltimmtes Rartenipiel $1907 \mathrm{Ettbm}$ unter Sonoratioren, jelten. Quş franzôf. piquet. (Fì gieidjlautenbes 280 ort ber 5eeresfipradee if uns fremb, bod) 5 fiebe Feldpikett. - Erl. 2, 28.

bifafcin (Eigid. : febr fein; pikfii unb pikfein 1916 Oberw. ( $($ a ft. $)$, neat eingebügett; aus bem Mieber: beutjøen. - stuge "s71.

vifieren (aแ franjô. piquer; meift im \$artizip)

40 fđrv. : 1) naseweise Wildente, biggirt uf Extra-Speise ,erpidt, begierig' (Eid) r o bt ${ }^{2} 124$. Bgl, pikant, - 2) pikitrt beteibigt Eenatird bf., şeibelb. 23gl. II Piek. -3) wirtlid biegungsiābig ift bas Wort in Jadipraden: a) Edneiber(imnen) pikieren einen $s$ ragen u. brgl. fie

45 tẳben mit gleiđmäsigen Ėtiđen Qeinmanb bort aul, wo

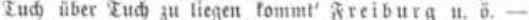
b) in Särtnerfprade: mit Bãngden ober ingetipizen gant junge, bidt febente Sälinge ausbeben unb fie lidgter fetsen $6 \mathrm{ttb} \mathrm{m}$ u. вे. Eolde (3uveilen mebruals

50 pifierte) Erblinge werben bann teurer verfauft und find

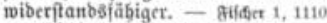

giftolo f.: bie ģidelflöte. Biggolo 8. Berner 9. 12; bigala, 9ebra. bigali oberiøeff. \&ub bem

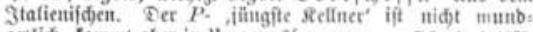
55 artid, tommit aber in llimgangsfpr. nor. - edfweis. 4 . 1079, gilaus w. feblt 1898 a n b f d.; if jetst burd Borfdubvereine uno Beitungen in victen Ortent belannt. - o. esfuts frembiob. i. $84 \mathrm{f}$.

\$itlatus m.: ,eine afte 28 etterregel fagt: Pilatus 60 geht nicht aus der Kirche, er richtet dann zuvor einen Spektakel an" Of derbote $19229 \mathrm{r}$. 85 . (Me: meint if bie flürmifde gsitterung vor Dptert; ber Beitumgs: fdoreiber vermutet, bais $P$ - eingetreten fei für Donar ober

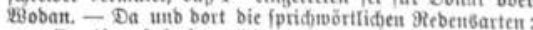

65 von Pontius [-ds-] zu Pilatus schicken; er ist dazu gekommen wie der Pontius ins Kredo. - Sdivets. 4. 1108. Edfweiger Botrathube 1984, 59

Bild) feblt. Siebe Siebenschldfer.

3iro bild (verfleinert bildle) $\mathrm{z}$ a uberbif fauptteil bes ganbes bild (ober bilt, bil'd), \&. 8. in

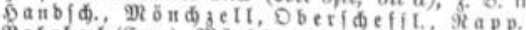

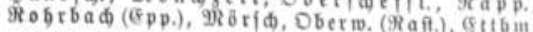

Abb. 3: Glattalphabetische Lemmaanordnung und gemeinsame Behandlung von $\mathrm{B}$ und $\mathrm{P}$ im Anlaut (Badisches Wörterbuch, Band 1: 227) 
Mühle(n)-stein mibldoi t 932 Seeb. (Wolfach); Miili15 schdià' Schonach, Gremmelsb. (neben Lindèschdài) m.: ,das Mühlespicl Fleig 104. Vgl. Mable( $n$ )spiel; Mablstein

Mūhle(n)straße f: FIN; milisidrōs Müllhm/W. Fi. scher 170; 1555 Milistraß Singen a.H. $N$. Schreiber

$20 \mathrm{Zw} .255$. - Fischer 4, 1789; Pfillz 4, 1453.- Mühle(n)tal n.: FIN; milidal, 1341 in múlital Wasenwlr/Roos 370. - Fischer 4, 1790. - Mühle(n)teich m., n.: FlN; miladiz Grieshm; 1496 dq milly tbúds Müllhm/W. Fischer 171. Wohl beides zu Deich, Teidh 2 Stellfalle'. -

25 Pfaly. 4, 1453; Südhess. 4, 806, - Mühle(n)tor n.: FIN; 1357 bi dem mílitor Radolf2./Hegau-Flurn. 7,53. Fischer 4, 1790. - Mühl(en)wasen m. FIN; 1344 muili alasen Freib.-Uffhsn, Wendlgn/Roos 370; 1476 míluasen Tengn/eb. - Fischer 4, 1790 . Mühl(en)-

30 wasser n.: FiN; 1327 muli masser Freib.-Uffhsn, Wendlgn/Roos 370f.; 1341 milt wasser Mundgn/ eb.; 145t míl/wasser Eichstet./cb.; 1494 mulle wasser Bötzgn/cb. - Mühle(n)weg m.: FIN; mehye, 1426 am maileacg Diershm/Bad. Flurn. 1 6, 32; 1772 Mableweg

35 Freib./Bad. Flurn. 1 3, 170; 1315 obe dem miliavere Munzgn/Roos 371; miliwaig, 1327 muliavg Tiengen (Freib.)/eb.; $134^{1}$ milit uxg Mengen/eb.; miliwä, 1368 múlinug Mundgn/eb.; 1423 miile arg Ebrgn/ eb.; 1429 aff den mályarg Schallst./eb.; muilinäg, 1495

40 milh weg Singen a.H. W. Schreiber Zw. 255; milnueg, 1618 vff der Millin avg Orsgn/Hegau-Flurn. 1, 41; millinajg Ebrgn/Hegau-Flurn. 4, 66. - Vgl. Mählueg. - Fischer 4, 1790, 1802; Sudhess. 4, 806. - Mühlenweingarten m. FIN; 1755 Müblenweingarth $\mathrm{Handsch./Bad.}$

45 Flurn. III 4,54, - Vgl. Fischer 4, 1790, - Mühle(n)wiese f: FN; 1561 ob der Meilly ars Hilzgn/E. Schneider Hilz, 165: 1644 br der Miblin $W_{\gamma} \beta$ Singen a.H. W. Schreiber Zw, $255 f ; 1749$ zw, der Mäblin ais Orsgn/ Hegau-Flurn. 1, 41; 1781 unten an die Mible wis $\mathrm{Böh}$ -

$50 \mathrm{rgn} / \mathrm{Hegau}-F l u r n .7,82 . \mathrm{Vgl}$. Mablenmatte, Maiblaie se(n). - Mühle(n)wuhr n: FIN; 1560 bei dem Mälin wuber Freib./Bad. Flurn. I 3, 170. Zu II'wer, Wehr', Fischer 4, 1790, - Mühle(n)zelgle n.: FIN; is müb tsprgli, 1693 in dew Mille Zelgle Singen a.H. NW. Schreiber

55 Zw, 256; militselgli, 1778 in Mülli Zelgle Riclasgn/ Hegau-Flurn, 2, 45, - Mühlenzinsgut n.: FIN; 1724 so genandten Millin Zinsguetios Singen a.H. W. Schreiber Zw. 256. - Mühle(n)zwingel m.: FIN?; Mühlen. graben'?; nengs on de Mieblezainge/ Ettlgn/Mein Hei-

60 matl. 3, 21 of. Wohl zu $\rightarrow$ Zaingel, Raum zw, äußerer Stadtmauer und Graben:. Vgl. Zuing(els)mäble. - Mühlereben $\mathrm{Pl}$., f: FIN; XIX $M$-Steinach/Bad. Flurn. III 3. 79 .

Mühle-roß milìnes Saig - Pl., n.: ,hölzerne Böcke aus

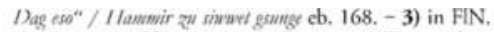
15 a) Sirbenduirken (PI.), d siebe bine Sruinach; Wiesengelände beim $\rightarrow$ Rinkeisenbübl, auf dem früher sieben Birken standen BAD. FLURes. III, 3, 94. - b) Siebenbnumucmbäidh (n.), mu. sfigabranghasli MÜNCHW./SCHWENDEMANN

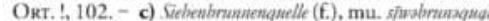

20 Müncinw,/Scarwendemann Ort. 1, 102, - d) Sieben Fideu (PI.), mu. splo diso Mourum; Aussichtspunkt, an dem eine Gruppe alter Eichen steht W. Fischer 199; vgl,

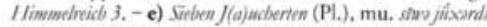

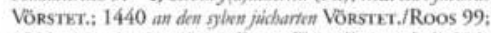

251808 Adker bri den 7 Jauch, Freis,/BAD. FurN, I, 3, 230; 1833 in den 7 Jasib, oder in Rothabfeld eb.; beruht auf dem alten Flächenmaß - Juschert. - f) Fieben Raine (PL). mu. siva nai Eıcuster.; simy nai NimBUre; 1287 silhen mi-

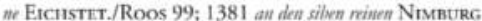

30 cb.; vgl. Rain 4a. - g) Sïlennänkel(surg (m.), amtl. Sïben-

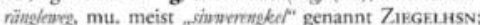
Waldpfad mit vielen Windungen BAD. Fwren. III, 6, 61; $\mathrm{zu} \rightarrow$ Ramk I, Wegbiegung, Kurve, Kehre: - h) Siebentogmain (m.) s, u. - Tagavm 2d. - i) Fichomarg (PL.), mu,

35 sima nư̆ Handsch.; Wegstern, -kreuzung Endin/E. H.

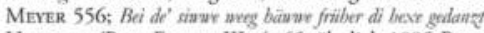
Handscit./Bad. Fuvrex. III, 4, 65, ähnlich 1895 BarI.GN/UMFr. - Ahd. sibun, mhd. silven, aus germ. "sebsus. Weiteres (in Auswahl) $\rightarrow$ Ablinch 1 , abmaschiern, Pfingst-

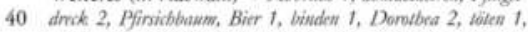

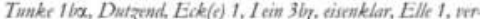
sclblagen 4a, remundt 2, Flab 1, rormittag, Gatgrn 1, gesdieit I.

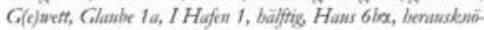

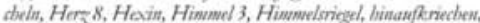

45 Kessellogern, Kind 1a, I Krupp 1, Knuz 1, 3 , Knopf 1a, Kubdrecte, -sebuanz 1 , III I app, I aus 1,1 ege Ia, I sute 1a, I I jicht 16 , liederlicb 2, Linse 1, Lippel 1, I Mas Ta. Maul 2a, Maus 1. Metwer 1, Monsen 2, nomerns, madb 12a, Nadib, Narm 2, nase avis(ive), oder 1a, I Rabe 1, Regenuetter 1, Renmer ta, Rock 3, I

50 Rog 1, Rübe ta, Rupffigel, Sark. Ta, Samstay I, II Saum, Schef

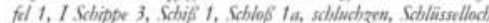

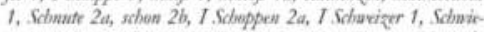
gerieute, Sergast, I sein 2d, selist 2, spanisch, Uhr, ungefithr, vgl,

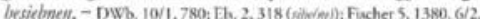

55 3112: PAilz, 6, 96: Schweiz, 7, 46: SDS II, 39. III, 248: SSA II/37.03. 11/170.02. IV/2.04: Scidhess. 5, 1018.

Sieben- in FIN s. $\rightarrow / /$ sieben 3.

Siebenbrunnen-birne $\mathrm{f}: \mathrm{PAN}$; eine früher häufige (Most-) Birnensorte', eig, $\rightarrow$ Kuaushinue A. WEBER OBsr.

60 48. - Laut A. Weber OBst. 48 ist die Birnensorte ein guter Saftspender, weshalb sie auch als Fassfiller bez. wird. Der Name $S$, beruhe darauf, dass sic nach volkstümlichem Glauben so viel Saft wie sieben Brunnen liefere, Vgl. Binumenbinte, - Fischer 6/2, 3112 (Eilmainumemiir).

Abb. 4: Abweichungen von der ansonsten glattalphabetischen Lemmaanordnung (links: Band 3, Seite 683 / rechts: Band 5/Lieferung 84, Seite 94)

\subsection{Mikrostruktur}

Jeder Wortartikel beginnt mit dem optisch leicht eingerückten und grundsätzlich voll ausgeschriebenen Stichwort in Fettdruck. Bei Komposita mit klar segmentierbarem Grund- und Bestimmungswort wird an der Wortfuge ein Bindestrich gesetzt, bei drei- oder mehrgliedrigen Komposita nur an der Hauptfuge. Mehrere Bindestriche kommen lediglich bei Fügungen wie Hopf-und-sitz, nach-der-hand oder Seppwie-meinsch zum Einsatz. Auch Präfixe werden durch Bindestriche abgetrennt, Suffixe dagegen nicht (mit Ausnahme von -bar, -lei und -lecht). Im Lemma können 
einzelne Buchstaben eingeklammert sein, wenn das Stichwort sowohl mit als auch ohne diese angesetzt werden kann, z. B. Seng-(n)essel, serbel(e)n, Seufz(g)er.

Seiler sälar Tauberbisch., WölchgN; sōlar Buch A. Ahorn, Riedhm (Konst.); sẹtar Hettgn; sëlar WERT-

15 HM, Schwetzgn, Billighm, Philippsburg; sãilp Rapp; sailv MÖrsch; sailr MÜNCHw.; sãilr REUTE (ЕMM.); seiler Elzach, Freib., Waldshut; sailor Schiltach, Schwerzen, Meersburg; spalar Schiltach, Gutmadgn, RaDOLFZ; sp̄plar MÖHRGN; soalor SINGEN A. H., Stockacher

20 Gegend, Reichenau; soelar Überl.gn a. R.; spelar Konst; sp̄alar Mimmenhsn; Pl.: sp̣̆lar BuCh A. AHORN. - m.: 1) ,Berufsbezeichnung für den Handwerker, der Hanfseile und Seilerwaren aller Art herstellt' Platz 295, Meis. WB. 147b, 1978 Schiltach, Schwendemann OrT. !fi,

25 57, Kramer Gutmadgn 275, Kirner 366, 1948 MimMENHSN, Orten. 1957, 215, ZfoMu. 1920, 103. 104. In Neuenburg A. RH. gehörten die Seiler gemäß des 1705 beginnenden Zunftbuches zur Zunft zum Riesen (vgl. Neuenb. Stadtr. XXVII). Zu den weiteren An-

30 gehörigen dieser Zunft s. $\rightarrow$ Reblente 1. 's Sailers Rädli springt; / loos, der Vogel singt HeBEL 46, 36; Ra.: arma Spjlar, armer Mensch' Kramer Gutmadgn 275; 's I jectit brennt fïr die arme Seiler, no kei Hanf bän, das Licht brennt überflüssigerweise ${ }^{4}$ MARKGR. 1993, 133. - 2) übertr.:

35 ,einer, der das Geschäft in die Länge zieht und doch rechtzeitig fertig wird' ELLENBAST 67. - 3) FN, bes. in Mittel- und Nordbaden verbr., vgl. KLAUSManN FN 62 u. Kt. 22. - Mhd. seiler. - Weiteres $\rightarrow$ arm 4a, I Buckel 5, Tocbter 2c, Läufer 4d; vgl. Lienen-, Magdalenen-, Wurfseiler;

40 vgl. Nestler, Seilmacber, Strickler. - DWb. 10/1, 221; Fischer 5 , 1328. 6/2, 3096; Pfalz 6, 39; Schweiz. 7, 761; Südhess. 5, 971.

Abb. 5: Der Artikel Seiler im „Badischen Wörterbuch“ (Band 5/Lieferung 83, Seite 42)

Dem Lemma können Hinweise zur Diachronie, Diatopik, Diastratik und Homonymik (I, II usw.) vorangestellt sein. Ist ein Stichwort nur historisch belegt (1850 und früher), wird dies mit einem Kreuz $(\dagger)$ gekennzeichnet. Wörter, die aufgrund ihrer Aussprache fränkisch wirken und nur im Norden des Arbeitsgebiets vorkommen,

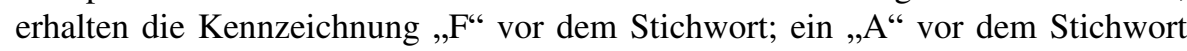
bedeutet, dass es sich um ein Wort handelt, das aufgrund seiner Aussprache alemannisch wirkt und nur für den Süden belegt ist. Rotwelsche Wörter werden durch ein „R“ vor dem Lemma markiert (vgl. Abbildung 3).

Auf das Stichwort folgt der so genannte „Lautkopf“. Dieser Artikelteil besteht aus den wichtigsten, in kursiver Schrift gedruckten Aussprachevarianten in phonetischer Transkription (nach Teuthonista, etwas vereinfacht) mit genauer Orts- oder einer Gebietsangabe, in jüngerer Zeit einheitlich in nordsüdlicher Reihenfolge. Nach den Angaben zur Grundform können ggf. die Aussprachevarianten flektierter Formen folgen. Die Ortsangaben wurden bis einschließlich Band 4 gesperrt gedruckt, seit Beginn des fünften Bandes werden sie in Kapitälchen gesetzt (siehe Abbildung 5, vgl. auch Abbildung 4). Bei ausschließlich historischen Belegen, oder wenn Angaben zur Aussprache fehlen bzw. nur in einer zweifelhaften Transkription vorliegen, entfällt der Lautkopf. Dies gilt in der Regel auch, wenn das Stichwort 
nur in einem einzigen Syntagma belegt ist, das später im Artikel noch als Beispiel gebracht wird.

Der nun folgende Grammatikteil besteht aus der Kennzeichnung der jeweiligen Wortart/grammatischen Kategorie des Stichworts. Bei Substantiven erfolgt dies durch die Angabe des Genus (in abgekürzter Form: m., f., n.), bei Verben durch „st.“ (= starkes Verb) oder „schw.“ (= schwaches Verb). Die anderen Wortarten werden überwiegend ebenfalls in abgekürzter Form angegeben, also „Adj.“ (= Adjektiv), ,Adv." (= Adverb) usw. ${ }^{5}$

Anschließend beginnt der Hauptteil, der die Darstellung der Bedeutung(en) enthält. Sind mehrere klar unterscheidbare Bedeutungen vorhanden, dann ist der Hauptteil semantisch - bei Synsemantika nach formalen oder grammatischen Kriterien - untergliedert. Für die Gliederung werden zunächst arabische Zahlen, für die weitere Untergliederung dann lateinische Buchstaben und - falls eine noch feinere Untergliederung notwendig ist - anschließend griechische Buchstaben verwendet. Die Wortbedeutung wird als definitorische Worterklärung (in einfachen Anführungszeichen) oder mittels klassifizierender Angaben (wie z. B. ,,verwunderter Ausruf") beschrieben und durch die Angabe von Quellen und/oder Orten (oft mit Datierung) belegt. Die Quellen- und Ortsangaben werden seit Beginn des fünften Bandes in Kapitälchen gedruckt (bis einschließlich Band 4 Sperrdruck). ${ }^{6}$ Wenn möglich, wird dann bei jeder Bedeutungsangabe der Wortgebrauch durch kursiv gedruckte Verwendungsbeispiele aus dem Material illustriert. Hier stehen zuerst historische Belege (chronologisch sortiert), dann Fügungen, Wendungen und Sätze, anschließend Redensarten und Vergleiche, Sprichwörter, Rätsel, Reime und Lieder sowie ggf. Informationen zu Volksbräuchen, Sitten etc.

Dem Darstellungsteil folgt der Etymologieteil mit Angaben zur Herkunft, Bedeutungsgeschichte und/oder Wortbildung. Im einfachsten Fall kann im Etymologieteil auch lediglich die mittelhochdeutsche Form des Stichworts aufgeführt werden (vgl. Abbildung 5). Bei Wortbestandteilen, deren Etymologie oder Wortbildung beim jeweiligen Grundwort behandelt wird, erfolgt ein entsprechender Verweis.

Da die Verwendungsbeispiele/Satzbelege aus Platzgründen möglichst nur einmal aufgeführt werden, wird in dem auf den Etymologieteil folgenden Verweisteil (mittels ,Weiteres $\rightarrow$ “) auf andere Artikel verwiesen, die einen Beispielsatz enthalten, in dem das im aktuellen Artikel behandelte Wort ebenfalls vorkommt. Im Verweisteil werden auch die Zusammensetzungen genannt, die auf das betreffende Wort enden; außerdem wird auf synonyme oder bedeutungsähnliche bzw. mit dem aktuellen Stichwort verwandte Wörter verwiesen (vgl. Abbildung 5).

Am Schluss eines jeden Artikels stehen schließlich Verweise auf die Nachbarwörterbücher und ggf. Sprachatlanten, in denen das aktuelle Stichwort ebenfalls

5 In Band 1 und Band 2 wurde statt „f.“ noch „w.“ (= weiblich) und statt „n.“ noch ,s.“ (= sächlich) verwendet. Auch bei anderen Wortarten weichen die Bezeichnungen in den ersten beiden Bänden teilweise ab, z. B. beim Adjektiv, das damals in den Wortartikeln noch als „Eigsch.“ (= Eigenschaftswort) bezeichnet wurde.

6 Die Gründe für diese typographische Umstellung werden in Kapitel 3 erläutert. 
behandelt wird, sowie in der Regel auf das GRIMMsche „Deutsche Wörterbuch“ als umfassendes gemeindeutsches Wörterbuch. Die Nennung der Nachbarwörterbücher liefert den Leserinnen und Lesern auch wichtige Informationen über die regionale Einbettung des behandelten Wortes innerhalb der Nachbardialekte. Kommt das Stichwort beispielsweise in allen Nachbarwörterbüchern vor, spricht dies für eine sehr weite Verbreitung. Wird es allerdings nur bei den nördlichen Nachbarn („Pfälzisches Wörterbuch“ und „Südhessisches Wörterbuch“) behandelt, deutet das auf ein Wort hin, das vor allem in fränkischen Dialekten gebräuchlich ist. Umgekehrt spricht zum Beispiel bei einem Wort, das in den beiden eben genannten Wörterbüchern nicht vorkommt, dafür aber sowohl im „Wörterbuch der elsässischen Mundarten“ als auch im „Schwäbischen Wörterbuch“ und im „Schweizerischen Idiotikon", vieles für eine gesamtalemannische Verbreitung dieses Wortes. Auch Hinweise auf eine kleinräumigere Verbreitung können diese Verweise liefern: Gibt es bei einem Lemma im „Badischen Wörterbuch“ beispielsweise nur Belege aus dem äußersten Südwesten des Arbeitsgebiets, und ist das Stichwort außer hier nur im „Schweizerischen Idiotikon“ belegt, so ist eine rein hochalemannische Verbreitung des Wortes damit zwar noch nicht bewiesen, jedoch sehr wahrscheinlich.

\section{TEXTTECHNOLOGISCHE ASPEKTE}

Die ersten Bearbeiter des Wörterbuchs schrieben die Wortartikel noch ausschließlich von Hand. KARL FRIEDRICH MÜLLER und anfangs auch GERHARD W. BAUR tippten ihre handschriftlich verfassten Artikel dann noch auf ,DIN-A5-Zettel in eine Schreibmaschine mit eingebauten Sonderzeichen“ (BAUR / SCHEER 1998: 265). Erst als die Arbeitsstelle 1973 eine Schreibangestellten-Stelle zugewiesen bekam, änderte sich das. Fortan wurde die Übertragung der weiterhin handschriftlich verfassten Wortartikel in ein maschinenschriftliches Manuskript von der Schreibangestellten übernommen.

Ende 1985 hielt der Computer Einzug in der Arbeitsstelle, „zunächst hauptsächlich als bequemes Schreibgerät" (BAUR / SCHEER 1998: 266). Trotzdem mussten auf den Ausdrucken der am Computer abgetippten Manuskripte noch Diakritika und Zeichen wie Schwa oder gestürztes $a$ von Hand eingesetzt werden. Einige Jahre später erfolgte ein Softwareumstieg von Word auf WordPerfect, das gute Möglichkeiten bot, Zeichenkombinationen zu erstellen. Für alle Sonderzeichen bzw. Zeichenkombinationen wurden Tastenkombinationen festgelegt, mittels derer das entsprechende Zeichen aus einem für die Wörterbucharbeit angepassten Zeichensatz in das Dokument eingefügt oder ein Makro aufgerufen wurde, das die entsprechende Zeichenkombination erstellte. Seit Mitte des Jahres 1990 war es schließlich möglich, dass aus BAURS weiterhin handschriftlich verfassten Wortartikeln über deren Eingabe in WordPerfect am Ende eine Postscript-Datei erstellt werden konnte, so dass dem Verlag das Manuskript fortan auf Diskette geliefert werden konnte. Das beschleunigte für den Verlag die Herstellung der jeweiligen Lieferung, wobei aufwändige Korrekturarbeiten dennoch nicht ausblieben (vgl. BAUR / SCHEER 1998). 
Neben diesen Aufgaben wurde der Computer unter der Leitung BAURS für die Erstellung einer elektronischen Lemmaliste, die bis heute vielfältige Suchmöglichkeiten im Rahmen der lexikographischen Arbeit gewährleistet, sowie für die Erstellung und Bearbeitung einer elektronischen Version des Quellenverzeichnisses des „Badischen Wörterbuchs“ genutzt (vgl. BAUR / SCHEER 1998: 271).

Deutlich ausgeweitet wurde der Computereinsatz, als RUdOLF Post 1998 die Leitung der Arbeitsstelle übernahm. Er hatte bereits für seine Arbeit am „Pfälzischen Wörterbuch“ einige Programme selbst geschrieben (vgl. Post 1998) und entwickelte nun auch für die Arbeit am „Badischen Wörterbuch“ verschiedene Hilfsprogramme. Diese ermöglichen eine komfortable Suche in der elektronischen Lemmaliste, die Erstellung einfacher Sprachkarten (vgl. auch Kapitel 1.3) sowie Recherchen in einem Korpus digitalisierter Mundartliteratur (vgl. ebenfalls oben Kapitel 1.3) und in Fragebüchern bzw. Kartenverzeichnissen von Sprach- und Volkskundeatlanten. PosT war auch der erste Bearbeiter, der die Wortartikel für das „Badische Wörterbuch" nicht mehr von Hand, sondern direkt am Computer - weiterhin in WordPerfect - verfasste. Darüber hinaus initiierte Post, der die Arbeit mit einem elektronischen Volltext bereits vom „Pfälzischen Wörterbuch“ kannte (vgl. PosT 1998: 215), eine Retrodigitalisierung aller nicht digital vorliegenden Wörterbuchlieferungen, d. h. der Bände 1 und 2 sowie rund die Hälfte des dritten Bandes. Mangels anderer Möglichkeiten und finanzieller Mittel schrieben studentische Hilfskräfte diese sicherlich mindestens 1500 Wörterbuchseiten in jahrelanger Arbeit bis ins Jahr 2011 Lieferung für Lieferung am Computer ab. Mithilfe eines Makros konnte der retrodigitalisierte Text in ein universales Format (ANSI-Text, in dem alle Sonderzeichen und Schriftauszeichnungen codiert sind) konvertiert werden, das auch andere Programme als WordPerfect verarbeiten können. Auf dieses Textformat greift insbesondere ein ebenfalls von RUDOLF POST geschriebenes VolltextSuchprogramm zu, das vielfältige Suchmöglichkeiten bietet und von großem Nutzen bei der lexikographischen Arbeit ist.

Nach dem Abschluss des vierten Bandes und dem Ausscheiden von RudoLF PosT im Jahr 2009 stellte sich allmählich die Frage, ob wohl auch noch der fünfte Band mithilfe von WordPerfect erarbeitet werden kann. Verwendet wurde damals die Version 8.0 (aktuell wäre 2009 die Version 14.0 gewesen), da die zahlreichen Makros usw. auf diese Version zugeschnitten waren. Auf einen Umstieg auf eine neuere Version von WordPerfect war bis dahin verzichtet worden, weil Versuche gezeigt hatten, dass die Makros usw. nicht kompatibel waren. Sie hätten somit alle neu programmiert werden müssen. Nun lief allerdings, wie sich zu Beginn der Arbeiten am fünften Band bald zeigte, WordPerfect 8.0 bereits unter Windows 7 nicht mehr völlig stabil. Da WordPerfect - ein in den 1990er-Jahren noch sehr verbreitetes und anerkanntes Textverarbeitungsprogramm - zwar von der Firma Corel noch vertrieben wurde, jedoch im Vergleich zu früher inzwischen von Konkurrenzprodukten weitgehend vom Markt verdrängt worden war, stellte sich die Frage, ob ein Wechsel zu einer neueren Version sinnvoll ist. Deshalb - und zusätzlich auch aus dem Bedürfnis nach einer Sicherung des Belegmaterials für den fünften Band wurde über einen Umstieg auf ein völlig anderes Redaktionssystem auf Datenbankbasis nachgedacht. 
Nach vielen Recherchen, Gesprächen und Kostenabschätzungen gelang es im Laufe des Jahres 2010, diese Idee zu verwirklichen. Sie wurde von ULI HELD, der für einige Monate als geprüfte Hilfskraft beschäftigt werden konnte, in Form einer Onlinedatenbank, die überwiegend auf OpenSource-Software basiert, technisch umgesetzt. In diese Datenbank werden seither sukzessive alle Informationen der Belegzettel für den fünften Band eingegeben (vgl. Kapitel 1.3). Um die Eingabe zu erleichtern, ist in der Datenbank eine Liste aller Orte und aller Quellen - jeweils mit korrekter Abkürzung, wie sie im Wörterbuch verwendet wird - sowie eine (für die noch zu bearbeitende Wortstrecke vorläufige) Lemmaliste hinterlegt. Für die Eingabe von Lautschrift/Sonderzeichen wurde mit der Software KbdEdit ein spezielles Tastaturlayout erstellt.

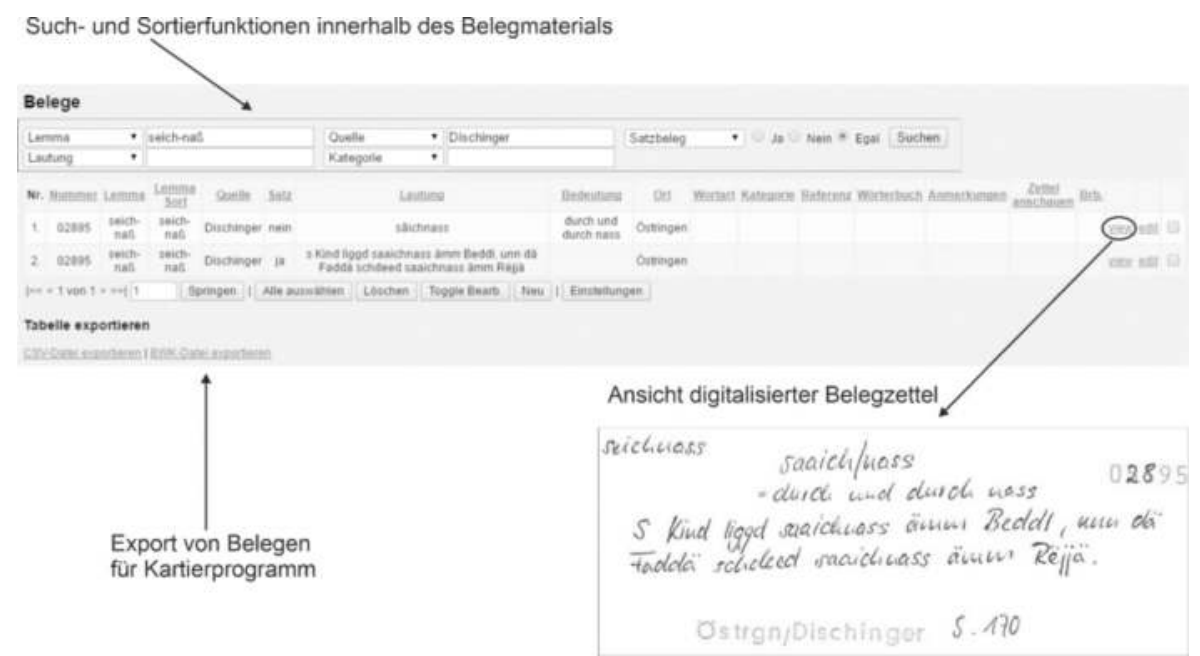

Abb. 6: Belegsuche in der Datenbank des „Badischen Wörterbuchs“ mit der Möglichkeit, ein Digitalisat des entsprechenden Belegzettels anzuzeigen

Die Datenbank bietet diverse Such- und Sortiermöglichkeiten innerhalb des Belegmaterials, verfügt über eine Funktion, mit der Belege für das Kartierprogramm exportiert werden können, und auch die Belegzettel selbst können in der Datenbank als Bilddateien angesehen werden (vgl. Abbildung 6 und Kapitel 1.3). Überdies beinhaltet sie einen Texteditor, in dem die Wortartikel erstellt werden. Beim Verfassen von Artikeln wird eine einfache Form der Textauszeichnung mittels einiger weniger selbst definierter Tags (wie z. B. , , $\{\mathrm{f}\}$ “ für fett gedruckten Text oder ,, $\{\mathrm{k}\}$ “ für kursiv gedruckten Text, vgl. dazu auch Abbildung 8) verwendet, was eine Konvertierung des Wörterbuchtexts in verschiedene Formate gewährleistet. Neben dem Texteditor ist immer eine - für das Auge besser lesbare - Vorschau des formatierten Artikels zu sehen (vgl. Abbildung 7 und Abbildung 8). Oberhalb des Texteditors werden die für das ausgewählte Lemma vorhandenen Belege angezeigt (vgl. Abbildung 7). Sie können in diesem Bereich der Datenbank ebenfalls anhand 
verschiedener Kriterien durchsucht und sortiert sowie auch kategorisiert werden. Außerdem besteht auch hier eine direkte Zugriffsmöglichkeit auf die digitalisierten Belegzettel (über ,view“, vgl. Abbildung 6).

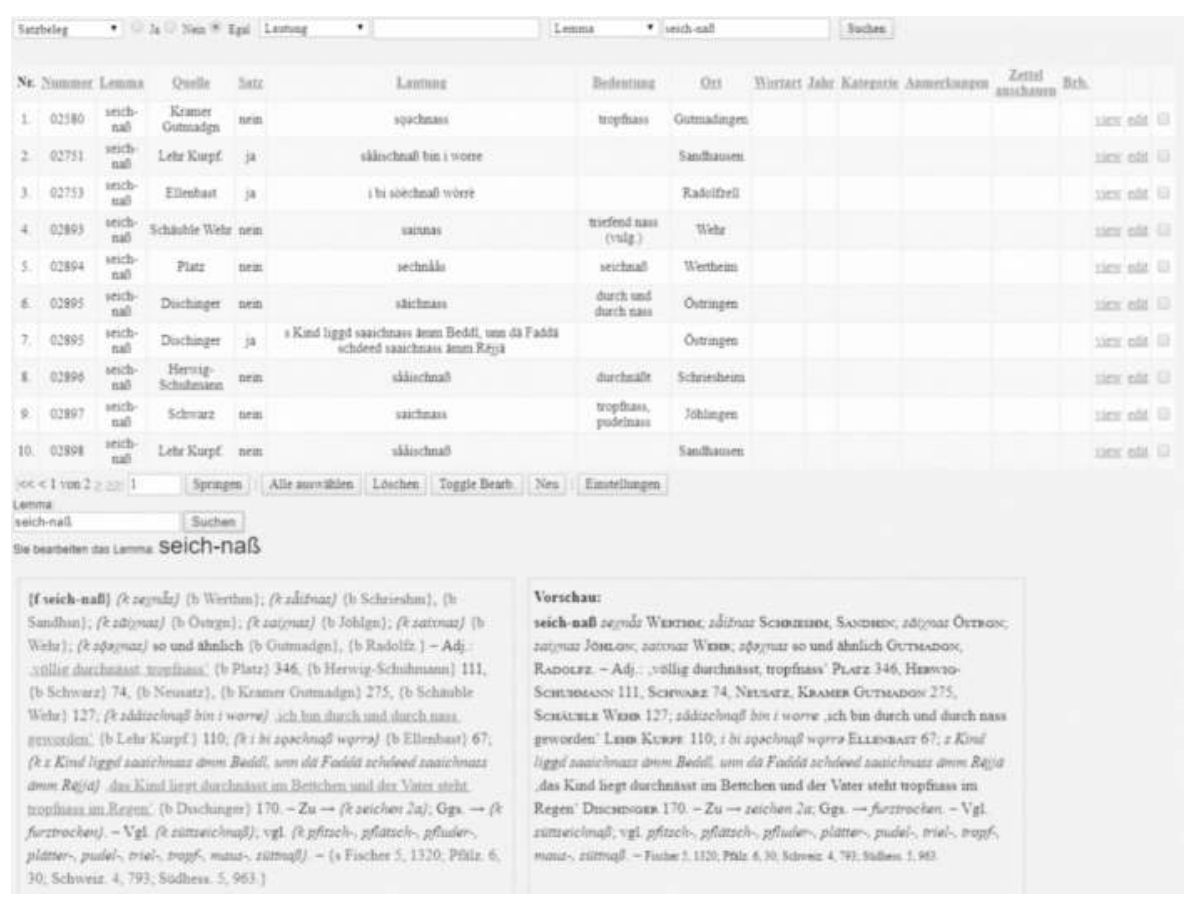

Abb. 7: Belegansicht (oben) und Artikeleditor (unten links) mit Vorschaufunktion (unten rechts) in der Datenbank des „Badischen Wörterbuchs“

Wie in Abbildung 8 (links unten) zu sehen ist, werden die in der Datenbank verfassten Artikel direkt mit einer Lieferungsnummer versehen. Dies gewährleistet, dass jederzeit alle für eine Lieferung bereits erarbeiteten Wortartikel aufgerufen und exportiert werden können, insbesondere für den Manuskriptsatz. Hat ein Artikel sämtliche Korrekturen durchlaufen und den druckfertigen Status erreicht, wird er entsprechend gekennzeichnet, indem ein Häkchen in einem Kontrollkästchen (,Der Artikel ist fertig“; vgl. Abbildung 8) gesetzt wird.

Da der Verlag die jeweils aktuelle Wörterbuchlieferung vertragsgemäß als druckfertige PDF-Datei erwartet, muss der gesamte Textsatz ebenfalls von der Wörterbucharbeitsstelle geleistet werden. 


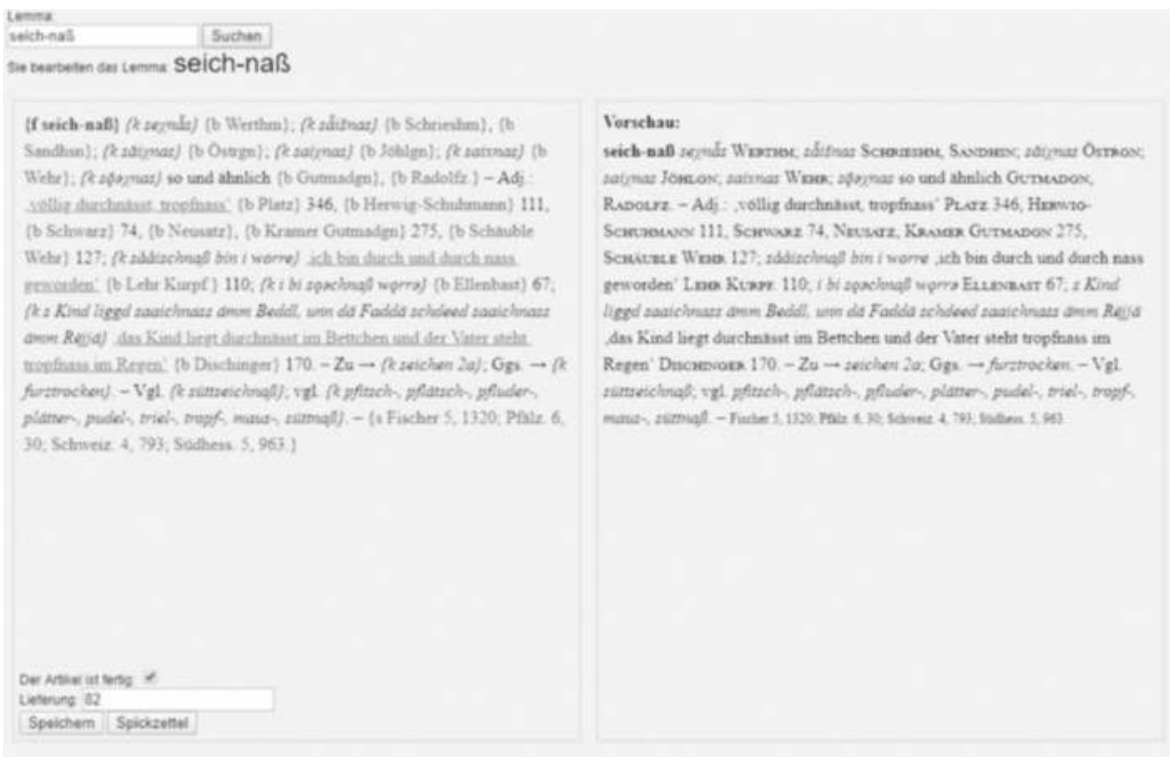

Abb. 8: Artikeleditor (mit Vorschaufunktion) und darunter das Kontrollkästchen für den Artikelstatus sowie die Zuordnung des Artikels zu einer Lieferung

Bald nach dem Umstieg auf die Artikelerstellung in der Datenbank erfolgte auch beim Textsatz eine technische Umstellung. DIETER STUDER-JOHO (Zürich) entwickelte für das „Badische Wörterbuch“ dankenswerterweise eine LaTeX-Vorlage, die das Layout der Wörterbuchseiten des vierten Bandes weitestgehend abbildet. Aus technischen Gründen wurde die Höhe des Satzspiegels geringfügig vergrößert. Außerdem wird nun aus typographischen Gründen auf sämtliche Sperrungen im Text verzichtet, da diese in den Testläufen für den LaTeX-Satz zu einem unschönen rechten Rand der Spalten führten (schlechter bzw. unregelmäßiger Randausgleich). ${ }^{7}$ Stattdessen werden die Quellen- und Ortsangaben seit Beginn des fünften Bandes (Lieferung 82) durch Kapitälchen ausgezeichnet, was insgesamt auch zu einem ruhigeren Druckbild führt. Auf der Basis der sehr detaillierten LaTeX-Vorlage von DiETER STUDER-JOHO erweiterte wiederum Uli HELD die Datenbank um eine Exportfunktion der Wörterbuchartikel als TeX-Code. Für den Satz einer Wörterbuchlieferung können nun alle Artikel, die der jeweiligen Lieferung zugeordnet sind, als TeX-Code exportiert werden. Ihre alphabetische Sortierung wird zuvor von der Datenbank weitgehend automatisch vorgenommen. Anschließend wird das Lieferungsmanuskript lokal auf dem Computer mit XeLaTeX unter Verwendung der erwähnten Vorlage gesetzt/kompiliert. Das Resultat ist ein PDF-Dokument.

7 Dieses Problem scheint in ähnlicher Weise auch schon früher aufgetreten zu sein (vgl. BAUR / SCHEER 199: 269). 
In den Jahren 2015 und 2016 wurde die Datenbank um zusätzliche Funktionen erweitert. Wiederum in Zusammenarbeit mit ULI HELD wurde zunächst die vorhandene, aber bisher mit einem gesonderten Programm durchsuchbare Lemmaliste (vgl. oben) mit Bedeutungs-, Wortartangaben u. a. in die Datenbank importiert und eine Suchmaske entwickelt, die alle wesentlichen Funktionen des externen Lemmasuchprogramms bietet. Weiterhin wurden die Suchfunktionen des ebenfalls externen Volltext-Suchprogramms (vgl. oben) in der Datenbank abgebildet. Im Laufe des Jahres 2016 wurde schließlich der gesamte - bereits als ANSI-Text vorliegende (vgl. oben) - Wörterbuchvolltext der Bände 1-4 in die Datenbank importiert, sodass nun alle bislang publizierten Artikel des „Badischen Wörterbuchs“ in einem einheitlichen und zeitgemäßen Format (unicodebasiert) verfügbar - und bei Bedarf wiederum im TeX-, XML-, CSV- oder ANSI-Format exportierbar (vgl. Abbildung 9) - sind.

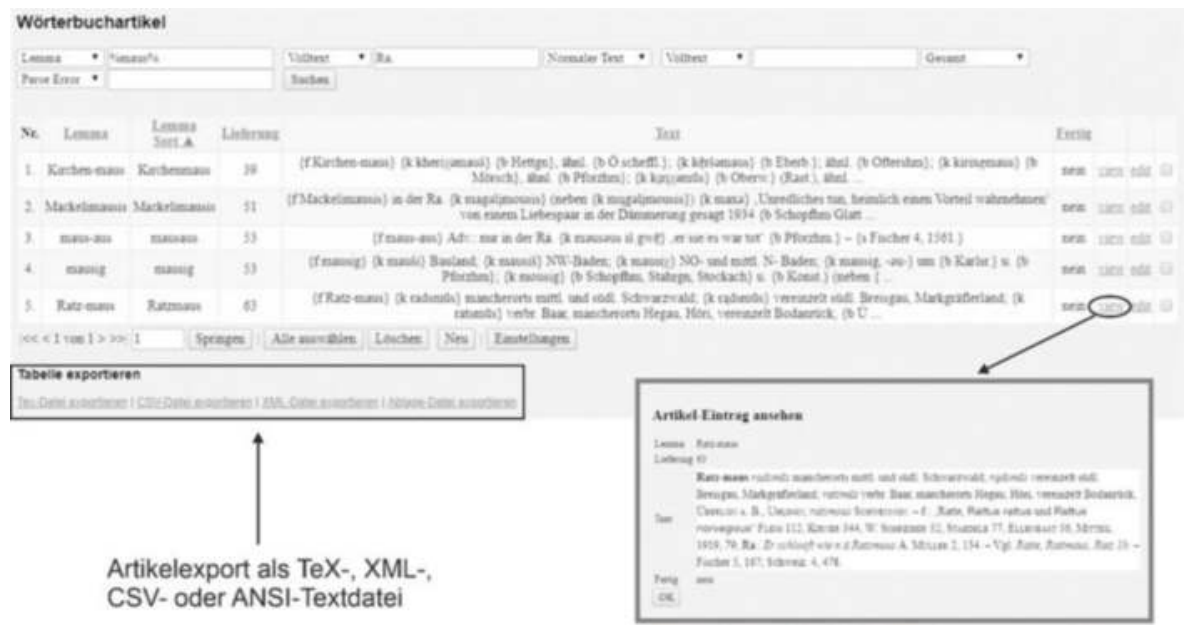

Abb. 9: Die Suchfelder und die Ergebnisliste einer Beispielsuche im Artikelbereich der Datenbank des ,Badischen Wörterbuchs“

Bei Recherchen im Volltext kann die Suche auf verschiedene Bereiche eingegrenzt bzw. durch die Kombination von Suchfeldern verfeinert werden, was recht differenzierte Suchanfragen ermöglicht. Die Suche kann also beispielsweise auf Bedeutungsparaphrasen, auf kursiv gedruckten Text (= Objektsprachliches und Verweise) oder Text in Groteskschrift (= lateinische Namen von Pflanzen, Tieren etc.) sowie auf verschiedene andere Bereiche eingeschränkt werden. Bei der Kombination von Suchfeldern wird eine UND-Verknüpfung verwendet. Verbindet man also zum Beispiel eine Suche nach \%maus\% (das Prozentzeichen dient als Platzhalter bei Suchanfragen) im Feld „Lemma“ mit der Suche nach „Ra.“ (die im Wörterbuch verwendete Abkürzung für Redensart) im Volltext, so erhält man als Ergebnis alle Wortartikel zu Stichwörtern, die die Zeichenfolge <maus> enthalten und für die das 
Vorkommen in einer Redensart im Wörterbuch belegt ist. ${ }^{8}$ Das Suchergebnis wird als Liste/Tabelle angezeigt; jeder gefundene Artikel kann mittels eines Klicks auf „view“ in einem eigenen Fenster in voller Länge angesehen werden. Abbildung 9 zeigt oben die Suchfelder und darunter die Ergebnisliste unserer eben beschriebenen Beispielsuche. In der Abbildung ist außerdem anhand des Beispiels Ratzmaus die Artikelansicht dargestellt. Des Weiteren sind unten links die Exportmöglichkeiten zu sehen.

Der retrodigitalisierte Teil des Wörterbuchvolltextes enthält unter anderem noch Tippfehler, die jedoch bei der Arbeit in der Datenbank inzwischen auf einfache Weise nach und nach „,nebenher" - d. h. immer dann, wenn ein Fehler entdeckt wird - korrigiert werden können. ${ }^{9}$ Für eine öffentlich zugängliche Onlineversion des „Badischen Wörterbuchs“ müsste dieser Teil des Volltexts selbstverständlich zunächst systematisch Korrektur gelesen werden. Abgesehen von diesem notwendigen - und sicherlich auch sehr zeitaufwändigen - Korrekturschritt sind beim „Badischen Wörterbuch“" seit spätestens 2011 (Fertigstellung der Retrodigitalisierung) recht gute Grundlagen für eine Onlineversion vorhanden. Alle bisherigen Bemühungen in diese Richtung fanden allerdings seitens des Verlages keinen Zuspruch.

Zusammenfassend kann festgehalten werden, dass das aktuelle Redaktionssystem des „Badischen Wörterbuchs“ verschiedene - vorher zum Teil über einzelne lokale Computerprogramme gewährleistete - Funktionen und auch Aufgabenbereiche in einem System zusammenbringt. Die Datenbank ermöglicht nicht nur die (prinzipiell arbeitsplatzunabhängige) Erarbeitung und Speicherung von Wörterbuchartikeln sowie deren Export, sondern sie gewährleistet auch eine moderne Belegverwaltung und -archivierung. ${ }^{10}$ Da nicht nur der Wörterbuchvolltext und die Lemmaliste durchsucht werden können, sondern auch alles bereits eingegebene Belegmaterial (für den fünften Band), bietet die Datenbank Recherchemöglichkeiten, die bereits zum jetzigen Zeitpunkt (wo die Eingabe des Materials noch nicht abgeschlossen ist ${ }^{11}$ ) deutlich über die Inhalte der publizierten Wortartikel hinausgehen.

8 Um nur diejenigen Lemmata zu finden, die auf $\{$ maus $\}$ enden (in unserem Beispiel Komposita mit $\{$ maus $\}$ als Grundwort), wird nach \%maus statt \%maus\% gesucht. Umgekehrt erbringt eine Suche nach maus\% alle Stichwörter, bei denen $\{$ maus $\}$ am Anfang steht.

9 Selbstverständlich wurden Fehler im retrodigitalisierten Volltext auch schon vor der Integration in die Datenbank notiert und korrigiert. Allerdings mussten sie in einer ANSI-Textdatei (die für das Auge schlecht lesbar ist, weil in ihr alle Sonderzeichen und Schriftauszeichnungen codiert sind) verbessert werden, und diese Datei musste dann auf jeden Computer, auf dem das Volltext-Suchprogramm verwendet wurde, kopiert werden.

10 Im Rahmen einer Masterarbeit (GERBER 2017) wurde die Datenbank einer ausführlichen und detaillierten Analyse unterzogen, die interessante und sinnvolle Vorschläge für zusätzliche Funktionen bzw. Anpassungen bereits vorhandener Funktionen erbrachte. In Abhängigkeit der verfügbaren finanziellen Mittel ist eine Erweiterung/Anpassung der Datenbank sicherlich durchaus lohnenswert.

11 Die Eingabe des Belegmaterials geht, auch aufgrund der personellen Situation der Arbeitsstelle, insgesamt natürlich schneller vonstatten als die deutlich komplexere Erarbeitung der Wortartikel. Allerdings wird sich auch die vollständige elektronische Erfassung des Belegmaterials für den fünften Band bei gleich bleibenden personellen Ressourcen noch längere Zeit hinziehen. 
Des Weiteren trägt das datenbankbasierte Redaktionssystem dazu bei, die recht geringen personellen Ressourcen der Arbeitsstelle möglichst optimal zu nutzen, indem die Eingabe des Belegmaterials, die Pflege der Lemmaliste, Korrekturarbeiten im Volltext oder auch bestimmte Vorarbeiten - wie z. B. das Recherchieren und Einpflegen externer Verweise (d. h. auf Nachbarwörterbücher und Sprachatlanten) etc. - von den entsprechend geschulten studentischen Hilfskräften und der „Schreibkraft“ übernommen werden können.

\section{LINGUISTISCHES POTENTIAL}

Beim „Badischen Wörterbuch“ wurde - wie in den vorangegangenen Kapiteln bereits erläutert - schon seit Jahren sehr vorausschauend auf eine Digitalisierung verschiedener Daten und Materialen gesetzt (vgl. z. B. BAUR / SCHEER 1998: 271). Dank jahrelanger Arbeit, die zu großen Teilen von studentischen Hilfskräften geleistet wurde, verfügt die Arbeitsstelle heute über einen digitalen Wörterbuchvolltext und eine elektronische Lemmaliste mit Angaben zur Wortart, zur Bedeutung sowie zusätzlichen Klassifizierungen wie Orts-, Flur-, Fluss-, Vor-, Familien-, Über-, Pflanzen-, Tiername oder Rotwelsch. ${ }^{12}$ Beides ist inzwischen - wie in Kapitel 3 dargestellt - in der Datenbank des „Badischen Wörterbuchs“ integriert und durchsuchbar. Hinzu kommen die ebenfalls bereits erwähnten Suchmöglichkeiten innerhalb des Belegmaterials für den fünften Band.

Obwohl die Datenbank in erster Linie als Redaktionssystem für die Artikelarbeit ausgelegt ist, bietet sie durchaus Möglichkeiten, die vorhandenen Daten für weitere linguistische Analysen zu nutzen. Da sie über eine Rechteverwaltung für Benutzerkonten verfügt, so dass z. B. der Zugriff auf bestimmte Bereiche begrenzt werden kann oder innerhalb eines Bereichs nur bestimmte Berechtigungen vergeben werden können, ist es prinzipiell möglich, auch Nutzerinnen und Nutzern von „außerhalb“ einen zeitlich begrenzten Datenbankzugang für eigene Recherchen zu gewähren. In Einzelfällen wurde dies in jüngster Zeit auch bereits praktiziert. Um auf der Grundlage der Datenbank ein „öffentlich“ zugängliches Recherchetool zu schaffen, müsste allerdings sicherlich noch einige Programmierarbeit geleistet werden. ${ }^{13}$ Die Suchmöglichkeiten könnten für so ein Vorhaben noch verfeinert werden; und idealerweise sollte dann auch eine eigene Oberfläche zur Verfügung stehen, so dass eine bessere Trennung zwischen Recherchetool und Redaktionssystem gewährleistet wäre. Anders als beim Wörterbuchvolltext und der Lemmaliste wäre die Suche auf Belegebene allerdings recht limitiert, da aktuell „nur“ das Belegmaterial

12 Für die noch nicht bearbeitete Wortstrecke sind die Angaben in der elektronischen Lemmaliste zunächst vorläufig. Sie wurden aus dem Zettelarchiv herausgearbeitet und werden grundsätzlich nach der Veröffentlichung einer Wörterbuchlieferung so aktualisiert, dass insbesondere die Bedeutungsangaben exakt denjenigen im jeweiligen Wortartikel entsprechen.

13 Außerdem müsste der retrodigitalisierte Teil des Wörterbuchvolltexts zunächst noch systematisch Korrektur gelesen werden (vgl. Kapitel 3). 
für den fünften Band - die Grundlage für die Erarbeitung der Wortartikel in der Datenbank - elektronisch erfasst wird. ${ }^{14}$

In Kapitel 3 wurde bereits ein Beispiel für die Suche im Wörterbuchvolltext illustriert. Wie oben erwähnt, kann die Suche auf verschiedene Artikelbereiche eingeschränkt werden: auf Bedeutungsparaphrasen, auf kursiv gedruckten (= Objektsprachliches und Verweise) oder fett gedruckten Text (Stichwörter), auf Text in Groteskschrift (= lateinische Namen von Pflanzen, Tieren etc.), in Kapitälchen (Quellenangaben und Ortsnamen) oder in Kleindruck (Verweise auf Nachbarwörterbücher und Sprachatlanten) sowie auf Text ohne besondere Auszeichnung. Die Suchfelder können einzeln verwendet oder (per UND-Verknüpfung, vgl. oben) kombiniert werden, was Suchmöglichkeiten eröffnet, die über die reine lexikalische Ebene hinausgehen. Es kann also beispielsweise gezielt nach einer bestimmten Lautung gesucht werden. Eine Suche nach \%hüs\% liefert alle Wortartikel, in denen $h \bar{u} s$ im Lautkopf oder in einem Verwendungsbeispiel (auch in Komposita und Ableitungen) vorkommt. ${ }^{15}$ Mit einer Suche nach \%les im Feld „Lemma“ und nach \%spiel\% in Bedeutungsparaphrasen (oder im gesamten Volltext) können alle auf -les gebildeten Bezeichnungen für (Kinder-)Spiele gefunden werden usw.

Je nach Analyseziel muss jedoch die Heterogenität der Daten im Blick behalten werden. So sind zum Beispiel viele Belege nicht datiert, zumindest nicht direkt im Wortartikel - der (ungefähre) Zeitraum, aus dem ein Beleg stammt, kann jedoch in der Regel über das Quellenverzeichnis erschlossen werden. Außerdem stammt das Material aus sehr unterschiedlichen Quellen, und es wird - je nach Analyseziel mitunter durchaus einen Unterschied machen, ob ein Beleg aus dem Volkskundefragebogen von 1894/1895, aus einer Ortsgrammatik des frühen 20. Jahrhunderts, aus einer Tonaufnahme aus den 1970er oder 1980er Jahren oder aus der Mundartliteratur stammt.

Auch die Lemmaliste bietet sehr gute Suchmöglichkeiten. Über sie können beispielsweise schnell alle Pflanzen-, Tier- oder Übernamen etc. gefunden werden. Da hier grundsätzlich zwei Suchfelder - relevant sind insbesondere die Felder „Lemma“, „Wortart“, „Bedeutung“ und „Hinweise“ (= die oben genannten klassifizierenden Angaben wie Pflanzen-, Tier-, Orts-, Übername usw.) - miteinander kombinierbar sind, kann die Suche entsprechend verfeinert werden. Sucht man also zum Beispiel nach \%bolle\% im Feld „Lemma“ und nach „PflN“ (= Pflanzenname) im Feld „Hinweise“ werden alle Lemmata gefunden, die die Zeichenfolge bolle oder bollen enthalten und Bezeichnungen für eine Pflanze sind. ${ }^{16}$ Abbildung 10 zeigt

14 Die Eingabe des bereits für die Bände 1-4 bearbeiteten Belegmaterials aus dem Zettelarchiv (und das Scannen der vielen tausend Belegzettel) wäre ein Unterfangen, das sich über etliche Jahre hinziehen würde bzw. vermutlich sogar im Rahmen eines zusätzlichen Projekts durchgeführt werden müsste. Da derzeit selbstverständlich die Erarbeitung des fünften Bandes im Vordergrund steht und die Datenbank in erster Linie als Redaktionssystem dient, ist klar, dass die elektronische Erfassung der bereits früher bearbeiteten Belegzettel aus aktueller Sicht auch nur von geringer Relevanz ist.

15 Wie oben in Fußnote 8 dargestellt, kann die Suche auch verfeinert werden.

16 Diese Suche könnte in ähnlicher Weise auch im Wörterbuchvolltext durchgeführt werden. Sie dauert allerdings länger, da erheblich mehr Text durchsucht werden muss. 
oben die Suchfelder und darunter die tabellarische Ergebnisdarstellung dieser Beispielsuche. In der Abbildung ist außerdem (unten links) zu sehen, dass das Suchergebnis im CSV-Format exportiert und somit zum Beispiel in einem Tabellenkalkulationsprogramm o. ä. weiterverarbeitet werden kann.

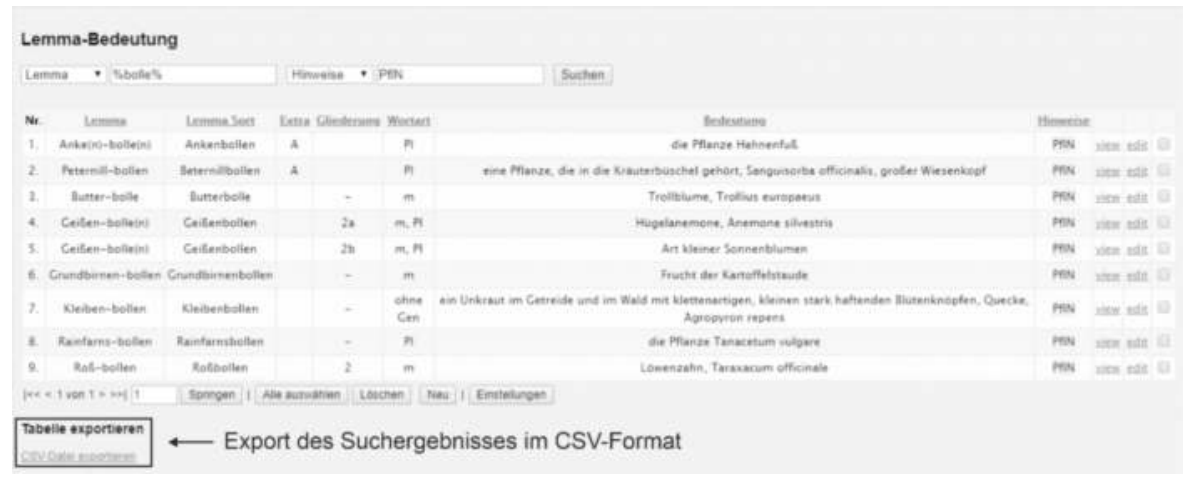

Abb. 10: Die Suchfelder in der Lemmaliste und die tabellarische Darstellung der Ergebnisse einer Beispielsuche in der Datenbank des „Badischen Wörterbuchs“

Für Wortbildungsanalysen bietet sich beispielsweise eine Kombination der Suchfelder „Lemma“ und „Wortart“ an. So können mittels einer Suche nach \%eln im Feld „Lemma“ und nach „schw“ (= schwaches Verb) im Feld „Wortart“ alle auf -eln endenden Verben gefunden werden. Durch die über das Feld „Wortart“ vorgenommene Sucheinschränkung wird vermieden, dass die Ergebnisliste auch Ortsnamen wie Minseln oder Substantive mit einem pluralischen Stichwortansatz wie Gänsnudeln ('Gänsekot') u. a. enthält.

Wie diese Beispiele zeigen, besitzen die Daten also einiges Potential für linguistische Analysen, die über die eigentliche lexikographische Arbeit hinausgehen. Allein die Tonaufnahmen, die allesamt digitalisiert und (in Form einer „Übersetzung“ ins Standarddeutsche) transkribiert sind und in der Online-Datenbank MOCA des Freiburger Lehrstuhls für Germanistische Linguistik komfortabel durchsucht werden können (vgl. Kapitel 1.2 und 1.3), bieten viele Stunden spontansprachlicher Dialektdaten. Im Rahmen von zwei Dissertationen (STRECK 2012 und SCHWARZ 2015) wurden diese auch bereits als Teilkorpus für die Untersuchung von phonologischem Dialektwandel in Südwestdeutschland genutzt. Das Potential dieser Daten ist selbstverständlich nicht auf die Phonologie begrenzt, sondern sie könnten beispielsweise auch für morphologische oder syntaktische Analysen verwendet werden. 


\title{
5 KONTAKTADRESSE, HOMEPAGE
}

\author{
Albert-Ludwigs-Universität Freiburg \\ Deutsches Seminar - Germanistische Linguistik \\ Arbeitsstelle Badisches Wörterbuch \\ Belfortstraße 14 \\ 79098 Freiburg \\ Deutschland
}

E-Mail: tobias.streck@germanistik.uni-freiburg.de

Homepage: <http://www.germanistik.uni-freiburg.de/badischwb/>

\section{LITERATURVERZEICHNIS}

BAUR, GERHARD W. (1976a): Das Badische Wörterbuch. In: FRIEBERTSHÄUSER, HANS (Hg.): Dialektlexikographie. Berichte über Stand und Methoden deutscher Dialektwörterbücher. Festgabe für LUISE BERTHOLD zum 85. Geburtstag am 27.1.1976. Wiesbaden: Steiner (Zeitschrift für Dialektologie und Linguistik. Beihefte. 17), 25-35.

BAUR, GERHARD W. (1976b): Mundartwörterbücher im alemannischen Sprachraum. In: Alemannisches Jahrbuch 1973/75 (Alemannica. Landeskundliche Beiträge. Festschrift für BRUNO BOESCH zum 65. Geburtstag), 28-85.

BAUR, GERHARD W. (1983): Das Badische Wörterbuch. In: Badische Heimat 63, 387-394.

BAUR, GERHARD W. / FRIEDEL SCHEER (1998): Vorzüge und Schwierigkeiten in der Nutzung des Computers bei der Arbeit am Badischen Wörterbuch. In: GROSSE, RUdOLF (Hg.): Bedeutungserfassung und Bedeutungsbeschreibung in historischen und dialektologischen Wörterbüchern. Beiträge zu einer Arbeitstagung der deutschsprachigen Wörterbücher, Projekte an Akademien und Universitäten vom 7. bis 9. März 1996 anläßlich des 150jährigen Jubiläums der Sächsischen Akademie der Wissenschaften zu Leipzig. Stuttgart/Leipzig: Hirzel (Abhandlungen der Sächsischen Akademie der Wissenschaften zu Leipzig, Philologisch-historische Klasse. 75, H. 1), 265-271.

GERBER, ANNA-LENA (2017): Lexikographische Arbeitsprozesse im Wandel. Vom Zettelkasten bis zur Datenbank beim Badischen Wörterbuch. Masterarbeit an der Albert-Ludwigs-Universität Freiburg i. Br.

LENZ, PHILIPP (1925/1926): Nachträge zu Lieferung 1 des Badischen Wörterbuches, bearbeitet von E. Ochs. In: Teuthonista 2, 286-289.

OCHS, ERNST (1921): Die Gliederung des Alemannischen. In: Germanisch-romanische Monatsschrift 9, 56-58.

OCHS, ERNST (1923): Proben des Badischen Wörterbuchs nebst Gliederung der badischen Mundarten. Mit Skizze. 2. Auflage. Karlsruhe: Müller (Vom Bodensee zum Main. 12).

OCHS, ERNST (1924): Grüße vom Badischen Wörterbuch. In: Zeitschrift für deutsche Mundarten 19, 210-212.

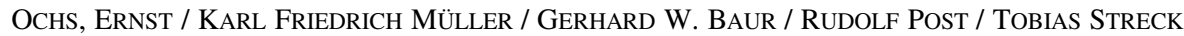
(1925 ff.): Badisches Wörterbuch. Herausgegeben mit Unterstützung des Ministeriums für Wissenschaft, Forschung und Kunst Baden-Württemberg. Vorbereitet von FRIEDRICH KLUGE, Alfred Götze, Ludwig SÜtTerlin, Friedrich Wilhelm, ERnSt OchS. Begonnen von ERNST OCHS, weitergeführt von KARL FrIEDRICH MÜller, GERHARD W. BAUR, RUdOLF POST, bearbeitet von TOBIAS STRECK. Berlin [u. a.]: de Gruyter. 1925-1999 im Verlag Moritz Schauenburg, Lahr, und 2000-2012 im R. Oldenbourg Verlag, München, erschienen. 
POST, RUdOLF (1998): Möglichkeiten der elektronischen Strukturierung, Vernetzung und Verfügbarmachung von lexikographischen Daten bei der Arbeit am Pfälzischen Wörterbuch. In: GROSSE, RUdOLF (Hg.): Bedeutungserfassung und Bedeutungsbeschreibung in historischen und dialektologischen Wörterbüchern. Beiträge zu einer Arbeitstagung der deutschsprachigen Wörterbücher, Projekte an Akademien und Universitäten vom 7. bis 9. März 1996 anläßlich des 150jährigen Jubiläums der Sächsischen Akademie der Wissenschaften zu Leipzig. Stuttgart/Leipzig: Hirzel (Abhandlungen der Sächsischen Akademie der Wissenschaften zu Leipzig, Philologisch-historische Klasse. 75, H. 1), 211-220.

Post, RUdolf (2001): Die Erhebungen zur Sammlung der Volksüberlieferungen Badens 1894/95 und die Anfänge des „Badischen Wörterbuchs“. In: BENTZINGER, RUDOLF / DAMARIS NÜBLING / RudOlf StefFens (Hg.): Sprachgeschichte, Dialektologie, Onomastik, Volkskunde. Wolfgang Kleiber zum 70. Geburtstag. Stuttgart: Steiner (Zeitschrift für Dialektologie und Linguistik. Beihefte. 115), 259-275.

POST, RUDOLF (2002): Sondersprachliches im Badischen Wörterbuch. In: SIEWERT, KLAUS (Hg.): Aspekte und Ergebnisse der Sondersprachenforschung II. Wiesbaden: Harrassowitz (Sondersprachenforschung. 7), 47-86.

Post, RUdOLF (2003): Badisches Wörterbuch. In: STÄDTLER, THOMAS (Hg.): Wissenschaftliche Lexikographie im deutschsprachigen Raum. Heidelberg: Winter, 319-322.

SCHEER-NAHOR, FRIEDEL (1998): Hebraismen im Badischen Wörterbuch. Magisterarbeit an der Albert-Ludwigs-Universität Freiburg i. Br.

SCHEER-NAHOR, FRIEDEL (2001): Wissenswertes, nicht nur zu Hudel und Schefe, im Badischen Wörterbuch. In: Alemannisch dunkt üs guet I-II/2001, 26-29.

SCHWARZ, CHRISTIAN (2015): Phonologischer Dialektwandel in den alemannischen Basisdialekten Südwestdeutschlands im 20. Jahrhundert. Eine empirische Untersuchung zum Vokalismus. Stuttgart: Steiner (Zeitschrift für Dialektologie und Linguistik. Beihefte. 159).

STRECK, TOBIAS (2008): Vom Scheßlong zum Boddschamber und retur ... Französische Entlehnungen in den badischen Mundarten. In: Alemannisches Jahrbuch 2005/2006, 261-315.

STRECK, TOBIAS (2012): Phonologischer Wandel im Konsonantismus der alemannischen Dialekte Baden-Württembergs. Sprachatlasvergleich, Spontansprache und dialektometrische Studien. Stuttgart: Steiner (Zeitschrift für Dialektologie und Linguistik. Beihefte. 148).

STRECK, TOBIAS (2015): Phonologischer Wandel am Beispiel der alemannischen Dialekte Südwestdeutschlands im 20. Jahrhundert. In: KEHREIN, ROLAND / ALFRED LAMELI / STEFAN RABANUS (Hg.): Regionale Variation des Deutschen. Projekte und Perspektiven. Berlin/Boston: de Gruyter, 157-171. 


\title{
HESSEN-NASSAUISCHES WÖRTERBUCH (HNWB)
}

\author{
Alexander Werth / Bernd Vielsmeier / Stefan Aumann
}

\section{GESCHICHTLICHES, RAHMENBEDINGUNGEN ZUM WÖRTERBUCH UND ZUR ARBEITSSTELLE}

Das „Hessen-Nassauische Wörterbuch“ (HNWb) dokumentiert den dialektalen Wortschatz der Provinz Hessen-Nassau des Königreichs Preußen (inkl. Kreis Herrschaft Schmalkalden), der Provinz Oberhessen des Großherzogtums Hessens, des Fürstentums Waldeck, des rheinischen Kreises Wetzlar und des westfälischen Kreises Wittgenstein in den Grenzen von 1914 (s. Abbildung 1). Sprachgeographisch betrachtet, werden die Dialekträume des Nord-, Zentral- und Osthessischen sowie Randgebiete des Westfälischen, Ostfälischen, Thüringischen, Ostfränkischen, Rheinfränkischen und Moselfränkischen erfasst (nach der Dialekteinteilung in WIESINGER 1983). Durch das Bearbeitungsgebiet nimmt das HNWb eine dialektgeographisch zentrale Stellung innerhalb der deutschen Dialektwörterbücher ein, wodurch sich mehrfach Überlappungen zu den Bearbeitungsgebieten anderer Dialektwörterbücher ergeben, etwa zum „Niedersächsischen Wörterbuch“, zum „Thüringischen Wörterbuch“ und zum „Rheinischen Wörterbuch“ (vgl. KÖNIG 2011: 138).

Das HNWb wurde 1911 von FERDINAND WREDE, dem damaligen Direktor des Deutschen Sprachatlas in Marburg, im Auftrag der Deutschen Kommission der Preußischen Akademie der Wissenschaften gegründet und seit 1927 in Einzellieferungen als „Hessen-Nassauisches Volkswörterbuch“ ${ }^{\text {publiziert. }}{ }^{1}$ Geldgeber waren zunächst die Akademie und - neben kleineren Zuwendungen - auch die Bezirksverbände Hessen und Nassau. Nach dem Zweiten Weltkrieg wurde die Finanzierung durch die Akademie eingestellt und vom Land Hessen übernommen. Das $\mathrm{HNWb}$ war bei seiner Gründung rechtlich und finanziell unabhängig von der Philipps-Universität Marburg, wurde aber später teilweise (als ,[m]it der Universität in Arbeitsgemeinschaft verbundene Anstalt") und ab 1960 zur Gänze in die Strukturen der Universität integriert. 1973 wurde die Arbeitsstelle schließlich mit dem Forschungsinstitut Deutscher Sprachatlas (später: Forschungszentrum Deutscher

1 Wie WREDE mehrfach selbst betont hat, gehen Idee und Initiative für ein „Hessen-Nassauisches Wörterbuch“" auf den Mediävisten Gustav RoETHE zurück, der u. a. Mitglied der Preußischen Akademie der Wissenschaften und ständiger Sekretär der Berliner Akademie der Wissenschaften war (vgl. z. B. Sitzungsberichte 1927: LV). Spätere Leiterinnen und Leiter des HNWbs sind (in chronologischer Reihenfolge): LUISE BERTHOLD, HANS FRIEBERTSHÄUSER, HEINRICH J. DiNGELDEIN und ALEXANDER WERTH (kommissarisch). 
Sprachatlas) zusammengelegt und unter dem Titel „Abteilung für Sprache in Hessen / Hessen-Nassauisches Wörterbuch“ weitergeführt (Forschungsberichte 19881992: 77).

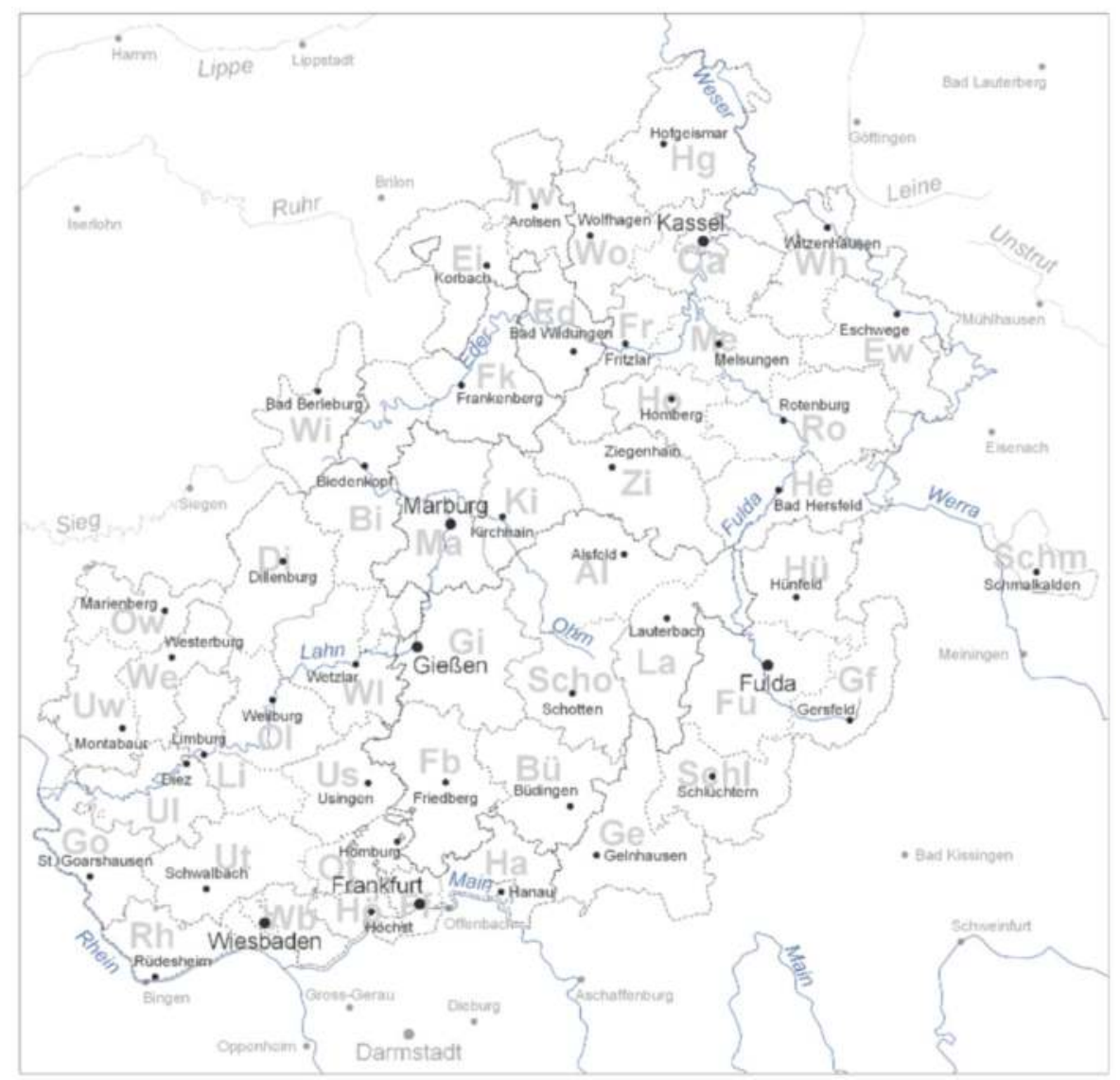

Abb. 1: Bearbeitungsgebiet des „Hessen-Nassauischen Wörterbuchs“

Über den Fortschritt des Projektes wurde ab 1912 in den „Sitzungsberichten der Preußischen Akademie der Wissenschaften“ (Rubrik: „Deutsche Commission“, kurz: Sitzungsberichte), ab 1954 dann sporadisch in den „Chroniken der PhilippsUniversität Marburg“ (kurz: Chroniken) und in den „Wissenschaftlichen Berichten des Forschungsinstituts für deutsche Sprache ,Deutscher Sprachatlas““ (kurz: Forschungsberichte) berichtet. Seit 2017 ist die Buchstabenstrecke $L-Z$ vollständig bearbeitet, der gedruckte Bestand umfasst drei Bände mit 45 Lieferungen à 32 Druckseiten und ist über das „Landesgeschichtliche Informationssystem Hessen“ (unter: <https://www.lagis-hessen.de>; Stand: 14.04.2020) über das Internet frei verfügbar (s. Kapitel 3). Mit der Bearbeitung der Einträge aus der Buchstabenstrecke $A-K$ 
wurde begonnen, eine Drucklegung ist zukünftig nicht mehr lieferungs-, sondern bandweise geplant.

Im Wörterbuch erfasst wird der dialektale (und auch umgangssprachliche) Wortschatz des oben skizzierten Gebietes, wie er im Erhebungszeitraum 1912 bis 1934 gebräuchlich war. ${ }^{2}$ Es handelt sich damit um ein primär synchron orientiertes Wörterbuch. Für die Wörterbucharbeit verzettelt und lemmatisiert wurden zunächst Bedeutungsangaben und Verwendungsweisen aus freien Einsendungen (Wortlisten, Bedeutungsparaphrasen, Satzbeispiele, Redewendungen), zu denen über Postkartenumfragen und über Aufrufe in Zeitschriften und Kalendern angeregt wurde (vgl. Sitzungsberichte 1920: 132). Hinzu kommen Exzerpte aus der Mundart- und Fachliteratur sowie historische Belege aus Urkunden, die für den Wörterbucheintrag aber erst ab 1800 berücksichtigt werden. ${ }^{3}$ Insgesamt umfasst das Archiv des HNWbs zirka 350000 lemmatisierte Belegzettel, die darauf verzeichneten linguistischen Informationen (mitunter finden sich verschiedene Typen von Angaben auf einem Zettel) lassen sich grob unterscheiden in Bedeutungsangaben, Herkunftsangaben, Wortbelege im (Satz-)Kontext, Formenbelege sowie morphologische und syntaktische Angaben (z. B. zu Genus, Numerus und Valenz).

Die zweite große Datenklasse des Wörterbuchs stellen Antworten von Gewährspersonen, meist handelte es sich dabei um Volksschullehrer, in Fragebogen dar. Kernbestand dieser Datensammlung bilden 79 Fragebogenrunden, die während der Jahre 1921 bis 1926 partiell im Wörterbuchgebiet erhoben worden sind. Sie wurden ergänzt um Fragebogen des „Rheinischen Wörterbuchs“, dessen Erhebungs- und Bearbeitungsgebiet sich in Teilen mit dem des HNWbs überschneidet (vgl. HNWb 2: IV). Zur Anwendung kamen dabei sowohl semasiologische als auch onomasiologische Fragestellungen, konkret wurden z. B. Wortlisten abgefragt (s. Abbildung 2) oder Skizzen, z. B. eines Webstuhls, vorgegeben, deren Bestandteile die Gewährspersonen benennen sollten (s. Abbildung 3). Schließlich ergab sich für die Bearbeiterinnen und Bearbeiter des Wörterbuchs auch mitunter die Möglichkeit, Wortformen in bestimmten Regionen direkt bei den Informantinnen und Informanten vor Ort abzufragen (vgl. z. B. Sitzungsberichte 1925: LXXII), um genauere Informationen über deren Verteilung im Raum zu erhalten. Ein entsprechender Auswertungsbogen mit den Wortformen in phonetischer Umschrift ist in Abbildung 4 dargestellt.

2 Genauer heißt es zur Auswahl des zu bearbeitenden Materials: „Das Volkswörterbuch hat nicht das gesamte Zettelmaterial des Hessen-Nassauischen Wörterbuchs verarbeitet. Es hat vielmehr daraus das ausgewählt, was vom Schriftdeutschen abweicht, d. h. also Mundartwörter, die diesem ganz fehlen oder ihm gegenüber in Form oder Bedeutung bemerkenswerte Abweichungen zeigen." (HNWb 2: V).

3 Die historischen Belege sollten den Grundstock für ein geplantes Wörterbuch der deutschen Urkundensprache für den Bereich Hessen und Nassau bilden, zu dem es aber bis heute nicht gekommen ist (vgl. FRIEBERTSHÄUSER 1976: 97). 


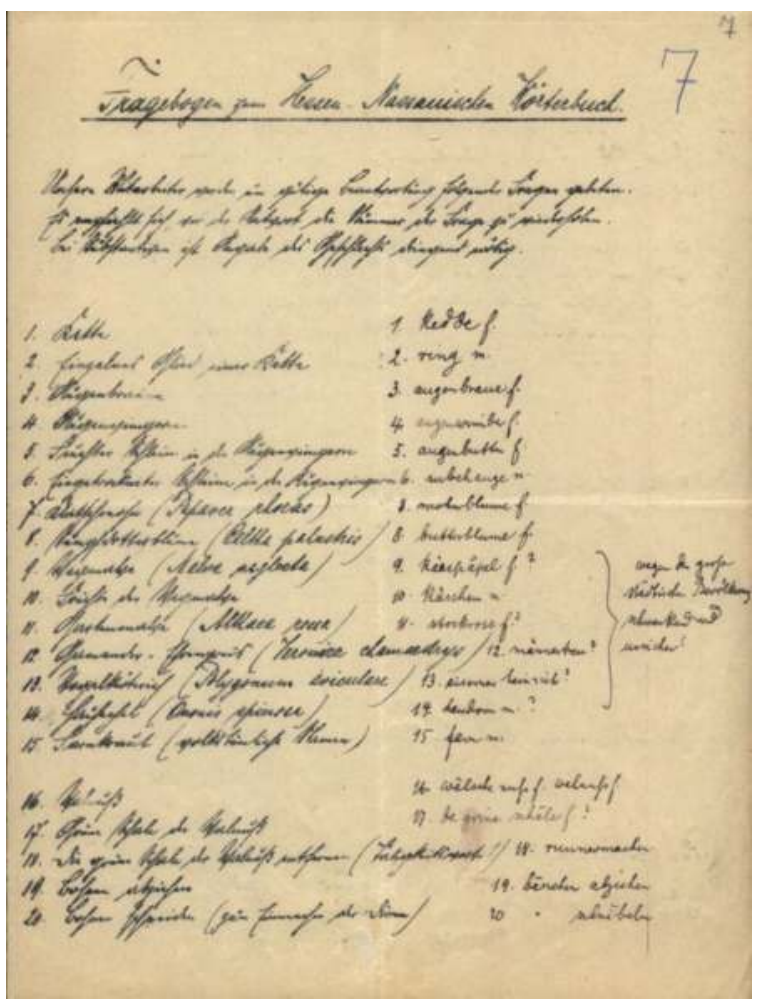

Abb. 2: Fragebogen mit abgefragter Wortliste

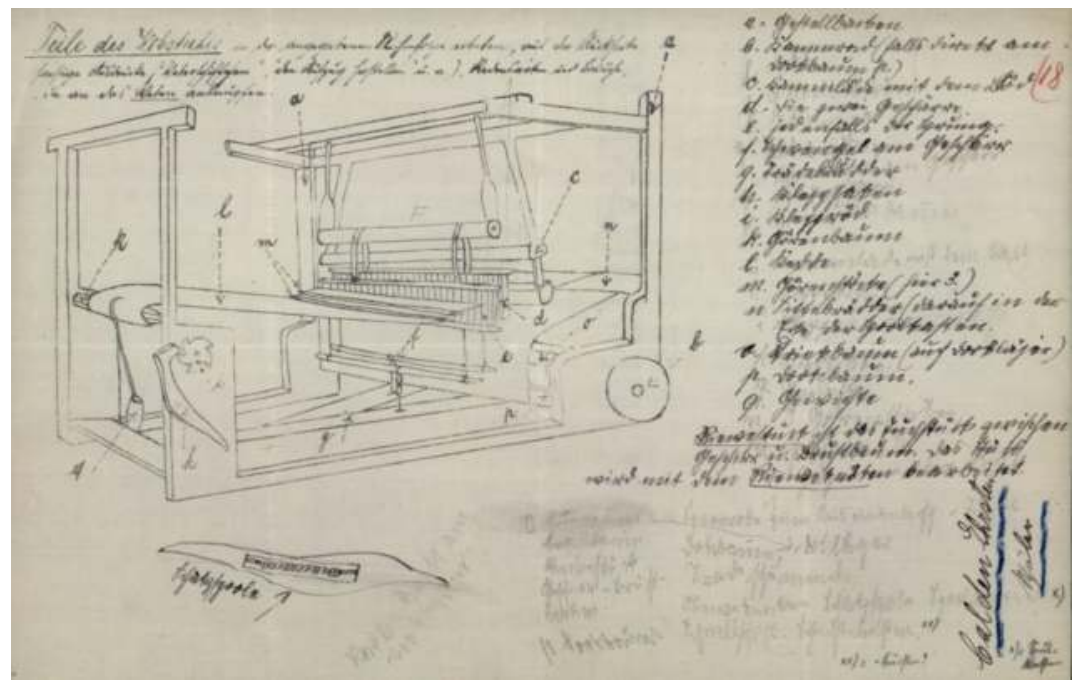

Abb. 3: Fragebogen mit zu benennenden Teilen eines Webstuhls 


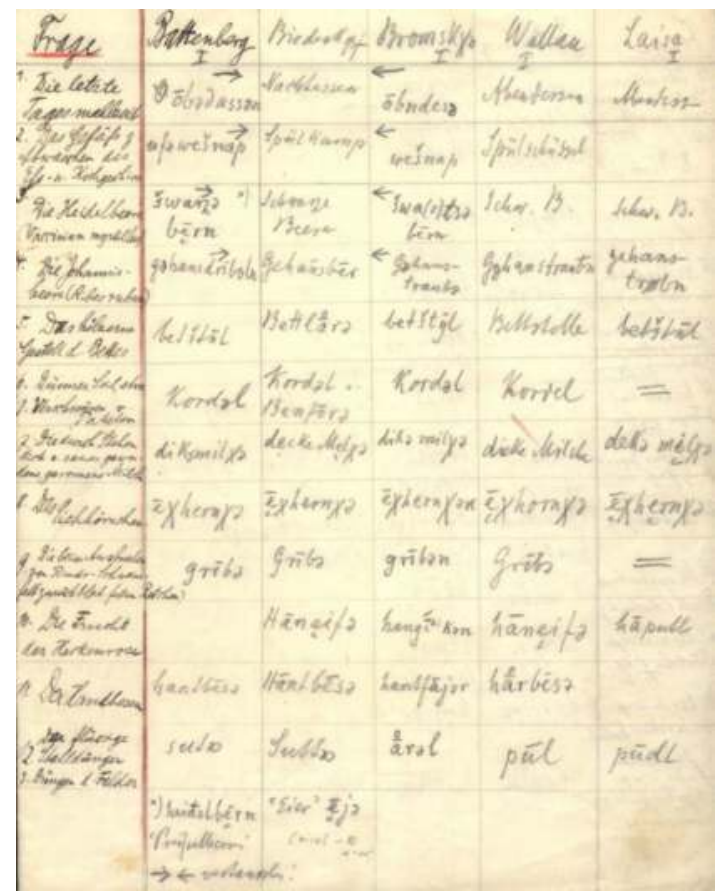

Abb. 4: Erhebungsbogen zu Wortformen aus einer direkten Erhebung

Der Gesamtbestand beläuft sich auf zirka 17700 ausgefüllte Fragebogenseiten. Diese sind von den Bearbeiterinnen und Bearbeitern des Wörterbuchs allerdings nur rudimentär verzettelt worden, sodass für ein konkretes Wort alleine der Verweis auf einen Fragebogen im Belegzettel vermerkt ist, nicht aber die Information aus dem Fragebogen selbst. (Dies führt für die Wörterbucharbeit zu dem ungünstigen, weil zeitaufwändigen und arbeitsintensiven Umstand, dass jeder Verweis auf einen Fragebogen in den Originalen eigens geprüft werden muss.)

Eine wesentliche Triebfeder der Fragebogenerhebung - und auch des HNWbs insgesamt - bestand darin, die dialektgeographische Methode, welche am Deutschen Sprachatlas im Rahmen der Erhebung des Sprachatlas des Deutschen Reichs entwickelt worden ist („Marburger Schule“, dazu KNOOP et al. 1982; LAMELI 2014), auf die Lexikographie anzuwenden. Angaben zum Belegort stehen damit nicht nur als Quellenhinweis, sondern sind als Beschreibung des Geltungsbereichs des Lemmas zu verstehen. Dadurch sollte ein neuer und für die Forschung wegweisender Typus von Dialektwörterbuch entstehen. ${ }^{4}$ So heißt es in den Berichten:

4 Tatsächlich wurde das wortgeographische Prinzip später auch in anderen Wörterbüchern aufgegriffen, so z. B. im „Preußischen Wörterbuch“, im „Schlesischen Wörterbuch“, im „Mecklenburgischen Wörterbuch“, im „Niedersächsischen Wörterbuch“, im „Mittelelbischen Wörterbuch“ und im „Pfälzischen Wörterbuch“(dazu BERTHOLD 1955; 1965). 
Die Eigenmarke unseres Werkes wird in der starken Betonung des wortgeographischen Prinzips liegen. (Sitzungsberichte 1925: LXXII)

Und es ist nur folgerichtig, wenn auch unsere Veröffentlichung, das Hessen-Nassauische Volkswörterbuch, als eigentümliche Note die wortgeograpische zeigt. (Sitzungsberichte 1935: LXXVII)

Die Karten waren dabei nur der stärkste Ausdruck des wortgeographischen Prinzips, das der Lieferung auch sonst seinen Stempel aufdrückte. (Chroniken 1955: 415)

Den sichtbarsten wortgeographischen Ertrag der Fragebogenerhebungen stellt ein Bestand an zirka 150 Karten auf Transparentfolie dar (s. Abbildung 5). Auf den Karten ist mittels einer Kombination aus Formen- und Symbolkartierung dargestellt, welche Wortform die Gewährsperson für ein bestimmtes, im entsprechenden Fragebogen abgefragtes Lemma am Ort angegeben hat. Über eine Grundkarte, die ebenfalls als Transparentfolie vorliegt, und deren Ortsnetz über das Ortsregister des HNWbs erschließbar ist, lassen sich die Wortformen dann eindeutig dem jeweiligen Erhebungsort zuordnen. In überarbeiteter und meist vereinfachter Form werden die Karten im Anschluss in das Wörterbuch übernommen (s. Abbildung 6). Sie dienen außerdem für Verbreitungsangaben im Wörterbuchartikel.

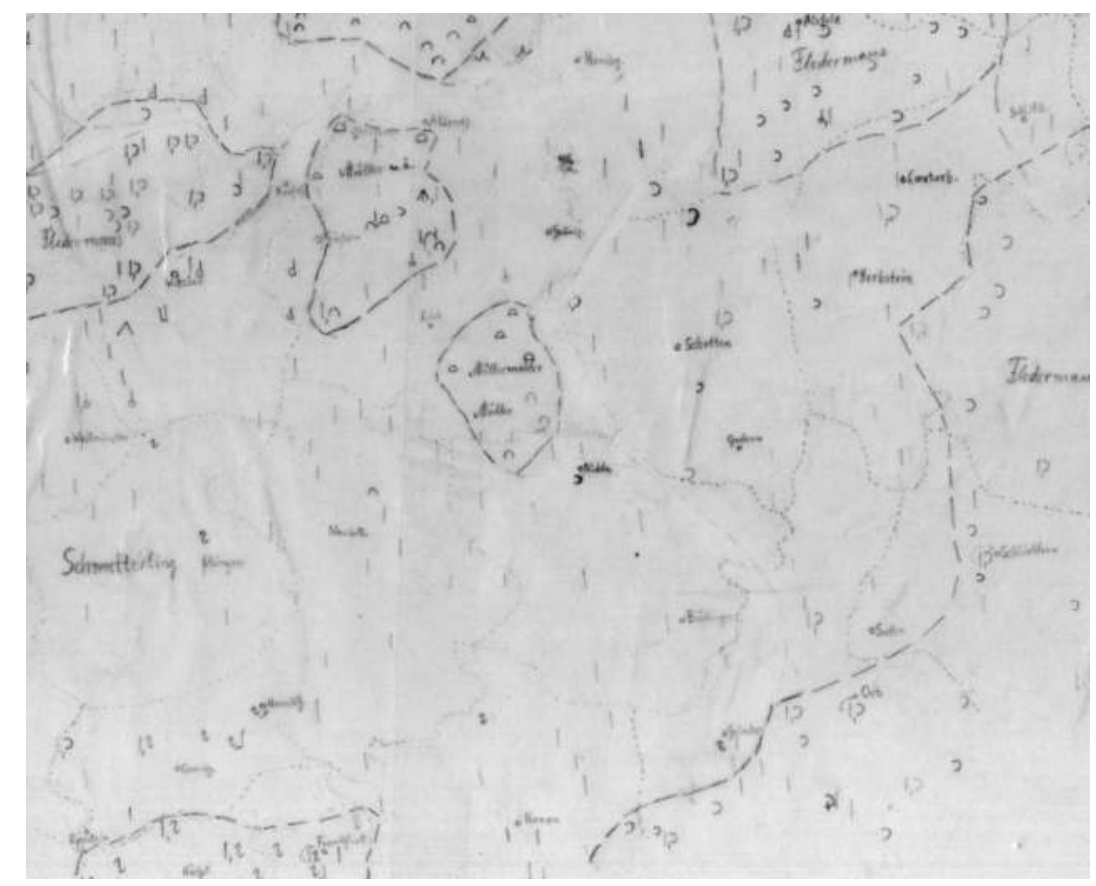

Abb. 5: Ausschnitt aus dem Kartenthema „Schmetterling“ 


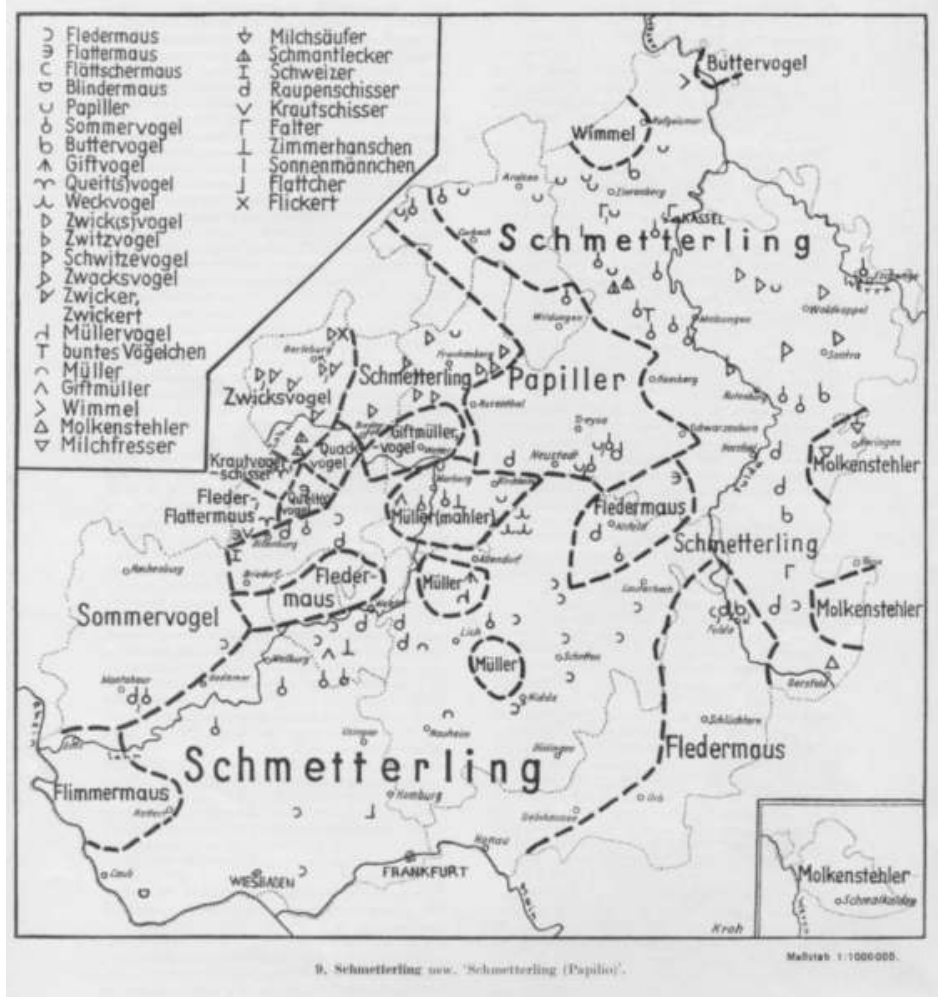

Abb. 6: Karte „Schmetterling“ aus HNWb 3: 291

\section{DIE WÖRTERBUCHARTIKEL: KONZEPTION, STRUKTUR, INHALT}

Schon die Titelei des Wörterbuchs ist programmatisch. Das „Hessen-Nassauische Volkswörterbuch“ ist nach den Angaben des Titelblattes von Band 2: $L-R$, der 1943 abgeschlossen war, eine Auswahl aus den Sammlungen des HNWbs. In ihrem Vorwort schreibt die Bearbeiterin LUISE BERTHOLD, dass ,,bei allem Bemühen um wissenschaftliche Genauigkeit eine allgemein verständliche Form angestrebt" wird. Die Wörterbuchartikel sollen ,,auch für den philologisch ungeschulten, aber ernsthaft fragenden Heimatfreund lesbar sein“ (BERTHOLD 1943: VI-VII). Lemma und Artikel sollen so angelegt sein, dass sie auch für Laien ohne Schwierigkeiten zu finden und zu verstehen sind. Das Abkürzungsverzeichnis enthält die Siglen für die Landkreise, die Städte und häufig genannte Belegorte, für die in der Regel umfangreiche Belegsammlungen vorliegen oder deren Wortschatz in einer Dissertation untersucht worden ist sowie die häufigen Grundbestandteile der zusammengesetzten Ortsnamen wie -bach, -dorf oder-heim. Einige wenige sonstige Abkürzungen und die älteren Mundartwörterbücher mit ihren Siglen folgen. Am Schluss stehen einige Angaben zur Lautschrift. Die in abgekürzter Form zitierten Quellen sind auch ohne 
ein Quellen- und Literaturverzeichnis leicht zu ermitteln. Die Benutzer/-innen des Wörterbuchs können daher ohne großen Aufwand gesuchte Wörter an der entsprechenden Stelle im Alphabet nachschlagen und die Angaben zu Orten und Quellen erschließen. Von mundartlich gebräuchlichen Formen wird auf das für den Artikel angesetzte Lemma verwiesen (z. B. wabbelig s. wappelig).

\subsection{Lemmatisierung}

Die Stichwörter der Wortartikel werden, soweit sie Bestandteil des standardsprachlichen Wortschatzes sind, in standardsprachlicher Form möglichst nach dem „Duden-Wörterbuch“ (Ausgabe von 1976-1981) angesetzt. Bei Wörtern aus dem hochund niederdeutschen Dialektgebiet, die nicht im Duden behandelt werden, orientiert sich der Lemmaansatz am „Deutschen Wörterbuch“ von JACOB GRIMM und WILHELM GRIMM sowie an dem „Niedersächsischen Wörterbuch“, „Rheinischen Wörterbuch“, „Südhessischen Wörterbuch“, „Thüringischen Wörterbuch“ und „Westfälischen Wörterbuch“ als benachbarte Mundartwörterbücher des Arbeitsgebiets. Auch Wörter, die nur mundartlich gebräuchlich sind, werden in einer an das Standarddeutsche angenäherten Form angesetzt (Konstruktlemma). So ist für die belegten mundartlichen Formen dremche, demmche, drempche und trempche 'stöhnen', 'sich träge besinnen' als Lemma trämpchen angesetzt worden. Wortgut aus Fremdsprachen, Geheim- und Sondersprachen sowie aus Namen werden behandelt, soweit sie Bestandteil der Mundart geworden sind (z. B. Schambes s. Jean Baptist).

Exemplarisch lässt sich dies für das in Hessen und Rheinhessen im Dialekt weit verbreitete Wort Hinkel für das 'Huhn' zeigen. Hinkel hat in dem Wort Hinkelstein für den 'Menhir' seit der zweiten Hälfte des 19. Jahrhunderts als Appellativum Eingang in den standarddeutschen Wortschatz gefunden. Im Duden stehen als Einträge Hinkel und Hinkelstein (vgl. Duden 3: 1250), hingegen fehlt Hünkel sowohl als Artikel als auch als Verweis. Das „Grimmsche Wörterbuch“ bringt unter Hinkel einen Verweis auf den Artikel Hünkel (vgl. DWB 4.2: 1444). Das „Südhessische Wörterbuch“ und das „Pfälzische Wörterbuch“, die als Dialektwörterbücher unmittelbar an das Bearbeitungsgebiet des HNWbs angrenzen, verweisen ebenfalls von Hinkel auf Hünkel. Der noch zu bearbeitende Artikel wird im „Hessen-Nassauischen Wörterbuch“ unter dem Lemma Hünkel angesetzt werden. Als Bestandteil in Zusammensetzungen wie Rebhünkel neben 'Rebhuhn' (Perdix perdix) und Zaunhünkelchen für den 'Zaunkönig' (Troglodytes troglodytes) wurde in diesem Fall der Lemmaansatz nicht nach Duden gewählt, sondern unter etymologischen Gesichtspunkten die verneuhochdeutschte Form Hünkel nach dem mittelhochdeutschen Huoninklin 'kleines Hühnchen', da Hünkel als Lemma genauer ist als Hinkel. ${ }^{5} \mathrm{Im}$ Wörterbuch wird von Hinkel auf den Artikel Hünkel verwiesen.

Unterschiede im Konsonantismus in den einzelnen Dialekträumen werden im Lemmaansatz berücksichtigt. Von der im Oberdeutschen verbreiteten Form Weiß für 'Weizen' wird auf den Artikel Weizen verwiesen. Diese oberdeutsche Form 
wird dem Lemma Weizen als eine der im Wörterbuchgebiet genannten Varianten nachgestellt (Weizen, Weiz(e), Weiß(e)). Die phonetisch ähnlich gesprochenen Anlaute $b / p, d / t, f / v, g / k$ werden nicht zusammengefasst, sondern als Lemma in der Reihenfolge des Alphabets eingeordnet. Weit verbreitet für den 'irdenen Topf' ist das im Westmitteldeutschen auftretende Dibbe, während im Ostmitteldeutschen Topf vorherrscht. ${ }^{6}$ Bei dem im Arbeitsgebiet nur selten bezeugten Topf wird auf den Artikel Tüpfen verwiesen. ${ }^{7}$ Ein Verweis von Dibbe auf den Artikel Tüpfen wird bei der Bearbeitung des Buchstabens $D$ gesetzt. Die im niederdeutschen Sprachgebiet entsprechende Bezeichnung dafür ist Pott. Bei niederdeutschen Wörtern wie Pott oder late 'spät' orientiert sich der Lemmaansatz des HNWbs an den niederdeutschen Wörterbüchern.

\subsection{Artikelstruktur}

\subsubsection{Lemma}

Die Lemmaformen sind vollständig ausgeschrieben und stehen in alphabetischer Reihenfolge. Substantive werden im Nominativ Singular angesetzt, Verben im Infinitiv, Adjektive und Adverbien im Positiv. Das Lemma steht im Druck recte und ist fett gesetzt. Die Umlaute $\ddot{a}, \ddot{o}, \ddot{u}$ werden in der alphabetischen Anordnung wie $a$, $o$ und $u$ aufgelistet. Lediglich bei Wörtern, die sowohl in nicht umgelauteter Form als auch in umgelauteter Form vorkommen, steht die umgelautete Form nach der nicht umgelauteten Form (Lacher vor Lächer). Wenn gleichberechtigt unterschiedliche Ansatzformen für ein Lemma möglich sind, werden einzelne Buchstaben oder Buchstabengruppen in Klammern gesetzt wie Welsch(e)nu $\beta$ 'Walnuss', das als Lemmaansatz die beiden möglichen Stichwörter Welschnu $\beta$ und Welschenu $\beta$ abbildet. Varianten eines Wortes folgen der Lemmaform im Druck in halbfettem und kursivem Satz (Ütsche, Utsche, $\ddot{U}(t) z e$ 'Kröte'). Bei Homonymen sind Verben, Adjektive und Adverbien dem Substantiv nachgestellt. Die homonymen Substantive stehen in der Reihenfolge: Femininum, Maskulinum, Neutrum. Diminutivformen, die synonym zu einem nicht diminuierten Wort sind, werden in einem gemeinsamen Artikel abgehandelt (Pfühl 'Unterkissen'). Bei Wörtern, die nur eine Pluralform (Pluraliatantum) und Diminutivform haben, werden diese in dieser Form als Lemma angesetzt (Leute, Maßliebchen). Bindestriche werden nur zur Kennzeichnung der Bestandteile eines Wortes (Plepp-pfeife, Sill-läppchen, Sommerhanschen) sowie bei Zusammensetzungen (Simon-Juda-Woche) und fremdsprachlichen Begriffen, die aus mehreren Teilen bestehen (vis-à-vis), gesetzt.

6 Vgl. DWA 8, Karte irdener Topf.

7 Zu Topf und Tüpfen vgl. DWB 11, I.I.: 814-835 (Topf), 846-850 (Töpfen, Tüpfen). 


\subsubsection{Angaben zur Wortform}

Nur bei Substantiven folgt in einer Abkürzung das Genus und bei Pluraliatantum (Mucken 'Schrullen') die Angabe „Pl.“ (für Plural) als zusätzliche Angabe. Bei den anderen Wortarten stehen zusätzliche Angaben nur, wenn dies im Artikel für die Gliederung nötig ist (vgl. weh: I. Adjektiv, II. Adverb, 1. weh tun, a. intransitiv, b. transitiv, c. reflexiv, III. Interjektion).

\subsubsection{Bedeutungsangabe(n)}

Auf der Basis des erhobenen und verzettelten Materials aus Befragungen und der ausgewerteten mundartlichen Literatur in der Zettelkartei wird die Bedeutung angegeben. Sind zwei oder mehr Bedeutungen klar erkennbar, wird das Bedeutungsspektrum eines Wortes semantisch beschrieben und entsprechend gegliedert. Dabei steht im Artikel der am häufigsten belegte Gebrauch zuerst. Die Bedeutungsangabe besteht aus einer möglichst kurzen, den Sachverhalt treffenden Formulierung, die in einfachen Anführungszeichen steht. Sind ausreichend viele Belege vorhanden, stehen Angaben zum Vorkommen und Gebrauch eines Wortes. Fragen aus den Fragebogen, die als Bedeutungsangabe in unveränderter Form in den Artikel übernommen sind (Suggestivformen), werden durch einen Asterisk (*) gekennzeichnet. Zur Gliederung werden Homonyme durch hochgestellte, halbfett gesetzte arabische Ziffern gekennzeichnet. Als erster Artikel steht das Wort mit der gebräuchlichsten Verwendung (Wurf ${ }^{1}$ 'Bewegung und Resultat des Werfens', Wurf ${ }^{2}$ 'Stil der Sense', Wurf ${ }^{3}$ 'Kette des Gewebes'). Bei komplexen Artikeln bilden die Großbuchstaben des lateinischen Alphabets die oberste Gliederungsebene, gefolgt von römischen Ziffern, Kleinbuchstaben des lateinischen Alphabets, arabischen Ziffern und Kleinbuchstaben des griechischen Alphabets wie im Artikel machen: A. I. c. 1. $\alpha-\zeta$ (vgl. HNWb 2: 203-204).

\subsection{Belegauswahl und Beleginterpretation}

Das Wörterbuchmaterial enthält neben den erhobenen Nachweisen des rezenten Wortschatzes für die Zeit von etwa 1850 bis 1920 aus den durchgeführten Befragungen auch Verweise auf die historischen hessischen Dialektwörterbücher vor 1900 sowie Belege aus ausgezettelten Quelleneditionen für ein Wörterbuch, das den historischen Wortschatz des Bearbeitungsgebietes beschreibt. Im bisher bearbeiteten Teil des HNWbs wurden historische Belege vereinzelt nur als Nachweis für das frühe Vorkommen eines Wortes angegeben. Dies erfolgte in den ersten Jahrzehnten in größerem Umfang als in den letzten Jahrzehnten. Ausschließlich historisch belegte Wörter sind nicht ins Wörterbuch aufgenommen worden.

Bei der Belegauswahl bleiben Wörter unberücksichtigt, die nur selten vorkommen und in ihren Bedeutungsangaben nicht über den schriftdeutschen Gebrauch 
hinausgehen, da davon auszugehen ist, dass es sich bei ihnen um keine Dialektwörter handelt, sondern diese aus dem Standarddeutschen übernommen und der regionalen Aussprache angeglichen wurden. Bei selten belegten Wörtern hängt die Aufnahme als Artikel ins Wörterbuch davon ab, wie gut die Bedeutungsangabe ist und ob das Wort mit anderen Präfixen oder als Wortbestandteil in vorhandenen Wörterbuchartikeln bereits vorkommt. Auf der Grundlage der vorhandenen Belege wird die Bedeutung ermittelt und angegeben. Zeigt sich für ein Wort ein Bedeutungsspektrum, werden die Unterschiede in den Verwendungsweisen im Wörterbuchartikel beschrieben. Dabei kommt die verbreitete Bedeutung zuerst, seltenere und vereinzelte in der Folge.

Sammlungen und Beschreibungen regionalen Wortschatzes aus neuerer Zeit (seit dem Zweiten Weltkrieg) werden in Auswahl als Belege mitberücksichtigt, z. B. das 1993 in dritter Auflage erschienene „Mittelhessische Wörterbuch“ von EMIL WINTER. Einige enthalten Beispielsätze, die sehr gut für die Wörterbuchartikel verwendet werden können, wenn sich im Material keine entsprechenden Texte befinden.

Der Bedeutungsangabe folgt in eckigen Klammern eine Angabe zum Vorkommen im Verbreitungsgebiet (z. B. Abend 4.a.). Die ausgewählten Belege im Anschluss daran sollen beispielhaft einerseits die verschiedenen Gebrauchsformen des Wortes in seiner Morphologie und Lautung präsentieren, andererseits die geografische Verbreitung aufzeigen. Diese Beispiele stammen aus den Fragebogen, der ausgewerteten mundartlichen Literatur und ausgewählten regionalen Wörterbüchern und Glossaren. Dies können kurze Sentenzen, ganze Sätze, feste Wendungen sowie Sprichwörter, Kinderreime, Wetterregeln oder Auszüge aus der mundartlichen Literatur sowie aus Gedichten und Liedern sein. Da für das HNWb von Anfang an Wert auf die Erhebung von volkskundlichen und sachgeschichtlichen Dingen gelegt worden ist, sind die Zitate im Belegteil zum besseren Verständnis oft eingebettet in volkskundliche, religiöse und historische Erläuterungen. Auch abgebildete Skizzen (z. B. Webstuhl) und Fotos (z. B. Weise ${ }^{2}$ ) dienen der Veranschaulichung, und Karten bilden wortgeografische und lautliche Verhältnisse innerhalb des Wörterbuchgebiets ab (z. B. weiß). In kleinerem Satz stehen am Ende eines Artikels unter der Überschrift „Formen“ philologische Angaben. Die belegten mundartlichen Formen, die in einer Laienschreibung oder phonetischen Transkription in der Teuthonista vorliegen, werden weitgehend unverändert übernommen. Die Auswahl der präsentierten Formen soll sowohl die verbreiteten als auch die davon im Konsonantismus und Vokalismus abweichenden dialektalen Formen präsentieren. Erläuterungen zum Verbreitungsgebiet, zu lautlichen Entwicklungen und zu etymologischen Angaben schließen sich daran an. Den Schluss bilden die Verweise auf dialektgeografische Untersuchungen und auf das Vorkommen des Wortes in anderen Wörterbüchern, die auch ausführliche Informationen zur Etymologie enthalten. 


\subsection{Verweissystem}

Verweise werden im Wörterbuch bei den Lemmata und innerhalb der Wortartikel gesetzt.

\subsubsection{Lemmata}

Bei den Lemmata wird bei einem einfachen Verweis mit „siehe“ (s.) von einer mundartlich verbreiteten Gebrauchsform eines Wortes (Vockediewes s. Vokativus) sowie bei einer Variante eines Wortes auf das Lemma des Wörterbuchartikels verwiesen, in dem es behandelt wird (Wase, Wäse s. Base), oder auf die genaue Fundstelle im Wörterbuch mit Angabe des Lemmas, des Bandes, der Spalte und Zeile (Wasenlämmchen N. = Maßliebchen (s. 2: 271, 21)). Weichen Lemmaansätze in den älteren hessischen Dialektwörterbüchern ab, wird unter Angabe der Fundstelle des älteren Wörterbuchs der Lemmaansatz im HNWb angegeben (Wassem (Pfister 330) s. Wasen).

Da sich durch die Reform der deutschen Rechtschreibung zwangsläufig Änderungen in der Schreibung ergeben haben, muss von den aktuell gültigen Schreibweisen auf die Artikel in den seit der Veröffentlichung des HNWbs 1943 angesetzten Lemmaformen verwiesen werden (fotografieren s. photographieren).

\subsubsection{Bedeutungsangaben}

Vielfältiger sind die Verweismöglichkeiten im Bereich der Bedeutungsangaben. Neben „siehe“ (s.), „siehe dort“ (s. d.), ,siehe oben“ (s. o.), ,siehe unten“ (s. u.) wird innerhalb des Wörterbuchs mit ,vergleiche“ (vgl.) auf andere Lemmata, sowie auf Wörterbücher und Literatur verwiesen und mit „Abbildung“ (Abb.) auf Karten, Fotos und Zeichnungen.

Im Artikel Zwitzvogel M. steht nach der Bedeutungsangabe 'Schmetterling' ein Verweis auf das Verbreitungsgebiet („Verbreitung s. Bd. 3, Abb. 9“) in der Wortkarte „9. Schmetterling usw. 'Schmetterling (Papilio)'“ (3: 291-292). Als Ergänzung sind vier weitere Belegorte für Zwitzvogel angegeben, in denen diese Bezeichnung neben Schmetterling genannt wurde (,nb. Schmetterling, s. 3, 289, 46ff“). Den Schluss des Artikels bilden Hinweise auf den Artikel Zwitzvogel im ,Idiotikon von Kurhessen“"von A. F. C. VILMAR aus dem Jahr 1868, auf den Artikel Schwitzevogel in Band 3 und den Artikel Zwitzvogel in Band 4 des HNWbs als Varianten: (,nb. Schmetterling; s. 3, 289, 46 ff.; vgl. V. 476, ferner 3, 543, 21 ff. und Zwicksvogel").

Im Artikel Zwirbelmutz als Bezeichnung für den 'Kreisel' als Kinderspielzeug, der nur in einem Ort belegt ist, wird nach einer ganz knappen Bedeutungsangabe auf den noch zu bearbeitenden Hauptartikel Dilltopf verwiesen sowie für die Erklärung der beiden Wortbestandteile Zwirbel und Mutz auf den Artikel Mutz ' 'Stumpf' in Band 2, zwirbelig 'unruhig', 'erregt', 'nervös' und auf Zwurbellatz als Variante 
in der gleichen Bedeutung: („,nb. Dilltopf; s. d.; s. 2, 404, 58 ff., zwirbeln und Zwurbellatz").

Zeichnungen und Fotos aus dem volkskundlichen Bereich konkretisieren Aussehen und Funktion von Geräten, Gegenständen, Kleidungsstücken und veranschaulichen heute in der Regel ausgestorbene Bräuche (s. Kapitel 1). Auf sie verweist wie auch bei den Karten die Abkürzung „Abb“. Im Artikel Platte wird unter der Bedeutungsangabe 8. mit Hilfe von drei Fotos die Stickerei am Frauenstrumpf der Schwälmer Tracht erläutert, die dort als Platte bezeichnet wird. Für die zahlreichen Bräuche an Pfingsten illustriert die Fotografie eines Umzugs durch ein Dorf in der Schwalm die Wörterbuchartikel Pfingstbier, Pfingstbügel und Pfingstmännchen („Abb. 67 Pfingstmännchen, Bd. 2, Sp. 611 f.“).

Sind Beispielsätze und Redensarten bereits in anderen Artikeln als Zitate verwendet worden, wird auf diese Stelle verwiesen wie am Ende des Artikels Abend (,weitere Rdaa. s. Morgengewitter, tagen“).

Vereinzelt finden sich nähere Angaben zum Verbreitungsgebiet wie bei Abend unter 4.a.

\subsubsection{Formen}

Die mundartlichen Formen stehen in kleinerem Satz am Ende eines Artikels. Anschließend folgen Hinweise und Erläuterungen zur Laut-, Wort- und Sprachgeografie, zur Wortbildung und zur Sprachgeschichte mit Verweisen auf die entsprechende für den Artikel benutzte und weiterführende Literatur. Es folgen die Stellenangaben des Wortes in den Wörterbüchern, die an das Gebiet des HNWbs unmittelbar angrenzen, in den historischen hessischen Wörterbüchern und in anderen grundlegenden Wörterbüchern sowie am Schluss Verweise auf andere Artikel im Wörterbuch wie bei dem Beispielartikel Abend (s. Kapitel 2.6).

\subsection{Lautschrift}

Für die Beschreibung der mundartlichen Formen wird als Lautschrift Teuthonista verwendet, soweit sie in Lautschrift im ausgewerteten Material transkribiert vorliegen. Laienschriftliche Belegformen werden auch als solche übernommen und durch die nachgestellte Anmerkung („Laienschr.“) gekennzeichnet. 


\subsection{Beispielartikel, der die aktuellen Artikel optimal repräsentiert:}

Abend M. 1. 'Zeitraum von Sonnenuntergang bis Mitternacht' [im gesamten Wbch.gebiet verbreitet]. Wenn he uns am Abed vom Feld kommen sah, do freute he sich [Friedewald-He: L. F. Werner, Aus einer vergessenen Ecke II, 1912, S. 110], De meste Spaß moichte mir sich off de Owed [Günter.-Bi], Eam Harbst 'Herbst' we(r)n die Oowede lenger ean die Doache ke(r)zer [Niederklein-Ki]. - 2. genauere zeitliche Bestimmungen. - a. allgemein 'am Abend' [Li Kohden$\mathrm{Bu}]$. Des Aweds, wenn ich schlafe gin [Mensfdn.-Li]. - b. 'heute A.' [Gi, Feudgn.-Wi Vasbeck-Tw, Schm Ro He Salzschlirf-Fu]. derre Owed 'diesen A.' [Feudgn.], de 'diesen' Owe(n)t gemer 'gehen wir' fort ens Wertshaus, betont haut, heit 'heute' Owe(n)t [Wsl.]. - c. 'morgen A.' moanse 'morgige' Owed [ebd.], mon frieh 'morgen früh' ze Nowed [Riebelsdf.-Zi]. - d. 'gestern A.' gasdr Owet u. nachds Owet [Obeb.] vgl. 2, 418, 35ff. u. Abb. 47. - e. 'Sonnabend A.' [Obeb.]. Sonoweds Owet [ebd.]. - f. 'heilig A.' [Obeb. Lhsn.]. as lit 'läutet' Haljowet [Obeb.]; der helge Owed 'Samstag' [bei Schotten]. - 3. 'Vortag', der Sonnabend ist der Tag vor dem Sonntag, der Veitsabend (14.6.) ist 'der Tag vor Sankt Veit' (15.6.) (C. 11). - 4. feste Wendungen - a. A. essen 'letzte Tagesmahlzeit' [im nördlichen Hessen: Eifa-Bi Laasphe-Wi Buhlen-Ed Rhena-Ei Burguffeln-Hg Weimar-Ka Lohre-Me Altersb.-Schm]. Mer winn ze Owend esse [Eifa], A. läuten [Wsl. Hesselb.-Wi Etlbn.]. Omend leide [Etlbn.]. 's lait tse owet/owent 'es lăutet den Abend ein' [Wsl.], es läutet heiliger Abend 'es läutet am Tag vor Sonn- oder Feiertagen' [Hesselb.]. - b. als Gruß guten A. [Großen-BuseckGi, Wiss. Mellnau-Ma Gudensbg.-Fr Klschmkd. Salzschlirf]. genowed [Gudensbg.-Fr], gurre Owed [Mellnau-Ma]. Spruch zur Kirchweihe: Haut i $\beta$ Kirb, $i^{e n} m o^{a} r n i^{e} \beta$ Kirb, $i^{m}$ biß Sunndoag Owend, woan aich zoau mei'm Schätzi komm; sahn aich: „Gudenowend, Gudenowend Lißßädsche, saah mer, wo dei" Bättche stieht". „Hinner'm Owe, linkerhand, bei dem griene Vorrang. " [Fauerb. v. d. Hōhe-Fb]: schönen A. [Rotenburg-Ro]. Rda. En schener Ove denove, den möcht ich mäh murgen frih mo bi Tage betrachten [ebd.]. - c. werktags A. 'der werktägliche A. [Großen-Buseck]. So mächd esch de Sondagsowed unn de Wärdagsowed 'so macht er's den Sonntag und den Werktagabend' [ebd]. - 5. Rda. Gott sei Dank, darret Owent eß, der Dag kömt van sälwer [Höhr-Uw], ähnl. Härr, le $\beta$ Owend wänn. Morje wädds von selwer [Schwalm], Herr laoss Aowend wean, meechlichsd noch bei Daoch [Gelnhsn.]; $r$ èas noch nerr aller Doge Owed die endgültige Entscheidung in einer Sache ist noch nicht gefallen' [Feudgn., ähnl. Gelnhsn.], mer deaffn Daoch nedd vorm Aowend lowe eine Sache nicht zu früh loben [Gelnhsn.], De Oowed we(r)n die Faule fleißich 'eine Sache vor sich herschieben bis zuletzt' [Niederklein]; als derbe, abweisende Antwort $D u$ kannst mich mal am A-bend besuchen [Langenseifen-Ut Lgstn. Steinwand-Gf]; weitere Rdaa. s. Morgengewitter, tagen.

Formen: dadwent Friedbg., alwed Dautphe. obend Nothfdn.-Wo, owend (Laienschr.) Buch-Go Kass., owat Niddawitzhsn.-Ew, PL. eweter, neben oweter (Laienschr.) NiederWeisel- $\mathrm{F}$, óawênd Kohden-Bū, ũowend Hemmighsn.-Fk, nowat, PL. nôwador Lhsn., omend Arzb.-Uw, ómd Bieber-Ge; Grußformel gurre Owed (Laienschr.) Mellnau, gundwod Klschmkd. gan awand Großen-Buseck, genowend (Laienschr.) Homburg v.d.H-Ot ganopwad, seltener gunoowad Wiss. - C. 10f. (Abed). - Vgl. Frankfurter Wbch. 1. 116f.. Südhessisches Wbch. 1, 17, 26ff. 6, 1103, Rheinische Wbch. 1, 17, 48ff.. Thüringisches Wbch. 1, 13f., Niedersächsisches Wbch. 1, 137ff. (avend). Westfalisches Wbch. 1, 346ff. (Äwend). Deutsches Wbch. 1, 121, 30ff. - Kluge ${ }^{2}$, 4, Collitz 8 (ôwant), - A. Grassow. Wörterb. d. Kasseler Mda., 1952, S. 62 (Owend), C. Hackler, Feudingen, 1974, S. A 1; O. Koch, Doas win mer nit vergèasse, 2009, S. 262 (Oowed), R. Post, Die Mundart von Bad Salzschlirf, 2013², S. 145 (Aäwed): E. Rückriegel, Niedergründauer Wörterb. ${ }^{2}, 2008$, S. 314 (Oowedd, Oowend), A. Seibig, Gellhâuser Deutsch, 1977, S. 11; E. Winter. Mittelhess, Wōrterb., 1993. S. 15: Wörterb. d. dt. Umgangsspr. 1, S. 32, 2, S. 38 u. 3, S. 29 . Zur Wortgeographie in Hessen zum Ende des 20. Jh.s vgl. Wortatlas zur Alltagsspr. der lāndl. Räume Hessen, 2010. Kt. 166 gestern Abend und zur umfassenden Wortgeographie Deutscher Wortatlas, Bd. 16. Kt. 2: gestern Abend. - S. auf, elınächt, gestern, gut, heilig, Heiligabend, heint, heute, lâuten, Morgen, Nacht, nächte. werktags?

Abb. 7: Artikel Abend abrufbar unter: <https://www.lagis-hessen.de/>; Stand: 14.04.2020. 


\section{TEXTTECHNOLOGISCHE ASPEKTE}

\subsection{Redaktionssystem und technische Grundlage}

Die Bearbeitung der Wörterbuchartikel erfolgt online über das „Landesgeschichtliche Informationssystem Hessen (LAGIS)“. Für das HNWb wurde ein Datenmodell entwickelt, das mehr als vierzig Deskriptoren für Angaben wie Lemma, Genus, Nebenformen, Wortart, Homonymzählung, Bedeutung, Formen usw. umfasst. Dabei stellt die starke Differenzierung eine Voraussetzung für die flexible Handhabung der Datenaufbereitung dar. Bietet sich für Korrekturzwecke etwa eine Darstellung in Listenform an, können für die Druckausgabe individuelle Formatierungsanweisungen für einzelne Deskriptoren oder Gruppierungen derselben ausgegeben werden. Die abschließende Lieferung des vierten Bandes, deren Lemmastrecke Zänkebock-Zyrese formulargestützt in LAGIS bearbeitet wurde, konnte so aus der Datenbank heraus exportiert werden, dass sich die erforderlichen Nachbearbeitungsschritte nahezu vollständig automatisieren ließen. Allein die Kopfzeilen mit Spaltenangaben und Kolumnentiteln mussten noch ergänzt werden.

\subsection{Digitale Daten}

Die datenbankgestützte Bearbeitung des Wörterbuchs und seine Einbindung in ein Informationssystem wie LAGIS bieten zahlreiche Vorteile. Dazu gehört die Bereitstellung zusätzlicher Materialien. So sind in einem von der Leifheit-Stiftung geförderten Drittmittelprojekt die Fragebogen des HNWbs aus konservatorischen Gründen und zur Beschleunigung der Artikelarbeit digitalisiert worden. Sie stehen ab 2019 im „Landesgeschichtlichen Informationssystem Hessen“ (LAGIS) unter <https://www.lagis-hessen.de> (Stand: 14.04.2020) zur Einsicht und Nutzung zur Verfügung. Wünschenswert wäre darüber hinaus die Digitalisierung der handschriftlichen Zettelkartei, die einen Zugriff auf das Gesamtmaterial des HNWbs ermöglicht. Sie bildet das Register zu ausgewerteten handschriftlichen Fragebogen sowie zu den Bänden der Arbeitsbibliothek des Wörterbuchs mit den ausgewerteten gedruckten Quellen und wissenschaftlichen Untersuchungen.

Kaum einer Erwähnung bedarf der Hinweis, dass neben den bisher im Druck erschienenen Wörterbuchartikeln der Buchstaben $L$ bis $Z$ auch die fertigen Artikel aus der ersten Hälfte des Alphabets praktisch unmittelbar nach Abschluss der Bearbeitung online bereitgestellt werden können. Aktuell geschieht dies - beginnend mit der Buchstabenstrecke $A$ - abzwicken - in Form von PDF-Dateien. Die bereits im Redaktionssystem intensiv genutzte Möglichkeit des Zugriffs über eine Volltextsuche soll künftig auch für die Lemmata $A$ folgende allen Nutzerinnen und Nutzern eröffnet werden. Der direkte Zugriff auf die betreffenden elektronischen Wörterbuchartikel wird auch durch die Anbindung an das Trierer Wörterbuchnetz (Onlineplattform des Kompetenzzentrums für elektronische Erschließungs- und Publikationsverfahren in den Geisteswissenschaften an der Universität Trier, unter 
<https://www.woerterbuchnetz.de>; Stand: 14.04.2020) angestrebt. Hierdurch sollen Vergleichsmöglichkeiten zu anderen Dialektwörterbüchern, z. B. zum „Rheinischen Wörterbuch“ und „Südhessischen Wörterbuch“, sowie zu historischen Wörterbüchern geschaffen werden.

\subsection{Publikationsformen}

LAGIS bietet von den bisher zur Verfügung stehenden Lexika und Themen ideale Anbindungsmöglichkeiten für das HNWb. Neben dem „Historischen Ortslexikon“, das fundierte Informationen zu den hessischen Siedlungen bereitstellt, besteht bereits eine Verknüpfung zu den hessischen Flurnamen, die für das gesamte heutige Landesgebiet als Daten vorliegen. Dies bietet eine gegenseitige Nutzungsmöglichkeit insbesondere für Namen und Namenbestandteile, die als Appellative heute nicht mehr in den Dialekten in Gebrauch sind und sich nur noch in Namen erhalten haben. Einen wichtigen Fundus zur Illustration der Beschreibung von Arbeitsvorgängen, Brauchtum oder Gegenständen und Gerätschaften bietet auch das Themenportal „Historische Bilddokumente“ (unter <https://www.lagis-hessen.de/bilddokumente>; Stand: 14.04.2020). Hier kann in den digital zur Verfügung stehenden Artikeln entweder auf ein entsprechendes Stichwort verwiesen werden oder ein Link führt zu einer ausgewählten Fotografie, die den Sachverhalt anschaulich abbildet.

In LAGIS stehen bislang die Wörterbuchartikel von A-abzwicken und Zänkebock-Zyrese als PDF-Datei sowie die im Druck erschienenen Artikel von L-Zankdiviensalat als Retrodigitalisate für Benutzer/-innen zur Verfügung. Die Strecke Accidenzercher-ezet ist zudem bereits als durchsuchbare Wortliste angelegt. Neben einer fortlaufenden Publikation der Wörterbuchartikel in LAGIS ist auch weiterhin ein Druck des Wörterbuches geplant, allerdings nicht mehr in Einzellieferungen, sondern bandweise. Nach der ursprünglichen Planung sollte das „Hessen-Nassauische-Volkswörterbuch“ in zwei Bänden veröffentlicht werden: Band 1 für die Buchstaben $A-K$ und Band 2 für die Buchstaben $L-Z$. Die bearbeiteten Buchstaben $L-Z$ mussten aufgrund des Textumfangs in drei Bänden (2-4) veröffentlicht werden. Daher wird Band 1 in zwei bis drei Teilbänden erscheinen.

\subsection{Texttechnologische Möglichkeiten}

Die elektronische Abfassung der Wörterbuchartikel ermöglicht eine rationellere Vorbereitung und Bearbeitung der einzelnen Wörterbuchartikel und trägt damit zu einer Beschleunigung der Wörterbucharbeit bei. Für die praktische Arbeit bietet die Volltextsuche in den bisher als Datei vorliegenden Wörterbuchteilen insbesondere bei der Suche nach bestimmten Begriffen einen zeitlich und inhaltlich nicht zu unterschätzenden Informationsgewinn. Darüber hinaus hat die Volltextsuche auch für die formale Artikelgestaltung Vorteile, etwa bei der Erstellung des Verweissys- 
tems: Die auf den Zetteln angegebenen Verweise müssen darauf hin überprüft werden, ob sie noch stimmen, oder ob sie im Zuge der Bearbeitung der Hauptartikel, auf den verwiesen werden soll, inzwischen unter einem anderen Lemma zu finden sind. Für Nutzerinnen und Nutzer, die Auskunft zu einem bisher noch nicht bearbeiteten Wort wünschen, und auch eine zitierfähige Quelle angeben möchten, besteht die Möglichkeit, dass dieser Artikel, innerhalb eines relativ kurzen Zeitraums bearbeitet, von einer ständigen Wörterbuchkommission redigiert und - quasi als Wörterbuchartikel ,on demand“ - in LAGIS publiziert wird. ${ }^{8}$

In Arbeit ist derzeit eine elektronische Ortspunktkarte für das gesamte Wörterbuchgebiet nach dem Gebietsstand von 1914 mit der preußischen Provinz HessenNassau und der hessischen Provinz Oberhessen. Mit Hilfe der Ortspunktkarte besteht die Möglichkeit, projektierte Sprachkarten zu erstellen, die sowohl das gesamte Arbeitsgebiet betreffen als auch Kartenausschnitte, die regional begrenzte Phänomene abbilden. Sie sollen auch die Grundlage für die Karten bilden, die später im Druck im ersten Wörterbuchteil von $A-K$ erscheinen. Über das SprachGIS der Online-Plattform <http://www.regionalsprache.de> (Stand: 14.04.2020) soll es zudem möglich sein, die Ortskarte mit sprachlichen und außersprachlichen Informationen, zum Beispiel mit Dialektgrenzen und alten Verkehrswegen, zu überblenden.

Die Bearbeitung des bisher unbearbeiteten Wörterbuchmaterials in elektronischer Form bietet durch die kontinuierliche Bearbeitung und die unmittelbare Publikation nach der Fertigstellung der Artikel für die Nutzerin / den Nutzer den Vorteil, dass ihr / ihm schneller die gewünschten Wörterbuchartikel in einer zitierfähigen Weise zur Verfügung stehen. Bis zum Druck der fertigen Artikel besteht auch immer noch die Möglichkeit, dass Fehler korrigiert und wichtige Informationen oder neue Untersuchungen ergänzt werden können.

\section{LINGUISTISCHES POTENTIAL}

Wie erwähnt beinhaltet der Materialbestand des HNWbs neben lexikographischen und lexikologischen Informationen mitunter auch Angaben zur Phonetik und Phonologie, zur Flexions- und Derivationsmorphologie, zur Syntax und zum Sprachgebrauch. So wurden in den 79 Fragebogenrunden unter anderem Fragen zur Aussprache, zum Genus („Aussprache und Geschlecht von Markt", Fragerunde 1) und zur Movierung (,,Spricht man von einer Frau namens Schneider als der Schneiderschen, der Schneidern, oder wie sonst?“, Fragerunde 5) gestellt, sowie Redensarten („Redensarten, die an das Getreide anknüpfen“, Fragerunde 14), Sprachgebrauch (,Scherzhafte Benennungen der Zigarre“, Fragerunde 27) und auch volkskundliche Informationen (,Was weiß der dortige Volksglaube noch vom Werwolf“, Fragerunde 15) erhoben. Doch gibt es bislang keine systematischen lin-

8 Stand April 2020 gehören zur ständigen Wörterbuchkommission: FRIEDHELM DEBUS, KURT GäRTNER (Vorsitz), RUDOLF POST und HANS RAMGE. 
guistischen Auswertungen der Fragebogen, was insofern bedauerlich ist, als zahlreiche der abgefragten Phänomene im Erhebungsgebiet arealbildend zu sein scheinen. Für die Mehrzahl der verzettelten Lemmata stehen darüber hinaus (in Teuthonista teils feinphonetisch transkribierte) Formen- und auch Satzbelege zur Verfügung. Hieraus lassen sich etwa Informationen zur Verbvalenz, zur Numerus- und Kasusmorphologie, zu Kongruenzerscheinungen sowie zur Wortstellung gewinnen. Die Daten zu dem nachfolgend diskutierten Phänomen, der Verwendung des neutralen Genus bei weiblichen Rufnamen, stammt aus Fragebogenrunde 29. Die Frage „Heißt es echt mundartlich die oder das (bzw. dat) Marie, Lisewitt usw.“ wurde von insgesamt 73 Gewährspersonen aus ebenso vielen Orten im Erhebungsgebiet beantwortet. ${ }^{9}$ Abbildung 8 weist die areale Verteilung der typisierten Varianten die oder das (bzw. dat) aus. Es lässt sich eine deutliche Zweiteilung des Erhebungsgebietes in der Art feststellen, dass im Norden und Westen ausschließlich neutrale Kongruenzformen verwendet werden (Ausnahme: Kassel), während im Süden, in der Mitte und im Osten die (standardkonforme) feminine Variante zu verzeichnen ist. Die Isoglosse, die das Areal mit neutralem vom femininen Genus trennt, verläuft von Südwesten nach Nordosten annähernd diagonal durch Hessen und stimmt damit weitgehend mit der Verteilung überein, wie sie LESER-CRONAU (2018) für die Genuskongruenz bei Referenz auf weibliche Personen im Rahmen der rezenten Erhebungen des „Syntaktischen Atlas hessischer Dialekte“ bestimmen konnte und wie sie sich auch durch die Erhebungen des „Atlas zur deutschen Alltagssprache“ (ADA, Frage 2e) andeuten. Weitergehende, insbesondere die Pragmatik betreffende Aussagen zum Phänomen (dazu NÜBLING / BuSLEY / DRENDA 2013), lassen sich aufgrund der vorliegenden Daten nicht treffen, doch weisen einzelne Antworten der Gewährspersonen beispielsweise darauf hin, dass die Wahl der Kongruenzform vom Namentyp abhängig sein könnte (Rufnamen mit neutralem, Familiennamen und Gesamtnamen mit femininem Genus) und die neutrale Kongruenzform in Teilen Hessens bereits zu Beginn des 20. Jahrhunderts von der (standardkonformen) femininen Variante verdrängt wurde.

9 Zwei Antworten, aus Niederzell und Bergen, sind uneindeutig und gingen nicht mit in die Auswertung ein. 


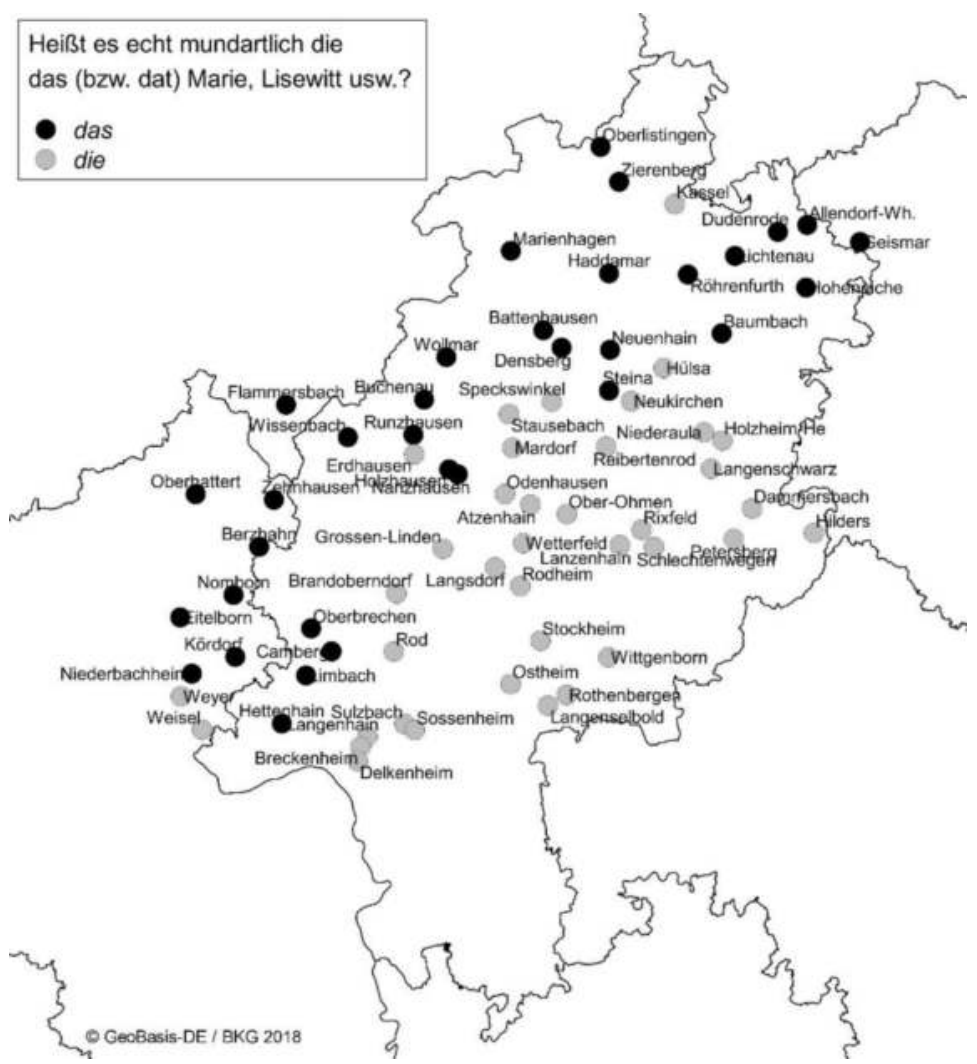

Abb. 8: Areale Verteilung der neutralen und femininen Kongruenzformen bei definitem Artikel vor weiblichem Rufnamen

\section{KONTAKTADRESSE, HOMEPAGE, WICHTIGE LINKS}

Forschungszentrum Deutscher Sprachatlas

Hessen-Nassauisches Wörterbuch

Pilgrimstein 16

35032 Marburg

Deutschland

E-Mail:

stefan.aumann@uni-marburg.de bernd.vielsmeier@uni-marburg.de

alexander.werth@uni-marburg.de

Homepage: <https://www.lagis-hessen.de/de/subjects/index/sn/hnwb> 


\section{LITERATURVERZEICHNIS}

ADA = ElSPASS, STEPHAN / ROBERT MÖLLER (Hg.): Atlas zur deutschen Alltagssprache. Unter: <https://www.atlas-alltagssprache.de>; Stand: 14.04.2020.

BERTHOLD, LUISE (1943): Vorwort. In: Hessen-Nassauisches Volkswörterbuch aus den für ein Hessen-nassauisches Wörterbuch von FERDINAND WREDE angelegten und verwalteten Sammlungen ausgewählt und bearbeitet von LUISE BERTHOLD. II. Band: $L-R$. Marburg: Elwert, III-VII.

BERTHOLD, LUISE (1955): Das wortgeographische Prinzip in den deutschen Mundartwörterbüchern. In: Orbis 4, 415-427.

BERTHOLD, LUISE (1965): Hessen-Nassauisches Wörterbuch. In: Zeitschrift für Mundartforschung $32,145-147$.

Chroniken der Philipps-Universität Marburg. Marburg.

Duden $=$ DROSDOWSKI, GÜNTHER (Hg.) (1976-1981): Duden. Das große Wörterbuch der deutschen Sprache in sechs Bänden. Hg. und bearb. vom Wissenschaftlichen Rat und den Mitarbeitern der Dudenredaktion unter Leitung von GÜNTHER DrosDOwSKI. Mannheim [u. a.]: Dudenverlag.

DWA = MitZKa, WALTHER / LudWIG ERICH SCHMitT (Hg.): Deutscher Wortatlas. Bd. 22. Ortsregister und Ergänzungskarten. Gießen: Schmitz.

DWB = GRIMM, JACOB / WILHELM GRIMM (Hg.) (1854-1961): Deutsches Wörterbuch. 16 Bde. in 32 Teilbänden. Leipzig: Hirzel. Quellenverzeichnis Leipzig 1971. ( $\left.{ }^{1} \mathrm{DWB}\right)$; Deutsches Wörterbuch von JACOB GRIMM und WILHELM GRIMM. Neubearbeitung. Hrsg. von der Akademie der Wissenschaften der DDR/Berlin-Brandenburgischen Akademie der Wissenschaften zu Berlin in Zusammenarbeit mit der Akademie der Wissenschaften zu Göttingen (1983-2006). Bd. 1-9. Leipzig und Stuttgart: Hirzel. $\left({ }^{2} \mathrm{DWB}\right)$.

Forschungsberichte des Forschungsinstituts für Deutsche Sprache „Deutscher Sprachatlas“. Marburg.

FRIEBERTSHÄUSER, HANS (1976): Hessen-Nassauisches Volkswörterbuch. In: FRIEBERTSHÄUSER, HANS (Hg.): Dialektlexikographie. Berichte über Stand und Methoden deutscher Dialektwörterbücher. Stuttgart: Franz Steiner (Zeitschrift für Dialektologie und Linguistik. Beihefte. 17), 91-103.

GRIMMsches Wörterbuch s. DWB

Idiotikon von Kurhessen = VILMAR, AUGUST FrIEDRICH ChristiAn (1868) (Hg.): Idiotikon von Kurhessen. Marburg [u. a.]: Elwert.

KLUGE, FRIEDRICH (2011): Etymologisches Wörterbuch der deutschen Sprache. 25. Aufl. Bearb. von ElMAR SEEBOLD. Berlin/New York: de Gruyter.

KNOOP, UlRICH / WOLFGANG PUTSCHKE / HERBERT ERNST WIEGAND (1982): Die Marburger Schule: Entstehung und frühe Entwicklung der Dialektgeographie. In: BESCH, WERNER / ULRICH KNOOP / WolfGAng PUTSCHKE / ERNST HERBERT WIEGAND (Hg.): Dialektologie. Ein internationales Handbuch zeitgenössischer Forschung. Bd. 1. Berlin [u. a.]: de Gruyter (Handbücher zur Sprach- und Kommunikationswissenschaft. 1.1), 38-92.

KÖNIG, WERNER (2011): dtv-Atlas deutsche Sprache. 17. Aufl. München: dtb.

LAMELI, ALFRED (2014): Schriften zum Sprachatlas des Deutschen Reichs. Bd. 3. Erläuterungen und Erschließungsmittel zu Georg Wenkers Schriften. Hildesheim [u. a.]: Olms (Deutsche Dialektgeographie. 111.3).

Lagis. URL: <https://www.lagis-hessen.de/>; Stand: 19.08.2019.

LeSER-CronaU, StePHANIE (2018): Neutrale Kongruenzformen für Personen. In: Fleischer, JÜrg / AleXandra N. Lenz / Helmut Weiss (Hg.): SyHD-Atlas. Marburg [u. a.] DOI: dx.doi.org/10.17192/es2017.0003.

Mittelhessisches Wörterbuch = WINTER, EMIL (1993): Mittelhessisches Wörterbuch: auf Grund des Gießener Landes zusammengestellt. 3. Aufl. Heuchelheim: Kulturring.

Niedersächsisches Wörterbuch. (1965-2017). Bd. 1-9. Neumünster: Wachholtz Verlag. 
NÜBling, DAmaris / Simone BuSley / JUliane Drenda (2013): Dat Anna und s Eva - Neutrale Frauenrufnamen in deutschen Dialekten und im Luxemburgischen zwischen pragmatischer und semantischer Genuszuweisung. In: Zeitschrift für Dialektologie und Linguistik 80, 152-196.

Regionalsprache.de. URL: <https://www.regionalsprache.de>; Stand: 19.08.2019.

Rheinisches Wörterbuch. Auf Grund der von FRANCK, J. begonnenen, von allen Kreisen des rheinischen Volkes unterstützten Sammlung bearb. u. hrsg. v. JOSEF MÜLLER (1928-1971). Bd. 1-9. Bonn: Klopp.

Sitzungsberichte der Preussischen Akademie der Wissenschaften. Philosophisch-historische Klasse (1922-1938). Berlin: Verlag der Akademie der Wissenschaften.

Südhessisches Wörterbuch. Begründet v. FRIEDRICH MAURER. Bearbeitet v. RUDOLF und ROLAND MulCH (1965-1985). Bd. 1-4. Marburg: Elwert.

SyHD = FLEISCHER, JÜRG / ALEXANDRA N. LENZ / HELMUT WEISS (2017): SyHD-atlas. Konzipiert von Ludwig M. Breuer. Unter Mitarbeit von Katrin KuHMichel, StePhanie LeserCronau, Johanna SchWAlm und Thomas Strobel. Marburg [u. a.].

Thüringisches Wörterbuch. Bearbeitet unter Leitung v. KARL SPANGENBERG (1974-2006). Bd. 16. Berlin: Akademie.

VILMAR, AUGUST FRIEDRICH CHRISTIAN (Bearb.) (1868): Idiotikon von Kurhessen. Marburg und Leipzig: Elwert.

Westfälisches Wörterbuch. Hrsg. von der Kommission für Mundart- und Namenforschung des Landschaftsverbands Westfalen-Lippe. Bearb. von HERMANN NIEBAUM, HANS TAUBKEN, PAul TeEPE u. FeliX WorTMANN (2015-2018). Bd. 1-4. Kiel/Hamburg: Wachholtz.

WiEsInger, PETER (1983): Die Einteilung der deutschen Dialekte. In: BESCH, WERnER / ULRICH KnOOP / WolfGang PutschKe / Herbert ERnST WiEGAND (Hg.): Dialektologie. Ein internationales Handbuch zeitgenössischer Forschung. Berlin [u. a.]: de Gruyter (Handbücher zur Sprach- und Kommunikationswissenschaft. 1.2), 807-900.

WINTER, EMIL (1993): Mittelhessisches Wörterbuch, auf Grund der Mundart des Gießener Landes zusammengestellt. 3. Aufl. Heuchelheim: Winter. 



\title{
DAS WESTFÄLISCHE WÖRTERBUCH (WWB)
}

\author{
Robert Damme
}

Das „Westfälische Wörterbuch“ (im Folgenden: WWb), herausgegeben von der Kommission für Mundart- und Namenforschung des Landschaftsverbandes Westfalen-Lippe, dokumentiert den Wortschatz der niederdeutschen Mundarten in Westfalen-Lippe und Nordhessen. Von dem in seiner Anlage am „Rheinischen Wörterbuch“ (RhWb) orientierten, im Wachholtz Verlag (Kiel/Hamburg) erscheinenden, auf fünf Bände angelegten und etwa 100000 Wortartikel umfassenden Werk liegen aktuell $^{1}$ vier Bände ( $A$ bis $S k$ ) vor; der Abschluss des fünften und letzten Bandes ist für Mitte 2021 geplant.

\section{GESCHICHTLICHER ABRISS}

\subsection{Gründung, institutionelle Einbindung, Stellensituation}

Das im Laufe des 19. Jahrhunderts gestiegene Interesse an einem Wörterbuch der westfälischen Mundarten machte sich Anfang des 20. Jahrhunderts die Historische Kommission für die Provinz Westfalen zu eigen und nahm 1906 die Bearbeitung eines Wörterbuchs in ihr Programm auf. Für die erste flächendeckende Erhebung mundartlichen Materials zeichnet THEODOR BAADER verantwortlich, der 1922 einen (auch in den „Heimatblättern der Roten Erde“, dem Organ des Westfälischen Heimatbundes, veröffentlichten) umfangreichen Fragebogen (mit 500 Fragen) verschickte und bis April 1924224 Antworten erhielt.

Nach BAADERS Berufung als Professor nach Nijmegen im Jahr 1923 und dem damit verbundenen Verlust eines Großteils des Materials für den Standort Münster bemühte man sich in Westfalen um einen Neuanfang. Dieser kann auf den 1. April 1927 datiert werden: An diesem Tag wurde ERICH NÖRRENBERG als erster hauptberuflicher Mitarbeiter des Wörterbuchs, genauer: als „Verwalter des Wörterbucharchivs" eingestellt. Die finanzielle Hauptlast (neben einem durch die Provinzverwaltung Westfalens übernommenen Zuschuss) trug die Preußische Akademie der Wissenschaften (bis 1945), die das (in Abgrenzung zu BAADERS Wörterbuch zunächst so genannte) „Westfälische Provinzial-Wörterbuch“ 1927 in die Reihe der von ihr geförderten Wörterbücher (neben dem „Rheinischen“ und dem „HessenNassauischen"u. a.) aufnahm. 
NÖRRENBERG, durch eine Dissertation über das westfälische Diminutiv als Kenner der westfälischen Mundarten ausgewiesen, hatte 1926 im Auftrag des Westfälischen Heimatbundes die Aufgabe übernommen, das 1882 posthum erschienene und seit Langem vergriffene „Wörterbuch der westfälischen Mundart“ ${ }^{\text {‘2 }}$ von FRIEDRICH WoEste zu überarbeiten und neu herauszugeben. Die Neuausgabe sollte den Sammlerinnen und Sammlern für das neue große Wörterbuch als Hilfsmittel dienen. In der Einleitung der 1930 erschienenen Neubearbeitung heißt es dann auch (WOESTE 1930, Einleitung: 5 [nicht gezählt]):

\begin{abstract}
Aber nicht nur Iserlohn ist zu kurz gekommen, sondern auch andre westfälische Gegenden, und zwar - wie eben schon angedeutet - diese in weit stärkerem Maße. Auch wird mancher Benützer an vielen Stellen des Wörterbuches die Stirne kraus ziehen: „So sprechen wir aber das Wort gar nicht aus!“ oder: „Bei uns bedeutet dieser Ausdruck aber etwas ganz andres!“ Auf solche Einwände ist der Herausgeber nicht nur gefaßt, nein, er erhofft sie und bittet, alle solche Ergänzungen mit genauer Angabe, wie das Wort richtig heißt und was es wirklich bedeutet und in welchem Ort es so vorkommt, einzusenden an die Geschäftsstelle des Westfälischen Provinzial-Wörterbuches, Münster i. W., Domplatz 20, wo jede solche Angabe ein Beitrag wird zu einem viel umfangreicheren Werk, das den gesamten Wortschatz aller niederdeutschen Mundarten Westfalens und der westfälisch sprechenden Grenzlandschaften umfassen soll.
\end{abstract}

Institutionell eingebunden war das Wörterbuch seit 1928 in die in diesem Jahr gegründete Volkskundliche Kommission für die Provinz Westfalen, die neben der Sammlung volkskundlichen Materials die Bearbeitung und Herausgabe des Wörterbuchs als Hauptaufgabe übernahm. Durch einen Vertrag vom 28. Februar 1931 zwischen der Preußischen Akademie der Wissenschaften und der Volkskundlichen Kommission wurde das WWb als gemeinsames Unternehmen der beiden Vertragspartner ausgewiesen.

Ab 1945 fielen die Zuschüsse der Preußischen Akademie durch deren Auflösung weg. Die Verwaltung des damaligen Provinzialverbandes Westfalen und heutigen Landschaftsverbandes Westfalen-Lippe (LWL) übernahm die gesamten sachlichen Ausgaben und richtete 1947 eine neue Stelle für einen wissenschaftlichen Mitarbeiter ein, die mit FELIX WORTMANn besetzt wurde. Seit Juni 1955 stellte die Arbeitsgemeinschaft für Forschung des Landes Nordrhein-Westfalen (NRW) Mittel für die Einstellung eines weiteren wissenschaftlichen Mitarbeiters und zweier studentischer Hilfskräfte bereit, beendete aber 1981 wegen des nur schleppenden Publikationstempos auf Empfehlung der Rheinisch-westfälischen Akademie der Wissenschaften die Förderung.

2 WOESTES posthum erschienenes Wörterbuch (WOESTE 1882) verzeichnet Wortschatz aus dem märkischen Sauerland sowie den angrenzenden, aber nicht-westfälischen bergischen und siegerländischen Mundarten; außerdem enthält es zahlreiche in der Mundart unbekannte Urkundenwörter. Hingegen fehlen vor allem nordwestfälische Ausdrücke. Es ist also eigentlich ein Wörterbuch des märkischen Sauerlands und angrenzender Gebiete. Der Titel „Wörterbuch der westfälischen Mundart" geht nicht auf WOESTE selbst zurück, sondern auf den herausgebenden „Verein für niederdeutsche Sprachforschung“, der mit diesem Titel wohl mehr Käufer locken wollte. Vgl. hierzu WOESTE (1930, Einleitung: 4-5 [nicht gezählt]). - Bei der Überprüfung und Ergänzung seiner Vorlage hat NÖRRENBERG besonderen Wert auf die Mundart seines Heimatortes Iserlohn gelegt, sodass die Neubearbeitung auch als Wörterbuch der Iserlohner Mundart angesehen werden kann; vgl. hierzu WOESTE (1930, Einleitung: 4 [nicht gezählt]). 
Seit 1972 gehört das WWb zum Aufgabenbereich der aus der Volkskundlichen Kommission hervorgegangenen Kommission für Mundart- und Namenforschung Westfalens (KoMuNa), einer von heute sechs durch den LWL geförderten wissenschaftlichen Kommissionen für westfälische Landeskunde. Bis 1974 stockte der LWL die Förderung auf insgesamt drei von ihm finanzierte Stellen auf, sodass das WWb von 1974 bis 1981 vier hauptamtliche Mitarbeiter hatte. Zehn Jahre nach dem Ausstieg des NRW-Wissenschaftsministeriums hatte das WWb zwei weitere Stellen verloren: 1989 wurde eine freigewordene Stelle zu einer anderen landeskundlichen Kommission abgezogen; und 1990 wechselte einer der beiden verbliebenen Mitarbeiter in die Geschäftsführung der KoMuNa, sodass nur noch ein hauptamtlicher Mitarbeiter (ROBERT DAMME) mit der Bearbeitung des WWb betraut war. An dieser Stellensituation hat sich bis heute nichts geändert.

\subsection{Publikation}

Am 10. August 1967, 40 Jahre nach der Gründung des WWb-Archivs im April 1927, wurde mit dem Wachholtz Verlag in Neumünster ein Vertrag abgeschlossen, in dem die Rahmenbedingungen für die Publikation festgelegt sind: Das Wörterbuch sollte fünf Bände à 1280 Spalten bzw. 640 Seiten umfassen und innerhalb von 25 Jahren fertiggestellt werden. Damit war das WWb in Bezug auf Umfang und Zeitrahmen klar definiert. Allerdings sei an dieser Stelle angemerkt, dass der Zeitrahmen bei damals nur zwei Mitarbeitern, die sich zwar in der westfälischen Mundartlandschaft hervorragend auskannten, aber noch keine Erfahrung im Artikelschreiben hatten, allzu optimistisch angesetzt war.

Als 1973 die erste Lieferung des WWb erschien, ging deren Autor FELIX WORTMANN in einem Zeitungsinterview noch von den im Verlagsvertrag genannten 25 Jahren aus, in denen das Wörterbuch abgeschlossen werden könnte. Die Voraussetzungen zur Erfüllung dieses Vorhabens hatten sich inzwischen deutlich verbessert, da sich die Personalausstattung bis Mitte der 1970er Jahre von zwei auf vier Mitarbeiter verdoppelt hatte. Doch es kam zunächst ganz anders. Die Geschichte der Publikation lässt sich in drei Phasen einteilen.

Für die erste Phase steht der erste Band, der seit 1973 in Lieferungen erschien. Die Arbeiten kamen nur schleppend voran. In den ersten Jahren wurde alle zwei Jahre eine Lieferung publiziert, später alle drei Jahre. Bis 1991 waren von den Bearbeitern des 1. Bandes (Hermann Niebaum, Hans TAubken, Paul TeEPE und FELIX WORTMANN) lediglich neun Lieferungen bis Bra- erschienen. Die Lieferungen zehn bis zwölf besorgte HERMANN NIEBAUM, der bis 1984 beim WWb gearbeitet hatte, im Anschluss an seine Pensionierung im Jahr 2010. Dabei orientierte er sich streng an der in den ersten neun Lieferungen praktizierten Vorgehensweise. 2015 konnte er den ersten Band $(A-C)$ abschließen.

Für die zweite Phase (hierzu DAMME 1997) steht der zweite Band: Da seit 1990 nur noch ROBERT DAMME als Bearbeiter zur Verfügung stand, wurde 1994 für die restlichen Bände eine Neukonzeption beschlossen, von der man sich eine 
Beschleunigung des Publikationstempos erhoffte. ${ }^{3}$ Diese sieht unter anderem - wie ursprünglich geplant ${ }^{4}$ - eine Beschränkung auf insgesamt fünf Bände, eine Verkleinerung des Bearbeitungsgebietes (vgl. Kapitel 2) und eine Vereinfachung der Verbreitungsangaben (vgl. Kapitel 4.5) vor. Der erste nach diesem Konzept erstellte Band ist der im Jahr 2011 abgeschlossene Band $2(D-G)$, dessen erste Lieferung im Jahr 1997 erschienen ist.

Das WWb ist ab dem zweiten Band in erster Linie ein Bedeutungswörterbuch, das neben der Form des mundartlichen Wortes vor allem dessen Bedeutung(en) beschreibt. Weitere zusätzliche, im Zettelarchiv vorhandene Angaben (volks- und sachkundlicher $u$. ä. Art) fließen nur noch in geringem Umfang in das Wörterbuch ein. Wem die im gedruckten Wörterbuch veröffentlichten Informationen nicht ausreichen, der kann das Archiv des WWb bei der KoMuNa in Münster einsehen.

Für die dritte Phase (hierzu DAMme 2013) stehen die Bände 3 bis 5: Nach einer Hochrechnung auf der Grundlage des zweiten Bandes war ein Abschluss des WWb nicht vor $2040 \mathrm{zu}$ erwarten. Anfang des Jahrtausends mit der Kulturverwaltung des LWL geführte Gespräche ließen befürchten, dass bei dieser Aussicht die Finanzierung des WWb durch den LWL auslaufen könnte. Unter diesen Voraussetzungen entschloss sich DAMME 2008, das Wörterbuch ohne Änderung der 1994 beschlossenen Konzeption als zeitlich befristetes Projekt zu organisieren, das 2020 mit seinem Renteneintritt abgeschlossen sein soll. Das bedeutete eine Steigerung um das Drei- bis Vierfache des bisherigen Publikationstempos. Durch Verbesserungen der Rahmenbedingungen (Befreiung des Bearbeiters von allen Tätigkeiten, die nichts mit dem WWb zu tun haben; Gewährung weiterer studentischer Hilfskräfte u. Ä.) sowie radikale Veränderungen in den Arbeitsabläufen gelang es, dieses überaus ambitionierte Vorhaben in die Tat umzusetzen. Ohne nochmalige Modifikation des Wörterbuchkonzepts erschienen 2015 der dritte Band $(H-L)$ und 2018 der vierte Band $(M-S k)$; der Abschluss des fünften Bandes $(S l-Y)$ ist für Mitte 2021 geplant.

Seit Mai 2009 steht dem Bearbeiter DAMME ein Team, bestehend aus einer wissenschaftlichen Volontärin bzw. einem wissenschaftlichen Volontär (mit 75-80 \% der Arbeitszeit) sowie drei bis vier studentischen Volontärinnen, zur Seite, das ihn von zahlreichen Aufgaben, die beim Artikelschreiben zu erledigen sind, entlastet: ${ }^{5}$

3 Vom 28. bis 30. September 1994 fand im Landhaus der Westfälischen Wilhelms-Universität Münster zu Wettringen (Kreis Steinfurt) eine Klausurtagung mit dem Ziel statt, eine Konzeption zu erarbeiten, mit der die Publikation des Wörterbuchs wieder in Gang kommen konnte. Neben den Vertretern der KoMuNa (JAN GoOsSEns, Hans TAUbKEN, GunTER MÜLleR, RoBERT DAMME) nahmen teil ELISABETH PIIRAINEN, HERMANN NIEBAUM und LUDGER KREMER als sachkundige Mitglieder der Kommission sowie als Bearbeiter bzw. Bearbeiterin anderer großlandschaftlicher deutscher Dialektwörterbücher: ULRICH SCHEUERMANN („Niedersächsisches Wörterbuch“), MARTIN SCHRÖDER („Preußisches Wörterbuch“), RENATE HERRMANNWINTER („Pommersches Wörterbuch“), JOACHIM WIESE („Berlin-Brandenburgisches Wörterbuch“) und RUDOLF POST („Pfälzisches Wörterbuch“). Es wurden grundsätzliche Vorgaben für eine vereinfachte, ab dem zweiten Band geltende Konzeption formuliert.

$4 \quad$ Vgl. den ersten Absatz von Kapitel 4.6.

5 Zwischen März 2009 und Februar 2019 gehörten die wiss. Volontärinnen MAREN BRAUN, AnNa FAnKhauser, Jennifer StaAR, CONSTANZE Wellendorf, der wiss. Volontär John 
Zum einen werden die Zettel in den Archivkästen nach bestimmten Kriterien markiert und vorsortiert, zum anderen wird durch Abschrift des Archivs eine Rohfassung des Wörterbuchtextes erstellt, die als Grundlage für die weitere redaktionelle Bearbeitung dient.

Um das Team für diese Aufgabe zu schulen und für die lexikografischen Probleme zu sensibilisieren, findet alle vierzehn Tage eine Wörterbuchsitzung statt, in der komplexere Artikel besprochen werden, die gerade in der Wortschatzstrecke eines Teammitglieds anstehen. Behandelt werden die Unterscheidung und Systematisierung der Bedeutungen sowie die Zuordnung der Verwendungsbeispiele und idiomatischen Wendungen. Die vom Team erstellte umfangreiche Rohfassung gleicht einem vorgeformten Holzklotz, den der Bildhauer noch in die endgültige Form bringen muss. Die Überarbeitung durch den Bearbeiter erfolgt unter Einbezug des Archivs. Jeder Archivzettel geht durch seine Hand; das heißt: Der vorbereitete bzw. schon leicht überarbeitete Artikel wird anhand des Archivs kontrolliert, verbessert, ergänzt oder gekürzt. Während der Text in den weniger umfangreichen, nicht selten nur auf einem Zettel beruhenden Wortartikeln oft übernommen werden kann, findet bei den umfangreicheren Wortartikeln eine umfassende Prüfung und in der Regel auch eine mehr oder weniger intensive Modifizierung des Entwurfs statt. Dies betrifft alle Bereiche. Zunächst wird sichergestellt, dass das Stichwort ${ }^{6}$ stimmt und dass nur Belege und Bedeutungsangaben aufgenommen werden, die auch zum betreffenden Stichwort gehören. Fallen hier Korrekturen an, müssen möglicherweise die Angaben zur Verbreitung und zur Bedeutung angepasst und Verwendungsbeispiele ausgetauscht werden. Sowohl bei Bedeutungsangaben als auch bei Verwendungsbeispielen wird kontrolliert, ob die Angaben auf den Zetteln richtig gelesen worden sind. Von den Zetteln übernommene Bedeutungserläuterungen sind oftmals bezüglich Formulierung und Wortwahl nicht geeignet, in einem Wörterbuch wiedergegeben zu werden, etwa, weil sie sich als nicht eindeutig erweisen oder nicht mehr gebräuchlichen Wortschatz enthalten. Es kommt auch vor, dass bestimmte Bedeutungen nicht erfasst worden sind. In all diesen Fällen muss nachgebessert werden. Zuweilen erweist sich auch die Auswahl der zitierten Beispiele und Redensarten als nicht besonders glücklich, weil sie etwa eine eher unübliche Verwendung des Wortes wiedergeben oder weil ein bestimmter Wortgebrauch in mehreren Varianten aufgenommen ist, während andere, wichtigere Belege fehlen. Auch wenn diese Überarbeitung mitunter recht zeitaufwändig ist, hat das Erstellen von Artikelentwürfen durch das Team doch eine enorme Entlastung für den Bearbeiter mit sich gebracht und so eine deutliche Beschleunigung der Publikation bewirkt.

Sturm, die stud. Volontärinnen LuISA BIER, DOROTHEE VON BRACHEL, JANA CORDES, SiNA Hub, VANessa Kossowski, Roswitha Meyer, AnNa Möllers, Nicole SCHLenKe, Marina Oeldemann, ANNIKa Pletowski, Melanie Reinelt, Bettina Schmidt, Linda Schwarzl und JULIa TwIEHOFF sowie die Praktikantin ViKTORIYa DukHNOva dem Team an. 


\section{BEARBEITUNGSGEBIET}

Ursprünglich wurde das Bearbeitungsgebiet des WWb durch einen historischen Westfalenbegriff bestimmt, der gemäß dem Beiband (1969: 16) folgende Gebiete umfasst:

die frühere Provinz Westfalen mit dem Land Lippe, also das Gebiet des heutigen Landschaftsverbandes Westfalen-Lippe, mit Ausnahme der Kreise Siegen und Wittgenstein, in denen hochdeutsche Mundarten gesprochen werden; weiter von der Rheinprovinz die ,westfälischen Randgebiete im Oberbergischen Kreis und in den Stadtgebieten von Essen und Oberhausen. Im Südosten wurde der niederdeutsche Teil von Waldeck und des Kreises Wolfhagen mit hinzugenommen. Im Nordosten sind die Kreise Grafschaft Schaumburg und Schaumburg-Lippe, dann der südliche Teil des Kreises Nienburg berücksichtigt worden. Im Norden gehören die südoldenburgischen Kreise Vechta und Cloppenburg (ohne das friesische Saterland) und der ganze Regierungsbezirk Osnabrück dazu, so dass der Wörterbuchraum im Norden bis nach Papenburg reicht.

Die Randgebiete im Südwesten fallen in das Bearbeitungsgebiet des RhWb, die hessischen in das des Hessen-Nassauischen Volkswörterbuchs (HnVwb) und alle nördlichen in das des Niedersächsischen Wörterbuchs (NdsWb), mit dem es die weitaus größte Überlappung gibt. Ab dem zweiten Band werden die bereits im $\mathrm{RhWb}$ und im NdsWb dokumentierten Mundarten nicht mehr berücksichtigt. Lediglich das niederdeutschsprachige Gebiet des hessischen Landkreises WaldeckFrankenberg wird weiterhin mitbearbeitet.

Das WWb definiert sich ab dem zweiten Band also weitgehend als ein Wörterbuch der niederdeutschen Mundarten in Westfalen-Lippe und Hessen. Sein Bearbeitungsgebiet erstreckt sich somit im Süden bis zur Lautverschiebungsgrenze, im Osten und Norden bis zur niedersächsischen Landesgrenze, im Nordwesten bis an die Staatsgrenze zu den Niederlanden und schließlich im Westen bis zum Rheinland.

\section{ARCHIV}

Das Archiv des WWb mit seinen etwa 1,5 Millionen Belegzetteln besteht aus zwei unterschiedlich geordneten Teilarchiven: dem lautschriftlichen und dem alphabetischen.

Das 48 Zettelkästen umfassende und nach Orten geordnete lautschriftliche Teilarchiv beinhaltet in Teuthonista-Lautschrift (vgl. Kapitel 4.4) notierte Belege von 45 Ortsmundarten. Sie stammen größtenteils von ERICH NÖRRENBERG, der über viele Jahre in ganz Westfalen Gewährspersonen vor Ort persönlich befragt hat.

Aus 15 Orten füllt das dort erhobene Datenmaterial mindestens einen Zettelkasten. Acht davon stammen aus dem Südwestfälischen: Iserlohn (Sigle ${ }^{7}$ : Isl Is, 6 Kästen), Altena (Alt Al, 3), Deilinghofen (Isl Dh, 3), Assinghausen (Bri Ah, 2),

7 Im Folgenden werden die im WWb verwendeten Siglen (mit) angegeben, damit sich die Leserin / der Leser dieses Beitrags bei Bedarf sofort im Wörterbuch orientieren kann. 
Elsey (Isl El, 2), Afferde (Unn Af, 1), Endorf (Arn En,1), Müschede ${ }^{8}$ (Arn Mü,1); fünf aus dem Münsterland: Vorhelm (Bek Vh, 5), Rheine (Stf Rh, 3), Mettingen (Tek Me, 2), Wessum (Ahs We, 2), Drensteinfurt (Lhs Dr, 1); und zwei aus Ostwestfalen: Sandebeck (Höx Sb, 3), Lüchtringen (Höx Lü, 1). Zu den Gewährspersonen im Münsterland zählen u. a. die bekannten Mundartautoren AUGUSTIN WIBBELT für Vorhelm (Bek Vh) und KARL WAGENFELD für Drensteinfurt (Lhs Dr). ${ }^{9}$

\subsection{Quellen}

Das Wörterbuch basiert auf dem Belegmaterial des aus 470 Kästen bestehenden alphabetischen Teilarchivs, des so genannten Hauptarchivs. Dieses geht auf unterschiedliche Quellen zurück:

1. Lautschriftliche Belege: Die lautschriftlichen Belege stammen zum großen Teil aus dem lautschriftlichen Teilarchiv, dessen Zettel inzwischen vollständig als Kopie in das alphabetisch sortierte Hauptarchiv integriert worden sind. Daneben gibt es einen großen Bestand an lautschriftlichen Belegen, die sofort in das alphabetische Archiv einsortiert worden sind. Die Daten des lautschriftlichen Archivs sind wie die im Beiband aufgelisteten Lauttabellen für die Wörterbucharbeit von unschätzbarem Wert. Sie gehen nicht auf schriftliche Erhebungen per Fragebogen zurück, sondern auf persönliche Gespräche, die besonders ERICH NÖRRENBERG und später FELIX WORTMANN in ganz Westfalen mit zahlreichen Gewährspersonen z. T. über einen längeren Zeitraum geführt haben. Anders als bei indirekten Erhebungen konnten sie so auch z. B. unterschiedliche Öffnungsgrade bei Vokalen erkennen und dokumentieren. Diese Belege des lautschriftlichen Archivs bilden den für lautliche Fragestellungen verlässlichsten Teil des Archivs und dienen dem Bearbeiter als wichtiges Korrektiv bei der Beurteilung laienschriftlicher Belege.

2. Wortsammlungen von freiwilligen Mitarbeiterinnen und Mitarbeitern: Nicht wenige engagierte Gewährspersonen haben - zum Teil sogar direkt auf Wörterbuchzetteln - Wortsammlungen ihrer Ortsmundart eingereicht. Als qualitativ wie quantitativ herausragende Sammlung sei hier die des Schlachthofdirektors Dr. AUGUST ECKARDT aus Dortmund-Wellinghofen (Dor Wl) genannt. Das WWb hat dieser Quelle die mit Abstand meisten Verwendungsbeispiele zu verdanken. Weitere erwähnenswerte Sammlungen stammen aus Altenrheine (Stf Ar), Bracht (Mes Br), Brockhagen (Hal Bh), Hagen (Arn Hg),

8 Vgl. hierzu auch Kapitel 7.

9 Es lässt sich eine geografische Gewichtung innerhalb des WWb-Archivs erkennen. Das Südwestfälische (vor allem der Westen, NÖRRENBERGS Heimat) ist gut bis sehr gut abgedeckt, das Ostwestfälische eher schlecht; das Münsterländische steht zwischen diesen beiden Polen. Die Auflistung der Gewährspersonen (Beiband 1969: 16) zeigt diese Tendenz besonders deutlich: 34 Gewährspersonen stammen aus dem Südwestfälischen, acht (gut verteilt) aus dem Münsterland und fünf aus Ostwestfalen (nur aus den Altkreisen Höxter und Büren). 
Niedersfeld (Bri Nf), um nur einige zu nennen. Eine Liste der wichtigsten Beiträger findet sich im Beiband (1969: 17-18). ${ }^{10}$

3. Wörterbücher

a) Unveröffentlichte Wörterbücher: Zahlreiche Wortsammlungen vor allem des 19. Jahrhunderts, die zum Zeitpunkt ihrer Entstehung aus Kostengründen nicht gedruckt werden konnten, gelangten als Manuskripte ins WWb-Archiv. Zu nennen sind hier vor allem das Wörterbuch von P. H. Holthaus (Enr Holthaus: „Materialien zu einer Schrift, betitelt: SüdWestfälisches Wörterbuch") und das von W. OESTERHAUS (Lippe OESTERH: „Wörterbuch der Lippisch-plattdeutschen Mundart“").

b) Im Druck erschienene Wörterbücher: Das (für die Bände 2 bis 5) älteste Wörterbuch ${ }^{11}$ im Archiv ist das 1790 erschienene „Ravensbergische Idiotikon“ von PETER Florens Weddigen (WEDDIGEN 1790). Weitere aufgenommene Werke sind u. a. das ,Wörterbuch der Mundart von Hahlen“ von CHRISTIAN FREDERKING (FREDERKING 1939), das „Plattdeutsche Wörterbuch in Istruper Mundart" von FRITZ Platenau (Platenau 1978), das „Wörterbuch der Soester Börde“ von HERMANN SCHMOECKEL und ANDREAS BLESKEN (SCHMOECKEL / BLESKEN 1952), das „Wörterbuch der Westfälischen Mundart“ von FrIEDRICH WOESTE (WOESTE 1882) und dessen Bearbeitung durch ERICH NÖRRENBERG (WOESTE 1930). Die jüngsten berücksichtigten Wörterbücher stammen aus dem Beginn der 1990er Jahre: das „Wörterbuch der westmünsterländischen Mundart“ von ELISABETH PIIRAinen und Wilhelm ElLiNG (PIIRAINEN / Elling 1992) sowie das „Wörterbuch des Kurkölnischen Sauerlands“ von REINHARD PILKMANNPoHL (PILKMANN-PoHL 1988).

4. Antworten auf Fragebogenerhebungen: Zwischen 1928 und 1975 führte die Wörterbuchkanzlei zahlreiche Fragebogenerhebungen durch: 23 gezählte sowie zusätzliche einzelne Abfragen zum Spezialwortschatz (u. a. Handwerk, Kartenspiele und Pflanzen). Darüber hinaus werden die Fragebogen des bislang unveröffentlichten „Niederdeutschen Wortatlasses“ (NWA I 1950 und NWA II 1965) und des „Deutschen Wortatlasses“ (DWA) sowie die (dem Archiv seit den 1960er Jahren als Kopie zur Verfügung stehenden) Fragebogen von THEODOR BAADER für das WWb ausgewertet. Das Fragebogenmaterial ist vollständig verzettelt, lemmatisiert und ins Hauptarchiv einsortiert. Die auf Fragebogenantworten basierenden Wörterbuchzettel haben statt der sonst üblichen Bedeutungsangabe einen Verweis auf die Fragebogensigle und die Nummer der Frage, die mit Hilfe einer - inzwischen digital im Excel-Format vorliegenden Frageliste zu entschlüsseln ist.

10 Auch hier zeigt sich die zuvor erwähnte geografische Gewichtung: Zwölf südwestfälischen Mitarbeiterinnen und Mitarbeitern stehen sieben ostwestfälische und sechs münsterländische gegenüber.

11 Für Band 1 ist es das „Idioticon Osnabrugense“ von Johann Christoph StrodtManN aus dem Jahre 1756. 
5. Belege aus mundartlicher Literatur finden sich vereinzelt. Da sie in den seltensten Fällen einem Ort exakt zugeordnet werden können, werden sie in der Druckfassung aber nur in Ausnahmefällen berücksichtigt.

\subsection{Zeitraum der Erhebung}

Sieht man von den frühen (z. B. WEDDIGEN 1790) und späten Wörterbüchern (PIIRAINEN / ElLing 1992 und PILKMANN-PoHL 1988) einmal ab, stammen bis auf wenige Ausnahmen die meisten Belege aus dem zweiten und dritten Viertel des 20. Jahrhunderts. Wegen fehlender personeller Ressourcen wurde 1994 die weitere Materialaufnahme gestoppt. Neuere Quellen werden seitdem nicht mehr erschlossen.

\subsection{Wörterbuchzettel}

Jeder der etwa 1,5 Millionen Zettel enthält mindestens vier Informationen: 1. den mundartlichen Wortbeleg - 2. die Angabe der Bedeutung (zuweilen ergänzt durch mundartliche Kontexte) bzw. (bei auf Fragebogenantworten basierenden Zetteln) den Verweis auf eine Fragebogenfrage - 3. die (aus einer dreistelligen Kreis- und einer zweistelligen Ortssigle bestehende) Sigle des Ortes, aus dem der Beleg stammt -4 . das Lemma, dem dieser Wortbeleg zugeordnet wird.

\subsection{Hilfsmittel für den internen Gebrauch}

In den 1990er Jahren ist das gesamte Zettelarchiv von studentischen Hilfskräften bezüglich Stichwort und Bedeutungsangabe digital erfasst worden. Darauf basiert ein 1998 fertiggestelltes zweibändiges Stichwortverzeichnis mit insgesamt 1412 Seiten, das im Gegensatz zu einem älteren Stichwortverzeichnis aus den 1960er Jahren mehr als 100000 Stichwörter enthält und diese zu Wortfamilien zusammenfasst. Zudem sind im Zuge der Bearbeitung die Kästen mit Kartenreitern versehen worden, die den Beginn eines neuen Stichworts oder einer neuen Wortfamilie markieren. Beide Maßnahmen haben die Orientierung im Zettelarchiv erheblich verbessert.

Für den internen Gebrauch existieren darüber hinaus einige Digitalisate, die das Artikelschreiben beschleunigen. So liegen seit einigen Jahren die von den Gewährspersonen ausgefüllten WWb- und NWA-Fragebogen in Form von PDF-Dateien vor und können jederzeit am Arbeitsplatz eingesehen werden. Das erspart das mühevolle Suchen in Aktenordnern im Archivkeller. Da die meisten zweifelhaften Belege aus Fragebogen stammen, wird dieses Digitalisat oft genutzt und erweist sich 
so als große Hilfe. Ferner liegen aus etwa 550 Orten $^{12}$ die im Beiband veröffentlichten Ortstabellen, die die normalen Entwicklungen der historischen Phoneme in der jeweiligen Ortsmundart dokumentieren, in Form von JPG-Dateien vor. Auch auf die auf der Grundlage der Lauttabelle noch von Hand gezeichneten Lautkarten kann man digital zugreifen. Dies ermöglicht eine schnelle Überprüfung bzw. Interpretation laienschriftlicher Belege sowie eine genaue Zuordnung lautschriftlicher Belege. Diese Lautkarten bilden die Grundlage für einen zu erstellenden Lautatlas zum WWb (vgl. Kapitel 4.7).

\section{WÖRTERBUCH}

\subsection{Lemmaansatz}

Beim Lemmaansatz konnte das WWb seinem großen Vorbild, dem RhWb, nicht folgen, da dieses - wegen des größtenteils hochdeutschen Bearbeitungsgebietes einen hochdeutschen Stichwortansatz hat. An dessen Stelle tritt beim WWb ein Ansatz mit niederdeutschem Konsonantismus. Im Vokalismus wäre die Orientierung an der am meisten verbreiteten Form oder der Form einer repräsentativen Mundart möglich gewesen. Man entschied sich aber für einen historischen Ansatz. Im Beiband (1969: 62) heißt es dazu: „Als Stichwort wird aus den heute gesprochenen Formen des Wortes die sprachhistorisch älteste, aus der also die andern hervorgegangen sind, ausgewählt".

Im Vokalismus erweisen sich die westfälischen Mundarten als sehr konservativ, indem sie im Gegensatz zu den meisten anderen niederdeutschen Mundarten z. B. die Unterscheidung alter Phoneme erhalten haben. So differenziert das Westfälische noch zwischen tonlangem $\bar{a}$ (māken, Wāter) und altlangem $\hat{a}$ (lâten, Schâp). Auch bei den alten Kurzvokalen in offener Silbe lassen sich vielerorts noch sieben Qualitäten unterscheiden.

Diese Umstände lassen einen historischen Ansatz als geeignet erscheinen, auf den sich alle oder zumindest die meisten der belegten Lautformen zurückführen lassen. Während z. B. das NdsWb im Stichwort eine Notation verwendet, die sich an der nordniedersächsischen Aussprache orientiert und daher nur bedingt Rückschlüsse auf zugrundeliegende historische Phoneme zulässt, ${ }^{13}$ verwendet das WWb im Haupttonvokal ein historisches Phonem, aus dem sich im Idealfall alle in den Mundarten bezeugten Laute ableiten lassen. ${ }^{14}$ Dieser historische und nicht selten von der gesprochenen Mundartform abweichende Lemmaansatz bewährt sich in der

12 Die Angabe bezieht sich auf das in Band 2 bis 5 gültige eingeschränkte Bearbeitungsgebiet. Vgl. zur Lauttabelle Kapitel 4.7.

13 NdsWb Band 3: VI: „Die im Stichwort zur Kennzeichnung eines Langvokals verwendete Kombination aus Buchstabe und Längestrich über ihm ermöglicht keine Erkenntnisse über die sprachgeschichtlich unterschiedliche Herkunft der so dargestellten Längen“.

14 Für das $\bar{o} \mathrm{im}$ Stichwort des NdsWb hat das WWb vier Ansätze: $\bar{o}^{1}$ (westgermanisch $\hat{o}$ ), $\bar{o}^{2}$ (westgermanisch $a u$ ), $u^{e}$ (altes kurzes $u$ in offener Silbe) und $o^{a}$ (altes kurzes $o$ in offener Silbe). 
lautlich sehr differenzierten westfälischen Mundartlandschaft: Die unterschiedlichen Mundartbelege eines Lexems lassen sich auf diese Art und Weise nachvollziehbar unter einem Lemma zusammenfassen.

\subsection{Makrostruktur}

Die Stichwortliste des WWb hat nach dem Muster des RhWb eine nestalphabetische Anordnung (vgl. Abbildung 1). Während bei den meisten großräumigen Dialektwörterbüchern alle Stichwörter gleichen Rang besitzen und folglich streng alphabetisch sortiert sind, unterscheidet das WWb mit dem RhWb zwei Arten von Stichwörtern: so genannte Haupt- und Nebenstichwörter. Hauptstichwörter stehen an der Spitze einer Wortfamilie, zu dem eine mehr oder weniger große Anzahl von Nebenstichwörtern gehört. Untereinander sind die Hauptstichwörter alphabetisch sortiert. Für die Reihenfolge innerhalb einer Wortfamilie gilt: Erst das Hauptstichwort bzw. Ursprungswort mit Diminutiv, dann die Zusammensetzungen, zunächst die Partikelkomposita (Komposita, bei denen „das Bestimmungswort ein Präfix, eine Präposition oder ein Wort ist, das sowohl Adverb wie Präposition sein kann“ (Beiband 1969: 63) $)^{15}$, dann die anderen, zuletzt die Ableitungen des Ursprungswortes, die meist alphabetisch aufeinander folgen.

Das nestalphabetische System hat immer wieder Anlass zur Kritik gegeben, da selbst geübte Benutzerinnen und Benutzer nicht selten Schwierigkeiten haben, das von ihnen gesuchte Wort zu finden. Aus diesem Grund ist die Makrostruktur auch 1994 bei den Beratungen für eine Neukonzeption des WWb diskutiert worden. Da eine Umsortierung des Zettelarchivs aber einen gewaltigen Zeitverlust mit sich gebracht hätte, entschied man sich für die Beibehaltung des alten Systems.

Neben zahlreichen Problemen sowohl für den Bearbeiter als auch für die Benutzerinnen und Benutzer bietet dieses System aber auch Vorteile, vor allem bezüglich der angestrebten Verschlankung in den Bänden 3 bis 5. Denn die einem Hauptstichwort untergeordneten Nebenstichwörter erhalten bestimmte Angaben nur noch dann, wenn diese entweder nicht bereits unter dem Hauptartikel zu finden sind oder von den dort gemachten abweichen. Vor allem Informationen des Lautund Formenteils lassen sich so auf das Hauptstichwort beschränken. Da die dort gemachten Angaben in der Regel auch für die gesamte Wortfamilie gelten, entfallen sie nun bei den Nebenstichwörtern. Verwendungsbeispiele, die die eigentliche Bedeutung eines Wortes illustrieren, finden sich im Bedeutungsteil von Nebenstichwörtern fast nur noch dann, wenn sie der Kontrastierung mehrerer Bedeutungen dienen.

15 Im Gegensatz zum RhWb, das nur die Verben so behandelt, weitet das WWb dieses Verfahren auf alle Fälle aus. 


\section{Westfälisches Wörterbuch: Makrostruktur}

Wortsippe mit
Hauptstichwort und
Nebenstichwörtern

Hauptstichwörter alphabetisch sortiert

Wortsippe mit Hauptstichwort und Nebenstichwörtern

Hauptstichwörter alphabetisch sortiert

Wortsippe mit Hauptstichwort und Nebenstichwörtern

Hauptstichwörter alphabetisch sortiert
Schō้

schōfel

Schö'fele

schoien

schäiken

Schok

Schok-rêde

Scho'ke

Schötkeken

Achter-scho"kes

Für

Ilinder

Under

scho'ken-dikke

Schotken-pot

swermer

Schöker

schökeren

Hauptstichwort

Diminutiv

Partikelkomposita

Schokelàde

Schokkele

schöks

'Scho'le

'Schotle

Schō'le

scholferen

'Scholle

'Scholle

schon

schö̀ne

schō'ne

'schö'nen

ber

fern

Schornc-tid

-tûg

Schơner

Schồ?nunge

Komposita

Ableitungen

Schop

Schō'p

'Schoppe

Schöppeken

rưr-schoppe

Schoppen-balke

vre'de

vdag

dak

$\checkmark d c^{*} l e$

vễ're

-hilde

Schöpperling

Schöpfel

Für-schōpBcl

Hauptstichwort

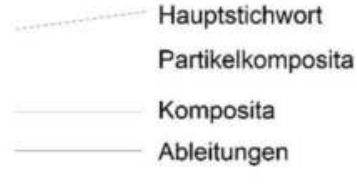

'Schoppe

iScho*'r

'Scho"r

Schôr

Schōre

Schôres

Schoßè
Hauptstichwort

Diminutiv

Partikelkompositum

Komposita

Ableitungen

Abb. 1: Makrostruktur des ,Westfälischen Wörterbuchs “ 


\subsection{Mikrostruktur}

Die Artikelgestaltung orientiert sich ab Band 2 weitgehend am NdsWb: Auf den umfangreichen Bedeutungsteil, der die Semantik des Stichworts beschreibt und durch Verwendungsbeispiele illustriert sowie Redensarten und Sprichwörter enthält, folgt - jeweils in Petit gesetzt - der Laut- und Formenteil sowie unter Umständen zwei Verweisteile. Im ersten, nach dem Vorbild des RhWb gestalteten Band befindet sich der zuweilen recht umfangreiche Lautteil noch prominent im Kopf des Artikels. Im Gegensatz zum ersten Band enthalten die Bände 2 bis 5 keine Sprachkarten, Abbildungen und Fotos, da sich das Erstellen von Zeichnungen und Karten in der Vergangenheit als sehr aufwändig und damit als nicht mehr vertretbar erwiesen hat.

Ein Wortartikel (vgl. Abbildung 2) setzt sich im Idealfall aus den folgenden Artikelteilen zusammen:

1. Stichwort (als Hauptstichwort halbfett, als Nebenstichwort gesperrt) ${ }^{16}$

2. Angaben zu Wortart bzw. Genus

3. Verbreitungsangabe (in eckigen Klammern)

4. Bedeutungsteil

5. Laut- und Formenteil (in Petit)

6. Verweise auf Stichwörter in anderen Wörterbüchern sowie ähnlich lautende Stichwörter mit identischer Bedeutung im WWb (in Petit)

7. Verweise auf im WWb bezeugte Zusammensetzungen mit dem Stichwort als Grundwort (in Petit) 


\section{Westfälisches Wörterbuch: Mikrostruktur}

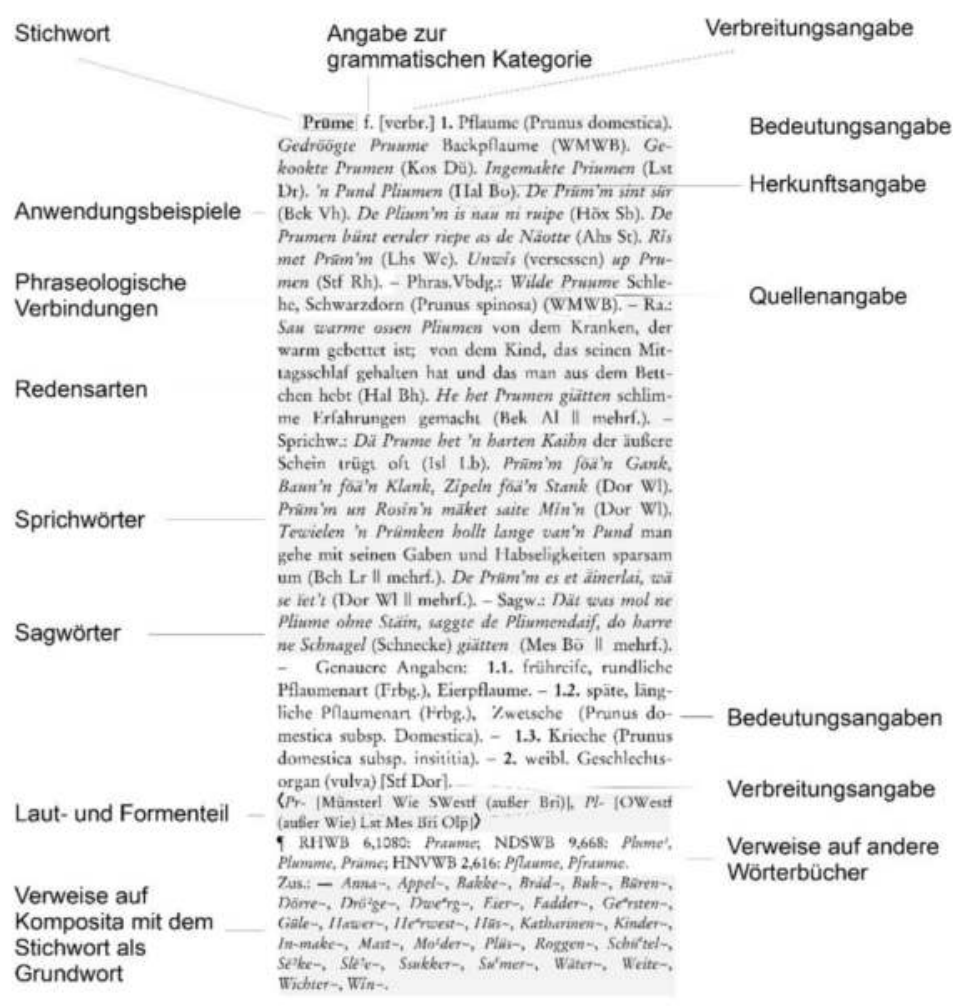

Abb. 2: Mikrostruktur des „Westfälischen Wörterbuchs“

Angaben zu Wortart bzw. Genus enthält ab Band 2 möglichst jeder Wortartikel. Für Substantive gilt folgende Regelung: Ein Genus wird nur dann angegeben, wenn es explizit genannt wird oder aus den Belegen eindeutig hervorgeht. Bei der Angabe mehrerer Genera wird der Befund im Formenteil erläutert; geschieht dies nicht, bedeutet dies, dass sich kein Verteilungsprinzip erkennen lässt.

Im Zentrum des Wortartikels steht der Bedeutung steil. Dort wird nicht mehr - wie in Band 1 - jede im Zettelarchiv vorhandene Bedeutungsangabe zitiert. Vielmehr werden ab Band 2 - vor allem bei Stichwörtern mit vielen Belegen - ähnliche Angaben in einer (möglichst umfassenden) Definition gebündelt. Formulierung und Wortwahl orientieren sich dabei häufig am NdsWb und am ,großen Wörterbuch der deutschen Sprache" vom Duden-Verlag; die gewählte Formulierung kann also von der der Gewährsperson oder der Quelle abweichen. Stammen Einzelbelege für ein Stichwort oder eine Bedeutungsangabe aus Fragebogenmaterial, sind sie mit 
dem Zusatz ,(Frbg.)“ versehen. Unterschiedliche Bedeutungen werden durch arabische Ziffern markiert. Gehen alle Bedeutungen eines Wortes auf eine Gewährsperson zurück, werden sie hingegen durch ,a)“, ,b)“ usw. eingeleitet.

Wenn vorhanden, werden die Bedeutungsangaben ergänzt durch unmittelbar folgende Verwendungsbeispiele, die den Gebrauch des Stichworts illustrieren, sowie Redensarten, Sprich- und Sagwörter, in denen das Stichwort das Kernwort ${ }^{17}$ bildet. Dabei werden aber nicht - wie in Band 1 - alle im Archiv vorhandenen Belege wiedergegeben, sondern nur eine möglichst repräsentative Auswahl. Volksoder sachkundliche Informationen werden im Bedeutungsteil nur noch in Ausnahmefällen angeführt.

Das WWb hat einen historischen Lemmaansatz; das Lemma spiegelt in der Regel nicht einen konkreten mundartlichen Beleg wider. In all den Fällen, in denen die mundartlichen Belege mehr oder weniger stark vom angesetzten Lemma abweichen, enthält der durch fette Spitzklammern eingeklammerte Laut- und Formenteil weiterführende Informationen. Er dokumentiert in erster Linie die unterschiedlichen Lauttypen der mundartlichen Belege. Darüber hinaus kann er Angaben zur Wortbildung, zum Genus, zur Koinzidenz von Wortform und Genus, von Wortform und (Teil-)Bedeutung u. Ä. enthalten. Ab Band 2 kommt er fast nur noch bei Hauptstichwörtern einer Wortfamilie vor. Außerdem führt er nicht mehr jede im Zettelarchiv belegte Lautform oder gar Schreibung an, sondern beschränkt sich weitgehend auf die den Belegen zugrunde liegenden und im Beiband dokumentierten Lauttypen bzw. die wichtigsten Varianten. Eine ausführliche formale Beschreibung der Belege kann bei Zusammensetzungen und Ableitungen entfallen.

Der mit dem Zeichen II eingeleitete Artikelteil enthält ab Band 2 Verweise auf andere Wörterbücher: Nicht mehr etymologische Erläuterungen wie in Band 1 stehen im Vordergrund, sondern Vernetzungen mit den Dialektwörterbüchern der umliegenden Gebiete: dem RhWb, dem NdsWb und dem HnVwb. Auf diese Weise wird auch die außerwestfälische Verbreitung eines Wortes dokumentiert. Fehlen Parallelen aus diesen Gebieten, kann auch auf das „Deutsche Wörterbuch“ (DWb) oder das „Mittelniederdeutsche Handwörterbuch“ (MndHwb) verwiesen werden. Ab Band 4 wird Lehnwortschatz jiddischen oder ähnlichen Ursprungs durch Verweis auf STERN (2000) markiert. Es finden sich darüber hinaus, markiert durch einen Pfeil, Verweise auch auf Stichwörter im WWb, die eine ähnliche Bedeutung aufweisen und in ihrer Lautung nur leicht abweichen.

Der mit ,Zus.:“ eingeleitete Artikelteil enthält Verweise auf Zusammensetzungen im WWb, in denen das Stichwort das Grundwort bildet.

17 In den meisten Fällen wird das erste auftretende Substantiv eines idiomatischen Ausdrucks als Kernwort angesehen. 


\subsection{Notation mundartlicher Belege}

Bezüglich der Notation mundartlicher Belege unterscheiden sich Bedeutungs- auf der einen und Laut- und Formenteil auf der anderen Seite. Im Bedeutungsteil werden mundartliche Belege entweder in Laienschrift oder - besonders im Bereich des Vokalismus - in der sogenannten Teuthonista-Lautschrift angeführt (TEUCHERT 1924/1925). Demnach wird eine Länge etwa mit einem Strich über dem Vokal, offene Qualität eines Langvokals durch ein unter das Vokalzeichen gesetztes Häkchen markiert. IPA wird nicht verwendet.

Im Laut- und Formenteil werden drei Notationen unterschieden: Kleine Spitzklammern (,,‘ und ,“" dass dieser als Haupttonvokal nicht einen konkreten mundartlichen Laut wiedergibt, sondern an dessen Stelle das vormittelniederdeutsche Phonem, aus dem sich die konkreten mundartlichen Laute entwickelt haben (vgl. zur Lauttabelle Kapitel 4.7). Es handelt sich wie im Stichwort um einen Lauttyp. Unter dem Stichwort lo $\bar{o}^{2}$ pen 'laufen' wird im Lautteil der Typ $>1 \bar{o}^{1}$ pen $<$ angeführt. Das bedeutet, dass in einigen (münsterländischen) Mundarten der Haupttonvokal sich bei diesem Wort nicht normal entwickelt hat, sondern abweichend wie der Vokal etwa in $>\mathrm{Fo}^{1} \mathrm{t}$ < 'Fuß'. Ein kursivierter Beleg bildet den bezeugten Vokalismus möglichst angemessen ab, während ein in Anführungsstriche eingeschlossener Beleg auf einen bezüglich der bezeugten Schreibung auffälligen Beleg hinweist, der unverändert zitiert wird. Wie die Lauttypen in den einzelnen Mundarten konkret realisiert sind, lässt sich den Ortstabellen des Beibandes entnehmen.

\subsection{Verbreitungsangaben}

Im ersten Band des WWb wird die Verbreitung einer Lautung oder Wortform sowie einer Bedeutung oder Bedeutungsnuance durch die Aneinanderreihung der Siglen der Orte angegeben, in denen diese belegt ist. Ab dem zweiten Band dienen Ortssiglen nur noch dazu, die Herkunft eines Belegs mitzuteilen. Die mitunter langen und unübersichtlichen Reihungen von Ortssiglen werden nicht mehr publiziert, sondern bilden die Grundlage für die Ermittlung von Verbreitungsangaben. Die kleinste Einheit bei den Verbreitungsangaben bildet nun die dreistellige Kreissigle. Herkunfts- und Verbreitungsangaben werden in der Darstellung dadurch unterschieden, dass erstere in runde und letztere in eckige Klammern eingeschlossen werden. Die übliche Herkunftsangabe ist eine Ortsangabe, die aus dreistelliger Kreis- und zweistelliger Ortssigle besteht; neben der Ortsangabe gibt es die Quellenangabe, die die Sigle des zitierten Werks - in einigen Fällen in Verbindung mit einer Kreis- oder anderen Sigle - in Kapitälchen anführt, z. B. Sos ScHMB (für das „Wörterbuch der Soester Börde“ von HERMANN SCHMOECKEL und ANDREAS BLESKEN) oder Lippe OESTERH (für das nicht publizierte Wörterbuch von W. OESTERHAUS).

Die im WWb verwendeten Kreissiglen referieren auf politische Einheiten, wie es sie vor der kommunalen Gebietsreform in Nordrhein-Westfalen (1966 bis 1974) 
gegeben hat; sie beziehen sich also auf sogenannte „Altkreise“. Der Beginn der Publikation des WWb (der Beiband, in dem die Siglen festgelegt wurden, erscheint 1969) fällt genau in die Zeit dieser Umgestaltung; eine Anpassung an die neuen Gegebenheiten ist zu diesem Zeitpunkt erstens nicht möglich und hätte zweitens zudem eine Änderung des gesamten Siglensystems und damit einen enormen Arbeitsaufwand erfordert. Dem Festhalten an den „Altkreisen“ lässt sich für das WWb sogar etwas Positives abgewinnen, nämlich, dass die Altkreissigle als kleinste Einheit für eine Verbreitungsangabe eine erheblich genauere geografische Zuordnung ermöglicht als die Orientierung an den heutigen Großkreisen, die meist mehrere Altkreise vereinen.

Eine in ganz Westfalen flächendeckende Bezeugung wird durch die Abkürzung „allg.“ (allgemein) angedeutet. Lediglich Fragebogenmaterial bietet für diese Angabe eine hinreichende Grundlage. Nicht flächendeckendes, aber für die meisten westfälischen Gebiete bezeugtes Vorkommen wird durch „verbr.“ (verbreitet) markiert. Ist die Bezeugung nur schwach und lässt sich keine klare Verbreitung erkennen, wird dies durch „,verstr.“ (verstreut) markiert. Angezeigt wird stets, aus welcher Region Belege im Archiv vorliegen, und nicht, in welcher Region Belege fehlen. Nur bei guter Materialgrundlage, also in der Regel bei Fragebogenmaterial, können durch „(außer ...)“ markierte Ausnahmegebiete genannt werden. Beschränkt sich die Bezeugung auf bestimmte westfälische Gebiete, so werden diese besonders genannt. Als größte westfälische Teilgebiete fungieren politisch definierte Räume, nämlich die drei westfälischen Regierungsbezirke: „Münsterl“ (Münsterland) steht für den Regierungsbezirk Münster, „OWestf“ (Ostwestfalen) für den Regierungsbezirk Detmold mitsamt dem hessischen Landkreis WaldeckFrankenberg sowie „SWestf“ (Südwestfalen) für den Regierungsbezirk Arnsberg. Zur Angabe von Teilgebieten in den Regierungsbezirken dienen neben den Kreissiglen der 37 westfälischen und zwei hessischen Altkreise die folgenden Siglen: „WMünsterl“ (Westmünsterland) für die Altkreise Ahs Bor, „Ravensbg“ (Ravensberg) für Hfd Hal Bie, „Lippe“ für Lem Det, „HPaderb“ (Hochstift Paderborn) für Pad Höx Bür Wbg, „Mark“ für Bch Wit Dor Unn, „Hellweg“ für Sos Lst, „MSauerl“ (märkisches Sauerland) für Enr Hag Isl Alt, „KSauerl“ (kurkölnisches Sauerland) für Arn Bri Mes Olp. Um unterschiedliche regionale Intensitäten anzuzeigen, kann den Siglen für die westfälischen Teilgebiete die Angabe „,allg.“, „,verbr.“”oder „verstr.“ vorangestellt werden.

Eine Verbreitungsangabe kann an verschiedenen Stellen des Artikels vorkommen: nach dem Stichwort, im Bedeutungsteil sowie im Laut- und Formenteil.

1. Die Verbreitungsangabe zu einem Stichwort befindet sich hinter der grammatischen Angabe bzw., wenn diese fehlt, direkt hinter dem Stichwort. Ist ein Wort nur von einer Gewährsperson bezeugt, entfällt eine Verbreitungsangabe. In diesem Fall befindet sich eine Herkunftsangabe am Ende des Artikels; diese gilt für alle Teile des Artikels. Die Verbreitung des Stichworts ist ebenfalls nicht angegeben, wenn sie sich aus der Kombination der Verbreitungs- bzw. Herkunftsangaben bei den Bedeutungen ergibt. 
2. In der Regel ist eine Bedeutung nur dann mit Verbreitungsangaben versehen, wenn sie sich von der Verbreitung des Stichworts unterscheidet oder unterschiedliche Bedeutungen in unterschiedlicher Gewichtung oder Verbreitung belegt sind. Singulär bezeugte Bedeutungen erhalten stets eine Herkunftsangabe.

3. Im Laut- und Formenteil werden Varianten in der Regel mit Verbreitungs- oder Herkunftsangaben versehen. Diese Angaben können jedoch entfallen, wenn sich klare Verteilungen nicht erkennen lassen.

\subsection{Umfang}

Die Bearbeiter des ersten Bandes haben sich nicht an die Vorgabe des Verlagsvertrages (vgl. Kapitel 1.2), der ein fünfbändiges Werk vorsieht, gehalten. Der Buchstabe $A$, der 2,5\% des Stichwortverzeichnisses ausmacht, kommt auf 358 Spalten, also 5,6 \% des geplanten Gesamtumfangs. Dieser Trend setzt sich im Buchstaben $B$ fort. Obwohl die Buchstaben $A$ bis $C$ nur 10,3 \% des Stichwortbestandes ausmachen, belegen sie bereits einen von fünf Bänden, d. h. ein Fünftel des Gesamtumfangs. Mit der Praxis des ersten Bandes hätte es das WWb bei Abschluss auf mindestens zehn Bände gebracht.

$\mathrm{Ab}$ dem zweiten Band wird der Umfang des WWb wieder auf die ursprünglich geplanten fünf Bände begrenzt. Die restlichen $90 \%$ des Stichwortbestandes müssen in $80 \%$ des Gesamtumfangs untergebracht werden. Die Bände 2 bis 5 enthalten also jeweils einerseits eine deutlich längere Wortschatzstrecke als der erste Band; andererseits steht für die Darstellung des Materials jeweils deutlich weniger Platz zur Verfügung. Diese Vorgaben erfordern Vereinfachungen und Kürzungen.

Legt man die Zahl der Seiten im Stichwortverzeichnis (vgl. Kapitel 3.4) und die Anzahl der Zettelkästen zugrunde, verteilen sich die Wortschatzstrecken der fünf Bände wie folgt:

- Band $1(A-C)$

145 Seiten $(10,3 \%) \quad 59$ Kästen $(12,5 \%)$

- Band $2(D-G)$

255 Seiten $(18,1 \%) \quad 95$ Kästen $(20,2 \%)$

- Band $3(H-L)$

316 Seiten $(22,4 \%) \quad 108$ Kästen $(23 \%)$

- Band $4(M-S k)$

344 Seiten $(24,3 \%) \quad 108$ Kästen $(23 \%)$

- Band $5(S l-Y)$

351 Seiten $(24,9 \%) \quad 100$ Kästen $(21,3 \%)$

Während der erste Band (fast) alle im Archiv vorhandenen Informationen an die Benutzerinnen und Benutzer weitergibt, indem nicht selten jede bezeugte Lautung bzw. Schreibung und jede bezeugte Bedeutungsangabe zitiert werden, beschränken sich die Bände 2 bis 5 weitgehend auf die Dokumentation der wesentlichen Informationen. Bezeugte Lautungen und Schreibungen werden, wie im Beiband vorgesehen, auf die zugrundeliegenden Lauttypen zusammengefasst; Entsprechendes gilt nun auch für die Bedeutungsangaben. 


\subsection{Beigaben}

Das fünfbändige Wörterbuch wird ergänzt durch zwei damit eng verbundene Publikationen, nämlich einen Beiband und einen Lautatlas.

Im Jahre 1969, vier Jahre vor der ersten Wörterbuchlieferung, erscheint der von FELIX WORTMANN bearbeitete Beiband. Er enthält den für das Verständnis des WWb erforderlichen wissenschaftlichen Apparat: Nach Ausführungen zur Geschichte des Unternehmens (von DIETRICH HOFMANN) folgen Abschnitte zum Bearbeitungsgebiet und Material, denen sich Abkürzungsverzeichnisse (besonders Kreis- und Ortssiglen) sowie ein Verzeichnis abgekürzt zitierter Quellen und Literatur anschließen. Dem Beiband beigegeben sind Karten der belegten Orte und der Orte, die in der Lauttabelle berücksichtigt sind, ferner eine Karte der westfälischen Länder von 1801. Wichtige Informationen für die Benutzerinnen und Benutzer bieten die Abschnitte zur Anlage und Schreibung der Wortartikel (Lemmaansatz, Makro- und Mikrostruktur, Notation) und die Erläuterungen zur Lauttabelle (Einführung, Benutzung, Defizite).

Den umfangreichsten und wohl wichtigsten Teil des Beibandes bildet die Lauttabelle, die für etwa 700 Orte des Wörterbuchgebietes ${ }^{18}$ in so genannten Ortstabellen (vgl. Abbildung 3) die normalen Vokalentwicklungen verzeichnet. ${ }^{19}$ Eine vollständige Ortstabelle unterscheidet sechs Kategorien: 1. Kurzvokale - 2. Kurzvokale in offener Silbe $-3 . \bar{a}$ und $\hat{a}$ mit Umlaut -4 . $\hat{e}$ - und $\hat{o}$-Laute mit Umlaut sowie Diphthonge $-5 . \bar{l}$ und $\bar{u}$ mit Umlaut -6 . Artikulation von $r$ sowie von anlautendem $g$ und $s$.

Jede Ortstabelle enthält eine mehr oder weniger umfangreiche Anzahl von Gleichungen: Links vom ,größer als“-Zeichen, das hier für ,,wird zu“ steht, ist das vormittelniederdeutsche Phonem angeführt, rechts von ihm die normale mundartliche Entsprechung dieses Phonems. Alle Belege, die die normale Entwicklung des im Lemma angesetzten Phomens zeigen, müssen im Laut- und Formenteil nicht eigens aufgelistet werden; nur die Abweichungen von diesem Lauttyp werden angeführt. Auf diese Art und Weise ist es möglich, die Wörterbuchartikel soweit wie möglich von z. T. komplizierten lautschriftlichen Angaben zu entlasten (vgl. Kapitel 4.3 Mikrostruktur).

18 Für das eingeschränkte Bearbeitungsgebiet der Bände 2 bis 5 gibt es immerhin noch etwa 550 Ortstabellen.

19 Hierzu ausführlicher DENKLER / NIEBAUM (2016). 


\section{Westfälisches Wörterbuch: Lauttabelle}

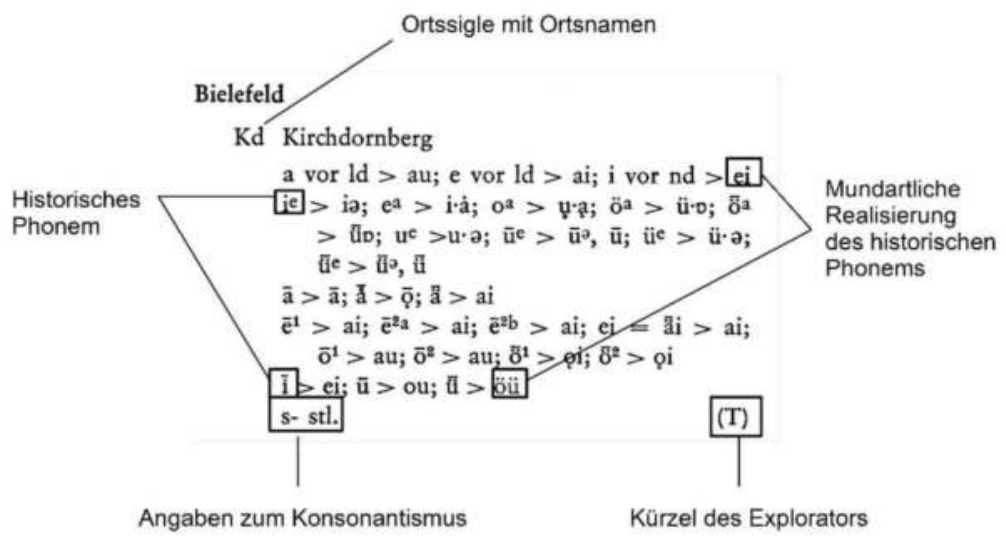

Abb. 3: Lauttabelle zum „,Westfälischen Wörterbuch“

Auf der Grundlage der im Beiband veröffentlichten Lauttabellen, also auf der Grundlage in mündlicher Befragung erhobener Daten, wird zurzeit ein Lautatlas zum WWb erarbeitet, der über die normalen Entwicklungen der wichtigsten historischen Phoneme und deren geografische Verteilung auf Sprachkarten informiert (hierzu DENKLER / NiEBAUM 2016).

\section{STAND DER PUBLIKATION}

Zurzeit liegen folgende Bände vor:

- Band 1: $A-C$, bearbeitet von Hermann Niebaum, Hans TAUbKen, Paul TEEPE, FELIX WortMANN, 2015 (12 Lieferungen 1973-2015)

- Band 2: $D-G$, bearbeitet von Robert DAMME, 2011 (13 Lieferungen 1997-2011)

- Band 3: $H-L$, bearbeitet von ROBERT DAMME, 2015

- Band 4: $M-S k$, bearbeitet von ROBERT DAMME, 2018

- Band $5(S l-Y)^{20}$ ist in Bearbeitung, seine Fertigstellung für Mitte 2021 geplant.

20 Dem hochdeutschen $z$ liegt im Niederdeutschen unverschobenes $t$ zugrunde. Lehnwörter aus dem Hochdeutschen sind im WWb unter $S$ (markiert durch „Ss“ bzw. „ss“) einsortiert. Die Wortschatzstrecke $Y$ enthält nur den Ortsnamen Ypern. Da Ypern das Kernwort einer Redensart ist, wird dieser Ortsname als Stichwort angesetzt und bildet nun das letzte Lemma im WWb. 


\section{TEXTTECHNOLOGISCHE ASPEKTE}

\section{Druckvorlage}

Bis zur achten Lieferung des ersten Bandes (Ende der 1980er Jahre) wurde die Druckvorlage per Schreibmaschine erstellt. In diesem Typoskript wurden die kursiven Passagen einfach, die halbfetten doppelt unterstrichen und die Diakritika der Teuthonista-Lautschrift per Hand eingefügt. Die Druckvorlage der neunten Lieferung war ein WordPerfect-Ausdruck, der die Sonderzeichen, Kursiven und Fettungen korrekt darstellte. Die anschließende Korrektur erfolgte Zeichen für Zeichen und war sehr aufwändig.

Für die ersten Lieferungen des zweiten Bandes erhielt der Verlag einen AsciiText mit Auszeichnungen für Kursive, Fettung und Sperrung. Die Sonderzeichen hatten spezielle Codes. Bei der von der Druckerei vorgenommenen Konvertierung des Ascii-Textes fielen jedoch nicht selten unvorhersehbare Fehler an, sodass auch hier oft Zeichen für Zeichen Korrektur gelesen werden musste.

Ab der zwölften Lieferung des zweiten Bandes, der zehnten Lieferung des ersten Bandes und für die Bände 3 bis 5 erhält der Verlag eine vollständig formatierte PDF-Datei, die sofort in den Druck gehen kann. Eine Korrektur ist nicht mehr nötig.

\section{WordPerfect}

Beim WWb erfolgt die Texteingabe und -verarbeitung seit Beginn der digitalen Publikation mit dem Textverarbeitungsprogramm WordPerfect. Eine spezielle lexikografische Software, z. B. ein Redaktionssystem, wird nicht verwendet. Da keine Zeit für Experimente bleibt, wird nach dem Motto „never change a running system" verfahren und schon seit Jahren mit der Version 12 (von 2004) gearbeitet. WordPerfect bietet durch Anzeige von Steuerzeichen für Textauszeichnungen u. Ä. die Möglichkeit, die Textformatierung für den Druck genau zu kontrollieren. Zudem ist in den vielen Jahren der Benutzung eine umfangreiche Makrobibliothek entstanden. Diese Makros dienen u. a. der Eingabe von Sonderzeichen, der Formatierung mit bestimmten Textvorlagen, dem Herausfiltern bestimmter Textpassagen bei der Korrektur, der Erstellung der Kolumnentitel der Druckvorlage. Der mit WordPerfect erstellte Text lässt sich auf diese Weise ohne Probleme in eine PDFDatei umformatieren, die dem Druck zugrunde liegt.

\section{Sonderzeichen}

Das WWb verwendet für seine Sonderzeichen nicht den Unicode. Die Sonderzeichen für die Teuthonista-Lautschrift sind vielmehr mit der Zeichensoftware CorelDraw erstellt und in einen vorhandenen Zeichensatz eingebunden worden, indem das neue Zeichen den Code eines anderen, für das WWb nicht genutzten Zeichens erhalten hat. So hat nun etwa das große $a$ mit Längenstrich $(\bar{A})$ den Ascii-Code 192. 
Dieses Verfahren hat den großen Vorteil, dass der gesamte Text des WWb mit nur einem Zeichensatz (Font) auskommt und sogar in einem einfachen Programm wie WordPad oder gar Editor korrekt dargestellt wird. Ein Wechsel des Zeichensatzes entfällt.

\section{Verbreitungskarten}

Seit Anfang 2016 ist dienststellenintern ein in VisualBasic for Applications programmiertes Excel-Makro im Einsatz, mit dem sich die geografische Verbreitung eines Stichworts auf einfache Art und Weise kartieren lässt. Karten dieser Art stehen für alle Artikel des fünften Bandes zur Verfügung. Sie lösen die für den dritten und vierten Band (vom Team) von Hand gezeichneten Karten ab. Diese Karten bilden die Grundlage für die Verbreitungsangabe im Artikelkopf.

\section{Datenbank}

Das oben in Kapitel 3.4 genannte, in den 1990er Jahren erarbeitete zweibändige Stichwortverzeichnis ist zeitnah in eine dBase-Datenbank überführt worden, die den gesamten Stichwortbestand mit Bedeutung und Verweisen enthält. Diese inzwischen nach Access und Excel konvertierte Datenbank erlaubt es unter anderem, Listen von Komposita oder Synonymen zu erstellen. Außerdem konnte mit Hilfe der Datenbank das Lemmatisieren der restlichen unlemmatisierten Zettelbestände auch an sprachhistorisch ungeschulte Volontärinnen delegiert werden. Eine Zuordnung zu einem bereits vorhandenen Lemma gelang sehr häufig, wenn die Bedeutung und die Anfangsbuchstaben des zu lemmatisierenden Wortes eingegeben wurden. Zwischen 1994 und 2008 konnten so mit Hilfe geschickter Abfragen große Zettelmengen abgearbeitet werden.

\section{Digitalisierung des Zettelarchivs}

An der Dienststelle wird erwogen, das Zettelarchiv zu digitalisieren und evtl. später im Internet allgemein zugänglich zu machen. Außerdem ließen sich - etwa mit einer Mustererkennung - von Gewährspersonen eigenhändig geschriebene Zettel zu einer Quelle zusammenfassen. Versuche dazu laufen bereits. Voraussetzung für eine solche Digitalisierung ist, dass sich das gesamte Archiv automatisch einlesen lässt, sodass keine zusätzliche Arbeit anfällt. In Frage kommt eine solche Digitalisierung aber erst nach der Fertigstellung der Druckversion ab 2021. 
Einbindung in ein digitales Wörterbuchnetz

Relativ einfach ließen sich die Bände 3 bis 5 sowie die Lieferungen 12 und 13 des zweiten Bandes in ein Wörterbuchnetz überführen, da für die einzelnen lexikografischen Kategorien bestimmte Marker verwendet wurden. Möglich ist eine Umsetzung auch für die ersten elf Lieferungen des zweiten Bandes; jedoch sind damals die Korrekturen nur in die Druckfahne und nicht in die Datei eingetragen worden. Hier wären Nacharbeiten notwendig. Die ersten neun Lieferungen des ersten Bandes sind nicht digital erfasst. Die Lieferungen 10 bis 12 stehen als WordPerfectDatei zur Verfügung, müssten aber für eine Einbindung in ein Wörterbuchnetz mit zusätzlichen Markern versehen werden.

\section{LINGUISTISCHES POTENTIAL}

Neben den üblichen, nicht WWb-spezifischen Möglichkeiten der Auswertung des Wörterbuchs unter bestimmten Aspekten ${ }^{21}$ bieten vor allem die im Archiv gesammelten Sprachdaten reichlich Potential für weiterführende Forschungen bzw. Auswertungen.

Das Archiv des „Westfälischen Wörterbuchs“ war ursprünglich nicht als umfassendes Archiv der westfälischen Mundarten geplant, hat sich aber zeitweilig so entwickelt. Das Vorhaben, möglichst viele, auch für das Wörterbuch nicht unbedingt relevante, Daten zu sammeln, behinderte einerseits die lexikografische Arbeit und war für das zeitweilig recht langsame Publikationstempo mitverantwortlich. Andererseits steht mit diesem Archiv nun ein Datenpool zur Verfügung, der weit über die von einem Wörterbuch, insbesondere dem aktuellen WWb, das sich auf die repräsentative Auswahl des Materials beschränkt, benötigte Datengrundlage hinausgeht. Dieser Datenpool bietet zahllose potentielle Auswertungsmöglichkeiten. Es seien hier nur einige wenige Beispiele genannt:

\subsection{Grundlage für lokale Mundartwörterbücher}

Das lautschriftliche Teilarchiv enthält lautschriftliches Material für 45 Orte und erweist sich vor allem für lautliche Fragestellungen als äußerst hilfreich. Darüber hinaus bieten die umfangreicheren Sammlungen eine weitere Möglichkeit der Nutzung: Sie könnten den Grundstock für ein lokales Mundartwörterbuch bilden. Diese bislang nur theoretische Möglichkeit ist 2016 zum ersten Mal in die Tat umgesetzt worden: Der „Arbeitskreis für Dorfentwicklung und Heimatpflege“ in Müschede (Hochsauerlandkreis) hat die vom ehemaligen Bearbeiter des WWb, FELIX WORTMANN, für seinen Heimatort Müschede (Arn Mü) gesammelten und im Archiv des

21 Viktoriya Dukhnova, eine ehemalige Praktikantin beim WWb, hat z. B. unter Verwendung von Band 3 des WWb eine Master-Arbeit zum Thema: „Genusschwankung in den westfälischen Dialekten“"geschrieben (Münster 2017). 
WWb vorhandenen Materialien zu einem Wörterbuch umgearbeitet und als Hauptteil in einem kleinen Büchlein herausgegeben: Erlebnis Mundart - Erinnerungen an den Müscheder Sprachwissenschaftler Dr. FELIX WoRTMANN (Erlebnis Mundart 2016).

Ein Großteil des alphabetischen Teilarchivs geht auf mehr oder weniger umfangreiche Sammlungen freiwilliger Mitarbeiterinnen und Mitarbeiter zurück. Manche Sammlung wie die von Dr. August ECKARDT aus Dortmund-Wellinghofen (Dor Wl) ist von der Gewährsperson auf Wörterbuchzetteln eingereicht und zeitnah ins Archiv einsortiert worden. Dieses Verfahren kommt der Wörterbucharbeit zugute, mit dem Nachteil allerdings, dass diese sowohl quantitativ als auch qualitativ äußerst wertvolle Sammlung nicht mehr als zusammenhängende Einheit besteht. Sollte das Projekt der Archiv-Digitalisierung (vgl. Kapitel 6) realisiert werden, ließen sich die im gesamten Zettelarchiv verteilten Dor Wl zugeschlagenen Belege zu einem umfangreichen Dortmund-Wellinghofer Wörterbuch vereinen.

\subsection{Vergleich unterschiedlicher Erhebungen}

Da der Sprachschatz der westfälischen Mundarten in etlichen Fragebogenerhebungen abgefragt und auf diese Weise auch dokumentiert worden ist, besteht grundsätzlich die Möglichkeit, das Material mehrerer Erhebungen zu einem Ort zu vergleichen und auf diese Art sprachliche Entwicklungen zu verfolgen. So bietet sich etwa ein Vergleich zwischen dem 1922 verschickten BAADER-Fragebogen und dem 1975 verschickten Fragebogen 23, dem von FELIX WORTMANN konzipierten, so genannten „Fragebogen für den Entwurf einer Karte der westfälischen Mundarten“, an. Nicht nur sprachliche Entwicklungen lassen sich so verfolgen, sondern auch stabile dialektale Raritäten: 1922 meldet eine Gewährsperson aus dem westmünsterländischen Ort Epe bei Gronau (Ahs Ep) bei der Frage nach „Flasche“ Pull und Fläske. Östlich von Epe herrscht das Lexem Pulle vor, außerdem die $e$-Apokope, westlich gilt Fläske. Dass in einem Ort Pulle mit und Fläske ohne $e$-Apokope bezeugt sind, erscheint zunächst als Momentaufnahme, die eine bestimmte Phase in der Entwicklung festhält. Wenn 53 Jahre später dieselbe Konstellation wieder gemeldet wird, ist zu konstatieren, dass es hier keine Entwicklung gegeben, sondern sich dieser Sonderfall gehalten hat. So können sich zeitlich bedingte Unterschiede herausstellen, aber auch Übereinstimmungen bei seltenen Phänomenen, die sich nur aus einer ganz bestimmten Kontaktsituation erklären lassen.

\subsection{Quellenkritik}

Das im Archiv vorhandene Datenmaterial kann auch zu einer Art Quellenkritik verwendet werden. Vergleicht man die in Fragebogenerhebungen gesammelten Belege eines Ortes mit den Belegen eingesandter Wortsammlungen desselben Ortes, entsteht häufig der Eindruck, dass die freien Mitarbeiter in ihren eingesandten Wortsammlungen bezüglich der schriftlichen Wiedergabe der Lautung anders verfahren 
als die Gewährspersonen von Fragebogenerhebungen. Während erstere die zu erwartende Lautung bzw. Schreibung liefern, scheinen letztere zu versuchen, mit ihren eigenen meist unzureichenden und schwer nachzuvollziehenden Methoden die lautliche Realität zu erfassen. Vielleicht bemühen sie sich auch, eine möglichst abweichende Variante zu erfassen bzw. wiederzugeben. Die Fragebogenbelege sind also in der Regel komplizierter und heterogener als die Belege aus Wortsammlungen. Es wäre die Aufgabe einer größeren Untersuchung, diesen Eindruck zu überprüfen.

7.4 Vorarbeit für ein etymologisches Wörterbuch des Niederdeutschen

Über die genannten Beispiele hinaus, bei denen andere (niederdeutsche) Wörterbücher bzw. deren Archive vermutlich ähnliches Potential aufweisen, kommt dem WWb eine besondere Eigenschaft zu. Das WWb dokumentiert eine sehr konservative, vormittelniederdeutsche Phonemunterschiede z. T. erhaltende Mundartengruppe und hat - nicht zuletzt aus diesem Grund - einen etymologisch begründeten, historischen Lemmaansatz (vgl. Kapitel 4.1). Ein mögliches etymologisches Wörterbuch des Niederdeutschen könnte davon in besonderem Maße profitieren. Denn die Vorarbeit, die Rekonstruktion des historischen Phonems, ist für einen großen Teil des niederdeutschen Wortschatzes durch die Bearbeiter des WWb bereits geleistet.

\section{KONTAKTADRESSE, HOMEPAGE}

Kommission für Mundart- und Namenforschung Westfalens

- Westfälisches Wörterbuch -

Schlossplatz 34

48143 Münster

Deutschland

E-Mail: robert.damme@lwl.org

Homepage: <www.mundart-kommission.lwl.org/de/projekte/westf_woerterbuch/> 


\section{LITERATURVERZEICHNIS}

Beiband (1969) $=$ Westfälisches Wörterbuch. Beiband. Einführung - Abkürzungen - Anlage der Artikel - Lauttabelle - Übersichtskarten. Bearbeitet von FELIX WORTMANN.

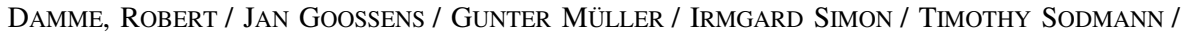
Hans TAUbKen / PAUl TEEPE (1988): Die Kommission für Mundart- und Namenforschung Westfalens. In: Westfälische Forschungen 38, 186-211.

DAMME, ROBERT (1997): Das Westfälische Wörterbuch. In: Niederdeutsches Wort 37, 13-20.

DAMME, ROBERT (2002): Aus der Werkstatt des Westfälischen Wörterbuchs: „Flasche“ und seine Grenzen in den westfälischen Mundarten. In: LEHMBERG, MAIK (Hg.): Wörter und Namen. Festgabe für ULRICH SCHEUERMANN zum 65. Geburtstag. Bielefeld: Verlag für Regionalgeschichte, 33-47.

DAMME, ROBERT (2013): Das Westfälische Wörterbuch als Projekt. Arbeitsoptimierung und Materialreduzierung. In: Niederdeutsches Wort 53, 7-19.

DenKLer, Markus / HeRmann Niebaum (2016): Vorüberlegungen zu einem Lautatlas für das Westfälische Wörterbuch. In: Verslagen \& Mededelingen van de Koninklijke Academie voor Nederlandse Taal- en Letterkunde 126, aflevering 1-2: Een leven lang taal- en literatuurwetenschap. Huldenummer voor JAN GoOSSENS, 167-179.

DWA = Deutscher Wortatlas (1951-1980). Hg. v. WALTER MITZKA und LUDWIG ERICH SCHMITT. Gießen: Wilhelm Schmitz Verlag.

$\mathrm{DWb}=$ Deutsches Wörterbuch von JACOB und WILHELM GRIMM. 16 Bände in 32 Teilbänden. Leipzig 1854-1961. Quellenverzeichnis Leipzig 1971.

Erlebnis Mundart - Erinnerungen an den Müscheder Sprachwissenschaftler Dr. FELIX WoRTMANN. (2016). Hrsg. vom Arbeitskreis für Dorfentwicklung und Heimatpflege Müschede/Balve.

FREDERKING, CHRISTIAN (1939): Plattdeutsches Dorfwörterbuch des Dorfes Hahlen bei Minden in Westfalen. Wortschatz. Spruchweisheit. Volkskunde. Bielefeld/Leipzig, unveränd. Neuaufl. Minden: Belhagen \& Klasing 2005.

HnVwb = Hessen-Nassauisches Volkswörterbuch (1943-2015). Bd. 2-4. Marburg: Elwert.

MndHwb = Mittelniederdeutsches Handwörterbuch (1956 ff.). Neumünster [u.a.]: Wachholtz Verlag.

$\mathrm{NdsWb}=$ Niedersächsisches Wörterbuch (1965 ff.). Neumünster/Kiel [u. a.]: Wachholtz Verlag.

PIIRAINEN, ELISABETH / WILHELM ELLING (1992): Wörterbuch der westmünsterländischen Mundart. Hrsg. vom Heimatverein Vreden unter Mitarbeit zahlreicher Gewährsleute. Vreden.

PILKMANN-POHL, ReINHARD (1988): Plattdeutsches Wörterbuch des kurkölnischen Sauerlandes. Hrsg. vom Sauerländer Heimatbund e. V. Arnsberg: Strobel-Verlag.

PlatenAU, Fritz (1978): Plattdeutsches Wörterbuch in Istruper Mundart. Im Auftrag des Lippischen Heimatbundes. Detmold.

RhWb = Rheinisches Wörterbuch (1928-1971). Bearb. und hg. von JOSEF MÜLLER, ab Bd. VII von Karl Meisen, HeinRICh DitTMaier und MatThias Zender. 9 Bde. Bonn/Berlin: Klopp.

SCHMOECKEL, HERMANN / ANDREAS BLESKEN (1952): Wörterbuch der Soester Börde. Ein Beitrag zur westfälischen Mundartenforschung. Soest (Soester wissenschaftliche Beiträge. 5).

STERN, HEIDI (2000): Wörterbuch zum jiddischen Lehnwortschatz in den deutschen Dialekten. Tübingen: De Gruyter.

STRODTMANN, JOHANN CHRISTOPH (1756): Idioticon Osnabrugense. Leipzig/Altona: Kortensche Buchhandlung.

TEUCHERT, HERMANN (1924/1925). Lautschrift des Teuthonista. In: Teuthonista 1, 5.

WEDDIGEN, M.P.F. (1790). Ravensbergisches Idiotikon. In: Historisch-geographisch-statistische Beschreibung der Grafschaft Ravensberg in Westphalen. Leipzig: Weidmannsche Buchhandlung, 269-332.

WOESTE, FRIEDRICH (1882): Wörterbuch der westfälischen Mundart. Norden/Leipzig (Wörterbücher. Hrsg. vom Verein für niederdeutsche Sprachforschung, I), Nachdruck: Bremen: Diedr. Soltau's Verlag 2010. 
WOESTE, FRIEDRICH (1930): Wörterbuch der Westfälischen Mundart. Im Auftrage des Westfälischen Heimatbundes neu bearbeitet und herausgegeben von ERICH NÖRRENBERG. Norden/Leipzig: Heinrich Soltau.

$\mathrm{WWb}=$ Westfälisches Wörterbuch. Herausgegeben von der Kommission für Mundart- und Namenforschung des Landschaftsverbandes Westfalen-Lippe. Kiel/Hamburg: Wachholtz Verlag.

Weitere Literaturhinweise unter

<https://www.mundart-kommission.lwl.org/de/projekte/westf_woerterbuch/literatur/> 



\title{
DAS NIEDERSÄCHSISCHE WÖRTERBUCH AUF DEM WEGE ZU SEINER FERTIGSTELLUNG
}

\author{
Maik Lehmberg
}

\section{GEGENSTAND, GESCHICHTE UND RAHMENBEDINGUNGEN}

\subsection{Abriss der Geschichte des Unternehmens}

Das „Niedersächsische Wörterbuch“ ist ein streng alphabetisch geordnetes Bedeutungswörterbuch der rezenten niederdeutschen Mundarten in den Bundesländern Niedersachsen und Bremen.

Erst verhältnismäßig spät wurde auf dem Gebiet des heutigen Bundeslandes Niedersachsen (und der Hansestadt Bremen) ein großlandschaftliches Mundartwörterbuch eingerichtet, erste Bestrebungen in diese Richtung sind erst ab Ende der 1920er Jahre nachweisbar (vgl. APPENZELLER 2011: 69). Diese „schmerzliche Lücke im Nordwesten" (MITZKA 1937: 95) zu schließen, gelang erst im Jahre 1935 mit der Gründung der „Geschäftsstelle für Mundartforschung“ an der Georg-August-Universität Göttingen und damit einer Wörterbucharbeitsstelle für die Vorgängerterritorien des Landes Niedersachsen. ${ }^{1}$ Bereits knapp drei Monate später wurde die „Geschäftsstelle“ dem Seminar für deutsche Philologie der Universität Göttingen angegliedert und in „Abteilung für niedersächsische Mundartforschung“ umbenannt. Tatsächlich war die Gründung des "Niedersächsischen Wörterbuches“ (Nds. Wb.) jedoch wohl bereits 1934 erfolgt. $^{2}$

Das Bearbeitungsgebiet des „Niedersächsischen Wörterbuches“ umfasst das Gebiet der alten Länder Braunschweig, Oldenburg, Schaumburg-Lippe, die PreuBische Provinz Hannover sowie die Freie Hansestadt Bremen, was den heutigen Bundesländern Niedersachsen und Bremen entspricht. Am Zuschnitt des Bearbeitungsgebietes änderte sich seither nichts Grundlegendes. ${ }^{3}$ Das bedeutet, dass im

1 „Offiziell nahm die Wörterbucharbeitsstelle am 1. Mai 1935 ihre Arbeit auf.“ (APPENZELLER 2011: 91).

2 „Am 8. Dezember war es dann soweit. Die ,Arbeitsstelle Niedersächsisches Wörterbuch wurde offiziell gegründet.“ (APPENZELLER 2011: 86).

3 Mit dem Wiederbeginn der Arbeiten am „Niedersächsischen Wörterbuch“ nach dem Zweiten Weltkrieg blieb das Gebiet des Amtes Neuhaus, das sich nun in der sowjetischen Besatzungszone befand, unberücksichtigt. Erst seit der Eingliederung des Amtes Neuhaus in den niedersächsischen Landkreis Lüneburg im Jahre 1993 wird dieser Bereich wieder berücksichtigt. Vgl. dazu Niedersächsisches Wörterbuch (1998: Vorwort). - Zum Amt Neuhaus ausführlich der Exkurs „Änderung des Bearbeitungsgebietes nach 1990“ bei APPENZELLER (2011: 229-231). 
Bearbeitungsgebiet des Nds. Wb.s alle drei großen westniederdeutschen Mundartverbände - Nordniedersächsisch, Ostfälisch und Westfälisch - vertreten sind, was wegen der enormen Vielfalt sowohl in der Lexik als auch vor allem hinsichtlich der Phonetik bzw. Phonologie nicht unerhebliche Auswirkungen auf die lexikographische Arbeit hat.

Die Konzeption des Nds. Wb.s folgte bei seiner Gründung in weiten Teilen den Vorgaben des sog. Wörterbuchkartells ${ }^{4}$; es war zunächst stark volkskundlich ausgerichtet. Erster Leiter des Nds. Wb.s wurde der Göttinger Germanist FRIEDRICH Neumann, die Aufgabe des ersten Bearbeiters erhielt der Mitzka-Schüler HANS JANSSEN, der zuvor Assistent am Deutschen Sprachatlas in Marburg gewesen war. ${ }^{5}$

Grundlage des Wörterbuch-Archivs ist mittels indirekter Fragebogenerhebung gewonnenes Material. HANS JANSSEN entwarf die ersten acht Fragebogen, die in der Zeit bis zum Zweiten Weltkrieg verschickt wurden, ${ }^{6}$ die Fragebogen 9 und 10 wurden nach dem Krieg von seinem Nachfolger WOLFGANG JUNGANDREAS konzipiert und verschickt. Die Fragebogenerhebung zielte schwerpunktmäßig auf die bäuerliche Lebensumwelt ab. Gefragt wird vorwiegend nach den mundartlichen Bezeichnungen für landwirtschaftliche Geräte, für den Bereich Arbeitswelt, Haus und Hof, Tier- und Pflanzennamen. ${ }^{7}$

Den anderen Strang des Wörterbuch-Archivs bildet das Zettelarchiv. Da klar war, dass Fragebogenerhebungen nur wenige Belegsätze ergeben würden, rief JANSSEN bereits während der Fragebogenerhebung wiederholt zur Mitarbeit am Wörterbuch auf, erbat Exzerpte aus regionalen Wörterbüchern, Sprichwortsammlungen und anderen Büchern, einschließlich literarischer Quellen, sowie die Einsendung vorhandener Mundartsammlungen.

Die beiden Stränge des Archivs des „Niedersächsischen Wörterbuches“ in der Übersicht:

- Fragenbogenarchiv: In 190 Formularkästen befinden sich die Rückläufer der Fragebogenerhebung der Bogen 1-8 aus den Jahren 1935-1938, des Bogens 9 (1947) und des Bogens 10 (1949). ${ }^{8}$ Die Bogen stammen aus mehr als 2200 Orten Niedersachsens und Bremens, die Gesamtzahl beträgt mehr als

4 „Hierunter versteht man einen losen Verbund deutschsprachiger diatopischer Dialektwörterbücher. Es wurde am 2. Oktober 1913 im Anschluss an eine Philologenversammlung von den Wörterbuchredaktionen Deutschlands, Österreich-Ungarns und der Schweiz auf Appell der Königlich-Preußischen Akademie der Wissenschaften und unter Leitung des Marburger Dialektologen Ferdinand Wrede gegründet.“(APPENZELLER 2011: 69)

5 Zur Gründungsphase des Nds. Wb.s. ausführlich der Abschnitt „Lexikografische Vorläufer und Gründung der Arbeitsstelle“ bei APPENZELLER (2011: 69-91).

6 Noch im Mai 1935 wurde bereits der erste Fragebogen versandt (vgl. APPENZELLER 2011: 100).

7 SCHEUERMANN (1976: 199): „Auf die persönlichen, allerdings auch dem Zeitgeist konformen Interessen Janßens ist der starke volkskundliche Einschlag zurückzuführen, den die damalige Konzeption des Nds.Wb. zeigt und der sich auch in den Fragebogen niederschlug.“

8 Der Fragebogen 10 wurde nicht mehr flächendeckend, sondern nur noch gezielt an 15 mundartkundige Gewährspersonen verschickt (vgl. LEHMBERG 2002: 19). 
20 000. Darin enthalten sind ca. 1,2 Millionen Belege zu (geschätzt) 40000 Stichwörtern. $^{9}$

- Zettelarchiv: Der Umfang beträgt insgesamt ca. 2,5 Millionen Belegzettel unterschiedlicher Provenienz. Den Grundstock bilden die von Laien eingesandten Sammlungen unterschiedlicher Größe (von einigen Hundert bis zu rund 17000 Zetteln). ${ }^{10}$ Daneben stehen insbesondere Kopien aus gedruckten (Teil-) Wörterbüchern und Sprichwortsammlungen. Mit ca. 350000 Zetteln sind auch Kopien zweier von der Universität Münster durchgeführter Sammlungen in das Zettelarchiv eingegangen. ${ }^{11} \mathrm{Zu}$ erwähnen sind noch die Ergebnisse der sog. Landschaftskorrektur. ${ }^{12}$ Ausgesondert wurden bei der Ordnung und Strukturierung des Archivs generell alle literarischen Belege sowie die handschriftlichen Exzerpte aus gedruckten Quellen. ${ }^{13}$

Daneben verfügt das Archiv noch über einige wenige Sammlungen und andere Quellen, die nicht mehr verzettelt werden konnten und deshalb nicht in das Zettelarchiv eingingen. Diese Quellen müssen jeweils in einem gesonderten Arbeitsgang in das Rohtyposkript eingearbeitet werden. ${ }^{14}$

Mit dem Wiederbeginn der Arbeiten am Nds. Wb. nach dem Zweiten Weltkrieg wurde die volkskundliche Ausrichtung zugunsten einer ausschließlich philologischen aufgegeben, was jedoch möglicherweise nicht abrupt erfolgte. ${ }^{15}$ Erst nach der Übernahme der Leitung des Nds. Wb.s durch HEINRICH WeScHE im Jahre 1954 wurde eine streng lexikologisch-dialektologische Wörterbuchkonzeption verfolgt.

9 Dazu ausführlicher auf der Homepage des Nds. Wb.s unter der URL: <http://www.unigoettingen.de/de/219496.html>; Stand: 29.03.2018. Die nachgestalteten Fragebogen können auch heruntergeladen werden, URL: <http://www.uni-goettingen.de/de/fragebogen/219741. html>; Stand: 29.03.2018.

$10 \mathrm{Zu}$ diesen sog. freien Sammlungen ausführlicher unter der URL: <http://www.uni-goettingen. de/de/219497.html>; Stand: 29.03.2018.

11 Einzelheiten zu den Fragebogenerhebungen APPENZELLER (2011: 201). Appenzeller nennt 325000 Zettel, die aus den Münsteraner Erhebungen stammen.

12 Zeitweise wurde das fertige Manuskript vervielfältigt und bis zu 100 kundigen Mundartsprechern im Bearbeitungsgebiet zur Durchsicht und Korrektur zugesandt. Dieses Verfahren wurde 1984 mit Ende des Buchstabens $B$ eingestellt. - Zu den Einzelheiten der Landschaftskorrektur ausführlich APPENZELLER (2011: 217-223).

13 Dazu ausführlicher auf der Homepage des „Nds. Wb.s“ unter der URL: <http://www.unigoettingen.de/de/219495.html>; Stand: 29.03.2018. Zu den gedruckten Quellen auch APPENZELLER (2011: 202-203).

14 Einige dieser Quellen wurden inzwischen digitalisiert und in das Datenbanksystem EFBA des Nds. Wb.s (s. dazu das Kapitel 1.3 Entwicklung und Einsatz eines Datenbanksystems) integriert.

15 „In der Folgezeit bis zur Übernahme der Arbeitsstellenleitung durch HeINRICH WeSCHE gab es ein Nebeneinander bzw. eine Mischung einer volkskundlichen und einer lexikologisch-dialektologischen Konzeption." (APPENZELLER 2011: 215) 


\subsection{Strukturierung, Neuordnung und EDV-gestützte Erschließung des Archivs}

Zwar wurden bereits 1945 die Arbeiten am Nds. Wb. wieder aufgenommen, doch wurde die systematische Erschließung und Ordnung des Archivs - durch Verzettelung des Fragebogenarchivs und Strukturierung des Zettelarchivs durch Lemmatisierungen und entsprechend folgende Sortierung zu leisten - versäumt. Vielmehr kam es zu einem völlig verfrühten Publikationsbeginn (1951). Auch als 1953 HANS NEUMANN auf WOLFGANG JUNGANDREAS als Leiter der Arbeitsstelle folgte, änderte sich an der Situation nichts. Wohl nicht zuletzt unter dem Druck der Geldgeber, die auf die Publikation weiterer Lieferungen drängten, unterblieb zunächst die Ordnung und Erschließung des Archivs.

Daran änderte sich erst Mitte der 1960er Jahre etwas, als sich der damalige Leiter HEINRICH WESCHE von seinem Assistenten GISBERT KESELING überzeugen ließ, dass nunmehr endlich die Ordnung des Archivs Vorrang vor der kontinuierlichen Publikation haben müsse, damit man anschließend umso besser werde schreiben können (vgl. LEHMBERG 2002: 20).

Zur Erschießung des Fragebogenarchivs machte sich das Nds. Wb. als erste dialektlexikographische Unternehmung schließlich die elektronische Datenverarbeitung (EDV) zu Nutze, die seinerzeit gerade für die Geisteswissenschaften gewissermaßen entdeckt wurde. Die Informationen sämtlicher mehr als 20000 Fragebogen wurden mittels Lochkarten maschinenlesbar gemacht ${ }^{16}$ und anschließend in einer Großrechenanlage verarbeitet. Dabei wurden unter verschiedenen Gesichtspunkten Listen generiert und gedruckt, die bei der täglichen lexikographischen Arbeit einen schnellen Zugriff ermöglichen. ${ }^{17}$ Vor allem wohl wegen des Pioniercharakters dieses Vorgehens war allerdings nicht absehbar, dass die gesamte Erschließung des Fragebogenarchivs rund zehn Jahre in Anspruch nehmen würde. ${ }^{18}$

Parallel zu der Erschließung des Fragebogenarchivs musste das seinerzeit aus ca. 800000 Zetteln bestehende Zettelarchiv geordnet werden, da die Zettel zuvor nur nach dem Anfangsbuchstaben grob vorsortiert waren. Dazu wurde jeder Zettel mit einem handschriftlichen Lemma versehen, unter dem der Beleg einzuarbeiten

$16 \mathrm{Zu}$ den Konventionen der Übertragung der sprachlichen Informationen auf Lochkarten besonders SCHEUERMANN (1974: 16-20).

17 „Wichtigstes Arbeitsmittel, das bei der im Hause als ,Ablochung‘ bezeichneten Prozedur durch den listenweisen Ausdruck der Informationen entstand, war der sog. Output. Dabei handelt es sich um gut 31000 Seiten, die, alphabetisch sortiert nach dem vergebenen Lemma und der Fragenummer, in zumeist codierter Weise das Lemma, den Belegort, die Fragebogen-Nummer, die Fragenummer, den Sprachbeleg selbst, Informationen grammatischer Art (etwa Wortart, Kasus, Numerus) sowie ggf. Hinweise zur Bedeutung enthalten. Jede dieser Seiten ist nur einmal vorhanden, so daß der Fortgang der Arbeiten auch immer durch einen Verlust dieser Listen bedroht war." (LEHMBERG 2009: 2-3); dort auch zu den anderen Listen (Heteronymenliste, Stichwortliste) sowie die ausschnittweise Abbildung einer Seite des sog. Outputs.

$18 \mathrm{Zu}$ den Einzelheiten der Erschließung des Fragebogenarchivs im Exkurs „Sortierungsarbeiten mittels EDV“ bei APPENZELLER (2011: 172-178). - Zum Verfahren der „Ablochung“ der Sprachdaten vgl. KESELING et al. (1970), zu grundsätzlichen Überlegungen zum Einsatz der EDV in der (Dialekt-)Lexikographie SCHEUERMANN (1974). 
ist. Die Sortierung erfolgte dann alphabetisch entsprechend der zuvor vermerkten Lemma-Form.

\subsection{Entwicklung und Einsatz eines Datenbanksystems}

Das Ergebnis der EDV-gestützten Erschließung des Fragebogenarchivs waren ausschließlich die unter bestimmten Kriterien generierten und so gedruckten Listen. Die gewonnenen Daten verblieben zunächst auf den verwendeten Magnetspulen der eingesetzten Großrechenanlage. Nachdem diese Geräte vom Universitätsrechenzentrum außer Dienst gestellt worden waren und Magnetspulen-Laufwerke nicht mehr eingesetzt wurden, galten die Daten als verloren.

Durch glückliche Umstände wurde jedoch nach der Auflösung des Lehrstuhls für Niederdeutsche Sprache und Literatur am Seminar für deutsche Philologie der Universität Göttingen (2005) ein Datenträger (CompactDisk) aufgefunden, auf den ein Mitarbeiter des Rechenzentrums vorsorglich die Banddaten kopiert hatte. ECKHARD EGGERS (Redakteur seit 2007) entwickelte ab 2008 aus den ursprünglichen Daten ein Datenbanksystem (,Elektronisches Fragenarchiv [EFBA]“ genannt), das komfortable Abfragen und Recherchen ermöglicht und in der täglichen Arbeit die Verwendung der mehr als 31000 Seiten umfassenden gedruckten Listen verzichtbar macht. ${ }^{19}$

Da für das EFBA ein proprietäres Datenbanksystem verwendet wird, das nicht mehr weiterentwickelt wird und auf neueren Computern in der Praxis teilweise nur sehr langsam läuft, nimmt EGGERS neben seiner eigentlichen Redakteursarbeit derzeit sukzessive eine Portierung der Datenbank auf ein zukunftssicheres MySQLSystem vor, für das die erforderlichen Abfrage- und Rechercheprogramme in der offenen Programmiersprache Python geschrieben werden.

Den Redakteuren steht zum einen ein wohlgeordnetes Zettelarchiv zur Verfügung, das nach wie vor den Ausgangspunkt für jeden Wortartikel bildet. Die Verarbeitung der Informationen aus dem Fragebogenarchiv kann durch das flexible und mächtige Abfragesystem der Datenbank nun allerdings gleichzeitig mit der Bearbeitung der Zettel erfolgen.

\subsection{Publikationsstand}

Zum jetzigen Zeitpunkt ${ }^{20}$ liegen neun Bände des Nds. Wb.s gebunden vor, es sind dies:

- Niedersächsisches Wörterbuch. Auf Grund der Vorarbeiten von HANS JANSSEN und unter Mitwirkung eines Arbeitskreises niedersächsischer Mundartforscher

19 Dazu der Abschnitt „Das Elektronische Fragebogenarchiv (EFBA)“ bei APPENZELLER (2011: 211-212). Ausführlicher, mit Anwendungsbeispielen und Screenshots des Datenbanksystems, EGGERS (2008) sowie LEHMBERG (2009).

20 Stand: Mai 2018. 
hg. von der Abt. für niedersächsische Mundartforschung des Seminars für Deutsche Philologie der Univ. Göttingen durch WoLfGANG JungANDREAS. Bd. 1: A bis bersen. Neumünster: Karl Wachholtz Verlag 1965. [Lfg. 1-7b, Erscheinungsbeginn: 1953].

- Niedersächsisches Wörterbuch. Auf Grund der Vorarbeiten von HANS JANSSEN $\uparrow$ und unter Mitwirkung eines Arbeitskreises niedersächsischer Mundartfreunde herausgegeben von der Abteilung für niedersächsische Mundartforschung des Seminars für Deutsche Philologie der Universität Göttingen durch HEINRICH WESCHE $\uparrow$ (bis biselig). Herausgegeben vom Institut für Historische Landesforschung der Universität Göttingen durch die Arbeitsstelle Niedersächsisches Wörterbuch (ab Biseljan). Bd. 2: Bertsche-Büzpott. Bearbeitet von Gisbert Keseling (bis biselig), Wolfgang Kramer, Ulrich ScheuERMANN. Neumünster: Karl Wachholtz Verlag 1985.

- Niedersächsisches Wörterbuch. Hg. von DIETER STELlMACHER, Institut für Historische Landesforschung der Universität Göttingen, Arbeitsstelle Niedersächsisches Wörterbuch. Bd. 3: $C$-exzēren. Bearbeitet von JENS VOLQUARD Gonnsen, Wolfgang Kramer, Ulrich Scheuermann, Peter Wagener. Neumünster: Karl Wachholtz Verlag 1993.

- Niedersächsisches Wörterbuch. Hg. von DieTER STEllmaChER, Institut für Historische Landesforschung der Universität Göttingen, Arbeitsstelle Niedersächsisches Wörterbuch. Bd. 4: $F / V$. Bearbeitet von UlRICH SCHEUERMANN. Neumünster: Wachholtz Verlag 1994.

- Niedersächsisches Wörterbuch. Hg. von DIETER STELlMACHER, Institut für Historische Landesforschung der Universität Göttingen, Arbeitsstelle Niedersächsisches Wörterbuch. Bd. 5: Gabbel-Haubön, bearbeitet von WOLFGANG Kramer, Karin Schade, Ulrich Scheuermann. Neumünster: Wachholtz Verlag 1998.

- Niedersächsisches Wörterbuch. Hg. von DIETER STELLMACHER, Institut für Historische Landesforschung der Universität Göttingen, Arbeitsstelle Niedersächsisches Wörterbuch. Bd. 6: Haubön-juxwisse. Bearbeitet von WOLFGANG Kramer, MaIK Lehmberg, Karin Schade, Ulrich ScheuermanN. Neumünster: Wachholtz Verlag 2003.

- Niedersächsisches Wörterbuch. Hg. von DIETER STELLMACHER, Institut für Historische Landesforschung der Universität Göttingen, Arbeitsstelle Niedersächsisches Wörterbuch. Bd. 7: ka-kü̈zen. Bearbeitet von MAIK LEHMBERG, ULRICH SCHEUERMANN. Neumünster: Wachholtz Verlag 2011.

- Niedersächsisches Wörterbuch. Hg. von DIETER STELLMACHER, Institut für Historische Landesforschung der Universität Göttingen, Arbeitsstelle Niedersächsisches Wörterbuch. Bd. 8: Lāb-Myrtenkranz. Bearbeitet von MARTIN SCHRÖDER. Neumünster: Wachholtz Verlag 2011.

- Niedersächsisches Wörterbuch. Hg. von ALBERT Busch, Seminar für Deutsche Philologie der Universität Göttingen, Arbeitsstelle Niedersächsisches Wörterbuch. Bd. 9: nā-quutschig. Bearbeitet von ECKHARD EGGERS, MARTIN SCHRÖDER. Kiel/Hamburg: Wachholtz Verlag Murmann Publishers 2017. 
Darüber hinaus liegen aktuell vier Lieferungen des 10. Bandes (Wortstrecke: $r$ Schāpsgarve) und drei Lieferungen des XI. Bandes (Wortstrecke: Slabāke-snīr) vor. $^{21}$

\subsection{Sonstige Publikationen zum Material}

Zwischen 1988 und 2010 führte die Arbeitsstelle Niedersächsisches Wörterbuch insgesamt elf „Regionaltreffen“ durch, bei denen sich die Mitarbeiter an verschiedenen Orten im Lande ihre Arbeit vorstellten. Die dabei zu verschiedenen Aspekten der Wörterbucharbeit bzw. zum Archiv gehaltenen Vorträge sind in sog. Berichtsheften dokumentiert worden.

Einzelheiten dazu (Tagungsorte, Vortragende, Vortragstitel, bibliographische Angaben zu den Berichtsheften) sind auf der Homepage der Arbeitsstelle dokumentiert. $^{22}$

\subsection{Neue Konzeption 2000 (Straffungskonzept)}

Unter anderem der 1999 bzw. 2003 vollzogene bzw. anstehende Wechsel der Bearbeitergeneration, vor allem aber die Notwendigkeit, die Bearbeitung des Nds. Wb.s in einem absehbaren und dem Geldgeber gegenüber vertretbaren Zeitrahmen abschließen zu können, gaben den Anlass, die zu Grunde liegende Konzeption so zu modifizieren, dass die Arbeiten zwar beschleunigt werden, die wissenschaftliche Qualität aber gewahrt bleibt und zudem kein Bruch innerhalb des Wörterbuches entsteht. So wurde im Jahr 2000 dem niedersächsischen Ministerium für Wissenschaft und Kunst (MWK) ein vom damaligen Leiter und Herausgeber DIETER STELLMACHER sowie den Redakteuren erarbeitetes Straffungskonzept vorgelegt. $^{23}$

Die wichtigsten Maßnahmen: Das Nds. Wb. wird als Dialektwörterbuch (nicht als Idiotikon) weitergeführt, das Thesaurus-Prinzip wird jedoch aufgegeben. Straffungen werden beispielsweise dadurch erreicht, dass Einzelbelege aus gedruckten Quellen und Spontanbildungen nicht mehr berücksichtigt werden, was auch für fach- und gruppensprachlichen Wortschatz gilt. Die Mikrostruktur wird vor allem durch vereinfachte Verbreitungsangaben, vereinfachte grammatische Angaben und

21 Eine jeweils aktualisierte Aufstellung aller erschienenen Wörterbuchlieferungen findet sich auf der Homepage. URL: <http://www.uni-goettingen.de/de/ver\%c3\%b6ffentlichungsfolge+\%28 lieferungsweise\%29/219541.html>; Stand: 03.04.2018.

22 URL: <http://www.uni-goettingen.de/de/198683.htmlde/berichtshefte/219711.html>; Stand: 04.04.2018.

23 Die „Konzeption 2000 (Straffungskonzept)“ blieb unveröffentlicht. 
Darstellungen von Lautverhältnissen entlastet, zudem wird in den Wortartikeln auf Satzbelege aus gedruckten (Teil-)Wörterbüchern verzichtet. ${ }^{24}$

Die erste nach dem Straffungskonzept erarbeitete Lieferung ist die 57. ( $L \bar{a} b-$ Leggelhenne), zugleich die erste Lieferung des achten Bandes (erschienen 2004). Vor allem während der Arbeit an dieser Lieferung wurden einige Maßnahmen der „Konzeption 2000“ nach Gesprächen der Redakteure an die praktischen lexikographischen Erfordernisse angepasst, so dass schließlich ein stimmiges Instrumentarium zur Verfügung steht, mit dem die fristgerechte und wissenschaftlich einwandfreie Fertigstellung des Nds. Wb.s gewährleistet wird. Die in der Praxis gesammelten Erfahrungen mit der Anwendung der „Konzeption 2000“ wurde 2003 in einem gemeinsamen Vortrag der Redakteure bei der 4. Arbeitstagung deutschsprachiger Akademiewörterbücher in Göttingen im Kollegenkreis vorgestellt. ${ }^{25}$

\subsection{Zeitplan bis zur Fertigstellung}

Nach organisatorischen Veränderungen im Jahre 2012 wurde von den Mitarbeitern des Wörterbuches ein verlässlicher Zeitplan erarbeitet, der sicherstellt, dass das Nds. Wb. in der Zeit von 2013 bis zur Pensionierung des jüngsten Mitarbeiters im Jahre 2029 fertiggestellt werden wird, sofern die dritte Redakteursstelle, die seit dem Jahre 2007 vom niedersächsischen Ministerium für Wissenschaft und Kunst (MWK) finanziert wird, bis zum Lebensarbeitszeitende des Stelleninhabers erhalten bleibt.

Grundlage der Berechnungen ist der bereits nach der „Konzeption 2000“ erarbeitete achte Band (Bereich $L-M$ ). Der Band 8 umfasst 7 1/2 Lieferungen, für die 120 Zettelkästen verarbeitet wurden, was durchschnittlich 16 Kästen pro Lieferung entspricht. Zum Zeitpunkt der Berechnungen waren insgesamt noch 550 Zettelkästen zu bearbeiten, was bei 16 Kästen pro Lieferung eine zu erarbeitende Restmenge von 35 Lieferungen bedeutet.

Vor dem Hintergrund des Befundes, dass die Arbeitsleistung von einer Lieferung pro Redakteur (Bearbeiter) und Jahr nicht gesteigert werden kann, wenn die nachweislich hohe wissenschaftliche Qualität des Nds. Wb.s erhalten bleiben soll, wurden die restlichen 35 Lieferungen auf die drei Bearbeiter mit deren jeweils noch verbleibenden Arbeitsjahren verteilt. Der auf diese Weise erarbeitete „Detaillierte Arbeitsplan 2013-2029“" wurde dem MWK vorgelegt und von diesem sowie dem Präsidium der Georg-August-Universität Göttingen genehmigt. Die Weiterfinanzierung der dritten Redakteursstelle und der Hilfskräfte wurde auf der Grundlage des Arbeitsplans zugesagt.

24 Die wichtigsten Maßnahmen finden sich ausführlicher bei LEHMBERG / SCHRÖDER (2004) (mit Beispielen und Veranschaulichungen). - Dort im Anhang als weiteres Beispiel der Wortartikel Kède 'Kette' im Vergleich nach vorheriger und nach neuer Konzeption.

25 Der Vortrag fand am 10.09.2003 statt. Er wurde (in leicht veränderter Form) später publiziert: LEHMBERG / SCHRÖDER (2004). 
Inzwischen erwies sich der Arbeitsplan durch die publizierten Lieferungen als in vollem Umfang tragfähig.

\subsection{Organisatorisches}

Seit dem 1. Januar 2013 gehört die Arbeitsstelle Niedersächsisches Wörterbuch nicht mehr zum Institut für Historische Landesforschung, sondern ist organisatorisch wieder dem Seminar für Deutsche Philologie der Universität Göttingen (SDP) zugeordnet.

Die Projektleitung hat der Leiter der Seminarverwaltung des Seminars für Deutsche Philologie apl. Prof. Dr. AlBERT Busch inne, der seither auch das Nds. Wb. herausgibt.

Die wissenschaftliche Verantwortung für das Nds. Wb. obliegt einem Wissenschaftlichen Beirat, ${ }^{26}$ dem derzeit fünf Personen angehören:

- Prof. Dr. HeInRICH DingELdEIN (Forschungszentrum Deutscher Sprachatlas, Marburg)

- Prof. Dr. Anthony Rowley (Bayerische Akademie der Wissenschaften, München)

- Prof. Dr. INGRID SCHRÖDER (Universität Hamburg)

- Prof. Dr. HANS-JOACHIM SolMS (Sächsische Akademie der Wissenschaften zu Leipzig)

- Prof. Dr. Dieter Stellmacher (Universität Göttingen, Sprecher des Beirates).

Das Nds. Wb. verfügt über drei Redakteure (Bearbeiter), von denen zwei fest angestellt bzw. beamtet sind:

- apl. Prof. Dr. ECKHARD EGGERS

- Dr. MAIK LeHMBERG

- Dr. MARTIN SCHRÖDER.

Unterstützt wird die Arbeit der Redakteure von derzeit zwei wissenschaftlichen Hilfskräften (mit zusammen 108 Stunden im Monat).

- Dr. UlF BORCHERS

- Dr. Peter StrathmanN.

26 Diese Konstellation gab es bereits in den Jahren 1973 bis 1982 (nach der Emeritierung HEINRICH WESCHES bis zur Übernahme der Leitung des Nds. Wb.s und der wissenschaftlichen Verantwortung durch DieTER STELLMACHER). Vgl. dazu ausführlich APPENZELLER (2011: 180189). 


\section{KONZEPTION, STRUKTUR UND INHALT DER WORTARTIKEL DES „NIEDERSÄCHSISCHEN WÖRTERBUCHES“}

\subsection{Lemmatisierung}

Jeder Zettel des Zettelarchivs wurde zeitlich parallel zu der EDV-gestützten Erschließung des Fragebogenarchivs mit einer möglichen Ansatzform versehen (vorlemmatisiert), nach der die Zettel schließlich sortiert wurden. Gleichfalls erfolgte die Erschließung des Fragebogenarchivs über jeweils eine mögliche Ansatzform für jeden Datensatz. ${ }^{27}$

Der Stichwortansatz des Nds. Wb.s erfolgt hinsichtlich der Lautung prinzipiell nach einem (maßvoll) historischen, gemäßigt phonologischen System, das sich an den Gegebenheiten des Nordniedersächsischen orientiert. Das bedeutet insbesondere, dass die alten Langvokale, die in vielen ost- und westfälischen Mundarten diphthongiert sind, als Monophthonge angesetzt werden und die Diphthonge der alten Kurzvokale der oberen Reihe, die sich in vielen westfälischen Mundarten finden (sog. Westfälische Brechung), unberücksichtigt bleiben. Die Ansätze sind somit weitgehend theoretische Konstrukte, stellen für weite Teile des Bearbeitungsgebietes aber dennoch tatsächlich gesprochene Formen dar. ${ }^{28}$

Das tatsächlich im Wörterbuch erscheinende Lemma kann in der Regel erst festgelegt werden, wenn sämtliche im Archiv vorhandenen Belege gesichtet sind. Bei der Schreibung orientiert sich das Lemma an den Regeln bzw. Gegebenheiten der hochdeutschen Standard-Orthographie, was der Lesegewohnheit der Benutzerinnen und Benutzer entgegenkommt und so auch ein leichteres Auffinden eines Stichwortes ermöglicht. Die Länge eines Vokales (bzw. Umlautes) wird sowohl in offener als auch in geschlossener Silbe durch einen über den Vokal (bzw. Umlaut) gesetzten Strich markiert. Bei vom Standard abweichender Betonung kann im Lemma ein Betonungszeichen über den Vokal der Silbe gesetzt werden, die den Ton trägt.

Das Lemma erscheint im Satz um jeweils $0,3 \mathrm{~cm}$ eingezogen und wird fett gedruckt. Gleichlautende Lemmata, die nicht durch Groß- bzw. Kleinschreibung unterschieden werden, werden mit einer hochgestellten Indexziffer versehen.

\subsection{Artikelstruktur}

Auf das Lemma folgt bei Substantiven (deren Wortart durch die Großschreibung nur implizit genannt ist) die Angabe des Genus, sofern es sich um ein Simplex han-

27 Vgl. das Kapitel 1.2 „Strukturierung, Neuordnung und EDV-gestützte Erschließung des Archivs".

28 Vgl. „Hinweise für den Benutzer“ im „Niedersächsischen Wörterbuch“ (1993: V). - In den „Hinweisen für den Benutzer“ zu den Einzelheiten der hinsichtlich des Ansatzes bzw. zur Schreibung der Stichwörter vorgenommenen Vereinheitlichungen. 
delt. Bei Komposita wird das Genus in der Regel nicht genannt, da davon auszugehen ist, dass es dem des Simplex entspricht. Im Einzelfall (vor allem bei von dem des Simplex abweichendem Genus) erfolgt jedoch eine Genusangabe.

Bei Nicht-Substantiven folgt auf das Lemma die Angabe der Wortart, wobei die Klassifizierung in Anlehnung an die Duden-Grammatik vorgenommen wird. Wie auch die Genusangabe erfolgt die Angabe der Wortart mit im Abkürzungsverzeichnis aufgeführten Abkürzungen. ${ }^{29}$

Nach der Sichtung sämtlicher im Archiv vorhandenen Belege zur Ermittlung des korrekten Lemmaansatzes kann in der Regel auch die Bedeutungsgliederung des zu schreibenden Wortartikels festgelegt werden. ${ }^{30}$ Hat das Lemma mehr als eine Bedeutung, folgt auf die Wortart- bzw. Genusangabe eine fett gesetzte Bedeutungsziffer. Handelt es sich um eine Pflanzen- oder Tierbezeichnung, folgt darauf die Kennzeichnung mit „PflN“ bzw. „TiN“. Bei Verben kann als weitere grammatische Angabe die Markierung als „transitiv“ (tr.), ,intransitiv“ (itr.), ,reflexiv“ (refl.) bzw. „reziprok“ (rezp.) erfolgen (mit der jeweiligen Abkürzung). Obligatorisch bei jedem Wortartikel ist das darauf folgende Interpretament (Bedeutungsangabe bzw. -beschreibung); eine gewisse Referenzfunktion erfüllt dabei das Duden-Wörterbuch.

Auf die Bedeutungsangabe folgt die Verbreitungsangabe, was bei bis zu zehn (in Ausnahmefällen bis zu zwanzig) Belegen durch die Nennung aller im Archiv vorhandenen Belege geschieht, denen das sog. Summenzeichen $(\Sigma)$ vorangestellt wird. In allen anderen Fällen folgt eine beschreibende Verbreitungsangabe, die die relative Häufigkeitsangabe ,selten“ (slt.), „,mehrfach“ (mf.), ,verstreut“(vst.), ,,verbreitet“ (vbr.) oder ,allgemein“ (allg.) mit der Mundartareal-Angabe „Nordniedersächsisch westlich der Weser“ (westl. nords.), „Nordniedersächsisch östlich der Weser“ (östl. nords.), „Ostfälisch nördlich der Aller““ (nördl. ofäl.), „Ostfälisch südlich der Aller“" (südl. ofäl.) oder „Westfälisch“ (wfäl.) verbindet. Liegen aus einem Mundartareal nur sehr wenige Belege vor, kann das durch Nennung der Belegorte mit der Angabe „ferner“ an die beschreibenden Angaben angeschlossen werden. ${ }^{31}$ Die verwendeten Siglen für die Belegorte und die gedruckten Quellen werden in der sog. technischen Lieferung (der 17.) aufgelistet. ${ }^{32}$ Diese Lieferung befindet sich am Anfang des dritten Bandes.

Es sei ausdrücklich darauf hingewiesen, dass es sich jeweils um relative Verbreitungsangaben handelt: Der Bearbeiter gewichtet für seine Angabe die absolute Anzahl der Belege im Archiv zum Zeitpunkt der Abfassung des jeweiligen Wortartikels, die Anzahl der Belege im einzelnen Mundartareal und die durchschnittliche Belegdichte in jedem der Mundartareale.

Auf die Verbreitungsangabe folgt der Belegteil, der in der Regel mit einem kurzen Strich vom Artikelkopf abgetrennt wird. Innerhalb des Belegsatzteils stehen

29 Das Abkürzungsverzeichnis wurde zuletzt aktualisiert für den 9. Band. Niedersächsisches Wörterbuch (2017: unpaginiert [5-7]).

30 Vgl. dazu Lehmberg (2014: 108-113). - Bei der Abfassung des Artikels Recht bot sich eine Bedeutungsgliederung nach Sachgruppen an.

31 Zu den Verbreitungsangaben im Nds. Wb. zuletzt SCHRÖDER (2014).

32 Niedersächsisches Wörterbuch (1993: XIV-LXII). 
zuerst die nicht-redensartlichen (nicht phraseologischen) Belege. Am Ende des nicht-phraseologischen Teils stehen Belege aus dem Bereich des Volksglaubens (,- Im Volksgl.:“") $)^{33}$ und Vergleiche (,,- In Vergleichen:“). Der phraseologische Teil schließt sich, eingeleitet mit der Markierung, ,- Phras.: “34, an.

Im nicht-redensartlichen Belegteil werden die Belege nach grammatischen Kategorien sortiert angeordnet, im phraseologischen Teil hingegen nach Textsorten:

- Adjektive, nicht phraseologisch: attributive Verwendung, prädikative Verwendung, adverbiale Verwendung, substantivierte Verwendung; Volksglaube, Vergleich. - Phraseologische Belege: (ohne bestimmte Textsorte), Sagwörter (SW), Bauernregeln (BR), Wetterregeln (WR), Volksreime (VolksR), Neckreime (NeckR), Tanzreime (TR), Abzählreime (AR), Kinderreime (KR), Wiegenlieder (WL), Bastlösereime (BlR), Rätsel (Rä.).

- Nomina/Substantive, nicht phraseologisch: ohne Artikel (Nominativ - Genitiv - Dativ - Akkusativ), mit bestimmtem Artikel (Kasus/Numerus), verneint, mit Präposition (alphabetisch), mit Präposition und Attribut (alphabetisch), Volksglauben, Vergleiche. - Phraseologische Belege: (ohne bestimmte Textsorte: sortiert wie nicht-phraseologische Belege), Sagwörter (SW), Bauernregeln (BR), Wetterregeln (WR), Volksreime (VolksR), Neckreime (NeckR), Tanzreime (TR), Abzählreime (AR), Kinderreime (KR), Wiegenlieder (WL), Bastlösereime (B1R), Rätsel (Rä.).

- Verben, nicht phraseologisch: Tempus - Person - Numerus jeweils mit DativObjekt, mit Akkusativ-Objekt, mit Präpositional-Objekt, Volksglauben, Vergleiche. - Phraseologische Belege: (Ohne bestimmte Textsorte), Sagwörter (SW), Bauernregeln (BR), Wetterregeln (WR), Volksreime (VolksR), Neckreime (NeckR), Tanzreime (TR), Abzählreime (AR), Kinderreime (KR), Wiegenlieder (WL), Bastlösereime (BlR), Rätsel (Rä.).

Auf den Belegsatzteil folgt am Ende eines Wortartikels der Verweisteil. Zunächst werden, mit einem Geviertstrich (-) abgesetzt, Parallelen im Mittelniederdeutschen genannt, bzw. wird auf Ähnlichkeiten hingewiesen (mit „Vgl. mnd.“). Gegebenenfalls folgt auf die kursive mittelniederdeutsche Entsprechung eine oder mehrere Ziffern, was bedeutet, dass die vorliegende Parallele zu der Bedeutung unter der jeweiligen Bedeutungsziffer des Wortartikels besteht. Referenz für die Parallelen im Mittelniederdeutsch ist das von AgATHE LASCH und CONRAD BorCHLING begonnene „Mittelniederdeutsche Handwörterbuch“, sofern der fragliche Wortschatzbereich dort bereits bearbeitet vorliegt. Anderenfalls dienen das „Mittelniederdeutsche Wörterbuch“ von KARL SCHILlER und AUGUST LÜBBEN bzw. das „Mittelniederdeutsche Handwörterbuch“ von AUgust LÜBBEN und CHRISTOPH WALTHER als Referenz.

Es kann der Verweis auf eine Heteronymensammlung folgen, d. h. auf das Wort, bei dem eine Sammlung der bedeutungsgleichen Wörter angelegt wurde bzw.

33 Bei nur einem Beleg, wird die Information, mit Komma getrennt, direkt an die Quelle angeschlossen, was auch für das Folgende gilt.

34 Bei nur einem Beleg, wird statt dessen, mit Komma getrennt, die Information ,redensartlich“ (ra.:) an die Quelle angeschlossen. 
werden wird. Solche Verweise können bei einem Stichwort mit mehreren Bedeutungen natürlich auf mehrere Heteronymensammlungen erfolgen. Auch Heteronymenverweise werden mit einem Geviertstrich abgesetzt (,,- Het.:“).

An letzter Stelle des Verweisteils stehen sonstige Verweise und mit „vgl./Vgl.“ versehene Hinweise (wiederum mit einem Geviertstrich abgesetzt). ${ }^{35}$

Den Wortartikel kann der sog. Formenteil beschließen; er wird stets Petit formatiert und wie das Lemma (in der ersten Zeile) um 0,3 cm eingezogen. Durch die Maßnahmen der „Konzeption 2000“ ergeben sich vor allem für die Formenteile erhebliche Reduzierungen, die zu großen Zeiteinsparungen führen. Gegenstand eines Formenteils sind beispielsweise Genusvarianz, die Flexion starker und unregelmäßiger Verben sowie die Varianz bei der Pluralbildung. Innerhalb der Formenteile gelten in besonderer Weise die Einschränkungen bei den Verbreitungsangaben, die sich aus den Bestimmungen der „Konzeption 2000“ ergeben. Für die rezente Realisierung der alten Langvokale war bereits für die sog. Technische Lieferung (die 17.) ein Satz von Karten erarbeitet worden, auf die bei Bedarf verwiesen werden kann (so auch im Beispielartikel unten geschehen: Für den Stammvokal /i:/ wird auf die Karte 9 ,Altniederdeutsch, Mittelniederdeutsch $\hat{i}$ “ verwiesen).

Hinweise auf die Herkunft eines Stichwortes aus einer anderen Sprache stehen, gleichfalls Petit gesetzt, in einer neuen Zeile unter dem Formenteil, sofern erforderlich und vorhanden, anderenfalls direkt unter dem Wortartikel.

\subsection{Belegauswahl und Beleginterpretationen}

In vielen Fällen liegen bei frequenten Wörtern zahlreiche Belege vor, aus denen eine sinnvolle Auswahl getroffen werden muss. Grundsätzlich wird dabei Belegen aus ungedruckten Quellen der Vorzug vor solchen aus gedruckten gegeben. Innerhalb der Belege aus gedruckten Quellen sollen vorzugsweise weniger frequente ausgewählt werden. Besonders im phraseologischen Bereich ergibt sich häufiger der Befund, dass bestimmte Redensarten in ganz ähnlicher Art aus mehreren oder gar zahlreichen Quellen belegt sind. In solchen Fällen wird lediglich ein möglichst exemplarischer Beleg aufgenommen. ${ }^{36}$

Durch die „Konzeption 2000“ ist die Belegauswahl dahingehend beschränkt, dass gedruckte (Teil-)Wörterbücher nicht mehr zitiert werden. Auf das Vorhandensein solcher Belege kann am Ende der jeweiligen Bedeutung durch einen senkrechten Pfeil ( $\uparrow)$, gefolgt von den Quellensiglen plus Seitenangabe, hingewiesen werden. Da durch die „Konzeption 2000“ die Formenteile erheblich reduziert werden, muss darauf geachtet werden, dass die aufgenommenen Belege bzw. Belegsätze möglichst das gesamte Spektrum der vorhandenen Formen abbilden.

35 Einen Sonderfall nehmen Wortartikel ein, die lediglich aus einem Verweis bestehen (sog. Verweisartikel). Solche Artikel sind obligatorisch für alle nicht-ersten Bestandteile von Mehrfachansätzen. Vgl. das Kapitel 2.5 Verweissystem.

36 Zur Belegauswahl am Beispiel des Artikels Recht vgl. LEHMBERG (2014: 114-116); vgl. ferner SCHEUERMANN (1988b: 39-40 und 42-46) zu den Artikeln Farken 'Ferkel', Färse 'junges Rind' bzw. Dēuvel 'Teufel'. 
Nicht immer ist die Bedeutung eines Beleges unmittelbar verständlich, was vor allem bei phraseologischen Belegsätzen gelegentlich der Fall ist. Es gilt dann für den Bearbeiter, über eine entsprechende Recherche in den einschlägigen Hilfsmitteln, ggf. auch bei sachkundigen Personen, die spezielle Bedeutung zu ermitteln und eine entsprechende Bedeutungsparaphrase an den Belegsatz anzuschließen. Wie das Beispiel der Rechtsregel De Aanten dragen hör Recht up de Puckel 'Fremde Enten darf man auch mit Gewalt von seinem Grundstück vertreiben' im Wortartikel Recht zeigt, führt die Ermittlung der Bedeutung ggf. zur Abtrennung einer eigenen Bedeutung (vgl. LEHMBERG 2014: 113 und Anmerkung 22 sowie 115-116).

Besonders in solchen Fällen, in denen ein Beleg ohne entsprechende Parallele im Standard ist, wird eine von der Gewährsperson gegebene Bedeutungserläuterung bzw. -paraphrase sinnvollerweise auch zitiert. Das geschieht nicht zuletzt vor dem Hintergrund der Dokumentationsaufgabe des Nds. Wb.s, damit auch künftigen Benutzerinnen und Benutzern die spezielle Bedeutung zugänglich bleibt.

\subsection{Karten und Abbildungen}

Das Nds. Wb. kann grundsätzlich sowohl Karten als auch Abbildungen enthalten. Beides dient der Benutzerin bzw. dem Benutzer zur Veranschaulichung und ergänzt so die Wortartikel.

Abbildungen werden vornehmlich eingesetzt, um nur schwer zu beschreibende Geräte oder andere Gegenstände zu zeigen, was vor allem heute kaum noch Bekanntes betrifft. Dieses gerät dort an seine Grenzen, wo der Druck einer entsprechenden Abbildung technische oder lizenzrechtliche Probleme aufwirft.

Der Einsatz von Karten im Nds. Wb. ist durch die „Konzeption 2000“ dahingehend eingeschränkt, dass keine Lautkarten mehr gemacht werden dürfen. Die nach der neuen Konzeption zu verwendenden Wortkarten veranschaulichen signifikante Verteilungen heteronymer Wörter innerhalb des Bearbeitungsgebietes. Grenzen des Karteneinsatzes sind durch die recht geringe Größe der gedruckten Karten bei gleichzeitig einer nicht selten hohen Zahl von Heteronymen gesetzt.

\subsection{Verweissystem}

Verweise können im Nds. Wb. einerseits am Ende eines Wortartikels auftreten (vgl. dazu das Kapitel 2.2 Artikelstruktur), andererseits aber auch innerhalb eines Wortartikels. Verweise werden entweder mit „siehe“ (s.) oder mit „,vergleiche“ (vgl.) vorgenommen. Das Verweissystem des Nds. Wb.s in der Übersicht:

- Innerhalb des Artikels:

- Statt einer expliziten Verbreitungsangabe kann auf eine Karte verwiesen werden (Beispiel: „Verbreitung s. K. ,Nebel““ im Artikel Nēvel).

- Im Falle fester Verbindungen, besonders aus Adjektiv und Substantiv häufiger bei Tier- und Pflanzennamen -, verfährt das Nds. Wb. traditionell 
so, dass im Wortartikel zum Bezugswort nur auf das Attribut verwiesen wird, wo die eigentliche Information erscheint. Inzwischen wurde das Verfahren dahingehend vereinheitlicht, dass solche Verweiseinträge mit einer eigenen Bedeutungsziffer jeweils am Ende eines Wortartikels stehen (Beispiel: ,in fester Vbdg. als Attr. in TiN: Blinne Slange Blindschleiche s. blind 6., Lüttjen Schlang Blindschleiche s. lüttig 1." als Ziffer 4. im Artikel Slange).

- Im Falle eines stark von der angesetzten Lemmaform abweichenden Beleges in einem Belegsatz kann - in runden Klammern - unter Nennung der Bedeutung auf diese Normalform verwiesen werden (Beispiel: „WTM $\mathrm{Ahs}^{37}$ De Schandarm hett hüm inspunnen (einsperren; vgl. inspinnen ${ }^{2}$ ). “ im Artikel Schandarm 'Schutzpolizist').

- Am Ende des Artikels:

- Verweis auf eine Heteronymen-Sammelstelle (vgl. Beispielartikel).

- Verweis auf Parallelen im Mittelniederdeutschen (vgl. Beispielartikel).

- Verweis auf eine Karte (am Ende des Beispielartikels mit Bezug auf den Stammvokal; häufig wird zusammen mit einem Heteronymenverweis auf eine Karte verwiesen, etwa „Het. u. K. s. Kummer.“ am Ende des Artikels Pannschutt 'Bauschutt').

- Verweis auf einen anderen Wortartikel, der eine bestimmte Parallele aufweist (Beispiel, am Ende des Artikels rachen": „Zu 1. vgl. marachen.“).

Zudem gibt es innerhalb des Verweissystems Artikel, die nur aus einem Verweis bestehen (Verweisartikel). Solche Artikel muss es von allen Lemmabestandteilen geben, die nicht an erster Stelle eines Mehrfachansatzes stehen. Diese Verweisartikel enthalten die Bedeutung des Stichwortes, auf das verwiesen wird, bzw. die Bedeutungen mit ihrer Bedeutungsziffer, auf die verwiesen wird.

Verweisartikel können auch auf solche Lemmata verweisen, bei denen das betreffende Stichwort nicht genannt ist (sog. Finde-Verweise). Diese Artikel erleichtern der Benutzerin bzw. dem Benutzer das Auffinden eines gesuchten Stichwortes, sie zu schreiben liegt im Ermessen des Bearbeiters.

Wenn sich eine gesamte Wortschatzstrecke an anderer Stelle befindet, können pauschale Verweise das Auffinden erleichtern (Beispiel: „rum- s. herüm-.“).

\subsection{Beispielartikel}

Schīve f. 1. von einem größeren Ganzen abgetrenntes flaches Stück (bei Lebensmitteln, bes. bei Brot, Wurst, Fleisch). Slt. östl. nords., südl. ofäl., ferner SY Hel, DK3,119, GA184, KLÖ2,142, STÜ354. - LG Bre 'n Schîf Brōd „,eine Scheibe Brot“. HM Hei Dä Beddelmann batt meck umme 'n Schieben Braut. GAN Ack ne Panne vull Scheuben „Bratkartoffeln in Scheiben geschnitten“. - Phras.: BRAU-Ölper Ne Schieve von afsnien „Beispiel nehmen“. GA184 Daor kaans di 'ne Schiiwe fan affschniin. NOM 
Son wie dee dat emoaket hä'ut, doa kannst Diu Deck ne Schaibe von afeschnai'en. NOM Son von dainen Brä'u'ere kannst diu deck emoal ne Schaiwe afeschnaien, wat dee farr en Tuignisse het. 2. flaches, häufig rundes Stück aus unterschiedlichem Material. 2.1. als Ziel beim Schießen dienender flacher Gegenstand (meist Papier oder Holz); Schießscheibe, Zielscheibe. $\Sigma$ HB Br-Bt, SY Hel, KüCK3,57, STÜ217, TEUT4,40. - GÖ Fri Hei schütt up dei Schiewe. WEM Ndh Sē hebt nō dĕ Schïf schōten. BRI64, in einem VolksR: Hört, ji Börgers und Börgerkinner, / Wer will mit genaiten / Na der Schibe schaiten, / Si hai Börger oder Börgerskind, / Harre hai Lüse oder Grint, / Dä kome hüte morgen / Klocke achtee / mit getogenen Gewehr / Nahn Rathuse her „Alljährlich im Sommer, am ersten Sonntag im Juli, ist das sogenannte Frischaiten“" 2.2. den Lichteinfall ermöglichendes Flachglasstück in Fenstern. Slt. südl. ofäl., ferner CLP Bar-Ba, EMDELarrelt, HB Br-Bt, OL Stu-Va, SY Hel, WEM Fle, DK3,119, KÜCK3,57, Teut4,40, Wes67. - SY Hel he hett de Schieben tweismäten. GÖ Fri Dei Reen pladdert na'n Schieben 'runder. OL Stu-Va De Schieben sund jo ganz. blaß, de möt nödig mal putzt weern. CLP Bar-Ba Dei Fensters schust du strieken ,(streichen)“, man nich dei Schieben un din Tüch mit Fawe beklackern. WEM Fle, ra.: Gah' mie uht'n Luch, dien Vadder is keen Gläsker wesen, anners her he die'n Schiew insett. 2.3. mit Löchern versehenes rundes Bauteil am Stampfer des Butterfasses. $\Sigma$ TEUT4,40. 2.4. Ring beim Ringreiten. $\Sigma$ OTT Hos Ken 6 Schieben op'm Dopp het, kann sick 'n Veergespann wünschen. 2.5. (mit Honig gefüllte) Wabe. Vst. östl. nords., slt. westl. nords., südl. ofäl., ferner BSB Epe, GF Gsw, Müd, UE Sud. 2.6. Rolle im Block eines Flaschenzuges bzw. Rolle in einem Kran. $\Sigma$ Kehd, OTT Frb, SY Hps, DK3,119, TeUT4,40. - Phras.: KeRn78 Dat geit over völ Schieven. Kehd Öbern annere Schie'w lopen ,einen anderen Ausgang nehmen“. Kehd Dat warrd wull öbern annere Schie'w lopen „die Angelegenheit wird wohl anders auslaufen“. 2.7. Span. $\Sigma$ OHA Kal Ne Scheiiwe von affsneien; ähnl. AUR Uth. 2.8. Pflugmesser in runder Bauform, Scheibensech. Slt. Ofriesl, ferner BRA Esh-K1, See-R1. 2.9. $\Sigma$ NIEK171 „Eiserne Scheibe, die vor einen verschlissenen Stoßring gesetzt wird, damit das Rad nicht klappert“. 3. kellenartiges Maurerwerkzeug (beim Verputzen von Wänden verwendet). $\Sigma$ NOM Bar. 4. Tisch. $\Sigma$ LÜ Erg3,75. 5. Kf. für ,Töpferscheibe‘. $\Sigma$ TeUT4,40. — mnd. schive 1., 2.1., 2.2., 2.6., 5. - Het. zu 2.8. s. Plōgmest.

Zum StV. vgl. K.9. 


\section{3 (TEXT-)TECHNOLOGISCHE ASPEKTE}

Seit 1987 werden die Wortartikel von den Bearbeitern unmittelbar mittels eines Personal Computers (PC) geschrieben. Zu verwendende Sonderzeichen und Textauszeichnungen (kursiv, fett, Petit, Kapitälchen) mussten aufgrund des eingesetzten Textverarbeitungsprogrammes codiert werden. Zur Weiterverarbeitung und für den Textsatz wurden die Dateien an den Verlag, später an einen externen Dienstleister, der den Satz durchführte, geschickt. ${ }^{38}$ Dieses Verfahren machte jeweils mehrere Korrekturdurchläufe mit Postversand zwischen Verlag, Dienstleister und Arbeitsstelle erforderlich, was neben einem hohen Zeitbedarf auch recht kostenintensiv war.

Der Wunsch, diese beiden Faktoren spürbar zu reduzieren, war einer der Gründe, derentwegen von mir ab Ende 1999 ein Verfahren entwickelt wurde, den kompletten Drucksatz der Wörterbuchlieferungen im Hause durchführen zu können. Dazu wurden zunächst die erforderlichen Sonderzeichenfonts erstellt, was auch die Artikelarbeit sehr erleichtert, da der gesamte Text am Bildschirm so dargestellt wird, wie er später im Druck erscheint. Die erste im Hause gesetzte Lieferung ist die Doppellieferung 44./45., die im Herbst 2000 erschien. ${ }^{39}$ Die Umstellung der Kartenerstellung auf eine digitales Verfahren nahm wegen der technischen Vorarbeiten etwas längere Zeit in Anspruch, die erste EDV-gestützt im Hause erstellte Karte ist schließlich bei Knicker ${ }^{l}$ 'kleine Spielkugel' erschienen. ${ }^{40}$

Während die Informationen des Fragebogenarchivs (wie oben beschrieben) inzwischen in einer Datenbank zur Verfügung stehen, liegt das Zettelarchiv lediglich in Form der Papierzettel vor und kann realistischerweise nicht digitalisiert werden. Aufgrund dieser Archivstruktur versprach der Einsatz eines speziellen Redaktionssystems zu keinem Zeitpunkt eine Beschleunigung oder Verbesserung der Artikelarbeit. Alle Wortartikel werden deshalb weiterhin mit einer handelsüblichen Textverarbeitung verfasst, die vor allem um die zur Sonderzeicheneingabe erforderlichen Shortcuts erweitert wurde. Zum Einsatz kommen in der Praxis zwei verschiedene Textverarbeitungsprogramme, da jeder Bearbeiter das von ihm bevorzugte Programm einsetzen kann.

Der Drucksatz wird von mir mit der bevorzugten Textverarbeitung vorgenommen; für die erforderlichen Konvertierungen aus dem anderen Programm stehen entsprechende Makros zur Verfügung, so dass der Zeitaufwand dafür nicht ins Gewicht fällt.

Sämtliche Aktivitäten der Arbeitsstelle sind darauf gerichtet, die Erarbeitung des Nds. Wb.s unter Einhaltung des erwähnten Arbeitsplans fristgerecht zum Abschluss zu bringen, und zwar in bewährter Weise in gedruckter Form. Eine digitale Publikation des Wörterbuches ist mit den vorhandenen personellen und finanziellen

$38 \mathrm{Zu}$ den Anfängen des PC-Einsatzes für die Erarbeitung des Nds. Wb.s vgl. SCHEUERMANN (1988a).

39 Zu den Einzelheiten vgl. LeHMBERG (2002: 23-27).

40 Zum eingesetzten Verfahren der Kartenerstellung und den erforderlichen Vorarbeiten ausführlich LEHMBERG (2003b). 
Ressourcen nicht zu leisten und deshalb auch nicht geplant. Daraus folgt, dass auch eine Veröffentlichung des Wörterbuches im Internet nicht geplant ist.

\section{LINGUISTISCHES POTENTIAL}

Das Archiv des Nds. Wb.s ist im Bereich des Niederdeutschen mit einigem Abstand das umfangreichste aller großlandschaftlichen Wörterbücher. Die große Zahl der vorhandenen Belege aus allen drei westniederdeutschen Mundartverbänden, viele davon auch im Satzzusammenhang, stellen ein einzigartiges Korpus für Forschungen zum Niederdeutschen dar. Zu denken ist dabei beispielsweise an etymologische, phonetisch-phonologische (synchron wie diachron) und syntaktische Fragestellungen. Zudem sind Untersuchungen zur Lehn- und Fremdwortsituation im Niederdeutschen oder - mit dem Archiv als Vergleichskorpus - zum Sprachwandel vielversprechend.

Das Archiv ist, wie auch die Arbeitsbibliothek, während der Arbeitszeiten der Wörterbuchbearbeiter zugänglich und kann dann selbstverständlich auch von externen Forscherinnen und Forschern genutzt und ausgewertet werden.

Während größere systematische Untersuchungen anhand des Archivmaterials bislang noch nicht durchgeführt wurden, sind von Mitarbeitern der Arbeitsstelle teils unabhängig von der eigentlichen Wörterbucharbeit, teils als ein Nebenprodukt derselben - einige Spezialfragen untersucht worden. Im Folgenden seien einige Beispiele genannt:

Etymologie: Ein zunächst rätselhafter Einzelbeleg aus Otterndorf im Land Hadeln (quon'n 'vertun, verschnökern' ließ PETER STRATHMANN der Frage nach einem möglichen etymologischen Anschluss nachgehen. Das Ergebnis ist in einer Miszelle im „Korrespondenzblatt des Vereins für niederdeutsche Sprachforschung“ dokumentiert (s. STRATHMANN 2017). Für das Wörterbuch resultierte in diesem Fall ein unmittelbarer Nutzen daraus, dass der Ansatz quānen als der etymologisch richtige plausibel gemacht werden konnte.

Fremdwort-/Lehnwortsituation: Aufgrund der günstigen Archivsituation konnte PETER STRATHMANN gewinnbringend der Frage nach der Etymologie von mndt. knîf, knîp 'Messer, besonders Schustermesser' im Niederdeutschen nachgehen und komplexe Lehnwort-Zusammenhänge plausibel machen. Diese ausführliche Untersuchung liegt gedruckt vor (s. STRATHMANN 2009).

Sprachwandel: Unabhängig voneinander und mit einigem zeitlichen $\mathrm{Ab}$ stand haben ULRICH SCHEUERMANN und MAIK LEHMBERG den rezenten niederdeutschen Wortschatz jeweils eines Ortes (Göttingen-Elliehausen bzw. Peine) mit dem im Fragebogenarchiv dokumentierten verglichen. Beide Untersuchungen liegen gedruckt vor (s. SCHEUERMANN 2001; LEHMBERG 2008).

Wortgeographie: Die interessante Wortgeographie der zum Teil hochfrequenten niederdeutschen Bezeichnungen für 'der Tag vor dem Sonntag' hat ULF BORCHERS untersucht (erschienen als Miszelle im „Korrespondenzblatt des Vereins für niederdeutsche Sprachforschung“" (s. BORCHERS 2017). 


\title{
5 KONTAKTADRESSE, HOMEPAGE
}

\author{
Arbeitsstelle Niedersächsisches Wörterbuch \\ Kreuzbergring 50 \\ 37075 Göttingen \\ Deutschland
}

E-Mail: ndswb@gwdg.de

Homepage:

<http://www.uni-goettingen.de/de/219054.html> bzw.

$<$ http://www.uni-goettingen.de/ndswb> (mit Weiterleitung)

\section{LITERATURVERZEICHNIS}

APPENZELLER, GERRIT (2011): Das Niedersächsische Wörterbuch. Ein Kapitel aus der Geschichte der Großlandschaftslexikografie. Stuttgart: Steiner (Zeitschrift für Dialektologie und Linguistik. Beihefte. 142).

BORCHERS, UlF (2017): „Der Tag vor dem Sonntag“ im Bearbeitungsgebiet des NdsWb. In: Korrespondenzblatt des Vereins für niederdeutsche Sprachforschung 124/1, 14-23.

DENKLER, MARKus / FRIEDEl HElga RoOlFS (Hg.) (2014): Studien zur Lexikographie und Lexikologie des Niederdeutschen. Festgabe für Robert Damme zum 60. Geburtstag. Münster: Aschendorff (Niederdeutsches Wort. 54).

EGGERS, ECKHARD (2008): Wörterbuch digital: Das elektronische Fragebogenarchiv (EFBA) des Niedersächsischen Wörterbuches. In: STELlMACHER, DiETER (Hg.): Das Niedersächsische Wörterbuch im Peiner Raum. Berichte und Mitteilungen aus der Arbeitsstelle. Bielefeld: Verlag für Regionalgeschichte (Göttinger Forschungen zur Landesgeschichte. 14), 43-64.

FAHNING, WOLFGANG (Hg.) (1988): Dialektlexikographie. Berichte und Analysen zur Arbeit an Dialektwörterbüchern. Jena: Abt. Wissenschaftl. Publ. der Friedrich-Schiller-Univ. Jena (Wissenschaftliche Beiträge der Friedrich-Schiller-Universität Jena. 17).

FRIEBERTSHÄUSER, HANS (Hg.) (1976): Dialektlexikographie. Berichte über Stand und Methoden deutscher Dialektwörterbücher. Festgabe für Luise Berthold zum 85. Geburtstag am 27.1.1976. Wiesbaden: Steiner (Zeitschrift für Dialektologie und Linguistik. Beihefte N.F. 17).

FRIEBERTSHÄUSER, HANS / HEINRICH J. DINGELDEIN (Hg.) (1986): Lexikographie der Dialekte. Beiträge zu Geschichte, Theorie und Praxis. Lexikographisches Kolloquium „Dialektlexikographie - Praxis, Theorie, Geschichte“ vom 18. bis 20. Februar 1985 Marburg. Berlin: de Gruyter (Germanistische Linguistik. 59).

GROSSE, RUDOLF (Hg.) (1998): Bedeutungserfassung und Bedeutungsbeschreibung in historischen und dialektologischen Wörterbüchern. Beiträge zu einer Arbeitstagung der deutschsprachigen Wörterbücher, Projekte an Akademien und Universitäten vom 7. bis 9. März 1996 anläßlich des 150jährigen Jubiläums der Sächsischen Akademie der Wissenschaften zu Leipzig. Stuttgart [u. a.]: Hirzel (Abhandlungen der Sächsischen Akademie der Wissenschaften zu Leipzig, Philologisch-Historische Klasse. 75,1).

HARM, VOLKER / Michael SCHLAEFER (Hg.) (2003): Wörterbuchstadt Göttingen. Göttingen: Selbstverlag

HAUSMANN, FRANZ JOSEF / OSKAR REICHMANN / HERBERT ERNST WIEGAND / LADISLAV ZGUSTA (Hg.) (1991): Wörterbücher. Ein internationales Handbuch zur Lexikographie = Dictionaries: an international encyclopedia of lexicography. Berlin: de Gruyter (Handbücher zur Sprach- und Kommunikationswissenschaft [HSK]. 5.3). 
JANSSEN, HANS (1935a): Aufruf zur Mitarbeit am „Niedersächsischen Wörterbuch“. In: Die Kunde. Zeitschrift für niedersächsische Archäologie. 3/9, 171-172.

JANSSEN, HANS (1935b): Richtlinien zum Ausziehen von Schrifttum. Göttingen: Selbstverlag.

KAMMERER, MATTHIAS (1995): Bildschirmorientiertes Abfassen von Wörterbuchartikeln. Dargestellt am Beispiel des Frühneuhochdeutschen Wörterbuches. Tübingen: Niemeyer (Lexicographica Series maior. 68).

Keseling, Gisbert / Bernd-Ulrich KetTner / Wolfgang Kramer / WolfGang PutschKe / MONIKA RÖsSING-HAGER / ULRICH SCHEUERMANN (1970): Richtlinien zur Ablochung und zentralen Speicherung mundartlichen Wortmaterials des Deutschen. In: Germanistische Linguistik 2, 179-242.

KnOWLeS, Francis E. (1991): The Computer in Lexicography. In: HAUSMANn, Franz JoSEF / OSKAR REICHMANN / HERBERT ERNST WIEGAND / LADISLAV ZGUSTA (Hg.): Wörterbücher. Ein internationales Handbuch zur Lexikographie $=$ Dictionaries: an international encyclopedia of lexicography. Berlin: de Gruyter (Handbücher zur Sprach- und Kommunikationswissenschaft [HSK]. 5.3), 1645-1672.

LASCH, AGATHE / CONRAD BORCHLING: Mittelniederdeutsches Handwörterbuch. Fortgeführt von GERHARD CORDES. Erster Band A - F/V. Neumünster: Wachholtz Verlag $1956 \mathrm{ff}$.

LEHMBERG, MAIK (2002): Zu Geschichte und Aufgaben des Niedersächsischen Wörterbuches. In: STELLMACHER, DiETER (Hg.): Niedersächsisches Wörterbuch. Berichte und Mitteilungen aus der Arbeitsstelle. 7. Regionaltreffen in der Gemeinde Amt Neuhaus am 2.3.2002. Göttingen: Selbstverlag, 6-32.

LEHMBERG, MAIK (2003a): Das Niedersächsische Wörterbuch. In: HARM, VOLKER / MichAEL SCHLAEFER (Hg.): Wörterbuchstadt Göttingen. Göttingen: Selbstverlag, 15-33.

LEHMBERG, MAIK (2003b): Kartenerstellung für das Niedersächsische Wörterbuch. In: LEHMBERG, MAIK (Hg.): Wörter und Namen. Festgabe für Ulrich Scheuermann zum 65. Geburtstag. Bielefeld: Verlag für Regionalgeschichte (Göttinger Forschungen zur Landesgeschichte. 7), 49-61.

LEHMBERG, MAIK (Hg.) (2003c): Wörter und Namen. Festgabe für Ulrich Scheuermann zum 65. Geburtstag. Bielefeld: Verlag für Regionalgeschichte (Göttinger Forschungen zur Landesgeschichte. 7).

LEHMBERG, MAIK (2004a): Kurzgefasste Projektdarstellung. Stand: Januar 2004. Göttingen: Selbstverlag.

LEHMBERG, MAIK (Hg.) (2004b): Sprache, Sprechen, Sprichwörter. Festschrift für Dieter Stellmacher zum 65. Geburtstag. Stuttgart: Steiner (Zeitschrift für Dialektologie und Linguistik. Beihefte. 126).

LeHMBERG, MAIK (2006): Die Sprachkarte als Mittel der Veranschaulichung sprachlicher Befunde. In: STELlMACHER, DiETER (Hg.): Das Niedersächsische Wörterbuch im Oldenburger Münsterland. Berichte und Mitteilungen aus der Arbeitsstelle. Bielefeld: Verlag für Regionalgeschichte (Göttinger Forschungen zur Landesgeschichte. 10), 9-36.

LEHMBERG, MAIK (2008): Niederdeutsch gestern und heute in Peine. Ein Vergleich des Wortschatzes nach 70 Jahren. In: STELlMACHER, DIETER (Hg.): Das Niedersächsische Wörterbuch im Peiner Raum. Berichte und Mitteilungen aus der Arbeitsstelle. Bielefeld: Verlag für Regionalgeschichte (Göttinger Forschungen zur Landesgeschichte. 14), 17-42.

LEHMBERG, MAIK (2009): Möglichkeiten und Grenzen neuer Techniken am Beispiel des Niedersächsischen Wörterbuches. 6. Arbeitstreffen deutschsprachiger Akademiewörterbücher Berlin 2. - 5. September 2009 [Online verfügbar unter der URL: <http://dwb.bbaw.de/tagung09/pdf/ Lehmberg.pdf >; Stand: 20.3.2018].

LEHMBERG, MAIK (2014): Der Artikel Recht im Niedersächsischen Wörterbuch. In: DENKLER, MARKUS / FRIEDEL HELGA RoOLFS (Hg.): Studien zur Lexikographie und Lexikologie des Niederdeutschen. Festgabe für Robert Damme zum 60. Geburtstag. Münster: Aschendorff (Niederdeutsches Wort. 54), 105-120. 
LEHMBERG, MAIK / MARTIN SCHRÖDER (2004): Kürzer, schneller - besser? Das neue Konzept des Niedersächsischen Wörterbuchs in der Diskussion. In: LEHMBERG, MAIK (Hg.): Sprache, Sprechen, Sprichwörter. Festschrift für Dieter Stellmacher zum 65. Geburtstag. Stuttgart: Steiner (Zeitschrift für Dialektologie und Linguistik Beihefte. 126), 341-359.

LOBENSTEIN-REICHMANN, ANJA (2016): Historische Lexikographie zwischen Tradition und Innovation. Berlin/Boston: de Gruyter (Studia Linguistica Germanica. 129).

LÜBben, August / CHRISTOPH WALTHER (1965): Mittelniederdeutsches Handwörterbuch. Darmstadt: Wissenschaftliche Buchgesellschaft. [Unveränderter Nachdruck der Ausgabe Norden 1888].

MitZKA, WALTHER (1937): Die landschaftlichen deutschen Mundartwörterbücher der Gegenwart. In: Zeitschrift für Mundartforschung 13, 91-99.

Mnd. WB. 1-5 = SCHILLER, KARL / AUGUST LÜBBEN (1875-1880): Mittelniederdeutsches Wörterbuch. Bd. 1-5. Bremen: J. Kühlmann's Buchhandlung.

Mnd. WB. 6 = SCHILLER, KARL / AUGUST LÜBBEN (1881): Mittelniederdeutsches Wörterbuch. 6. Bd. Bremen: Hinricus Fischer.

Nds. Wb. 1 = JUNGANDREAS, WOLFGANG (Hg.) (1965): Niedersächsisches Wörterbuch. Auf Grund der Vorarbeiten von Hans Janssen und unter Mitwirkung eines Arbeitskreises niedersächsischer Mundartforscher hg. von der Abt. für niedersächsische Mundartforschung des Seminars für Deutsche Philologie der Univ. Göttingen durch WOLFGANG JUNGANDREAS. Bd. 1: A bis bersen . Neumünster: Wachholtz Verlag. [Lfg. 1-7b, Erscheinungsbeginn: 1953].

Nds. Wb. 2 = WESCHE, HEINRICH (Hg.) (1985): Niedersächsisches Wörterbuch. Auf Grund der Vorarbeiten von Hans Janssen $\uparrow$ und unter Mitwirkung eines Arbeitskreises niedersächsischer Mundartfreunde herausgegeben von der Abteilung für niedersächsische Mundartforschung des Seminars für Deutsche Philologie der Universität Göttingen durch HEINRICH WESCHE $†$ (bis biselig). Herausgegeben vom Institut für Historische Landesforschung der Universität Göttingen durch die Arbeitsstelle Niedersächsisches Wörterbuch (ab Biseljan). Bd. 2: BertscheBüzpott. Bearbeitet von GISBERT KESELING (bis biselig), WOLFGANG KRAMER, UlRICH SCHEUERMANN. Neumünster: Wachholtz Verlag.

Nds. Wb. 3 = STEllmacher, DieTER (Hg.) (1993): Niedersächsisches Wörterbuch. Bd. 3: $C-$ exzēren. Bearbeitet von JENS VOLQUARD GONNSEN, WOLFGANG KRAMER, ULRICH SCHEUERMANN, PETER WAGENER. Institut für Historische Landesforschung der Universität Göttingen, Arbeitsstelle Niedersächsisches Wörterbuch. Neumünster: Wachholtz Verlag.

Nds. Wb. 4 = STELlMACHER, DieTER (Hg.) (1994): Niedersächsisches Wörterbuch. Bd. 4: F/V. Bearbeitet von ULRICH SCHEUERMANN. Institut für Historische Landesforschung der Universität Göttingen, Arbeitsstelle Niedersächsisches Wörterbuch. Neumünster: Wachholtz Verlag.

Nds. Wb. 5 = STELLMACHER, DIETER (Hg.) (1998): Niedersächsisches Wörterbuch. Bd. 5: Gabbel Haubön, bearbeitet von WOLFGANG KRAMER, KARIN SCHADE, ULRICH SCHEUERMANN. Institut für Historische Landesforschung der Universität Göttingen, Arbeitsstelle Niedersächsisches Wörterbuch. Neumünster: Wachholtz Verlag.

Nds. Wb. 6 = STELLMACHER, DIETER (Hg.) (2003): Niedersächsisches Wörterbuch. Bd. 6: Haubönjuxwise. Bearbeitet von WOLFGANG Kramer, MAIK LEHMBERG, KARIN SCHADE, UlRICH SCHEUERMANN. Institut für Historische Landesforschung der Universität Göttingen, Arbeitsstelle Niedersächsisches Wörterbuch. Neumünster: Wachholtz Verlag.

Nds. Wb. 7 = STELlMACHER, DieTER (Hg.) (2011a): Niedersächsisches Wörterbuch. Bd. 7: $k a-$ küzen. Bearbeitet von MAIK LEHMBERG, ULRICH SCHEUERMANN. Institut für Historische Landesforschung der Universität Göttingen, Arbeitsstelle Niedersächsisches Wörterbuch. Neumünster: Wachholtz Verlag.

Nds. Wb. 8 = STELLMACHER, DiETER (Hg.) (2011b): Niedersächsisches Wörterbuch. Bd. 8: LābMyrtenkranz. Bearbeitet von MARTIN SCHRÖDER. Institut für Historische Landesforschung der Universität Göttingen, Arbeitsstelle Niedersächsisches Wörterbuch. Neumünster: Wachholtz Verlag. 
Nds. Wb. 9 = BUSCH, AlBeRT (Hg.) (2017): Niedersächsisches Wörterbuch. Bd. 9: nā -quutschig. Bearbeitet von ECKHARD EGGERS, MARTIN SCHRÖDER. Seminar für Deutsche Philologie der Universität Göttingen, Arbeitsstelle Niedersächsisches Wörterbuch. Kiel/Hamburg: Wachholtz Verlag Murmann Publishers.

PETERS, ROBERT / HoRST P. PÜTZ / UlRich Weber (Hg.) (2001): Vulpis Adolatio. Festschrift für Hubertus Menke zum 60. Geburtstag. Heidelberg: Winter (Germanistische Bibliothek. 11).

POST, RUDOLF (1988): Komputereinsatz in der Dialektwörterbucharbeit. In: FAHNING, WOLFGANG (Hg.): Dialektlexikographie. Berichte und Analysen zur Arbeit an Dialektwörterbüchern. Jena: Abt. Wissenschaftl. Publ. der Friedrich-Schiller-Univ. Jena (Wissenschaftliche Beiträge der Friedrich-Schiller-Universität Jena. 17), 131-151.

POST, RUDOLF (1998): Möglichkeiten der elektronischen Strukturierung, Vernetzung und Verfügbarmachung von lexikographischen Daten bei der Arbeit am Pfälzischen Wörterbuch. In: GROSSE, RUdOLF (Hg.): Bedeutungserfassung und Bedeutungsbeschreibung in historischen und dialektologischen Wörterbüchern. Beiträge zu einer Arbeitstagung der deutschsprachigen Wörterbücher, Projekte an Akademien und Universitäten vom 7. bis 9. März 1996 anläßlich des 150jährigen Jubiläums der Sächsischen Akademie der Wissenschaften zu Leipzig. Stuttgart [u. a.]: Hirzel (Abhandlungen der Sächsischen Akademie der Wissenschaften zu Leipzig, Philologisch-Historische Klasse. 75,1), 211-220.

SCHEUERMANN, UlRICH (1974): Linguistische Datenverarbeitung und Dialektwörterbuch. Dargestellt am Beispiel des Niedersächsischen Wörterbuches. Mit einer Dokumentation: Automatische Stichwortliste. Wiesbaden: Steiner (Zeitschrift für Dialektologie und Linguistik. Beihefte. N.F. 11).

SCHEUERMANN, UlRICH (1976): Niedersächsisches Wörterbuch. In: FRIEBERTSHÄUSER, HANS (Hg.): Dialektlexikographie. Berichte über Stand und Methoden deutscher Dialektwörterbücher. Festgabe für Luise Berthold zum 85. Geburtstag am 27.1.1976. Wiesbaden: Steiner (Zeitschrift für Dialektologie und Linguistik. Beihefte. N.F., 17), 195-210.

SCHEUERMANN, ULRICH (1986): Zettel oder EDV? Probleme bei der Materialaufbereitung zu einem Dialektwörterbuch. In: FRIEBERTSHÄUSER, HANS / HEINRICH J. DINGELDEIN (Hg.): Lexikographie der Dialekte. Beiträge zu Geschichte, Theorie und Praxis. Lexikographisches Kolloquium „Dialektlexikographie - Praxis, Theorie Geschichte“ vom 18. bis 20. Februar 1985 Marburg. Berlin: de Gruyter (Reihe Germanistische Linguistik. 59), 103-114.

SCHEUERMANN, UlRICH (1988a): Tradition und Fortschritt: Der Personal Computer in der Wörterbucharbeit. In: STELLMACHER, DIETER (Hg.): Berichte und Mitteilungen aus der Arbeitsstelle. 1. Regionaltreffen in Rotenburg (Wümme). Göttingen: Selbstverlag, 37-51.

SCHEUERMANN, UlRICH (1988b): Von Z bis A oder: Wie unser Wörterbuch entsteht. In: Zeitschrift für Dialektologie und Linguistik 55/1, 26-48.

SCHEUERMANN, Ulrich (2001): „Wie heißt oder heißen in Ihrer Mundart...?“ Das Original und seine Wiederholung nach sechs Jahrzehnten am Beispiel von Elliehausen GÖ / Göttingen-Elliehausen. In: Peters, Robert / Horst P. PÜtZ / Ulrich Weber (Hg.): Vulpis Adolatio. Festschrift für Hubertus Menke zum 60. Geburtstag. Heidelberg: Winter (Germanistische Bibliothek. 11), 639-657.

SCHRÖDER, MARTIN (1997): Brauchen wir ein neues Wörterbuchkartell? Zu den Perspektiven einer computerunterstützten Dialektlexikographie und eines Projektes „Deutsches Dialektwörterbuch". In: Zeitschrift für Dialektologie und Linguistik 64, 57-66.

SCHRÖDER, MARTIN (2014): Wie allgemein ist eigentlich ,allg."? Frequenzangaben im Niedersächsischen Wörterbuch. In: DENKLER, MARKUS / FRIEDEL HELGa ROOLFS (Hg.): Studien zur Lexikographie und Lexikologie des Niederdeutschen. Festgabe für Robert Damme zum 60. Geburtstag. Münster: Aschendorff (Niederdeutsches Wort. 54), 121-134.

SPEER, HEINO (1994): DRW to FAUST. Ein Wörterbuch zwischen Tradition und Fortschritt. In: Lexicographica. International Annual for Lexicography / Revue Internationale de Lexicographie / Internationales Jahrbuch für Lexikographie 10, 171-213. 
STÄDTLER, THOMAS (Hg.) (2003): Wissenschaftliche Lexikographie im deutschsprachigen Raum. Heidelberg: Winter.

STELLMACHER, DiETER (Hg.) (1988): Berichte und Mitteilungen aus der Arbeitsstelle. 1. Regionaltreffen in Rotenburg (Wümme). Göttingen: Selbstverlag.

STELlMACHER, DiETER (Hg.) (1990): Niedersächsisches Wörterbuch. Berichte und Mitteilungen aus der Arbeitsstelle. 2. Regionaltreffen in Aurich. Göttingen: Selbstverlag.

STELLMACHER, DIETER (Hg.) (1992): Niedersächsisches Wörterbuch. Berichte und Mitteilungen aus der Arbeitsstelle. 3. Regionaltreffen in Bad Iburg am 7.11.1992. Göttingen: Selbstverlag.

STELLMACHER, DIETER (Hg.) (1994): Niedersächsisches Wörterbuch. Berichte und Mitteilungen aus der Arbeitsstelle. 4. Regionaltreffen in Schöningen am 19.11.1994. Göttingen: Selbstverlag.

STELLMACHER, DIETER (Hg.) (1998): Niedersächsisches Wörterbuch. Berichte und Mitteilungen aus der Arbeitsstelle. 5. Regionaltreffen in Bückeburg am 4.7.1998. Göttingen: Selbstverlag.

STELLMACHER, DIETER (Hg.) (2000): Niedersächsisches Wörterbuch. Berichte und Mitteilungen aus der Arbeitsstelle. 6. Regionaltreffen in Herzberg/Harz am 8.4.2000. Göttingen: Selbstverlag.

STELlMACHER, DiETER (2002a): Dialektlexikographie am Scheideweg. In: WIESINGER, PETER (Hg.): Akten des X. Internationalen Germanistenkongresses Wien 2000 ,Zeitenwende - Die Germanistik auf dem Weg vom 20. ins 21. Jahrhundert“. Bd. 2, unter Mitarbeit von HANS DERKITS. Bern [u. a.]: Lang (Jahrbuch für Internationale Germanistik Reihe A, Kongressberichte), 257-262.

STELLMACHER, DiETER (Hg.) (2002b): Niedersächsisches Wörterbuch. Berichte und Mitteilungen aus der Arbeitsstelle. 7. Regionaltreffen in der Gemeinde Amt Neuhaus am 2.3.2002. Göttingen: Selbstverlag.

STELLMACHER, DIETER (Hg.) (2004): Das Niedersächsische Wörterbuch im Ammerland. Berichte und Mitteilungen aus der Arbeitsstelle. Bielefeld: Verlag für Regionalgeschichte (Göttinger Forschungen zur Landesgeschichte. 9).

STELLMACHER, DIETER (Hg.) (2006): Das Niedersächsische Wörterbuch im Oldenburger Münsterland. Berichte und Mitteilungen aus der Arbeitsstelle. Bielefeld: Verlag für Regionalgeschichte (Göttinger Forschungen zur Landesgeschichte. 10).

STELLMACHER, DiETER (Hg.) (2008): Das Niedersächsische Wörterbuch im Peiner Raum. Berichte und Mitteilungen aus der Arbeitsstelle. Bielefeld: Verlag für Regionalgeschichte (Göttinger Forschungen zur Landesgeschichte. 14).

STELlMACHER, DIETER (Hg.) (2011): Das Niedersächsische Wörterbuch in Bad Fallingbostel. Berichte und Mitteilungen aus der Arbeitsstelle. Bielefeld: Verlag für Regionalgeschichte (Göttinger Forschungen zur Landesgeschichte. 19).

STRATHMANN, PETER (2009): 'n Knief mutt ma up' $n$ Lann ümmer in t' Tasch hemm'. Überlegungen zur Herkunft der Sippe von mnd. knîf, knîp 'Messer, besonders Schustermesser' im Niederdeutschen. In: Jahrbuch des Vereins für niederdeutsche Sprachforschung 132, 69-127.

STRATHMANN, PETER (2017): Quoon'n 'vertun, verschnökern' (Otterndorf (Land Hadeln)). Auch zu engl. squander, scatter, shatter, norw. skvette, schwed. skvätta etc. In: Korrespondenzblatt des Vereins für niederdeutsche Sprachforschung 124/1, 7-14. 



\title{
DAS MITTELELBISCHE WÖRTERBUCH
}

\author{
Ulrich Wenner
}

\section{GESCHICHTLICHES, RAHMENBEDINGUNGEN ZUM WÖRTERBUCH UND ZUR ARBEITSSTELLE}

\subsection{Abriss der Geschichte des Wörterbuchs ${ }^{1}$}

Nachdem bereits der größte Teil der großlandschaftlichen Dialektwörterbücher des Deutschen die Arbeit aufgenommen hatte, galt es, auch für die verbliebenen weißen Flächen Lösungen zu finden. Zu diesen Territorien zählte auch der Regierungsbezirk Magdeburg der preußischen Provinz Sachsen. Nach vielfältigen Bemühungen erhielt KARL BISCHOFF 1935 von WALTHER MitzKA den Auftrag, ein solches Wörterbuch zu etablieren, das den Namen „Ostfälisches Wörterbuch“ erhielt. Das zunächst rein niederdeutsche Bearbeitungsgebiet wurde 1940 um südlich davon gelegene Territorien mit ostmitteldeutschen Mundarten (nordthüringisch, nordobersächsisch/anhaltisch) erweitert, um die Lücke zum Thüringischen und zum Obersächsischen Wörterbuch zu schließen. Diese Ausdehnung war ein Grund, die Unternehmung in „Mittelelbisches Wörterbuch“ (MEWB) umzubenennen.

BISCHOFF, damals in Magdeburg im höheren Schuldienst tätig, begann das Archiv aufzubauen, u. a. indem er Fragebogen entwarf und versandte sowie Literatur unterschiedlicher Art exzerpierte. Während im Zweiten Weltkrieg das Material eingelagert war, wertete BISCHOFF in Archiven historische Quellen aus. Mit seiner Berufung als Professor für Deutsche Philologie an die Martin-Luther-Universität Halle-Wittenberg zog auch die Arbeitsstelle nach Halle um. Obwohl die Wörterbucharbeit weiterhin eher seiner Freizeit vorbehalten blieb, erweiterte BISCHOFF das Archiv, entwarf Karten und analysierte die sich aus dem Material abzeichnenden sprachlichen Verhältnisse und ordnete sie sprachhistorisch ein.

Den Bedrängungen und Überwachungen, denen er als bekennender Christ an der Universität ausgesetzt war, entzog er sich zum Jahreswechsel 1958/1959 durch Flucht in den Westen. Das Material musste er zurücklassen. Es wurde an der Akademie der Wissenschaften in Berlin ,eingesargt“ (BISCHOFF 1984: 5), eine wie auch immer geartete Weiterarbeit war nicht möglich. Kurz vor seinem Tod verfasste BISCHOFF einen Beitrag über das „Mittelelbische Wörterbuch“ (postum 1984 erschienen), um es vor dem Vergessen zu bewahren. Vielfältige Bemühungen (u. a. von der Familie Bischoff, MANFREd LEMMER, Professor an der Universität Halle)

1 Vgl. zur Geschichte des Projekts u. a. Bischoff (1984), Kettmann (1997), Kettmann (1998a), KetTMAnN (2004), KetTMAnN (2008), Solms (2008), Stellmacher (2008). 
führten nach der Wiedervereinigung dazu, dass 1992 eine durch die Sächsische Akademie der Wissenschaften zu Leipzig getragene Arbeitsstelle unter der Leitung des BISCHOFF-Schülers GERHARD KETTMANN in Halle errichtet wurde - je zur Hälfte durch den Bund und das Land Sachsen-Anhalt finanziert (bis 1998). Möglich wurde dies nur, weil die Familie BISCHOFF das Archivmaterial, den Gesamtbestand an der eingelagerten Bibliothek sowie Teile der Privatbibliothek KARL BISCHOFFS dem Projekt als Schenkung überließ, zu deren institutioneller Sicherung 1994 das Karl-Bischoff-Archiv am Germanistischen Institut der Universität Halle gegründet wurde.

Am Beginn stand die Erarbeitung einer Konzeption, die 1993 durch die Sprachwissenschaftliche Kommission der Sächsischen Akademie bestätigt wurde. Mit dem bereits vorhandenen Material sollte der Mundartwortschatz des Bearbeitungsgebiets in Bezug auf die Bedeutung, Lautung und räumliche Verbreitung umfassend beschrieben werden. Die Reaktionen auf eine 1995 verfertigte „Probelieferung“ (Kabache-Klatsche), die Fachkollegen vorgelegt wurde, führten zu letzten Spezifizierungen der Konzeption. Bereits 2002 erschien der Band $H-O, 2008$ folgte der Band $A-G$.

Nach dem Auslaufen der Bund-Land-Mischfinanzierung förderte Sachsen-Anhalt das Projekt noch bis 2003. Danach konnten Mittel der Fritz-Thyssen-Stiftung und der Lotto-Toto GmbH Sachsen-Anhalt eingeworben werden. Von 2009 bis 2018 gab es keine institutionelle Förderung des Projekts mehr. Für zwei Jahre hat das Land Sachsen-Anhalt nun finanzielle Mittel bewilligt (2019) bzw. in Aussicht gestellt (2020).

\subsection{Bearbeitungsgebiet}

Das Bearbeitungsgebiet ${ }^{2}$ umfasst die nördlichen zwei Drittel des heutigen Bundeslandes Sachsen-Anhalt (vgl. Abbildung 1). Dabei nehmen die niederdeutschen Dialekte den größten Raum ein (vgl. Tabelle 1: Gebietsziffern 1-3). Innerhalb des heterogenen niederdeutschen Gebiets lassen sich nordwestaltmärkische (1), nordund mittelbrandenburgische (2) sowie (elb-)ostfälische Mundarten (3) unterscheiden (vgl. Tabelle 1). Das Territorium des sich südlich anschließenden Nordthüringischen und Anhaltischen (4a und 4b) ist dadurch gekennzeichnet, dass hier der Sprachwechsel $^{3}$ vom Niederdeutschen zum Ostmitteldeutschen stattfand, so dass diese Mundarten niederdeutsche Relikte aufweisen.

2 Zur sprachlichen Genese und sprachhistorischen Charakterisierung des Gebietes vgl. BISCHOFF (1967), einen Überblick über die niederdeutschen Gebiete gibt MÖHRING (2000), zu Merkmalen des Anhaltischen sowie zur Problematik dieser Sprachraumbezeichnung vgl. WENNER (2001).

3 Seit der Mitte des 14. Jahrhunderts begann Mitteldeutsches in die Schreibsprache einzudringen. In einigen Orten am Nordrand hielten die Sprecher bis zur Mitte des 19. Jahrhunderts am Niederdeutschen fest, vgl. WENNER (2001: 74). 


\begin{tabular}{|c|c|c|c|c|}
\hline Merkmal & $\begin{array}{l}1 \text { nordwest- } \\
\text { altmärkisch }\end{array}$ & $\begin{array}{l}2 \text { branden- } \\
\text { burgisch }\end{array}$ & 3 elbostfälisch & $\begin{array}{l}4 \text { ostmittel- } \\
\text { deutsch }\end{array}$ \\
\hline mnd. $\hat{o}^{l}$ & $-a u-$ & $\begin{array}{l}\text { nbrdb.: }-\bar{o}- \\
\text { mbrdb.: }-\bar{u}(\mathrm{e})-\end{array}$ & $-a u-$ & $-\bar{u}-$ \\
\hline mnd. $\hat{e}^{4}$ & $-e i-$ & $\begin{array}{l}\text { nbrdb.: }-\bar{e}- \\
\text { mbrdb.: }-\bar{l}(\mathrm{e})\end{array}$ & $-e i-$ & $\overline{-\bar{l}-}$ \\
\hline mnd., mhd. $\hat{\imath}$ & $-\bar{l}^{-}$ & $-\overline{\mathbf{1}}-$ & $-\bar{l}-$ & $\begin{array}{l}\text { nthür.: }-\bar{l}- \\
\text { anhalt.: }-e i-\end{array}$ \\
\hline$e$-Apokope & ja & $\begin{array}{l}\text { nbrdb.: ja }{ }^{4} \\
\text { mbrdb.: nein }\end{array}$ & nein & nein \\
\hline $\begin{array}{l}\text { Partizip II: } \\
\text { Präfix }\end{array}$ & - & $\begin{array}{l}\text { nbrdb.: - } \\
\text { mbrdb.: } ә-, j \partial-\end{array}$ & $\partial-$ & jo- \\
\hline
\end{tabular}

Tab. 1: Binnengliederung des „Mittelelbischen Wörterbuchs“ mit einigen distinktiven Sprachmerkmalen

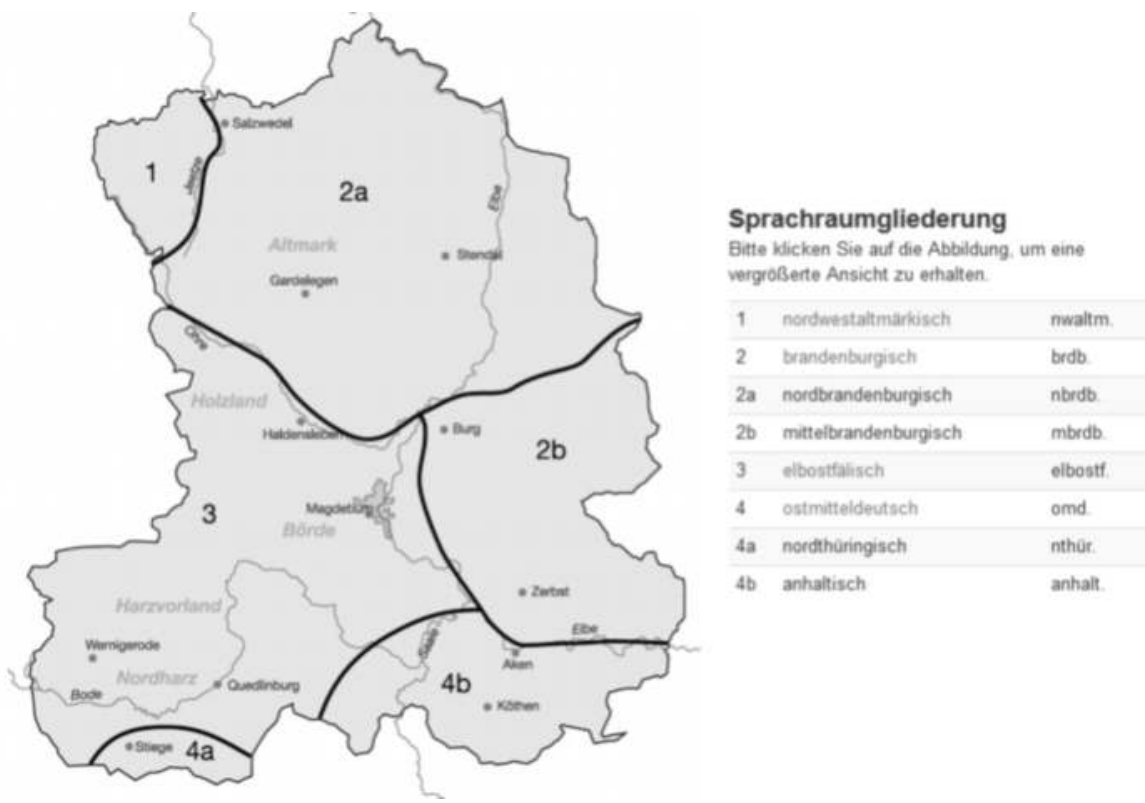

Abb. 1: Sprachraumgliederung des Bearbeitungsgebiets des „Mittelelbischen Wörterbuchs“

\subsection{Charakterisierung des verwendeten Materials}

Wie bei den meisten derartigen Unternehmungen bildet die indirekte Spracherhebung durch Fragebogen das Rückgrat des Archivmaterials, weil es auf diese Weise

4 Die $e$-Apokope ist in der Altmark (außer Südrand) und im Nordteil des Jerichower Landes (östlich der Elbe) zu finden. 
möglich ist, relativ schnell an flächendeckendes Material zu gelangen. Zwischen 1936-1939 wurden neun verschiedene Bogen mit je 50 Fragen versandt, nach dem Zweiten Weltkrieg noch einmal zwei (Nr. 10 mit 114 und Nr. 11 mit 122 Fragen). Durch diese überwiegend onomasiologisch ausgerichteten Abfragen wurden hauptsächlich Benennungen von Gegenständen, Dingen und Tätigkeiten des dörflichen Alltags sowie Eigenschaften, besonders von Personen, erhoben, in geringerem Umfang zielten sie auf Sachverhalte (z. B. Fragen nach gitterartigem Einsatz am Erntewagen oder nach besonderen Arten von Zäunen), Bräuche und Volksglauben (z. B. Frage nach Versen, die gesprochen werden, wenn ein Marienkäfer auf dem Finger sitzt). Nur relativ wenige Fragen befassten sich mit Lauterscheinungen (so z. B. zur Entrundung, zur Palatalisierung von $s$ vor $t$ sowie vor $l, m, n, w$, zur Länge bei $a f$ 'ab' oder an). Ebenfalls nur vereinzelt mussten ganze Sätze übertragen werden (z. B. ich gehe zum Großvater; wir haben noch 5 Zentner Pflaumen stehen; ein bisschen Essen ist noch übrig). Wenn auch das Prinzip ein Ort - ein Fragebogen galt, war BISCHOFF bemüht, auch diastratische Unterschiede zu erfassen. Im Kopf der Fragebogen heißt es: „Werden Wörter nur von älteren Leuten gebraucht, so merken Sie das bitte an und nennen Sie dann auch die dafür bei der Jugend üblichen Bezeichnungen!“'Diese Aufforderung wurde jedoch von den Gewährspersonen zumeist ignoriert.

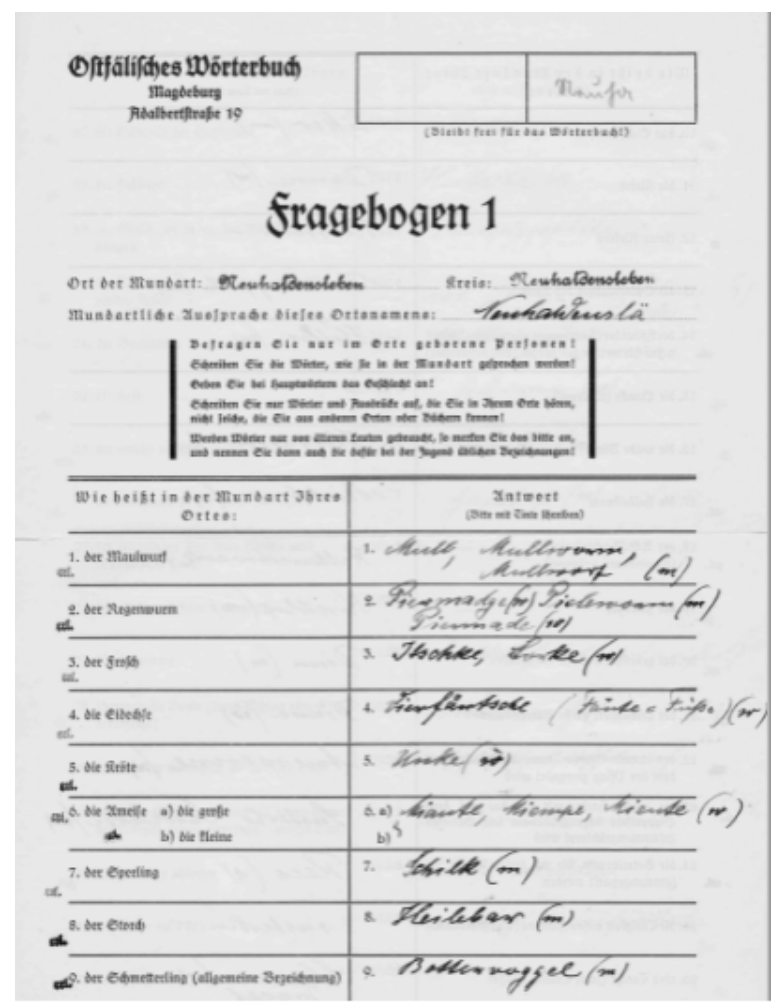

Abb. 2: Fragebogen des „Mittelelbischen Wörterbuchs“ 
Der Geschichte des Wörterbuchs ist es geschuldet, dass insgesamt nur elf verschiedene Fragebogen ausgewertet werden konnten. Aus diesem Grund ist Quellenmaterial anderer Herkunft und Art für das „Mittelelbische Wörterbuch“ von besonderer Relevanz. In das Archiv gelangten beispielsweise Fragebogen anderer Unternehmungen, so der benachbarten großlandschaftlichen Dialektwörterbücher (Niedersächsisches, Brandenburg-Berlinisches, Obersächsisches und Thüringisches) sowie der Sammelstelle für ostfälische Mundart- und Flurnamenforschung des Braunschweiger Landesmuseums.

Die Nachteile der Erhebung durch Fragebogen sind oft beschrieben worden, sie liegen vor allem in der ungenauen Darbietung der Lautformen. Hinzu kommt, dass die Einsender das Genus der Substantive häufig durch das Hinzufügen des bestimmten Artikels angaben. Durch die weitgehende lautliche Identität von maskuliner und femininer Form ( $d e$ bzw. dei) im niederdeutschen Teil des Bearbeitungsgebiets sind deshalb diesbezügliche Aussagen nicht immer zweifelsfrei möglich. Nur in Teilen der Altmark, in Anhalt sowie in Einzelorten konnten direkte Erhebungen durchgeführt werden. Die Ergebnisse liegen in Lautumschrift nach Teuthonista vor. Vereinzelt wurden dem Archiv private Wortsammlungen von sprachinteressierten Laien zur Verfügung gestellt.

In das Zettelarchiv ist eine Vielzahl von exzerpierten Werken eingeflossen, die einen Zeitraum von der Mitte des 19. bis zur Mitte des 20. Jahrhunderts abdecken. Dabei handelt es sich um Idiotika, Wörterbücher und Grammatiken einzelner Orte oder Landschaften, um Beiträge verschiedener Art zu vielfältigen Aspekten, die Mundarten, aber auch Brauch und Volksglaube betreffend, und mundartliche Literatur. Einbezogen wurde auch Material aus gedruckten oder handschriftlichen archivalischen Quellen. Insgesamt umfasst das Archiv ca. 250000 Zettel mit über 1 Million Belegformen.

\subsection{Datenaufbereitung, Zugänglichkeit}

Der Datenbestand ist bisher nicht digitalisiert worden, es existieren auch keine Pläne hierzu. Die Materialsammlung (u. a. Fragebogen, Wortsammlungen, Zettelkartei) ist als Teil des Karl-Bischoff-Archivs Interessenten (nach vorheriger Anmeldung) zugänglich. Erstellt wurde dagegen eine Datenbank, die u. a. alle Stichwörter mit den jeweiligen Bedeutungen enthält (ausf. vgl. Kapitel 3.1).

Die erarbeiteten Artikel liegen als Textverarbeitungsdateien vor (WordPerfect), die beiden veröffentlichten Bände sind darüber hinaus vollständig gescannt worden und als PDF-Dateien verfügbar. Auf die geplante Online-Version dieser Bände wird unter Kapitel 3.2 näher einzugehen sein. 


\subsection{Publikation}

Die Publikation des „Mittelelbischen Wörterbuchs“ ist auf drei Bände ausgelegt, wovon zwei bereits erschienen sind. 2002, also bereits zehn Jahre nach Beginn der direkten Arbeiten am Sprachmaterial, konnte der Band $H-O$ vorgelegt werden. Es wurde mit dieser Alphabetstrecke begonnen, um nicht gleich zu Beginn lange Artikelreihen mit den Verbpartikeln / Präfixen af-, an-, (auf-), be-, dorch-, (ein-) abhandeln zu müssen, die nicht die Vielfalt der mundartlichen Benennungen hätten zeigen können. 2008 wurde der Band $A-G$ - wie sein Vorgänger - im Akademie Verlag (jetzt zu de Gruyter gehörend) publiziert. Der Situation der Arbeitsstelle (vgl. Kapitel 1.6) ist es geschuldet, dass dieses hohe Tempo nicht beibehalten werden kann. Vom abschließenden Band 3 liegt bisher die Strecke $P-S e$ vor. Die erarbeiteten Artikel werden in Form von internen Lieferungen (bisher vier: $P$-sarpig) einem kleineren Interessentenkreis zugänglich gemacht. Ein Termin, zu dem das Wörterbuch vollendet sein wird, ist weder festgelegt worden, noch konkret zu benennen, hängt er doch von der zukünftigen Lage der Arbeitsstelle ab. Aufgrund der noch verbleibenden Artikelstrecke ist es aber absehbar, dass mit einem Abschluss nicht in den kommenden Jahren zu rechnen ist.

\subsection{Wörterbuchstelle}

Von 2000-2019 war das Projekt an den Lehrstuhl „Altgermanistik“ der MartinLuther-Universität Halle-Wittenberg angebunden, dessen Inhaber, Prof. Dr. HANSJoACHIM Solms, nach dem Tod von Prof. Dr. GERHARD KETTMANN (2009) als Projekt- und Arbeitsstellenleiter fungiert. Da ab 2009 keinerlei finanzielle Mittel zur Verfügung standen, konnte die Arbeit am Wörterbuch durch den verbliebenen wissenschaftlichen Mitarbeiter, Dr. ULRICH WENNER, nur neben dessen Verpflichtungen am Institut weitergeführt werden. Mit der erneuten Förderung durch das Land Sachsen-Anhalt (2019/2020), durch die eine Vollzeitstelle finanziert werden kann, ist die Arbeitsstelle mitsamt dem Material des Karl-Bischoff-Archivs an der Stiftung Leucorea und der Forschungsbibliothek Deutsche Sprachgeschichte in Wittenberg ${ }^{5}$ angebunden. Träger der Forschungsbibliothek sind neben der Stiftung Leucorea u.a. das Aninstitut Deutsche Sprache und Kultur (Direktor: Prof. Dr. HANS-JOACHIM SOLMS). Dort steht eine umfangreiche Arbeitsbibliothek zur Verfügung. Die Ausstattung mit Computertechnik ist so, dass alle notwendigen Arbeiten in der entsprechenden Qualität bewältigt werden können. 


\section{DIE WÖRTERBUCH-ARTIKEL: KONZEPTION, STRUKTUR, INHALT ${ }^{6}$}

\subsection{Konzeption}

Nach der Wiederbegründung des Projekts 1992 stand zunächst die Erarbeitung einer Konzeption im Vordergrund. KARL BISCHOFF hatte zwar ein Manuskript $A-$ bedöwen hinterlassen, ohne jedoch seine genauen Vorstellungen hinsichtlich der Gestaltung präzise darzulegen. Die Analyse dieses Manuskriptes ergab ein Bild davon, welche Schwerpunkte zu setzen waren. Zugleich zeigte sich, dass BISCHOFF bei der Realisierung einzelner Punkte noch verschiedene Möglichkeiten im Blick hatte. Die neu erarbeitete Konzeption versucht(e) nun, BISCHOFFS Grundanliegen mit dem aktuellen Stand der Dialektlexikografie in Übereinstimmung zu bringen, d. h. in den Wörterbuchartikeln finden sich alle Informationen, die von einem modernen großlandschaftlichen Dialektwörterbuch erwartet werden (vgl. Kapitel 2.3). Daneben wird der Fokus u. a. auf volkskundliche Aspekte und auf Hinweise zu siedlungsgeschichtlich relevantem Wortgut gerichtet, die für BISCHOFF von besonderem Interesse waren.

\subsection{Stichwortansatz ${ }^{7}$}

Schwierigkeiten beim Stichwortansatz resultieren aus zweierlei Gegebenheiten: einmal aus dem Nebeneinander von nieder- und ostmitteldeutschen Mundarten und zum anderen aus der Heterogenität des Niederdeutschen im Bearbeitungsgebiet. Die zur Anwendung kommenden Prinzipien sollen kurz illustriert werden:

\section{Nur niederdeutsch belegte Lexik}

Das Stichwort repräsentiert nicht die Lautform, wie sie sich in einem der niederdeutschen Dialektgebiete findet, sondern stellt ein Konstrukt dar, das sich am Mittelniederdeutschen orientiert, z. B.:

Stichwort Peddik 'Mark in Pflanzen, bes. in Holunder', zu mnd. ped $(d) i k$, peddek 'inneres Gewebe aus organischem Material, Mark' (vgl. LASCH / BORCHLING (2: 1429), Lautformen u. a.: Peddik / Päddik / Peddek, Pettk / Pittk, mit Wandel d > r: Pirk(s), Pääk (mit Vokalisierung des r).

6 Um Raum genug für eine Darstellung der Spezifika des „Mittelelbischen Wörterbuchs“ zu haben, wird nachfolgend bewusst auf eine Auseinandersetzung mit theoretischen Positionen der (Dialekt-) Lexikografie verzichtet. Beispielhaft sei nur auf folgende Werke verwiesen: APPENZELLER (2011), FRIEBERTSHÄUSER (1976) und (1986), GROSSE (1998), SUTTER (2017), ZILZ (2010).

7 Vgl. dazu auch die Einleitung MEWB (2: XI-XII). 
Im Gegensatz zu den Lemmata in hochdeutscher Gestalt wird die Vokallänge bei niederdeutschen Stichwörtern durch einen waagerechten Strich über dem Vokal gekennzeichnet, z. B.:

Brīchen 'Wasserdampf in der Küche' (MEWB 1: 531).

Werden verschiedene Varianten eines Lexems zu einem Lemma zusammengefasst, wird der variable Teil in runde Klammern gesetzt (gilt nicht nur für niederdeutsche Stichwörter), z. B.

Klūt(en) 'Klumpen', 'Kloß' (MEWB 2: 564); Afstēke(r)forke 'zweizinkige Gabel mit langem Stiel zum Reichen der Garben' (MEWB 1: 98); Metz(er) 'Messer' (MEWB 2: 2028).

2. Niederdeutsch und mitteldeutsch belegte Lexik

Wie bei 1. wird ein Stichwort in normalisiertem Niederdeutsch angesetzt, von der hochdeutschen Entsprechung wird auf das niederdeutsche Lemma verwiesen, z. B.:

Stichwort Brōder 'Bruder' (MEWB 1: 537)

zu mnd. brôder (vgl. LASCH / BORCHLING 1: 350).

Die Realisierung des Stammvokals (mnd. $\hat{o}^{l}$ ) erfolgt hier in den niederdeutschen Mundarten des Gebiets regelhaft, so dass diese Spracherscheinung die Grundlage der vorgenommenen Binnengliederung (vgl. Tabelle 1) bildet:

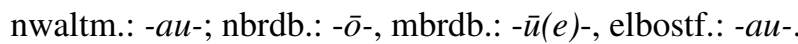

Als hochdeutsches Verweis(lemma) wird eingesetzt:

Bruder(-) $\rightarrow$ Brōder(-) (MEWB 1: 549).

Die in Klammern gesetzten Bindestriche geben zu erkennen, dass der Verweis hochdeutsch-niederdeutsch auch für Komposita mit Bruder/Brōder als Erstglied gilt.

3. Nur mitteldeutsch belegte Lexik

Hier fungiert die hochdeutsche / verhochdeutschte bzw. standardsprachliche Form als Lemma, z. B.:

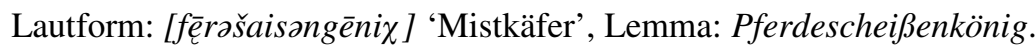

Der allgemeinen lexikografischen Praxis gemäß erhalten Homonyme jeweils ein eigenständiges Stichwort. Eine Unterscheidung erfolgt durch hochgestellte arabische Ziffern vor dem Lemma, z. B.:

${ }^{l}$ Fessel f. 'Band, Kette zum Fesseln'

${ }^{2}$ Fessel f. 'Teil des Beins über dem Huf des Pferdes' (MEWB 1: 952). 


\subsection{Artikelstruktur $^{8}$ \\ 2.3.0 Überblick über die Artikelstruktur}

Der Umfang eines Wörterbuchartikels hängt von der Art des verfügbaren Materials ab. Die Maximalstruktur umfasst folgende Positionen:

1. Stichwort im Fettdruck

2. Grammatische Angabe

3. Bedeutungsangabe

4. Verbreitungsangabe und -häufigkeit

5. Belegteil

6. Sachbeschreibungen oder Informationen zu Brauch und Volksglauben

7. Synonymenzentrale / Wortverbreitungskarte

8. Lautformenteil

9. Etymologische Angabe

\subsubsection{Stichwort}

Ausführungen dazu sind im Kapitel 2.2 zu finden.

\subsubsection{Grammatische Angabe}

Die grammatische Angabe beschränkt sich auf das Aufführen der Wortart bzw. bei Substantiven des Genus, z. B.:

Droppenfall m. 'Dachtraufe' (MEWB 1:784), Kūle f. 'Grube' (MEWB 2: 766), Rapphōn n. 'Rebhuhn'

pfietschen $\mathrm{Vb}$. 'ein schmatzendes, quietschendes Geräusch hervorbringen' gistern Adv. 'gestern' (MEWB 1: 1172).

Sind für ein Substantiv mehrere Genera belegt, erscheinen diese dann im Artikelkopf, wenn beide in nennenswertem Umfang vorkommen, z. B.:

Quell(e) m., f.

Ist ein Substantiv nur im Plural belegt, wird diese Numerusform als Lemma angesetzt und die grammatische Angabe lautet demzufolge Pl., z. B.:

Dünnen Pl. 'seitliche weiche Teile des Körpers' (MEWB 1: 813). 


\subsubsection{Bedeutungsangabe}

Die in einfache Häkchen gesetzte Bedeutungsangabe ist das Ergebnis einer umfangreichen Merkmals- oder Kontextanalyse. ${ }^{9}$ Das gewählte lexikografische Verfahren bei der Bedeutungspräsentation ist u. a. von der Art des Denotats, von der Wortart, aber auch vom „Weltwissen“ der gedachten Benutzer/-innen des Wörterbuchs abhängig. In vielen Fällen genügt das Aufführen eines oder mehrerer Synonyme, vor allem dann, wenn es für die Benutzer/-innen um die Identifizierung von Bekanntem bzw. um die Reaktivierung von Wissen geht, z. B.:

Damper m. 'Dampfschiff' (MEWB 1: 636).

Besonders wenn es sich um Tier- oder Pflanzennamen handelt, genügt die Aufführung der standardsprachlichen Benennung, z. B.:

Grüttblome PflN 'Wiesenschaumkraut' (MEWB 1: 1264).

Bei niederdeutschen Stichwörtern kann deshalb bereits die Nennung der standardsprachlichen Entsprechung ausreichend sein, bei Übereinstimmung mit dem Schriftdeutschen der Hinweis ,wie Standardspr.“, z. B.:

dwēr Adv. 'quer' (MEWB 1: 832),

Blitz m. 1. wie Standardspr. (MEWB 1: 440).

Vorteilhaft erscheint hier die Kürze und Prägnanz der Bedeutungsangabe, eine Gefahr besteht darin, dass der semantische Gehalt oder auch die Konnotation der verwendeten Synonyme nicht immer deckungsgleich sind. Statt eines Synonyms können zur Verdeutlichung auch zwei verwendet werden, z. B.:

anklātern $\mathrm{Vb}$. '(sich) anziehen, ankleiden' (MEWB 1: 174).

Besteht eine Bedeutungsangabe aus mehreren gleichartigen Gliedern, müssen hierbei nicht alle mit dem Lemma bedeutungsgleich sein, sie können auch den Bedeutungsumfang umreißen, z. B.:

nissig 'zänkisch, bösartig, gehässig' (MEWB 2: 1198).

Zur Präzisierung bzw. zur Bestimmung des Bezugsbereichs kann außerhalb der eigentlichen Bedeutungsangabe eine Erläuterung stehen, z. B.:

duff 'matt, dumpf', bes. von Farben oder Tönen (MEWB 1: 801).

Auf diese Weise werden auch Informationen zur kommunikativen, sozialen oder zeitlichen Geltung mitgeteilt wie „Schimpfwort“, „Kinderspr.“ oder „,veralt.“.

9 Verschiedentlich ist eine solche Analyse aber nicht möglich, vor allem dann, wenn die Gewährspersonen für ihre Bedeutungsangabe lediglich ein Synonym verwenden, das den Bedeutungsgehalt nur ungenau wiedergeben kann, z. B. wird passlich mit 'passend' umschrieben, es bleibt unklar, ob 'maßgerecht, gut sitzend', 'für jmdn., etw. geeignet' oder 'angenehm, recht, zusagend' damit gemeint ist, da eine Einbindung in einen Kontext fehlt. 
Erfordert das Denotat eine komplexere Umschreibung, wird auf die Paraphrasiermethode zurückgegriffen, bei der in einem möglichst kurzen Syntagma die wichtigsten und kennzeichnenden Merkmale genannt werden, z. B.:

Dēlige 'kleines abgeteiltes Ackerstück' (MEWB 1: 669),

Kulpōge 'stark hervortretendes, starr blickendes Auge' (MEWB 2: 769),

gletschern 'flache Steine so auf eine Wasserfläche werfen, dass sie mehrmals springen' (MEWB 1: 1178).

Eine formale Festlegung besteht nicht, bei Substantiven bietet sich die Methode von „genus proximum“ und „differentia specifica“ an, z. B.:

Brand 9. 'durch Pilze hervorgerufene Krankheit, die die Getreideähren (bes. bei Weizen) staubig zerfallen lässt' (MEWB 1: 513).

Bei Deckungsgleichheit in den Bedeutungen mehrerer Lexeme, die im Alphabet nicht weit voneinander stehen, wird ab dem zweiten Lexem die Bedeutungsangabe durch das in der Regel platzsparende ,dass. wie $\rightarrow$ “ ersetzt, z. B.:

${ }^{2}$ Röre 'hölzernes Gerät zum Rühren des kochenden Pflaumenmuses'

Rörrer dass. wie $\rightarrow{ }^{2}$ Röre

Rörrholt dass. wie $\rightarrow{ }^{2}$ Rörre.

Erläuterungen, die über die eigentliche Bedeutung eines Wortes / Semems hinausgehen (z. B. bei Lexik, die sich auf (sach-)volkskundliche Gegebenheiten bezieht), werden entweder direkt im Anschluss an die Bedeutungsangabe oder nach dem Belegteil präsentiert.

Sind einem Formativ mehrere Bedeutungen zuzuordnen, werden diese durch arabische Ziffern unterschieden, weitere Spezifizierungen sind durch kleine Buchstaben möglich. Für die Reihenfolge gelten folgende Prinzipien: konkrete Bedeutung steht vor übertragener, häufiger belegte vor seltener vorkommende, z. B.:

\section{Rulle}

1a. 'walzen- oder radförmiger Gegenstand, worauf etw. gerollt wird oder etw. gleitet', auch als Teil von Geräten.

1b. 'Wäschemangel (mit Drehrad)'.

2a. 'etw. Zusammengerolltes'.

2b. 'zusammengerolltes Bettzeug des $\rightarrow$ Haupters 1', Schifferspr.

2c. 'Wulst unter den Haaren'.

3. 'Purzelbaum'.

4. 'von einem Schauspieler dargestellte Gestalt'.

\subsubsection{Verbreitungsangabe und -häufigkeit}

Die areale Lagerung einzelner Lexeme / Bedeutungen stellt jeweils einen Baustein zur Charakterisierung der Mundarträume dar, die innerhalb eines großlandschaftlichen Dialektwörterbuchs beschrieben werden. Deren Angabe bildet somit ein wei- 
teres Kernstück der lexikografischen Arbeit. In Korrelation dazu ist die Beleghäufigkeit zu betrachten, die absteigend durch die Termini „allg.“, „,verbr.“, „,verstr.“ und ,vereinz.“ (bis zu drei oder vier Belege, die ungleich verteilt sein können) charakterisiert wird. Sie beziehen sich auf die Mundartgebiete (vgl. Kapitel 1.2), sowie auf geografische (z. B. Altmark, Harzvorland, Nordharz) oder administrative Einheiten (z. B. die einzelnen Landkreise ${ }^{10}$ ). Auf der untersten Ebene genügt das Aufführen von Ortssiglen, die aus Kürzeln für den Kreis und den Ort bestehen (z. B. WO-Ro für Kreis Wolmirstedt, Ort Rogätz). Den einzelnen Mundartgebieten wird jeweils eine arabische Ziffer vorangestellt (1: nordwestaltmärkisch, 2: brandenburgisch, 3: elbostfälisch, 4: ostmitteldeutsch), so dass für die Benutzerin bzw. den Benutzer eine schnelle Zuordnung möglich wird (2: WO-Ro). Die in diesen Ziffern zum Ausdruck kommende Reihenfolge von 1 bis 4 (von Nordwesten nach Südosten) findet sich dann auch bei der Verbreitungsangabe wieder.

\subsubsection{Belegteil}

In diesem Teil des Wortartikels wird das Einzelwortprinzip durchbrochen, indem hier ein Wort bzw. eine Bedeutung in Verbindung mit anderen Lexemen gezeigt wird. Ziel hierbei ist es, die Semantik, den kommunikativen Anwendungsbereich sowie den grammatischen Gebrauch aufzuzeigen. Die Wörterbuchbenutzerin bzw. der Wörterbuchbenutzer wird somit in die Lage versetzt, die aufgeführte Bedeutungsangabe anhand der Belege zu verifizieren. Sie bilden die Vielfältigkeit des Sprachgebrauchs ab und umfassen einfache und umfangreichere Syntagmen, Sätze, Redensarten (als Gliederungssignal Rda.), Sprichwörter (Sprw.), Reime, Verse, Rätsel (in dieser Reihenfolge werden die Belege im „Mittelelbischen Wörterbuch“ in der Schreibung der Quelle präsentiert). Redensarten und Sprichwörter vermögen sehr anschaulich, Einblicke in die Denkweise und in die Wertsysteme der Mundartsprecher des berücksichtigten Zeitraums ${ }^{11} \mathrm{zu}$ vermitteln. Eine Erläuterung einzelner Redensarten oder Sprichwörter wird dann beigefügt, wenn dies dem Bearbeiter sinnvoll bzw. notwendig erscheint, vgl. den Auszug aus dem Wortartikel zu Kind (MEWB 2: 485):

Rda.: Hei kann keen Kind vertär'n (verärgern) oder hei mütt'n in'n Hinnersten kniepen. von einem friedfertigen Menschen gesagt, BewohnerAltm 1,343; Dao schall man wol de
Kinner mit to Bett jaogen. von einem hässlichen Menschen gesagt, WbAltm 100; Kinder ät't langsam, da lett sick feel bipacken. Wb-Holzl 34; in Ausrufen: Kinner ne, wi kann denn

10 Die Kreiseinteilung basiert auf dem Stand zur Zeit der Wörterbuchgründung. Angesichts verschiedener Gebietsreformen in Sachsen-Anhalt, die jeweils zu Veränderungen der Ortszugehörigkeit und zu Reduktionen der Anzahl der Kreise führten, erscheint dieses Vorgehen angemessen.

11 Die Belege entstammen der Zeit von der Mitte des 19. bis zur Mitte des 20. Jahrhunderts (vgl. Kapitel 1.3). Diese zeitliche Einordnung ist wichtig, um Einstellungen z. B. zu Frauen, Minderheiten oder zu bestimmten Berufsgruppen richtig einordnen zu können. 
man so was ānjēn! CA-Ak; Kinner ok, wat hebb ich mei dunn freut, dätt kunn ick keen' seng'! Hausfr-Altm 1925,54; Sprw.: Een Kind - keen Kind, twee Kinner - Spölkinner, dree Kinner - völ Kinner. Spr-Altm 14; en Kinneken en gut Stünneken 'die Betreuung eines Kleinkindes entbindet von harter körperlicher Arbeit' WE-Dee; Kinder un Narren segget de Wahrheit HANo; Kinner un oll Lü' seng'n die Wahrheit. Bewohner-Altm 1,344; Kinder un Besopene spreken de Wahrheit Sprw-Börde; Kinder daun wie se klauk sind Sprw-Börde; wenn $t$ Kinner still sind dän mōkns wat 'wenn Kinder leise sind, machen sie meist Dummheiten' STE-KIMö; Wecker sin Kind lew hät, de hölt et unner de Tucht. Spr-Altm 14; auch: Je leewer Kind, je schärper Ro' (Rute). Bewohner-Altm 1,343; Wer sin Kinnern gewt dät Brod un litt naoher sülwst Nod, de is wert, dät'n met'n Kül sleit dod. 'Wer den Kindern den Besitz übergibt, sollte sich ein ausreichendes Altenteil sichern.' a.a.O. 1,344.

Der Präsentation von Sprachmaterial des Untersuchungszeitraums können historische Belege folgen, um früheres Vorkommen eines Lexems im Bearbeitungsgebiet aufzuzeigen. Nach Möglichkeit sollen sie aussagekräftige oder typische Verwendungsweisen abbilden. Von Interesse kann daneben die Lautgestalt oder der Umstand sein, dass das entsprechende Lexem einem Areal entstammt, in dem es in der Gegenwart nicht mehr belegt ist. Bei welchen Lexemen und in welchem Umfang das praktiziert wird, liegt im Ermessen des Bearbeiters. ${ }^{12}$

Neben dem Idealfall, dass der Verfasser eines Artikels aus einer Vielzahl von Belegen diejenigen auswählt, die besonders typisch, treffend oder signifikant sind und dabei auch alle vertretenen Mundartgebiete berücksichtigt, sieht die lexikografische Praxis nicht selten so aus, dass nur wenige Belege zur Verfügung stehen, die überdies nicht sehr erhellend sind. Dieser Fall tritt häufig bei Adjektiven auf, die Personen charakterisieren: prädikative Verwendung nach dem Muster: hel hei is ...

Da per Fragebogen kaum Sätze abgefordert wurden, werden Belege zumeist anderen Quellen wie den Orts- und Landschaftswörterbüchern, den Privatsammlungen oder der Mundartliteratur entnommen.

\subsubsection{Sachbeschreibungen oder Informationen zu Brauch und Volksglauben}

Um die Arbeits- und Lebenswelt der Mundartsprecher/-innen noch plastischer hervortreten zu lassen, werden u. a. Aufbau und Funktionsweise von Geräten, Arbeitsabläufe, Kleidung, Heilverfahren, aber auch Bräuche sowie Vorstellungen, die mit einzelnen Sachverhalten verbunden sind, dargestellt. Diese Angaben schließen sich direkt an den Belegteil an, eingeleitet werden sie durch die Kürzel „Brauch“ und „Volksgl.“, die - wenn es sachlich geboten ist - auch zusammen-gefasst werden

12 Während im Band $H-O$ solche historischen Belege noch häufiger aufgenommen wurden, ist die Zahl im nachfolgend publizierten Band $A-G$ aus Gründen der Platzersparnis reduziert worden. 
können. Die Menge der vorliegenden Informationen bestimmt den Umfang der Ausführungen, die sich auf einen Satz beschränken (vgl. den Artikel Nāmiddagsbrūt), aber auch mehrere Spalten (z. B. beim Artikel Hochtīt) umfassen können.

Nāmiddagsbrūut f. 'Braut, die später als 12 Uhr am Ort der Trauung er- scheint' - Volksgl.: Die N. hat Unglück in der Ehe zu erwarten. 2: Hochzeit-Altm 23 (MEWB 2: 1154).

Besondere Aufmerksamkeit wird den zentralen Ereignissen im Lebens(z. B. Döpe, Hochtīt, Dōd) oder Jahreslauf (z. B. Nījār, Fastelāwend, Pingsten, Erne 'Ernte') geschenkt, vgl. den Artikel Fīstenmeier.

Fìst(en)meier m. 'in Laub gehüllte, verkleidete Gestalt im Pfingstumzug', \ Pingsten, 2: WbAltm* 53, verbr. mittlere/s Altm. (auBer WO, nur WO-San), 3: verstr. s GA. - Brauch: Der Junge, der als F. zusammen mit anderen Gestalten im Pfingstumzug auftritt (ausf. $\rightarrow$ Pings ten), wird mit Stricken umwickelt und dann vollständig in Maiengrün eingehüllt, so dass er nicht mehr zu erkennen ist (GA-Da). Als Verkleidung dient auch eine mit Grün und Blumen behängte Kiepe, die dem F. übergestülpt wird. (SA-GrGe). Im s Teil von GA setzt man ihm außerdem eine Blumenkrone mit langen Bändern auf den Kopf. Brauch-wAltm 62; Heischevers:

Fiestmeier,

Schock Eier!

Geft uns kene fulen Eier!

Bo'm in de Höste

hangt de langen Wöste ... Brauch-wAltm 116 (CALV-Zo) (MEWB 1: 978).

Erläutert werden auch Gegenstände, Werkzeuge und Arbeitsverfahren aus dem Bereich der Sachvolkskunde (vgl. den Artikel Grūde)

Grūde f. 1a. 'feine Erde, Staub' 3: vereinz. w elbostf., 4: Wb-Nharz 65 (BLA-Sti) - de akker is sau fìn wī grūe a.a.O. 65. - 1b. 'heiße, glühende Asche' 3: Spr-Asch 39. - 2. dass. wie $\rightarrow$ Grūdekōks - mōrjen missmor Jrūde hō'l 4: DE-Ca. - 3. 'ummauerte Feuerstelle, in der Speisen mit glühender Asche langsam gegart oder warm gehalten werden', veralt., 2: vereinz. nbrdb., 3: verstr. elbostf., 4: vereinz. anhalt. - in de Grūd kaok'n Wb-Altm 71; Låß de Jrūse nich ausjēn! Wb-Ak 81; Wer wett hütigen Da'es noch wat von Pott in de Gru'e setten? ... Wenn de Lü'e et morjens na'n Felle jungen, sette de Fru en Pott in de Grue ... un make en groten Hupen glu'e Asche op'n Pottdeckel. Un wenn se denn et Midda's no Hus keimen, denn word de Pott ut de Gru'e krejjen, de Zuppe war gar ... WEDDE 1938,12. - Die G. war zu jeder Tages- und Jahreszeit in Gebrauch. Sie befand sich neben dem Ofen oder aber in der oberen Hälfte des Küchenherdes, als Brennmaterial dienten zunächst Stroh, Sägespäne, Laub oder Mohn- und Kartoffelkraut, später dann Koks. Die Töpfe mit dem vorbereiteten Essen wurden (morgens) in die glühende Asche gestellt und mit dieser vollständig bedeckt, so dass die Gerichte ohne Aufsicht garen und dabei nicht anbrennen konnten. 
ausf. u.a. Gesch-Ro 45, Speisen-

Börde 386, Wb-Ak 81

(MEWB 1: 1255).

\subsubsection{Synonymenzentrale / Wortverbreitungskarte}

Ein Ort, um im semasiologisch aufgebauten Wörterbuch das onomasiologische Prinzip, das den meisten Abfragen zugrunde liegt, anzuwenden, sind die Synonymenzentralen, die sich in den meisten großlandschaftlichen Dialektwörterbüchern finden lassen. Im „Mittelelbischen Wörterbuch“ werden alle erfassten Synonyme / landschaftlichen Heteronyme unter dem Lexem mit der größten räumlichen Verbreitung am Ende des Artikels oder des jeweiligen Semems aufgeführt, eingeleitet durch einen schräg nach oben gerichteten Pfeil, der als optisches Signal dient, z. B. Artikel Pīrmāde 'Regenwurm':

Pīrmāde f. TiN 'Regenwurm', z.T. veralt., 1: SA-GrGra, 2: verbr. n/mittlere Altm. (außer w Rand, dort vereinz.), GA-Po, vereinz. $n$ WO nö JE2, Dialektgeogr-Elbe/Saale 119 (ZE$\mathrm{Ze}$ ), 3: verbr. $\mathrm{n}$ elbostf. (außer nw Rand), OSCH-Crot, vereinz. n WA Rda.: Män nich so ängstlich, sä de

Haohn to de Piermao', dao fratt häi se up. Bewohner-Altm 1,338. > Dauworm ${ }^{I}$ Lork(e) Lorkworm Māde Pīr(e) Pìrlātsche Pīrlork(e) Pīrworm Rēgenmāde Rēgensnāke Rēgenworm Slingmāde ${ }^{l}$ Snāke, die wichtigsten Synonyme s. Kt.

Von den einzelnen Gliedern der Synonymenzentrale wird mit diesem Pfeil, der dort nach der Bedeutungsangabe erscheint, wiederum auf die Zentrale verwiesen, z. B.:

Dauworm m. TiN 'Regenwurm', \ Pīrmäde (MEWB 1: 658).

Nicht immer sind die einzelnen aufgelisteten Benennungen semantisch deckungsgleich. Mit einem kurzen Kommentar wird zu Beginn der Aufzählung darauf hingewiesen ${ }^{13}$ oder innerhalb der Zentrale erfolgt eine weitere Untergliederung, wie in der Zentrale Born 'Brunnen', in der die Aufzählung geordnet nach Brunnentypen (Zieh-, Dreh-, Wippbrunnen) vonstattengeht (MEWB 1: 485).

Oftmals lassen sich aus der Lagerung der einzelnen Benennungen dialektgeografisch interessante oder relevante Aussagen herleiten, z. B. zu sich abzeichnenden Bewegungsrichtungen, Kontaminationsformen oder zur arealen Verteilung von Wortschatz, der auf bestimmte Siedlergruppen zurückzuführen ist wie im Fall von Pìrmāde, wo das Kompositionsglied $P \bar{l} r$ auf die niederländischen ${ }^{14}$ Siedler des

13 Die Zentrale balgen (MEWB 1: 294) wird mit folgendem Hinweis eingeleitet: „Der Bedeutungsumfang bei den Benennungen reicht vom leichten spielerischen Ringen bis zum ernsthaften Prügeln.“

14 Dieser Terminus ist ungenau, da er sich auf Gebiete bezieht, die heute in Deutschland, den Niederlanden, Belgien und Nordfrankreich zu lokalisieren sind. 
Hochmittelalters weist. Solche Sachverhalte können mit Hilfe von Wortverbreitungskarten - im „Mittelelbischen Wörterbuch“ als Kombination von Flächen- und Punktsymbolkarte - visualisiert werden. Sie finden sich in unmittelbarer Nähe zu dem Stichwort, das als Zentralartikel dient, am Ende der Synonymenzentrale gibt es einen Hinweis darauf (vgl. Artikel Pīrmāde).

Pīrmāde [Regenwurm]

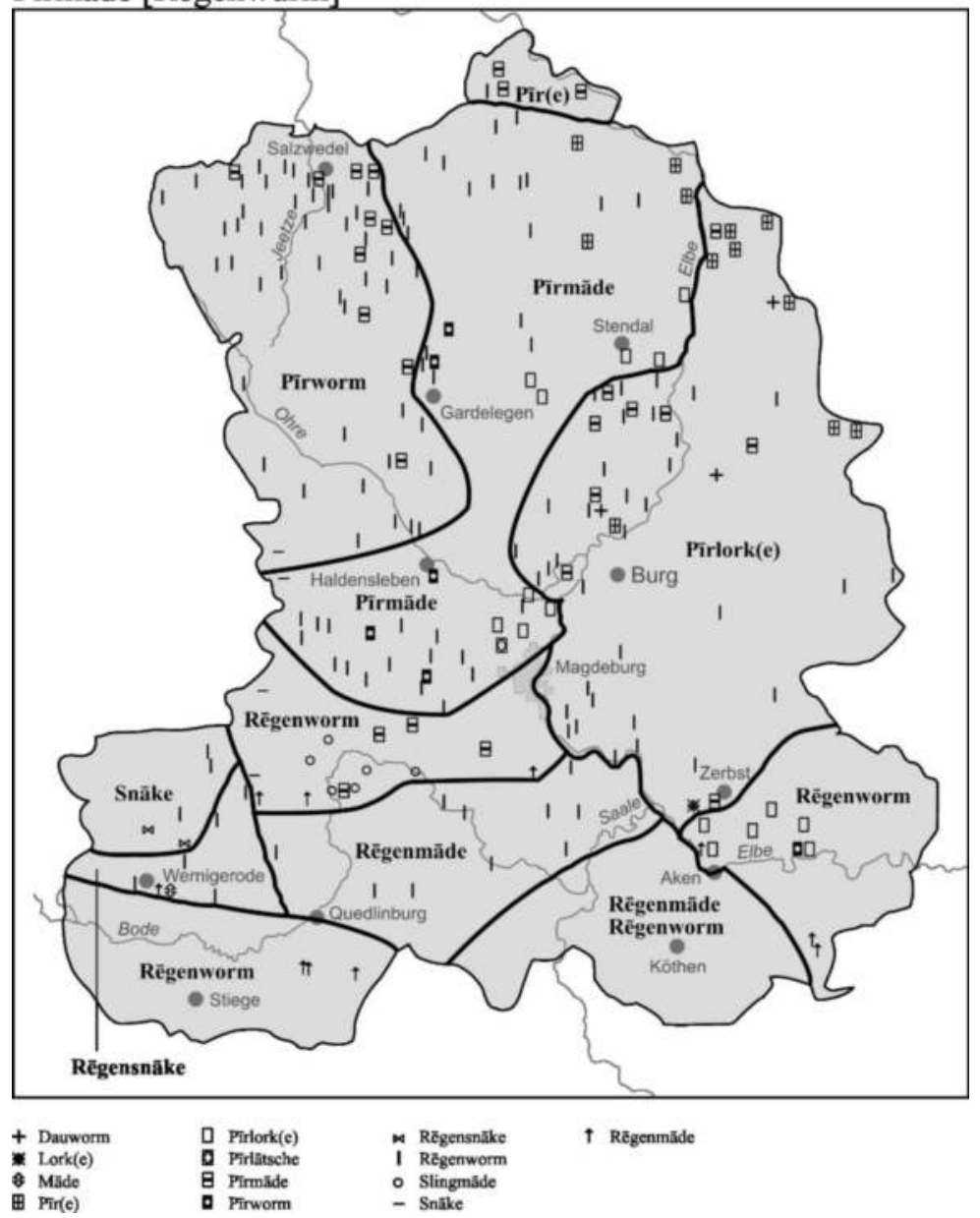

Abb. 3: Wortverbreitungskarte Regenwurm

\subsubsection{Lautformenteil}

Unter 2.2 ist erläutert worden, dass das Lemma ein Konstrukt darstellt, das sich von den tatsächlich belegten Lautformen unterscheiden kann. Der typografisch vom Hauptartikel abgesetzte Lautformenteil (eingeleitet durch: „Lautf.“) ist der Ort, an 
dem diese mit ihrer jeweiligen Verbreitung (und Häufigkeit) beschrieben werden. Ausgangspunkt ist immer die Form, die dem Lemma am nächsten steht. Es werden die Schreibungen der Quellen übernommen, was zu einem Nebeneinander von lautlich ungenauerer Schreibweise mit den Mitteln des Alphabets und Lautumschrift (nach Teuthonista) führt. Mutmaßlich identische Lautformen ${ }^{15}$ in unterschiedlicher Notation werden durch Komma, differierende durch Semikolon abgetrennt, z. B. Lautformenteil zum Artikel Grūde (vgl. den Artikel hierzu in Kapitel 2.3.6):

Lautf.: Grude, Jrude, [jrūda] JE2-Scho, verstr. s elbostf., vereinz. anhalt.; Grud, [grūd] vereinz. Altm.; Gruhe HA-Bo Neu; Grue, Jrue, [jrūa] Hbl-Ohre 1926 Nr. 6 (CALV-Zo), verstr. w elbostf., QUE-Hau; Gru, grū vereinz. w elbostf., QUE-Di; Jrūse, [jrūzə] Wb-Ak 81, CADo; jrōde Wb-Nharz 65 (BLA-Sti). - Etym.: zu mnd. grūde 'heiße Asche, Rückstände von einem Strohfeuer, Strohkohlen', vgl. HWb-Mnd 2,176.

Stärker vom Lemma abweichende Formative oder unter dem Stichwort subsumierte Wortbildungsvarianten erhalten den Hinweis Nebenform (,Nbf.“), z. B.:

bētsch Adj. 'bissig', vgl. bissig, 2: Id-Altm, Wb-Altm 12 und 18, 3: Wb-We 17 und 18.

Lautf.: betsch Wb-We 17; bätsch, bätsch Id-Altm, Wb-Altm 12; Nbf.: bītsch a.a.O. 18, WbWe 18 (MEWB 1: 395).

Die regionale Verteilung wird wie bei der Verbreitungsangabe des Lemmas / der Bedeutung von Nordwest nach Südost (in der Reihenfolge der vier großen Mundarträume) präsentiert. Für die Abhandlung grammatischer Besonderheiten steht ein eigener Gliederungspunkt („Gram.“) zur Verfügung.

Artikel dörschen 'dreschen' (MEWB 1: 740)

Gram.: Part. Prät.: wird weitgehend sw. gebildet, Stammvokal dann wie Inf.; bei dreschen neben sw. Part. Prät. -drescht bes. im s Nd. Omd. auch st. -droschen.

Bei einem engen Zusammenhang zwischen Lautform und grammatischer Erscheinung (z. B. bei lautlicher Differenz verschiedener Genera eines Substantivs) können beide Artikelpositionen aber zusammengefasst werden, z. B.:

Artikel Rabüntje(n) PflN 'Rapunzel, Feldsalat'

Lautf., Gram.: f.: Rabüntje Blicke-Drömling 2,156; rabintje Wb-Nharz 151; Rebintje Wb-Holzl 159 (WA-KIWa); n.: Rabüntjen verstr. elbostf. (außer sö); Rabüntchen GA-Ev; Rabünchen HA-Bee; Rabünjen HA-Wa; Rapüntjen HA-Oh; Rapünzchen DE-Vo.

15 Nicht immer ist aus der Laienschreibung die genaue Lautform klar ersichtlich. Verschiedentlich setzten die Gewährsleute nicht alle zu erwartenden Lauterscheinungen um, beispielsweise die Entrundung, die Realisierung von $/ g /$ als Frikativ oder die Palatalisierung von $/ s /$ vor $/ p /$ oder /t/. Das ist auch die Ursache dafür, dass auf eine stärkere Abstraktion bei der Darstellung verzichtet wurde. 


\subsubsection{Etymologische Angabe}

Etymologische Hinweise sind fakultativ, sie erscheinen vorwiegend dann, wenn die Herkunft des Lexems Aufschlüsse über die zugrunde liegende Bedeutung oder die Bedeutungsentwicklung geben kann sowie bei Herleitung aus anderen Sprachen. Das ist im Untersuchungsgebiet vor allem für Lexik von Interesse, die Rückschlüsse auf Siedlungsvorgänge zulässt. Dazu zählen slawisches Substrat oder Wortgut der niederländisch / niederfränkischen Siedler des 12. Jahrhunderts. Aber anders als in etymologischen Wörterbüchern wird die Wortgeschichte nicht bis zum Ursprung verfolgt, Bezugspunkt ist zumeist das Vorkommen im Mittelalter. Der Umfang differiert, je nachdem, ob die bloße Angabe der Herkunft ausreichend ist oder ob es Erläuterungen bedarf. Zur Überprüfbarkeit und zur Vertiefung wird den etymologischen Angaben ein Hinweis auf die entsprechende Literatur beigegeben, z. B. Gr $\overline{\mathbf{u}}$ de Lautformenteil mit etymologischer Angabe siehe in Kapitel 2.3.8.

Moll 'Maulwurf' (MEWB 2: 1069)

Etym.: Moll im Brdb. und Anhalt. mit Entsprechungen im rhein.-ndl.-fries. Sprachgebiet; Mull könnte als Kontamination von Moll und westelbischem $\rightarrow$ Mūlworm aufgefasst werden, allerdings hält Bischoff ein ehemals nach $\mathrm{O}$ und $\mathrm{W}$ ausgedehnteres Mull-Gebiet auf nordseegerm. Grundlage für möglich, vgl. ausf. Studien-Elbostf 41f., TEUCHERT ${ }^{2} 1972$, 334ff., LERCHNER 1965, 206ff., SANDERS 1967, $18 \mathrm{ff}$.

\section{TEXTTECHNOLOGISCHE ASPEKTE}

\subsection{Technologische Grundlagen der Wörterbucharbeit}

Die Erstellung der einzelnen Wörterbuchartikel erfolgt noch auf traditionellem Wege: Die auf Zetteln befindlichen Belege (vgl. Kapitel 1.4) werden durch den Bearbeiter gesichtet, geordnet, inhaltlich ausgewertet, so dass daraus der Wörterbuchartikel entsteht, der in ein Textverarbeitungsprogramm (WordPerfect) eingegeben wird. Bei den beiden bisher erschienenen Bänden wurde dann das fertige Manuskript einer Satzfirma übergeben, die es in eine Druckvorlage umwandelte. Im Zusammenhang mit der Generierung der Online-Ausgabe der Bände $A-G$ und $H-O$ (vgl. Kapitel 3.2) wurde zwar auch die Versuchsversion eines Redaktionssystems erarbeitet, eine Integration in den Arbeitsalltag fand bisher jedoch nicht statt. ${ }^{16}$

Um sich die Lagerung der einzelnen Lexeme / Bedeutungen bzw. Lautformen anzeigen zu lassen, wird ein Kartierungsprogramm verwendet, das RUDOLF POST der Arbeitsstelle Anfang der 1990er-Jahre unentgeltlich zur Verfügung stellte. Damit werden durch den Redakteur auch die Vorlagen für die Karten angefertigt, die publiziert werden sollen. Ihre endgültige Gestalt erhalten sie durch eine Grafikerin.

16 Hauptgrund dafür ist, dass die bisher vorliegenden Teile des dritten Bandes auf die beschriebene Weise mit einem Textverarbeitungsprogramm erstellt wurden. 
Besonders für die Zusammenstellung von Synonymenzentralen, aber auch für das Auffinden von Verweisen, Parallelstellen usw. ist die in den 1990er-Jahren begonnene und inzwischen vervollständigte Lemmadatenbank unentbehrlich. Mit dem Programm paradox erstellt und nun auch als Excel-Tabelle verfügbar, enthält sie alle Stichwörter mit den jeweiligen Bedeutungen, Fragebogennummern sowie Verweise auf andere Stichwörter.

\subsection{Digitale Version der Bände $A-G$ und $H-O$}

In Zusammenarbeit mit dem Institut für Informatik der Martin-Luther-Universität Halle-Wittenberg ${ }^{17}$ wurde eine digitale Version der beiden bisher veröffentlichten Bände entwickelt. Die Freischaltung ist im November 2018 erfolgt (<https://mew.uzi.uni-halle.de/>; Stand: 20.04.2020). Im Folgenden soll das Potential anhand einiger Funktionen und Beispiele angedeutet werden. ${ }^{18}$

Über eine einfache Lemma-Suchfunktion findet man den gewünschten Wörterbuchartikel, der als zusammenhängender Text - so wie in der Druckfassung - erscheint (vgl. Abbildung 4). Durch einen Klick auf das Lemma erhält man die Möglichkeit einer Strukturansicht, die die einzelnen Artikelpositionen voneinander abhebt. Zudem kann man durch Ansteuerung der oben befindlichen Reiter eine Auflösung der verwendeten Siglen erreichen (vgl. Abbildung 5). Besteht die Bedeutungsangabe wie bei Bornstange 2. aus der Information 'dass. wie $\rightarrow$ ' gelangt man ebenfalls durch Anklicken des blau hervorgehobenen Verweislemmas direkt zum entsprechenden Artikel mit der Bedeutung 'Hebebaum am Wippbrunnen'. Existiert zu einem Wörterbuchartikel eine Karte, ist diese über einen Reiter ansteuerbar.

Wörterbuchartikel

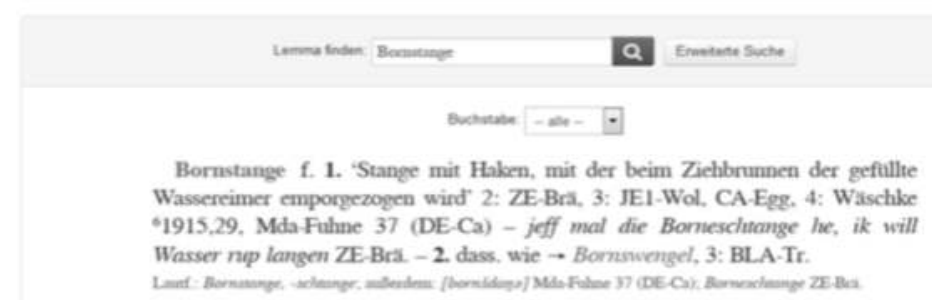

Abb. 4: Wörterbuchartikel Bornstange in der Online-Version: Normalansicht

17 Verantwortlich dafür waren Prof. Dr. PAUl Molitor, Dr. JÖRG RITTER und MARTIN ANDERT.

18 Die für die Wörterbuchtagung in Wien im November 2017 geplante Vorstellung der digitalen Fassung musste leider krankheitsbedingt entfallen. 
Wörterbuchartikel: Bornstange

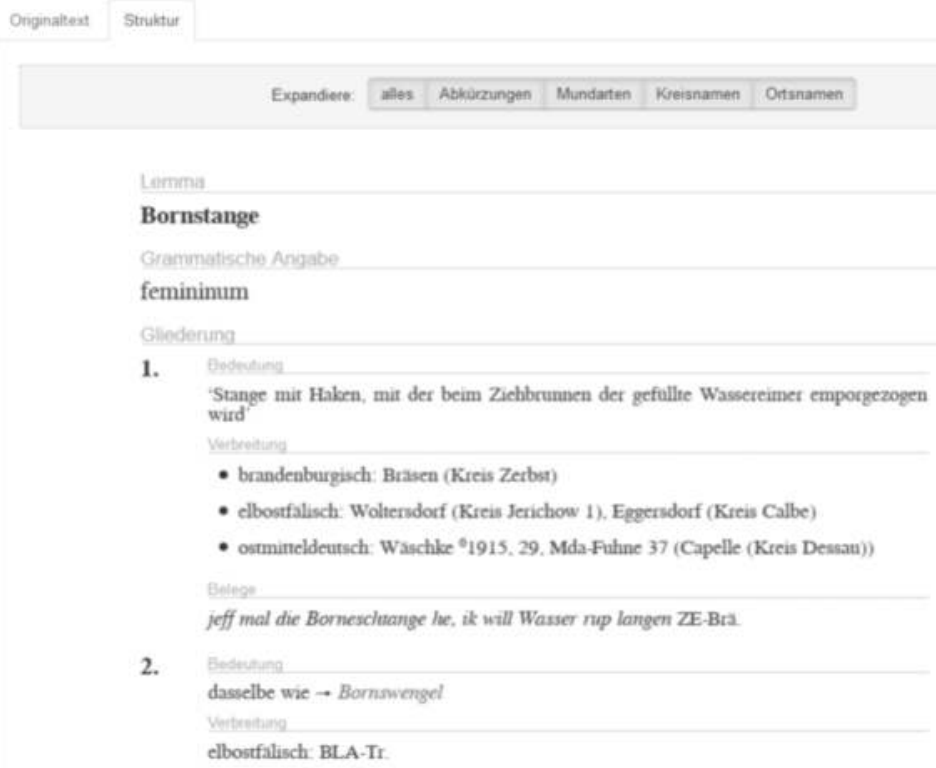

Abb. 5: Wörterbuchartikel Bornstange in der Online-Version: Strukturansicht mit aufgelösten Siglen

Eine erweiterte Suche ist in verschiedene Richtungen hin möglich: nach einem Wort(teil), nach der Wortart, nach der Bedeutung, nach Belegen und der Verbreitung (vgl. Abbildung 6). Alle Abfrageparameter lassen sich miteinander verknüpfen.

\section{MITtELEL BISCHES WORTERBUCH}

\section{Erweiterte Suche}

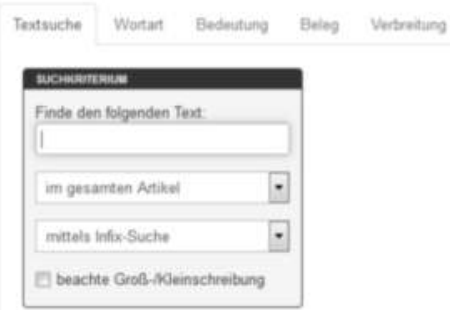


Hinsichtlich der Textsuche hält das Programm unterschiedliche Optionen bereit. So ist die Suche nach einem exakten Wort oder nach Wortbestandteilen (Präfixen, Infixen, Suffixen) möglich, gesucht wird entweder im gesamten Artikel, im Lemma, in der Bedeutungsangabe oder im Belegteil. Bei der Suche nach 'Wasserdampf' in der Bedeutungsangabe erhält man als Treffer Substantive mit der entsprechenden Bedeutung ${ }^{19}$ (Brōdem, Brīchen, Brīten, Dunst, Damp) sowie Verben mit der Bedeutung 'dampfen, Wasserdampf bilden' (bräsen, brīchen, briten, dampen) oder 'sich mit Wasserdampf beziehen, anlaufen' (beslāgen). Lemmata mit bestimmten Struktur- oder Wortbildungselementen lassen sich zusammenstellen, so z. B. durch die Suche nach dem Suffix bzw. der Suffixerweiterung -haftig (Lemmata: an-, bōs-, lām-, līf-, abend-, düwel-, èrnst-) - eine komfortable Grundlage für einschlägige Analysen (vgl. Kapitel 4.2).

Es kann nach allen Wortarten gesucht werden, so finden sich beispielsweise 32 Lemmata innerhalb der Artikelstrecke von $A-O$ mit der grammatischen Angabe „Indefinitpronomen“. Bei Substantiven ist eine weitere Untergliederung nach den einzelnen Genera vorgesehen.

Unter dem Reiter „Bedeutung“ verbergen sich Angaben zur kommunikativen oder semantischen Eingrenzung wie Kindersprache, Schimpfwort, Neck-, Orts-, Flur-, Pflanzen- oder Tiername.

Das Feld „Beleg“ ermöglicht die Suche nach solchen Texteinheiten, die im Belegteil gesondert gekennzeichnet werden wie u. a. Redensart, Sprichwort, Reim, Rätsel, aber auch Brauch, Volksglaube (vgl. Kapitel 2.3.5).

Zuletzt sei noch die Rubrik „Verbreitung“ in den Blick zu nehmen, die die Suche einerseits nach Häufigkeitsangaben (allgemein, verbreitet, verstreut, vereinzelt), andererseits nach Mundartgebieten, Kreisen und Orten sowie die Verknüpfung beider Kategorien erlaubt: 33 Stichwörter erfüllen z. B. die Kriterien „verstreut" und „,nordthüringisch“.

Spezifizierungen sind durch Kombinationen der angebotenen Abfrageoptionen zu erreichen. Hier wird das enorme Potential der digitalen Version des „Mittelelbischen Wörterbuchs" für die Auswertung des zugrunde liegenden Materials besonders deutlich. Die Bandbreite kann an dieser Stelle nur angedeutet werden:

Die Suche nach Wörtern mit der Bedeutung mürrisch und der Wortart „Substantiv" liefert 37 Artikel, bei denen es sich um Personenbezeichnungen handelt. Eine weitere Eingrenzung ist dadurch erreichbar, dass man sich nur Feminina anzeigen lässt. Das Interesse kann auf ein bestimmtes Mundartgebiet gelegt werden, z. B. durch die Verknüpfung der Bedeutung mürrisch mit der Wortart "Substantiv“ und dem Mundartgebiet „elbostfälisch“: 29 Treffer (vgl. Abbildung 7). 


\section{Erweiterte Suche}

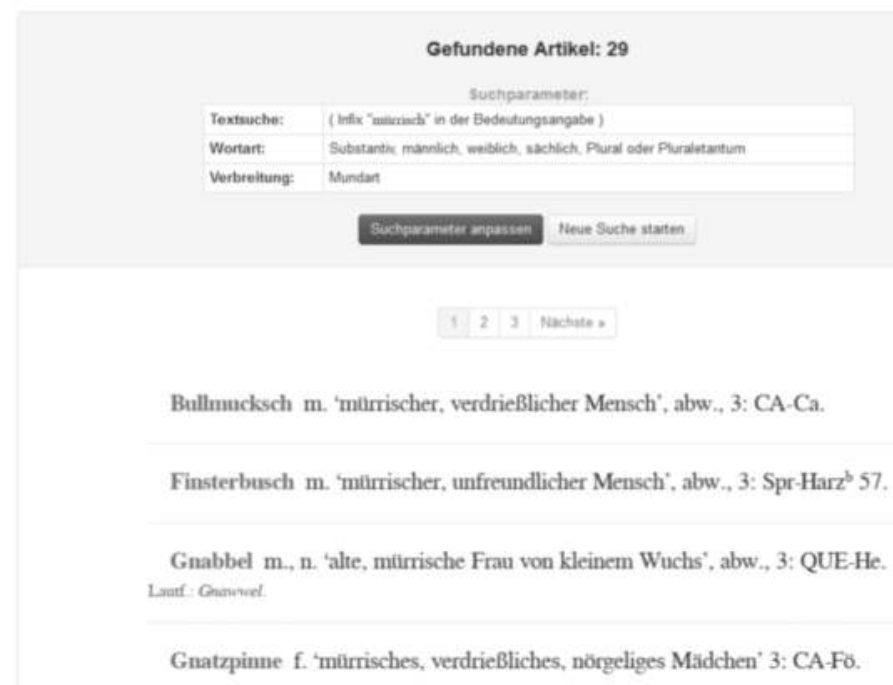

Abb. 7: Erweiterte Suche in der Online-Version: mürrisch + „Substantiv“ + ,elbostfälisch“

Es existieren 87 Stichwörter mit Hund (Text) im Lemma (bei Großschreibung: als Erstglied). Bei einer Einschränkung auf das Verbreitungsgebiet „brandenburgisch“ bleiben noch 42 Treffer. Bildungen mit Hund als Zweitglied werden gefunden, wenn man sich bei der Suchoption für die Kleinschreibung (hund) entscheidet.

Die Suche nach Hund im Belegteil von Lemmata, die „Adjektive“ (Wortart) sind und im „Brandenburgischen“(Verbreitung) belegt sein müssen, ergibt 19 Treffer, z. B. den Artikel alert mit der Bedeutung 'flink, schnell' und dem Beleg dei Hund is höllisch alart $\mathrm{Wb}-\mathrm{We} 4$.

Allerdings soll nicht verschwiegen werden, dass der Suche auch Grenzen gesetzt sind und dass die Ergebnisse nicht immer den Abfrageintentionen entsprechen müssen. Die als Treffer erscheinenden Wörterbuchartikel erfüllen zwar die Bedingungen, die mit einer kombinierten Abfrage gestellt werden, der Bezugsrahmen ist aber immer der Gesamtartikel: z. B. die Verknüpfung von Marienkäfer (Text), „Bedeutungsangabe“ (Artikelposition) und „Volksglaube“ (Beleg) bringt u. a. als Resultat den Artikel ,Engel“, der unter 3. die gewünschte Bedeutung enthält, das Textsegment „Volksglaube“ findet sich jedoch unter 1. 'meist mit Flügeln gedachtes, überirdisches Wesen' ${ }^{20}$ Gefunden werden nur Belege mit der angegebenen Grafie. Besonders bei der Suche innerhalb des Belegteils sind bei Wörtern mit großer Lautvarianz daher mehrere Abfragen erforderlich, wenn man bei der Erfassung nach

20 Ähnlich ist es bei der oben aufgeführten Kombination von Text (Hund), Artikelposition (,,Belegteil“), Wortart („Adjektiv“) und Verbreitung (,brandenburgisch“). Die Bedingungen sind alle erfüllt, der wiedergegebene Belegsatz stammt aber aus dem Elbostfälischen. 
Vollständigkeit strebt. Naturgemäß kann die Suche nach bestimmten Buchstabenfolgen (Infix-Suche) zu Ergebnissen führen, die nicht dem Ziel der Abfrage Genüge tun (z. B. die Suche nach hund erbringt auch hundert). Abhilfe schafft in vielen Fällen die Eingrenzung auf die Präfix-, Suffix-, die exakte Suche oder die Berücksichtigung der Groß- oder Kleinschreibung.

Der Inhalt der digitalen Version geht aber über die dargestellten Abfragemöglichkeiten hinaus, denn zum Angebot gehören weiterhin Informationen zum Projekt sowie die den Bänden beigegebenen Verzeichnisse. Unter dem Punkt „Info“ erfahren die Benutzer/-innen u. a. etwas über die Geschichte und die Aufgaben wie auch zum Stichwortansatz und Artikelaufbau des Wörterbuchs. ${ }^{21}$ Die „Sprachräume“ sind anhand einer Karte zu lokalisieren. Als Mehrwert gegenüber der Printausgabe werden darunter zusätzlich zum herkömmlichen Kreis- und Ortsverzeichnis (unter „Kreise \& Orte“, dort flankiert von einer entsprechenden Übersichtskarte) alle vorkommenden Orte den einzelnen Mundartgebieten zugeordnet. Noch stärker ins Gewicht dürfte aber die Gegebenheit fallen, dass man durch Klicken auf einen gewünschten Ort alle Wörterbuchartikel aufgelistet bekommt, in denen dieser Ort (als Ortssigle) erscheint. Das gilt gleichermaßen für alle „Siglen" der im Wörterbuchtext verwendeten Literatur (vgl. Abbildung $8^{22}$ ).

\section{Sigle: Heimatgesch-Bad 20 Arlike)}

\footnotetext{
Aftrëder m. 'Abtreter' 3: Wedde 1938,56, Heimatgesch-Bad 23, 4: Wb-Ak 12. Wb-Be - Taun Glicke lag de dicke Afträ'r an de Treppe ... Wedde 1938,56.

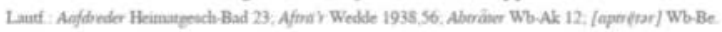

Āwendschoppen m. 'geselliges Beisammensein am Abend, abendlicher Umtrunk' 2: Hausfr-Altm 1929,23 (STE-KIMo), 3: Lindaub o.J. 44, HeimatgeschBad 50 - Lug Oto salut met sien'n Kumpan'n bien Lamme ten Abendschoppen a.a.O. 50.

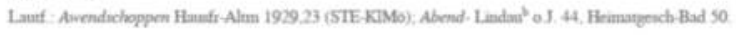

Déglaken n. 'Tuch, in dem Brot- oder Kuchenteig zum Băcker gebracht wird' 3: Wb-Nharz 39, Heimatgesch-Bad 33.

Laut Deighalim, dricidiliom.

Flēgenfänger m. 'Fliegenfänger' 3: Heimatgesch-Bad 50, 4: Krause 1964,40 Da sielt Otto von der Decke en Fleienfänger häng 'h... Heimatgesch-Bad 50. Lauf: Fleienfanger Heinargeach Bad 50, Fine in- Kame 1964.40
}

Abb. 8: Suche nach allen Wörterbuchartikeln, die die Sigle „Heimatgesch-Bad“ enthalten

21 Der dort zu findende Text ist dem Band 2 (X-XIV) entnommen.

22 Bei der Quelle handelt es sich um folgendes Werk: KLOCKE, FRITZ (1961): 1000 Jahre Badeborn. Ballenstedt. 
Obwohl mit dem Inhalt der Druckausgaben weitgehend identisch, besitzt die digitale Fassung einen ungleich höheren Gebrauchswert. Die Vielzahl an unterschiedlichen Zugriffs- und Suchmöglichkeiten führt nicht nur zu einem größeren Komfort für die Benutzer/-innen, sondern bietet ein völlig neues Instrumentarium für die Erschließung und die Analyse des Materials (vgl. dazu Kapitel 4.2).

\section{LINGUISTISCHES POTENTIAL}

\subsection{Die Forschungen KARL BISCHOFFS}

Eine umfangreiche Auswertung des Materials ist durch den Begründer des „Mittelelbischen Wörterbuchs“, KARL BISCHOFF, vorgenommen worden. Der Titel seines Hauptwerks „Sprache und Geschichte an der mittleren Elbe und unteren Saale“ (BISCHOFF 1967) steht dabei programmatisch für seine Forschungen, die dadurch gekennzeichnet waren, dass er durch die Analyse des gewonnenen Sprachmaterials der Gegenwart ${ }^{23}$ unter Hinzuziehung der greifbaren überkommenen Überlieferung, die zur Geschichte des Territoriums in Bezug gesetzt wurde, zu Aussagen zu Siedlungs- und Kulturräumen sowie zu Art und Richtung von sprachlichen Veränderungen gelangte. Dabei entwarf er ein Bild der diachronen Entwicklung der einzelnen Teile des Bearbeitungsgebiets und legte die verschiedenen sprachlichen Schichtungen offen, die hier kurz mit den folgenden Stichworten benannt werden sollen: östliches (Nieder-)Sächsisch südlich der Ohre und westlich der Elbe (bis zur Unstrut reichend), nordseegermanische Einflüsse, slawisches Substrat, die sprachlichen Auswirkungen der Besiedlungsvorgänge des Hochmittelalters in den heute zum Brandenburgisch-Märkischen und zum Anhaltischen gehörenden Gebieten, der frühe mitteldeutsche Einfluss auf den Südosten des Niederdeutschen mit der sprachlichen Sonderstellung des Elbostfälischen sowie die Auswirkungen der zu unterschiedlichen Zeiten stattfindenden Einbeziehung von Teilen des Territoriums in den brandenburg-preußischen Staat.

Besonderes Augenmerk legte BISCHOFF auf die sprachlichen Hinterlassenschaften der niederfränkisch-niederländischen ${ }^{24}$ Siedler des 12 . Jahrhunderts. Im Mittelpunkt standen die Identifizierung des Wortgutes als niederländisch (in Abgrenzung zu [gemein]niederdeutscher Lexik), die Lokalisierung möglicher Herkunftsgebiete, die Feststellung der neu besiedelten Areale sowie Prozesse des sprachlichen Austauschs und Ausgleichs. ${ }^{25}$ Ein Teil der Fragebogenabfragen war auf dieses Ziel hin ausgerichtet. Gemäß der in der ersten Hälfte des 20. Jahrhunderts

23 KetTManN (1998b: 68) weist in seinem Aufsatz zu den Auswertungsmöglichkeiten darauf hin, dass vom Material aus Aussagen sowohl zur Gegenwart als auch zur Vergangenheit möglich seien.

24 Zum Terminus vgl. Kapitel 2.3.7.

25 Vgl. neben BISCHOFF (1967) u. a. auch BISCHOFF (1938), (1958). Zur diesbezüglichen Forschungsgeschichte und zur wissenschaftlichen Auseinandersetzung mit HERMANN TEUCHERT vgl. WENNER (2004). 
dominierenden sprachgeografischen Methode wurden Ergebnisse auch in Form von Wortkarten dargeboten, die die Lagerung dieses Wortbestands zeigten.

Die Karten anderer Fragebogenabfragen dokumentier(t)en zwei für das Untersuchungsgebiet charakteristische Entwicklungsrichtungen: das Vorrücken von (mitteldeutscher) Lexik von Süden nach Norden (z. B. Hutsche 'Fußbank' verdrängt Fōt-, Fautbank) und von Osten nach Westen (z. B. Kant(en) 'Endstück des Brots' verdrängt Knūst).

\subsection{Nutzung des Wörterbuchmaterials für die verschiedenen Ebenen des Sprachsystems}

KetTMAnN hebt in seinem Beitrag zu Auswertungsmöglichkeiten des Wörterbuchs die das Bearbeitungsgebiet prägende Tendenz des Zurückweichens des Niederdeutschen hervor. Anhand des konkreten Sprachbestands könnten durch „theoretische Verallgemeinerung [...] immanente Züge“ dieses Prozesses ,verdeutlicht werden“ (KeTTMANN 1998b: 68).

Eine Kontrastierung von neu erhobenen Sprachdaten mit dem mehrheitlich aus der ersten Hälfte / der Mitte des 20. Jahrhunderts stammenden Wörterbuchmaterial brächte Erkenntnisse darüber, ob es sich dabei um eine kontinuierliche, weiter andauernde Entwicklung handelt, und wenn ja, in welchem Umfang und welcher Geschwindigkeit sie weiter vonstattengegangen ist. Für das Bearbeitungsgebiet fehlt bisher eine übergreifende Darstellung des Laut- und Formeninventars. Wenn auch die aus den Fragebogen gewonnenen Daten prinzipiell nur begrenzt Aussagen zur Phonologie zulassen, so stehen doch für große Teile des Areals verlässliche lautschriftliche Belege verschiedener Herkunft zur Verfügung.

Auf BISCHOFFS Interesse an der Volkskunde ist unter 2.1 hingewiesen worden. Erst die Einbeziehung von volkskundlichen Elementen und Themen und deren Verknüpfung mit mundartlicher Lexik vermag ein plastisches Bild von der Alltagskultur der Menschen zu geben, die sich der mundartlichen Varietät bedien(t)en. Nach KETTMANN (1998b: 73) gewährt das Wörterbuch so „Einblicke in die regionale und soziale Ausformung allgemein ausgeübter Bräuche“ und dient so als „Quelle der Traditionsforschung“ (KETTMANN 1998b: 67).

Nicht unbedingt zu den Kernbereichen der klassischen Dialektologie gehört die Wortbildung, hier bestehen erhebliche Forschungsdefizite. ${ }^{26}$ Mit dem unter 3.2 vorgestellten Instrumentarium der digitalen Version des „Mittelelbischen Wörterbuchs“ wird es einfacher, für derartige Studien benötigtes Material zusammenzustellen, gehört zu den Recherchemöglichkeiten doch die Suche nach einzelnen Wortkonstituenten. Die Inventarisierung der in einem Sprachraum vorzufindenden Wortbildungselemente für einzelne Wortarten kann dabei nur ein erster Schritt sein, dem weitere Analysen zu folgen haben, die die Einbeziehung sprachlicher Kontexte erfordern. In einer synchron angelegten funktional-semantischen Untersuchung sind von WENNER (2018) die im Bearbeitungsgebiet vorzufindenden suffigierten 
Adjektive der Artikelstrecke $A-S$ analysiert und semantisch determinierten Funktionsgruppen zugeordnet worden. Durch den Vergleich mit der Standardvarietät konnten Spezifika der einbezogenen Mundarten herausgestellt werden.

Nutzbringend einzusetzen sind die verschiedenartigen Zugriffe der digitalen Ausgabe auf die Inhalte der Wörterbuchbände zweifellos ebenso auf der Ebene der Lexikologie / Semantik. Das gilt einerseits für paradigmatische Beziehungen wie Synonymie, Heteronymie, Antonymie oder die Untersuchung von Wortfeldern und Wortfamilien. Durch verknüpfende Abfragen wird man überdies in die Lage versetzt, lexikalisch-semantische Relationen zwischen einzelnen Lexemen aufzuzeigen. Speziell die Lexik und deren Zusammenspiel in Syntagmen und Phraseologismen könnten genauer in den Fokus genommen werden. Viele Ansätze in unterschiedliche Richtungen ließen sich verfolgen: z. B. Erfassung der von der Standardsprache abweichenden Genera bei Substantiven oder statistische Auswertungen (z. B. Anteil einzelner Wortarten am Gesamtbestand oder die quantitative Auslastung bestimmter Wortbildungstypen).

Damit wäre das linguistische Potential des „Mittelelbischen Wörterbuchs“ sicher noch nicht umfassend beschrieben. Ein Gewinn für die weitere dialektologische Forschung wäre es, die in einem Bearbeitungsgebiet erbrachten Ergebnisse mit denen anderer Areale zu vergleichen, um so die Spezifika einzelner Mundartgebiete von dialektimmanenten Faktoren und Entwicklungen abheben zu können. Für gebietsüberschreitende Forschungen wäre ein Zugreifen auf die Inhalte der anderen großlandschaftlichen Dialektwörterbücher, wie es teilweise durch das „Wörterbuchnetz" (<http://www.woerterbuchnetz.de>; Stand: 20.04.2020) bereits möglich ist, zweifelsohne ein Gewinn.

\section{KONTAKTADRESSE, HOMEPAGE, WICHTIGE LINKS}

\section{Stiftung LEUCOREA}

Mittelelbisches Wörterbuch

Collegienstraße 62

06886 Lutherstadt Wittenberg

Deutschland

E-Mail:

hans-joachim.solms@germanistik.uni-halle.de

ulrich.wenner@germanistik.uni-halle.de

Homepages:

Mittelelbisches Wörterbuch, URL: http://www.germanistik.uni-halle.de/ altgermanistik/forschungsschwerpunkte/mittelelbisches_woerterbuch/ Seite Leucorea: https://leucorea.de/forschung/mittelelbisches-woerterbuch/ Online-Version, URL: <https://mew.uzi.uni-halle.de>

Karl-Bischoff-Archiv, URL: <http://www.germanistik.uni-halle.de/ altgermanistik/forschungsschwerpunkte/bischoffweb/> 


\section{LITERATURVERZEICHNIS}

APPENZELLER, GERRIT (2011): Das Niedersächsische Wörterbuch. Ein Kapitel aus der Geschichte der Großlandschaftslexikografie. Stuttgart: Steiner (Zeitschrift für Dialektologie und Linguistik. Beihefte. 142).

BISCHOFF, KARL (1935): Studien zur Dialektgeographie des Elbe-Saale-Gebietes in den Kreisen Calbe und Zerbst. Marburg: Elwert (Deutsche Dialektgeographie. 36).

BISCHOFF, KARL (1938): Zur Westgrenze der niederfränkischen Wörter Ostdeutschlands. In: Zeitschrift für Mundartforschung 14, 199-223.

BISCHOFF, KARL (1940): Zur mittelelbischen Wortgeographie. In: Sachsen und Anhalt. Jahrbuch der Historischen Kommission für Sachsen-Anhalt 16, 164-204.

BISCHOFF, KARL (1954): Elbostfälische Studien. Halle: Niemeyer (Mitteldeutsche Studien. 14).

BISCHOFF, KARL (1958): Sprachliche Beziehungen zwischen niederdeutschem Altland und Neuland im Bereich der mittleren Elbe. Berlin: Akademie-Verlag (Berichte über die Verhandlungen der Sächsischen Akademie der Wissenschaften zu Leipzig. Philologisch-historische Klasse. Bd. 103. Heft 4).

BISCHOFF, KARL (1967): Sprache und Geschichte an der mittleren Elbe und unteren Saale. Köln/Graz: Böhlau (Mitteldeutsche Forschungen. 52).

BISCHOFF, KARL (1984): Das Mittelelbische Wörterbuch. Wiesbaden: Steiner (Abhandlungen der Akademie der Wissenschaften und der Literatur Mainz. Geistes- und sozialwissenschaftliche Klasse. Jg. 1984. Nr.7).

ELSEN, HILKE / SASCHA Michel (2011): Wortbildung im Spannungsfeld zwischen Sprachsystem und Sprachgebrauch. Zur Einführung in diesen Band. In: ELSEN, HILKE / SASCHA MICHEL (Hg.): Wortbildung im Deutschen. Zwischen Sprachsystem und Sprachgebrauch. Perspektiven - Analysen - Anwendungen. Stuttgart: Ibidem, 1-15 (Perspektiven Germanistischer Linguistik. 5).

FRIEBERTSHÄUSER, HANS (Hg.) (1976): Dialektlexikographie. Berichte über Stand und Methoden deutscher Dialektwörterbücher. Festgabe für Luise Berthold zum 80. Geburtstag am 27.01.1976. Wiesbaden: Steiner (Zeitschrift für Dialektologie und Linguistik. Beihefte 17).

FRIEBERTSHÄUSER, HANS (Hg.) (1986): Lexikographie der Dialekte. Beiträge zu Geschichte, Theorie und Praxis. Tübingen: Niemeyer (Reihe Germanistische Linguistik. 59).

GROSSE, RUDOLF (1998) (Hg.): Bedeutungserfassung und Bedeutungsbeschreibung in historischen und dialektologischen Wörterbüchern. Beiträge zu einer Arbeitstagung der deutschsprachigen Wörterbücher, Projekte an Akademien und Universitäten vom 7. bis 9. März 1996 anläßlich des 150jährigen Jubiläums der Sächsischen Akademie der Wissenschaften zu Leipzig. Stuttgart/Leipzig: Hirzel.

KETTMANN, GERHARD (1997): Das Mittelelbische Wörterbuch - Geschichte und Stand der Arbeiten. In: Sachsen und Anhalt. Jahrbuch der Historischen Kommission für Sachsen-Anhalt 20, 155173.

KeTTMANN, GERHARD (1998a): Das Mittelelbische Wörterbuch: Stationen seines Weges nach 1992. In: GROSSE, RUDOLF (1998) (Hg.): Bedeutungserfassung und Bedeutungsbeschreibung in historischen und dialektologischen Wörterbüchern. Beiträge zu einer Arbeitstagung der deutschsprachigen Wörterbücher, Projekte an Akademien und Universitäten vom 7. bis 9. März 1996 anläßlich des 150jährigen Jubiläums der Sächsischen Akademie der Wissenschaften zu Leipzig. Stuttgart/Leipzig: Hirzel, 199-208.

KETTMANN, GERHARD (1998b): Das Mittelelbische Wörterbuch als Quelle für die Traditionsforschung (Auswertungsmöglichkeiten eines Mundartwörterbuches). In: Zeitschrift für deutsche Philologie 117, 67-81 (Sonderheft: Regionale Sprachgeschichte).

KeTtMANN, GERHARD (2004): Das Mittelelbische Wörterbuch - die problemreiche Geschichte eines notwendigen Forschungsprojektes. In: Mitteldeutsches Jahrbuch für Kultur und Geschichte $11,27-34$. 
KetTMann, GeRHARD (2008): Das Mittelelbische Wörterbuch. Generelle Anmerkungen zur Arbeit an einem Dialektwörterbuch. In: Sachsen und Anhalt. Journal für Natur- und Heimatfreunde, Heft 2 / 2008, 5-8.

LASCH, AGATHE / CONRAD BORCHLING (1956-2019): Mittelniederdeutsches Handwörterbuch. Fortgeführt von GERHARD CORDES, DIETER MÖHN und INGRID SCHRÖDER. Bde. 1-3. Neumünster [u.a.]: Wachholtz Verlag.

MEWB = Mittelelbisches Wörterbuch (2002 / 2008): Begründet von KARL BISCHOFF, weitergeführt und herausgegeben von GERHARD KETTMANN. Unter der Leitung des Herausgebers bearbeitet von HANS-JÜRgEN BADER, JÖRG MÖHRING (nur Band 2), Ulrich WENNER. Band 2: H-O. Band 1: A-G. Berlin: Akademie-Verlag.

MÖHRING, JÖRG (2000): Zur Sprachgeographie des elbostfälisch-brandenburgischen Gebietes an der mittleren Elbe. In: Jahrbuch des Vereins für niederdeutsche Sprachforschung 123, 85-101.

SOLMS, HANS-JOACHIM (2008): Eine Synopsis sozialen Wissens. Grußwort zur Präsentation des Mittelelbischen Wörterbuches. In: Sachsen und Anhalt. Journal für Natur- und Heimatfreunde, Heft 2 / 2008, 3-5.

STELlMACHER, DIETER (2008): Das Mittelelbische Wörterbuch und warum wir es brauchen. In: Sachsen und Anhalt. Journal für Natur- und Heimatfreunde, Heft 2 / 2008, 9-10.

SuTTER, PATRIZIA (2017): Diatopische Variation im Wörterbuch. Theorie und Praxis. Berlin/Boston: de Gruyter (Studia linguistica Germanica. 27).

WenNER, UlRICH (2001): Das Anhaltische. In: Die Mundarten des Harzgebietes in Ton und Text. Hg. v. Lutz Wille. Clausthal-Zellerfeld: Piepersche Druckerei und Verlag GmbH, 70-78.

WENNER, UlRICH (2004): Hermann Teuchert, Karl Bischoff, Max Bathe - Auf der Suche nach niederländischen Sprachspuren im Mittelelbegebiet. In: STELLMACHER, DIETER (Hg.): Sprachkontakte niederländisch, deutsch und slawisch östlich von Elbe und Saale. Frankfurt a. M. [u. a.]: Lang, 101-131 (Wittenberger Beiträge zur deutschen Sprache und Kultur. 3).

WENNER, ULRICH (2018): Suffixale Adjektivderivation in den Mundarten des Bearbeitungsgebiets des Mittelelbischen Wörterbuchs. Sandersdorf-Brehna: Renneritz-Verlag.

WIEsINGER, PETER (2000): Die deutsche Dialektologie zwischen Tradition und Neuansätzen. In: STELLMACHER, DIETER (Hg.): Dialektologie zwischen Tradition und Neuansätzen. Beiträge der Internationalen Dialektologentagung, Göttingen, 19.-21. Oktober 1998. Stuttgart: Steiner (Zeitschrift für Dialektologie und Linguistik. Beihefte. 109), 15-33.

ZILZ, WILFRIED (2010): Das Ortswörterbuch von Eltze in der Region Hannover. Praktische und theoretische Studien zur syntopischen Lexikographie. Bielefeld: Verlag für Regionalgeschichte (Veröffentlichungen des Instituts für Historische Landesforschung der Universität Göttingen. $55)$. 


\title{
DAS POMMERSCHE WÖRTERBUCH
}

\author{
Matthias Vollmer

\section{GESCHICHTLICHES UND RAHMENBEDINGUNGEN}

\subsection{Zur Geschichte des Wörterbuchs}

Das „Pommersche Wörterbuch“(PWB) ist ein großlandschaftliches Dialektwörterbuch, das den niederdeutschen Wortschatz der ehemaligen preußischen Provinz Pommern in den Grenzen von 1936 in zwei umfangreichen Bänden dokumentiert. Das Vorhaben hat mittlerweile eine nahezu hundertjährige, sehr wechselvolle und keinesfalls immer glückliche Geschichte hinter sich. Gegründet wurde die Arbeitsstelle 1925 an der Universität Greifswald von WOLFGANG STAMMLER, unter dessen Leitung das Wörterbuchprojekt allerdings nur geringe Fortschritte machte, was nicht zuletzt mit den wissenschaftlichen Interessen und Schwerpunktsetzungen STAMMLERS zu tun hatte, unter denen die pommersche Dialektlexikographie nur eine unbedeutende Rolle spielte. Als STAMMLER 1937 aus politischen Gründen in den Ruhestand versetzt wurde, war der bis dahin realisierte Umfang des Archivs mehr als bescheiden, konnte man doch nach zwölfjähriger Sammelarbeit lediglich etwa 20000 Belegzettel vorweisen.

Neue und fruchtbare Impulse erhielten die Arbeiten anschließend durch das große Engagement von HANS-FRIEDRICH ROSENFELD, der noch 1937 die Nachfolge des Lehrstuhls von STAMMLER antrat und entscheidende Weichenstellungen vornahm, die sich für das „Pommersche Wörterbuch“ außerordentlich positiv auswirkten. Leider entwickelte sich jedoch zunächst eine höchst unerfreuliche Auseinandersetzung um die Leitung des Wörterbuchs, die auch von KURT MISCHKE beansprucht wurde, der als ehemaliger Assistent von STAMMLER und NSDAP-Mitglied sogar nicht davor zurückschreckte, nahezu das gesamte Archivmaterial in seine Wohnung nach Gristow in der Nähe von Greifswald zu überführen und zu seinem Privateigentum zu erklären. Erst als MiSCHKE 1942 im Krieg gefallen war, konnte ROSENFELD schließlich die Leitung des Wörterbuchs offiziell übernehmen, ohne dabei jedoch das von MiscHKE entwendete Material zurückzuerhalten, weil dessen Witwe nicht gesprächsbereit war und die Rückgabe kategorisch verweigerte. Zwangsläufig musste ROSENFELD deshalb mit einer neuen Materialsammlung beginnen. In der kurzen Zeitspanne bis zum Kriegsende gelang es ihm dennoch, einen Bestand von immerhin ca. 30000 Zetteln aufzubauen, wobei dafür vor allem niederdeutsche Handschriften aus dem Staatsarchiv Stettin und der Greifswalder Universitätsbibliothek herangezogen wurden. Diese Sammlung musste in den letzten Kriegsmonaten auf amtlichen Befehl nach Lübeck ausgelagert werden. Nach dem 
Kriegsende bemühte sich ROSENFELD erfolgreich darum, wenigstens einen Teil dieser ausgelagerten Bestände nach Greifswald zurückzuholen. Darüber hinaus konnten schließlich doch noch ungefähr 6000 ältere Zettel aus MisCHKES Wohnung in Gristow auf fast abenteuerliche Weise im Austausch gegen Schreibpapier für die dortige Dorfschule gerettet werden.

Nachdem das Wörterbuch 1948 eine Arbeitsstelle der Ostberliner Akademie der Wissenschaften geworden war, arbeitete ROSENFELD in Greifswald zusammen mit einer kleinen Gruppe von Helferinnen und Helfern an der Ausarbeitung von insgesamt gut 450 Fragelisten, die zur wesentlichen Grundlage der Datensammlung wurden, und dem Aufbau eines Netzes von Gewährspersonen aus allen Dialektregionen Pommerns. Besonders schwierig war die Datenerhebung naturgemäß für die nach dem Krieg abgetretenen Gebiete östlich der Oder, also für den Großteil der ehemaligen mittelpommerschen und die Gesamtheit der ostpommerschen Dialekte. Für diese Sprachräume konnten nur noch Sprecherinnen und Sprecher befragt werden, die es als Heimatvertriebene nach Mecklenburg oder Vorpommern verschlagen hatte. Eine annähernd flächendeckende Erfassung dieser historischen Sprachräume war deswegen nicht mehr möglich.

Als ROSENFELD 1958 die Lehrerlaubnis an der Universität Greifswald und zugleich die Leitung des Wörterbuchs aus politischen Gründen entzogen wurde, übernahm IRMGARD REHFELD die kommissarische Führung der Arbeitsstelle, die bereits 1952 in „Niederdeutsches Wörterbuch“ umbenannt worden war. Unter ihrer Leitung, die bis 1964 dauerte, wurde die Datenerhebung fortgesetzt. Zu diesem Zeitpunkt waren bereits über eine Million Belegzettel erstellt worden. Anschließend übernahm HELMUT SCHÖNFELD die Leitung des Wörterbuchs, die er bis 1966 bekleidete. Zu den Schwerpunkten der Arbeiten in dieser Zeit gehörten die Lemmatisierung der Archivzettel und die Anfertigung einer umfangreichen Synonymenkartei, um onomasiologische Fragestellungen bearbeiten zu können. Schließlich wurde 1967 RENATE HERRMANN-WINTER zur Leiterin des Wörterbuchs ernannt. Zwei Jahre später, als schon erste Probeartikel ausgearbeitet waren, wurde die Arbeitsstelle jedoch vor dem Hintergrund politischer Entscheidungen geschlossen. Das umfangreiche Archiv verblieb aber glücklicherweise in Greifswald.

Nach der deutschen Wiedervereinigung konnten die Arbeiten, die über zwanzig Jahre geruht hatten, wieder aufgenommen werden. RENATE HERRMANN-WINTER war es gelungen, die Arbeitsstelle 1992 zunächst bei der Sächsischen Akademie der Wissenschaften zu Leipzig zu etablieren. Das neue Konzept sah zwar einschneidende Kürzungen und Modifikationen gegenüber den ursprünglichen Planungen vor, die sich sowohl auf die Makro- als auch auf die Mikrostruktur des Wörterbuchs auswirken. Doch nur auf diese Weise schien es möglich, das großlandschaftliche „Pommersche Wörterbuch“ in einem überschaubaren Zeitrahmen und mit gesicherter Finanzierung zu realisieren.

Festgelegt wurde damals ein Umfang für das „Pommersche Wörterbuch“ von lediglich noch zwei Bänden mit strikt-alphabetischer Makrostruktur. Die Publikation der ersten Lieferung mit einer umfangreichen Einleitung der Herausgeberin erfolgte im Jahr 1997. Seit Anfang 1999 wird das Wörterbuch nur noch vom Bundesland Mecklenburg-Vorpommern finanziert, zugleich erhielt es seine neue und 
alte Heimat an der Universität Greifswald. Der erste Band des Wörterbuchs, der die Buchstabenstrecke $A-K$ umfasst, konnte 2007 mit der 13. Lieferung fertiggestellt werden. 2014 übernahm MatTHIAS VOLLMER die Leitung des Projekts. Mittlerweile sind bereits zehn Lieferungen des zweiten Bandes erschienen, die bis zum Stichwort Treck reichen. Damit fehlen noch drei Lieferungen bis zur endgültigen Fertigstellung, mit der Mitte 2025 zu rechnen ist.

\subsection{Materialkorpus}

Das Materialkorpus für das „Pommersche Wörterbuch“ ist insgesamt als heterogen zu charakterisieren, was jedoch in ganz ähnlicher Weise auch für andere großlandschaftliche Dialektwörterbücher des Deutschen gilt. Im Zentrum der Materialerhebung für das Vorhaben stand die Ausarbeitung von über 450 Fragebögen, die onomasiologisch ausgerichtet waren. Auf diese indirekte Form der Datenerhebung ist letztlich der überwiegende Teil der Belege zurückzuführen, die in Form von Belegzetteln im Format DIN A7 und alphabetisch lemmatisiert Aufnahme in das Wörterbucharchiv gefunden haben. Diese Erhebungen fanden vor allem zwischen 1948 und 1962 sowie in den Jahren 1966 und 1967 statt. Die folgende Abbildung zeigt einen aus den fünfziger Jahren stammenden Archivzettel des „Pommerschen Wörterbuchs" zum Stichwort Schnack. Die Signatur unten links verweist auf die Frage 61 des Fragebogens 275. Rechts unten ist eine Sigle („Pa“) für den Ortspunkt Pasewalk zu erkennen.

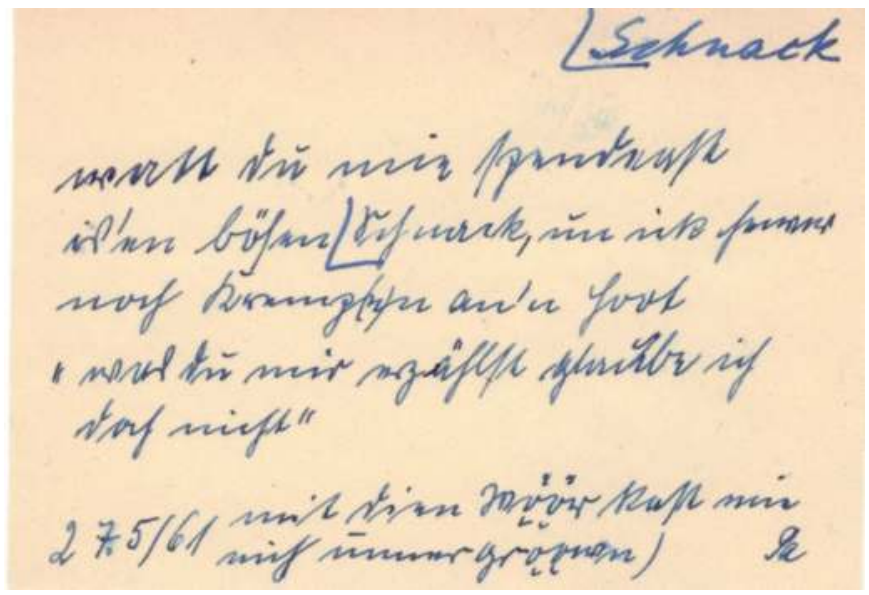

Abb. 1: Archivzettel des „Pommerschen Wörterbuchs“

Von den an der Arbeitsstelle entwickelten Vorkriegsfragebögen sind hingegen nur noch drei bekannt, die allesamt zwischen 1929 und 1935 ausgegeben worden sind, ohne dabei allerdings nennenswerten Rücklauf erzielt zu haben. Die nachfolgende Abbildung zeigt einen Ausschnitt der Vorderseite eines Fragebogens aus den frühen 
dreißiger Jahren des vergangenen Jahrhunderts, der von dem Dialektschriftsteller HEINRICH BANDLOW ausgefüllt worden ist. Der Vermerk links oben auf dem Fragebogen bezieht sich auf die Einarbeitung in das Zettelarchiv des Wörterbuchs, die erst 1963 erfolgte.

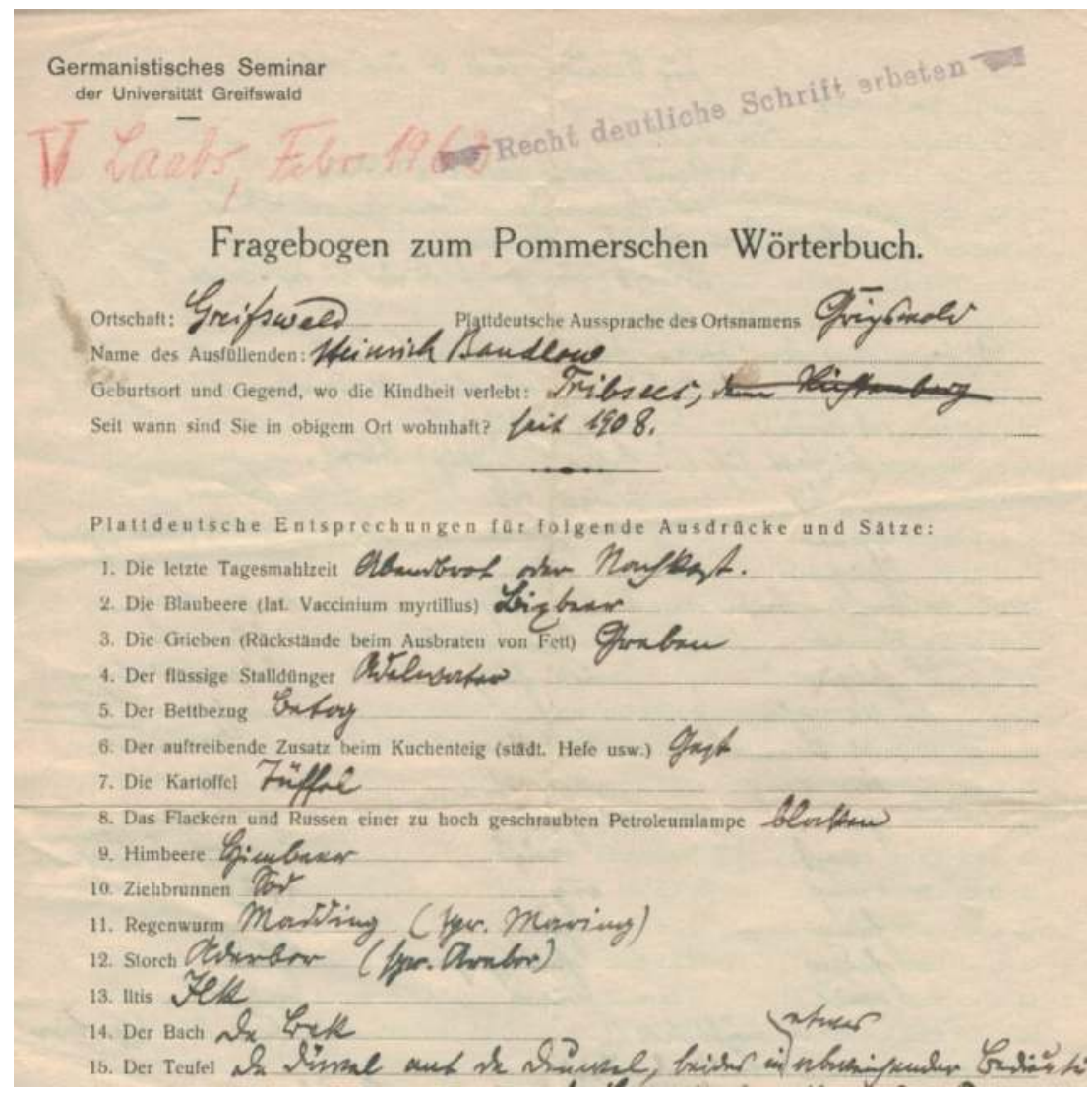

Abb. 2: Fragebogen des „Pommerschen Wörterbuchs“ aus den 1930er Jahren

Daneben sind aber noch weitere wichtige Quellengruppen zu nennen. Dazu zählen zunächst direkte Befragungen von Gewährspersonen, aber auch Material, das auf der dialektalen Sprachkompetenz ehrenamtlicher Mitarbeiter beruht. Ausgewertet wurden zudem alle greifbaren wissenschaftlichen Beiträge zu den pommerschen Dialekten, besonders Lautlehren und Grammatiken. Hierzu gehören vor allem die Arbeiten von Brose (1955), KüHL (1932), MAhnKe (1931), MischKe (1936), Pfaff (1898), Pirk (1928), Priewe / Teuchert (1927/1928), Stritzel (1937), TITA (1965) und WARNKROSS (1912). Zu nennen sind weiterhin gedruckte und ungedruckte Orts- und kleinlandschaftliche Wörterbücher für den pommerschen Sprachraum, besonders JOST (1993), LAABS (1988) und LAUDE (1995). Außerdem wurde auch die umfangreiche pommersche Mundartliteratur berücksichtigt, die in 
der Handbibliothek des „Pommerschen Wörterbuchs“ gut vertreten ist. Schließlich sind noch Tonaufnahmen pommerscher Dialekte und deren Transkriptionen zu nennen. Insgesamt umfasst das Archiv des „Pommerschen Wörterbuchs“ ca. 1,2 Millionen Belegzettel, die in 526 Archivkästen verwahrt werden.

\subsection{DIE WÖRTERBUCHSTELLE}

Seit der Wiedereinrichtung der Arbeitsstelle musste das „Pommersche Wörterbuch" zweimal innerhalb von Greifswald umziehen. War es zunächst in drei Räumen in der Rudolf-Petershagen-Allee 1 untergebracht, erfolgte 2001 ein erster Umzug in die benachbarte Rudolf-Breitscheid-Straße 27. Seit 2006 ist das „Pommersche Wörterbuch“" schließlich in zwei Räumen im Gebäude der Domstraße 14 in Greifswald in unmittelbarer Nähe zum Institut für Deutsche Philologie, an dem es institutionell verankert ist, untergekommen. Die personelle Situation hat sich leider kontinuierlich verschlechtert. Verschiedene Stellenkürzungen haben im Lauf der Zeit dazu geführt, dass von den ehemals drei vollen Mitarbeiterstellen seit Mai 2016 nur noch die des Leiters der Einrichtung verblieben ist, der gleichzeitig eine Lehrverpflichtung von vier Semesterwochenstunden am Institut für Deutsche Philologie der Universität Greifswald zu erfüllen hat. Als langjährige Sekretärin arbeitet MANUELA DITTMANN momentan zudem vier Stunden pro Woche für das Wörterbuchprojekt.

Folgende Redakteurinnen und Redakteure waren seit der Wiedereinrichtung der Arbeitsstelle nach der deutschen Wiedervereinigung am „Pommerschen Wörterbuch" in Greifswald tätig:

- Dr. JÜRGEN GRAMbOW (von 1993 bis zu seinem Tod 2003)

- Frank SchnibBEn (von 1993 bis 1997)

- Dr. WolfGang FedDERS (von 1994 bis 1998, verstorben 2003)

- Dr. Ulf-Hermann BADER (von 1998 bis 2008)

- PD Dr. MATTHIAS Vollmer (seit 1998)

- Ute Neumann (von 2008 bis 2012)

- Katharina Oelze (von 2012 bis 2016)

\section{DIE WÖRTERBUCHARTIKEL}

\subsection{Struktur der Wortartikel}

Die Wortartikel des „Pommerschen Wörterbuchs“ entsprechen in ihrer Struktur den in der Fachwelt gemeinhin anerkannten Vorgaben für großlandschaftliche Dialektwörterbücher, wie sie z. B. von HERMANN NIEBAUM formuliert worden sind (vgl. NiEBAUM 1986). Jeder der insgesamt gut 60000 Wortartikel wird mit dem fett gedruckten Lemma eingeleitet. Danach folgen noch im Artikelkopf grammatische Angaben, zu denen in erster Linie die obligatorische Wortartangabe zählt. Bei substantivischen Stichwörtern sind zudem Hinweise zu Form und Gebrauch des Plurals 
möglich. Falls weitere grammatische Angaben nötig erscheinen, werden diese in einen am Schluss des Wortartikels befindlichen Petit-Absatz verlagert. Fakultativ werden im Artikelkopf außerdem abgekürzte Symptomwertangaben vermerkt, die vor allem der stilistisch-pragmatischen Zuordnung dienen. In diesen Zusammenhang gehören auch Markierungen, die fachsprachlichen, aber auch veralteten oder bereits historischen Wortschatz kennzeichnen. Eventuell vorhandene Laut- und Formvarianten schließen sich an und beenden den Artikelkopf. Nur wenn mehr als drei dieser Laut- und Formvarianten belegt sind, werden sie zusammenhängend in einem Petit-Absatz am Ende des jeweiligen Wortartikels aufgelistet.

Den zentralen Teil der Wortartikel bildet naturgemäß der dem Artikelkopf folgende Bedeutungsteil. Bei polysemen Wörtern werden die einzelnen Lesarten durch arabische Ziffern voneinander getrennt. Dabei stehen in aller Regel hochfrequente Bedeutungen vor gering belegten und allgemeinere vor speziellen. Die möglichst knapp gehaltenen Bedeutungserläuterungen können abhängig von dem zu behandelnden Stichwort unterschiedlichen Definitionstypen entsprechen. Dazu zählen besonders logische, paradigmatische, morpho-semantische, funktionsbezogene und taxonomische Definitionen. Enzyklopädische Angaben ergänzen die Bedeutungserläuterungen, falls immer dies notwendig erscheint. Die Bedeutungserläuterungen werden nach Möglichkeit durch aussagekräftige Sprachgebrauchsbeispiele illustriert, denen entweder eine Ortssigle oder eine Literatursigle zugeordnet wird. Dabei stehen Belege für freien Sprachgebrauch vor solchen, die idiomatisch gebunden sind. Gliederungssignale oder metasprachliche Kommentare trennen beide Gruppen von Sprachgebrauchsbeispielen. Liegt ein besonders umfangreicher phraseologischer Gebrauch eines Lexems vor, kann ebenfalls ein Petit-Absatz gebildet werden, in dem die entsprechenden Phraseologismen genannt werden. Übersetzungen objektsprachlicher Belege werden aus Platzgründen nur dann vorgenommen, wenn sie in Gänze oder teilweise als unverständlich einzuschätzen sind.

Jede einzelne Bedeutung wird mit einer Verbreitungsangabe versehen, die grundsätzlich der Bedeutungserläuterung folgt und ausschließlich die Beleglage des Wörterbucharchivs abbildet. Nur wenn allgemeine Verbreitung eines Lexems oder einer bestimmten Bedeutung angenommen werden kann, entfällt ein solcher Hinweis. Angaben zur Belegdichte (verbreitet, verstreut, vereinzelt, selten) können sich sowohl auf den gesamten Sprachraum als auch auf Teile davon beziehen. In letzterem Fall treten sie nur in Kombination mit Sprachgebiets- oder Kreissiglen auf.

Ergänzt werden können die Wortartikel durch kurze etymologische Angaben, wenn die Zuordnung zu einem Etymon erklärungsbedürftig ist. Dies gilt grundsätzlich für Lehnwörter in den pommerschen Dialekten, falls diese nicht bereits durch standardsprachliche Parallelen bekannt sind. Für diese etymologischen Angaben ist eine feste Position am Ende der Wortartikel vorgesehen.

Onomasiologische Informationen werden durch entsprechende Verweise auf Heteronyme und vor allem bei besonders reichhaltiger Synonymik durch die Einrichtung von Raumsynonymenzentralen vermittelt. Davon gibt es im „Pommerschen Wörterbuch“ insgesamt 245. 
Anschließend sollen zur Veranschaulichung zwei Artikel aus dem Wörterbuch präsentiert werden, von denen der erste eine der angesprochenen Raumsynonymenzentralen im abschließenden Petit-Block enthält. Von den Wortartikeln der dort genannten Heteronyme wird natürlich jeweils auf das Stichwort verwiesen, das die Raumsynonymenzentrale enthält.

Stamptüffel Pl. Kartoffelbrei verbr. VPom, verstr. HPom. Stamptïffel mit Borrermelk, dat is 'n pommersch Gericht Sch/Ac. Scherzh. von einer Schwangeren: Dei hett tau väl Stamptüffel äten $\mathrm{Fra} / \mathrm{Br}$. - Sagw.: Schlicht Läben hett ' $n$ End, säd de Fru un schmeert sich Stamptüffel up't Brot Stral.

Rsyn.: Maustüffel, Quetsch(pan)tüffel, Quetschtüften, Riefnudeln, Rögtiüfke, Stamptüften, Stukes, Stuktüften.

Abb. 3: Wortartikel mit Raumsynonymenzentrale

Der folgende Wortartikel stellt ein Beispiel für einen typischen Artikel mit mittlerer Länge dar.

Speck ${ }^{1}$ n./m., Spack Lau/Ke, Spetch verstr. ${ }^{\circ}$ Slo, Spetsch Slo/La. 1. Schweinespeck. Dat gifft fetten un dörchwossen Speck Gwd/Ba; gräunen Speck roher, nicht geräucherter Speck Gri/Gm; utlåten Speck ausgebratener Speck Gwd/Da; witt Speck fetter Speck Dra/Bu; Speck in'n Wiemen hängen Gri/Go. Wi äten hüt Speck mit Arften Rüg/Zi. Speck un Brot, dat schmeckt got Pyr/Lt. Dat kann em nich fett naug warn, hei lecht sich Speck up'e Bodder Gwald. Speck gifft Murr [Kraft] in'e Knåken Dem/De. Giff $m i$ ' $n$ bäten Speck för'e Musfall! Dem/Kt. In fester Vbdg.: Speck mit Ogen Salzhering VPom, sonst vereinz. Aufmunternder Zuruf: Nu man ran an'n Speck! allg. Lob fetten Essens: Ganz eengal, Speck orre Aal, wenn't man fett is! HuMGwD 8,51,8. - Sprw.: Speck un Brot verdriwwt den Dôt BLFPVK 4,74. Mit Speck fängt man Müs' Ank/Br. Wo dei Fru gaut wirtschaften deit, wasst dat Speck an'n Balken Gwd/Nu. - Sagw.: Jedereen nåh sienen Schmack, säd de Jung', Vadder, ät du denn $K o h l$, ik ät denn Speck Gwald, ähnl. HUMGWD 74,177f.,1. De Hunger ward 't woll indriewen, säd de Jung un schmeert sick Bodder up 'n Speck HUMGWD $8,16,2$. Dat kümmt wedder, säd de Bur, don fodert he sien Schwien mit Speck ${ }^{\circ}$ Rüg. Ik sitt gaud, saed de Katt, da satt sei up'n Speck Rum/Gl BLFPVK 10,43. - 2. Fettpolster. Dei Kierl hett väl Speck up'e Ribben Dem/Tp. Ral.: Dat gifft wat up'n Speck! es gibt eine Tracht Prügel! Stral. Spruch: Speck sett'/giffi werrer Speck wer Speck ißt, setzt schnell Fett an $\mathrm{Fra} / \mathrm{Br}$, ähnl. verbr. - 3. harziges Kiefernholz vereinz. 


\subsection{Auswahl und Ansatz der Stichwörter}

Das „Pommersche Wörterbuch“ ist ein semasiologisches Dialektwörterbuch mit einer strikt alphabetisch geordneten Stichwortreihung. Im Zentrum der Bemühungen steht die Dokumentation des dialektalen Allgemeinwortschatzes Pommerns im 20. Jahrhundert. Die nach der deutschen Wiedervereinigung beschlossene Neukonzeption sah gegenüber den ursprünglichen Plänen einen fast vollständigen Verzicht auf die Bearbeitung historischer Lexik vor, die nach dem Jahr 1800 nicht mehr belegt ist, auch wenn entsprechendes Material für die davorliegende Zeit im Wörterbucharchiv in ausreichender Menge vorhanden ist. Eigennamen werden nur dann als Stichwort angesetzt, wenn Sie auch appellativisch gebraucht werden oder in phraseologischen Kontexten eine Rolle spielen. Einschränkungen gelten auch für die Berücksichtigung rein fachsprachlicher Bezeichnungen, also vor allem für die Fachterminologie der Bauern, Handwerker, Fischer und Seeleute, die nur in Auswahl vorgenommen werden konnte. Okkasionelle Bildungen sind grundsätzlich nicht als Stichwort angesetzt worden. Das gilt auch für Komposita, die offenkundig nicht fest lexikalisiert sind. Neologismen in den vorpommerschen und in den verbliebenen mittelpommerschen Dialekten nach 1945 sind nur dann berücksichtigt worden, wenn idiolektaler Gebrauch ausgeschlossen werden kann.

Der Stichwortansatz erfolgt in ostniederdeutscher Form auf der Grundlage mecklenburgisch-vorpommerscher Lautung. Davon weichen nur mittelpommersche und ostpommersche Stichwörter ab, die keine lautliche Entsprechung in den Dialekten Vorpommerns haben. Sie werden in normalisierter Form der jeweiligen Herkunftsregion angesetzt.

\subsection{Karten}

Um die Wortartikel von umfangreichen und unübersichtlichen Aufzählungen von Lautvarianten zu entlasten, sind in der ersten Lieferung des „Pommerschen Wörterbuchs“ 14 Lautkarten abgedruckt worden, die wichtige lautgeografische Realisierungen vorrangig im Bereich des Vokalismus für den gesamten Untersuchungsraum darstellen. Auch in den weiteren Lieferungen sind nach Möglichkeit Karten abgedruckt worden, die entweder der Laut-, der Formen- oder der Wortgeografie gewidmet sind. Bislang sind auf diese Weise im „Pommerschen Wörterbuch“ insgesamt 26 Lautkarten, fünf Formenkarten und 15 Wortkarten veröffentlicht worden. Diese Karten werden auf großformatigen Grundkarten per Hand von den Redakteurinnen und Redakteuren entworfen und anschließend von einem kartografischen Unternehmen am Computer bearbeitet und für den Druck vorbereitet. Beispiele für eine Wortkarte und eine Formenkarte sind den beiden folgenden Abbildungen zu entnehmen. 


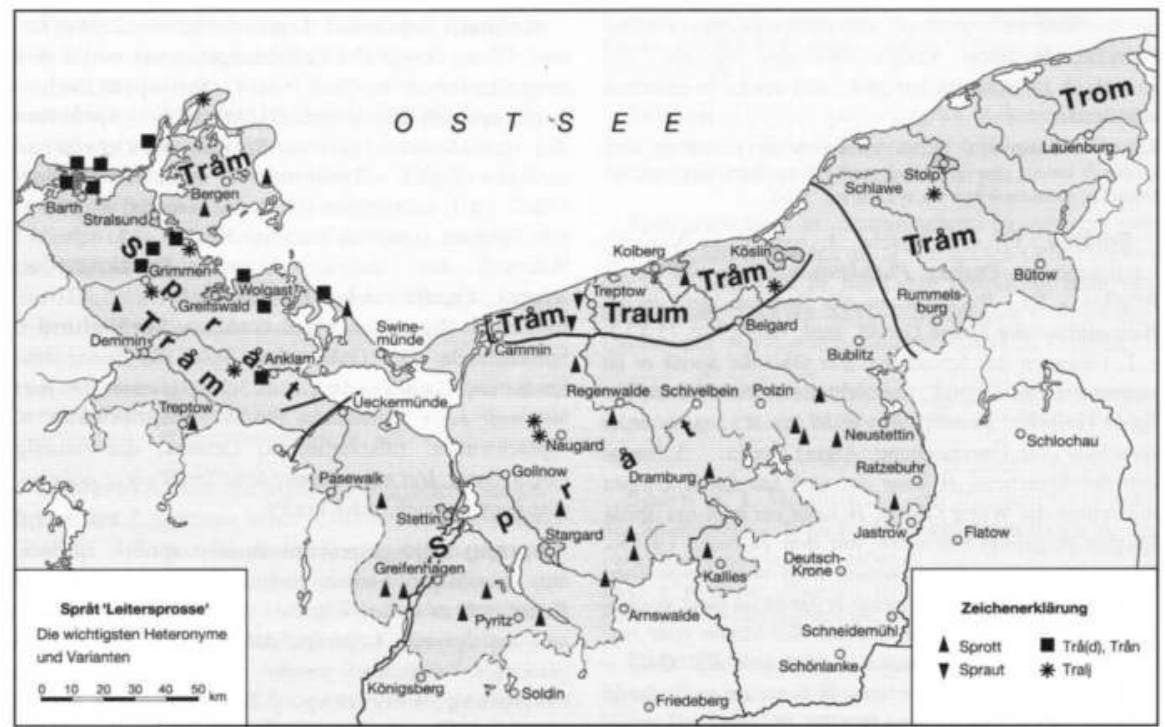

Abb. 5: Wortkarte zur Bedeutung 'Leitersprosse'

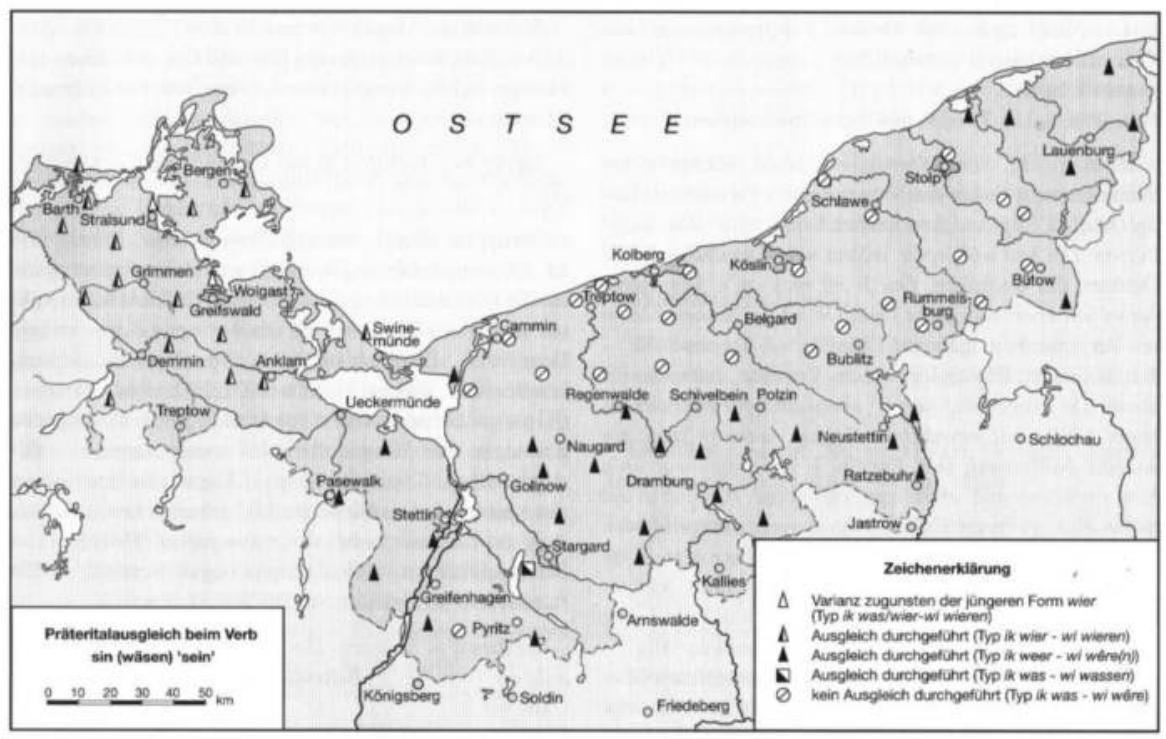

Abb. 6: Formenkarte zum Präteritalausgleich 


\section{TEXTTECHNOLOGISCHE ASPEKTE}

Seit der Neueinrichtung der Arbeitsstelle in Greifswald arbeiten die Redakteurinnen und Redakteure mit der Textverarbeitung Word unter Microsoft Office. Grundlage der lexikographischen Arbeit sind die lemmatisierten Belegzettel im Greifswalder Wörterbucharchiv, die nicht digitalisiert vorliegen. Die fertigen Typoskripte werden anschließend durch ein Satzunternehmen bearbeitet und für den Druck vorbereitet.

Feste Digitalisierungspläne gibt es zum jetzigen Zeitpunkt noch nicht. Es ist derzeit noch nicht entschieden, ob und wann nach Abschluss der Print-Publikation eine Veröffentlichung im Internet erfolgen kann. Entsprechende Absprachen sind zudem mit dem betreuenden Verlag zu treffen.

\section{LINGUISTISCHES POTENZIAL}

Die Belege im Wörterbucharchiv sind selbstverständlich auch außerhalb der Ausarbeitung von Wortartikeln für das großlandschaftliche niederdeutsche Dialektwörterbuch für sprachwissenschaftliche Zwecke verschiedener Art nutzbar und bereits genutzt worden. Diverse wissenschaftliche Beiträge konnten bislang von Daten des Archivs profitieren und wären ohne diese kaum denkbar gewesen. Dazu zählen vor allem WinTER (1967), HERRMANN-WinTER (2003), VOLLMER (2008), HANSEN (2009), HERRMANN-Winter (2013), VolLmer (2016), VolLMER (2017) und VOLLMER (2018).

Wertvolle Materialien stellt das „Pommersche Wörterbuch“ auch in Bezug auf namenkundliche Fragestellungen bereit. Besondere Relevanz für die onomastische Forschung haben dabei derzeit die im Archiv befindlichen Informationen zu pommerschen Flurnamen. Für das seit Mai 2016 von der Deutschen Forschungsgemeinschaft für die Dauer von vier Jahren geförderte Projekt mit dem Titel „Digitales vorpommersches Flurnamenbuch", das am Pommerschen Wörterbuch durchgeführt wird, sind die archivalischen Flurnamenbelege eine willkommene und wertvolle Ergänzung zu anderen gedruckten und ungedruckten Quellen. Als Beispiel dafür soll die folgende Abbildung dienen, die einen Archivzettel mit dem niederdeutschen Flurnamen Düwelsbrauk zeigt, der aus dem Ortspunkt Born auf dem Darß stammt und durch eine Nummernsigle auf einer Flurkarte exakt verortet werden kann. 


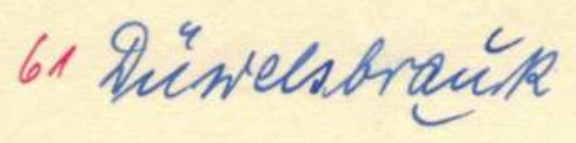

\section{Pe Dars}

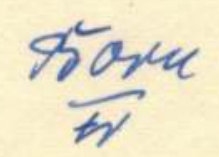

Abb. 7: Belegzettel zu einem niederdeutschen Flurnamen

Ein Beispiel für die Aussagekraft des Archivmaterials für Fragen der historischen Wortgeografie, die wesentlich von namenkundlichen Archivdaten profitiert, bietet die folgende Wortkarte. Sie zeigt die Verbreitung des Appellativs Tanger in der Bedeutung 'kleiner Nadelwald' in den pommerschen Dialekten in der ersten Hälfte des 20. Jahrhunderts. Der Geltungsbereich des Wortes, das über das Ostmitteldeutsche und die Mark Brandenburg nach Norden vermittelt worden ist, beschränkt sich zu dieser Zeit auf den mittelpommerschen und südpommerschen Raum. Dagegen offenbart der im Archiv des Wörterbuchs dokumentierte namenkundliche Befund, der sich auf das Vorkommen des Wortes in historischen Flurnamen bezieht, dass das Appellativum noch im 17. Jahrhundert eine deutlich größere räumliche Verbreitung gehabt haben muss, denn auch auf der Insel Usedom, die auf der Karte durch einen Kreis markiert ist, sind Flurnamen mit Tanger für diesen Zeitraum gut belegt. 


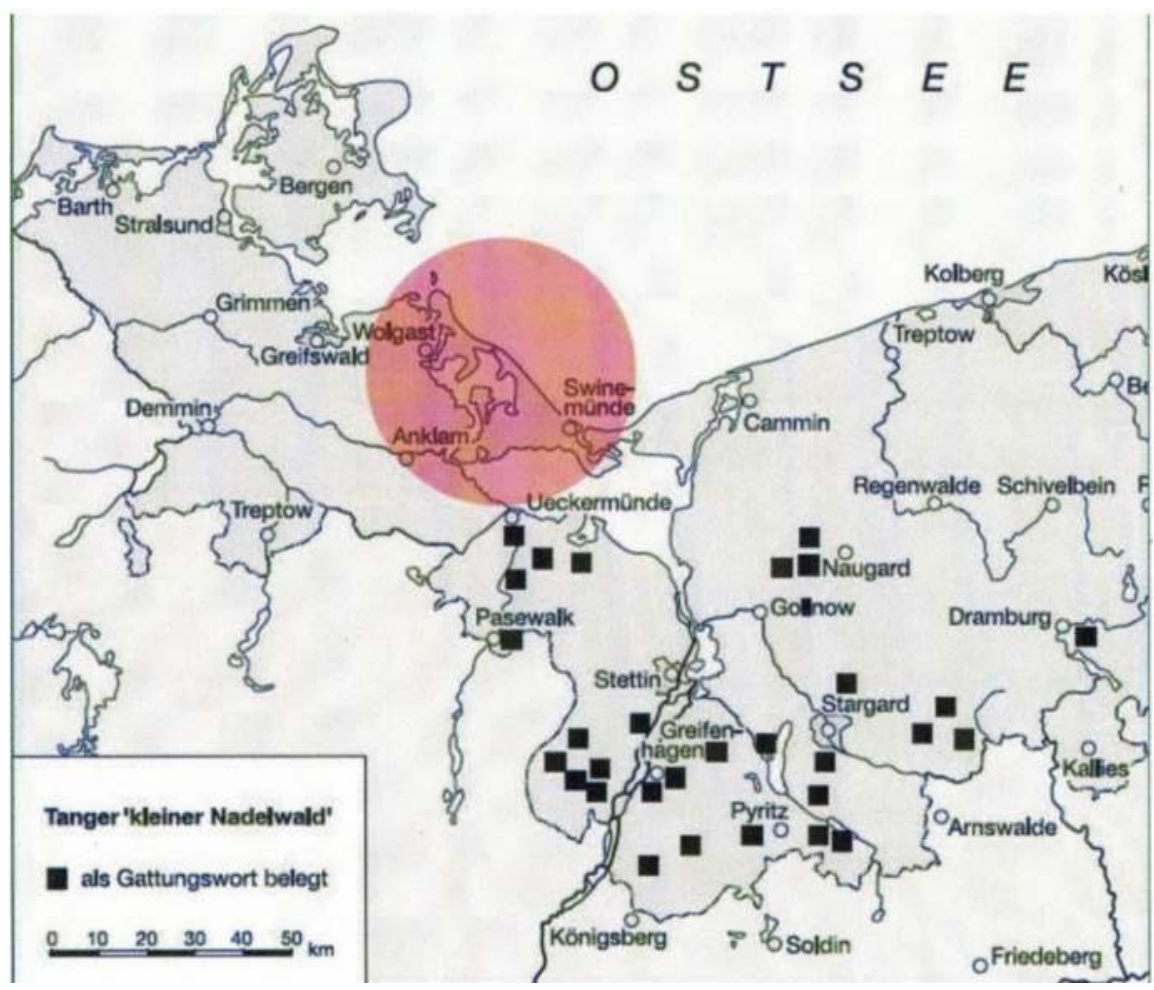

Abb. 8: Wortkarte zum Appellativ Tanger, 'kleiner Nadelwald'

Die Ergiebigkeit des Wörterbucharchivs für Fragen, die im weitesten Sinn mit Sprachwandelvorgängen zu tun haben, soll nachfolgend an einigen Beispielen verdeutlicht werden, bei denen metasprachliche Kommentare von Gewährspersonen auf den Archivzetteln vermerkt sind. Dazu gehören vorrangig Angaben der Informant/-innen, die sich auf das Nebeneinander, vor allem aber auf das Vordringen oder die Zurückdrängung von linguistischen Varianten beziehen, die keineswegs immer auf die lexikalische Ebene beschränkt sind. Deswegen sind auch Vermerke auf den Belegzetteln wie „,noch vereinzelt von den Alten im Gebrauch“ oder ,nur in der jüngeren Generation“" keine Seltenheit.

Thematisiert werden durch solche Kommentare verschiedentlich grammatisch relevante Phänomene. Im Bereich der Morphologie ist etwa das Schwanken zwischen älterer unregelmäßiger und jüngerer schwacher Konjugation zu nennen. So hat eine Gewährsperson von der Insel Rügen zur Beugung des Verbs trecken 'ziehen’ zuerst nur unregelmäßige Formen notiert, um dann zu ergänzen: „geht auch schwach."

Das Archiv des Wörterbuchs enthält auch Stellungnahmen von Gewährspersonen zu syntaktischen Aspekten, zu denen etwa die Frage der Stabilität der doppelten 
Verneinung in den pommerschen Mundarten zählt. Deren Relevanz im sprachlichen Alltag wird von einigen Informanten geradezu als entscheidendes und unverzichtbares dialektales Kennzeichen herausgestellt. Eine Gewährsperson hat dabei versucht, diese subjektive Spracheinstellung dadurch zu untermauern, dass sie in sehr kleiner Schrift eine sehr umfangreiche Liste von entsprechenden Sprachgebrauchsbeispielen auf die Vorder- und Rückseite eines Belegzettels geschrieben hat.

Schließlich sind dem archivalischen Material auch metasprachliche Aussagen zur soziologischen Schichtung dialektaler Varietäten in Pommern zu entnehmen. Dabei offenbart sich beispielsweise für die früheren sprachlichen Verhältnisse auf dem vorpommerschen Festland ein von verschiedenen Gewährspersonen reflektierter Unterschied zwischen einer prestigeträchtigen dialektalen Varietät der städtischen und stadtnahen Regionen und einer davon primär durch lautliche Differenzen abgehobenen Basismundart der ländlichen Gebiete mit deutlich geringerem Ansehen. Auf solche vertikalen Schichtungen des Varietätenspektrums in der Region haben übrigens schon im 18. Jahrhundert zeitgenössische Stimmen hingewiesen (vgl. VOLLMER 2016: 59-62). Das wichtigste Unterscheidungskriterium vor diesem Hintergrund ist eine lautliche Entwicklung in den vorpommerschen Dialekten, die sich auf bestimmte historische Langvokale bezieht. Konkret betroffen sind die mittelniederdeutschen Langvokale $\hat{e}^{2 b}$ (z. B. in Been, Bein 'Bein'), $\hat{e}^{4}$ (etwa in leef, leif 'lieb'), $\hat{o}^{l}$ (z. B. in Koken, Kauken 'Kuchen') und der Umlaut von $\hat{o}^{l}$ (z.B. in Föt, Fäut 'Füße'). Während diese Langvokale in den städtischen Prestigedialekten ihre monophthongische Struktur bewahrt haben, sind sie in den Basismundarten diphthongiert worden.

\section{KONTAKTADRESSE, HOMEPAGE}

Universität Greifswald

Pommersches Wörterbuch

PD Dr. Matthias Vollmer

Domstr. 14

17487 Greifswald

Deutschland

E-Mail: mvollmer@uni-greifswald.de

Homepage: <https://germanistik.uni-greifswald.de/forschung/einrichtungen/ pommersches-woerterbuch/> 


\section{LITERATURVERZEICHNIS}

BROSE, GERHARD (1955): Zur Dialektgeographie der pommersch-neumärkischen Grenzzone. In: Zeitschrift für Mundartforschung 23, 22-57.

HANSEN, MARTIN (2009, unveröffentlicht): Zum Wandel des Niederdeutschen auf der Insel Rügen zwischen dem 19. und 21. Jahrhundert. Masterarbeit an der Universität Greifswald.

HERRMANN-Winter, RENATE (1999): Das Pommersche Wörterbuch. Vorläufer - Ansätze - Konzepte. In: SCHMIDT, RODERICH (Hg.): Tausend Jahre pommersche Geschichte. Köln [u. a.]: Böhlau, 281-306.

HERRMANN-WINTER, RENATE (2003): Plattdeutsch-hochdeutsches Wörterbuch für den mecklenburgisch-vorpommerschen Sprachraum. 5. Auflage. Rostock: Hinstorff.

HERRMANN-WINTER, RENATE (2013): Sprachatlas für Rügen und die vorpommersche Küste. Rostock: Hinstorff.

JOST, FRANZ (1993): Hinterpommersches Wörterbuch der Mundart von Groß Garde (Kreis Stolp). Auf Grund der von FRANZ JOST (1887-1958) gesammelten Materialien bearbeitet und zu einem Wörterbuch gestaltet von HANS-FRIEDRICH ROSENFELD. Köln [u. a]: Böhlau.

KÜHL, KARL (1932): Die Saatzig-Dramburger Mundart. Ein Beitrag zur niederdeutschen Sprache in Ostpommern. Greifswald: Universitätsverlag L. Bamberg.

LAABS, KURT (1988): Belbucker Wörterbuch. Der Wortschatz der ehemaligen Abtei Belbuck und einiger Randgebiete. Murnau: Selbstverlag.

LAUde, RoBerT (1995): Hinterpommersches Wörterbuch des Persantegebietes. Hg. von DiETER STELLMACHER. Köln [u. a]: Böhlau.

MAHNKE, GEORG (1931): Die Schlawer Mundart. Sprachgeschichtliche und dialektgeographische Untersuchung. Greifswald: Universitätsverlag L. Bamberg.

MISCHKE, KURT (1936): Rummelsburger und Bütower Mundart. Greifswald: Universitätsverlag L. Bamberg.

NIEBAUM, HERMANN (1986): Lemma und Interpretament. Zur Problematik der Artikelgestaltung in Dialektwörterbüchern. In: FRIEBERTSHÄUSER, HANS (Hg.): Lexikographie der Dialekte. Beiträge zu Geschichte, Theorie und Praxis. Tübingen: Niemeyer, 125-143.

Pfaff, Hermann (1898): Die Vocale des mittelpommerschen Dialects. Labes: Buchdruckerei Straube.

PIRK, KURT (1928): Grammatik der Lauenburger Mundart. Ein Beitrag zur niederdeutschen Sprache in Ostpommern. Greifswald: Ratsbuchhandlung L. Bamberg.

PRIEWE, OTTO / HERMANN TEUCHERT (1927/1928): Dialektgeographische Forschungen östlich der unteren Oder. In: Teuthonista 4, 130-159, 221-262.

PWB $=$ Pommersches Wörterbuch. Begründet von WOLFGANG STAMMLER. Fortgeführt von HANSFRIEDRICH RosENFELD. Herausgegeben von RENATE HERRMANN-WinTER und MatTHIAS VOLLMER. Band $1(A-K)$. Berlin 2007: Akademie-Verlag.

StRITZEL, HERBERT (1937): Die Gliederung der Mundarten um Lauenburg in Pommern. Marburg: Elwert.

TITA, FRITZ (1965): Die Bublitzer Mundart. Für den Druck bearbeitet von ALFRED SCHÖNFELDT. Marburg: Elwert.

VOLLMER, MATTHIAS (2008): Zur Dialektlexikographie in Pommern. Kosegartens Wörterbuch der Niederdeutschen Sprache älterer und neuerer Zeit. In: Jahrbuch des Vereins für niederdeutsche Sprachforschung 131, 113-132.

VOLLMER, MATTHIAS (2016): Niederdeutsche Wortschatzsammlungen in Hinterpommern am Ende des 19. Jahrhunderts. In: Baltische Studien. Neue Folge 102, 199-208.

VOLLMER, MATTHIAS (2017): Anmerkungen zu den sprachlichen Verhältnissen Vorpommerns in der ersten Hälfte des 19. Jahrhunderts. In: ARENDT, BIRTE / ANDREAS BIEBERSTEDT / KLAAS-HINRICH EHLERS (Hg.): Niederdeutsch und regionale Umgangssprache in Mecklenburg-Vorpommern. Strukturelle, soziolinguistische und didaktische Aspekte. Frankfurt a. M.: Lang, 55-70. 
VOLLMER, MatThias (2018): Das pommersche Wörterbuch von Georg Gotthilf Jacob Homann (1774-1851). Eine Sammlung pommerisch-deutscher Wörter und Redensarten. Berlin: Verlag Peter Lang.

WARNKROSS, JULIUS (1912): Die Lautlehre des Wolgaster Platt. Greifswald: Abel.

WINTER, RENATE (1967): Suffixe der slawischen Lehnwörter im Pommerschen und ihr Einfluß auf die niederdeutsche Wortbildung. In: Jahrbuch des Vereins für niederdeutsche Sprachforschung $90,106-121$. 



\title{
KURZPRÄSENTATION DES SUDETENDEUTSCHEN WÖRTERBUCHS ${ }^{1}$
}

\author{
Isabelle Hardt
}

\section{DEFINITION, AUFGABE UND ZIEL}

\begin{abstract}
Als Arbeitsstelle des Collegium Carolinum in München ist das Sudetendeutsche Wörterbuch (SdWb) an der Justus-Liebig-Universität Gießen ein derzeit von Prof. Dr. OTFRID EHRISMANN herausgegebenes und von ISABELLE HARDT und BETTINA HOFMANN-KÄS bearbeitetes wissenschaftliches Dialektwörterbuch, das die verschiedenen deutschen Mundarten der geographischen Regionen Böhmen, Mähren und Sudetenschlesien synchron auf deren Sprachstand um 1945/46 dokumentiert. Damit bietet es nicht nur Linguist/-innen, Historiker/-innen und interessierten Laien einen guten Zugang zu den betreffenden Varietäten, sondern trägt auch dazu bei, dass diese als wichtiges Kulturgut der deutschen Geschichte in den böhmischen Ländern nicht in Vergessenheit geraten. Im November 2018 konnte der V. Band mit den Buchstaben $H, I$ und $J$ veröffentlicht werden; neue Einzellieferungen von 80 Seiten mit der jeweils an die Vorlieferung anschließenden Wortstrecke erscheinen im Abstand von ca. 9-12 Monaten.
\end{abstract}

\section{DOKUMENTATIONSGEGENSTAND 2}

Da die vom Sudetendeutschen Wörterbuch abgedeckten Gebiete keine einheitliche Sprachlandschaft bilden, gibt es nicht d e n sudetendeutschen Dialekt. Dies bedeutet, dass das Projekt vor der besonderen Aufgabe steht, ganze fünf verschiedene Grundmundarten nebst einiger Mischdialekte in einem Werk - meist sogar in einem Wortartikel - abzubilden. Zu diesen zählen:

1 Bei der in diesem Rahmen erfolgten Darstellung des Sudetendeutschen Wörterbuchs handelt es sich lediglich um eine Kurzpräsentation als Abriss einer ausführlichen Vorstellung des Projekts, die in einem eigenen Tagungsband zu der 2018 von dieser Arbeitsstelle selbst ausgerichteten LexikoNet 2 vorgenommen wird. Detaillierte Informationen und Erläuterungen zu Geschichte, Anliegen, Material(verarbeitung), Artikelstruktur und Potential dieses Wörterbuchs sind dem Beitrag „Das Sudetendeutsche Wörterbuch - Aufgaben, Ziele, Quellenbasis und Artikelgestaltung“ (Arbeitstitel) von ISABELLE HARDT zu entnehmen, der in dem zu dieser Tagung herausgegebenen Band mit dem Titel „Das Sudetendeutsche Wörterbuch - Bilanzen und Perspektiven. Beiträge zum Abschluss des V. Bandes“ enthalten ist (erscheint voraussichtlich 2020).

2 Vgl. hierzu auch ENGELS / ENGLISCH (1988: V-VI). 
- das Mittelbairische in Südmähren, im unteren und mittleren Böhmerwald, im Schönhengst sowie im Bereich der Budweiser, der Wischauer, der Brünner und der Olmützer Sprachinsel;

- das Nordbairische oder Oberpfälzische in Westböhmen und im Bereich der Iglauer Sprachinsel (hier auch - je nach Gebiet - im Mischdialekt mit dem Obersächsischen);

- das Ostfränkische von Nordwestböhmen über das Erzgebirge, auch mit Einflüssen auf das mittlere Nordböhmen und den Schönhengst;

- das Obersächsische in Nordböhmen sowie im Mischdialekt mit dem Nordbairischen im Bereich der Iglauer Sprachinsel;

- das Schlesische in Ostböhmen und Nordmähren.

Daneben finden auch Pflanzennamen, Austriazismen und der deutsch-slawische Lehnwortaustausch sowie bestimmte Soziolekte und Fachsprachen (u. a. die Imkerund die Jägersprache) Eingang in das Wörterbuch. Alle in das Werk aufgenommenen Lemmata werden in ihrer gemäß dem „Duden“33 verneuhochdeutschten Form streng alphabetisch angeordnet.

\section{DIE URSPRÜNGE DES WÖRTERBUCHS ${ }^{4}$}

Ein erstes Vorhaben, die sudetendeutschen Dialekte in einem Mundartenwörterbuch systematisch zu erfassen, war, angeregt durch den Leipziger Germanisten THEODOR FRINGS, bereits im Jahr 1930 an der deutschen Karlsuniversität in Prag unter der Leitung der beiden Germanisten ERICH GIERACH und ERNST SCHWARZ vorangetrieben worden. Die hierfür unter der Mitarbeit von FRANZ JOSEF BERANEK bis zum Zweiten Weltkrieg erstellte Sammlung von ca. 1,2 Mio. Belegzetteln und 800 Verbreitungskarten verschwand jedoch in den Wirren des Krieges und gilt seither als verschollen.

Von neuem offiziell begonnen werden konnte das Projekt als „Sudetendeutsches Wörterbuch“ ab 1957, nachdem FrANZ JOSEF BERANEK im Rahmen einer Dozentur für Jiddistik an die Justus-Liebig-Universität nach Gießen gekommen war. Die abermals notwendigen Vorarbeiten zu diesem Projekt leistete er zunächst von zu Hause aus, bis ihm 1959 seitens der Universität kostenlos Räume für dieses Vorhaben zur Verfügung gestellt wurden. Im Zuge dieser Vorbereitungen ließ FRANZ JOSEF BERANEK einige Aufrufe in den sudetendeutschen Heimatzeitschriften ergehen, über die sowohl Mitarbeiter als auch v. a. Gewährspersonen rekrutiert werden sollten, die die neu entworfenen Fragelisten beantworten mochten. So sind es in der Hauptsache diese ca. 650 Gewährsleute, denen das Projekt die Basis seines im Folgenden kurz umrissenen, umfangreichen Materialbestands zu verdanken hat.

3 Für das Wörterbuch maßgeblich ist die Ausgabe DROSDOWSKI (Hg.) (1976-1981).

4 Vgl. hierzu auch ENGELS / ENGLISCH (1988: VI-VII) und ENGELS (1983: 349-352). 


\section{VOM SUDETENDEUTSCHEN WÖRTERBUCH ERFASSTE MATERIALIEN UND QUELLEN ${ }^{5}$}

Eine der unmittelbarsten und somit wichtigsten Quellen für das Sudetendeutsche Wörterbuch stellen die unter der Leitung von FRANZ JOSEF BERANEK entworfenen und von ca. 650 Gewährspersonen ausgefüllten 100 Fragelistenkategorien mit ihren je 4060 Einzelfragen dar. Ergänzungsfragelisten zu 20 essentiellen Themen, die auf diese Weise vertieft worden sind, bereichern diesen Datenfundus genauso wie die Sonderfragelisten zu über 150 verschiedenen Sachthemen (darunter auch berufsgruppenbezogene Listen und solche zu Brauchtum und Volksglaube) und die unzähligen freien Einsendungen derjenigen Gewährsleute, die zu verschiedenen Abläufen, Gegebenheiten und Geräten des täglichen Lebens individuelle und damit nicht selten anrührend persönliche Texte verfasst haben. In das Werk einbezogen werden auch Stichwörter aus der „Bayerischen Wortliste ${ }^{66}$ und der ,Schlesischen Frageliste ${ }^{67}$ sowie solche aus Heimat- und Stadtchroniken, aus Heimatbüchern und Heimatzeitschriften sowie aus wissenschaftlichen Publikationen (z. B. Mundartmonographien) zu den vom „Sudetendeutschen Wörterbuch“ abgedeckten Gebieten. Aus den Namensbeständen des Archivs (u. a. Orts-, Flur- und Personennamen) werden lediglich männliche und weibliche Rufnamen aufgenommen, da diese für zahlreiche Bräuche, Redensarten und Wetterregeln von Belang sind, die u. a. ebenfalls in die einzelnen Artikel eingearbeitet werden. Die aus den genannten Quellen exzerpierten Stichwörter, Wendungen und Redensarten liegen in Form von ca. 2,6 Millionen alphabetisch geordneten Zetteln im Hauptkatalog der Redaktion vor, der die Basis zur Erstellung der Wortartikel bildet. Eine Aufstellung der bei der redaktionellen Arbeit herangezogenen Literatur sowie der im Wörterbuch erscheinenden Belegorte wurde zuletzt 2008 in einem „,Vorläufigen Belegorte- und Literaturverzeichnis “8 veröffentlicht. Sowohl dieses Verzeichnis als auch alle bisher erschienenen Lieferungen und Bände des Wörterbuchs sowie sämtliche seiner Quellen liegen bisher ausschließlich in Papierform vor. Eine Digitalisierung dieses Materials (oder einiger wesentlicher Teile daraus) wird erwogen, ist allerdings zum jetzigen Zeitpunkt noch nicht konkret in Planung.

5 Vgl. hierzu auch ENGELS / ENGLISCH (1988: VII).

6 Die Dokumente der Bayerischen Wortliste wurden von ca. 20 Gewährspersonen desjenigen bairischsprachigen Raums ausgefüllt, der die auch für das „Sudetendeutsche Wörterbuch“ relevanten Gebiete betrifft. Sie wurden der Redaktion von den Kollegen des „Bayerischen Wörterbuchs" in München zur Verfügung gestellt.

7 Die Dokumente der Schlesischen Frageliste zur Abdeckung des vom „Sudetendeutschen Wörterbuch" erfassten mährisch-schlesischen Raums wurden der Gießener Arbeitsstelle von den Kollegen des „Schlesischen Wörterbuchs“ in Marburg überlassen.

Vgl. Collegium Carolinum (Hg.) (2008). 


\section{KONTAKTADRESSE, HOMEPAGE}

JLU Gießen Zeughaus

Senckenbergstraße 3

35390 Gießen

Deutschland

E-Mail: sdwb.cc@collegium-carolinum.de

Homepage: <http://www.collegium-carolinum.de/institut/sammlungen-sdwb/ sudetendeutsches-woerterbuch.html>

\section{LITERATURVERZEICHNIS}

Collegium Carolinum (Hg.) (2008): Sudetendeutsches Wörterbuch. Wörterbuch der deutschen Mundarten in Böhmen und Mähren-Schlesien: Vorläufiges Belegorte- und Literaturverzeichnis. München: Collegium Carolinum (Selbstverlag).

Duden = DROSDOWSKI, GÜNTHER (Hg.) (1976-1981): Duden. Das große Wörterbuch der deutschen Sprache in 6 Bänden. Mannheim [u. a.]: Bibliographisches Institut.

Engels, HeINZ (1983): Aufbau und Anliegen des Sudetendeutschen Wörterbuchs. In: Bohemia 24/2, 348-357.

ENGELS, HeINZ / NORBERT ENGLISCH (1988): Vorwort zum ersten Band. In: ENGELs, HeINZ (Hg.): Sudetendeutsches Wörterbuch. Bd. I: A. München: Oldenbourg, V-VIII.

HARDT, ISABELLE (erscheint 2020): Das Sudetendeutsche Wörterbuch - Aufgaben, Ziele, Quellenbasis und Artikelestaltung. In: EHRISMANN, OTFRID / ISABELLE HARDT (Hg.): Das Sudetendeutsche Wörterbuch - Bilanzen und Perspektiven. Beiträge zum Abschluss des V. Bandes. Berlin: 2020 (DigiOst).

Sd. WB. 1 = ENGELS, HeINZ (Hg.) (1988): Sudetendeutsches Wörterbuch. Bd. I: A. München: Oldenbourg.

Sd. WB. 2 = EHRISMANN, OTFRID / HEINZ ENGELS (Hg.) (1996): Sudetendeutsches Wörterbuch. Bd. II: B/P. München: Oldenbourg.

Sd. WB. 3 = EHRISMANN, OTFRID (Hg.) (2002): Sudetendeutsches Wörterbuch. Bd. III: C, D/T, E. München: Oldenbourg.

Sd. WB. 4 = EHRISMANN, OTFRID (Hg.) (2010): Sudetendeutsches Wörterbuch. Bd. IV: F/V, G. München: Oldenbourg.

Sd. WB. 5 = EHRISMANN, OTFRID (Hg.) (2018): Sudetendeutsches Wörterbuch. Bd. V. H, I, J. München: Collegium Carolinum. 


\title{
WÖRTERBUCH DER UNGARNDEUTSCHEN MUNDARTEN (WUM)
}

\author{
Elisabeth Knipf-Komlósi / Márta Müller
}

\section{GESCHICHTLICHES UND RAHMENBEDINGUNGEN ZUM WÖRTERBUCH UND ZUR ARBEITSSTELLE}

Die sprachlichen Entwicklungen bei Sprachinselmundarten zeigen ein vielfältiges, auf jeden Fall sehr komplexes Bild, das zahlreiche Facetten der Sprachgebrauchsweise einer Sprachinselminderheit aufweisen kann (z. B. Spracherosionen, Sprachkontaktphänomene wie Sprachwechselerscheinungen, Codeswitching- und Codemixingformen, Mischformen, typische und atypische Transferenzen etc.). Befindet sich die Sprachinselminderheit im Abbau, ist es aus mehrfacher Sicht geboten und zu erwarten, dass der Wortschatz dieser Minderheit aus dialektologischen, sprachhistorischen, kontaktologischen und sprachkulturellen Aspekten erfasst und festgehalten wird.

Die Sprachinselforschung (vgl. WIESINGER 1980: 491) der ungarndeutschen Minderheit (vgl. HUTTERER 1991: 178) blickt insbesondere seit der zweiten Hälfte des 20. Jahrhunderts auf eine längere Geschichte und auf mehrere Herangehensweisen von Untersuchungen zurück, doch bislang ist kein wissenschaftliches dezidiert lexikographisches Projekt zur Dokumentation und Erfassung des Wortschatzes dieser Minderheit entstanden.

Die Wichtigkeit der Kodifizierung des Wortschatzes ungarndeutscher Sprecher führte zuerst TAFFERNER 1941 in den wissenschaftlichen Diskurs ein. Auf diesen Gedanken griffen MOLLAY (1986: 111) und HUTTERER (1991: 188) in den 1960erJahren zurück, indem beide die Erstellung der klassischen Datensammlungen - einen Sprachatlas, ${ }^{1}$ ein Wörterbuch und ein Tonarchiv - für die ungarndeutschen Mundarten forderten. HUTTERER stellte sich das „Ungarndeutsche Wörterbuch“ dreibändig vor: Die einzelnen Bände hätten die Wortschätze der drei Siedlungsräume präsentiert (vgl. KNIPF-KOMLÓSI / ERB / MÜLLER 2012: 22).

Aufgrund der schwindenden Dialektkompetenzen der ungarndeutschen Sprecherinnen und Sprecher wurde die Dringlichkeit der Erstellung eines Wörterbuchs immer größer, sodass die Vorbereitungsarbeiten des „Wörterbuchs der Ungarndeutschen Mundarten“ (WUM) mit einer einmaligen Unterstützung des Ungarischen 
Wissenschaftlichen Fonds zwischen 2010-2015 beginnen konnten. ${ }^{2}$ Das Wörterbuch setzt sich zum Ziel, den Wortschatz jener mittel- und oberdeutschen (Siedlungs-)Mundarten zu dokumentieren, die auf dem Gebiet des heutigen Ungarn gesprochen werden. ${ }^{3}$

Das ungarndeutsche Wörterbuch ist primär ein synchrones, diatopisches, dokumentationslexikographisches Bedeutungswörterbuch, dessen Korpus vornehmlich auf einem bäuerlich-handwerklichen Wortschatz mit Sprachinselcharakter fußt. Darüber hinaus hat es einen sekundären sachlexikographischen Schwerpunkt (zu den Anforderungen und Überlappungen von Sprach- und Sachlexikographie vgl. NIEBAUM 1986: 128; WIEGAND 1998: 47-49; SPREE 2012: 549).

Die Arbeitsstelle des „Wörterbuchs der Ungarndeutschen Mundarten“ ist am Germanistischen Institut der Eötvös-Loránd-Universität angesiedelt. An der Entwicklung des Konzeptes, an der Datensammlung und -verarbeitung sowie an der Erstellung von Wörterbucheinträgen wirk(t)e außer den Kollegen in Budapest (Eötvös-Loránd-Universität) auch eine Kollegin von der Germanistik der Universität Pécs mit. ${ }^{4}$

Als Gegenstand unseres Wörterbuches betrachten wir den überwiegend im 20. Jahrhundert und in der Gegenwart gesprochenen Wortschatz deutscher Provenienz dieser Minderheit. ${ }^{5}$ Somit sehen wir unsere erstrangige Aufgabe darin, dass wir eine reichhaltige Materialbasis aufbauen.

Die historischen Wurzeln der heute in Ungarn lebenden deutschstämmigen Bevölkerung gehen auf das ausgehende 17. bzw. auf das 18. Jahrhundert zurück, als teils aus wirtschaftlich-sozialen, teils aus religiösen Gründen deutsche Siedlerinnen und Siedler überwiegend aus Mittel-und Süddeutschland in drei Wellen ins Karpatenbecken, auf das Gebiet des ehemals osmanischen Ungarn zogen. Sie ließen sich in Transdanubien (Ungarisches Mittelgebirge, Umgebung der Städte Pesth-Ofen [heute Budapest]), in einigen Teilen der Ungarischen Tiefebene (Saboltsch, Bekesch, welche heute bereits weitgehend madjarisiert sind), in den Regionen des Nördlichen Mittelgebirges (Hewesch, Zemplin) und auf Gebieten südlich des Plattensees (Branau, Tolnau, Schomodei, Batschka, Banat) nieder (vgl. SEEWANN

2 Ungarisch Országos Tudományos Kutatási Alapprogramok (OTKA), Projektnummer 81342, unter der Leitung von ElISABETH KNIPF-KoMLÓSI. Seit 2015 werden die lexikographischen Arbeiten individuell von drei Kolleginnen durchgeführt.

3 Zwischen 2010-2015 wurden Konsultationen mit namhaften, traditionsreichen Wörterbuchkanzleien durchgeführt. An dieser Stelle wollen wir uns bei den Mitarbeiterinnen und Mitarbeitern für ihre Hilfsbereitschaft (Ratschläge, Erläuterungen, Literaturhinweise) bedanken, die sie dem WUM-Projekt entgegengebracht haben, namentlich bei EDITH BURKHART-FUNK, HEINRICH J. DINGELDEIN, INGEBORG GEYER, MANFRED GLAUNINGER, SIGRID HALDENWANG, AlEXANDRA N. LENZ, ANTHONY ROWLEY, ANDREA SCHAMBERGER-HIRT, CHRISTINA SCHRÖDL, PhILIPP STÖCKLE, EVELINE WANDL-VoGT sowie den Mitgliedern des LexikoNetNetzwerkes.

4 In alphabetischer Reihenfolge: MARIA ERB, REgInA HESSKY, ELISABETH KNIPF-KomlósI, KARL MANHERZ, Éva MÁrKUS, MARTA MÜLler, KATHARINA WILD.

5 Selbstverständlich kann und muss z. B. bei etymologischen oder volkskundlichen Kommentaren auch auf ältere Belege Bezug genommen werden. 
2012a und 2012b; FATA / DroBAC 2013; KRAUSs 2014). Als Ergebnis der zwangsmäßig ablaufenden sprachlichen wie kulturellen Ausgleichsprozesse innerhalb der jeweiligen Siedlungen bzw. Regionen ${ }^{6}$ entstanden ostdonaubairische, bairisch-fränkische, fränkische, hessische, pfälzische, fuldische, ostfränkische und schwäbische Mischmundarten, deren synchron erhebbare lexikalische Einheiten auch in das WUM Eingang finden.

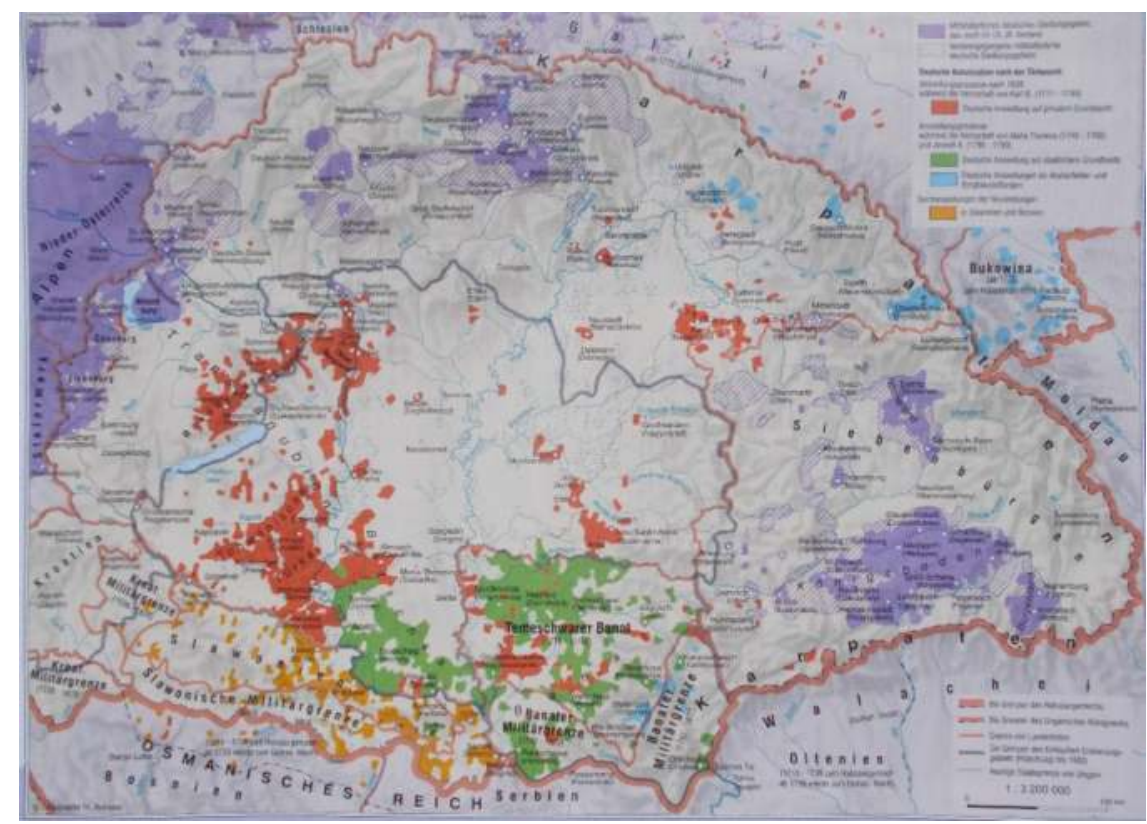

Abb. 1: Deutsche Siedlungsgebiete im 18. Jahrhundert in Ungarn (SchA 2016: 149)

Genealogisch nicht zu den nachtürkischen Siedlungsmundarten gehören das Ostdonaubairische (Ostmittelbairische) in Nordwestungarn (Wieselburger Heide/Mosoni-síkság, Ödenburg/Sopron und Umgebung) und das Ostdonaubairisch-Steirisch-Südbairische im Raab-Lafnitztal/Rába-Lapincs-völgy. Aufgrund der frühen Ansiedlung (12. Jahrhundert) stellt die im Nördlichen Ungarischen Mittelgebirge befindliche Mundart der Ortschaft Deutschpilsen/Nagybörzsöny zu den nachtürkischen Mischmundarten eine Ausnahme dar. Sie zeigt eine besondere, nicht leicht verständliche Mischung aus bairischen und mitteldeutschen Elementen sowie Entlehnungen aus den benachbarten Sprachen (Ungarisch, Slowakisch) (vgl. MÁRKUS 2014). Beide kleineren Mundartinseln - die der Voralpendeutschen an der österreichisch-ungarischen Grenze als auch die der Deutschpilsener - werden in die Korpusbildung des WUM einbezogen. 


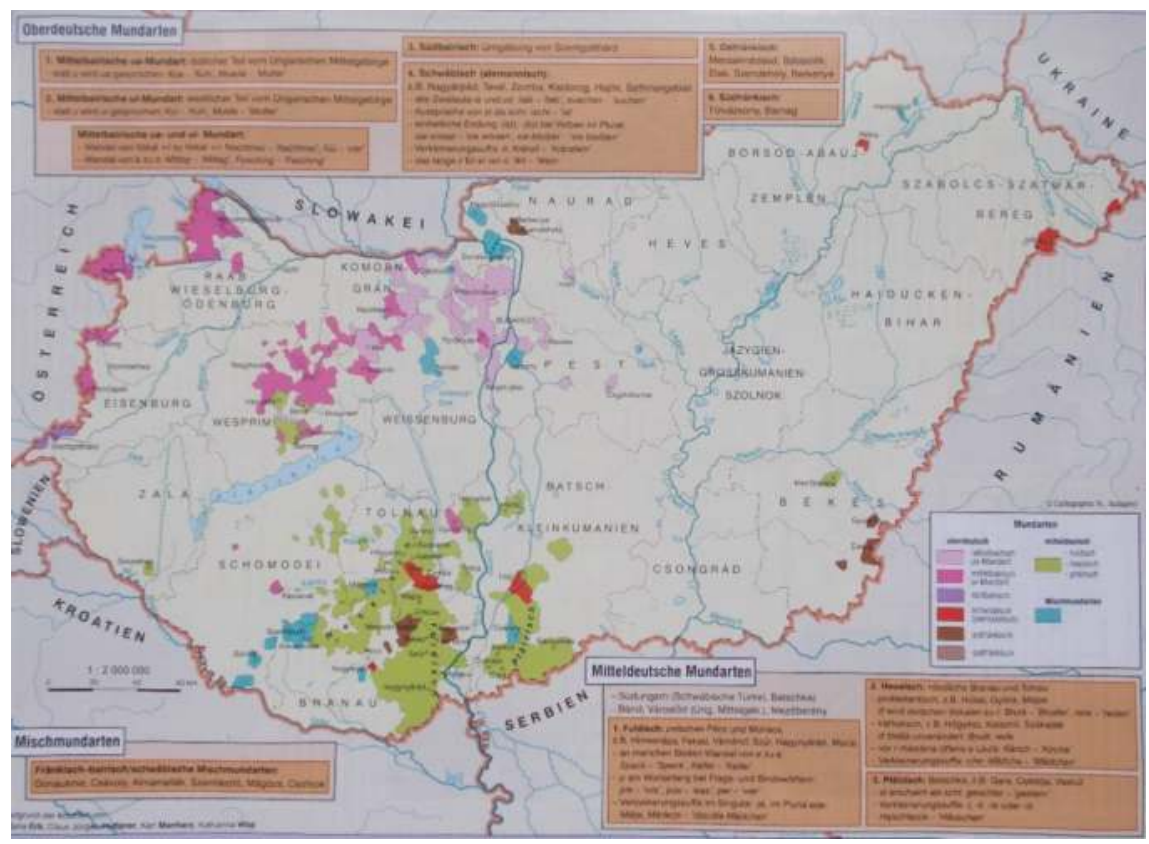

Abb. 2: Deutsche Mundarten in der Gegenwart in Ungarn (SchA 2016: 151)

Das Bearbeitungsgebiet und der Geltungsbereich des WUM wird dementsprechend nicht durch sprachliche Grenzen umschlossen - wie z. B. die $i k$-ich-Linie im Falle des THWB (vgl. NIEBAUM / MACHA 1999: 110) -, sondern durch politische Grenzen der Gegenwart.

Die deutsche Sprachinselminderheit in Ungarn erwies sich über einen längeren als in der Fachliteratur beschriebenen Zeitraum hinweg als vital (vgl. PARRILLO 1994: 526-527; HAMERS / BlANC 2003: 176): Die Abbauprozesse im Dialektgebrauch der deutschsprachigen Bevölkerung haben sich - trotz der in Ungarn eigentlich seit dem 19. Jahrhundert spürbaren Tendenz und des Druckes zur Assimilierung und Madjarisierung der Deutschen - erst nach dem Zweiten Weltkrieg v. a. durch die gravierenden historischen Umwälzungen, ferner durch die Industrialisierung, Urbanisierung und die damit verbundene gesellschaftlich-geografische Mobilität beschleunigt. Diese Veränderungen zeigen sich auch in den aktuellen Sprachpräferenzen der Ungarndeutschen (vgl. KNIPF-KoMLÓSI 2011: 45-53; 2012: 61-74; KNIPF-KOMLÓSI / MÜLLER 2019: 533-540).

Zum potentiellen Benutzerkreis des WUM gehören über die Gruppe der Expertinnen und Experten hinaus (Dialektologinnen und Dialektologen, Kontaktlinguistinnen und -linguisten, Historikerinnen und Historiker, Volkskundlerinnen und Volkskundler, Kulturhistorikerinnen und Kulturhistoriker) in erster Linie jene Laienbenutzerinnen und -benutzer (Erwachsene, Schülerinnen und Schüler), die 
ungarndeutsche Mundarten aktiv oder passiv beherrschen, ferner nichtmundartkundige Benutzerinnen und Benutzer, die sich entweder in der dialektalen Vielfalt der deutschen Sprache kundig machen wollen oder sich für kulturhistorische Kommentare interessieren.

Folgendes Schema stellt die möglichen Zielgruppen der Ungarndeutschen in der Gegenwart dar sowie den Nutzen eines Wörterbuchs bezogen auf ihre Generationen:

\section{Generations- und Sprachkontinuum}
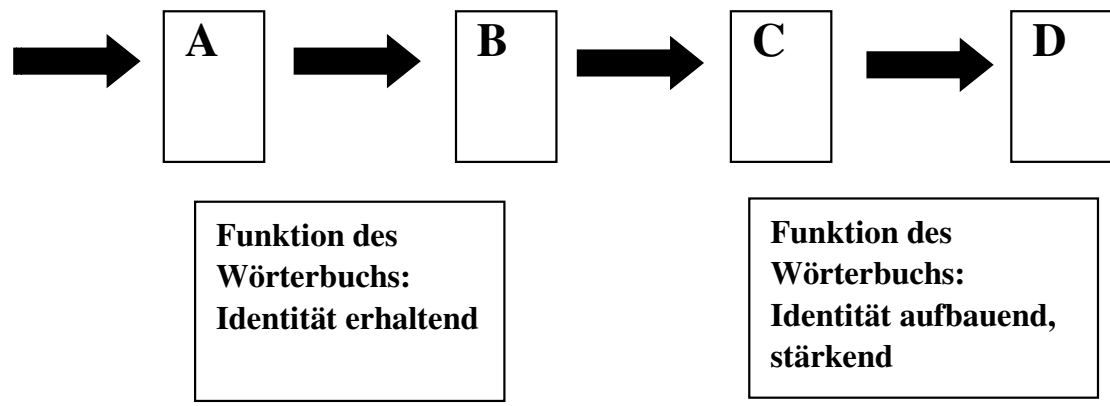

Abb. 3: Sprecherbezogene Funktionen des WUM nach KNIPF-KoMLóSI (2011: 45-53)

Die Erstellung eines Sprachinselwörterbuchs würde bei den noch autochthonen Sprecherinnen und Sprechern (Generationen A und B, geboren spätestens in den 1950er Jahren) zur Stärkung der Dialektkenntnisse und der Identität, und bei jenen Generationen, die die Mundart passiv oder rudimentär verstehen (Generationen C und D, geboren ab den 1960er Jahren), zum Aufbau der sprachlichen Vielfalt und zur Stärkung der Identität beitragen.

Das Belegmaterial der WUM-Datenbank lässt sich den Sachbereichen Volksnahrung, Sitten und Bräuche, Geburt/Taufe, Eheschließung/Hochzeit, Tod/Bestattung, Verwandtschafts- und Personenbezeichnungen, Flora und Fauna sowie Gegenstände des Alltags zuordnen (vgl. Abbildung 4): 


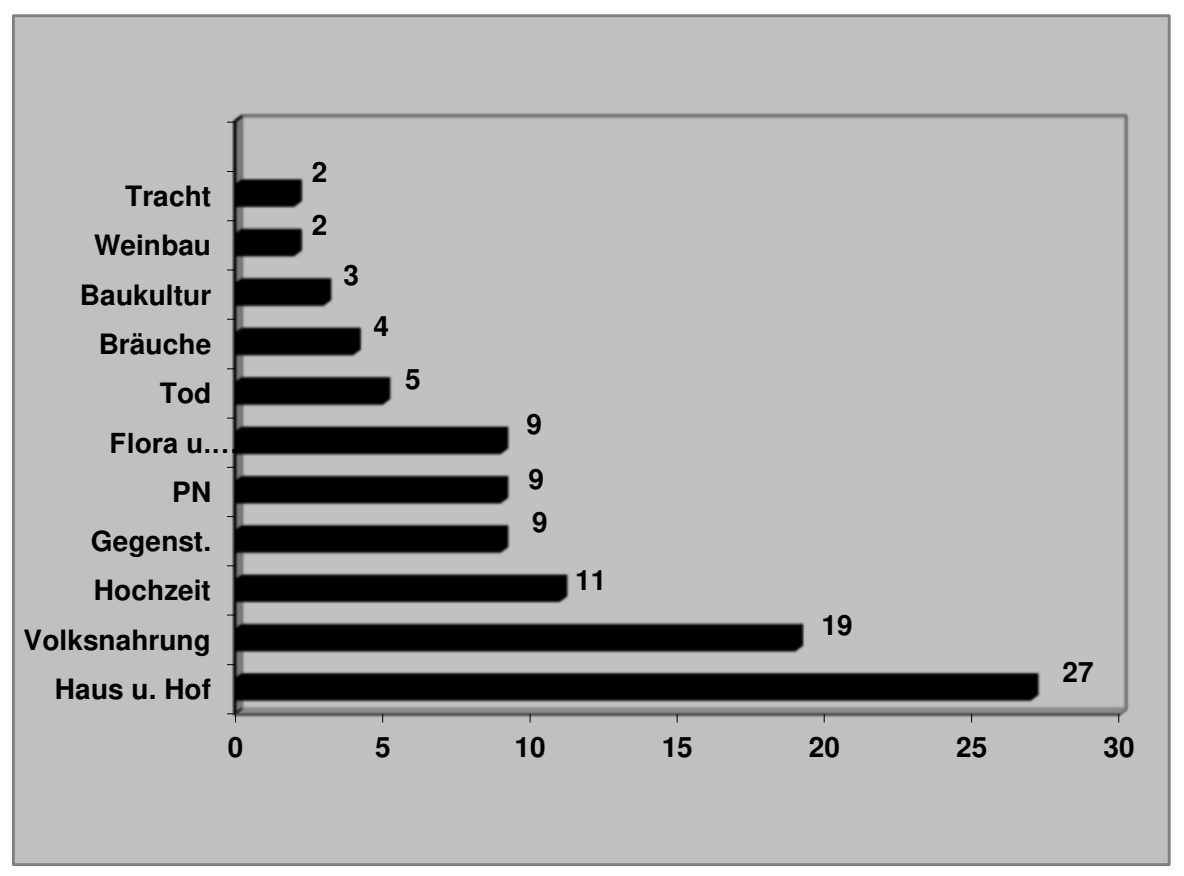

Abb. 4: Exzerpierte Quellen nach Sachbereichen (Angaben in Prozent) in der WUM-Datenbank (Stand: 05.08.2018)

Einen beträchtlichen Teil des Belegmaterials bilden - wie auch in den großlandschaftlichen Dialektwörterbüchern des Deutschen - Simplizia, Komposita und derivierte Wortformen, darüber hinaus attributive Konstruktionen, Kollokationen, seltener Redewendungen, Sprichwörter, Sprüche, Bauernregeln und kurze (Kinder-) Reime.

\section{KONZEPTION, STRUKTUR UND INHALT DER WÖRTERBUCHARTIKEL}

\subsection{Lemmatypen}

Da das WUM den gegenwärtigen von ungarndeutschen Sprecherinnen und Sprechern noch gebrauchten Wortschatz von alphabetisch aufgelisteten standarddeutschen Stichwörtern ausgehend dokumentiert und kommentiert, kann bei der Zusammenstellung der endgültigen Lemmaliste des WUM auf die Stichwörter der deutschen und österreichisch-deutschen einsprachigen Großwörterbücher rekurriert werden. Obwohl die Sammeltätigkeit der Lemmata und die Erstellung der Datenbank der Lemmaliste noch nicht als abgeschlossen betrachtet werden kann, zeichnet 
sich aufgrund bisheriger Forschungen ab, dass das zu lemmatisierende Sprachmaterial vier Lemmatypen zugeordnet werden kann (vgl. KNIPF-KOMLÓSI / MÜLLER 2017: 147-150):

- 1. Lemmatyp: standarddeutsches Lemma - dialektale Entsprechung (= formale Dialektwörter);

- 2. Lemmatyp: standarddeutsches Lemma - dialektale Entsprechung mit Bedeutungsunterschied in bestimmten Basisdialekten;

- 3. Lemmatyp: Dialektwörter im engeren Sinne (= echte Dialektwörter);

- 4. Lemmatyp: Kontaktphänomene

- a. Bezeichnungsentlehnungen,

- b. Lehnprägungen und -übersetzungen;

- c. Hybride.

Zum ersten Lemmatyp gehören über die formalen Dialektwörter hinaus die dialektalen Komposita, bei denen bestimmte Konstituenten (meistens die Grundwörter) mit den dementsprechenden Konstituenten in ihren standarddeutschen Äquivalenten übereinstimmen, andere Konstituenten (meistens die Bestimmungswörter) mit den entsprechenden Konstituenten in ihren standarddeutschen Äquivalenten aber nicht wie Altfator (fränkisch) vs. Groß $\underline{\text { vater }}$ (standarddeutsch). ${ }^{7}$ Auch jene Mundartwörter werden dem ersten Lemmatyp zugeordnet, die eine grammatische Varianz z. B. im Genus, Numerus oder in der Flexion aufzeigen wie der Butter (pfälzisch) oder der/die Krumbire (pfälzisch, unter „Grundbirne“), oder das Bett mit der dialektalen Mehrzahlform Better (pfälzisch).

Dem zweiten Lemmatyp werden jene Dialektwörter zugeordnet, die in einigen (oder einzelnen) Ortsmundarten in ihren Bedeutungen von den standarddeutschen Entsprechungen abweichen wie in den südungarischen (pfälzisch-hessisch-fränkischen) Ortsmundarten z. B. Fledermaus, welches einerseits 'Fledermaus', andererseits in manchen Ortschaften auch 'Schmetterling' bedeutet.

Als dritter Lemmatyp werden die echten Dialektwörter betrachtet, d. h. jene lexikalischen Einheiten, die in einsprachigen Wörterbüchern der deutschen Gegenwartssprache nicht (mehr) verzeichnet sind oder durch spezifische (z. B. „,mitteld.“), gegebenenfalls unspezifische (z. B. „landschaftl.“, „regional“, „mundartl.“) Markierungstechniken diatopisch gekennzeichnet sind (NIEBAUM 1989: 662-668). Manche der Belege, die wir als echte Dialektwörter erachten, sind Einzelmeldungen, die aus Kleinregionen oder aus einzelnen Ortsmundarten stammen. Nicht selten hängt dieser Lemmatyp mit den Spendersachbereichen Flora und Fauna zusammen wie z. B. bei Pounehipser 'Heuschrecke/r' oder Hääpian 'zur Zeit der ersten Heuernte (meist Ende Juni, Anfang Juli) reifende, kleinförmige Frühbirnesorte' (beide bairisch). Ist dies nicht der Fall, so bezeichnen die echten Dialektwörter oft sach- oder kulturhistorische Denotate, die für ungarndeutsche Verhältnisse charakteristisch waren wie z. B. Hanikel 'Kalvinist' oder aafremme 'Anzug, Kleid nach $\mathrm{Maß}$ anfertigen lassen' (beide pfälzisch).

7 Das Lemma im Falle von Altfatər wird als „Altvater“ angesetzt, doch wird es wegen des auch im Standarddeutschen existierenden Grundwortes zum ersten Lemmatyp gezählt. 
Dem vierten Lemmatypus werden die usualisierten, lexikalisierten Übernahmen wie z. B. Punda 'Pelzmantel' (bairisch < ungarisch bunda 'Pelzmantel') und die hybriden Bildungen wie z. B. das Holzvályú 'Holztrog' (bairisch $<\mathrm{Holz}+$ ungarisch vályú 'Trog') zugeordnet.

\subsection{Auswahl der Lemmata}

Bei der Auswahl der Lemmata wird stets danach getrachtet, dass im WUM neben den (vor allem in Südungarn recht) unterschiedlichen Dialektarealen auch - sofern dies möglich ist - den unterschiedliche Wortschatzschichten Rechnung getragen werden soll. Die genealogische Vielfalt impliziert v. a. eine angemessene Kennzeichnung der Heteronyme in den Artikeln. Die Einrichtung von Zentralartikeln (vgl. NIEBAUM / MACHA 1999: 111) für den Überblick aller Heteronyme desselben Stichwortes hat sich auch für unser Wörterbuch bewährt. Es werden zwar die Heteronyme des WUM-Korpus in selbstständigen Artikeln (unter den entsprechenden Etyma) behandelt, doch werden sie auch in dem den Heteronymen zugeordneten Zentralartikel, im Artikelfuß, aufgelistet: Da den ungarndeutschen Dialektwörtern Gaul und Ross schriftsprachlich Pferd entspricht, finden sich Verweise auf Gaul und Ross im Artikelfuß des Eintrags Pferd, und im Artikelfuß von Gaul und Ross ebenfalls Verweise auf das Stichwort des Zentralartikels Pferd. Auf diese Weise wird den Leser/-innen gezeigt, wo sie weitere Informationen zur Form- und Inhaltsseite des Denotats finden können. Im WUM-Korpus sind v. a. die Sachbereiche Pflanzen und Tiere reich an Heteronymen.

Verkleinerungsformen werden entsprechend der Praxis in den Dialektwörterbüchern entweder unter der nicht diminuierten Grundform (z. B. im BWB), oder als selbstständiges Lemma angeführt, wenn sich ihre Bedeutungen von den Bedeutungen der nicht-verkleinerten Formen unterscheiden wie z. B. Kätzchen ('Blüte der Weise') vs. Katze ('Mäuse fangendes domestiziertes Tier'). In den WUM-Einträgen werden sie zunächst als selbstständige Lemmata behandelt, erst nach dem Abschluss der Erstellung aller Artikel einer Wortfamilie werden die verkleinerten Formen unter die Grundformen eingefügt.

Zusammensetzungen werden entweder im Eintrag zum jeweiligen Grundwort (z. B. im WBÖ), oder nach dem Eintrag des Grundwortes (z. B. im BWB), oder aber - wenn sie eine große Verbreitung zeigen - selbstständig lemmatisiert (z. B. SHWB, PFWB, SSWB, WBBDM). Der vorherrschenden lexikographischen Praxis entsprechend erhalten die Zusammensetzungen in unserem Wörterbuch einen eigenen Ansatz.

Das Ordnungsprinzip unseres Wörterbuchs ist vorrangig semasiologisch, im Mittelpunkt der Einträge stehen die den jeweiligen streng alphabetisch aufgelisteten Stichwörtern zugeordnete Bedeutungen und Belege sowie ihre Erhebungsregionen und -orte. Zwar können die Zusammenhänge zwischen Wörtern desselben Wortfeldes, zwischen Komposita und Ableitungen, die dasselbe Grundwort haben, durch die nischen- oder nestalphabetische Anordnung oder durch onomasiologische Anordnungsprinzipien besser berücksichtigt werden (vgl. HASS 2015: 498), doch ist 
eine streng alphabetische Stichwortliste leichter erweiterbar, den Leser/-innen auch übersichtlicher, selbst dann, wenn bei der Erstellung strikt alphabetischer Artikel Wiederholungen und Mehrfachnennungen nicht zu vermeiden sind (vgl. SPREE 2012: 552).

Beim Lemmaansatz unseres Wörterbuchs stehen Substantive im Nominativ Singular (mit der Ausnahme der Pluraliatantum wie z. B. Heupatschen), Adjektive werden in der Positivform, Verben in der Infinitivform, ${ }^{8}$ Pronomen unter dem Nominativ lemmatisiert. ${ }^{9}$

Kollokationen und längere Phraseologismen wie Sprichwörter werden innerhalb der Wörterbuchartikel nach ihrer Basis (vgl. HAUSMANN 2007: 218) geordnet.

Bei Homonymen werden in unserem Wörterbuch nur diejenigen homonymen Dialektwörter getrennt lemmatisiert, deren hochdeutsche Stichwörter auch in der deutschen Schriftsprache als Homonyme gelten, wie z. B. im Falle des Homonympaares Arm und $\mathrm{arm}$.

In der Lemmaliste sowie bei der Aufzählung der Belege in den Einträgen werden die umgelauteten Formen mit den nichtumgelauteten Formen gleichgesetzt: $\ddot{a}$ und $a, \ddot{o}$ und $o, \ddot{u}$ und $u$ ferner $\beta$ und ss sind gleich. Bei homographen Stichwörtern kommt in der Stichwortliste das Substantiv zuerst (Großschreibung kommt vor Kleinschreibung): Zapfen 'Verschluss eines Gefäßes, besonders eines Fasses' steht in der Stichwortliste vor zapfen 'Teile eines Gegenstandes durch Zapfen miteinander verbinden'. ${ }^{10}$

\subsection{Zur Informationsgliederung der Mikrostruktur im WUM}

Die Wörterbuchartikel müssen primär zwei Erwartungen entsprechen: den Zielsetzungen des Wörterbuch-Projektes und seinen Benutzerinnen und Benutzern. Die Mikrostruktur muss bereits vorab präzise erarbeitet werden, denn wenn die Publikation angelaufen ist, lassen sich die Bearbeitungsprinzipien nur schwer berichtigen oder straffen (vgl. NIEBAUM 1986: 125).

Zur Schaffung einer vielseitig anwendbaren Wissensstruktur (Informationsgliederung) auf der Mikroebene, gehen wir sowohl deduktiv als auch induktiv vor: Die deduktive Vorgehensweise verlangt, dass die Praxen benachbarter oder ähnlicher Wörterbuchprojekte studiert und das lexikographische Potenzial des Belegmaterials eingeschätzt werden muss, um das theoretische Wissen durch die Induktion, d. h. durch das Verfassen von Artikeln zu bestätigen oder - falls erforderlich - zu revidieren. In unserer Mikrostruktur wurden bei den Wortartikeln das Lemma, der grammatische Kommentar (Wortart, Genus, Angabe der Konjugationsklasse), die Bedeutungserklärungen mit mindestens einem Beleg sowie die Herkunft der Belege (Verbreitung, Erhebungsregion oder -ort) als obligatorisch bestimmt. Als optional

8 Suppletivformen werden unter den Grundformen gebracht.

9 Possessivpronomen werden selbständig lemmatisiert (unter mein, dein, sein usw.).

10 In Anlehnung an die Regeln der alphabetischen Sortierungsnorm DIN 5007-1 s. unter URL: <www.din.de> DIN Deutsches Institut für Normung e.V. (Stand: 22.07.2018). 
wurden die Angaben zur Flexion, ferner Verwendungsbeispiele und Phraseologismen, Angaben zur Etymologie (nur bei Kontaktphänomenen), zur Sach- und Volkskunde, zur Synonymik und die Angabe der - die Artikel schließenden - ungarischsprachigen Äquivalente erachtet (vgl. Abbildung 5).

stand.dt. Lemma / [Lemma nach Mundartform] / usualisiertes Lehnwortlemma Wortklassenzuordnung, bei Substantiven nur Genusangabe, bei Verben die Konjugationsklasse (st./schw.) 1. 'Bedeutungsangabe in einfachen .. Anführungsstrichen (bei Pflanzenund Tierbezeichnungen lat. Äquivalent)' (Stilebene, Sach- oder Fachbereich): Belege aus dem A-Gebiet in alphabetischer Reihenfolge, wenn abweichend vom Lemma grammatische Kategorien (beim Substantiv: Genus, Plur.tant/Sing.tant, Plural; beim Verb: Konjugationsklasse [sw., st., unr.], Hilfsverb im Perfekt, Partizipform; beim Adjektiv: Steigerungsformen), (A: Erhebungsregion, -ort abgekürzt, Datierung der Erhebung [optional: nur bei Belegen vor 1945]); B: Belege aus dem B-Gebiet in alphabetischer Reihenfolge [...]; C: Belege aus dem C-Gebiet in alphabetischer Reihenfolge [...]; Belege aus den Streusiedlungen in alphabetischer Reihenfolge [...]. Syntagmawertiges Verwendungsbeispiel (Verwendungsbeispiele ( $\mathrm{Vb}$ ) mit einem Substantiv, Vb mit einem Verb, $\mathrm{Vb}$ mit einem $\mathrm{Adj}, \mathrm{Vb}$ mit einem $\mathrm{Adv}, \mathrm{Vb}$ mit einer Präp, $\mathrm{Vb}$ mit einer Konj, $\mathrm{Vb}$ mit einem Fragepronomen, $\mathrm{Vb}$ mit passivischer Struktur, Vb mit Partizipien) aus dem A-Gebiet (Erhebungsregion, -ort, Datierung der Erhebung [optional: bei Belegen vor 1945]) deutsche Übertragung. $\diamond$ Kulturhistorischer Kommentar (optional). Syntagmawertiges Verwendungsbeispiel aus dem B-Gebiet [...]. Syntagmawertiges Verwendungsbeispiel aus dem C-Gebiet [...]. Syntagmawertiges Verwendungsbeispiel aus den Streusiedlungen [...]. Satzwertiges Verwendungsbeispiel aus dem A-Gebiet (Erhebungsort, Datierung der Erhebung [optional: nur bei Belegen vor 1945]) deutsche Übertragung. $\diamond$ Kulturhistorischer Kommentar (optional). Satzwertiges Verwendungsbeispiel aus dem B-Gebiet [...]. Satzwertiges Verwendungsbeispiel aus dem C-Gebiet [...]. Satzwertiges Verwendungsbeispiel aus den Streusiedlungen [...]. 2. Bedeutungsangabe [...]. Phras.: Syntagmawertige Phraseologismen (Phraseologismen mit einem Substantiv, Phr. mit einem Verb, Phr. mit einem Adj, Phr. mit einem Adv, Phr. mit einer Präp, Phr. mit einer Konj, Phr. mit einem Fragepronomen, Phr. mit passivischer Struktur, Phr. mit Partizipien) aus dem A-Gebiet (Erhebungsort, Datierung der Erhebung [optional: bei Belegen vor 1945]) deutsche Übertragung, Bedeutung in einfachen Anführungsstrichen. $\diamond$ Kulturhistorischer Kommentar (optional). Syntagmawertige Phraseologismen aus dem B-Gebiet [...]. Syntagmawertige Phraseologismen aus dem C-Gebiet [...]. Syntagmawertige Phraseologismen aus den Streusiedlungen [...]. Satzwertige Phraseologismen aus dem A-Gebiet (Erhebungsort, Datierung der Erhebung [optional: bei Belegen vor 1945]) deutsche Übertragung, Bedeutung in einfachen Anführungsstrichen. $\diamond$ Kulturhistorischer Kommentar (optional). Satzwertige Phraseologismen aus dem B-Gebiet [...]. Satzwertige Phraseologismen aus dem C-Gebiet [...]. Satzwertige 


\begin{abstract}
Phraseologismen aus den Streusiedlungen [...]. Etym.: Etymologische Herleitung im Falle der usualisierten Lehnwortlemmata. Verweis auf dialektgeografische Referenzwerke (UDSA I.1 Identifikationsnummer der Leitform, UDSA I.2 Identifikationsnummer der Leitform). $\rightarrow$ Verweis auf im WUM lemmatisierte Komposita deren zweite Konstituente das Stichwort ist. - 1. ung. Äquivalent(e) der Bedeutung 1 2. ung. Äquivalent(e) der Bedeutung 2 [...].
\end{abstract}

Abb. 5: Obligatorische und fakultative Informationsklassen im WUM

Den Wortartikeln wird meistens nur ein Stichwort vorangestellt. Die Stichwörter der Lemmatypen I und II (formale Dialektwörter, Dialektwörter mit Bedeutungsunterschied) werden im WUM grundsätzlich im Fettdruck angesetzt. Die echten Dialektwörter (Lemmatyp III) erscheinen darüber hinaus unter einem dem Standarddeutschen angeglichen Stichwort in eckigen Klammern wie z. B. „,[Altschneider] m. 'Junggeselle': Oid/tschnäida (A: $\left.\mathrm{OB}^{11}\right)$. - agglegény". Die verschiedenen Typen der Kontaktphänomene (Lemmatyp IV) werden durch kursive Schriftart gekennzeichnet. Bezeichnungsentlehnungen (vor allem aus dem Ungarischen und evtl. aus dem Slawischen) werden in der Schreibweise der Spendersprache angegeben und die entlehnten Substantive werden - der deutschen Orthographie entsprechend - großgeschrieben wie „Tanácsháza 'Bürgermeisteramt' < ung. tanácsháza“ oder „Ámbit 'offener Gang an der langen Seite eines Bauern- oder größeren Landhauses' < ung. Ámbitus“. Hybride Lehnübersetzungen werden ihrer gemischten Morphemstruktur entsprechend in (zum Teil standardisierter) deutsch-dialektaler Form angesetzt wie z. B. „Wintertschismen 'Winterstiefel' < ung. csizma 'Stiefel'،".

Dem Stichwort folgt in der Regel der grammatische Kommentar, der Angaben zur Wortart, Flexion und Syntax des Stichwortes bietet. Im „Wörterbuch der ungarndeutschen Mundarten" werden die grammatischen Informationen an drei Stellen angeführt: 1) entweder im Artikelkopf (Genusangabe bei Substantiven, Konjugationsklasse bei Verben) oder wenn die grammatischen Eigenschaften der Belege von denselben des Stichwortes abweichen, 2) oder nach jenem Beleg, auf die sie sich beziehen. ${ }^{12}$ Darüber hinaus finden sich 3) in den Verwendungsbeispielen und Phraseologismen Informationen zur Grammatik der Belege. Manche grammatischen Merkmale werden nicht explizit gekennzeichnet, da sie aus den Bedeutungen bzw. Verwendungsbeispielen eindeutig erschließbar sind wie z. B. die Transitivität der Verben. Das Genus der substantivischen Zusammensetzungen wird immer angegeben, weil das Genus eines Grundwortes nicht unbedingt gleich ist mit dem Genus jener Zusammensetzungen, die aus demselben Grundwort gebildet wurden: So besitzt z. B. das Wort Joa ('Jahr') im Ofner Bergland neutrales Genus, das Wort

$11 \mathrm{OB}=$ Ofner Bergland.

12 Beim Substantiv das abweichende Geschlecht, Plural, Pluralia- und Singulariatantum; beim Verb die ablautende Form im Präsens, die Perfektform mit Hilfsverb; beim Adjektiv: die Steigerungsformen. 
Fruajoa ('Frühjahr') jedoch alterniert in derselben Region zwischen neutralem und maskulinem Geschlecht.

Eine lexikographische Aufgabe taucht bei der Wortartangabe jener Stichwörter auf, die nach zwei (oder mehreren) Wortarten kategorisiert werden können. In den WUM-Einträgen werden die semantischen Teile durch römische Ziffern (I., II. usw.) getrennt wie im Falle von akkurat:

akkurat Adj. u. Adv. I. Adj. 'ordentlich, präzise': ak'rat (A). Ti Schwoom saan akrati Lääd. Die Schwaben sind Leute (Wr). Des is a akkurati Arweit. Das ist eine Arbeit (Gara). II. Adv. 'genau': ak'rat (A). Ak'rat taun khumt ti Liisl ums Aik und si heat wos mia krait haam. Genau dann kommt die Liesl ums Eck und sie hört was wir geredet haben (OB). - I. akkurátus, precíz II. pontosan (abban a pillanatban).

Abb. 6: Trennung der semantischen Teile im Artikelbeispiel akkurat im WUM

Im WUM werden bei substantivischen Lemmata die Abkürzung des Genus (m./f./n.), ansonsten die Abkürzung(en) der jeweiligen Wortart(en), und nach der Wortartangabe nur die Konjugationsklasse (st./schw.) der Verben angegeben.

Bei der Erstellung eines Dialektwörterbuchs hat die Semantik den Vorrang vor Grammatik (vgl. WIEGAND 1989a: 434-435) - so auch im ungarndeutschen Wörterbuch, wo die Bedeutungserläuterung(en), Verwendungsbeispiele, Datierungsund Quellenangaben, Fach- oder Sachbereichsangaben, Stilschichtangaben, Phraseologismen, Synonyme (ungarische Äquivalente der Bedeutungen), Etymologie sowie Verweise auf wörterbuchinterne oder -externe Informationsstellen erscheinen.

Bei mehr als einer Bedeutungserläuterung werden die Bedeutungen im WUM mit arabischen Ziffern nummeriert und zur Untergliederung eines Bedeutungsteils Kleinbuchstaben benutzt.

Von den möglichen Bedeutungserklärungen (vgl. SCHLAEFER 2002: 97-98) werden im WUM die taxonomische, logische, morpho-semantische, paradigmatische und die Einwortdefinition verwendet. Die Bedeutungsangabe wird, falls erforderlich, um eine ergänzende Erläuterung in runden Klammern vervollständigt.

Die Bearbeitung eines Artikels gestaltet sich bei polysemen Stichwörtern ziemlich komplex. In den Dialektwörterbüchern werden die Bedeutungsangaben bei polysemen Stichwörtern entweder nach dem historisch-etymologischen Aspekt oder dem Häufigkeitsaspekt sortiert. Im ungarndeutschen Wörterbuch wird die Reihenfolge der Bedeutungen nach einem pragmatischen, dem Häufigkeitsaspekt, gebildet (vgl. Abbildung 7). 


\begin{abstract}
Blatt n. 1. 'flächiger (meistens grüner) Teil von Pflanzen, der zur Atmung und Wasserverdunstung dient': Blat (A: Ra, Pl. Plaat, Plädr B: Kig, Kier), Plåt (Pl. Plaal) (A: Bana), Platt (Pl. Pleetl A: Hak, Plaal A: Wiell, Bläite A: Gll, Plaaln A: Tschok), Plot (A: OB). Ti platl fliäng ooli roo. (Wr) Die Blätter fliegen alle runter (im Herbst). 2.a 'rechteckig geschnittenes Papier': a Plot Popia (A: OB) ein Papier. 2.b 'Seite eines Buches, Heftes': Tu teafst khaa Blätr aos'm Heft aosraisn (Ög). Du darfst keine Blätter aus dem Heft ausreißen. 3. 'Zeitung': Plot (Pl. Pleeda A: Sirtz). Mäi Fooda hod as Plot in Wiatshåås klaisn. (OB) Mein Vater hat das im Wirthaus gelesen. 4. 'Spielkarte': Päm Khoatnschpü̈̈nj a Khäinich is a kuades Plot, täis muas ma kschait ååsschpüünj. (OB) Beim Kartenspiel(en) ein König ist ein gutes $\sim$, das muss man gescheit ausspielen. 5. (Handw.) 'flacher Teil eines Werkzeugs oder Gerätes mit scharfer Kante': äs Plot fn Soog (Wr) das der Säge. 6. (Fleisch.) 'Schulterstück des Rindes': A Plot is a Schuidjaschtikl päm Rint (Wr). Ein ist ein Schulterstück(erl) beim Rind. Phras.: ds Plot hod si umkheert (Ög) das hat sich umgekehrt ('eine Situation hat sich geändert'). - 1. levél 2.a papírlap 2.b oldal (könyvé, füzeté) 3. lap (újság) 4. (kártya-)lap 5. lap (szerszámé) 6. lapocka (marháé). (É.M., M.M.)
\end{abstract}

Abb. 7: Wörterbucheintrag Blatt im WUM

Zur besseren Abgrenzung der Bedeutungen der polysemen Stichwörter werden zu den Bedeutungsangaben Glossen und Markierungen, wo es möglich und nötig ist, auch einige Illustrationen hinzugefügt. Glossierungstechniken mit ihren erklärenden und spezifizierenden Funktionen unterliegen keiner einheitlichen Regelung (vgl. LÁSZLó 1996: 38), in den Artikeln des WUM haben wir sie möglichst kurz und eindeutig gehalten. In unserem Projekt wird von Glossen an zwei Stellen Gebrauch gemacht: Einerseits werden sie in die Bedeutungserklärung eingeschoben, andererseits erscheinen sie in der artikelschließenden letzten Position der ungarischsprachigen Stichwort-Äquivalente.

Eine diachronische Markierung wird im WUM nur bei Mundartwörtern und Belegen angegeben, bei denen bereits die Quellen einen entsprechenden Befund oder eine Anmerkung (wie veraltet) beinhalten. Stammen die Belege aus der Zeit vor 1945, wird die im Zusammenhang mit den Belegen genannte Jahreszahl der Quelle nach der Position der Verbreitungsangabe angegeben. Im WUM werden Dialektwörter, die im Verschwinden sind, durch Pfeile $(\downarrow)$ markiert, die nach unten zeigen, Dialektwörter, die neuere Formen sind, durch Pfeile $(\uparrow)$ markiert, die nach oben zeigen. ${ }^{13}$

Das WUM hat die Aufgabe, die genealogisch verschiedenen Mundarten dreier Dialektlandschaften zu dokumentieren. Aus diesem Grunde wird das Belegmaterial nach dem geografischen Prinzip - der HUTTERERSCHEN Gliederung der ungarndeutschen Dialekte (vgl. HUTTERER 1963: 52-54) - angeordnet: Es werden Belege zuerst aus dem A-Gebiet (Westabschnitt und Ostabschnitt des Ungarischen Mittelge-

13 Vgl. dazu eschtimiere statt einschätzen in den pfälzischen Mundarten der Batschka. Ausführlicheres dazu in Kapitel 4. 
birges samt Agglomerationsgebiete um Budapest mit Donauknie und Plattenseeoberland), gefolgt von Belegen aus dem B-Gebiet (Südungarn, mit den Komitaten Branau / Baranya, Schomodei / Somogy, Tolnau / Tolna und Batschka / Bácska), schließlich Belege aus dem C-Gebiet (Westungarn, Ödenburg / Sopron, Güns / Köszeg und Sankt Gotthard / Szentgotthárd mit Umgebung) aufgeführt. Bei der Angabe der Erhebungsorte werden die deutschsprachigen Ortsbenennungen verwendet. ${ }^{14}$

Bei Dialektwörtern mit allgemeiner Verbreitung werden nur die Großbuchstaben der Siedlungsräume (also: $A, B$ und $C$ ) angegeben. Die kleinere Verbreitung wird durch Siglen der geographischen Kleinregionen (z. B. OB für Ofner Bergland), der Komitate (z. B. Bran für Branau, Toln für Tolnau), der Bezirke (z. B. Kf für Krottendorf) oder der einzelnen Ortschaften (z. B. SbO für Sanktiwan bei Ofen) dargestellt.

Diastratische Informationen der einzelnen Lemmata werden im „Wörterbuch der ungarndeutschen Mundarten“" unter dem volkskundlichen Kommentar (eingeführt durch eine Raute) angegeben (vgl. Abbildung 8):

Grammel f. 1. 'Rest beim Ausbraten von Speckwürfeln' 2. 'Ausschlag am
Mund': Kra(a)ml (A: Donauknie, südl. Buchenw.), Kraamü (A: OB),
Kraamü(l) (A: OB), Kraamüi (A: OB), Krame (A: südwestl. Schildg., west-
nördl. Buchenw), Kroml (A: Donauknie, südl. Buchenw.); Krammel (B: nord-
westl. Schelitz, um Petschw, Bohl, Tschaw); Graaml verbr. (C), Kraimü (C),
Kramü (C), Kreamü (C). $\diamond$ Der Fettspeck wurde in Bohl in Würfel (Wiarfl)
geschnitten, im Kessel unter ständigem Rühren ausgekocht und mit einem Kar-
toffeldrücker ausgedrückt. Das Fett kam in Eimer (Ambe), nach dem Ausküh-
len in Fetttöpfe (Fettese 'Fettdosen'). ist im Gebiet B in Ortschaften mit bair.,
z.T. ostfr. Siedlern sowie mit zahlenmäßig hoher Handwerkerschicht ge-
bräuchlich. Hutterer 1963: 259-260; Mihályi 1981: 31; Manherz 1977: 120;
SchwäbWb III, 1911. - 1. tepertö 2. kiütés szájon (herpesz). (K.W., M.M.)

Abb. 8: Wörterbucheintrag Grammel im WUM

Die diatechnischen Markierungen haben wir in unserem Wörterbuch bescheiden gehalten (z. B. „fachspr.“), doch im Laufe der Arbeit versuchen wir im Sinne der Anmerkungen der Gewährspersonen oder Sammlerinnen und Sammler diese wortkargen Markierungen durch feiner spezifizierende Marker (z. B. durch Fach- und Sachbereichsangaben, Berufsbezeichnungen etc.) zu ersetzen. Diaevaluative Angaben können sich über Generationen ändern, sie können von den Sprecherinnen und Sprechern nicht einheitlich empfunden werden oder gar gruppenspezifisch sein (vgl. PÜSCHEL 1989: 694-699). Die gehobene Stilschicht (,geh.“) wurde im WUMKorpus bisher überwiegend durch Wörter belegt, die dem sakralen Bereich entstammen, wie

14 Die deutschsprachigen Ortsbenennungen wurden übernommen von: Die Deutschen in Ungarn. Landkarte mit den deutschen Ortsnamen (2004). Budapest: Neue Zeitung Stiftung. 
Antlitz n. 1. 'Gesicht' geh.: Auntlits (A: OB, Bogr; C: Hbn) 2. 'Angesicht' (Gottes): foa Kots Auntlits niidakniin (A: Marka) vor Gottes $\sim$ niederknien. $\diamond$ Die neutrale, im gesamten Bearbeitungsgebiet verbreitete Bezeichnung für $\sim$ ist Gesicht. -1. orca 2. (isten) színe. (É.M.)

Abb. 9: Wörterbucheintrag Antlitz im WUM

Auf den stilistischen Zusatz der Bedeutung hinzuweisen ist v. a. bei derben, frivolen Bedeutungen zweckmäßig und nötig, diese werden am häufigsten durch die Markierungsprädikate „Schimpfwort“, „derb“, ,grob“ oder „,obszön“ gekennzeichnet. Im WUM wird durch die Marker „pej.“ oder „derb“ auf die Elemente niederer Wortschatzschichten hingewiesen wie bei der dritten Bedeutung des Dialektwortes Sau:

Sau n./f. 1. 'Schwein': Sa/ou (A; B; C) 2. 'Mutterschwein': Såå (A: Wr), Sau/ao (A: Getz, Sirtz; C), Soo (A: Deun), Sau (B: Bera, Jg, Nr, Wigsch; C: Rf), Sou (B: Ras). 3. 'widerwärtige Person' pej.: Ta Mainsch is a Såå. (Wr) Der Mensch ist eine 'unaufrichtig, unredlich'. (...). $\diamond$ Die Bezeichnung $\sim$ wird oft für abstoßende, unangenehme weibliche Personen gebraucht, das männliche Pendant heißt Sååpea ('Saubär'). - 1. disznó 2. koca 3. visszataszító, ellenszenves személy (többnyire nö). (É.M., M.M.)

Abb. 10: Wörterbucheintrag Sau im WUM

Die Belege in den WUM-Artikeln erbringen den Nachweis dafür, dass eine Bezeichnung mit einer bestimmten Bedeutung in einem Ortsdialekt, einer Mikro-, Meso- oder Makroregion, existiert. Unser Belegmaterial ist z. Z. wegen der Erhebungsarten, des Bearbeitungsgebietes und der Dauer der Sammelaktion recht heterogen, die Reihe der Belegmaterialsorten reicht von direkt oder indirekt erhobenem Material, privaten Einsendungen und Sammlungen, Materialien von Orts- oder Gebietswörterbüchern, Exzerpten aus wissenschaftlichen Monographien bis hin zu Materialien aus historischen Quellen.

Die Verwendungsbeispiele werden - über die alphabetischen und geografischen Ordnungsprinzipien hinaus - danach gegliedert, ob sie Syntagmen oder ganze Sätze sind. Längere Verwendungsbeispiele werden nicht ins Standarddeutsche übertragen, damit der Artikelkörper nicht überladen wird.

Die phraseologischen Mehrworteinheiten werden von den allgemeinen Verwendungsbeispielen abgegrenzt behandelt. In Sprachinselmundarten kommen ziemlich häufig in der Mundart verfasste Reime, Gedichte, Gebete vor, die in der Sprachgemeinschaft tradiert und oft mit Ritualen der Sprachgemeinschaft oder einer kleineren Region verbunden sind, und die ebenfalls zu den phraseologischen Einheiten gezählt werden (vgl. BURGER 2003: 15). Im Gegensatz zu den leicht kategorisierbaren Reimen, Gedichten und Gebeten erfordert die Identifizierbarkeit und die Einteilung der mundartlichen phraseologischen Mehrwort-Lexeme, v. a. der (teilidiomatischen) Kollokationen eine genauere Überprüfung (vgl. HESSKY 1987: 13-40). Der Unterschied zwischen (teilidiomatischen) Kollokationen und echten Idiomen liegt darin, dass die Bedeutung einer Kollokation dekompositionell 
(aufgrund der Konstituentenbedeutungen) hergeleitet werden kann, während dies bei echten Idiomen wegen der übertragenen Bedeutung gerade nicht möglich ist. Die dialektalen Kollokationen im WUM-Korpus sind einerseits lautliche Varianten des binnendeutschen Sprachschatzes wie wiinicha Hunt (bairisch) 'Taugenichts', andere sind wiederum (formal und semantisch) dialekttypische Kollokationen wie kfellt Paprika (fuld.) 'mit Hackfleisch, Reis, Eiern, Zwiebeln, Paprika, Salz und Pfeffer gefüllte Paprikaschote'. Im Sinne der Ordnungsprinzipien der partiell integrierten Mikrostruktur (vgl. HAUSMANN / WERNER 1991: 2748-2750; WIEGAND 1989b) werden im WUM die Kollokationen, die transparent genug sind, um sie wörtlich zu interpretieren, bei den Verwendungsbeispielen untergebracht (vgl. LÁSZLó 1996: 41). Das, was sich nicht einordnen lässt, wird in einer gesonderten Position, in der zweiten Hälfte des Artikels dargestellt, damit erstens die Artikelstruktur durchschaubar bleibt, zweitens die Auflistung der Belege nach Dialektlandschaften sie nicht auseinander reißt, und damit drittens die Benutzer/-innen wissen, dass die nach der Abkürzung „Phras.“ folgenden lexikalischen Einheiten eine andere Qualität und Bedeutung als die einfachen Verwendungsbeispiele haben.

Im WUM werden zuerst die syntagmawertigen, dann die satzwertigen Phraseologismen aufgelistet. Bei ihrer alphabetisch-geografisch-morphologisch gesteuerten Anordnung ist das erste Substantiv ausschlaggebend, wenn dies nicht vorhanden ist, steht der Phraseologismus unter dem ersten sinntragenden Wort. ${ }^{15}$ Den Phraseologismen werden Angaben über ihren Erhebungsort, die Datierung der Erhebung (optional bei Belegen aus der Zeit vor 1945), ihre schriftdeutsche Übertragung (optional), die Bedeutung sowie sach- oder kulturhistorische Kommentare (optional) hinzugefügt (vgl. dazu Abbildung 11):

Zaun m. 'zum Abgrenzen des eigenen Gartens von dem des
Nachbarn bzw. von der Gasse dienende Vorrichtung': Tsaa (A:
Bana), Tsaoo, Tsae Pl. (A: Marka; C: Hbn), Tsau (A: Gt, Sirtz,
Wr; C; B: Fed), Tsaun Tsoine Pl. (B: Nasch), Tsoo (A: Deun).
Ta Tsau is aas Lottn. (Sirtz) Der ist aus Latten. Hindan Tsau
saan olawöö Puschn. (Wr) Hinter dem sind immer Blumen.
Phras.: Tia, wii tea Tsaa (Bana, Mádl 2008: 131) Dürr, wie der
(aus Eschenruten zusammengetrocknete) 'sehr dünn'. A aajts
Weep is a Tsaa ums Haas. (Bana, Mádl 2008: 179) Ein altes
Weib ist ein Zaun ums Haus 'es passt auf alles auf, weiß über
alles Bescheid'. $\rightarrow$ Bretter $\sim$, Brettl $\sim$, Eisen $\sim$ Garten , Gärtl $\sim$
Holz , Latten $\sim$ Die $\sim$ e, im OB vor dem Zweiten Weltkrieg
wurden meistens aus Naturstein, Lehm, Holz oder aus getrock-
neten Pflanzen oder Pflanzenteilen wie Fliederstauden, Teu-
felzwirn oder Maisstengel gebaut. Erst in den 1960er und

15 In Ermangelung eines Substantivs wird ein Phraseologismus unter dem ersten Autosemantikum (Verb, Adjektiv, Adverb) lemmatisiert. Die Einreihung der phraseologischen Einheiten unter mehreren autosemantischen Konstituenten wird ebenfalls praktiziert (vgl. dazu das WBBDM). Die Phraseologismen im WUM werden in alphabetischer Reihenfolge nach Gebietszuordnung (A, B, C) und Wortklassenzuordnung gebracht, in jener Nennform wie sie der WUM-Datenbank entnommen wurden. 
1970er Jahren kam es in Mode, dass die Garten e aus Kunststein, aus Eisen gebaut wurden. An der Grenze zwischen zwei Gärten wurden Steinmauern aufgezogen. Steinmauern wurden geweißelt. Der eiserne und die Kellertür wurden im OB traditionell grün gestrichen. Andere Farben wurden zum Streichen nicht verwendet. Über die blaue Farbe sagte man z. B. dass sie nicht deutsch, sondern typisch slowakisch sei. In den $\sim$ n gab es zwei Öffnungen: ein großes Tor und eine kleine Tür. Durch die kleine Tür ging man zu Fuß. Das große Tor hatte zwei Flügel und war so breit angelegt, dass ein Wagen oder die Dreschmaschine durch das Tor passte. Vor dem Kriege gab es noch eine Reihe von Gärten im OB, die an der Straßenfront oder zwischen zwei Gärten gar keine e gehabt haben. In Deun war der vor dem Zweiten Weltkrieg eine Pfahlhecke. - kerítés. (É.M., M.M.)

Abb. 11: Phraseologische Belege mit sachkundlichem Kommentar sub voce Zaun im WUM

Informationen über Zusammensetzungen, in denen das Lemma das Grundwort ist, etymologische Herleitungen sowie ergänzende Angaben zu den lautlichen und morphologischen Merkmalen des Lemmas, finden im Artikelfuß ihren Platz, gemäß den Konventionen der jeweiligen diatopischen Wörterbuchkanzleien (vgl. MüLLER 2016: $147-155) .^{16}$

Der Artikelfuß umfasst im ungarndeutschen Wörterbuch vier optionale Positionen: jeweils eine für die Wortherkunft, für Verweise auf dialektlexikographische Referenzwerke, für den Verweis auf die im WUM lemmatisierten Wortzusammensetzungen deren Grundwort das Stichwort ist, sowie für die Angabe der ungarischsprachigen Äquivalente (wenn das Lemma nicht ein ungarischsprachiges Lehnwort ist) der Stichwortbedeutungen.

Auf die Etymologie des Stichwortes wird nur bei echten Dialektwörtern und bei usualisierten Lehnwörtern eingegangen. Bei den anderen Lemmatypen erübrigt sich die etymologische Herleitung wegen der Übereinstimmung des Formativs der Stichwörter mit dem ihrer dialektalen Äquivalente (vgl. CARSTENSEN 1989: $668-672)^{17}$

16 Diatopische und Sprachinselwörterbüchern kennzeichnen typographisch den Artikelfußteil (durch einen Geviert- oder Halbgeviertstrich, in neue Zeile gesetzt) und seine Teile (durch „Ltg.“ für Lautungsbesonderheiten oder „Et./Etym.“ für die Wortherkunft) im Großen und Ganzen genauso gleich wie sie Etymologie und Verweise auf allgemeine deutsche Wörterbücher und auf benachbarte Dialektwörterbücher behandeln. Im Gegensatz zu den diatopischen Dialektwörterbüchern werden Lautbesonderheiten in kleineren Sprachinselwörterbüchern im Lautkopf untergebracht, möglicherweise auch wegen des Umfangs des zur Verfügung stehenden Belegmaterials. Darüber hinaus gleichen sich die Sprachinselwörterbücher auch darin, dass sie auf Komposita am Ende des Artikels verweisen. Eine Position für die Äquivalente in der überdachenden Landessprache gibt es nur in der Mikrostruktur des WUM (vgl. Abbildung 5).

17 Etymologische Herleitungen sind nur bei Lehnwortlemmata nötig, alle anderen Stichwörter kann man in einschlägigen Standardwerken des Deutschen nachschlagen. 
Die ungarischsprachigen Äquivalente sollen einerseits das WUM für ungarische Muttersprachlerinnen und -sprachler benutzerfreundlicher machen, andererseits lassen sie die Rolle des Deutschen als eine der bedeutendsten Geber- bzw. Vermittlersprache des Ungarischen deutlich hervortreten. ${ }^{18}$ Finden sich keine ungarischsprachigen Äquivalente, werden ungarischsprachige Bedeutungsumschreibungen angeführt.

\section{TEXTTECHNOLOGISCHE ASPEKTE}

Zum Exzerpieren werden überwiegend Quellen aus dem 20. Jahrhundert herangezogen, denn viele der im 19. Jahrhundert über ungarndeutsche Ortsmundarten verfassten Arbeiten behandeln Ortschaften oder Regionen, die heute jenseits der Grenzen von Ungarn liegen und aus diesem Grunde heute nicht zu unserem Bearbeitungsgebiet gehören. ${ }^{19}$

Manuell digitalisiert wurden in der ersten Phase der Materialsammlung die Inhalte des äußerst bescheidenen Zettelkatalogs des Ungarndeutschen Forschungszentrums (UDFZ). Die Katalogzettel beinhalten Simplizia, Syntagmen, ganze Sätze, Sprichwörter mit deutscher Übertragung und Bedeutungserklärungen (vgl. den Katalogzettel mit dem Dialektwort Morgensregen (Morgenregen, aus Großmanok / Nagymányok) auf der folgenden Abbildung):

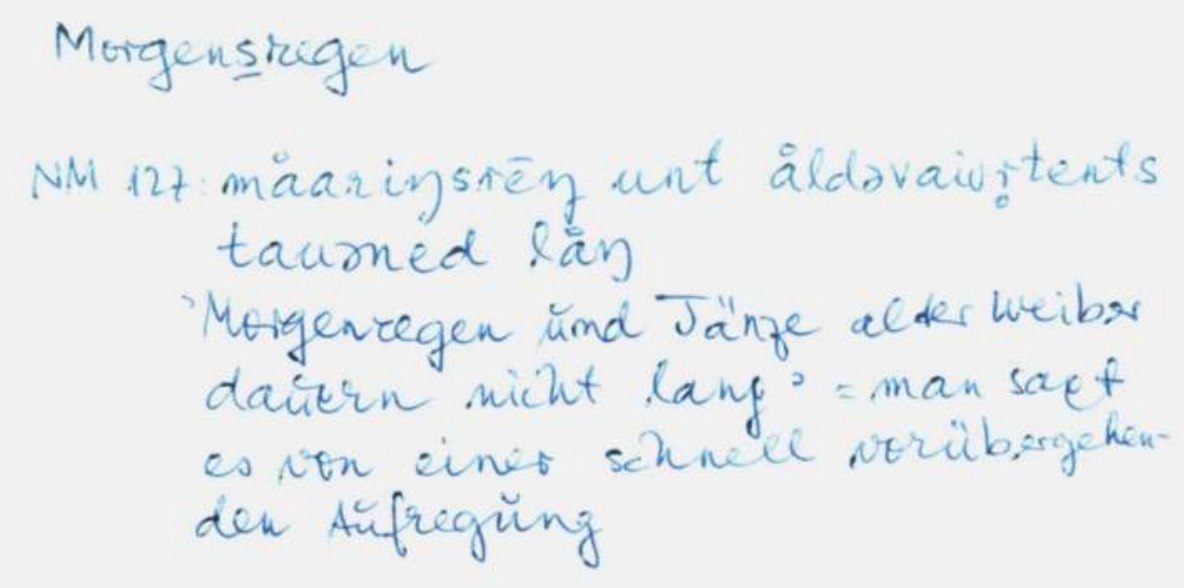

Abb. 12: Katalogzettel Morgensregen (Großmanok/Nagymányok) aus dem Archiv des WUM

18 Über den intensiven lexikalischen Austausch der Sprachen im östlichen Mitteleuropa und die dadurch entstandene geistige Verwandtschaft vgl. NYOMÁRKAY (2012: 1-13).

$19 \mathrm{Zu}$ den Anfängen der wissenschaftlichen Erforschung der in Ungarn gesprochenen deutschen Mundarten vgl. HUTTERER (1960: 44-49). 
Unter den Zetteln finden sich auch welche, auf denen Reime zu lesen sind:

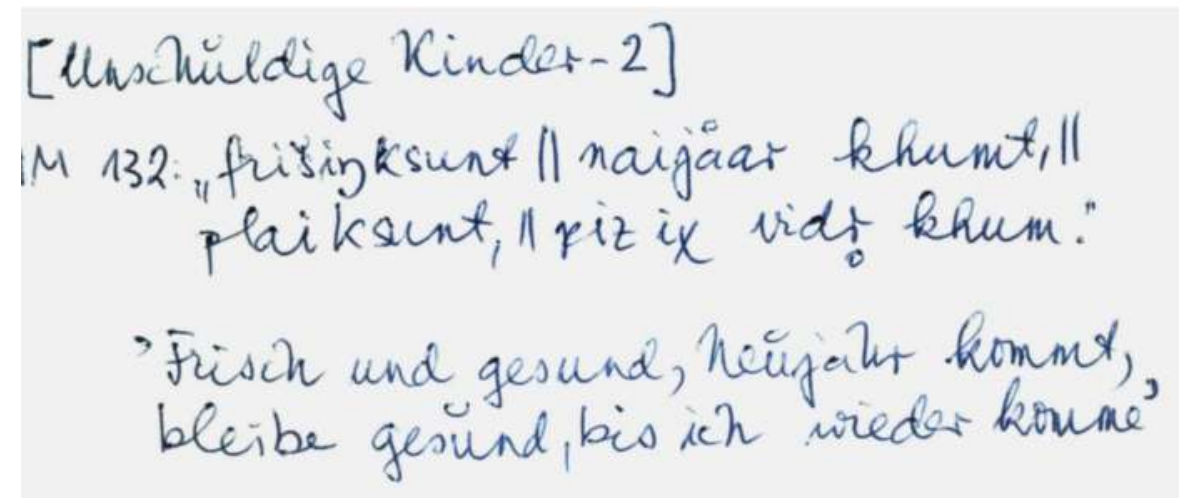

Abb. 13: Katalogzettel unschuldige Kinder - 2 aus Großmanok/Nagymányok aus dem Archiv des $W U M$

Stichwörter werden häufig auch aus volkskundlicher Sicht erklärt (vgl. Abbildung 14):

\section{Neülicht \\ NM 89: 2. (Aberglaube) Wenn man in N weist (vaizlt), bekomint man Spinnen. $\rightarrow$ weikeln}

Abb. 14: Katalogzettel Neulicht aus Großmanok/Nagymányok aus dem Archiv des WUM

Eine überaus wichtige Aufgabe ist für uns zur Erweiterung unserer Lemmaliste das kontinuierliche Exzerpieren von Dissertationen und wissenschaftlichen Beiträgen, die sich auf eine Belegsammlung stützen oder volkskundliche Themen aus dem Alltag dieser Sprachgemeinschaft behandeln. Wir exzerpieren ferner Hauskalender, Zeitungsartikel und Werke der ungarndeutschen Mundartliteratur (schöngeistige Mundartliteratur und Volksdichtungen).

Über die genannten Quellen hinaus wurden jene thematischen Fragebogen über Volkstracht, Haus und Hof sowie Weinbau digitalisiert, die noch in den Jahren 1963 
und 1964 von C. J. HutTERER und K. MANHERZ vorbereitet und an Privatpersonen der drei Mundartgebiete ausgesandt wurden (vgl. MüLlER 2016: 26). Parallel zu unserem WUM-Projekt werden auch weiterhin private Erhebungen und Veröffentlichungen von interessierten Laien oder Heimatvereinen gesammelt (vgl. HIDASMÁRIN et al. 2014; „Hajoscher Wörterbuch“ 2016).

Um den minderheitenspezifischen Alltagswortschatz möglichst breit zu erfassen, ist es notwendig, eine landesweite Erhebung durchzuführen. Teils abgeschlossen, teils in Vorbereitung sind Fragebogen zu folgenden Sachbereichen:

- Ackerbau, Feld- und Gartenarbeit, Waldarbeit;

- Weinbau, Schnapsbrennerei, Bierbrauerei;

- Nutztiere, Haustiere, Wildtiere, Fischerei, Jagd;

- Speisen, Getränke, Essgewohnheiten;

- menschlicher Körper, Hygiene, Krankheiten, Heilmittel;

- Wendepunkte des Lebens: Geburt, Taufe, Hochzeit, Tod;

- Sakrale Orte und Handlungen: Feiertage, Kirche, Messe, Religionsunterricht, Seelsorge, Sakramente;

- Frauenarbeiten: Wäsche, Flicken, Stricken, Häkeln, Nähen, Brotbacken;

- Witterungsverhältnisse, Zeitmessung, temporale Adverbien, Lokaladverbien, Zahlen. $^{20}$

Durch die systematische Exzerpierarbeit ist zurzeit aus 267 Siedlungen und Kleinregionen zu insgesamt 12467 Lemmata dialektales Material in der Datenbank auf den Servern der Eötvös-Loránd-Universität abgespeichert. ${ }^{21}$

Die Datenbank spiegelt die diatopische, diastratische und die diafunktionale Vielfalt des ungarndeutschen Sprachschatzes wider, da in ihr Belege aus genealogisch verschiedenen Dialekten (mittelbairisch, westmitteldeutsch, ostfränkisch, schwäbisch) von Sprecher/-innen unterschiedlicher soziokultureller Herkunft (städtisches Milieu in der Agglomeration um Budapest herum vs. ländlicher Lebenswandel in südungarischen Kleindörfern) ${ }^{22}$ und unterschiedlicher Berufe (Bauern, Handwerker, Kaufleute) gespeichert sind.

Bei der Planung und Durchführung der Exzerpierarbeit sowie bei der Auswahl von geeigneten Beiträgen wird über ihre Authentizität hinaus auch darauf Wert gelegt, dass die mundartlichen Bezeichnungen möglichst vielen Quellen - und dadurch vielen Regionen und Siedlungen - entstammen. Die Digitalisierung wird auch zum Zeitpunkt der Erstellung vorliegenden Beitrags fortgeführt. Aufgrund des

20 Die Zusammenstellung des Themenkatalogs und der einzelnen Fragebogen wurden durch die freundliche Hilfe der Wörterbuchkanzlei des BWB - allen voran Prof. ANTHONY ROWLEY und Dr. EDITH FUNK - möglich.

21 Homepage der WUM-Datenbank, URL: <http://wum.elte.hu>; Stand: 22.07.2018.

22 Die überwiegende Mehrheit der Belege kommt aus dem basisdialektalen Bereich. Der Wortschatz der höheren sozialen Schichten (z. B. bildungsnaher Ungarndeutscher) war und ist nur in der Nähe von Budapest bzw. in Ofen, in Fünfkirchen und Baja sowie in den Kleinstädten entlang der österreichisch-ungarischen Grenze zu erheben. 
bisher gesammelten Korpus wurden bis 2017700 Probeartikel geschrieben, v. a. mit Stichwörtern aus den reich belegten Sachbereichen „Haus und Hof“, „Tod und Bestattung“ sowie „Verwandtschaftsbezeichnungen“. Beim Vorhandensein nötiger finanzieller Mittel wird beabsichtigt, die Lieferungen in Printausgaben und darauf folgend online zu publizieren.

Das Wörterbuchkorpus des WUM wird in einer passwortgeschützten, internetgestützten Datenbank <http://wum.elte.hu> gespeichert. Die Daten werden in die WUM-Datenbank manuell eingegeben. Auf der Hauptseite der Datenbank (vgl. Abbildung 14) findet sich die Liste jener Lemmata, zu denen in der Datenbank digitalisiertes Material aufbewahrt wird. Die Stichwörter werden durchnummeriert, jede Änderung wird mit einem Zeitstempel dokumentiert.

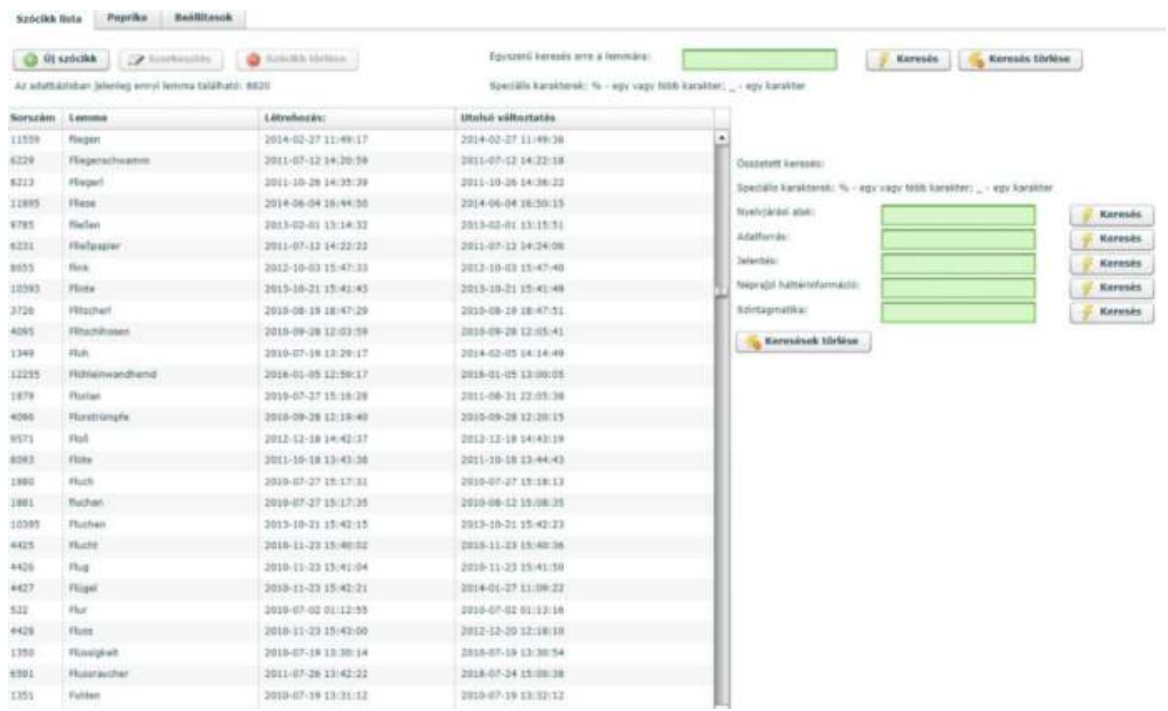

Abb. 15: Hauptseite der WUM-Datenbank mit Stichwortliste

Beim Anklicken eines Stichwortes öffnet sich die dazugehörende Seite mit den zum Stichwort abgespeicherten objekt- und metasprachlichen Informationen (vgl. Abbildung 16). 


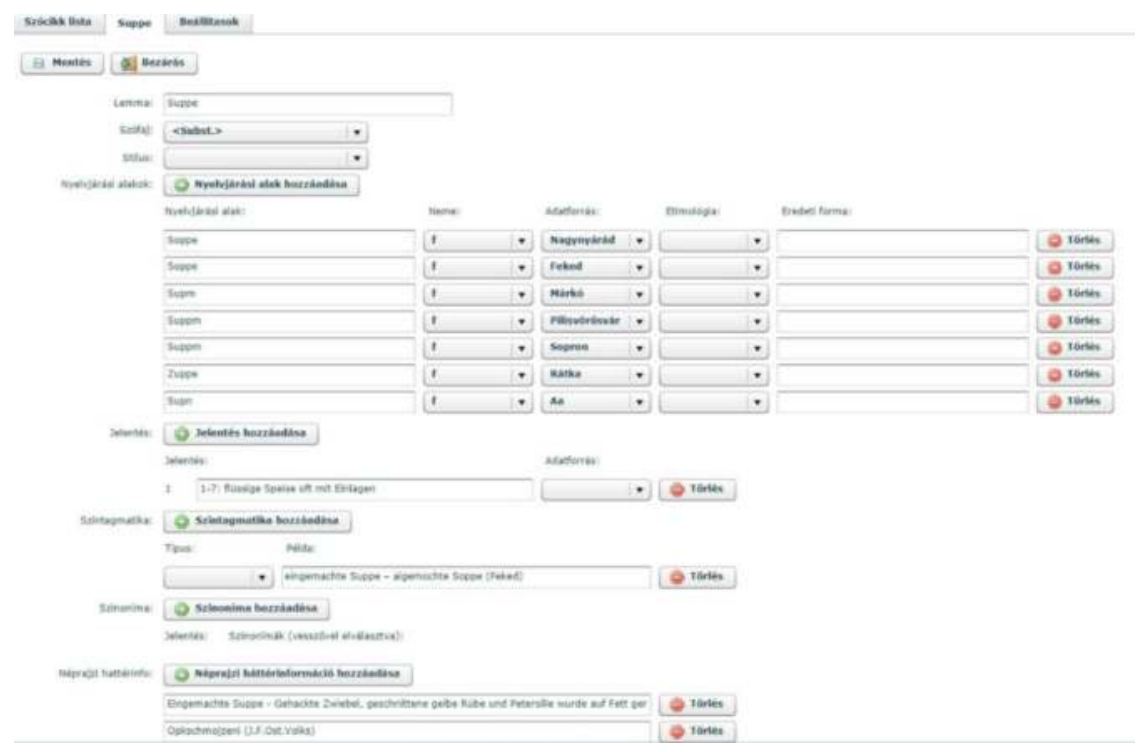

Abb. 16: Meta- und objektsprachliche Informationen zum Stichwort Suppe in der WUM-Datenbank

Eine Suche kann nach allen Informationsklassen und allen konkreten objekt- sowie metasprachlichen Informationen durchgeführt werden. ${ }^{23}$

Die Datenbank ist für zugelassene User/-innen via Internet von überall aus zugänglich. Die Probeartikel wurden mit Word erstellt, manuell formatiert und korrigiert. Zur Erstellung von Wörterbuchartikeln in großer Anzahl wird z. Z. eine Maske entworfen, mithilfe derer die zu einem Stichwort geordneten Informationen der Reihenfolge der Informationsklassen im WUM entsprechend aus der Datenbank gewonnen werden können.

\section{LINGUISTISCHES POTENTIAL}

Da sich das WUM-Korpus noch im Aufbau befindet, lassen sich hinsichtlich seines linguistischen Potenzials nur eingeschränkt Aussagen machen.

Unter unseren Sprachdaten finden sich viele, die die Mundarten einer ganzen Region in prototypischer Form widerspiegeln wie Aantn ('Ente'), rästn ('rösten'), liew ('lieb') (alle Belege aus dem bairischen Dialektgebiet Aa, östlich der Moorer Senke bis hin zu Budapest und Umgebung). Für die Untersuchung des Wortschatz-

23 Die Datenbank wurde für das WUM-Korpus erstellt, aber durch sie können weitere, nach bestimmten Kriterien gefilterte Korpora gewonnen werden u. a. auch siedlungs- und regionentypische Dialektwortregister. Ein eindeutiges Interesse ungarndeutscher Heimatvereine ist in den letzten Jahren zu verzeichnen (vgl. HIDAS-MÁRIN et al. 2014). 
wandels sind jene Sprachdaten von Interesse, deren Denotate wegen der wirtschaftlich-soziokulturellen Umwälzungen des 20. Jahrhunderts nicht mehr existieren, oder in der alltäglichen Konversation nur zufällig vorkommen wie z. B. Schawesma(a)t (pfälzisch) 'bei jüdischen Familien als ständige oder gelegentliche Aushilfe dienende (meist junge) Frau'. Ein weiteres Beispiel stellt das Wort Dienstag dar, das im Ofner Bergland von der Generation A (vor dem Zweiten Weltkrieg geboren) als Iaridoog ('Eritag') realisiert wird, von Sprecher/-innen der Nachkriegszeit bereits als Dinsdoog. Diese Belege repräsentieren zwei verschiedene Entwicklungsstadien des Sprachgebrauchs der Ungarndeutschen: Einerseits das Stadium der Vollsprache mit vollwertigen kommunikativen Funktionen, andererseits das Stadium der Verdrängung, der Erosion, der zunehmenden Mischung, des Domänen- und Sprecherverlusts (MüLLER 2016: 45).

Für die Untersuchung diastratischer Variabilität eignen sich Belege, die aus derselben Kleinregion oder Ortschaft stammen und unterschiedlichen Gruppensprachen und Stilschichten zugeordnet werden können wie Ä̈̈skhostn ('Eisschrank') und Fridschidea ('Frigidaire') aus dem Ofner Bergland. Gegenwärtig konkurriert mit der älteren Bezeichnung Ä̈̈skhostn das vornehmere im Ungarischen gebräuchliche Fridschidea (ungarisch fridzsider), beide in der Bedeutung 'Kühlschrank'. Letzteres wurde durch Frauen verbreitet, die bei (Wienerisch sprechenden, betuchten) Bürgern Ofens (Budapest) als Dienstmagd oder Haushälterin angestellt waren.

Die Sprachdaten des WUM-Korpus können sich auch bei etymologischen Herleitungen ungarischer Wörter als dienlich erweisen. Zwar ist die durch das Deutsche vermittelte Schicht des ungarischen Wortschatzes ausführlich erforscht und kodifiziert (vgl. KOBILAROV-GöTZE 1972; GERSTNER 1998; 2003: 153-159; HEWBU), doch finden sich Nischen mit Klärungsbedarf. Durch die Belege bzw. die ungarischen Äquivalente am Ende der WUM-Artikel ist es möglich, die deutsche Herkunft mancher ungarischer Wörter aufzudecken wie bei ung. szajmóka 'in Schweinsdarm gefüllte, ca. $60 \mathrm{~mm}$ breite, $40 \mathrm{~mm}$ lange aus Schweinefleisch, ev. Kartoffeln, Sauerkraut oder anderen Gemüsesorten angefertigte, mit Salz, Pfeffer, scharfem Paprika, Knoblauch, Majoran, Kümmel und Muskatnuss gewürzte, geräucherte Salami'. Diese Salamisorte war unter den Deutschen in der Batschka und Tolnau (Südungarn) unter dem Namen Saumagen bekannt und beliebt, wurde wegen ihres guten Geschmacks auch von den Ungarn übernommen. Die bairische Bezeichnung Sååmoogn wurde als szajmóka ['spimo:kp] madjarisiert.

Historischer Wortschatz ist im WUM-Korpus insofern involviert, als die - noch vor dem Zweiten Weltkrieg entstandenen - ausgezettelten wissenschaftlichen Arbeiten (vgl. BAKONYI 1940; FOLLÁTH 1941; BEDI 1912; EsZTERLE 1929) teilweise auch historische Bezeichnungen beinhalten. Die rezente Belegsammlung reicht aber nicht aus, dieses Sprachgut differenziert in das Wörterbuch aufzunehmen. 


\title{
5 KONTAKTADRESSEN, HOMEPAGE
}

\author{
Eötvös-Loránd-Universität \\ 1088 Budapest \\ Rákóczi út 5 \\ Ungarn \\ E-Mail: \\ knipf.erzsebet@btk.elte.hu \\ muller.marta@btk.elte.hu
}

Homepage der WUM-Datenbank: 〈http://wum.elte.hu>

\section{LITERATURVERZEICHNIS}

BAKONYI, JÁNOS (1940): Márkó telepítése és nyelvjárása [Siedlungsgeschichte und Mundart von Marka]. Budapest (Német nyelvészeti dolgozatok. 5).

BEDI, REZSÖ (1912): A soproni hienc-nyelvjárás hangtana [Lautlehre der Ödenburger Hienz-Mundart]. O.O.: Romwalter.

BRENNER, KOlOMAN / MARIA ERB / KARL MANHERZ (Hg.), in Zusammenarbeit mit HeINRICH J. DINGELDEIN (2008): Ungarndeutscher Sprachatlas (UDSA). Südungarn. Erster Halbband. Budapest: ELTE Germanistisches Inst.

BURGER, HARALD (2003): Phraseologie. Eine Einführung am Beispiel des Deutschen. Berlin: Erich Schmidt Verlag.

BWB = Bayerisches Wörterbuch (2002). Bd. I. München: R. Oldenbourg Verlag.

CARSTENSEN, BRODER (1989): Die Markierung von Entlehnungen im allgemeinen einsprachigen Wörterbuch. In: HAUSMANN, FRANZ JOSEF / OSKAR REICHMANN / HERBERT ERNST WIEGAND / LADISLAV ZGUSTA (Hg.): Wörterbücher. Ein internationales Handbuch zur Lexikographie. Bd. 1. Berlin/New York: de Gruyter. (HSK. 5.1).

Die Deutschen in Ungarn. Landkarte mit den deutschen Ortsnamen (2004). Budapest: Neue Zeitung Stiftung.

DIN Deutsches Institut für Normung e.V.. URL: 〈www.din.de>; Stand: 22.07.2018.

ERB, MARIA (Hg.), in Zusammenarbeit mit HEINRICH J. DINGELDEIN (2012): Ungarndeutscher Sprachatlas (UDSA). Südungarn. Zweiter Halbband. Budapest: ELTE Germanistisches Institut.

EsZTERLE, MÁRIA EDIT (1929): A budakeszi német nyelvjárás hangtana [Lautlehre der deutschen Mundart in Wudigeß]. Budapest. (Német philologiai dolgozatok. 41).

FATA, MÁRTA (Hg.) / KATHARINA DrobAC (Mitarb.) (2013): Migration im Gedächtnis. Auswanderung und Ansiedlung im 18. Jahrhundert in der Identitätsbildung der Donauschwaben. Stuttgart. (Schriftenreihe des Instituts für donauschwäbische Geschichte und Landeskunde. 16).

FOLLÁTH, FERENC (1941): Szóképzés a budakörnyéki német nyelvjárásban [Wortbildung der deutschen Mundart im Ofner Bergland]. Pécs (Német nyelvészeti dolgozatok. 6).

GERSTNER, KÁROLY (1998): A német vonatkozású elemek újabb etimológiai szótárainkban [Elemente mit deutschem Bezug in unseren neueren etymologischen Wörterbüchern]. Budapest: Akadémiai Kiadó.

GERSTNER, KÁROLY (2003): Karl Mollay und die ungarische Lehnwortforschung. In: MANHERZ KARL (Hg.): Gedenktagung zu Ehren von Claus Jürgen Hutterer und Karl Mollay. Budapest: ELTE Germanistisches Institut (Budapester Beiträge zur Germanistik. 38), 153-159.

Hajoscher Wörterbuch / Hajós sváb-magyar szótár. (2016) Emberi Erőforrások Minisztériuma. 
HAMERS, Josiane F. / Michel H. A. BlanC (2003): Bilinguality and Bilingualism. Cambridge: University Press.

HASS, UlRIKE (2015): Das Wort in der Lexikographie. In: HASS, UlRIKE / PetRA STORJOHANN (Hg.): Handbuch Wort und Wortschatz. Berlin/Boston: de Gruyter, 492-516.

HAUSMANN, FRANZ JoSEF (2007): Die Kollokationen im Rahmen der Phraseologie. Systematische und historische Darstellung. In: Zeitschrift für Anglistik und Amerikanistik 3/55, 217-234.

HAUSMANN, FRANZ JOSEF / REINHOLD WERNER (1991): Spezifische Bauteile und Strukturen zweisprachiger Wörterbücher: eine Übersicht. In: HAUSMANN, FRANZ JOSEF / OSKAR REICHMANN / HERBERT ERNST WiEgAND / LAdisLav ZGUSTA (Hg.): Wörterbücher. Ein internationales Handbuch zur Lexikographie. Bd. 3. Berlin/New York: de Gruyter, 2729-2769.

HESSKY, REGINA (1987): Phraseologie. Linguistische Grundfragen und kontrastives Modell deutsch $\rightarrow$ ungarisch. Tübingen: Max Niemeyer Verlag.

HEWBU = A magyar nyelv történeti-etimológiai szótára [Historisch-etymologisches Wörterbuch der ungarischen Sprache]. I. 1967, II. 1970, III. 1976. Budapest: Akadémiai Kiadó.

HidAs-MÁRIN, MAGDOLNA / JózSEF HómANn / ERZSÉBET HómANN-FRIEDRICH / ERZSÉBET PETZ / ANTAL SCHEIBL / ISTVÁN SChUSTER / ISTVÁNNÉ SCHUSTER / JÁNOS VIKKER (Hg.) (2014): Császártöltési Sváb Szótár [Tschasarteter Schwäbisches Wörterbuch]. Császártöltés: Manuskript.

HUtTERER, Claus JÜRGEN (1960): Geschichte der ungarndeutschen Mundartforschung. Berlin: Akademieverlag.

HUtTERER, Claus JÜRgen (1963): Das Ungarische Mittelgebirge als Sprachraum. Historische Lautgeographie der deutschen Mundarten in Mittelungarn. In: FRINGS, THEODOR (Hg.): Mitteldeutsche Studien 24. Halle a./S.: Max Niemeyer Verlag.

HutTerer, Claus JÜrgen / MANHERZ, KARL (1963-1964): Fragebogen über Volkstracht, Haus und Hof sowie Weinbau (unveröffentlichtes Manunskript).

HUTTERER, ClAUS JÜRGEN (1975): Die deutsche Volksgruppe in Ungarn. In: BALASSA, IVÁN / Claus KlotZ / Karl ManherZ (Hg.): Beiträge zur Volkskunde der Ungarndeutschen Bd. 1. Budapest.

HUTTERER, ClAus JÜRgEN (1991): Geschichte der ungarndeutschen Mundartforschung. In: HuTTERER, ClAuS JÜRGEN (Hg.): Aufsätze zur deutschen Dialektologie. Budapest: Tankönyvkiadó (Ungarndeutsche Studien. 6), 123-200.

KNIPF-KOMLÓSI, ELISABETH (2011): Wandel im Wortschatz der Minderheitensprache. Am Beispiel des Deutschen in Ungarn. Stuttgart: Steiner Verlag.

KNIPF-KOMLÓSI, ELISABETH (2012): Wortschatzdynamik im Sprachkontakt (Am Beispiel der deutschen Minderheitensprache in Ungarn). In: KNIPF-KomLÓsI, ElisabETH / ClaUdiA MARIA RIEHL (Hg.): Kontaktvarietäten des Deutschen synchron und diachron. Wien: Praesens, 61-74.

KNIPF-KOMLÓSI, ElISABETH / MARIA ERB / MÁRTA MÜLLER (2012): Wörterbuch der Ungarndeutschen Mundarten. Forschungsstand. Budapest: Germanistisches Institut.

KNIPF-KOMLÓSI, EliSABETH / MÁRTA MÜLLER (2017): „Ein unermäßliches Land von Begriffen.“ Sichtbar gewordene Wörter der Ungarndeutschen. In: MAUERER, CHRISTOPH (Hg.): Mehrsprachigkeit in Mittel-, Ost- und Südosteuropa. Gewachsene historische Vielfalt oder belastendes Erbe der Vergangenheit. Regensburg: Pustet (Forschungen zur deutschen Sprache in Mittel-, Ost- und Südosteuropa. 4), 147-150.

KNIPF-KOMLÓSI, ElISABETH / MÁRTA MÜLLER (2019): „Deutsche sind hilfsbereit, aber verschlossen. Affektive Komponenten der Einstellung ungarndeutscher Jugendlicher zur deutschen Sprache und ihrer Sprecher“. In: KegYeS, ERIKA / RENATA KRISTON / MANUELA SCHÖNBERGER (Hg.): Sprachen, Literaturen und Kulturen im Kontakt. 25. Fachtagung der GeSuS. Hamburg: Dr.-Kovač-Verlag (Sprache und Sprachen in Forschung und Anwendung), 533-540.

KoBILAROV-GÖTZE, GUDRUN (1972): Die deutschen Lehnwörter der ungarischen Gemeinsprache. Wiesbaden (Veröffentlichungen der Societas Uralo-Altaica. 7). 
KrAUSS, KARL-PETER (Hg.) (2014): Normsetzung und Normverletzung. Alltägliche Lebenswelten im Königreich Ungarn vom 18. bis zur Mitte des 19. Jahrhunderts. Stuttgart (Schriftenreihe des Instituts für donauschwäbische Geschichte und Landeskunde. 19).

LÁSZló, SAROLTA (1996): Probleme der Mikrostruktur. In: HESSKY, REGINA (Hg.): Lexikographie zwischen Theorie und Praxis. Das deutsch-ungarische Wörterbuchprojekt. Tübingen: Max Niemeyer Verlag, 21-48.

MÁRKUS, ÉVA (2014): Die deutsche Mundart von Deutschpilsen/Nagybörzsöny. Wien: Praesens Verlag.

MOLLAY, KARL (1986): Das Wörterbuch des Frühneuhochdeutschen in Ungarn. In: ÁGEL, VILMOS / RAINER PAUl / LAJOS SZALAI (Hg.): Beiträge zur historischen Lexikographie. Bp. (Budapester Beiträge zur Germanistik. 15), 111-112.

MüLlER, MÁRTA (2016): „Ein unermäßliches Land von Begriffen.“ Dialektlexikographische Konzeptionen im Vergleich. Budapest: ELTE Germanistisches Institut (Budapester Beiträge zur Germanistik. 74).

NIEBAUM, HERMANN (1986): Lemma und Interpretament. Zur Problematik der Artikelgestaltung in Dialektwörterbüchern. In: FRIEBERTSHÄUSER, HANS (Hg.): Lexikographie der Dialekte. Beiträge zur Geschichte, Theorie und Praxis. Tübingen: Max Niemeyer Verlag, 125-143.

NIEBAUM, HERMANN (1989): Diatopische Markierungen im allgemeinen einsprachigen Wörterbuch. In: Hausmann, Franz J. / OSKar ReICHMANN / Herbert ERnST WiEGAND / LAdislaV ZGusta (Hg.): Wörterbücher. Ein internationales Handbuch zur Lexikographie. Bd. 1. Berlin/New York: de Gruyter (HSK. 5.1), 662-668.

NiEBAuM, HeRmann / JÜRGEN MACHA (1999): Einführung in die Dialektologie des Deutschen. Tübingen: Max Niemeyer Verlag.

NYOMÁRKAY, ISTVÁN (2012): A közép-kelet-európai nyelvek szellemi rokonsága [Die geistige Verwandtschaft der Sprachen in Mittelosteuropa]. In: Magyar Nyelvőr 2012/1, 1-13.

PARRILlo, VinCENT N. (1994): Diversity in America: A Sociohistorical Analysis. In: Sociological Forum 9/4, 523-545.

PFWB = Pfälzisches Wörterbuch (1965-1968). Bd. 1. Wiesbaden: Franz Steiner Verlag.

PÜSCHEL, ULRICH (1989): Evaluative Markierungen im allgemeinen einsprachigen Wörterbuch. In: HAUSMANN, FranZ J. / OSKAR REICHMANN / HERBERT ERNST WIEGAND / LADISLAV ZGUSTA (Hg.): Wörterbücher. Ein internationales Handbuch zur Lexikographie. Bd. 1. Berlin/New York: de Gruyter (HSK. 5.1), 694-699.

SchA = Schulatlas für die ungarndeutschen Nationalitätenschulen (2016). Budapest: Cartographia Tankönyvkiadó Kft.

SCHLAEFER, MiCHAEL (2002): Lexikologie und Lexikographie. Eine Einführung am Beispiel deutscher Wörterbücher. Berlin: Erich Schmidt Verlag.

SEEWANN, GERHARD (2012a): Geschichte der Deutschen in Ungarn. Band 1. Von Frühmittelalter bis 1860. Marburg: Verlag Herder-Institut (Studien zur Ostmitteleuropaforschung. 24/1).

SEEWANN, GERHARD (2012b): Geschichte der Deutschen in Ungarn. Band 2. 1860 bis 2006. Marburg: Verlag Herder-Institut (Studien zur Ostmitteleuropaforschung. 24/2).

SHWB = Südhessisches Wörterbuch (1965-1968). Bd. 1. Marburg: N. G. Elwert Verlag.

SPREE, ULRIKE (2012): Wörterbücher und Enzyklopädien. In: KUHLEN, RAINER (Hg.): Grundlagen der praktischen Information und Dokumentation. 6. Auflage. Berlin/Boston: de Gruyter Saur, $550-559$.

SSWB = Siebenbürgisch-Sächsisches Wörterbuch (2006). Bd. 9. Neubearbeitung (Q - R). Bukarest: Rumänischer Akademie Verlag u. Köln [u. a.] : Böhlau Verlag.

TAFFERNER, ANTAL (1941): Vértesboglár, egy hazai német település leírása [Vértesboglár, eine deutsche Siedlung in Ungarn]. Budapest: Dunántúli Pécsi Egyetemi Könyvkiadó és Nyomda R.-T.

THWB = Thüringisches Wörterbuch (1991). Bd. 1. Jena: Akademie Verlag.

WBBDM = Wörterbuch der Banater Deutschen Mundarten (2013). Bd. I. München: IKGS Verlag. 
WBÖ = Wörterbuch der bairischen Mundarten in Österreich (1970, 1976, 1983, 1998, 2015). 5 Bde. Wien: Verlag der österreichischen Akademie der Wissenschaften.

WiegAND, HERBERT ERNST (1989a): Der Begriff der Mikrostruktur: Geschichte, Probleme, Perspektiven. In: HAUSMANN, Franz JOSEF / OSKAR REICHMANN / HERBERT ERNST WIEGAND / LADISLAV ZGUSTA (Hg.): Wörterbücher. Ein internationales Handbuch zur Lexikographie. Bd. 1. Berlin/New York: de Gruyter (HSK. 5.1), 409-462.

WIEGAND, HERBERT ERNST (1989b): Formen von Mikrostrukturen im allgemeinen einsprachigen Wörterbuch. In: HAUSMANN, FRANZ JOSEF / OSKAR REICHMANN / HERBERT ERNST WIEGAND / LAdislav ZGUSTA (Hg.): Wörterbücher. Ein internationales Handbuch zur Lexikographie. Bd. 1. Berlin/New York: de Gruyter (HSK. 5.1), 462-501.

WIEGAND, HERBERT ERNST (1998): Wörterbuchforschung. Untersuchungen zur Wörterbuchbenutzung, zur Theorie, Geschichte, Kritik und Automatisierung der Lexikographie. 1. Teilband. Berlin/New York: de Gruyter.

Wiesinger, Peter (1980): Deutsche Sprachinseln. In: Althaus, Hans Peter / Helmut HenNe / HERBERT ERNST WIEGAND (Hg.): Lexikon germanistischer Linguistik. Band 3. Tübingen: Niemeyer, 491-501. 



\title{
SIEBENBÜRGISCH-SÄCHSISCHES WÖRTERBUCH
}

\author{
Sigrid Haldenwang
}

\section{EINFÜHRUNG: DIE SIEBENBÜRGISCHE SPRACHLANDSCHAFT; WESENSZÜGE DES SIEBENBÜRGISCH-SÄCHSISCHEN DIALEKTS}

Die siebenbürgisch-sächsischen Mundarten dieser mittelalterlichen deutschen Sprachinsel ${ }^{1}$ Siebenbürgen innerhalb des Karpatenbogens haben, getrennt vom deutschen Sprachraum, durch Mischung und Ausgleich im Laufe von über 850 Jahren eine eigenständige gut überschaubare Entwicklung durchgemacht. Die größte Schwierigkeit bei der Erforschung der siebenbürgisch-sächsischen Mundarten und ihren Entwicklungsphasen rührt nicht nur von dem Mangel an echten alten Sprachquellen her, sondern auch von der Unmöglichkeit, früher vollzogene sprachliche Wandlungen im Einzelnen aufzudecken und zu datieren. Die langjährigen Forschungen zwecks genauer Ermittlung des Auswanderungsgebietes unserer Vorfahren haben zu der Einsicht geführt, dass ein Vergleich mit heutigen Sprachverhältnissen im deutschen Sprachraum und in den Gebieten der Ostsiedlung zu nur bedingt oder teilweise gültigen Ergebnissen führen kann, da diese Mundarten selber verschiedentlich großen Veränderungen unterworfen waren. Das SiebenbürgischSächsische, dem 243 Ortsmundarten angehören, ist ein mittelfränkischer Dialekt mit moselfränkischem, linksrheinischem Gepräge. Die meisten Gemeinsamkeiten hat dieser Dialekt mit den Mundarten, die im deutschen Sprachraum zwischen Köln und Trier gesprochen werden, sowie mit dem Luxemburgischen. Zu der westfränkischen Grundlage kamen im Laufe der Ansiedlung ostmitteldeutsche und oberdeutsche Sprachelemente hinzu (vgl. REIN 1963: 24-64). Bemerkenswert sind zudem Einflüsse österreichischer Umgangssprache während der Zugehörigkeit zur Habsburgermonarchie. Das jahrhundertelange „Miteinander“ der Sachsen, Rumänen und Ungarn in derselben Heimat spiegelt sich auch im Wortschatz wieder.

Ein auffallender Wesenszug des siebenbürgisch-sächsischen Dialekts ist die Vielfalt und große Verschiedenheit der einzelnen Ortsmundarten, die - in den Lautformen des Vokalismus - von Ort zu Ort oft in erheblichem Maße voneinander abweichen. Eine gewisse Einheitlichkeit des Dialekts ist durch den Konsonantismus gesichert (vgl. CAPESIUS 1990a: 122-138). Die siebenbürgisch-sächsische Sprachlandschaft ist nicht einheitlich, man unterscheidet das südsiebenbürgische ${ }^{2}$

1 Vgl. WIESINGER (2012: 151-164).

2 Das südsiebenbürgische Mundartgebiet kann im Großen folgendermaßen gegliedert werden: das Zwischenkokelgebiet, anschließend das Mittlere Siebenbürgen, dazu gehört Schässburg 
und das nordsiebenbürgische Mundartgebiet ${ }^{3}$ (vgl. RICHTER 1986: VII-X), die ihrerseits nicht einheitlich sind. Gemeinsamkeiten zeigen sich in der grammatischen Struktur und im Wortschatz (vgl. auch zu obigen Erläuterungen: HALDENWANG 2009: 11-14 und NÄGLER 1979: 168-173).

\section{ZUM STANDARDWERK DER SIEBENBÜRGISCH-SÄCHSISCHEN SPRACHFORSCHUNG: DAS SIEBENBÜRGISCH-SÄCHSISCHE WÖRTERBUCH - MATERIALIEN, PUBLIKATIONSSTAND, GESCHICHTE UND ZIELE}

Das „Siebenbürgisch-Sächsische Wörterbuch“ ist ein schon weit zurückreichendes Unterfangen der siebenbürgisch-sächsischen Sprachforschung und gleichzeitig das älteste und wichtigste verlegerische Projekt des „Forschungsinstituts für Geisteswissenschaften in Hermannstadt - Rumänien“.

Der siebenbürgische Volkskundler, Pfarrer, Sprachforscher und Lexikograf AdOLF SCHULLERUS (geb. 1864, $\uparrow 1928$ ) hat in seiner „Vorgeschichte des siebenbürgisch-deutschen Wörterbuchs" diesem großlandschaftlichen Wörterbuch eine doppelte Bestimmung zugedacht. Aus seiner Sicht sollte es nicht nur der Wissenschaft als Baustein zum Aufbau der deutschen Sprachwissenschaft dienen, sondern auch ein Bild des Volkslebens der Siebenbürger Sachsen im Spiegel der Sprache sein (vgl. SCHULLERUS 1894/1895: 44). Das Wörterbuch ist ein Werk mehrerer Generationen von Wissenschaftlern, Wortgutsammlern, Gutachtern, Chronisten, Dichtern, Schriftstellern sowie zahlreicher Gewährspersonen aus allen Teilen des Mundartgebietes, die Auskunft zur Aussprache, Wortbedeutung, zu Sitte und Brauchtum, Orts- und Flurnamen geleistet haben. Das Wörterbuch will den Wortschatz der rund 243 Ortsmundarten in ihrer lautlichen, grammatischen und bedeutungsmäßigen Eigenart möglichst weitgehend erfassen und nach wissenschaftlichen Grundsätzen bearbeitet wiedergeben.

Es belegt den Allgemeinwortschatz dieser Ortsmundarten in allen bäuerlichen Lebensbereichen: im Alltag und bei festlichen Gelegenheiten (es handelt sich z. B. um Brauchtumsbeschreibungen oder Ansprachen bei Taufe, Hochzeit, Beerdigung, Zusammenkünfte der Nachbarschaften, der Bruder-, Schwesterschaften und der

und Umgebung, weiter östlich das Keisder Gebiet, anschließend das Repser Gebiet und südöstlich das Burzenland; ganz südlich das Gebiet zwischen Harbach und Alt, anschließend links gelegen Hermannstadt mit den umliegenden Dörfern; im Westen letztlich der Unterwald mit Mediasch und Umgebung.

3 Dem nordsiebenbürgischen Mundartgebiet gehören 48 Ortsmundarten an (das Nösnerland [Bistritz und Umgebung] und das Reener Ländchen mit den umliegenden Ortschaften). Die tiefste Trennungslinie zwischen dem Norden und dem Süden ergeben zwei Auslauterweichungen: die „Eifler Regel“ oder der $n$-Abfall in Satzphonetik und Wortzusammenhang [kātəštä ] 'Kettenstich', die heute ausschließlich in Südsiebenbürgen wirksam ist, und die regressive Erweichung stimmloser Verschlusslaute vor stimmhaften [bagdbruit] 'bäckt Brot', [hạgbret] 'Hackbrett' als nordsiebenbürgische Eigentümlichkeit (vgl. dazu: CAPESIUS 1990a: 132-133 und THUDT 1968: 19-36). 
Zünfte); berücksichtigt die mundartliche Volks- und Kunstdichtung, bringt Redensarten, Sprichwörter, Vergleiche, Zaubersprüche, Heilsegen, Rätsel, Kinderspiele, Handwerkwortschatz sowie aus dem Rumänischen, Ungarischen und anderen Sprachen übernommene Entlehnungen. Hinzu kommen von den Eigennamen alle toponomastischen Bezeichnungen im weitesten Sinn (aber keine Personennamen, auBer solchen Vornamen, die zahlreiche lautliche Varianten aufweisen oder auch im Sinne von Gattungsnamen auftreten) sowie Pflanzennamen, oft verbunden mit Ausführungen zu Volksmedizin und Aberglauben.

Eine Besonderheit des Wörterbuchs besteht darin, dass auch die siebenbürgische Urkundensprache [alte Sprache $=$ a. Spr.] berücksichtigt wird. Es handelt sich um deutsche Belege in siebenbürgischen Quellen von der Mitte des 13. Jahrhunderts bis zur Mitte des 19. Jahrhunderts, die unter dem Gesichtspunkt ihrer Bedeutung für den mundartlichen Einfluss auf die deutsche Schriftsprache in Siebenbürgen oder für die Geschichte der deutschen Sprache gebracht werden.

\subsection{Materialgrundlage, das Wörterbucharchiv}

Ein Wörterbuch kann ohne eine sinnvoll angelegte Materialgrundlage nicht zustandekommen. Das Wörterbucharchiv enthält etwa eine Million Zettel, die in alphabetischer Reihenfolge in 155 Zettelkästen im Raum der Wörterbuchstelle aufbewahrt werden. ${ }^{4}$

Das Archiv beinhaltet:

- Das im Laufe von über hundert Jahren von geschulten und ungeschulten freiwilligen Sammlern wie auch von einzelnen Bearbeitern zusammengetragene Zettelmaterial. Diesem sind auch Exzerpte aus den handschriftlichen Wörtersammlungen (zweite Hälfte des 18. Jahrhunderts bis einschließlich der ersten Hälfte des 19. Jahrhunderts) und Exzerpte aus gedruckten siebenbürgisch-sächsischen Wörterbuchproben und -vorarbeiten sowie aus beginnend mit der zweiten Hälfte des 19. Jahrhunderts folgenden zu den siebenbürgisch-sächsischen Mundarten gehörigen Facharbeiten des 20. Jahrhunderts ${ }^{5}$ zuzuordnen.

4 Vgl. HaldeNWANG (1995: 134-139).

5 Die angeführten Wortschatzsammlungen und Facharbeiten liegen größtenteils in der Arbeitsstelle des Wörterbuchs vor, sie sind exzerpiert worden und gehören zum Zettelarchiv des Wörterbuchs. Auf Wortschatzsammlungen, von denen im Wörterbucharchiv nur Exzerpte auf Zetteln vorliegen, wird unter Kapitel 5.1 aufmerksam gemacht, ihr Standort ist meist unbekannt. 
- Direktaufnahmen, die um 1960 von geschulten Mitgliedern des damaligen Wörterbuchteams ${ }^{6}$ auf Geländefahrten in den meisten Ortschaften des Mundartgebietes durchgeführt wurden ${ }^{7}$ und dann auch von den einzelnen Mitarbeitern in den 70er und 80er Jahren für weniger gut belegte Wörter noch eingebracht werden konnten. ${ }^{8}$

- Indirekte Aufnahmen durch Fragebogenversendung (in den Jahren 1935-1942) sowie durch Listen von Wörtern und Wendungen, die freiwillige Helfer einsandten.

- Ausschriften aus schöngeistiger Mundartliteratur.

- Ausschriften aus historischen Quellen und Archiven von der Mitte des 13. Jahrhunderts bis zur Mitte des 19. Jahrhunderts.

- Der in zwei Zettelkästen in alphabetischer Reihenfolge angelegte siebenbürgisch-sächsische Pflanzennamenbestand.

- Die Hermannstädter Wörterbuchstelle beherbergt auch „Das Handschriftliche Namenverzeichnis der siebenbürgisch-sächsischen Familiennamen“, zusammengestellt von ALBERT KLEIN. ${ }^{9}$ Es wurde aufgrund der Taxenträger der evangelischen Kirche A.B. vom Jahre 1924 erarbeitet. Das Namenverzeichnis ist mit einer Anleitung zur Benützung der Karthothek versehen, die Familiennamen sind alphabetisch geordnet. Es ist und bleibt für das Wörterbuch ein nützliches Auskunftsmaterial, da manche siebenbürgisch-sächsische Flurnamenbezeichnung anhand dieses Namenverzeichnisses zu dem entsprechenden Familiennamen gestellt werden kann, auf den sie zurückgeht.

\subsection{Bearbeitungs-/ Publikationsstand}

SSWB = Siebenbürgisch-Sächsisches Wörterbuch. Bisher sind 10 Bände erschienen: Bd. $1(A-C) 1924$ bearb. von ADOLF SCHULLERUS [erste Lieferung schon ab 1908, KARL J. TRÜBnER], Bd. 2 ( $D-F) 1926$ bearb. von AdOlF Schullerus, FRIEDRICH HOFSTÄDTER und GEORG KEINTZEL, Bd. 5 (R-Salarist [alte Folge = SSWB 5 A. F.] 1929-1931 bearb. von JOHANN ROTH, GUSTAV GÖCKLER [FRIEDRICH KRAUSS Hg.]. Berlin/Leipzig: Walter de Gruyter. Bd. 3 (G) 1971 bearb. von Anneliese Thudt, Annemarie BIEsselt-Müller, Arnold PANCRATZ, BernHARD CAPESIUS, GISELA RICHTER, Bd. $4(H-J) 1972$ bearb. von ANNELIESE THUDT, BERNHARD CAPESIUS, Gisela RiCHTER, ROSWITHA BRAUN-SÁNTA, Bd. 5 ( $K$ [neue Zählung]) 1975 bearb. von ANNELIESE THUdT, GiSElA Richter, SigRID HALDENWANG, Roswitha BRAUn-SÁNTA (Bukarest: Rumänischer Akademie-Verlag und Berlin: Walter de Gruyter. Bd. 6 (L) 1993 bearb. von ANNELIESE THUdT, GiSELA

6 AnNeliese Thudt, Gisela RichteR; auch HEINRICH MANTSCH und RuTH KisCH vom Linguistikinstitut Bukarest.

7 Vgl. dazu: Richter, Gisela / THUDT, ANNELIESE (1964: 91-108).

8 Die große Auswanderungswelle der Siebenbürger Sachsen nach der Wende in Rumänien hat dazu geführt, dass Mundartaufnahmen, von „um 1990“ und später nur noch im Rahmen der Möglichkeit gezielt fortgesetzt werden konnten und können.

9 Von 1969 bis 1990 Bischof der Evangelischen Landeskirche A.B. in Rumänien. 
Richter, Sigrid Haldenwang, Bd. 7 (M) 1998 bearb. von AnNELIESE THUdT, SigRID HALDENWANG, Ute MAURER [unter Mitarbeit von IsOLDE HubER und MALWINE DENGEL], Bd. $8(N-P) 2002$ bearb. von ANNELIESE THUdT, SIGRID HALDENWANG, Stefan Sienerth, Ute MAURER [unter Mitarbeit von MALWINE DENGEL], Bd. $9(Q-R) 2006$ bearb. von Isolde Huber, MALWINE DENGEL, Sigrid HALDENWANG, Stefan Sienerth, Ute MAURER, Bd. 10 (S-Schenkwein) 2014 bearb. von MALWINE DENGEL, SigRID HALDENWANG. Bukarest: Rumänischer Akademie-Verlag und Köln/Weimar/Wien: Böhlau.

Ab Band $6(L)$ hat der Rumänische Akademie-Verlag das Verkaufsrecht für Rumänien und Südosteuropa und der Böhlau-Verlag für Westeuropa.

\subsection{Geschichtlicher Werdegang}

\subsubsection{Von Wortschatzsammlungen und -proben zum eigentlichen Wörterbuch}

Eine Liste mit 33 siebenbürgisch-sächsischen Wörtern, die den altgermanischen (gotischen) Ursprung des Dialektes und damit die Ansässigkeit der Sachsen in Siebenbürgen seit dem Altertum beweisen sollten, veröffentlichte vor genau 353 Jahren JOHANNES TRÖSTER $(\dagger 1670)$ in seinem Buch „Das Alt- und Neu- Teutsche Dacia. Das ist: Neue Beschreibung des Landes Siebenbürgen [...]" (TRÖSTER 1666: 160-161).

So bleibt dem Buch sein guter Ruhm, daß es [...] als Beispiel für die vielen in der Mundart noch vorhandenen, 'heut zu Tag in Teutschland ungebräuchliche Wörter, so von der alten Teutschen Sprach überblieben' die erste Sammlung siebenbürgischer Idiotismen $\left[{ }^{10}\right]$, mit zum Teil ganz respektabeln etymologischen Erläuterungen und guten Bemerkungen über den mundartlichen Unterschied der einzelnen Gegenden des Landes, enthält (SCHULLERUS 1924: X-XI).

Das Zustandekommen des eigentlichen „Siebenbürgisch-Sächsischen Wörterbuchs" geht auf den bedeutenden deutschen Philosophen, Historiker und Sprachforscher GOTTFRIED WILHELM LEIBNIZ (geb. 1646, † 1716) zurück, der Ende des 17. Jahrhunderts den Wunsch geäußert hatte, eine Sammlung siebenbürgisch-sächsischer Wörter zu erhalten, die er zur Klärung wichtiger geschichtlicher Fragen für notwendig hielt. Die erste handschriftliche Sammlung siebenbürgisch-sächsischer Wörter haben wir erst aus der zweiten Hälfte des 18. Jahrhunderts, von JOSEPH TEUTSCH (geb. 1720, $†$ 1770). Es ist zunächst ein „Verzeichnis von rund 80 AltDacisch-Teutschen Wörtern, welche unter den Sachsen in Burzenland noch im Gebrauche sind", das erst im 19. Jahrhundert gedruckt wurde (TEUTSCH 1899: 24). Ebenso blieb seinerzeit ungedruckt eine umfangreiche Liste sächsischer Idiotismen, ${ }^{11}$ die MARTIN FELMER (geb. 1720, $†$ 1767) in seine unvollendet gebliebene „Abhandlung von dem Ursprung der Sächsischen Nation“ aufgenommen hatte, die

10 Idiotismus 'ein Mundartwort, das in der hochdeutschen Schriftsprache nicht bezeugt ist'.

11 Mit ausdrücklichem Nachweis dessen, dass sie keinesfalls gotisch seien, sondern eine deutsche Mundart darstellen. 
dann später unter einem andern Titel „Die Martin Felmer-Handschrift. Eine Darstellung der Geschichte und Volkskunde der Siebenbürger Sachsen aus dem Jahre 1764" von GOTTLIEB BRANDSCH herausgegeben wurde (BRANDSCH 1935). Im gleichen Sinn sammelte JOHANN SEIVERT (geb. 1735, † 1785) sächsische Idiotismen und vereinigte sie in zwei handschriftlichen Verzeichnissen „Idiotikon. Handschriftliche Sammlungen v. Hermannstädter Mundartwörtern, 1760-1780“ (SEIVERT 1760-1780) und „Versuch (eines Siebenbürgisch-sächsischen Wörterbuchs). Handschriftliche Sammlung v. Hermannstädter Mundartwörtern 1773“ (SEIVERT 1773). ${ }^{12}$ Diese Verzeichnisse ergänzte Johann BINDER (geb. 1767, $\dagger$ 1805) durch eigene Sammlungen und wollte sie zusammen herausgeben, wozu es aber infolge seines frühen Todes nicht kam. Seine „Handschriftliche Sammlung siebenbürgischsächsischer Wörter und Redensarten“ (meist aus Schässburg), „schriftsprachlich aufgezeichnet, 1861“13 (BINDER 1861), hat ausschließlich eine semasiologische Bestimmung.

In der ersten Hälfte des 19. Jahrhunderts kamen dann viele handschriftliche Wörtersammlungen zustande. Von bleibendem Wert ist die Sammlung von DANIEL JOSEF LEONHARD (geb. 1786, $\uparrow 1853$ ) ,Verzeichnis einiger siebenb.-sächsischer eigenthümlicher Wörter oder Idiotismen. Handschrift, 1826“14 (LEONHARD 1826) und die von Josef TRAuSCH (geb. 1795, † 1871) „Wörterbuch SiebenbürgischSächs. Idiotismen. Handschrift, 1816-1868“ (TRAUSCH 1816-1868). ${ }^{15}$ Schließlich gehört in diese Reihe auch der Beitrag des Mundartdichters VIKTOR KäSTNER (geb. 1826, † 1857): „Idiotikon. Handschriftliche Idiotismensammlung, Mitte 19. Jh.“ (KÄSTNER 1850). Es enthält eine Reihe von Redewendungen sowie Sprichwörtern und deren Parallelen aus andern Mundarten.

Gedruckte siebenbürgisch-sächsische Wörterbuchproben und -vorarbeiten sind erst für die zweite Hälfte des 19. Jahrhunderts bezeugt. Auf südsiebenbürgischem Gebiet wollen wir die Arbeiten von JohanN CARL SCHUller (geb. 1794, $\uparrow$ 1865), JOSEF HALTRICH (geb. 1822, $\uparrow$ 1886), und JOHANN WOLFF (geb. 1844, $\uparrow 1893$ ) hervorheben, auf nordsiebenbürgischem Gebiet die Arbeiten von GEORG BERTLEFF (geb. 1833, $\dagger$ 1876), Friedrich KraMER (geb. 1843, $\dagger$ 1911), GEORG KEINTZEL (geb. $1858, \uparrow 1925$ ) und GUSTAV KISCH (geb. 1869, $\uparrow$ 1938). Es ist zunächst JoHANN CARL SCHULLER, der eine Sammlung von 3000 Wörtern unter dem Titel „Siebenbürgische Etymologien und Analogien“ der Wiener Akademie der Wissenschaften einreichte, die vom ersten Professor für Germanistik an der Wiener Universität und späteren Präsidenten der Akademie TH. von KARAJAN (geb. 1810, $\uparrow 1873$ ) in der Sitzung vom 7. XI. 1849 (KARAJAN 1849: 227-236) scharfe Kritik ernten musste, da Beigaben von Belegstellen fehlten und die Auseinanderlegung der Idiotismen nach Örtlichkeiten. Durch diese Kritik entmutigt, ließ SCHULLER die Wörterbucharbeit zunächst liegen und erst 1865 kurz vor seinem Tod ging er an die

12 Ehemalige Sammlung des Brukenthalmuseums, heute im Nationalarchiv Hermannstadt unter der Signatur EE 1-4 Nr.43. Von beiden Sammlungen sind im Archiv des Wörterbuchs nur Exzerpte auf Zetteln vorhanden.

13 Im Archiv des Wörterbuchs nur Exzerpte auf Zetteln vorhanden.

14 In der handschriftlichen Abschrift JOSEF HALTRICHS.

15 In der handschriftlichen Abschrift JOSEF HALTRICHS. 
Veröffentlichung seiner Wörterauswahl, die aber erst nach seinem Tod unter dem Titel „Beiträge zu einem Wörterbuche der siebenbürgisch-sächsischen Mundart“ (SCHULLER 1865) erschienen ist. Die Aufgabe des Wörterbuchs sah er darin:

jedes mundartliche Wort durch ein dem Sinne nach vollständig oder annäherungsweise entsprechendes hochdeutsches Wort zu übersetzen oder aber die Wurzel, den Ursprung desselben nachzuweisen (CAPESIUS 1990c: 234).

Schließlich hatte der „Verein für siebenbürgische Landeskunde“ das Voranbringen der Wörterbucharbeiten zu seiner eigenen gemacht und beauftragte 1861 JOSEF HALTRICH mit der Ausarbeitung eines Plans für ein eigentliches Wörterbuch, das dann unter dem Titel „Plan zu Vorarbeiten für ein Idiotikon der siebenbürgischsächsischen Volkssprache“ (HALTRICH 1865) veröffentlicht wurde. Zu seinen Veröffentlichungen gehört auch die Abhandlung „Negative Idiotismen der siebenbürgisch-sächsischen Volkssprache“ (HALTRICH 1865/1866). Er entfaltete durch Versendung des Plans und Aufforderung zur Sammeltätigkeit eine rege Werbung, die aber ohne starken Widerhall und Erfolg blieb.

Dazu kommen unabhängig vom Landeskundeverein zwei Veröffentlichungen in Nordsiebenbürgen, die für die Wörterbucharbeit wichtiges Material brachten. Es sind GEORG BERTLEFFS „Beiträge zur Nösner Volkssprache“ (BERTLEFF 1866/1867: 4-49; 1867/1868: 1-23). Sie enthalten neben einer Reihe volkstümlicher Erzählungen in verschiedenen Ortsmundarten etwa 600 Wörter ebenfalls in sechs verschiedenen Ortsmundarten bzw. Mundartgruppen des Nösnerlands. ${ }^{16}$ BERTLEFFS Anliegen war in erster Linie phonetischer Natur. Er wollte aus den „sich abschleifenden Dorfmundarten [...] retten, was noch gerettet werden kann" (CAPESIUS 1990c: 236). FRIEDRICH KRAMERS Veröffentlichung „Idiotismen des Bistritzer Dialectes. Beitrag zu einem siebenbürgisch-sächsischen Idiotikon“ (KRAMER 1875/1876) enthält Bistritzer bzw. nösnische Ergänzungen zu dem von HALTRICH geplanten Wörterbuch (das nur Rücksicht auf die Mundarten von Hermannstadt, Mühlbach, Mediasch, Schässburg) nimmt. Im Vordergrund steht die Etymologie im Dienste der Herkunftsfrage. ${ }^{17}$

Die Wörterbucharbeit wurde auch durch eine Reihe von Publikationen gefördert, die vor allem das Nordsiebenbürgische in den Vordergrund treten ließ. Zunächst sind die Arbeiten von GeORG KeINTZEL zu nennen: „Die Lautlehre der Mundarten von Bistritz und Sächsisch-Reen. Mit Berücksichtigung abweichender Lautverhältnisse in den sächsischen Ortsdialekten der Umgebung“ (KEINTZEL 1894: 133-222), „Zum Vokalwandel im Siebenbürgisch-Sächsischen seit der Einwanderung“ (KEINTZEL 1924: 161-172) sowie „Nösner Idiotismen“ (KEINTZEL 1897: 47-80). Von den Arbeiten GusTaV KISCHS führen wir an: „Nösner Wörter und Wendungen. Ein Beitrag zum siebenbürgisch-sächsischen Wörterbuch“

16 Zweitname der Stadt Bistritz; etymologisch zum luxemburgischen Familiennamen Nies, Niesen [Patronymikum im Genitiv] gestellt.

17 Eine wichtige Triebfeder der siebenbürgisch-sächsischen Mundartforschung jener Zeit war immer wieder die Frage, woher die deutschen Siedler/-innen Siebenbürgens stammen, welches ihre eigentliche „Urheimat“ ist, die man mit Hilfe der Sprachwissenschaft zu lösen suchte. 
(KISCH 1900), „Vergleichendes Wörterbuch der Nösner (siebenbürgischen) und moselfränkisch-luxemburgischen Mundart [...]“" (KISCH 1905: 5-273).

Inzwischen widmete sich der südsiebenbürgische Mundartforscher JOHANN WOLFF (geb. 1844, $\uparrow 1893$ ) der Aufgabe, das Erbe HALTRICHS systematisch zu ordnen. Während HALTRICH mehr oder weniger gelegentliche Beobachtungen verwertet hatte, exzerpierte WOLFF alles, was bisher in Druckschriften veröffentlicht oder handschriftlich gesammelt worden war, auf einzelne Zettel und ordnete sie nach bestimmten Gesichtspunkten. Das ganze Zettelmaterial legte er in 26 Mappen an, die rund 10000 Zettel enthalten. Als Proben, wie er sich die Wörterbuchkonzeption dachte, arbeitete er einige Wortartikel aus und veröffentlichte sie unter dem Titel „Vorarbeiten zum siebenbürgisch-deutschen Wörterbuch“ (WOLFF 1897: 587650). Es sind dafür meist solche Artikel des Wörterbuchs ausgewählt worden, die einen breiten, weite Perspektive ermöglichenden kulturgeschichtlichen Hintergrund haben und die zugleich für andere Fragen, so für die nach der Herkunft der Siebenbürger Sachsen, maßgebend sind (vgl. SCHULLERUS 1924: XXVIII).

Nach WoLFFs frühem Tode übernahm der „Verein für Siebenbürgische Landeskunde" das Erbe am Wörterbuchmaterial und gab es an ADOLF SCHULLERUS weiter, der dann 35 Jahre lang die Wörterbucharbeit als treibende Kraft, führender Geist und fruchtbarster Ausarbeiter maßgebend bestimmt hat. ${ }^{18}$ Die Bearbeitung der Wortartikel der einzelnen Buchstaben wurde verschiedenen Wissenschaftlern anvertraut. ${ }^{19}$ Aufgrund des vorhandenen relativ umfangreichen Materials und nach Veröffentlichung von Proben ausgearbeiteter Artikel wurden dann die Grundsätze für die weitere Ausarbeitung festgelegt und schließlich die Herausgabe des Wörterbuchs beschlossen.

In den 1905 beschlossenen Grundsätzen für die Ausarbeitung des Wörterbuchs trat zum ersten Mal die Ansicht auf, dass das Wörterbuch nicht ein Idiotikon sein soll, das nur der Mundart eigentümliche Wörter, Wendungen sowie Namen enthält, sondern den ganzen Sprachschatz, sofern er sich durch sichere Belege als in volkstümlichem Gebrauch befindlich erweist. Damit ging die neue Ausarbeitung über alle bisherigen Vorläufer weit hinaus und vergrößerte den Umfang des Werkes in beträchtlichem Maß. Auch wurde zum ersten Mal die Heranziehung urkundlichen Materials nach sprachlichen und kulturgeschichtlichen Gesichtspunkten ins Auge gefasst. Zudem sollte das Wörterbuch sich auch mit der Behandlung von materiellem und geistigem Volksgut beschäftigen. Von dieser Einstellung ausgehend hat SCHULLERUS weitgehend den volkskundlich-enzyklopädischen Charakter, der von ihm ausgearbeiteten und unter seiner Leitung erschienenen Bände bestimmt. In den vom Redaktionsausschuss angenommenen Grundsätzen wurden ferner der hochdeutsche Ansatz der Stichwörter in streng alphabetischer Anordnung, die Schreibung der mundartlichen Belege in einer der Schriftsprache angenäherten Lautschrift, die Bezeichnung der Quellen usw. festgelegt. Infolge der Klärung all dieser

18 Sein Nachlass mit siebenbürgisch-sächsischen Mundartwörtern von 'um 1900' ist auf Zetteln exzerpiert worden und gehört auch zur Materialgrundlage des Wörterbuchs.

19 Die Namen der Bearbeiter sind dem Bearbeitungs- / Publikationsstand zu entnehmen (siehe: Kapitel 2.2). 
Fragen konnte die erste Lieferung 1908 in der Bearbeitung von ADOLF SCHULLERUS im Verlag Karl J. Trübner, Strassburg (nach 1918 Walter de Gruyter, Berlin/Leipzig) erscheinen. Sie enthält auch ein Vorwort des Bearbeiters mit einer ausführlichen Geschichte des Wörterbuchs und Lauttafeln, die 144 nach dem Vokalismus ausgewählte Wörter in 51 Ortsmundarten aus verschiedenen Gegenden Siebenbürgens bringen (vgl. SCHULLERUS 1924: XLVII-LXXII), sodass in der Zeitspanne 1924-1931 die Bände $A-C, D-F$ und $R$-Salarist [alte Folge $=$ A. F.] erscheinen konnten. Positiv soll hervorgehoben werden, dass es das bleibende Verdienst des Sprachforschers und Lexikografen ADOLF SCHULLERUS ist, die endlosen Vorarbeiten durch eine entschlossene Tat dem Anfang einer Verwirklichung zugeführt zu haben, auch wenn in diesen Bänden einige Mängel zu vermerken sind. ${ }^{20}$

Nach dem Tod der Mitarbeiter erfolgte eine zeitweilige Unterbrechung der Wörterbucharbeit.

\subsubsection{Wiederaufnahme der Wörterbucharbeiten, dank eines Kooperationsabkommens}

Aufgrund eines Kooperationsabkommens zwischen der Akademie der Rumänischen Volksrepublik und der Deutschen Akademie der Wissenschaften zu Berlin kam es im Jahr 1955 zur Wiederaufnahme der Wörterbucharbeiten. Im Rahmen dieses Abkommens wurde beschlossen, dass die Rumänische Akademie die Schirmherrschaft für die Ausarbeitung und den Druck des Wörterbuchs übernimmt, die Durchsicht der Manuskripte aber die Aufgabe der Deutschen Akademie ist. Von deutscher Seite war THEODOR FRINGS (geb. 1886, † 1968) von rumänischer Seite EMIL PETROVICI (geb. 1859, †1968) verantwortlich. Das Abkommen sah vor, dass FRIEDRICH HOLZTRÄGER (geb.1888, † 1970), der 1934 vom Verein für siebenbürgische Landeskunde zur Leitung der Wörterbucharbeiten bestimmt worden war, und der zwischen 1945 und 1955 die Arbeit eigenständig fortgesetzt hatte, im Auftrag der Rumänischen Akademie die begonnene Arbeit weiterführen und koordinieren solle. Im Oktober 1957 trat Holzträger von der Leitung des Wörterbuchs zurück. In demselben Jahr wurde auf Vorschlag der beiden Akademien eine Wörterbuchkommission ins Leben gerufen, der BERNHARD CAPESIUS (geb. 1889, † 1981) (Vorsitzender), CARL GÖLlNER (geb. 1911, † 1995) FRIEDRICH HOLZTRÄGER (bis 1959),

20 Es fehlen bei den Stichwörtern:

- Der vorangestellte, sogenannte Lautkopf, mit Anführung aller vorhandenen Lautformen;

- Die Datierung der Mundartbelege und oft auch die Ortsangabe;

- Ein System in der Anlage von Synonymen- und Vergleichszentralen sowie bei Verweisen;

- Ein System bei etymologischen Angaben (der Etymologie wurde im Hinblick auf die Lösung der „Urheimatfrage“ eine unverhältnismäßig hohe Bedeutung beigelegt, zahlreiche Hinweise auf andere Mundartwörterbücher erfolgten, selbst wenn die Etymologie fraglich und auch durch die vielen Hinweise nicht befriedigend zu klären war).

- Stichwörter größeren Umfangs, wie Substantive, Verben sind der Darstellungsweise entsprechend von erschwerten hochsprachlichen volkskundlichen Exkursen durchwoben. (Siehe dazu: „Das Siebenbürgisch-Sächsische Wörterbuch im Urteil der germanistischen Fachgenossen“ KLEIN 1963: 40-44). 
ARNOLD PANCRATZ (geb. 1859, $\uparrow$ 1939) und HeLMUT PROTZE ${ }^{21}$ angehörten. Ihr oblag die praktische Durchführung der aus dem Akademieabkommen für das Wörterbuch erwachsenden Aufgaben. Die Leitung wurde 1957 BERNHARD CAPESIUS übertragen, der sie bis zum Erreichen der Altersgrenze im Jahre 1963 innehatte. Ursprünglich war das Wörterbuch im Rahmen der Hermannstädter Abteilung der Klausenburger Filiale der Akademie der Rumänischen Volksrepublik erarbeitet worden. Im Jahr 1965 wurde die Arbeitsstelle des „Siebenbürgisch-Sächsischen Wörterbuchs“ der von der Akademie der Sozialistischen Republik Rumäniens neu gegründeten Sektion für Germanistik unter der Leitung von MIHAI ISBĂȘESCU (geb. 1915, $\uparrow$ 1998) angegliedert. Dem Wörterbuchteam der ersten Stunde gehörten damals an: BERNHARD CAPESIUs als wissenschaftlicher Berater, GiSELA RichteR (ab 1956), ANNELIESE ThudT (ab 1956) und Roswitha Braun (ab 1960). Schließlich gründete die Deutsche Akademie der Wissenschaften zu Berlin die Abteilung Siebenbürgisch-Sächsisches Wörterbuch in Leipzig, in deren Rahmen HeLmUT PROTZE die wissenschaftliche Revision der von dem Hermannstädter Wörterbuchteam erarbeiteten Typoskripte oblag. Ab 1965 koordinierte GERHARD ISING ${ }^{22}$ die Zusammenarbeit. ${ }^{23}$ (vgl. zu diesem Abschnitt der Wörterbuchgeschichte CAPESIUS (1990b: 98-104) und PROTZE (1959: 210-218).

Im Jahr 1957 wurden neue Grundsätze für die Darstellung des Wörterbuchs erarbeitet, nach langjähriger Beratung der damaligen zuständigen Stellen. ${ }^{24}$ Für die Neubearbeitung des Buchstabens $G$, der schon von FRIEDRICH HOLZTRÄGER bearbeitet vorlag, doch den neuen Grundsätzen nicht entsprach, musste zunächst der Zettelkatalog neu geordnet und das Wortgut mit Neuaufnahmen ergänzt werden, die auch den mit dem ,sozialistischen Aufbau“ verbundenen Wortschatz Rechnung tragen mussten. BERNHARD CAPESIUS koordinierte die Ausarbeitung der Stichwortstrecke der Buchstaben $G$ bis einschließlich $J$. Von 1963 bis 1977 leitete GISELA RICHTER (geb. 1931, $\uparrow$ 1977) die Wörterbucharbeiten. In dieser Zeitspanne wurde dieselbe Stichwortstrecke überarbeitet und die Wortartikel des Buchstabens $K$ ausgearbeitet und redigiert sowie die Ausarbeitung der Wortartikel des Buchstabens $L$ begonnen. Die Bände $G, H-J$ und $K$ wurden herausgegeben. Auf GisELA RICHTER folgte ANNELIESE THUDT (geb. 1927, $\uparrow 2018$ ). Unter ihrer Leitung wurde das Typoskript der Stichwortstrecke des Buchstabens $L$ für den Druck fertiggestellt und die Typoskripte der Stichwortstrecken $M$ bis einschließlich $O$ und ein Teil der Stichwortstrecke des Buchstabens $P$ erarbeitet. Als ANNELIESE THUDT 1986 in Rente ging, übernahm SIGRID HALDENWANG die Wörterbuchleitung. Die Typoskripte der Stichwortstrecken der Buchstaben $M$ bis einschließlich $O$ wurden revidiert, der weitere Teil der Stichwortstrecke des Buchstabens $P$ und die Stichwortstrecke des

21 Lebensdaten unbekannt.

22 Lebensdaten unbekannt.

23 Im Jahr 1980 trat an Stelle der Deutschen Akademie der Wissenschaften die Sächsische Akademie zu Leipzig. Mit dem Erscheinen des 8. Bandes $(N-P) 2002$ wurde das rumänisch-deutsche Abkommen hinfällig. Im Jahr 1970 wurde die Hermannstädter Sektion zu einer selbstständigen Einrichtung unter der Schirmherrschaft der Akademie für Soziale und Politische Wissenschaften der Sozialistischen Republik Rumänien.

24 Siehe dazu: Bearbeitung aus methodischer Sicht (Kapitel 3). 
Buchstabens $Q$ wurde ausgearbeitet und anschließend auch diese Typoskripte revidiert. Außerdem wurde die Stichwortstrecke des Buchstabens $R$ des fünften Bandes ( $R$-Salarist [A. F.]) zum Großteil überarbeitet und ergänzt. ${ }^{25}$

\subsubsection{Neue Perspektiven bezüglich Publikation der Wörterbuchbände}

Nach der politischen Wende 1989, mit der Umbenennung unserer Forschungseinrichtung in Forschungsinstitut für Geisteswissenschaften - Hermannstadt, ergaben sich für dieses Standardwerk des Siebenbürgisch-Sächsischen neue Perspektiven, die dem Abbau der vorliegenden Typoskripte den Weg bahnten. Um die beträchtliche Lücke, die seit dem Erscheinen des fünften Bandes $(K)$ eingetreten war, zu schließen und weil der Verlag der Rumänischen Akademie zu jenem Zeitpunkt die Drucklegung des Wörterbuchs finanzieren konnte, wurde das L-Typoskript 1990 der Kronstädter Druckerei übergeben, doch konnte der Band nach Druckschwierigkeiten und aufgrund editorischer Missverständnisse erst 1993 erscheinen. Von 1996-2001 einschließlich wurde das Wörterbuch durch ein von der Volkswagenstiftung (Bundesrepublik Deutschland) gefördertes Programm unterstützt, das den Aufbau der Germanistik an der Lucian-Blaga-Universität in Hermannstadt zum Ziel hatte. Die Förderung fand im Rahmen einer wissenschaftlichen Zusammenarbeit zwischen der Universität Hermannstadt und der Mainzer Akademie der Wissenschaften und der Literatur statt. Das Wörterbuch war als Teilprojekt miterfasst. Die Arbeitsstelle war außerdem auch als Dokumentationsstätte für Studierende in den Germanistiklehrgang der Universität eingebunden.

Die Unterstützung ermöglichte die Gründung eines wissenschaftlichen Beirats für das Wörterbuch, dem namhafte Professoren angehörten. ${ }^{26}$

Dank der Förderung konnte das $M$-Typoskript in der Bundesrepublik Deutschland im Auftrag des Wörterbuchbeirats einer Gegenlesung unterzogen werden. Damit sind GiSEla Richter (Gundelsheim/Neckar) und HELMUT PROTZE (Leipzig) beauftragt worden. Außerdem wurde SIGRID HALDENWANG 1996 ein Aufenthalt in Kaiserslautern beim damaligen Leiter des 1997 abgeschlossenen Pfälzischen Wörterbuchs, RUDOLF POST, ermöglicht, der die Zeichensätze erstellt hat, die alle bei der Bearbeitung des Wörterbuchs benötigten 30 Sonderzeichen enthalten.

Diesen Voraussetzungen zufolge konnte die Wörterbucharbeit auf Computertechnik umgestellt werden. Mittels der Förderung konnte der siebente Band $(M)$ 1998 gedruckt werden sowie das Ende der 80er Jahre erarbeitete $N-P$-Typoskript EDV-erfasst und der achte Band $(N-P) 2006$ publiziert werden. Es folgte die langwierige, durch noch ungelöste Fragen und hinzugekommenes Mundartmaterial erschwerte wissenschaftliche Revision der Stichwortstrecke der Buchstaben $Q-R$ sowie die Bearbeitung und anschließende wissenschaftliche Revision der Wortstrecke

$25 \mathrm{Zu}$ entsprechenden Erläuterungen, siehe: Bearbeitung aus methodischer Sicht (Kapitel 3).

26 Professor Dr. Werner Besch (Bonn), Professor FrIEDhelm Debus (Kiel), Professor Dr. Dr. h.c. Siegfried Grosse (Bochum), Professor Dr. Rudolf Grosse (Leipzig), Professor Dr. WolfGang Kleiber (Mainz), Dr. Dr. Dr. h.c. HARALD ZimMERMANN (Tübingen). 
$S-$ Schenkwein. Die Gegenlesung der EDV-erfassten Typoskripte $N-P, Q-R, S-$ Schenkwein wurde von Frau GrETE KLASTER-UngurEANU (geb. 1927, † 2015), Hochschuldozentin für deutsche Sprache (damals in Rente), durchgeführt. Dank Förderung der Gemeinnützigen Hermann-Niermann-Stiftung in Düsseldorf und der Botschaft der Bundesrepublik Deutschland - Bukarest konnte auch die Drucklegung des neunten Bandes $(Q-R) 2006$ erfolgen. Zusätzliche Förderung zwecks Gegenlesung und Durchführung technischer Abschlussarbeiten ist STEFAN SIENERTH zu verdanken, der in der Zeitspanne 2005 bis 2013 Leiter des Instituts für Deutsche Kultur und Geschichte Südosteuropas (IKGS) an der Ludwig-Maximilians-Universität München war. Da die Bearbeitung und wissenschaftliche Revision der Stichwortstrecke $S-$ Schenkwein von nur zwei verbliebenen Mitarbeiterinnen bewältigt werden musste, konnte der zehnte Band (S-Schenkwein) erst 2014 mittels Förderung desselben Instituts sowie dem Demokratischen Forum der Deutschen in Hermannstadt gedruckt werden.

\subsubsection{Aktuelle personelle Besetzung wissenschaftlicher Mitarbeiter und Hilfskräfte}

Im Laufe der Zeit hat es zahlreiche Personalwechsel beim „Siebenbürgisch-Sächsischen Wörterbuch“ gegeben. Im Jahr 1990, nach der politischen Wende in Rumänien, sind die Mitarbeiter, Ute MAURER und STEFAN SIENERTH in die Bundesrepublik Deutschland ausgewandert. ${ }^{27}$ Hinzu kamen 1990 IsOLDE HUBER und 1991 MALWINE DENGEL. Sie haben Korrekturen für den 1993 erschienenen 6. Band $(L)$ gelesen, Hilfsarbeiten für die in Typoskripten vorliegende Stichwortstrecken der Buchstaben $N, O, P, Q$ durchgeführt, das schon vorhandene Zettelmaterial des Buchstabens $S$ mit Verzettelungen schöngeistiger Mundartliteratur, urkundlicher Quellen sowie mit Fachliteratur zur Mundart ergänzt und die Zettel innerhalb der Zettelkästen geordnet. Besonders hervorzuheben ist ihr Einsatz bei der Neubearbeitung des Literatur- und Ortsnamenverzeichnisses. ${ }^{28}$ Ende des Jahres 1998 hat ISOLDE HUBER die Wörterbuchstelle verlassen. Im Oktober 2002 konnte ELKE MARION CINĂZAN eingestellt werden, ist aber Ende Juni 2006 ausgeschieden. Sie hat für den 9. Band $(Q-R)$ teilweise Quellenkontrollen der urkundlichen Belege durchgeführt, das Zettelmaterial für die Stichwortstrecken der Buchstaben $T, U, W$ in Ordnung gebracht, Probeseiten für den Buchstaben $S$ geliefert, sowie verschiedene technische Arbeitsgänge geleistet. Von Ende Dezember 2006 bis Ende Juli 2007 besetzte ROLF AUNER probeweise die Stelle eines wissenschaftlichen Assistenten beim Wörterbuch. Von Anfang Dezember 2008 bis Ende Januar 2012 konnte AGNES MATTIS-TEUTSCH als wissenschaftliche Assistentin eingestellt werden. Sie war mit Hilfsarbeiten für den 10. Band (S-Schenkwein) betraut (z. B. mit der Ergänzung der schon angelegten Synonymenzentralen für den Buchstaben $S$ anhand der ab dem 7. Band $(M)$ erschienenen Bände) sowie mit technischen Arbeiten (dem Fotografieren des noch zu bearbeitenden Zettelmaterials des Wörterbuchs, als Grundlage für 
eine zukünftige Digitalisierung). Von Anfang Oktober 2013 war sie mit einer halben Arbeitsnorm eingestellt, um weitere technische Arbeitsgänge durchzuführen.

Die Bearbeitung des 10. Bandes ( $S$-Schenkwein) haben zwei Lexikografinnen bewältigt, SigRID HALDENwang (Mitarbeiterin seit 1971) und MALwine DeNGEL (Mitarbeiterin seit 1991), die seit Juni 2011 im Ruhestand ist, doch für die Erstellung dieses Bandes weiter ehrenamtlich gearbeitet hat. Zur Zeit führt SIGRID HALDENWANG $^{29}$ die Wörterbucharbeiten weiter.

\section{GESTALTUNG DER WORTARTIKEL AUS METHODISCHER SICHT}

Methodisch betrachtet wird heute die von ADOLF SCHULLERUs begonnene Wörterbuchanlage beibehalten, doch unterscheidet sich die Ausarbeitung seit 1957 in anderer Hinsicht von der alten Auffassung, da sie das Sprachliche in den Vordergrund stellt.

- Die Gestaltung des Wörterbuchs ist hauptsächlich auf Bedeutungsanalyse, lautlich-grammatische Fragen, Wortbildungsmuster und Synonymik ausgerichtet.

- Bemühungen um die Etymologie bleiben nicht aus (auch können ungeklärte Etymologien offen bleiben).

Um den 1957 festgelegten, neuen methodischen Grundsätzen gerecht zu werden, denen entsprechend die Redaktion der vorangegangenen Bände erfolgt ist, wurde schon vor Jahren eine Neubearbeitung der Stichwortstrecke der Buchstaben $\mathrm{R}-\mathrm{Sa}$ larist sowie der folgenden Stichwortstrecke Salarist in Fortsetzung bis einschließlich Saumwerk beschlossen, was sich als sinnvoll erwiesen hat: ${ }^{30}$ Die vielen kultur-

29 Sie ist seit dem 1. Januar 2016 mit einer halben wissenschaftlichen Norm eingestellt.

30 Bezüglich des Buchstabens $R$ handelt es sich um eine Überarbeitung und Ergänzung eines schon erstellten Werkes, das in zwei Lieferungen erschienen ist (erste Lieferung bearbeitet von JOHANN ROTH, aus dem Nachlass herausgegeben und ergänzt von FRIEDRICH KRAUSS: $R-$ Reussner 1929; zweite Lieferung bearbeitet von JOHANN ROTH, aus dem Nachlass herausgegeben und ergänzt von FRIEDRICH KRAUSS. Diese Lieferung enthält auch den Anfang der von FRIEDRICH HOFSTÄDTER teilweise erarbeiteten Stichwortstrecke des Buchstabens $S$, aus dem Nachlass herausgegeben von GUSTAV GÖCKLER: Reusswald-Salarist [Seite 301-320] 1931. Die gesamte Bearbeitung ist in dem Band $(R$-Salarist) zusammengefasst, der als 5 . Band alte Folge [abgekürzt: SSWB 5 A. F.] in die Wörterbuchgeschichte eingegangen ist. Nach dem Tod des Agnethler Sprachforschers JOHANN ROTH (geb. 1842, $\uparrow$ 1923) hat ADOLF SCHULLERUS den wissenschaftlichen $R$-Nachlass seines ehemaligen Lehrers übernommen. Er war es auch, der in den ersten Bogen die nötigen Ergänzungen eingefügt und die Herausgabe des Bandes in die Wege geleitet hat. Nach SchULLERUS' Tod ist das Werk von dem nordsiebenbürgischen Sprachforscher FRIEDRICH KRAUSS zu Ende geführt worden. Die Stichwortstrecke Salarist in Fortsetzung bis einschließlich Saumwerk [bis Seite 352] wurde gedruckt, doch nicht ausgeliefert. Sie wird als Einzelexemplar in der Siebenbürgischen Bibliothek in Gundelsheim/Necker aufbewahrt. Eine Xerokopie derselben ist der Hermannstädter Wörterbuchstelle freundlicherweise von GISELA RICHTER †, der einstigen Leiterin des abgeschlossenen „Nordsiebenbürgisch-Sächsischen Wörterbuchs", überwiesen worden. Diesen Erläuterungen zu Folge handelt 
geschichtlichen sowie volkskundlichen Exkurse sind weggeblieben, ohne die persönliche Note der Darstellungsweise des Bearbeiters außer Acht zu lassen. Dementsprechend wird z. B. bei bestimmten Stichwörtern zwar nicht beschreibend auf Brauchtum, Aberglauben bzw. Volksglauben oder Heilkunde eingegangen, doch fallweise innerhalb der semantischen Gliederung kurz auf Quellen verwiesen, in denen darüber nachzulesen ist.

Eine Bereicherung bringen die schon erwähnten auf Geländefahrten in den meisten Teilen der siebenbürgischen Mundartlandschaft eingebrachten Mundartbelege, dann auch noch gezielt ergänzt mit Mundartbelegen für Stichwörter die weniger gut belegt waren von Malwine DENGEl und SigRID HaldENwang. Dazu kommt auch das Flurnamenmaterial, das ANNELIESE THUDT in Rente aus ihrem persönlichen Flurnamenarchiv zur Verfügung gestellt hat und die Exzerpierung der Flurnamen aus Ortsmonografien.

\subsection{Lemmaansatz}

- Die Stichwörter werden grundsätzlich in schriftsprachlicher Form angesetzt.

- Lehnwörter, die in die deutsche Schrift- oder Umgangssprache Siebenbürgens eingegangen sind, haben schriftsprachlichen Ansatz, z. B. Ratze (m.) 'im Mittelalter in Sold stehender Serbe, wohl auch Slawe überhaupt'; Sabathheiduck (m.) $\dagger$ 'Freibeuter'.

Mundartansatz erfolgt:

- Bei Entlehnungen aus dem Rumänischen ${ }^{31}$ und Ungarischen ${ }^{32}$ übernommenen

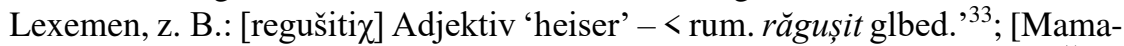
liga] (f.) 'Maisbrei'; 'energieloser Mensch' - < rum. mămăligă 'glbed.'; [Šaszrō] (m.) 'durch Schrecken od. Grämen entstandene Krankheit' - < rum. ceas rău 'böse Stunde'; [Rētəš] (m.) 'Gebäck aus feinem Hefeteig, Art Strudel' - < ung. rétes 'glbed.'; [Šatərt] (m.) 'Zelt der Wanderzigeuner'; 'Verkaufs-, Jahrmarktsbude' - < ung. sátor 'Zelt, Marktbude';

- Bei Dialektwörtern, für die keine vertretbare deutsche etymologische Form gebildet werden kann, mit ungeklärter Herkunft (mit einem Verweis auf verschiedene Dialektwörterbücher), z. B.: [Rāksz] (f.) 'weinerliche Person'; [rēkli $]$ Adjektiv 'sauber, nett, ansehnlich, stattlich, niedlich, munter, nur v. Mädchen' [Rätšəl] (n.) 'nach oben sich verjüngende Wasserkanne'; [Šapōrəl] (---) (m.) 'Hemdzipfel, der kleinen Jungen, der häufig bei den hinten geknöpften Höschen heraushing'.

es sich bezüglich des 10. Bandes ( $S$-Schenkwein) teilweise um die Überarbeitung und Ergänzung eines schon erstellten Werkes. (vgl. dazu auch: Bearbeitungs-/Publikationsstand, Kapitel 2.2).

31 Rumänisch $=$ rum

32 Ungarisch $=$ ung.

33 Gleichbedeutend $=$ glbed . 
Ansatz von Mischformen:

- In zusammengesetzten Stichwörtern erscheinen zuweilen auch schriftsprachlich-mundartliche bzw. mundartlich-schriftsprachliche Mischformen, z. B.: Hasen [makrišs ${ }^{34}$ (m.) Pfl.N. ${ }^{35}$ 'Gemeiner Sauerklee'; Riemer [šatərt] (m.) 'Verkaufszelt des Riemers, das anders beschaffen ist als das anderer Handwerker'; [Mät $\left.{ }^{36}\right]$ kirsche (f.) 'Frucht des Sauerkirschbaums'; [Šaszrō]gekräutig (n.) 'Heilpflanze gegen die Krankheit [Šaszrō]'; [Šatərt]zigeuner (m.) 'Zeltzigeuner'.

Die Anordnung der Stichwörter erfolgt alphabetisch. Die bei mundartlichen Ansätzen verwendeten Lautschriftzeichen werden dabei folgendermaßen eingeordnet: $[\chi]$ und $[\mathrm{x}]=c h ;[\gamma]=g ;[\mathrm{y}]=n g ;[\check{\mathrm{s}}]$ und $[\check{z}]=s c h ;[\mathrm{sz}]=\operatorname{stimmloses} s(s s$ bzw. $\beta)$; es wird immer nach stimmhaftem $s$ eingeordnet; $[\mathrm{ts}]=z$. Das Wörterbuch verwendet nicht die internationale, sondern eine eigene, an der Teuthonista orientierte Lautschrift.

Nach dem Stichwort folgt gewöhnlich zuerst die Hermannstädter, darauf die Bistritzer Lautform. Ist ein Wort in den beiden genannten Orten nicht belegt, wird möglichst je ein Beleg aus einem anderen südsiebenbürgischen ${ }^{37}$ bzw. nordsiebenbürgischen ${ }^{38}$ Ort gebracht. Ist ein Wort im Südsiebenbürgischen nicht belegt, so steht die Bistritzer Form an erster Stelle mit der Angabe „nur nsbg.“, außer bei den ausschließlich aus „KRAUSS, Hwspr.“ belegten Wörtern. Die Angabe „,nsbg. nicht belegt" steht bei Wörtern, die nur im Südsiebenbürgischen vorkommen. Bei kurzen, übersichtlichen Artikeln, die nicht mehr als drei Belege aufweisen, wird in beiden Fällen auf diese Angaben verzichtet. Besteht der Artikel nur aus einem einzigen südsiebenbürgischen oder nordsiebenbürgischen Satzbeispiel, so gilt die am Ende des Satzes angegebene Quelle auch für die angeführte mundartliche Form des Stichwortes, z. B. Rahmkanne, Reitermaschine, Samklee, schaukelmarig.

Nach der mundartlichen Form des Stichwortes werden Substantive durch die Angabe des Genus und Verben durch die Angabe der Konjugation als solche gekennzeichnet. Ist bei Substantiven das Genus nicht feststellbar, steht „o.A.d. ${ }^{39} \mathrm{Ge}$ nus“. Ist bei einem Verb die Konjugation nicht feststellbar, steht gleich nach dem Stichwort nur die Bezeichnung „Vb.“ Die Grundformen werden nur bei starken und unregelmäßigen Verben angegeben. Bei Adjektiven stehen die Steigerungsstufen, wenn diese nicht regelmäßig gebildet werden.

[Makriš] - < rum. macriş 'glbed'.

Pflanzenname $=$ Pfl.N.

[Mätx] - < ung. megy 'Sauerkirsche'.

Südsiebenbenbürgisch $=$ ssbg.

38 Nordsiebenbürgisch $=n s b g$.

39 Ohne Angabe des = o.A.d. 


\subsection{Zur Gliederung der Wörterbuchartikel}

Die Artikelstruktur gliedert sich hauptsächlich in 2 Teile:

I. Phonetik:

- Dem Lemma folgt die Lautform für H (als ssbg. Vertretung) und B (als nsbg. Vertretung). Die folgende Phonetik illustriert, immer in den Grenzen des vorhandenen Materials, ein Lautkopf der Vokal- und Konsonantenvarianten für das Mundartgebiet mit Ortssigel (bei Stichwörtern mit zahlreich belegten Lautvarianten [besonders bei Verben, Substantiven] werden ab Band $6(L)$ - wenn möglich - die Vokalvarianten ihrer lautgeografischen Verbreitung entsprechend gebietsweise erfasst, z. B. Stichwort Schaf: - Lautvariante [-ō-] verbr. ${ }^{40}$ H u. Umgebung., z. T. Med u. Umgebung, westliches u. mittleres Zwischenkokelgebiet usw.); daran schließen im Mundartgebiet verstreute Einzellautungen mit Ortssigel in runder Klammer an. Um eine bessere lautgeografische Übersicht zu erhalten, wird bei umfangreichen Lautköpfen auch die Einteilung in „ssbg.“ und „nsbg.“ gemacht. Bei Substantiven stehen fallweise auch die Plural- und Diminutivformen im Lautkopf. Bezeichnet ein Pflanzenname mehrere Pflanzenarten, die dasselbe Genus haben, erfolgt die Genusangabe gleich nach „Pfl.N.“ vor der Aufzählung der Pflanzenarten, z. B.: Safranschwamm.

II. Strukturierung nach semantischen Gesichtspunkten mit jeweiligen Synonymenund Vergleichshinweisen und fallweise mit anschließender Aufzählung der Komposita:

- Sie erfolgt durch arabische Ziffern, Unterabteilungen tragen kleine lateinische, bei weiterer Aufgliederung auch kleine griechische Buchstaben. Die Bedeutungsanalyse erfolgt vom konkreten zum übertragenen Wortgebrauch. Die Gliederung kann auch nach nichtsemantischen Gesichtspunkten gemacht werden, z. B.: richten.

- Die Synonymen- und Vergleichshinweise stehen anschließend an jede Bedeutung. In der Synonymenzentrale (werden sinngleiche Wörter aufgezählt, in der Vergleichszentrale die nur sinnverwandten: z. B. stehen unter [Prəpədit] ${ }^{41}(\mathrm{~m}$.) i.S.v. ${ }^{42}$ 'Taugenichts', 'Lump' die Synonyme: [Kalik] ${ }^{43}$, [Newoie ${ }^{44}$, Nichtsgutziger, Nichtsnutz, Nichtsnütziger, Niemandnütz, [Podlogār $]^{45}$, [Purligār $]^{46}$; unter $[\text { Rētəš }]^{47}$ (n.) i.S.v. 'Gebäck aus feinem Hefeteig, Art Strudel mit Rosinen

40 Verbreitet $=$ verbr.

41 - < rum. prăpădit 'Lump, Taugenichts'.

42 Im Sinne von = i.S.v.

43 - < rum. calic 'armer Mensch'.

44 - < rum. nevoie 'glbed.'; in der Verbindung om de nevoie 'erbärmlicher, nichtsnutziger Kerl'.

45 - < rum. potlogar 'Gauner, Hochstappler, Nichtsnutz, Spitzbube'.

46 - zu rum. purligar 'Wortvariante zu potlogar (s.d.)' (veraltet u. regional) 'glbed.' (IORDAN, Dlr. 8, 4, $\left.{ }^{1} 146\right)$.

47 - < ung. rétes 'glbed.' 
und Nuss- oder Mohnfüllung (Festgebäck an Feiertagen und festlichen Veranstaltungen)' die Vergleiche: Kolatsche, Magestriezel, Nussstrudel, [Nutšə]strudel..$^{48}$

- Die Synonymenzentralen werden dann bei einem Wort angelegt, das am Ende des Alphabetes steht, wenn alle sinngleichen Wörter aufgefunden werden konnten. So steht z. B. unter dem Stichwort [prəpədin] ${ }^{49}$ (Vb.): Synonyme siehe unter zugrundegehen (wo dann alle entsprechenden Synonyme aufzufinden sind).

- Aus Mangel an Übersicht konnten beim Anlegen der Zentralen in den bisher erschienenen Bänden nicht immer alle synonymen und sinnähnlichen Wörter berücksichtigt werden. Deshalb gibt es Doppelzentralen: z. B. werden bei Sabbatalie (2) (f.), mdal. [szabatalən] (_-_--) Pl. i.S.v. 'Siebensachen, Habseligkeiten' folgende Synonyme angeführt: [Buländre $]^{50}(-\iota)(1)$, Gebachtsel $(3 b)^{51}$, $\left[\right.$ Gəbulendər] ${ }^{52}$, Gezatter $^{53}$ (siehe unter Gezatter 1), [Halubi] ${ }^{54}$, [Hapərlēkn] ${ }^{55}$,

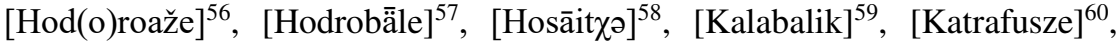
[Kosnōtən] $(1)^{61}$, [Merinde] (-s-) (2) ${ }^{62}$, [Negots] $(2)^{63}$, Sack u. Pack (siehe unter Sack 1d), sieben Bilsen (siehe unter Bilse 3f), sieben gebackene Birnchen (siehe unter Birne 3), sieben Sachen (siehe unter Sache 1aß), [Taramtutzər] ${ }^{64}$ u. unter sieben Zattern ${ }^{65}$ (siehe unter Zatter 2). Vgl. Gehader, Gepritze ${ }^{66}(1)$, Gerümpel (1), Gezeug (2f), Kikes [kākəszkər]. ${ }^{67}$

Am Ende des Wortartikels stehen die Komposita (mit dem Stichwort als Grundwort, z. B. Hase 'Grundwort', Zstzgn ${ }^{68}$ : Gebirgs-, Herbst-, Stall-, Wald-).

48 Bestimmungswort - < rum. nuci, Pl. von Sg. nисӑ 'Nuss'.

49 - < rum. a se prăpădi 'zugrundegehen'.

50 - < rum. bulendre, Pl. von Sg. buleandră 'Lumpen, Plunder'.

51 - Zu mhd. bāt 'Unrat' (LeXER, Hwb., Bd. 1: 9).

52 Bildung zu buländre 'glbed.'

53 - Zu Zatter, i.S.v. 'Fetzen'.

$54-$ Herkunft ungeklärt.

55 - < ung. aprolék 'Keinigkeit, Abfall'.

56 - < rum. hodoroagă 'schlechtes Pferd'.

57 - < rum. hodrobele 'Kleinigkeiten'.

58 - Herkunft ungeklärt: könnte zu ung. haza 'nach Hause' od. zu hozzá 'zu ihm', 'für ihn' gehören; das Suffix scheint allerdings rum. Ursprungs.

59 - < rum. calabalic ( $<$ türkisch kalabalik) 'Habseligkeiten'.

60 - < rum. catrafuse 'Siebensachen'.

61 - Vgl. Kissenaten 'Kuchen- und Brotreste' (Rhein. Wb., Bd. 4: 458).

62 - < rum. merinde 'Essvorrat; Lebensmittelvorrat'.

63 - < rum. negot 'Handel, Ware'.

64 - Wohl zu ung. teremtö 'Schöpfer, Gott' u. tudja 'weiss es', also etwa 'nur Gott weiss es (was da alles ist), wieviel Krempel, Plunder' (vgl. NSSWB, Bd. 5: 536).

65 Zatter $=$ Fetzen .

66 - Vgl. Pritzel 'allerlei Hausrat von geringem Wert' (GRIMM, Dwb., Bd. 7: 2133).

67 - Zum Bestimmungswort vgl. Kickeschen 'etwas Winziges' (Rhein. Wb., Bd. 4: 458).

68 Zusammensetzung, -en = Zstzg, Zstzgn. 
Lokalisierung der Belege durch Ortssigel: Die Belege werden anschließend durch Ortssigel lokalisiert. Gibt es aus mehreren Orten Belege, die in Wortlaut und Sinn, nicht aber phonetisch identisch sind, wird der relevanteste ausgewählt, sein Ortssigel angeschlossen und dahinter werden mit ,auch“ die übrigen Sigel angeführt, z. B.: [dä hürt dət szakszəš fäöütərąsər af] ${ }^{69}$ Bei nur sinnverwandten Belegen werden nach dem Ortssigel des relevantesten Beispiels die übrigen Sigel mit ,ähnl.“ angeschlossen, z. B.: [dər dētərnäszt štä $\chi$ dij än sąk] ${ }^{70}$. Beide Fälle können auch kombiniert auftreten.

3.3 Einbezug deutscher Belege aus der siebenbürgischen Urkundensprache (siehe: a. Spr.):

Berücksichtigt werden:

- Mundartlich gefärbte Wortformen, in verschiedenen Schreibweisen, besonders O.N. ${ }^{71}$ und Fl. ${ }^{72}$;

- Lexeme lateinischer Herkunft, über die Sprache der österreich-ungarischen Kanzleien übernommen, z. B. Intimation [f.] 'Veranlassung, Zuschrift' ${ }^{73}$, intimieren $\mathrm{Vb}$. 'veranlassen,74;

- Kulturgeschichtlich interessante Begriffe, z. B. Einrichtungen, Funktionen, die im deutschen Sprachraum nicht belegt sind, wie Königsboden 'bis 1876 dem ungarischen König unterstelltes, von den Sachsen selbstverwaltetes Gebiet'; Königsrichter 'Comes Nationis, Judes regius, bis 1795 das Oberhaupt der Verwaltung des Königsbodens; Nachbarschaft 'in verschiedenen Bedeutungen und die entsprechenden Zstzgn'.

3.4 Die Datierung der innerhalb einzelner Bedeutungsabschnitte folgenden Satzbeispiele nach Perioden in chronologischer Folge

Einem Satzbeispiel mit Datierung „um 1960“ mit Ortsangabe beispielsweise folgen Belege ohne Jahresangabe, die sechziger Jahre werden schließlich mit dem letzten Satzbeispiel, z. B. 1969, mit Ortssigel angeschlossen. Damit ergeben sich Anhaltspunkte sowohl für das Leben eines Wortes als solches als auch für den Wandel der sozialökonomischen Grundlage im Laufe der Zeit. Wenn etwa die Stichwörter: Kollektiv (f.), mdal. [kolektīf] i.S.v. 'Kollektivwirtschaft' (ab 1949), später 'Landwirt-

69 'Da hört das sächsische Vaterunser auf'; von Gemeinden gesagt, die am Rande des siebenbürgisch-sächsischen Siedlungsgebietes liegen (um 1930 Schaas, auch De, Gr-Prdf, Nst/Kr).

70 'Der Tutdirnichts steckt dich in den Sack'; Drohung an Kinder (um 1960 Tkdf, ähnl. Au, Jaad, Tatsch Tre).

71 Ortsname $=$ O.N.

72 Flurname $=$ Fl.N.

73 - Zu lat. intimatio 'Ankündigung'.

74 - Zu lat. intimare 'mitteilen'. 
schaftliche Produktionsgenossenschaft (LPG)' (1960 H); die damit gebildeten Zusammensetzungen, wie Kollektivross (n.), mdal. [koläktīfrōsz] i.S.v. 'Ross der LPG' (1960 B); Kollektivgüter P1., mdal. [kolektīfgetər] 'Vieh der LPG' (1965 Hamr) bis etwa 1989 zum Wortschatz des Siebenbürgisch-Sächsischen gehören, bestehen die genannten Einrichtungen nach der Wende in Rumänien nicht mehr und damit sind auch die dazu gebildeten Begriffe aus der Mundart verschwunden.

Angaben zur Etymologie erfolgen bei Wörtern, die keine klar erkennbare Entsprechung in der allgemein gebrauchten neuhochdeutschen Schriftsprache haben, sowie bei Herkunft aus anderen Sprachen.

Es handelt sich um:

- Wörter, die auf das Mittelhochdeutsche zurückgehen (deren sprachgeschichtliche Entwicklung auch verfolgt werden kann), z. B. Poje, mdal. [poiən] (P1.) 'Fußfessel, (Fuß)eisen ${ }^{75}$; schauchern (schw. $\left.{ }^{76}\right)$, mdal. [šoxərn] schw. (H), [šauchxərn] (B) 'Angst, Abscheu, Entsetzen, Grausen empfinden'77;

- Wörter, deren Herkunft ungeklärt ist, z. B. [Šäszənāltði] (--_--) (n.) (nsbg.) 'ein Nichtschen' (etwas Wertloses) ${ }^{78}$;

- Lehnwörter, z. B.: [Šemər] (m.) 'Herpes, die fieberhafte Empfindung, von welcher man nach zu hastigem Genusse, besonders von fetten Speisen, befallen wird"79; [Sabą̄le] (--s) o.A.d. Genus 'entzündete, wunde Mundwinkel' ${ }^{\prime 80}$.

Die Zusammensetzungen mit dem Stichwort als Bestimmungswort erhalten grundsätzlich jede einen eigenen Ansatz. Auf nähere grammatische Angaben (z. B. Grundformen bei starken Verben oder Plural bei Substantiven wird ebenso verzichtet wie auf Lautvarianten und Angaben zur Etymologie, siehe z. B. Jahrmarkt[šatərt] (m.) 'Jahrmarktbude' und Schell[kąląp] ${ }^{81}$ (m.) 'Schellober im Kartenspiel' (Grundwort der Lexeme in dieser Wortform belegt).

Belege aus der alten Sprache (die deutsche Sprache in siebenbürgischen Quellen von der Mitte des 13. bis zur Mitte des 19. Jahrhunderts) werden unter dem Gesichtspunkt ihrer Bedeutung für den mundartlichen Einfluss auf die deutsche Schriftsprache in Siebenbürgen oder für die Geschichte der deutschen Sprache gebracht. Die angeführten Belege bringen das betreffende Wort in gesperrter Schrift und werden in Anführungszeichen gesetzt.

Mundartbelege des 18. Jahrhunderts werden sowohl kursiv geschrieben, als auch in Anführungszeichen gesetzt. Ab dem 19. Jahrhundert werden Mundartbelege - auch Zitate aus der Fachliteratur und Belletristik - in die Lautschrift des Wörterbuchs übertragen.

75 - Zu mhd. boije, boye, boie [lat. bojae] Fessel (LEXER, Hwb., Bd. 1: 323); vgl. Boie 'Halseisen, Kette' (GRIMM, Dwb., Bd. 2: 229).

76 schwach $=$ schw.

77 - Zu mhd. schiuchen 'Scheu empfinden, sich scheuen' (LEXER, Hwb., Bd. 2: 760f.).

$78-\mathrm{Zu}$ etymologischen Erwägungen vgl. SSWB, Bd. 10: 250.

79 - < ung. csömör 'der Ekel, die Unlust'.

80 - < rum. zăbale (Pl.) von zăbală (Sg.) 'faule Mundwinkel'.

81 Das Grundwort zu ung. kalap 'Hut'; im ungarischen Kartenspiel trägt der Ober einen Hut. 
Ausdrücke und Wendungen, die oft nur ein Hochdeutsch in siebenbürgischsächsischer Lautung darstellen, dem würdigen Kanzelton nacheifern und sich daher auf Rede und Gegenrede bei feierlichen Anlässen beschränken, werden von denen in echter Mundart abgehoben; sie erhalten den vor- oder nachgestellten Vermerk „geh. Spr.“82, z. B. Sachsenherz.

\subsection{Zum Verweissystem}

\subsubsection{Verweise vom Wortartikel auf andere Wörterbücher:}

- Auf GRIMM, Dwb. und entsprechende deutsche Mundartwörterbücher wird bei Lexemen verwiesen, die in der allgemein gebrauchten neuhochdeutschen Schriftsprache fehlen, im deutschen Sprachraum aber in derselben oder ähnlichen Wortform in besagten Wörterbüchern belegt sind. Der Verweis erfolgt am Ende des Wortartikels mittels Gedankenstrich (-) und anschließendem ,Vgl.“, z. B. Schauke, mdal. [šōk] (f.) (Kr), [šok] (B) 'Schaukel'. - Vgl. Schocke f. '[glbed.]' (GRIMM, Dwb., Bd. 9: 1435) u. Schocke (f.) (1b) 'glbed.' (Rhein. Wb., Bd. 7: 1697).

- Auf das NSSWB ${ }^{83}$ wird verwiesen: wenn bei einem Stichwort Bedeutungen auftreten, die das Archiv des SSWB nicht belegt; bei unterschiedlichem Ansatz desselben Wortes in dem jeweiligen Wörterbuch; wenn bei einem Stichwort

82 Gehobene Sprache $=$ gehob. Spr.

83 Von besonderer Bedeutung für die siebenbürgisch-sächsische Wörterbuchgeschichte ist das in Gundelsheim/Neckar zwischen 1986 und 2006 in fünf Bänden erarbeitete „Nordsiebenbürgisch-Sächsische Wörterbuch“. Es handelt sich um die Mundart der Bewohner/-innen von 48 Ortschaften, meist Dörfern, in den Kleinräumen Nösnerland und Reener Ländchen. Für das Nösnerland ist Bistritz, auch Nösen genannt, ,die Stadt“ mit starker Ausstrahlung auf die umliegenden Orte, für das südlichere Reener Ländchen ist es Sächsisch-Regen oder Reen. Eine Begründung, für die Erstellung dieses Wörterbuchs, das nicht als ein Parallelunternehmen zum „Siebenbürgisch-Sächsischen Wörterbuch“ betrachtet werden darf, sondern ein Pendant zu demselben ist, bringt GISELA RICHTER †, die federführende Kraft des Wörterbuchs, in der Einleitung des 1.Bandes: XVII.:

Die Weiterführung und Herausgabe des Nordsiebenbürgischen Wörterbuchs, zu dem ein so umfangreiches, vorgeordnetes Zettelgut vorlag, erschien nach reiflicher Überlegung aus mehreren Gründen sinnvoll: einmal, weil die Dörfer und die beiden Stadtgemeinden von Nordsiebenbürgen nirgends mehr in der früheren Geschlossenheit existieren, zweitens, weil die Ortschaften, die das Nordsiebenbürgische Wörterbuch festhält, im Verschwinden begriffen sind und drittens, weil das „Siebenbürgisch-Sächsische Wörterbuch“ den nordsiebenbürgischen Mundartraum zwar miterfasst, ihn aber, trotz seiner prekären Lage, nicht stärker berücksichtigen kann als die übrigen Kleinräume des ganzen sächsischen Mundartgebietes; das „Siebenbürgisch-Sächsische Wörterbuch“ muss schließlich rund 243 Ortschaften einbeziehen, davon gehören zu Nordsiebenbürgen heute nur 48.

Das Nordsiebenbürgische Wörterbuch behandelt demnach die Mundarten einer kleineren Sprachinsel innerhalb der großen siebenbürgisch-sächsischen Sprachinsel. Diese lexikographische Symbiose zeigt sich auch bei der Bearbeitung des siebenbürgisch-sächsischen Wortgutes der beiden Wörterbücher, die in bestimmten Fällen aufeinander verweisen. 
nur urkundliche Belege gebracht werden können, das NSSWB aber Mundartbelege bringt; wenn das NSSWB urkundliche Belege bringt (z. B. bei Fl.N.), die im Archiv des SSWB fehlen; ab Bd. 10 (S-Schenkwein) wird auch auf nordsiebenbürgische Lautformen verwiesen, die im Archiv des SSWB nicht belegt sind sowie auf Fl.N. (auch Namen von Brunnen, Quellen), die nur in Nordsiebenbürgen bezeugt sind.

\subsubsection{Verweise außerhalb der Wortartikel:}

- Zur besseren Orientierung der Benutzer/-innen wird von schwer verständlichen mundartlichen Lautvarianten auf das betreffende Stichwort verwiesen, z. B. [Saox] siehe Sache; [Sāōt], [Säot] siehe Saat; [Sarlər] siehe Sattler. Nur bei besonders stark abweichenden Schreibvarianten der a. Spr. wird auf den entsprechenden schriftsprachlichen Ansatz verwiesen, z. B.: Qottember s. Quatember; Rabber s. Radbbahre; Santh s. Sankt; Sars s. Scharosch (O.N.).

- Verweise bei hochdeutschen Stichwörtern, die der Mundart fehlen, erfolgen, wenn es sich um sehr gebräuchliche Wörter handelt, z. B. Quarz fehlt der Ma. ${ }^{84}$, siehe Kraderstein; schellen fehlt der Ma., siehe klingeln, läuten ${ }^{2}$ (1), tingeln, zimbeln, zingeln; Saturei fehlt der Ma., siehe Eisabat.

\subsection{Zum Klammersystem}

\subsubsection{In runde Klammern werden gesetzt}

- Schwer verständliche Mundartwörter in etymologisch entsprechender Form un-

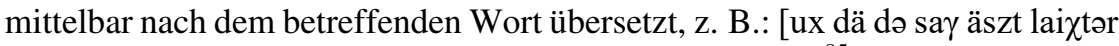
niən, ändem sə si $\chi$ net gle $\chi$ bəgiən] (beeindrucken lassen) ${ }^{85}$; [də giunts są āsz mər nau āltsi $\chi$ (widerwärtig) gəwōrn] ${ }^{86}$; [də hą̄ōšrak ku šuərənwāisz ąnt sə frāszn də gą̄nts fruxt (Getreide) uə] ${ }^{87}$; steht das zu übersetzende Mundartwort nicht in seinem Stichwortansatz bzw. Grundform, erfolgt auch deren Angabe, z.B.: [hieltsəra sậltsbiszkər fum boją̧̌šn] (siehe Boiāš = Zigeuner, der Löffel, Tröge, Körbe u. Ä. herstellt u. damit handelt). ${ }^{88}$

84 Mundart $=$ Ma.

85 'Auch die die Sache etwas leichter nehmen, in dem sie sich nicht gleich beeindrucken lassen' (SCHUSTER 1921: 3).

86 'Die ganze Sache ist mir jetzt widerwärtig geworden' (Win).

87 'Die Heuschrecken kommen scharenweise und sie fressen das ganze Getreide ab' (Wall).

88 'Salzbüchschen aus Holz vom Zigeuner gefertigt' (Alz). 


\subsubsection{Schräge Klammern stehen:}

- Wenn das entlehnte Mundartwort durch ein sinngleiches schriftsprachliches Wort übersetzt wird, z. B. [sen szuszpin /schwerer Atem/ wird əm ux šuədən] ${ }^{89}$; ['d äs əm än turmə/Herde/ šōf geblim $]^{90}$;

- Bei hochdeutschen Ergänzungen bzw. Erklärungen innerhalb eines Mundartbelegs, z. B.: /die Mädchen lecken an den Fingern, um zu sehen/ōf sə/die Finger/ nō $\gamma$ än ${ }^{y}$ əsi garts sen fun dəm weiszə saft /des Löwenzahns/ $]^{91}$;

- Hochdeutsche Ergänzungen bzw. Erläuterungen zu weniger verständlichen Textstellen bei (Satz)beispielen, die urkundlichen Quellen entnommen sind, z. B.: ,/Sie/ sagte zu meiner Dienstmagd, sie sollte gemelte Sachen /Pelz, Hemd, Schuhe usw./ nur da lassen" (1692: Cap. Cib.);

- Die hochdeutsche Entsprechung der Wörter, deren Wortform stark vom Hochdeutschen abweicht, z. B.: ,wy vil ellen Samat ein herr sal nemen zw eyner schwben /Schaube s.d./“ Schneiderartikel (1499, MüLLER, Sprdm. 144).

\subsection{Sonstige Angaben:}

Die Angabe „fehlt SSWB“ kann bei den angeführten Zusammensetzungen oder Synonymen eines Simplexes in folgenden Fällen stehen:

- Bei Belegen, die beim Exzerpieren urkundlicher Quellen noch hinzugekommen sind, zur Zeit der Bearbeitung des betreffenden Wortartikels nicht belegt waren, siehe z. B. Sache: Zstzgn Gericht- (a. Spr.) fehlt SSWB, Bd. 3; Grund- (a. Spr.) fehlt SSWB, Bd. 3;

- Bei Wörtern, die bei mundartlichen Erhebungen noch hinzugekommen sind, siehe z. B. Schelle (1b): Zstzgn Kuh- (fehlt SSWB 5), Ross- (fehlt SSWB 9); Schaf: Syn. Gemein (d)e- (fehlt SSWB 3);

- Bei Wörtern, die hauptsächlich dem nordsiebenbürgischen Fachwortschatz, dem nordsiebenbürgischen Pflanzennamenbestand, aber auch anderem mundartlichen Wortschatz angehören, da die entsprechende Fachliteratur (siehe z. B. Krauss, Hwspr.; Krauss, Pfl.N.; KraUSS, Tre Wb.) zur Zeit der Bearbeitung noch nicht erschienen war. Vgl. z. B. Zstzgn zu Säge (1a): Absatz- (fehlt SSWB, Bd. 1; siehe Krauss, Hwspr. 14), Band- (fehlt SSWB, Bd. 1; siehe Krauss, Hwspr. 107); Zstzg zu Salat (1): Enten- (fehlt SSWB, Bd. 2; siehe KraUSS, Pfl.N. 473). Dazu kommen vereinzelt auch die im Laufe der Zeit beim Exzerpieren von pflanzenkundlichen Beiträgen hinzugekommenen Pflanzennamen, siehe z. B. Zstzg zu Salat (1): Lamm- (fehlt SSWB, Bd. 6; siehe SchUlLERUS 1916: 179).

89 'Sein schwerer Atem wird ihm auch schaden'(Holz).

90 'Es ist ihm eine Schafherde geblieben' (Bud).

91 'Die Mädchen lecken an den Fingern, um zu sehen, ob sie, die Finger, von dem weißen Saft des Löwenzahns immer noch so bitter schmecken '(SCHULLERUS 1904: 43). 
Die Angabe „fehlt i.d. Bed. ${ }^{92}$ SSWB“ kann in folgenden Fällen stehen:

- Bei Wörtern, die dem Fachwortschatz angehören oder Pflanzennamen sind, die in den bisher erschienenen Bänden zwar aufzufinden sind, doch konnte zur Zeit der Bearbeitung die hinzu gekommene Bedeutung nicht belegt werden, siehe z. B. Schaufelkloben: Syn. Bügel (fehlt i.d. Bed. SSWB, Bd. 1; siehe KraUSS, Hwspr. 181 [1b])); Salbei (2): Syn. Eidechsengekräutig (fehlt i.d. Bed. SSWB, Bd. 2; siehe Krauss, Pfl.N. 514).

Die Angabe ,a.d.“93 erscheint häufig bei der „Lautkopfgestaltung“ neben den einzelnen Vokalvarianten:

- Es handelt sich meist um Verben oder Substantive. Das ist darauf zurückzuführen, dass bei den Direktaufnahmen derselbe Laut unterschiedlich gehört und so notiert wurde oder haben die verschiedenen Gewährspersonen dasselbe Wort anders ausgesprochen.

- Bei Angaben von Zitaten aus Wörterbüchern und der mundartlichen Fachliteratur werden die bei der Wörterbuchbearbeitung benutzten Abkürzungen beibehalten.

\subsection{Begleitinformation}

Seit dem Erscheinen des dritten Bandes $(G)$ konnte die für die Benutzer/-innen so wichtige Begleitinformation (da sie den Kostenpreis erhöht hätte) nicht mehr gebracht werden. Unter den neuen Bedingungen wird sie ab dem siebenten Band $(M)$ wieder mitgegeben. Die Begleitinformation ist dem eigentlichen Wörterbuchteil vorangestellt. Sie umfasst die Hinweise zur Benutzung des Wörterbuchs, die Erläuterungen zur verwendeten Lautschrift, das Abkürzungs-, das Literatur- und das Ortsnamenverzeichnis.

Das Literaturverzeichnis zu Band $G$ wurde überarbeitet, dabei auch ergänzt. Hinzugekommen sind: Fachliteratur, deutsche Mundartwörterbücher, rumänische sowie ungarische Wörterbücher, historische Quellen bezüglich siebenbürgischsächsischer Ortsnamen und Ortsmonografien. Die Hinweise zur Benutzung des Wörterbuchs sind nicht unverändert aus dem dritten Band $(G)$ übernommen worden. Die Begriffe sind eingehender erklärt worden, und die Erläuterungen werden bei jedem Band von anderen anschaulichen Beispielen begleitet, um das Wörterbuch benutzerfreundlicher zu gestalten. Zur besseren Orientierung der Benutzer/innen wird auch ab dem siebenten Band $(M)$ das Verweissystem zweckentsprechender angelegt.

Zur Illustration bringen wir zwei Beispielseiten (Abbildung 1 und Abbildung 2), die dem zehnten Band (S-Schenkwein) entnommen sind:

92 In der Bedeutung $=$ i. d. Bed.

93 Auch dies = a.d. 
Sch Laut u. Buchstabe: $d x t s(\mathrm{H}, \mathrm{B})$. Zum Wesen des Lautes s.: BERTLEFF G. 2, 20; KRAUSS, Hwspr. XVII; Huss, Lautl. 163-166; 171-182; KeINTZEL, Lautl. 215; KisCH, Ma. 64; KLeIN, B Ma. 64-65; MAURER 60-61; SCHEINER, Ma. 162; ders., Med Ma. 140-141; RoTH, Lautl. 15-16; WoLfF, Kons. 52-53; i.d. Fachliteratur z.T. unter $\mathrm{S}$ behandelt. 1. Stimmloser alveolarer Spirant a) steht in best. Konsonantenvbdg. $\boldsymbol{\alpha}$ ) für westgermanischen Spiranten s: $\boldsymbol{\alpha} \boldsymbol{\alpha}$ ) im Anlaut, i.d. Vbdg.: - sl für sl: z.B. šlōfon (H), šlōfm (B) mhd. sla f e n schlafen; släkan (H), släkn (B) mhd. slukken schlucken; $s l o ̄ s z(H, B)$ mhd. sloz Schloss; - šm für sm: z.B. Śmaiszan (Bgbg), క̌maiszn (B) mhd. s mizen schmeißen; šmüal (H), šmual (B) mhd. s m a l schmal; క̌moiran (Bgbg), šmairn (B) mhd. $\mathrm{s}$ mir ( w e ) n schmieren; - šn für sn: z.B. šnegdan (Bgbg, H), šnaidn (B) mhd. sn id a n schneiden; šnaryan (H), śmuarxan (Bgbg), šmuarzn (B) mhd. sn a r c h e n schnarchen; క̌muawal (H), šnuabal (B) mhd. s n a bel Schnabel; - ¿̌p für sp: z.B. späjal (H), špaigal (B) ahd. s pi a ga I Spiegel; špoit (Bgbg), špēt (B) mhd. s p ae te spăt; śpilan (H), śpiln (B) mhd. spiln spielen; - క̌w für sw: z.B. šwānts (H, B) mhd. s w a n z Schwanz; క̌wejan (H), ふ̌waigy (B) mhd. sw eig en schweigen; - šp für sw (verbr. im Bzld, durch schriftsprl. Einfluss städt. im Verschwinden; nsbg. nur in Tkdf): z.B. špen (Heldf) mhd. $\mathrm{s}$ w i n Schwein; špuart (Bart) mhd. s w a r t Schwarte; špurrts (Wdf $/ \mathrm{Kr}$ ) mhd. sw arz schwarz (vgl. dazu: SCHEINER, Bzld. 5; Sbg. Maa. 70 (Fuß-

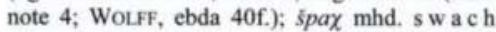
schwach; ‘̆piawal mhd. s.w ë v a I Schwefel; špeesszt mhd. sweiz Schweiß (Tkdf, KEINTZE, ebda

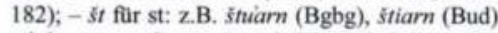
mhd. stern Stern; štiwal (H), śtibal (B) mhd. st i va l, st tiv e I Stiefel; šsōn (H), štō (B) mhd. st e n stehen; štuf (H, B) mhd. st u be Stube, Wohnraum. $\beta \beta$ ) im In- u. Auslaut, i.d. Vbdg. $r \dot{s}$ für rs (allg.): z.B. biršt (Schbg), biršt (Tkdf) zu mhd. b or s t 'Borste' Bürste; duršt (Kr), düršt

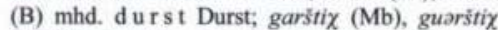
(Mttdf) zu mhd. ga r s t 'verdorben schmeckend' garstig; giarš́t (Alz), giāršt (Schbk) mhd. gërste Gerste; hĩars $(\mathrm{Hb})$, hỉr $(\mathrm{Ky})$ mhd. hirse Hirse; āndorš (H, B) mhd. anders anders; in Zstzgn, im Auslaut des Bestw.: z.B. milaršbir (Pi) Müllersbirne; nōbaršhausz (B) Nachbarhaus; nōbars'lait (B), auch mit r-Ausfall nōbaślä ' (FröMM, Jaad 23) Nachbarsleute; farškänt (Fell), auch foarzškaint (Ur) Pfarr(er)skind; in O.N.: z.B. Pitaršdarf (Pdf/Mb), Pitaršdurf (Mb), Pãtaršdrof (O-Neudf) Petersdorf; vgl. dazu auch $\mathrm{R}(1 \mathrm{~h} \alpha)$. B) bei Fremdwörtern, im An-, In- auch Auslaut: i.d. Vbdg. - šp für sp: im Anlaut, z.B. spalï (H, B) Spalier, Gitter an einer Mauer, an der Kletterpflanzen gezüchtet werden; špagat $(\mathrm{H}$, B) Spagat, Bindfaden; im Inlaut, z.B. inśpäktar (H) Inspektor; rešpäka (Seid) Respekt; inšpitsiran $(\mathrm{Wu})$ inspizieren; $-\grave{s t}$ für st: im Anlaut, z.B. $s ̌ t a-$ tsion (H, B) Station, Haltestelle, Bahnstation; śtudirran (H), śtudirn (B) studieren; im Inlaut. z.B. inštalìran (H) instalieren; inštigìrn (B) instigieren; inštrumant $(\mathrm{Hb})$, inštrumänt (Krew) Instrument; $-\dot{s}(k)$ für sk: im Anlaut, z.B. Šiarts (Rs, Schbg), šerts (B) Schörze zu mlat. s cort i a m (Baum)rinde; škandāl (H, Bgbg); skandal (Tkdf) Skandal; im Inlaut, z.B. diškuroiran (Bgbg), diškurirn (B) diskurieren veralt; auch im Auslaut

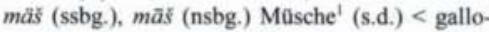
romanisch $\mathrm{mus}$ c i o Sperling, lat. $\mathrm{mu} \mathrm{s} \mathrm{c}$ a Fliege. b) kann im Anlaut vor Vokal zu $t \tilde{s}$ werden (nur nsbg.): z.B. Salasztar neben tsalasztar (B. Bgh, Nd-Wall) Elster; šatart neben $t$ šatart (B) Marktbude; šok neben $t s ̌ o k$ (B) Schauke 'Schaukel'; šipaln (B), tšipaln (KI-B, Krew) schüppeln d.h. 'wälzen, rollen'. Vgl. dazu Krauss, Tre Wb. 1018. c) für $s$ im Anlaut (selten): z. B. $s a$ aral (KI-A) Sattel, sararaln (ebda) satteln. 2. Stimmhafter Spirant a) im Anlaut (nur in einigen Wörtern) sch $>$ z: Zabarn (H, B) schabbern 'schwatzen, viel reden'; $\operatorname{tatarn}(\mathrm{H})$, zadarn (B) schattern glbed.; $\mathbf{s}>$ $z$ (selten): $z \tilde{a} a s ̌$ săuisch (Ur), Zuəf Sophia (V.N.) (Alz); \&äp (H, B) Kleidertasche < ung. z s e b glbed.; auch zagodi ( $-\alpha)$ (B) Teufel, nur in einer Verwünschung < rum. jig o die Hundekrankheit; zap! Int. (Tre) plumps! < rum. j a p, ahmt das Aufschlagen eines Gegenstandes nach. b) im Inlaut, nach stimmhaftem Konsonanten $\mathrm{r} u$. meist folgendem Vokal kann stimmhaftes sch stehen: z.B. mirzal (H), mêrzal (B) Mörser 'Gefaßß zum Zerkleinern harter Stoffe mit dem StöBel'; drukata (Boo, Tre), drukatn (B) drucksen 'umarmen, herzen'; grapazan (Gr-Schenk), grapaza (Reen) grapschen 'zugreifen'; firzäpk (nsbg. verbr.) Pfirsich; auch im Fremdwort perzaln $(\mathrm{H})$, pärżaln $(\mathrm{B})$ versengen < ung. perzse In i glbed. 3. Buchstabe: šreif mar wirtar af mät dam baxštuawa 5 ! (um $2000 \mathrm{H}$ ); ta terfszt dat $\breve{s}$ ned asi gritšaln (kritzeln = klein u. schlecht leserlich schreiben)! (ebda). 4. Int: $\dot{s}$ a) Scheuchnuf für Geflügel u.a. Vögel (allg): $s-s$. ana mad ix! husch, hinein mit euch! (um 2010

Abb. 1: Beispielseite „,Sch“ aus dem ,Siebenbürgisch-Sächsischen Wörterbuch“ 
Ur); häšso, $\tilde{s}$ dohär! husch, husch, weg v, dort!

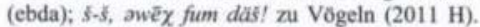
b) zum Einwiegen v. Kleinkindern: $\bar{s}, \bar{s}, \delta$ me geanaltxan /od./ mẽdaškan, stōf nea higs,, men on'altxan! Wiegenspruch (um $2010 \mathrm{Ur}$ ); auch zu Kleinkindern vor dem Schlafengehen: $\hat{\xi}, \hat{S}, m \bar{\alpha} x$ da kukaltxar (s. Guckelchen = Auglein) $t s a \overline{,}$, stlōf $n \bar{a}$, mei inija! $(2011$ H). Vgl. NSSWB 4, 710.

Šã s. Schau.

Šäax s. Schuh.

Schaaf, Schăaff s. Schaf.

Šäafs. Schaf.

Šäpyan s. Schuh.

Schaal O.N. Sual (H), rum. Soala, ung. Sálya, Kreis H: „S a a l“" (1331, Ub. 1, 441; vgl. SCHWARZ, Herk. 184); „S a u li“" (1359, ebda 3, 217); „.Nicolai plebani de S ca li is" (1414, ebda 597); „Nicolai de $\mathrm{S}$ c hal is" (1414, ebda 599 u. 600; vgl. SCHWARz, ebda); ,Nicolaus, plebanus in $\mathrm{S} \mathrm{cal}^{-1}(1415, \mathrm{~V} . \mathrm{A}$. $21,308) ;$.. Clemens von der $\mathrm{S} \mathrm{c}$ h a l e n" Register der Johannes-Bruderschaft (um 1500, ebda 16, 360); „S c hall e n hat vyrt $50^{\circ}(1532, \mathrm{Qu} . \mathrm{Kr} 2$. 283); „S all a" (1733, Suctu 2, 174); , S c ho a la" (1750, ebda); Sual (ssbg., Kisch, Vgl. Wb. 192); en dar Sól, auch ken da Sól sagt der Martinsdorfer (um 1900 Mart); gəfleitsta (geflöBten = ausgewaschenen, s. flőßen 1) bodam hu mar ă Śuāl (1962 Schaal). Dazu Adj.: im Fl.N, bām Sãalnar Wüar (Weiher) (1965 Wu). Vgl. FeLMER 90/201; WAGNER 376.

- 'Wohl dt., als Fl.N. mitgebracht (Schaal liegt am Schaalbach)' (SCHEINER, O.N. 128); v. Kisch a.a.O. zum mslff. F.N. 'Schaal' gestellt.

Schaal(n)er šalnar m. (Schaal), KısCH, Sbg. 220 ebenso; Bewohner v. Schaal: ,also das sye denn hattert kegen die $\mathrm{S}$ chaler zu bawen nicht bedorfft haben" (1570, Arch.); ,.kommen ... $\mathrm{S}$ c holn e r zu uns in die herberg sambt weibern" (1609/15, ebda); śalnar (um 1900 Arb); di halt sij a śalnar, di halt sij a kleax mänš (1983 Schaal).

Schaas O.N. Sãsz (H; Dr, ScheINER, O.N. 128), Săazz (Schaas), rum. Saes, ung. Segesd, Kreis Mureș: „terrae Sugus" (1301, Ub.1, 220); ..plebanus de Z e gi s" (1309, ebda 251); „S e g h es din u m" (1309, ebda 273); .Z e gu s" (1309, ebda 292; vgl. Sprdm. 9); „S e gu s (d )" (1349, ebda 2, 69; vgl. V.A. 2, 153); „comes Georgius de S chais" (1372, Ub, ebda 371); .S e ge s" (1373/75, ebda 415); „Andreas Wencz de S cheg s" (1393, ebda 3, 48); „S ch ey s" (1393, Sprdm. 30); ..S che ges" (vor 1500, Sprdm. 143); . S c he sz habet hospites $107^{\prime \prime}$ (um 1500 ,
Berger O.N. 68); ,S c h e e z" (1504 Schbg, Qu. Gesch. 395 u. 396; vgl. Kbl. 2, 11); ,S che es" (1506 ebda, ebda 404; vgl. Kbl., ebda); , dy /Komkaule/ hat eyn man gefolt fom s c h e s" Verzeichnis der Kornkaulen des Schässburger Dominikanerklosters (1515, Sprdm. 167; vgl. V.A. 5, 34); ,herr Barthmes von s c h e s s" (1522, Sprdm. 176); "s c h ce s" (1572 Honteruskarte); . S c h ey s z" (1532, Qu. $\mathrm{Kr} 2,283)$; , an der stroßen die ... kem s c h a a s gehet" (1563, Arch.); „legerten sich ihn dass Dorf $\mathrm{S}$ c h e e s s, so eine halbe Meill von der Stadt lieget" (Kraus, Chron. 2, 217); ..S c ha a B" (Tróster, Dacia 405); „ein frembder Mann vom S chäs s" (1672, SchotsCH 27); dea am fu Prîdən /O.N. ux fu Sãsz sä /zwei Knaben/ aff gümnasium brōxt tsea āsz (Ẽwend 39). In Sage (hd.) vgl. MOLer, Sagen 222. Dazu Adj.: ..2 S ch es s er pauren" (KRAUS, ebda 237); scherzh. sãsar hinxan Speise aus Maisbrei u. Käse, s. Käsepalukes, auch nur Maisbrei (um 1900 Schbg: auch HALTRICH, Plan 105 u. 103; H.-W. 141) vgl. Hennchen (2), Syn. Ausdrüke (scherzh.) s. unter Hünkel (1), Knoche(n) (1b) u. unter Palukes (1a); dar šâsar tseixan (s. Gemein[d]ezeichen = Ortsmarke, die man dem Vieh einbrennt) (um 1930 Schaas); saāsar špẫsz bezieht sich auf einen Schässburger Bürger, der verurteilt war, auf dem hölzernen Schandesel zu reiten; als er einen vorbei gehenden Schaaser Bauern fragte, weshalb er ihn so angaffe, antwortete dieser: na wô at dom häran net jafalt, sa ret dar här än jen jasz li.d. andere Gasse/! scherzh. (vgl. H.-W. 147; auch SSWB 5 A.F. 138); vgl. reiten (2); im Bachnamen "S c hesser bach f." (Kraus, ebda 1, 118); da Šãsar Bāx führt Ton, Mergel (Schbg. SSWB 1. 365 u. 369; vgl. Fronius, Flora 30 u. 38); in De /O.N $/$ heißt ders. Libāx (Leibach s.d.) (HALTRıCH, Plan 83); in Fl.N. Ŝãsar Hil (Hōhle) (1965 Pesch); dat Sãsar Felt Sumpfland (1969 Schbg); auch zsgez. Sãsarfêlt (MOLLR, Id. I; vgl. FroNIUS, ebda 16, 23 u. 34; Monografie Schaas 52); Şāsar Mäuar (Mar 2 = Moor, Sumpfland) (1965 Schaas); vgl. Monografie ebda 26f., 32 u. 53f.; da Šãsar Wan'art Weingarten (1972 Baa); Śäsar Watar (Wasser) mit unverschobenem -t-, in Pesch (KısCH, Sbg. 45 u. 142; ders., in: Kbl. 64, 182), auch zsgez. kễm (gegen) Sãsanwatar (um 1900 Pesch); vgl. auch Sbg. Maa. 63; im G.N. 'Schaaser Gasse' in Schbg (HoFstÃDTER, Nachl.), vgl. Schaas(en)gasse. Vgl. WAGNER 360.

$-<$ ung. seg es Adj. (v. s é g Hugel [Ung.

Etym. 3, 507]); vgl. dazu: SCHEINER, ebda; 


\section{BEDEUTUNG UND ZUKUNFTSPERSPEKTIVE}

Das „Siebenbürgisch-Sächsische Wörterbuch“ wird deshalb als das wohl bedeutendste Werk siebenbürgisch-sächsischer Sprachgeschichte eingeschätzt, da das darin bearbeitete Sprachgut das Volksleben der Siebenbürger Sachsen in seiner Vielfalt dokumentiert.

Geht man von der Tatsache aus, dass die Berücksichtigung alter Sprachinselmundarten der neueren Forschung eine bessere und differenziertere Rekonstruktion mittelalterlicher binnendeutscher Laut- und Wortgrenzen ermöglicht und damit der Sprachgeografie dient, lässt sich daraus auch die Bedeutung und die Wichtigkeit des Wörterbuchs herauslesen. Die im Untergang begriffenen rund 243 siebenbürgisch-sächsischen Mundarten haben, neben der Berücksichtigung des Lehnwortguts aus dem Rumänischen und Ungarischen, viele altertümliche Sprachzüge und Reliktwörter bewahrt, die im deutschen Sprachraum nicht mehr bezeugt sind.

Da man zu Recht die Frage stellen kann, weshalb das ,Siebenbürgisch-Sächsische Wörterbuch" nach 100 Jahren immer noch nicht abgeschlossen ist, wurde versucht, das Auf und Ab in der Geschichte dieses so groß angelegten Werkes in obigen Ausführungen zu verdeutlichen. Die konzeptionellen Überlegungen im Wandel der Zeit, die etappenweise erfolgten methodischen Neuerungen, die sozial-politischen Ereignisse, denen die Lexikografen bei der Ausarbeitung der Wortartikel Rechnung tragen mussten, die administrativ-finanziellen Schwierigkeiten, verbunden mit der Suche nach Förderungen, um die einzelnen Bände in Druck zu geben, der etappenweise Personalwechsel, sowie letztlich im positiven Sinn - nach der Wende in Rumänien - die ganzen Vorarbeiten, die eine Umstellung auf Computertechnik ermöglicht haben, um mit den Dialektwörterbüchern des deutschen Sprachraums Schritt halten zu können, sind ein Beweis dafür. Das sind die vielen ,Rädchen und Räder", die ein Vorankommen gebremst, dann aber auch wieder in Bewegung gebracht haben. Eine Vorschau bezüglich in der Zukunft Liegendes kündet ein nicht zu unterschätzendes Maß an lexikografischer Arbeit an. Zu bewältigen ist noch ein Großteil der Stichwortstrecken von Schenkweinbis einschließlich der Stichwortstrecke des Buchstabens Z. Trotz aller Bemühungen konnten bisher keine bleibenden Mitarbeiter/-innen gewonnen und eingeschult werden. Das ist darauf zurückzuführen, dass nach der Massenauswanderung der Siebenbürger Sachsen 1989 meist rumänische Jugendliche Germanistik studieren, die nicht Mundartsprecher/-innen sind. Dazu kommen die niedrigen Anfangslöhne. Doch haben sich bisher immer wieder Lösungen zur Weiterführung des Wörterbuchs gefunden, deshalb wird die Suche nach Nachwuchskräften nicht aufgegeben.

Abschließend kann gesagt werden, dass das „Siebenbürgisch-Sächsische Wörterbuch“ ein Nachschlagewerk für die allgemeine deutsche Sprachgeschichte und Mundartforschung, für die Siedlungsforschung, für Studien zum Sprachausgleich, zum Wesen der Sprachkontakte, Interferenzen mit Nachbarsprachen, für volkskundliche Untersuchungen und anderes mehr ist. Es richtet sich an Sprachwissenschaftler/-innen, Volkskundler/-innen, Historiker/-innen und an alle an dieser deutschen Sprachinselmundart Interessierte. 


\section{KONTAKTADRESSE}

Träger, Leitung: Rumänische Akademie Bukarest - Rumänien

Arbeitsstelle: Forschungsinstitut für Geisteswissenschaften - Hermannstadt / Sibiu, der Rumänischen Akademie unterstellt

Direktor des Forschungsinstituts für Geisteswissenschaften:

Prof. Dr. Paul Niedermaier

Adresse:

Forschungsinstitut für Geisteswissenschaften

(Institutul de Cercetări Socio-Umane Sibiu)

Bdul Victoriei 40

550024 Sibiu

Rumänien

E-Mail: sigridhaldenwang@yahoo.de

\section{LITERATURVERZEICHNIS}

BERTLEFF, GEORG (1866/1867; 1867/1868): Beiträge zur Kenntnis der Nösner Volksssprache. In: Programm des evangelischen Obergymnasiums und der damit verbundnen Lehranstalten in Bistritz. Schuljahr 1866 / 1867, 4-46; Schuhljahr 1867 / 1868, 1-23.

BINDER, JOHANN (1861): Handschriftliche Sammlung siebenbürgisch-sächsischer Wörter u. Redensarten (meist aus Schässburg) hochdeutsch aufgezeichnet, 1861.

BRANDSCH, GOTTLIEB (1935): Die Martin Felmer-Handschrift. Eine Darstellung der Geschichte und Volkskunde der Siebenbürger Sachsen aus dem Jahre 1764. Quellen zur Deutschen Volkskunde. 5. Heft. Berlin/Leipzig: de Gruyter.

Cap. Cib. = Capitulum Cibiniensis. Abschriften aus den Verhandlungsberichten des Hermannstädter Kirchenbezirks betreffend das 17.-18. Jh. Nationalarchiv Hermannstadt.

CAPESIUS, BERNHARD (1990a): Wesen und Werden des Siebenbürgisch-Sächsischen. In: KelP, Helmut (Hg.): Bernhard Capesius, Linguistische Studien. 1. Auflage. München: Verlag des Südostdeutschen Kulturwerks, 122-138.

CAPESIUS, BERNHARD (1990b): Das Siebenbürgisch-Sächsische Wörterbuch. In: KelP, HeLMUT (Hg.): Bernhard Capesius, Linguistische Studien. 2. Auflage. München: Verlag des Südostdeutschen Kulturwerks, 98-104.

CAPESIUS, BERNHARD (1990c): Siebenbürgisch-Sächsisches Wörterbuch. Dritter Band „G““. Vorwort. In: KelP, Helmut (Hg.): Bernhard Capesius, Linguistische Studien. 3. Auflage. München: Verlag des Südostdeutschen Kulturwerks, 234-243.

GRIMM, Dwb. = GRIMM, JAKOB / WILHELM GRIMM (1984-1999): Deutsches Wörterbuch. 16 Bde. plus Quellenverzeichnis $=33$ Teile. Hg. v. der Preußischen [später: Deutschen] Akademie der Wissenschaften zu Berlin [später: Akademie der Wissenschaften der DDR. (Abgeschlossene Originalausgabe)]. Fotomechanischer Nachdruck der Erstausgabe 1854-1971 Leipzig. Leipzig/Stuttgart.

HALDENWANG, SigRID (1995): Sammlungen der Hermannstädter Wörterbuchstelle. In: SCHENK, ANNEMIE (Hg.): Europäische Kulturlandschaft Siebenbürgen. Reflexion einer wissenschaftlichen Dokumentation. Thaur bei Innsbruck: Wort und Welt (Kulturdenkmäler Siebenbürgens. Band 3), 134-139. 
HALDENWANG, SIGRID (2009): Das Siebenbürgisch-Sächsische in der Sprachlandschaft Siebenbürgen. In: KRIEGLEDER, WYNFRID / ANDREA SEIDLER / Jozef TANCER (Hg.): Deutsche Sprache und Kultur in Siebenbürgen. Bremen: edition lumière, 11-22.

HALTRICH, JOSEF (1865/1866): Negative Idiotismen der siebenbürgisch-sächsischen Volkssprache. In: Programm des evangelischen Gymnasiums in Schässburg und der damit verbundenen Lehranstalten. Hermannstadt: S. Filtsch.

HALTRICH, JOSEF (1865): Plan zu Vorarbeiten für ein Idiotikon der siebenbürgisch-sächsischen Volkssprache. Kronstadt: Johann Gött.

IORDAN, Dlr. = IORDAN, IORGU / ALEXANDRU GRAUR / ION COTEANU (1980): Dicționarul limbii române (serie nouă) tomul 8, partea a 4-a (P). București: editura Academiei Republicii Socialiste, 1146.

KARAJAN, THEODOR VON (1849): Wiener-Sitzungsberichte, phil.-hist. Klasse 1849, Heft 9, 227 236.

KÄSTNER, VIKTOR (1850): Idiotikon. Handschriftliche Idiotismensammlung, Mitte 19. Jh.

KEINTZEL, GEORG (1894): Lautlehre der Mundarten von Bistritz und Sächsisch Regen. Mit Berücksichtigung abweichender Lautverhältnisse in den sächsischen Ortsdialekten der Umgebung. In: Archiv des Vereins für siebenbürgische Landeskunde Bd. 26, 133-222.

KeINTZEL, GEORG (1897): Nösner Idiotismen. In: Festgabe der Stadt Bistritz. Bistritz: Verlag der Stadt Bistritz, 47-80.

KEINTZEL, GEORG (1924): Zum Vokalwandel im Siebenbürgisch-Sächsischen seit der Einwanderung. In: Archiv des Vereins für siebenbürgische Landeskunde Bd. 42, 161-172.

KISCH, GuSTAV (1900): Nösner Wörter und Wendungen. Ein Beitrag zum siebenbürgisch-sächsischen Wörterbuch. Beilage zum Programm des ev. Obergymnasiums A.B. in Bistritz (Siebenbürgen). Bistriz: Verlag des Bistritzer Gymnasiums.

KISCH, GUSTAV (1905): Vergleichendes Wörterbuch der Nösner (siebenbürgischen) und moselfränkisch-luxemburgischen Mundart nebst siebenbürgisch-niederrheinischem Orts- und Familienverzeichnis sowie einer Karte zur Orientierung über die Urheimat der Siebenbürger Deutschen. In: Archiv des Vereins für siebenbürgische Landeskunde Bd. 33, 5-273.

KLEIN, KARL KURT (1963): Adolf Schullerus und das siebenbürgisch-sächsische Wörterbuch. In: Transsylvanika. Buchreihe der Südostdeutschen Historischen Kommission. Band 12. München: R. Oldenbourg, 36-52.

KRAMER, FRIEDRICH (1875 / 1876): Idiotismen des Bistritzer Dialectes. Beitrag zu einem siebenbürgisch-sächsischen Idiotikon. In: Programm des evangelischen Obergymnasiums A.B. in Bistritz und der damit verbundenen Lehranstalten (Schujahr 1875/1876).

KRAUSS, Hwspr. = KRAUSS, FRIEDRICH (1957): Wörterbuch der nordsiebenbürgischen Handwerkssprachen. Siegburg: F. Schmitt.

KRAUSS, Pfl.N. = KRAUSS, FRIEDRICH (1943): Nösnerländische Pflanzennamen. Ein Beitrag zum Wortschatz der Siebenbürger Sachsen. Bistritz: Carl Csallner.

KRAUSS, Tre Wb. = KRAUSS, FRIEDRICH (1970): Treppener Wörterbuch. Ein Beitrag zum Nordsiebenbürgischen Wörterbuch. Marburg: N.G. Elwert.

LEONHARD, DANIEL JOSEPH (1826): Verzeichnis einiger siebenb.-sächsischer eigenthümlicher Wörter oder Idiotismen. Handschrift, 1826.

LEXER, Hwb. = LEXER, MATTHIAS VON (1872-1878): Mittelhochdeutsches Handwörterbuch. 3 Bde. Bd. 1 (A-M) 1872, Bd. 2 (N-U) 1876, Bd. 3 (P-Z) 1878. Leipzig. Unveränderter Nachdruck. Stuttgart 1974: S. Hirzel.

MANTSCH, HEINRICH (2002): Siebenbürgisch-Sächsisches Wörterbuch. 8 Band: Buchstaben N-P. In: Zeitschrift für Siebenbürgische Landeskunde 25/2. Köln [u. a.]: Böhlau, 294-296.

MANTSCH, HEINRICH (2008): Siebenbürgisch-Sächsisches Wörterbuch. Neunter Band: Buchstaben Q-R. In: Zeitschrift für Siebenbürgische Landeskunde 31. Köln [u. a.]: Böhlau, 211-214.

MÜLLER Sprdm. = Verein für Siebenbürgische Landeskunde (Hg.) (1864): Deutsche Sprachdenkmäler aus Siebenbürgen. Aus schriftlichen Quellen des zwölften bis sechzehnten Jahrhunderts gesammelt v. FRIEDRICH MÜLLER. Hermannstadt: Theodor Steinhaussen. 
NÄGLER, THOMAS (1979): Die Ansiedlung der Siebenbürger Sachsen. Bukarest: Kriterion.

NSSWB = Nordsiebenbürgisch-Sächsisches Wörterbuch. 5 Bde. Bd. 1 (A-C) von FRIEDRICH KRAUSS, bearb. von GiSElA RICHTER, 1986, Bd. 2 (D-F) 1990. Köln / Wien: Böhlau. Bd. 3 (H-M) 1993, Bd. 4 (N-Sch) 1995, Bd. 5 (Se-Z) 2006. Köln [u. a.]: Böhlau. Bd. 2-4 bearb. von Gisela RICHTER aufgrund der nachgelassenen Sammlungen von FRIEDRICH KRAUSS, Bd. 5 bearb. von Gisela RICHTER ( $\dagger$ ) und Helga Fessler [unter Mitarbeit von URSUla GalsTERER] aufgrund der nachgelassenen Sammlungen von Friedrich KRAUSS.

Protze, Helmut (1959): Das Siebenbürgisch-Sächsische Wörterbuch. In: Forschungen zur Volksund Landeskunde 1, 210-218.

REIN, KURT (1963): Der bairische Anteil am Siebenbürgisch-Sächsischen Wörterbuch nach den Karten des siebenbürgisch-sächsischen Sprachatlasses. In: Südostdeutsches Archiv 6, 24-64.

Rhein. Wb. = Rheinisches Wörterbuch. 9 Bde: Bd. 1 (A-D) 1928, Bd. 2 (E-G) 1931, Bd. 3 (H-J) 1953, Bd. 4 (K) 1938, Bd. 5 (L-M) 1941, Bd. 6 N-Q) 1944 bearb. und hrsg. von MülLER, JosEF. Berlin: Fritz: Klopp. Bd. 7 (R-Sch) 1948-1958 bearb. von MüLLER, JOSEF † [unter Mitarbeit von MatThias ZENDER und HeInRICH DitTMAIER], hrsg. von KARL Meisen; Bd. 8 (Se-T) 1958-1964 bearb. von Josef Müller † [unter Mitarbeit von HEINRICH DITTMAIER], hrsg. von KARL MEISEN; Bd. 9 (U-Zyriakus) 1964-1967 nach den Vorarbeiten von JOSEF MÜLLER $\dagger$ bearb. von HEINRICH DITTMAIER. Berlin: Klopp.

RICHTER GISELA / THUDT ANNELIESE (1964): Ergebnisse der mundartlichen Neuaufaufnahmen im Unterwald (südwestlicher Teil Südsiebenbürgens). In: Forschungen zur Volks- und Landeskunde 7/1, 91-108.

RICHTER, GiSELA (1986): Einleitung (zur nordsiebenbürgisch-sächsischen Mundart). In: Nordsiebenbürgisch-Sächsisches Wörterbuch, Bd. 1, VII-X.

SCHULlER, JOHANN CARL (1865): Beiträge zu einem Wörterbuche der siebenbürgisch-sächsischen Mundart. Prag: F. A. Credner.

SCHULLERUS, ADOLF (1894 / 1895): Die Vorgeschichte des siebenbürgisch-deutschen Wörterbuchs. In: Programm des theologisch-pädagogischen Seminars der ev. Landeskirche A.B. in Hermannstadt. Am Schluss des Schuljahrs 1894/1895 veröffentlicht von Dr. Friedrich Teutsch. Hermannstadt, 3-44.

SCHULlERUS, AdOLF (1924): Vorwort. In: Siebenbürgisch-Sächsiches Wörterbuch, Bd. 1. Berlin/Leipzig: de Gruyter, IX-LXXII.

SCHULLERUS, ANNA (1904): Hīmwih. Kleine sächsische Erzählungen. Hermannstadt: W. Krafft.

SCHUllerus, PAUline (1916): Pflanzen in Glaube und Brauch der Siebenbürger Sachsen. In.: Vereins-Ausschuss (Hg.): Archiv des Vereins für siebenbürgische Landeskunde Bd. 40, 1. Heft. Hermannstadt: Franz Michaelis, E. Dück.

SCHUSTER Dutz (1921): Eos mënger Ährevakanz. Lastich Gedichtcher. Medwesch: G. A. Reissenberger.

SEIVERT, JOHANN (1760-1780): Idiotikon. Handschriftliche Sammlungen v. Hermannstädter Mundartwörtern.

SEIVERT, JOHANN (1773): Versuch (eines Siebenbürgisch-sächsischen Wörterbuchs). Handschriftliche Sammlung v. Hermannstädter Mundartwörtern.

SSWB = Siebenbürgisch-Sächsisches Wörterbuch (siehe Bearbeitungs- / Publikationsstand, Kapitel 2.2).

TEUTSCH, JOSEPH (1899): Alt-Dacisch-Teutsche Wörter, welche unter den Sachsen in Burzenland noch im Gebrauche sind. In: Korrespondenzblatt für Siebenbürgische Landeskunde $22 \mathrm{Jg}$. Hermannstadt: W. Krafft, 24-25.

ThudT, ANNELIESE (1968): Das Gesetz der Auslauterweichung im Siebenbürgisch-Sächsischen. In: Forschungen zur Volks-und Landeskunde 11/1, 19-36.

TRAUSCH, JOSEF (1816-1868): Wörterbuch Siebenbürgisch-Sächs. Idiotismen. Handschrift, 18161868.

TRÖSTER, JOHANN (1666): Das Alt- und Neu-Teutsche Dacia. Das ist: Neue Beschreibung des Landes Siebenbürgen [...]. Nürnberg: Johann Kramers, 161-162. 
WIESINGER, PETER (2012): Sprachinsel versus Sprachminderheit. In: GLAUNINGER, MANFRED MICHAEL / BETTINA BARABAS (Hg.): Wortschatz und Sprachkontakt im Kontext oberdeutscher Wörterbücher, Sprachatlanten und Sprachinseln. (Werner Bauer zum Geburtstag). Beiträge zur Sprachinselforschung, Band 21. Wien: Praesens Verlag, 151-164.

WOLFF, JOHANN (1897): Vorarbeiten zum siebenbürgisch-deutschen Wörterbuch. In: Archiv des Vereins für siebenbürgische Landeskunde Band 27, 587-650. 
Die Autorinnen und Autoren des Bandes widmen sich der germanistischen Dialektlexikographie zu Beginn des 21. Jahrhunderts und bieten einen Überblick über sämtliche aktive Wörterbuchprojekte zu Dialekten des Deutschen.

Die Beiträge enthalten Informationen zur Geschichte der Wörterbuchprojekte, ihren wissenschaftshistorischen Kontexten und institutionellen Rahmenbedingungen sowie den empirischen Materialien. Daneben werden konzeptionelle Fragen der Wörterbucharbeit diskutiert und anhand von Beispielartikeln illustriert. Ein weiterer Schwerpunkt des Bandes liegt auf texttechnologischen Aspekten, die sowohl die elektronische Aufbereitung und Bereitstellung von vorhandenen Materialien als auch das digitale Publizieren von Wörterbuchartikeln umfassen. Exemplarische Fallstudien verdeutlichen die linguistischen Potenziale der zugrundeliegenden Materialien und deren Verwertbarkeit über die klassische Wörterbucharbeit hinaus. 